\title{
LASER-FUSION PROGRAM
}

SEMIANNUAL REPORT - JULY-DECEMBER 1973

Prepared for U.S. Atomic Energy Commission under contract No. W-7405-Eng-48
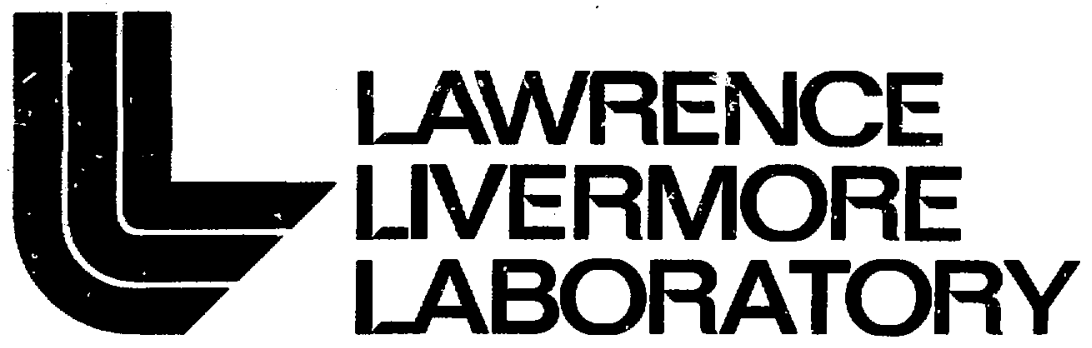

University of California/Livermore

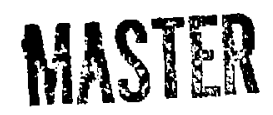




\section{NOTICE:}

"This report was prepared as an aecount of work sponsores by the United States Government. Neither the United States nor the United States Aiomic Encray Conimission, not any of their employecs, nor any of their contrectors, subcontractors, or their employeus, makes any warraniy. express or implied, or assumes any lezal liability or responsibility for the aceuracy, complereness or usefulness of any information, apparatus, product or precess disclosed, or represents that ins use would nol infringe privat cly. owned rights."

Printed in the United States of America

Available from

National Technical Information Service

U.S. Department of Commerce

5285 Port Royal Road

Springfield, Virginia 22151

Price: Printed Copy \$7.60; Microfiche $\$ 1.45$ 


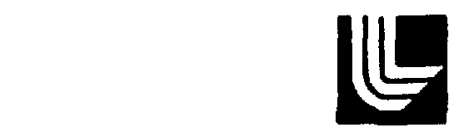

\section{LAWRENCE LNERMOAE LABOAATOFY}

Uniersity ol Cavionia Livermore, Celitomia 94550

\section{UCRL-50021-73-2 \\ LASER-FUSION PROGRAM \\ SEMIANNUAL REPORT - JULY-DECEMBER 1973}

MS. datc: Apri? 10. 1974

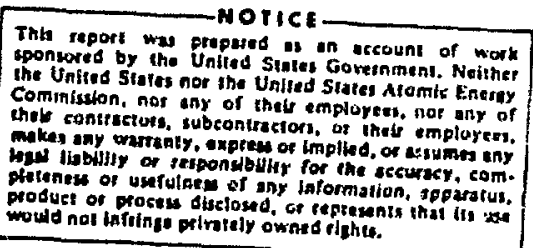

This report wh plupated os in account of nowk he United States nor the States Govenmen. Neither Coministon, nor any of their employe domik tretily

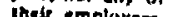

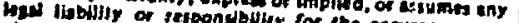
ofetenes of untutar af anr for the sccuracy, com. would nol infotnge disefosed, Ge represents that its sed 


\section{Foreword}

This report covers progress in the laser-fusion program at Lawrence Livermare Laboratory during the period July-December 1973 - the first half of fiscal year 1974. All the work described here is unclassified. Whenever there is classified work to be reported it is covered in a separate classified supplement.

Some of the articles in this report make reference to company or product names. Such reference does not imply approval or recommendation of the product by the University of California or the U.S. Aton: Energy Commission to the exclusicn of others that may be suitable.

Inquiries about the information presented in this report should be addressed to the Director's Office, Lawrence Livermore Laboratory, Livermore, California 94550, attention Scientific Editor, Lase1-Fusion Program Semiannual Report.

Both printed and microfiche copies of this report are available from the National Technical Information Service, as described on the inside front cover. 


\section{Contents}

1. INTRODUCTION AND PROGRAM OVERVIEW (A. C. Haussmann and J. L. Emmet+1) . . . . . . . 1

Laser Program Goals . . . . . . . . . . . . . . . . . . . . . . . . . . . . . . . . 3

Glass Laser Program . . . . . . . . . . . . . . . . . . . . . . . . . . . 4

Gas Laser Program . . . . . . . . . . . . . . . . . . . . . . . . 5

Other Laser Studies . . . . . . . . . . . . . . . . . . . . . . . . . . . . . . . 6

2. SOlID.STATE LASER PROgRAM (W. F. Krupke) . . . . . . . . . . . . . . . . . . . . . . 8

Master Oscillator . . . . . . . . . . . . . . . . . . . . . . 8

Rod Amplifiers . . . . . . . . . . . . . . . . . . . . . . . . . 12

Disk Ainplifiers . . . . . . . . . . . . . . . . . . . . . . . . . . . . . 13

Performance of the Prototype System (Cyclops) . . . . . . . . . . . . . . . . . 19

3. DESIGN ANALYSIS (A, J. Glass and J. B. Trenholnie) . . . . . . . . . . . . . . . . . . . . 45

Temporal Pumping Simulation . . . . . . . . . . . . . . . . . . . 45

Reflector Optimization . . . . . . . . . . . . . . . . . . . . . . 46

ZAP Progress . . . . . . . . . . . . . . . . . . . . . . . . . 446

Small-Scale Growth .. . . . . . . . . . . . . . . . . . . . . . . . 47

Whole-Beam Distortions . . . . . . . . . . . . . . . . . . . . . 49

4. COMPONENT DEVELOPMENT (G. W. Leppelmeier) . . . . . . . . . . . . . . . . . . . . 50

Faraday Isolators . . . . . . . . . . . . . . . . . . . . . . 50

Polarizers . . . . . . . . . . . . . . . . . . . . . 52

Apodizing Apertures . . . . . . . . . . . . . . . . . . . . . . 52

Calorimeters . . . . . . . . . . . . . . . . . . . . . . . . . 54

10.6- $\mu \mathrm{m}$ Detectors . . . . . . . . . . . . . . . . . . . . . . . 55

5. DIAGNOSTICS (L. W. Coleman) . . . . . . . . . . . . . . . . . . . . 56

Laser-Plasma Facility . . . . . . . . . . . . . . . . . . . . . . . 56

Compact Streak Camera . . . . . . . . . . . . . . . . . . . . . . 56

X-Ray Streak Camera . . . . . . . . . . . . . . . . . . . . . . . . . . . . . . . 57

Optical Kerr Effect Detection System for 10.6- $\mathrm{m}$ Pulses . . . . . . . . . . . . . . . . . 59

Holocamera for Diagnostics of Wavefront Distortion in Lasing Media . . . . . . . . . . . . . 59

Holograplic Microinterferometry of Laser-Produced Plasmas . . . . . . . . . . . . . . . . 61

Ion Spectroscopy of Luser-Produced Plasmas . . . . . . . . . . . . . . . . . . . . . . 61

6. LASER FUSICN (J. H. Nuckolis) . . . . . . . . . . . . . . . . . . . . . . . . . . . . 69

Development of LASNEX Computer Code . . . . . . . . . . . . . . . . . . . . . . . 69

Experimental Clieck of LASNEX Code . . . . . . . . . . . . . . . . . . . . . . 72

2-D Calculations to Study Synimetry and Stability of Solid Spherical DT Targets . . . . . . . . 75

Laser Targets Requiring Minimum Laser Power . . . . . . . . . . . . . . . . . . . . . 87

7. LASER-PLASMA EXPERIMENTAL (S. W. Mead) . . . . . . . . . . . . . . . . . . . . . . 93

X-Ray Microscopy . . . . . . . . . . . . . . . . . . . . . . . . . . . . 93

X-Ray Spectroscopy . . . . . . . . . . . . . . . . . . . . . . . . . . . . . . . . 94

The High-Energy X-Ray Spectrum . . . . . . . . . . . . . . . . . . . . . . . . . . 94

Liser Beam Intensity Profiles . . . . . . . . . . . . . . . . . . . . . . . . . . . . 96

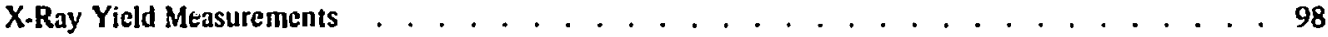

Luser Light Reflected from the Target . . . . . . . . . . . . . . . . . . . . . . . . . 100 
8. LASER-PLASMA THEORY (W. L. Kruer) . . . . . . . . . . . . . . . . . . . . . . 102

Absorptive Processes Near the Critical Density . . . . . . . . . . . . . . . . . . . 102

Control of Non-Maxwellian Heated Distributions . . . . . . . . . . . . . . . . . . . . . 104

Reflection and Self-Focusing in the Underdense Plasma . . . . . . . . . . . . . . . . 108

Summary Remarks . . . . . . . . . . . . . . . . . . . . . . . . . 110

9. BASIC STUDIES AND ADVANCED CONCEPTS (A. J. Glass and M. J. Weber) . . . . . . . . . . 111

Index Nonlinearity and Self-Focusing . . . . . . . . . . . . . . . . . . . 111

Frequency Conversion and Pulse Compression . . . . . . . . . . . . . . . . . 116

Laser Materials Studies . . . . . . . . . . . . . . . . . . . . . . . . . 120

Research Plans . . . . . . . . . . . . . . . . . . . . . . . . 122

10. ISOTOPE SEPARATION (B. B. Snavely) . . . . . . . . . . . . . . . . . . . . . . . . 123

Spectroscopic Facilities and Expenments . . . . . . . . . . . . . . . . . 123

Kinetics of Uranium Reactions . . . . . . . . . . . . . . . . . . . . . . . . . 126

Uranium Vapor Sources . . . . . . . . . . . . . . . . . . . . . . . . . 127

Development of a High-Power Tunable Laser . . . . . . . . . . . . . . . . . . . . . . 128

Photochemical Separation of ${ }^{202} \mathrm{Hg}$ from Natural Mercury . . . . . . . . . . . . . . . . 130

11. PROPAGATION (J. A. Fleck, Jr.) . . . . . . . . . . . . . . . . . . . . . . . . . . . . . . . 134

Propagation Code for Disk Laser Systems . . . . . . . . . . . . . . . . . . . . . . . 134

Nonlinear Effects and the 1-kJ Laser Design . . . . . . . . . . . . . . . . . . . . . . 134

Nonlinear Self-Focusing of Elliptical Beams . . . . . . . . . . . . . . . . . . 136

12. GAS LASER RESEARCH AND TECHNOLOGY (L. P. Bradley, T. Kan, and C. K. Rhodes) . . . . . . 139

Bound-Free Systems . . . . . . . . . . . . . . . . . . . . . . . . . . . . . . . . 139

Pulsed Power Technology and Electron Transport Studies . . . . . . . . . . . . . . . . . 142

Theoretical Studies . . . . . . . . . . . . . . . . . . . . . . . . 145

Conclusions . . . . . . . . . . . . . . . . . . . . . 148

13. $\mathrm{CO}_{2}$ LASER PROGRAM (K. Manes) . . . . . . . . . . . . . . . . . . . . . . . . . . . . 149

Studies of Rapid Relaxation in $\mathrm{CO}_{2}$. . . . . . . . . . . . . . . . . . . . . . . . 149

Theoretical Modeling of Pulse Propagation and Energy

Extraction in $\mathrm{CO}_{2}$ Amplifiers . . . . . . . . . . . . . . . . . . 152

$\mathrm{CO}_{2}$ System Status . . . . . . . . . . . . . . . . . . . . . 153

System Control and Diagnostics . . . . . . . . . . . . . . . . . . . . 156

Facilities . . . . . . . . . . . . . . . . . . . . . 156

Conclusions .......................... 156

14. PROGRAM ResOURCES (R. A. Berg) . . . . . . . . . . . . . . . . . . . . . . . . . . 157

Manpower .......................... . 157

Operating Costs . . . . . . . . . . . . . . . . . . . . . . . 157

Capital Equipment . . . . . . . . . . . . . . . . . . . . . . . . 157

Facilities ........................ . . . 157

REFERENCES ................................159

BIBLIOGRAPHY OF RECENT PUBLICATIONS . . . . . . . . . . . . . . . . . . . . . . 163 


\section{INTRODUCTION AND PROGRAM OVERVIEW}

This report reflects the activities and progress of the laser fusion program at Lawrence Livermore Laboratory during the period July through December 1973. Its function is to provide a description of the objectives, scope, and achievements of the LLL program in a coherent and readable manner. References to other material have been freely incorporated to furnish additional detail. We begin with a historical note.

Livermore's interest in laser technology began in the early 1960 s, shortly after the laser was invented. Perhaps the most noteworthy property of the laser, from LLL's viewpoint, is its ability to produce an energy concentration in space and time even greater than that produced in the heart of a nuclear explosion. Since cnergy concentration is central to LLL's overall mission-nuclear energy research-iasers from their inception have been regarded here as interesting energy sources that may be competitive with, and supplemental to, nuclear fission and fusion in this aspect.

In parallel with developments elsewhere in the world, LLL's early laser years were spent in developing an understanding of fundamental laser science and technology, and in considering the widening applications potential, both military and civil. During this period we learned that the time scale for bringing laser applications to fruition is sometimes a long one and that the "ante in" fee-the entry cost and size of the team required to make rapid progress-is large. During the late sixties and early seventies we expressed our optimistic views to both the LLL and the AEC managements; they concurred in an expansion of the laser fusion program, which to that time had simply been a program of physics evaluation at a relatively modest level of effort. Most of this original expansion was militarily oriented in that it was related to our nucleac explosives and military applications programs. Now that interest in possible civil applications is beginning to expand rapidly, these areas too are being funded and pursued in their own right.

The AEC-sponsored laser program can be sumniarized by three primary objectives:

- To demonstrate the scientific feasibility of initiating thermonuclear burn in a fuel pellet by irradiating it with a high-power, short-duration laser pulse.

- To exploit the knowledge gained in achieving the first objective to develop military and commercial applications (the military applications first because of economic constraints, with the commercial applications following later). A broad level of advanced laser technology is being established at LLL and other laboratories in the process of attaining these first two objectives.
- To apply this laser technology to various nonfusion applications of interest to the AEC and to other governmert agencies.

Much of our laser-program science and technology base is derived from the nuclear explosives research and development of the AEC and from the laser experience of the world. The chart in Fig. 1 shows the influence of nuclear weapons research on fusion and outlines some of the major potential applications of both lasers and laser fusion.

The funding of the LLL laser applications program for fiscal years 1974 and 1975 is shown in Table 1.

Table 1. Operating costs of LLL laser applications program for FY 1974-75

Costs (millions of $\$)^{\mathrm{a}}$

FY $1974 \quad$ FY 1975

\begin{tabular}{|c|c|c|}
\hline $\begin{array}{l}\text { Laser fusion: } \\
\text { Military } \\
\text { Civil (energy applications) }\end{array}$ & $\begin{array}{l}18.1 \\
- \\
\end{array}$ & $\begin{array}{l}18.1 \\
2.3(4.3) \\
\end{array}$ \\
\hline Subtotal & 18.1 & $20.4(22.4)$ \\
\hline Isotope separaiion ${ }^{b}$ & 1.0 & $2.6(4.0)$ \\
\hline Total laser applications & 19.1 & $23.0(26.4)$ \\
\hline
\end{tabular}

\footnotetext{
${ }^{a}$ Figures in parenthesis are amounts requested by LLL.

${ }^{b}$ An additional $\$ 0.5$ million is allocated to isotope separation in the laser fusion portion of the budget for both years.
}

Livermore's program, which represents approximately one-half of the AEC's total laser effort, has two major sections: (1) laser fusion, and (2) isotope separation. Systems studies of potential new applications are also included. Laser fusion research and development is further separated (somewhat arbitrarily) into military and civil components.

Anticipated growth in the near and intermediate term is substantial. The laser fusion effort is expected to expand over the next five years from its current level of 250 directly assigned people to about 450 (the level we feel is necessary for research and, most important, for timely pursuit of appications evaluations), and the laser photoseparation program should grow from its current level of about 25 people to some 250 .

Two major laser-program facihities it LLL have been authorized. The first, authorized in FY 1973, is a \$6.8-million laser laboratory containing both light and heavy optics laboratories and space for 200 people. Figure 2 shows how this building will look when com- 


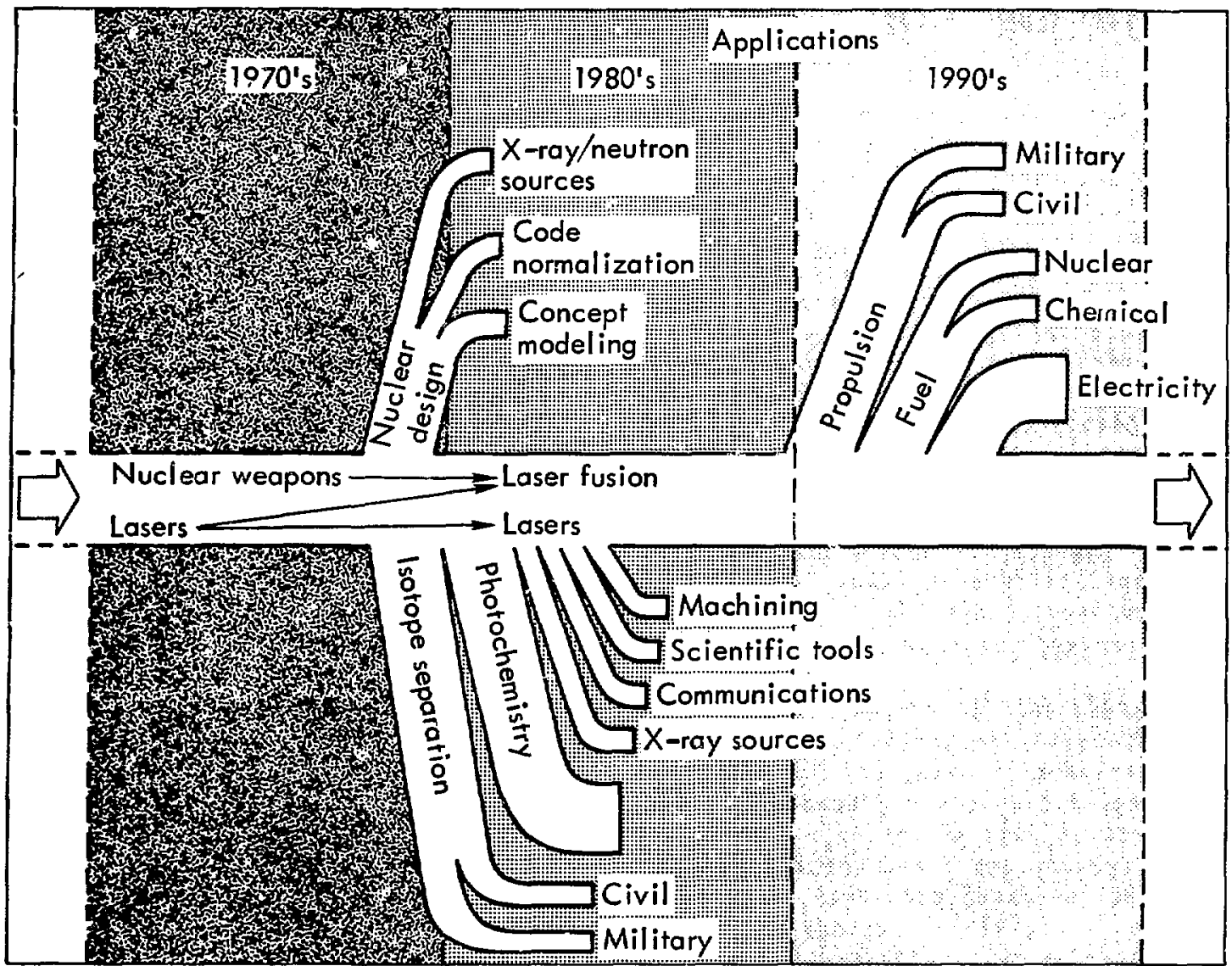

Fig. 3. Some of the potential applications seen for lasers and laser fusion.

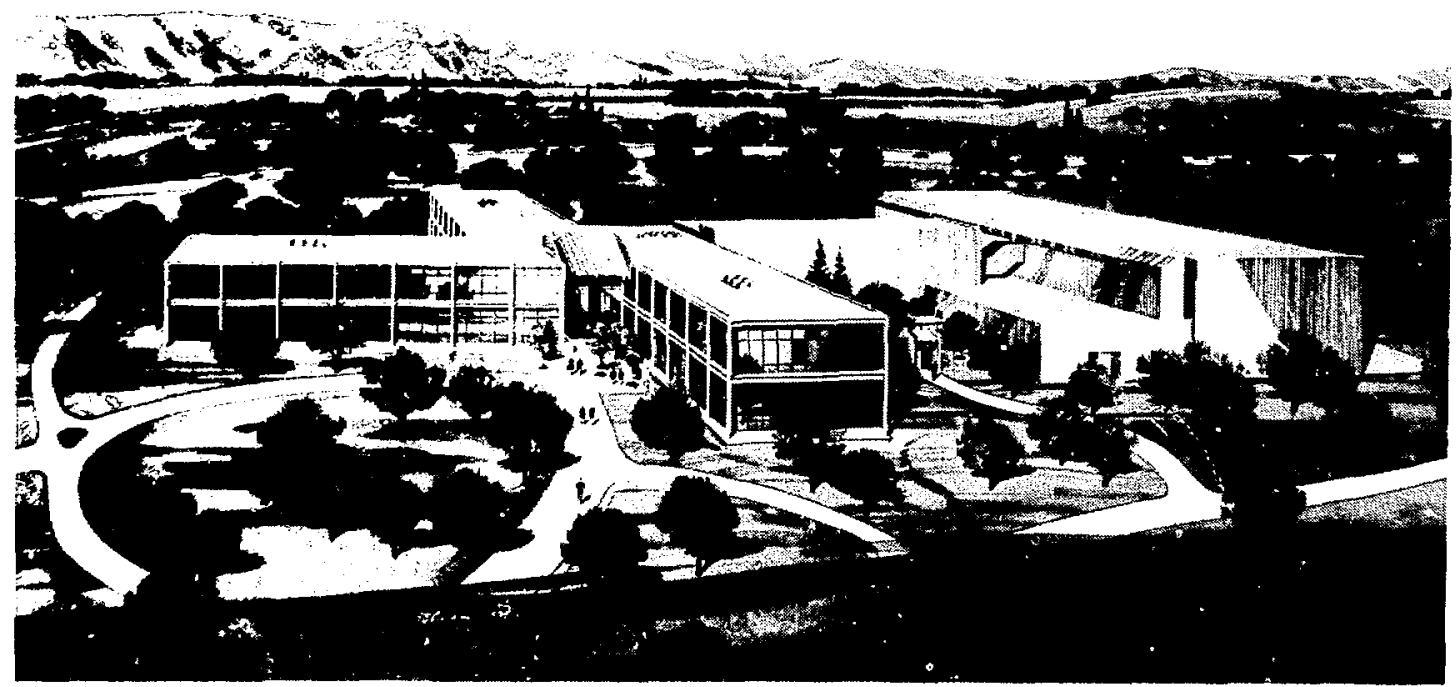

Fig. 2. The LLL Laser Fusion Laboratory is scheduled for occupancy beginning about midyear 1974. 
pleted. Occupancy will begin in June 1974. The second, authorized in FY 1974, is a high-energy laser ir radiation facility with a design goal of $10 \mathrm{~kJ}$ (kilojoules) of laser energy delivered to a target in . fraction of a nanosecond from multiple $\mathrm{Nd}$ :glass laser chains. This 10-kJ system and the building required to house it represent a $\$ 20$-million project. The facility is scheduled for completion in early 1977. We expect to demonstrate significant thermonuclear burn, and possibly scientific break-even, from pellets imploded by this facility. A model illustrating the general configuration of the laser system is shown in Fig. 3.

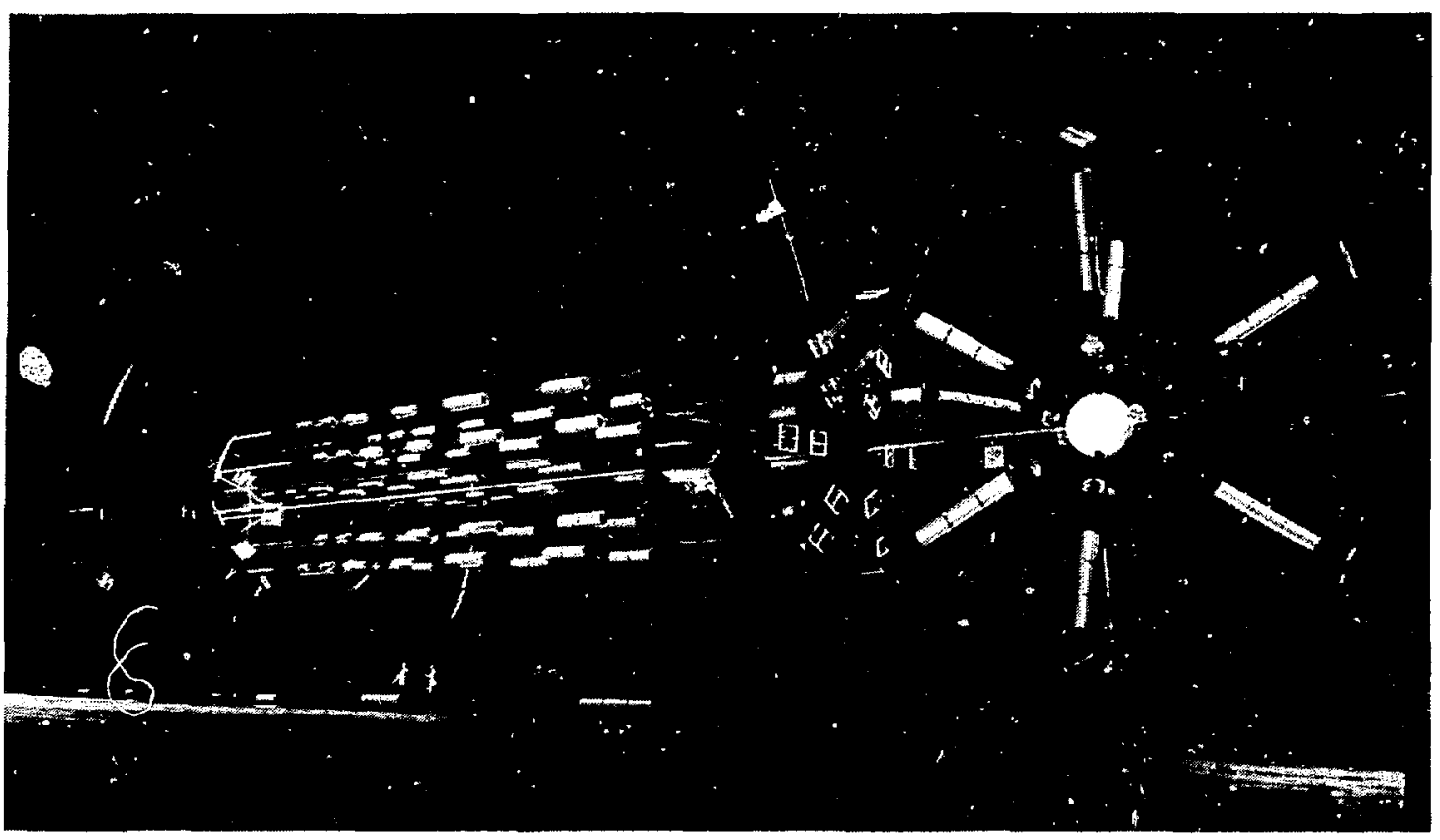

Fig. 3. Model of the 10-kJ laser irradiation facility under development at LLL.

\section{LASER PROGRAM GOALS}

We have set for ourselves five milestones by which to reckon our progress in achieving laser-induced fusion:

1. Reaching an adequate understanding of the physics of the interaction between laser light and plasma.

2. Derronstrating a laser-induced implosion (defined to occur when compressions greater than tenfold and fusion energy outputs greater than $1 \%$ of the light energy pulse from the laser are attained).

3. Achieving significant thermonuclear burn (defined to occur when compressions greater than a hundredfold and fusion energy outputs greater than $10 \%$ of the laser light energy are attained).

4. Achieving light energy break-even, by raising the pellet burn efficiency so that the fusion energy produced is of the same magnitude as the light energy in the laser pulse.
5. Achieving net energy gain, by raising the system efficiency to the point where the fusion energy output substantially exceeds the energy input to the whole laser system.

Our present estimates of the laser technology needed to reach these milestones are given in Table 2 .

To achieve the first milestone of the laser fusion program we need to determine the kind of laser output pulse-in terms of energy, pulse duration and shape, wavelength, coherence properties, and symmetry-that will be required to compress a pellet of thermonuclear fuel to burn conditions, and then to design and build a laser that will produce this output pulse.

For the next few years (FY 1975-1977), the Laboratory will continue to perform basic laser/matter experiments using well-characterized lasers of moderate size. Pulse energies will range from tens of joules to about $1 \mathrm{~kJ}$, and pulse lengths from the low nanosecond 
Laser requirements

\begin{tabular}{lccc} 
Milesione & $E_{\text {fusion }} / E_{\text {light }}$ & Energy (kJ) & Pulse length (psec) $^{\mathrm{a}}$ \\
\hline 1. Light/plasma interactions & - & $0.01-0.1$ & $10-1000$ \\
2. Laser-indi ed implosion & 0.01 & $0.1-1.0$ & $50-200$ \\
3. Significant thermonuclear burn & 0.1 & $1-10$ & $100-1000$ \\
4. Light energy breakeven & 1 & $10-100$ & $50-1000$ \\
5. Net energy gain & $\geqslant 10$ & $100-1000$ & $\begin{array}{c}\text { (with shaping) } \\
\text { (with shaping) }\end{array}$ \\
\hline
\end{tabular}

\footnotetext{
ame to deliver about the last $50 \%$ of the laser energy. The early part of the pulse, of gradually increasing intensity, may have a duration of tens of nanoseconds.

${ }^{b}$ Assuming a $5 \%$ laser system efficiency.
}

region to tens of picoseconds. The main purpose of these experiments will be to provide a data base for comparison with theoretical calculations, in order to develop the capability to predict with high confidence the key laser and target parameters necessary to obtain thermonuclear burn. We hope to attain the first and second milestones during this period. Concurrently, the laser required to achieve the third milestone-significant thermonuclear burn-will be constructed. Research leading to the development of the laser systems necessary to support the last two milestones-light energy break-even and net energy gain-will also be intensively pursued. In the FY 1977-78 era we expect to demonstrate significant thermonuclear burn! As a concurrent and strongly related objective the Laboratory will also work on obtaining sources of laserproduced $\mathrm{x}$-rays which would be useful in conducting experiments simulating the intense $x$-roy output of nuclear weapons.

\section{GLASS LASER PROGRAM}

Neodymium-doped glass lasers (Nd:glass) are the best understood high-energy, short-pulse lasers now available. With Nd:glass lasers we will be able to thoroughly investigate the physics of laser/plasma coupling, which is so fundamental to the eventual success of laser fusion. We are confident that our glass laser effort offers the most reliable path to achieving the first three milestones, and perhaps even the fourth one (light energy break-even). However, the high energy and high average power eventually required for controlled thermonuclear power generation preclude the use of solid-state lasers as we now conceive them.

For several years LLL has had in operation a laser with Nd:glass disks (the Long Path Laser) which produces $200 \mathrm{~J}$ in $5 \mathrm{nsec}$ and $100 \mathrm{~J}$ in $2 \mathrm{nsec}$. Currently under development is a much higher performance system that will produce a $1-\mathrm{kJ}$ pulse of $100 \mathrm{psec}$ duration (see Fig. 4). It will be constructed in four successive increments, with outputs of approximately 5, 50, 250 , and $1000 \mathrm{~J}$. Several replicas of the lower-energy increments will be built and used to study the details of the laser/plasma interaction. Modular design will make these devices extremely flexible; they will be able to provide useful energy output at pulse durations from 20 psec to 1 nsec.

The 1-kJ system, which will be almost entirely constructed from disk laser amplifiers, will form the module basis for the development of the $10-\mathrm{kJ}$, 100-psec spherical irradiation facility to be used for the first milestone experiments. Twelve $1000-J$ or possibly twenty 500-J lasers will be operated in parallel, driven by a common oscillator, to comprise the $10-\mathrm{kJ}$ laser facility.

$\mathrm{Nd}$ :glass lasers operate at a wavelength of $1.06 \mu \mathrm{m}$. However, certain theoretical considerations indicate that shorter wavelengths would be more useful fo: plasma heating. Thus, the 10-kJ facility will, in the: long term, incorporate the capability of generating the second and fourth harmonics as well as Stokes-shifted radiation in $\mathrm{H}_{2}$, allowing covcrage of the wavelength region from 0.265 to $1.9 \mu \mathrm{m}$. At all of these wavelengths, total energies in excess of $2 \mathrm{~kJ}$ will be available. Thus we will have a flexible facility for pursuing the second and third milestone experiments (laserinduced implosions and significant thermonuclear burn). 


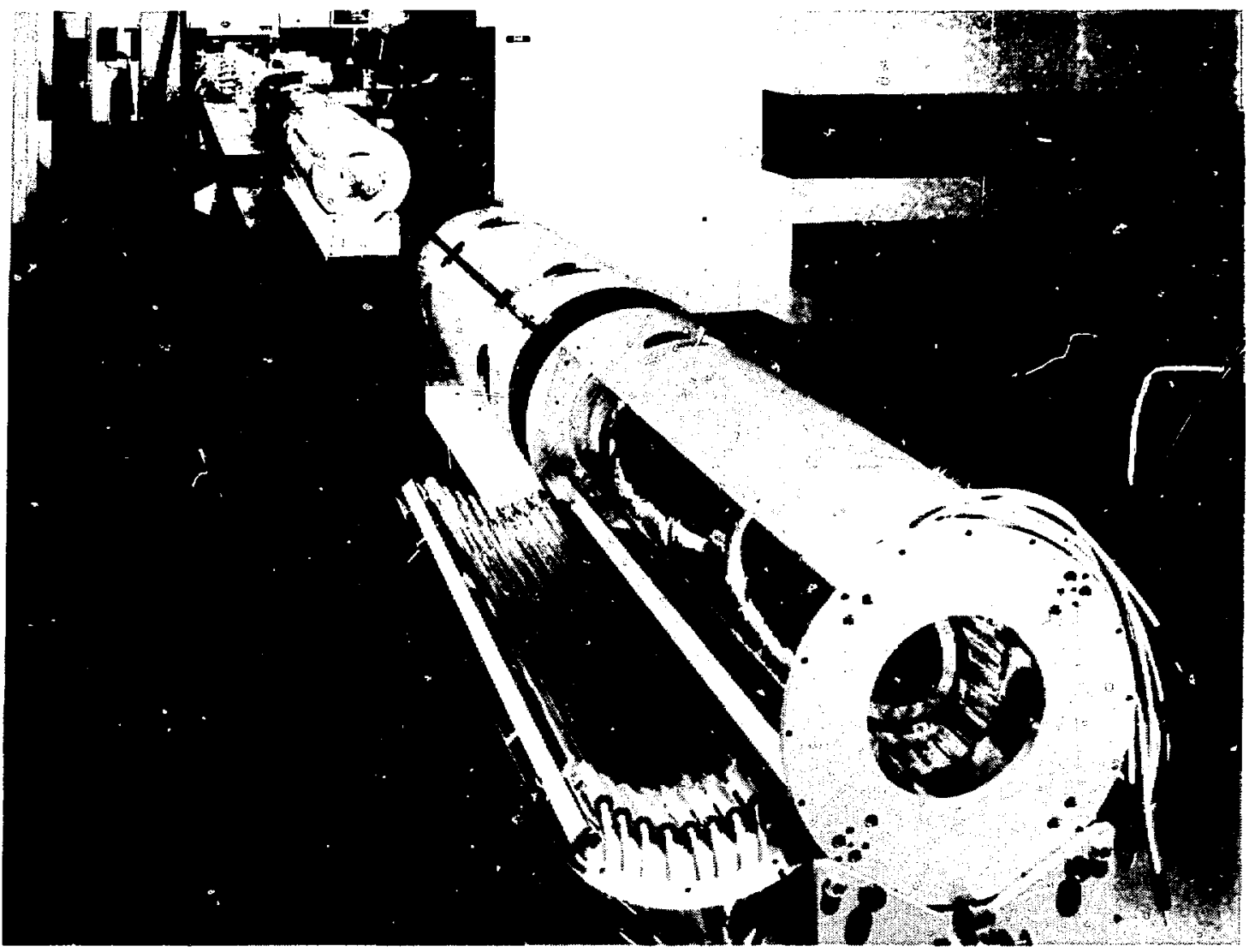

Fig. 4. P- tions of the 1-kJ Nd:glass hase chain being developed at LLC. for use in the 10-kJ laser irradiation facility.

\section{GAS LASER PROGRAM}

Gas lasers appear to provide greaier long-term potential for the generation of energies in the $10^{4} \cdot 10$ $10^{6}-\mathrm{J}$ region at high efficiency. The $\mathrm{CO}_{2}$ laser operating at $10.6 \mathrm{\mu m}$ has demonstrated the ability to generate I-nsec pulses with an efficiency of 5\%. Development of a shorl-pulse $\mathrm{CO}_{2}$ laser is presently under way at LLL. wit! the goal of a 50-10-100-J. I-nsec output. It is expected to be operating by the end of FY 1974 . Details are shown in Fig. 5. This will primarily be an irradiation facility, one that is amenalyle en experimentation and numerical modeling.

Energy decoupling and target preheating effects, which can preven: successful implosion. lave been found, and these effects may make it less desirable to use laser radiation of as long a wavelength as $10.6 \mu \mathrm{m}$. Both problems, it appears, may be alleviated io a significant extent by using radiation of shorter wavelength. An extensive program is in progress at LLL to deveiop short-wavelength, high-efficiency gas laser media which could share many of the advantages of the $\mathrm{CO}_{2}$ system, but which operate at more desirable wavelengths. We have observed, for example, ultraviolet laser action from molecular xenon $\left(\mathrm{Xe}_{2}^{*}\right)$,molecular krypton $\left(\mathrm{Kr}_{2}^{*}\right)$, and nolecular argon $\left(\mathrm{Ar}_{2}\right)$. Similar experiments with other van der Waals type molecules are under way. Shorl-wavelength laser systems using both resonant and nonresonant stimulated anti.Stokes Raman scaltering are also under development. 


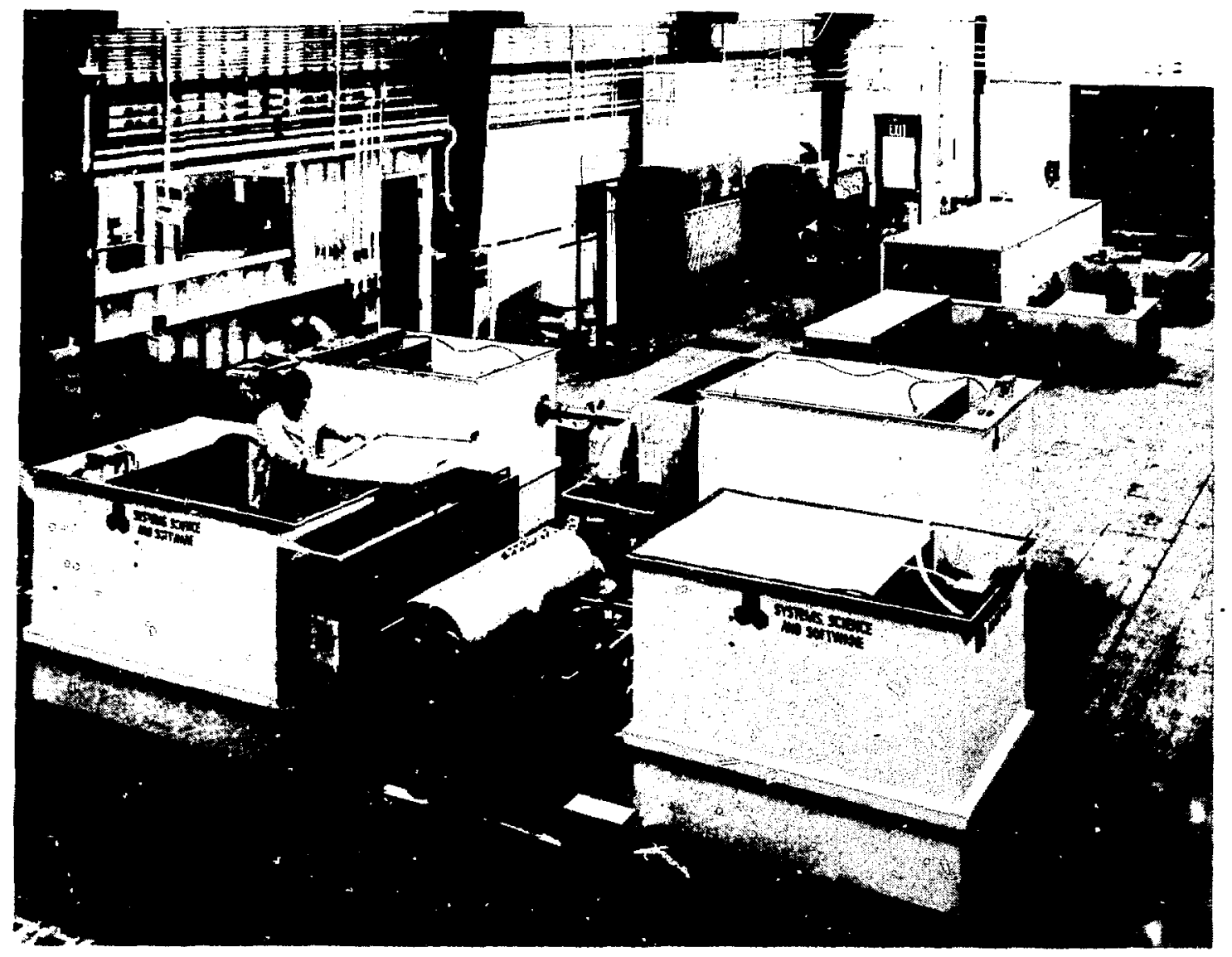

Fig. 5. Short-pulse $\mathrm{CO}_{2}$ laser under construction at LLL will produce 50 to $100 \mathrm{~J}$ in a pulse length of 1 nsec.

\section{OTHER LASER STUDIES}

Pulsed chemical lasers appear to offer the greatest potential for weapon applications ultimately, because-in theory, at least-they can store large amounts of energy in a reasonably small volume and weight. We are still in the very early research stages with these concepis, and plan only a mndest level of effort in the near future.

For any laser system, diagnostic capability is of paramount importance, especially for measuring the properties of light pulses of subnanosecond duration. Our diagnostic program addresses these needs. We have developed a streak camera with 10-psec resolution and are extending its capability 101 psec. An x-ray photocathode has been developed which allows time-resolved $x$-ray spectroscopy on the laser-produced plasmas. In general. we are working to extend the time resolution of all conventional diagnostics for lasers and plasmas to the 1-10-1000-psec regine.
The extensive laser development effort in support of the laser fusion program will clearly have applications in areas other than fusion. A program directed generally at optical separation of isotopes and specifically at uranium isotopes has been initiated. We expect that measurable quantities of uranium iubstantially enriched in ${ }^{235} \mathrm{U}$ will be separated in this way during FY 1974 and that several photoseparation concepts will be experimentally investigated during FY 1975. The technology required for process scale-up will also be pursued.

Table 3, which compares diffusion and laser separation costs, illustrates the potential savings in both money and uranium ore that photoseparation would introduce over the next 25 years while providing for power-reactor fuel needs. Other laser applications will be explored as the needs appear and the technologies become available. 
Table 3. Comparative estimated costs of gaseous diffusion process and laser photoseparation process in meeting U.S. requirements for enriched uranium in the period 1980-2000

\begin{tabular}{|c|c|c|c|c|c|}
\hline & $\begin{array}{c}\text { Capifal } \\
\text { investment } \\
\left(\$ 10^{\circ}\right)\end{array}$ & $\begin{array}{c}\text { Ore } \\
\text { Isquired } \\
\left(10^{6} \text { tons }\right)\end{array}$ & $\begin{array}{c}\text { Ore } \\
\text { cost } \\
\left(510^{\circ}\right)\end{array}$ & $\begin{array}{c}\text { Separation } \\
\cos 1^{2} \\
\left(\$ 10^{\circ}\right)\end{array}$ & $\begin{array}{c}\text { Tots } \\
\text { cost } \\
\left(510^{\prime}\right)\end{array}$ \\
\hline Diffusion process & 20 & 2.4 & 70 & 65 & 135 \\
\hline Laser separation & 2 & 1.4 & 30 & 8 & 38 \\
\hline
\end{tabular}

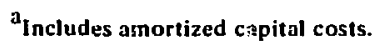

We close by observing that the LLL laser program is broadly based in the total laser technology while also strongly accenting the specific applications of laser fusion and isotupe separation. The basic motif of research, development, and engineering in the context of the overall applications establishes, we believe, the most conducive atmosphere for the successful conclusion of the desired objectives. The following sections of this report furnish details on the full range of these activities. 


\section{SOLID-STATE LASER PROGRAM}

The primary goal of the solid-state laser program is the developnient and construction of a $10 . \mathrm{kJ}$ Nid:glass laser facility for high-enesgy laser fusion experintents. Secondary goals include the construction of lowerenergy single- and multiple.beam Nd:glass lasers for laser/plasma coupling and fusion experinents. The technical approach taken to achieve these goals in a reasonably short time is characterized by the use of extensive computer simulation of both laser conponents and component arrays, an aspect which is developed in Sec. 3 of this report, "Design Analysis." To minimize engineering design load and spare parts inventories, and to maximize system reliability, we have adopted a modular concept of laser hardwarc. Thr details of many of these elements are contained in this section and in Sec. 4. "Component Development."

The 10-kJ high-energy laser facility (HELF) received Congressional approval as a \$20-million line item with an appropriation of S2 million in FY 1974. Figure 6 shows a conceplual design of lite fecility, including a 10.kJ laser array, target bay and clamber, energy storage area, and control and diagnostics rooms. Pre-Title I facility design studies were initiated during the reporting period and are well under way. The $10-\mathrm{kJ}$ laser array shown in Fig. 6 consists of 12 identical l-kJ amplifier thains. driven by a single master oscillator. Designs using 20 amplifier chains, with a correspondingly smaller amount of energy per chain, are aiso receiving intensive study. We are now in the midst of the development of the $[-k J$ protolype amplifier chain from which the hardware for the 10-kJ array will derive. Figure 7 shows the staging of the $I-k J$ chain and the four basic disk-amplifier modules, $A, B, C$, and $D$, with nominal aperture diameters of $5,10,20$, and $30 \mathrm{~cm}$. Progress during the first tralf of FY 1974 is reported below, under separate headings dealing with the master oscillator, rod amplifiers, disk amplifiers, performance of the prototype system (Cyclops), and efforts relating to supporting technologies.

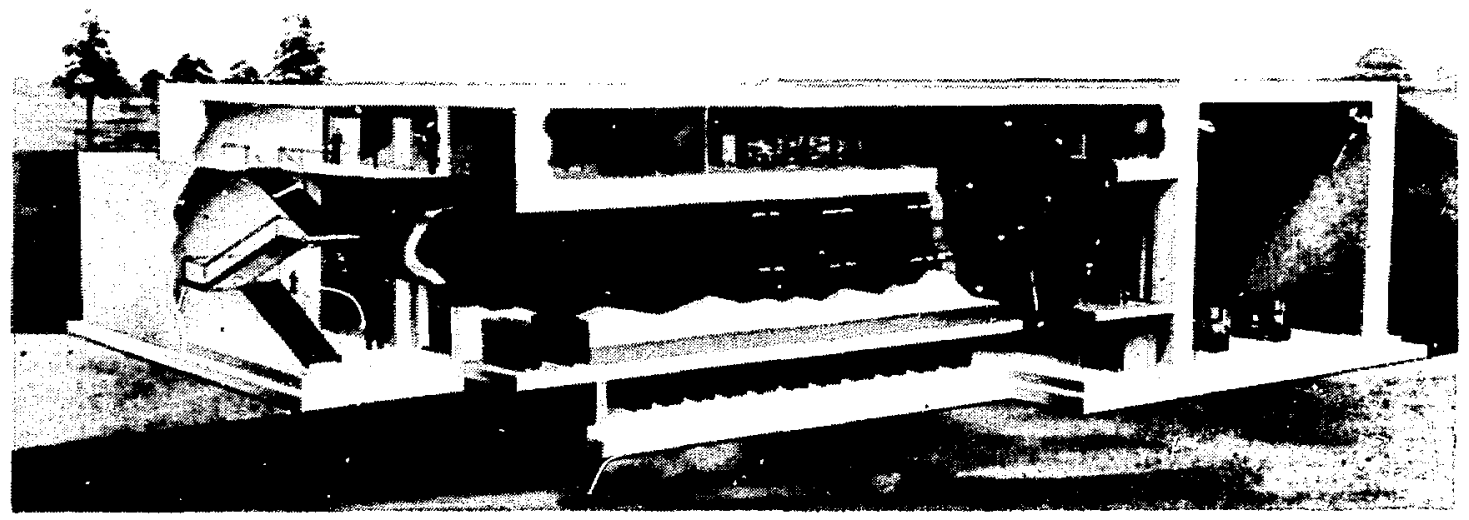

Fig. 6. Coneeptual detign of HELF (figh Energy Laser Facility), which will contain the I0-kJ laser irmadiation facility.

\section{MASTER OSCILLATOR}

A reliable high-quality master oscillator is an essential element of a properly designed high-energy amplifier system. The master oscillator developed for the LLL single-arm $1 \cdot k J$ laser chain is shown in place in Fig. 8. The master oscillators for the twin-arm and 12 -arm $1 \cdot k J$ chains will be similar to this one. The design of the oscillator has been presented previousiy, but we will briefly recapilulate it here.
In cross section the oscillator chassis is basically H-shaped, with cylindrical Invar rods mounted near the four corners of the top half of the $H$. The rescnator mirrors are referenced to the Invar rods, while all of the otiner components (excluding connectors) are mounted to the horizontal central meinber. This structure is inlerently thermally stable. The mirror mounts are constructed totally from stainless steel, specifically 


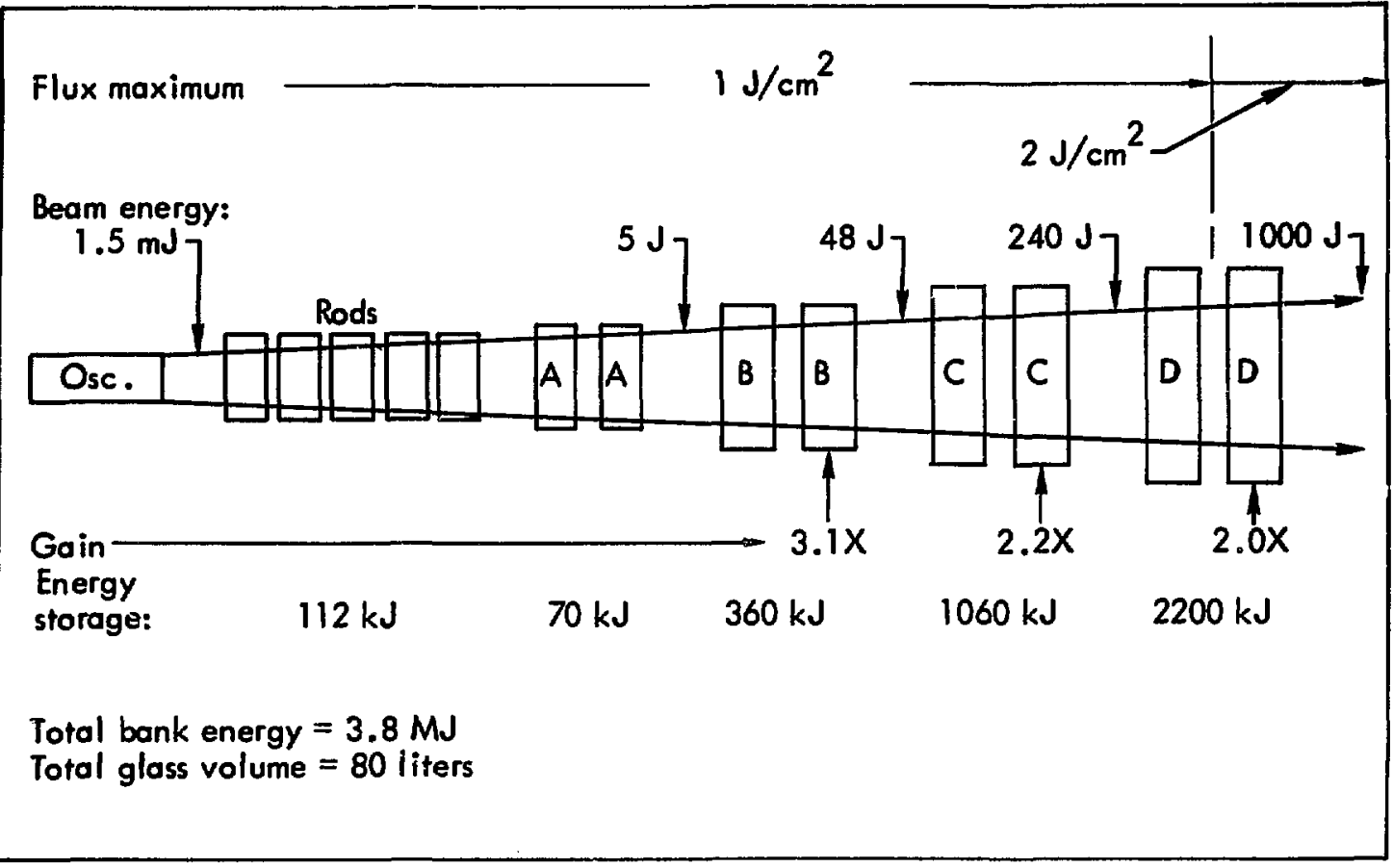

Fig. 7. Staging design for the 1-kJ chain, 12 of which would be combined in the 10-kJ laser irradiation facility.

including the adjustment mechanisms, to preclude misalignment due to thermal bimetallic effects. The resonant cavity is semiconfocal.

The oscillator is mode-locked with a flowing bleachable dye. The temperature of the dye and of the intracavity bandwidtl-limiting etalon is held within $\pm 0.1^{\circ} \mathrm{C}$ by one closed-cycle coolant loop, while the temperature of the rod is maintained within $\pm 1^{\circ} \mathrm{C}$ by a second loop.

The laser head for this oscillator, shown in Fig. 9, is the result of a compromise between uniform pumping and moderate repetition rate. Its afocal double-ellipse geometry is shown in Fig. 10, and the results of a normalized gain distribution calculation are shown in Fig. 11. Since the rod is operated at Brewster's angle (which implies that the beam inside the rod occupies a $33.4^{\circ}$ ellipse) and since the beam's $e^{-2}$ intensity diameter just outside the rod is about $2 \mathrm{~mm}$, the segments of interest are numbers 14 through 18 (particularly 15 and 16).

The basic oscillator emits a train of subnanosecond pulses, one of which is switched out to the preamplifiers and amplifiers (see Fig. 12). The parameters of the basic oscillator are presented in Table 4 . Single pulse selection is accomplished by the switchout assembly,

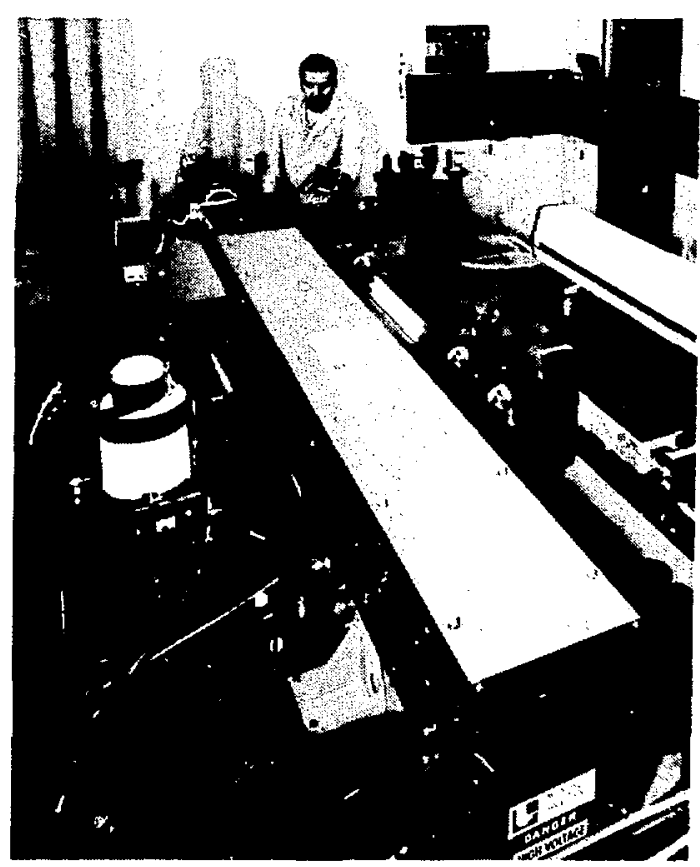

Fig. 8. Master oscillator for the $1-\mathrm{kJ}$ chain, in place. 


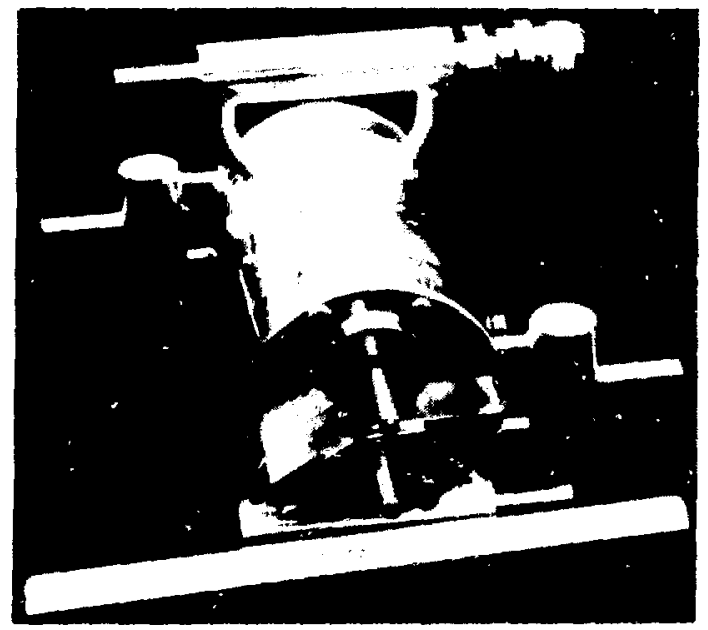

Fig. 9. Laser head for the master oscillator.

which is contained in the switchout chassis (the smaller box in Fig. 8) and in the front fifth of the oscillator chassis. The switchout chassis also provides for the sele_tion of the pulses just preceding and just following
Tahle 4. Parameters of the master oscillator
Outjut mirzot reflectance

Dye ank solvent

Dye cell thickness

Dyc Iransmission

Filsthlamp capacilor

Flasilamp inductor

Laser tiresliolu

Operating point

Pulso spacing

Pulse train energy

Linergy of peak pulse

Missing pulse train tate

Double pulse train sate
$25 \% n$

Eastnian $13183 \mathrm{dyc}$ in 13187 solvent

I5 mils (0.38 mm)

$40 \% \mathrm{r}\left(\mathrm{o}=25 \mathrm{~cm}^{-1}\right)$

$17.4 J(997 \mathrm{~V})$

$19.0 \mathrm{~J}(1042 \mathrm{~V})$

$10 \mathrm{nsec}$

$10 \mathrm{~mJ}(0=5 \%)$

$2.2 \mathrm{ml}(\mathrm{\sigma} \approx 6 \%)$

$<1 \%$

$<2 \%$
$35 \mu F \mid \cdot 3 t=112 \mu \sec$

the output pulse; these subsidiary pulses are emitted from the switchout chassis, to be processed and used for double-pulse holography at the target. Threshoiding and switching are presently both performed by an

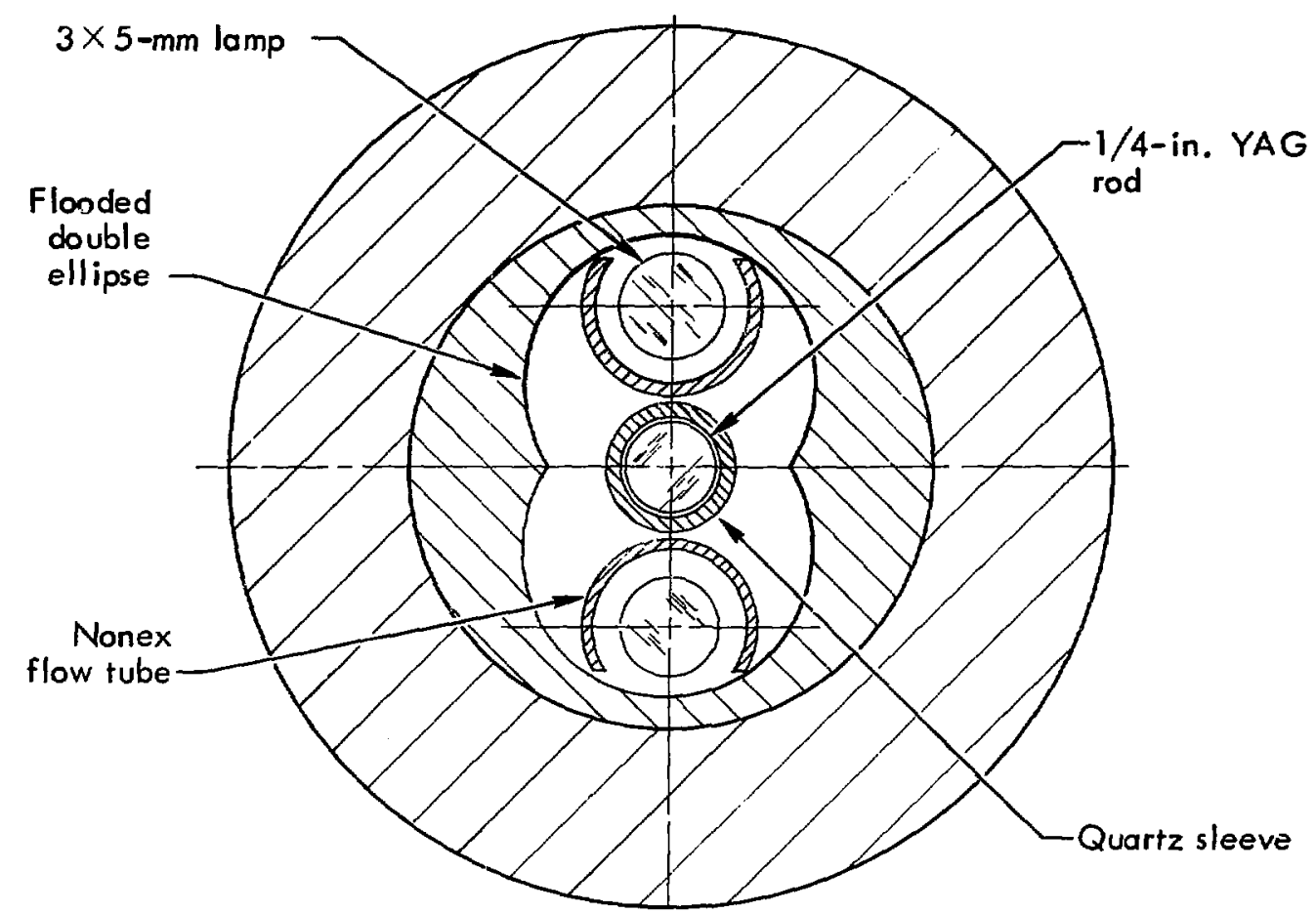

Fig. 10. Cross section of master oseillator's laser head. 
uptically triggered spark gap, which degrades the reproducibility of the single-pulse output from the performance one would expect based cn Table 4; the statistics are presented in the systcm test and integration section of this report. Work is presently in progress that slıculd separate the thresholding and switching functions and/or improve the spark gap.

A smooth well-characterized spatial profile is an important requirement of the oscillator system. Figure 13 shows the transverse beam profile at various points between the basic cscillator and the single-pulse output port. These photos, taken with a multispot camera ${ }^{2}$ using a high-sensitivity low-dyramic-range film, show some beam degradation due to the switchout Pockels cell, but do not show the high-spatialfrequency modulation imposed by one of the switch. out Glan-air polarizers. Both of these effects appear to be eliminated by the spatial filter located just after the master oscillator (see the system test and integration section).

Neither thermal stability nor mechanical stability have been checked by formal testing, but indirect evidence indicates that both are satisfactory. Alignment did not change because of the system's move from the oscillator development lab to the kilojoule laser lab, nor within either lab, so that existing ambient temperature variations do not affect it. Alignment only rieeded "tweaking" (the laser operated, but not optimally) after an impromptu vibration test in

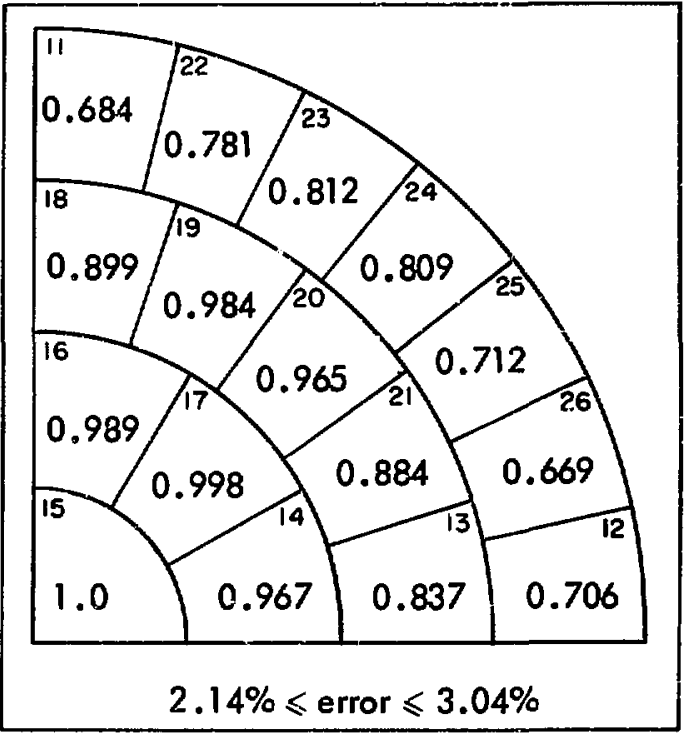

Fig. 11. Calculated gain distribution in rod of master oscillator's laser head.

which two 5/8-in. holes were drilled through the 1/2-in.-thick horizontal mounting plate with the aid of a hand-held $1 / 2$-in. drill motor, and was unaffected by the laser's being rolled over through $360^{\circ}$ to take photographs of it.

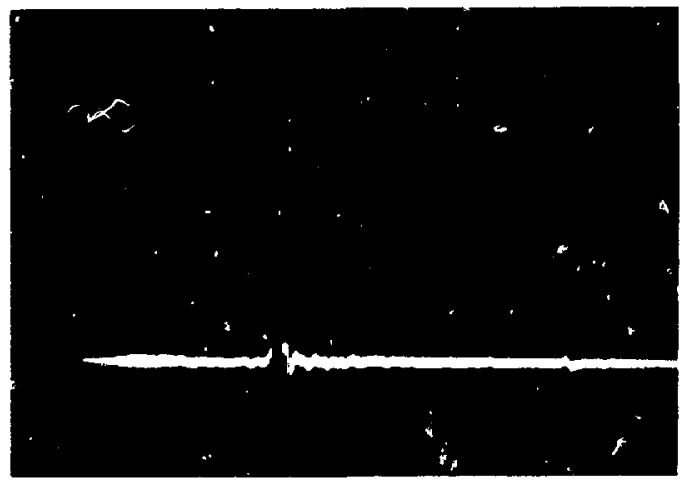

(a)

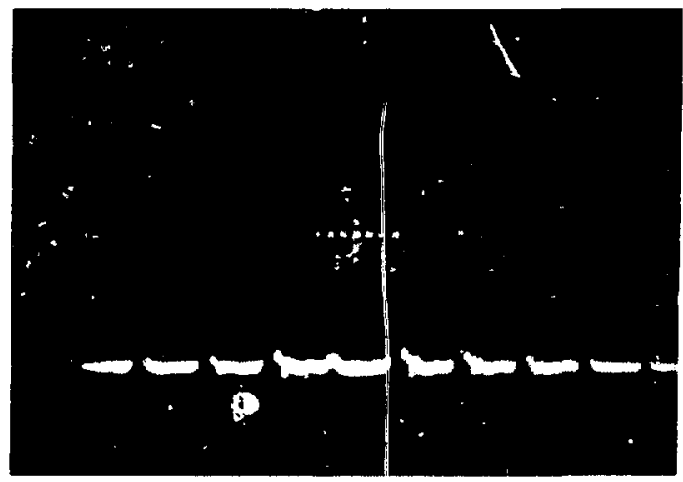

(b)

Fig. 12. Master oscillator output, showing (a) the switched-out pulse, and (b) the rejected portion of the pulse train. Scale is 10 nsec/division. Note: Amplitudes and temporal locations are not comparable between (a) and (b) because different detectors and cable lengths are involyed. 
For the amplification of short pulses of about 0.1 nsec duration and total energies up to the 1.J level, solid rods are most effective because of their high energy storage capability for diameters up to $25 \mathrm{~mm}$. For beam diameters above $25 \mathrm{~mm}$, however, the gain coefficient for disks can be maintained at a higher level than for rods because of pumping limitations.

For further amplification of the laser beam after passing through the apodized aperture in the $1-\mathrm{kJ}$ laser system, three 1-in.-diam. rod amplifiers were chosen, with nominal small-signal gains of $10 \mathrm{~dB}$ each. These amplifiers consist of 1 -in.-diam. by 10-in.-long rods with AR-coated end faces normal to the optical axis. The rods are made from 1.0-wt\%-Nd-doped ED-2 laser glass and have a fine ground cylinder $s$. fface to insure uniform pumping. They are submersed in an aqueous solution of sodiu.n nitrite for uv protection and for reduction of rod heating by pumplight which does not contribute to population inversion. The rods are pumped by six linear tlashlamps with a bore diameter of $13 \mathrm{~mm}$ and an arc length of $8 \mathrm{in}$. Three of these flashlamps are connected in series to a single-mesh 5-kV energy-storage bank with $240 \mu \mathrm{F}$ capacitance and

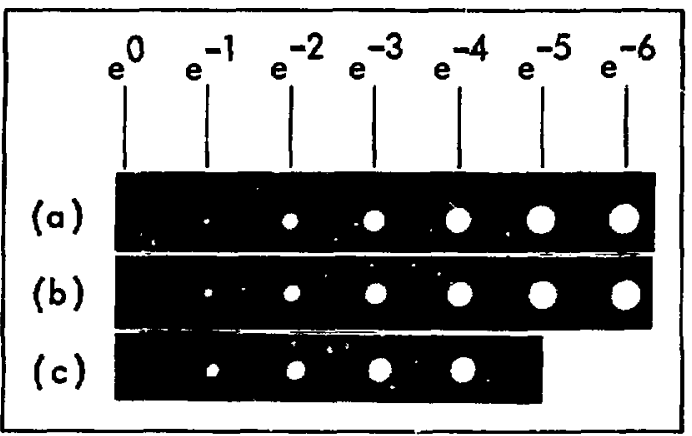

Fig. 13. Transverse beam profile for the master oscillator at various points between the basic oscillator and the single-pulse output port. (a) Pulse train after switchout polarizer. (b) Pulse train after switchout Pockels cell. (c) Single switched-out pulse after switchout analyzer. Film is about $2 \mathrm{~m}$ from output mirror.

$300 \mu \mathrm{H}$ inductance. The pump cavity is formed by six thin silver-plated brass reflectors in a close wrap configuration. A cross-sectional view of this amplifier is shown in Fig. 14.

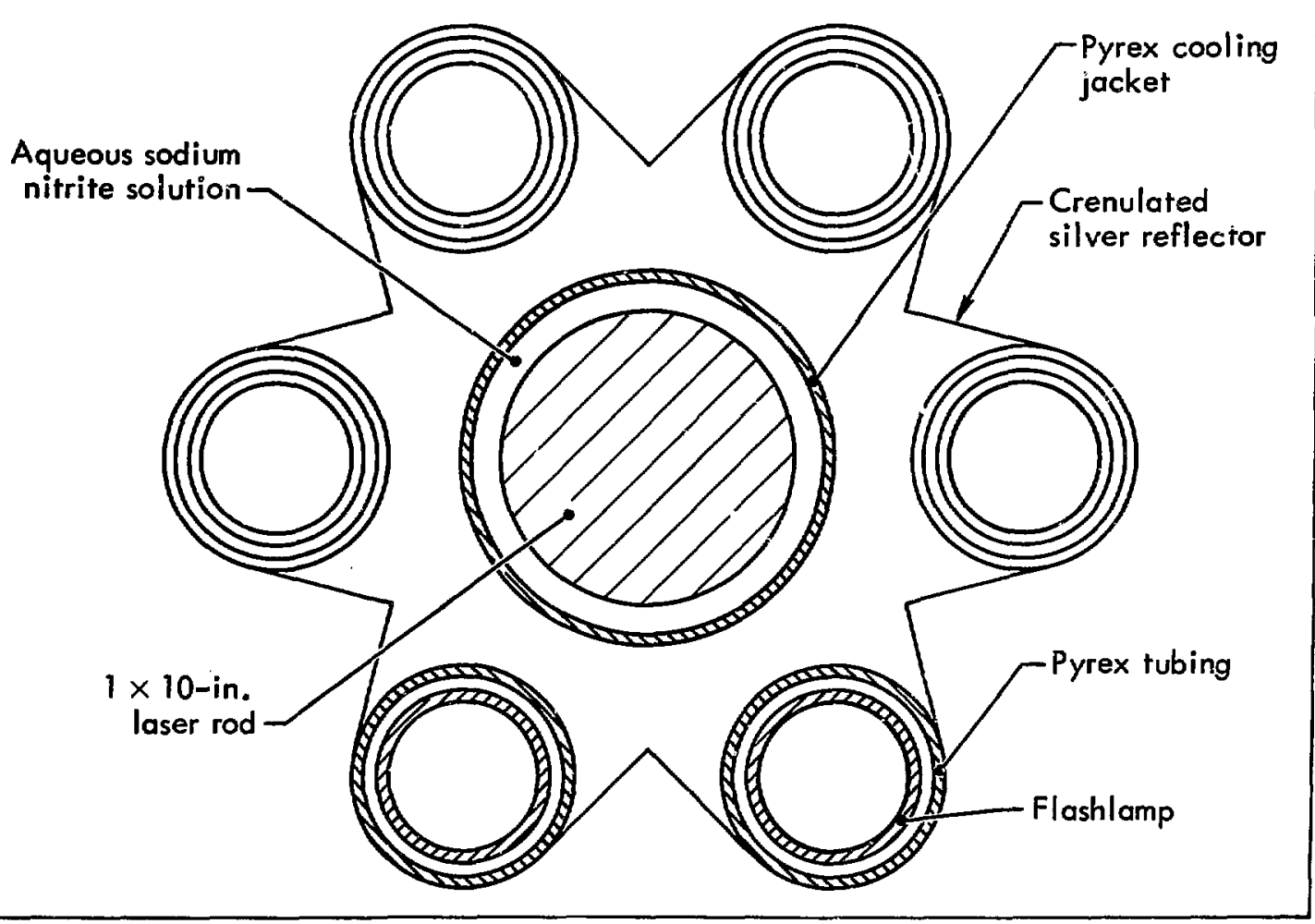

Fig. 14. Cross section of rod amplifier for the $1-\mathrm{kJ}$ laser chain. 
The gain distribution for this geometry was computed with the ZAP code for 1.0 - and $1.5 \% \mathrm{Nd}$-doped laser rods, with the results shown in Fig. 15. From this analysis, the gain is uniform to within $\pm 2 \%$ over the central $75 \%$ of the rod's diameter, which agrets well with experimental results taken on rod amplifiers with similar pump geometries.
The small-signal gain was measured with a cw YAG oscillator, and the results are shown in Fig. 16. The initial slope of this gain curve corresponds to a pumping efficiency of $2.2 \%$, which compares favorably with respect to other rod and disk amplifiers. The calculated Jesign goal of $10 \mathrm{~dB}$ gain at $6 \mathrm{~kJ}$ input was met within the experimental errors of the measurement.

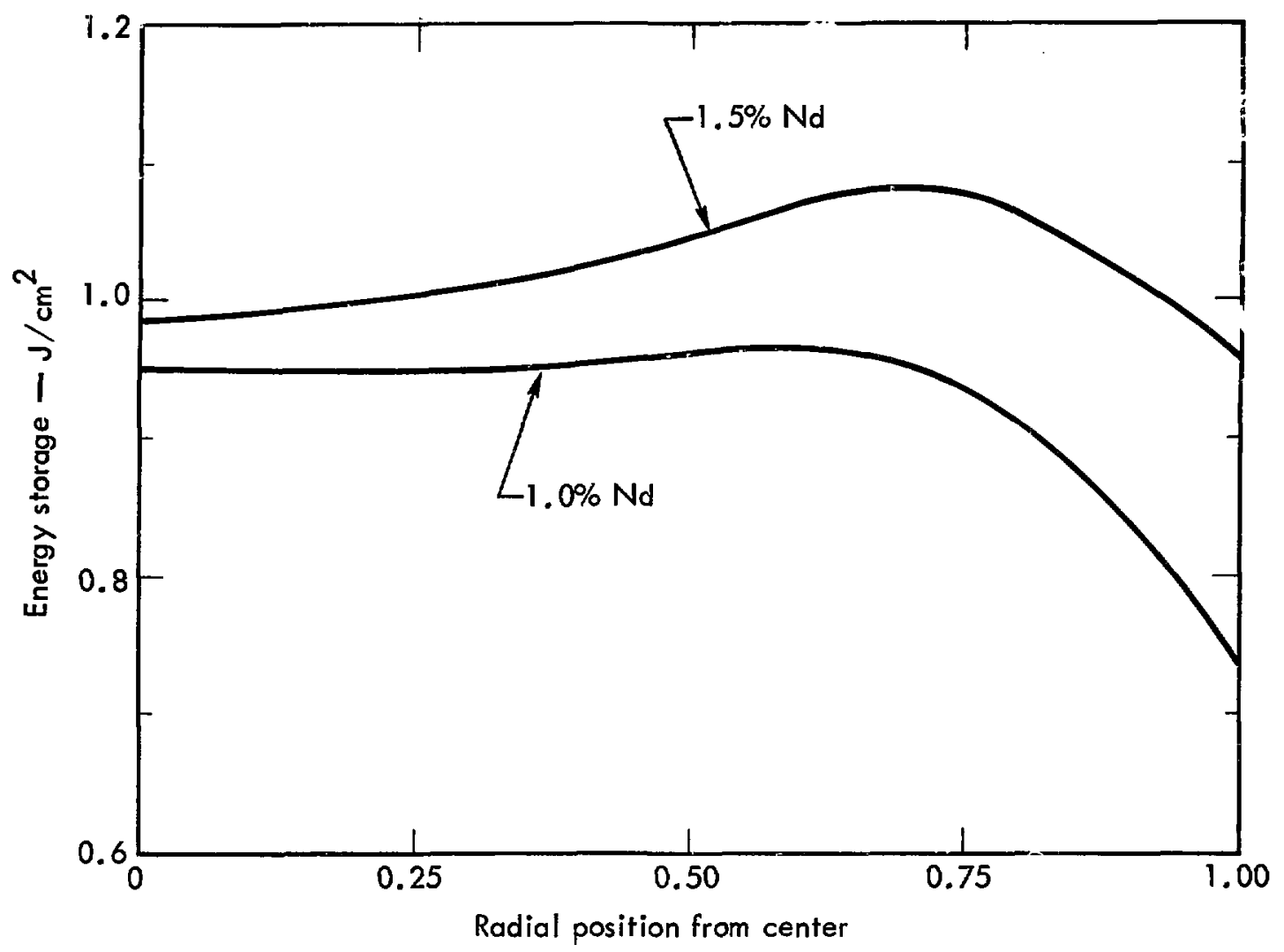

Fig. 15. Calculated gain distribution in the rod amplifier of Fig. 14 for 1.0 and $1.5 \%$ Nd doping.

\section{DISK AMPLIFIERS}

For short laser pulses with energies above approximately the 1-J level, disk amplifiers are required for optimum performance. The general design considerations for the disk amplifiers were described in the last semiannual report. ${ }^{3}$ At that time the design of the hardware for the $A$ and $B$ amplifieis was just completed. Since then, these amplifiers have been built and tested.

During operation of the $A$ and $B$ disk amplifiers, several criticai areas have been identified which need further development and improvement. One of the most critical problems with disk lasers is to maintain the disk assembly as clean as possible in order to avoid damage of the disk surfaces, and our present assembly area is not clean enough to insure dust- and lint-free assembly. A clean room will be installed to improve this situation. Another problem we had was with the Pyrex tubing ' 'sed to shield the disk assembly; it deteriorated under the high uv exposure from the flashlamps, causing additional surface damage to the disks. We will replace the Pyrex tubing with quartz tubing for all further tests. 


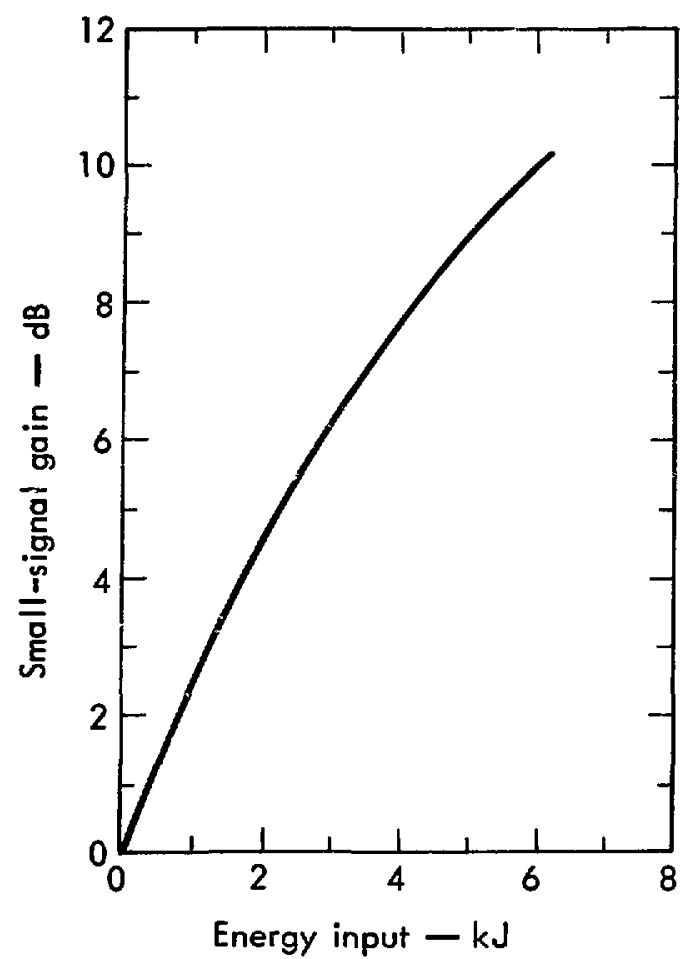

Fig. 16. Measured small-signal gain for the rod amplifier of Fig. 14.

A third source of contamination resulted from deterioration of the edge coatings on the disks, despite shielding of the edges from direct pumplight. This problem might be circumvented for the $A$ and $B$ amplifiers by use of the old G- 9000 edge coating, which has been previously successfully tested. But the G-9000 coating has a relatively high index and so cannot be used for the $C$ and $D$ amplifiers.

We have also experienced some difficulties with the electrical insulation of the flashlamps due to mechan:cally weak insulation of the flexible lead on the flashlamps. Three vendors have been working on this problem and will provide alternate solutions to the insulation of the flashlamps. In addition we have purchased flashlamps without insulation in order to investigate our own soluticins.

\section{- A Amplifier}

The $A$ amplifier consists of six elliptical disks with major axis of $84 \mathrm{~mm}$, minor axis of $48 \mathrm{~mm}$, and 'jickness of $15 \mathrm{~mm}$. The nominal useful aperture of this anplifier is $35 \mathrm{~mm}$. The disks are mounted in individually removable holders which shield the disks' edge coating from direct pumplight to avoid deterioration.
The disk holders incorporatc flow channels as shown in Fig. 17, which concentrate the nitrogen flow over the disk surface through a narrow gap between the mask and the disk. A high gas-flow velocity along the disk surface is desirable for better cooling and to keep the gas consumption within reasonable limits. The disks and disk holders can be individually removed from the disk array for ease of maintenance. Surrounding the disk array is uv-absorbing filter tubing to protect the disks from excessive uv exposure and to keep the disk assembly clean. Pyrex tubing was used in early tests before other more appropriate material was available. In order to prevent contamination and thermo-optic beam distortions, the optical patil of this laser system is completely sealed off. A positive nitrogen pressure in the disk assembly insures a steady outflow of gas from the disk assembly into the flashlamp pump cavity. This nitrogen flow also serves to cool the device. Typical $\mathrm{N}_{2}$ flow rates of about $20 \mathrm{cfm}$ are required for a repetition rate of $0.1 \mathrm{ppm}$ (pulse per minute).

The $A$ disk assembly is pumped with linear flashlamps of 22-in. arc length. Either 16 lamps with $10-\mathrm{mm}$ bore diameter or 12 lamps with $15-\mathrm{mm}$ bore diameter are used. In both cases, these flashlamps are connected in groups of four in series, which provides alternating current path through adjacent flashlamps and results in a reduction of the magnetic forces exerted on the flashlamps. The 10-mm-bore lamps are connected to four energy storage banks with $87 \mu \mathrm{F}$ capacitance and $1050 \mu \mathrm{H}$ inductance each, while the $15 \cdot \mathrm{mm}$-bore lamps are connected to three energy storage banks with $117 \mu \mathrm{F}$ capacitance and $775 \mu \mathrm{H}$ inductance each. Both of these arrangements provide a total input energy of $70 \mathrm{~kJ}$ to the device at $20 \mathrm{kV}$ in a pump pulse of about $0.9 \mathrm{msec}$ duration. The flashlamp characteristics at

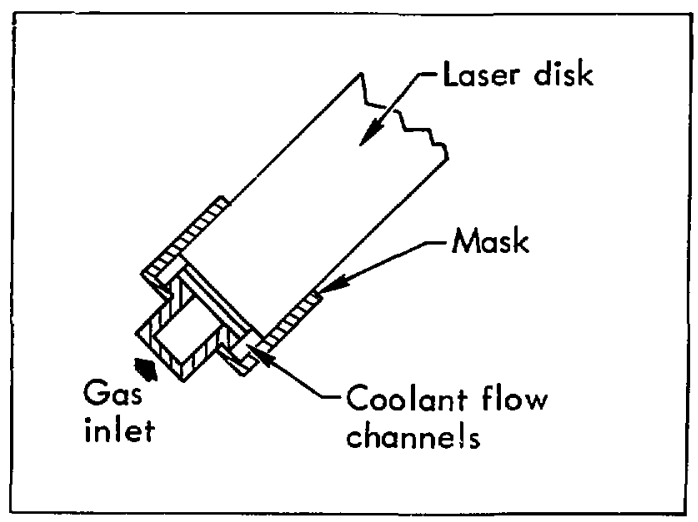

Fig. 17. Disk holders for the $A$ amplifiers of the $1-k J$ chain incorporate flow channels for the nitrogen coolant gas. 
nominal performance levels of the device are summarized in Table 5. Figur 18 shows the different components of the A amplifies. Two different reflectors are used to form the pump cavity; they are shown in Fig. 19. The gain distribution with the cylindrical reflector is in general lower in the center of the disk than at the edge. With the present crenulated reflector the pumplight is directed more toward the center of the disk, resulting in a somewhat higher gain in the center than at the edge of the disk. This allows design of a crenulated reflector that provides almost uniform gain distribution. In addition, the crenulated silver reflector was folind to be appreciably more efficient than the cylindrical gold reflector.

The performance characteristics of the $A$ amplifier are shown in Fig. 20 for different lamp and reflector configurations. These data were taken with 2.5\%-Nd-doped ED-H disks, which were found to be about $15 \%$ more efficient than 3.5\%-Nd-doped ED-2 disks. The best performance was obtained with 15-mm-bore lamps and crenulated silver reflectors, giving a gain curve whose initial slope corresponds to a pumping efficiency of $0.75 \%$. Figure 20 shows that the 15-mm-bore lamps are about $20 \%$ more efficient than the 10-mm-bore lamps and that the crenulated silyer reflector is about $60 \%$ more efficient than the cylindrical gold reflector, which results in an overall improvement of about a factor of 2 over the original design.

\section{$B$ Amplifier}

The six elliptical Jisi: of the $B$ amplifier have dimensions of $23.5 \times 100 \times 184 \mathrm{~mm}$ respectively for the thickness and the minor and major axes. These disks are mounted essentially in the same way as the $A$ disks, described in the previous section. The $B$ ampli-

Table 5. Flashlamp characteristics for the $A$ amplifier

Bore diameter (mm)

Arc length (in.)

Number of lamps per head

Number of lamps connected in series

Number of energy storage banks

Capacitance per bank $(\mu F)$

Inductance per bank $(\mu \mathrm{H})$

Pulse duration $=3 \sqrt{I \bar{C}}$ (msec)

Lamp impedance $(\Omega)$

Free-air explosion energy per lamp (kJ)

Encrgy required for gain of $3.8(\mathrm{~kJ})$

Operating voltage $(\mathrm{kV})$

Loading factor (\%)

Damping factor

Peak current density $\left(\mathrm{kA} / \mathrm{cm}^{2}\right)$

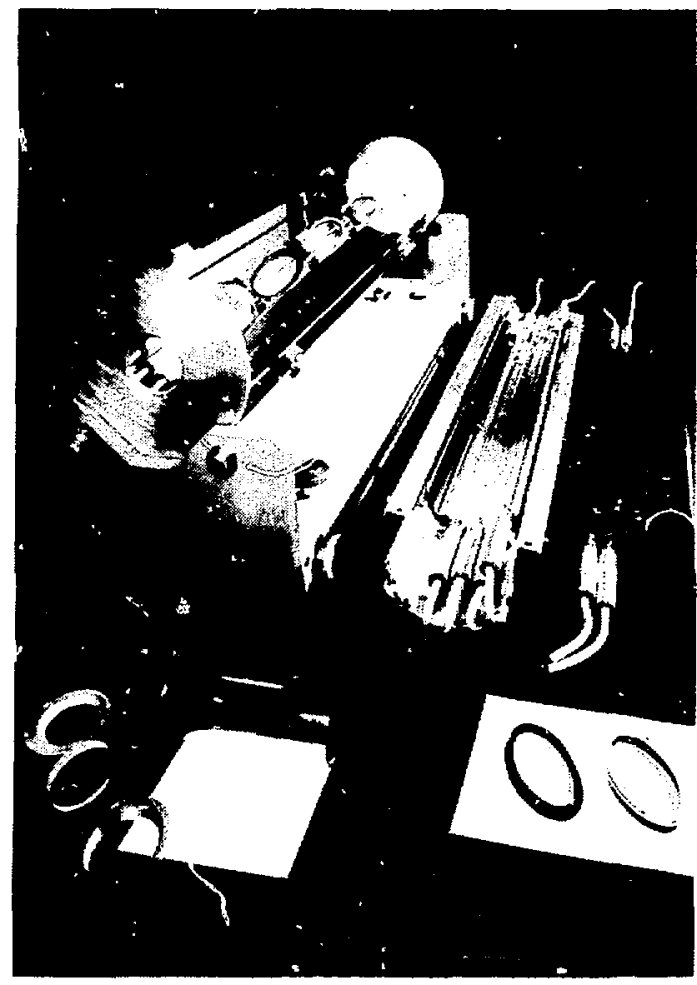

Fig. 18 Components of the $A$ amplifier.

fier provides a useful aperture of $85 \mathrm{~mm}$. Its disk assembly is pumped with either 24 linear flashlamps of $10-\mathrm{mm}$ bore or 20 lamps with $15-\mathrm{mm}$ bore. In both cases two of the 44-in.-arc-length lamps are connected in series for a better match to the $20-\mathrm{kV}$ energy storage banks and to reduce the magnetic forces exerted on the flashlamps. The 10-mm-bore lamps are connected to 12 single-mesh energy storage vanks with $72 \mu \mathrm{F}$ capacitance and $1050 \mu \mathrm{H}$ inductance each, while the $15-\mathrm{mm}$ lamps are connected to 10 banks with $87 \mu \mathrm{F}$ capacitance and $775 \mu \mathrm{H}$ inductance each. In both cases a total input energy of $175 \mathrm{~kJ}$ is delivered to the device at $20 \mathrm{kV}$ in a pump pulse of about $0.8 \mathrm{msec}$ duration. The flashlamp characteristics are summarized in Table 6. Again either a cylindrical gold reflector or a crenulated silver reflector is used to form the pump cavity. Figure 21 shows the $B$ amplifier assembled and installed in the $1-\mathrm{kJ}$ laser system. The amplifier is mounted on an adjustable base, which allows alignment of the amplifier with the laser beam. The HV terminal boxes on either side of the device provide the connections between the heavy cables from the energy storage banks and the insulated flexible leads of the flashlamps. 


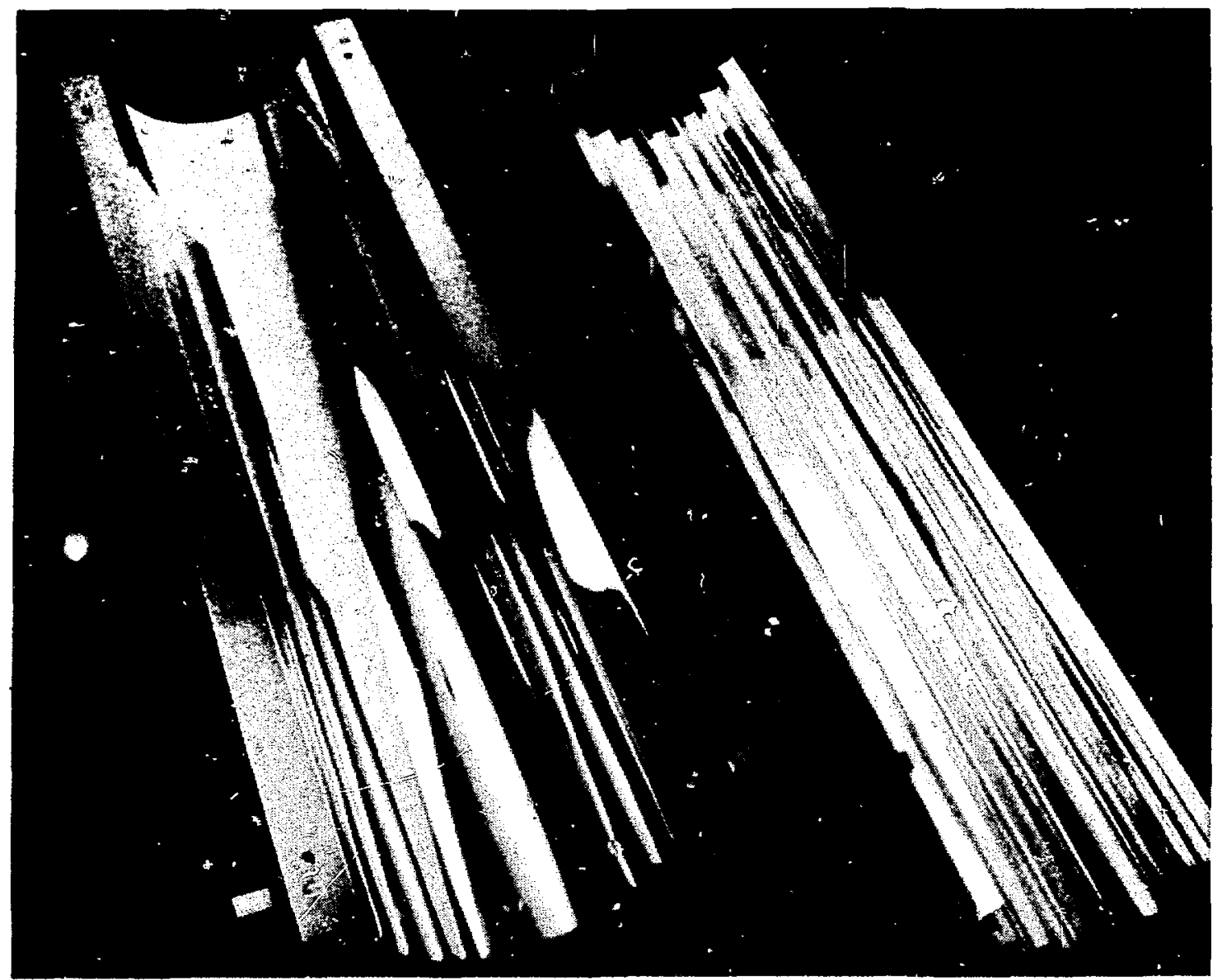

Fig. 19. Two types of reflectors for use in the pump cavity of the $A$ amplifier: cylindrical gold reflector (left), and crenulated silver rellector (right).

Table 6. Flashlamp characteristics for the $B$ amplifier

Bore diameter $(\mathrm{mm})$

Arc iength (in.)

Number of lamps per head

Number of lamps connected in series

Number of energy storage banks

Capacitance per bank ( $\mu F)$

Inductance per bank ( $\mu \mathrm{H})$

Pulse duration $=3 \sqrt{L C}$ (msec)

Lamp impedance $(\Omega)$

Free-air explosion energy per lamp (kJ)

Energy required for gain of $3.8(\mathrm{~kJ})$

Operating voltage ( $\mathrm{kV}$ )

Loading factor (\%)

Damping factor

Peak current density $\left(\mathrm{kA} / \mathrm{cm}^{2}\right)$
Figure 22 shows the $B$ amplifier with one reflector module removed. Here the flashlamps and reflector are exposed for inspection and cleanir g withont handling of the flashlamps. The flashlamps are also incorporated in a module of their own. This modular construction ailows exchange of flashlamps without disturbing the clean environment of the disk assembly cr the alignment of the amplifier. The disk assembly is also exposed for inspection and cleaning.

The performance characteristic of the $B$ amplifier with 10-mm-bore flashlamps is shown in Fig. 23. These data were taken with $2 \%$-Nd-doped ED-2 disks, with and without edge coatings. Without edge coatings, the gain of the device was found to be limited by parasitic oscillations to about $3.5 \mathrm{dE}$, which corresponds to a gain coefficient of $0.047 \mathrm{~cm}^{-1}$. The product of this gain coefficient and the major axis of the disk is about 


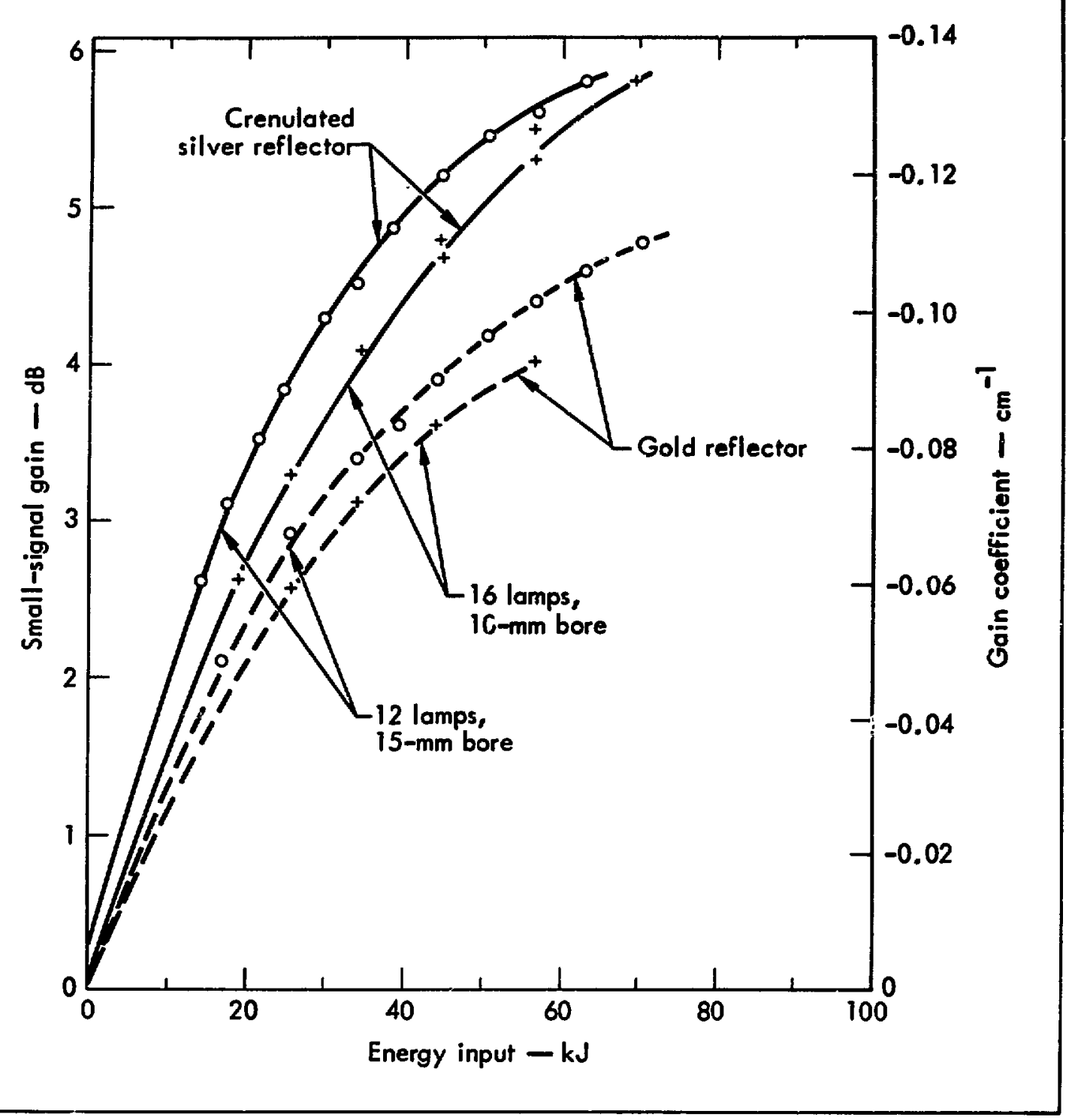

Fig. 20. Performance characteristics of the $A$ ampitier for different reflectors and lamps.

0.86 , which seems to be typical for disks with ground edges. The performance of the crenulated silver reflector shows again about a $50 \%$ improvement in efficiency over the cylindrical gold reflector. The initial slope of the gain curve with the crenulated silver reflector corresponds to a pumping efficiency of about $1.8 \%$. There is still some further improvement expected with the use of 15 -mm-bore flashlamps similar to that observed on the $A$ amplifier, which would bring the pumping efficiency of the $B$ amplifier close to the pumping efficiency typically obtained for solid rods.

\section{$C$ and $D$ Ampifiers}

The design of the $C$ amplifier has been completed and the components are presently in fabrication. The first layout of the $D$ amplifier is also in progress. The $C$ and $D$ modules will consist of three and two disks respectively. The dimensions for the $C$ and $D$ disks are $25 \times 206 \times 392 \mathrm{~mm}$ and $35 \times 306 \times 584 \mathrm{~mm}$, providing clear apertures of 200 and $300 \mathrm{~mm}$ respectively. Both modules are pumped with the same flashlamps as used for the $B$ module, with a bore diameter of $15 \mathrm{~mm}$ 


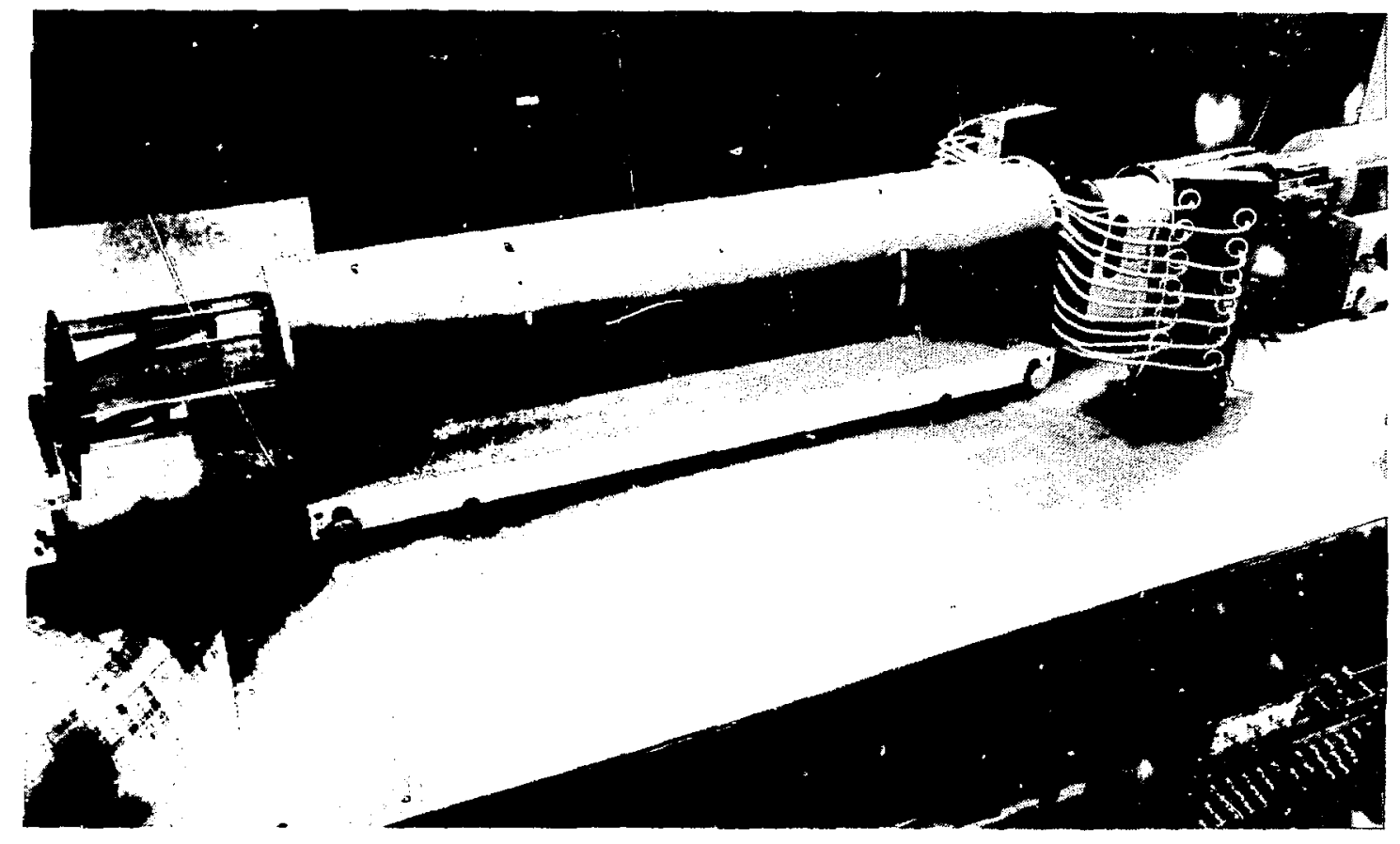

Fig. 21. The $B$ amplifier installed in the $1-k J$ chain.

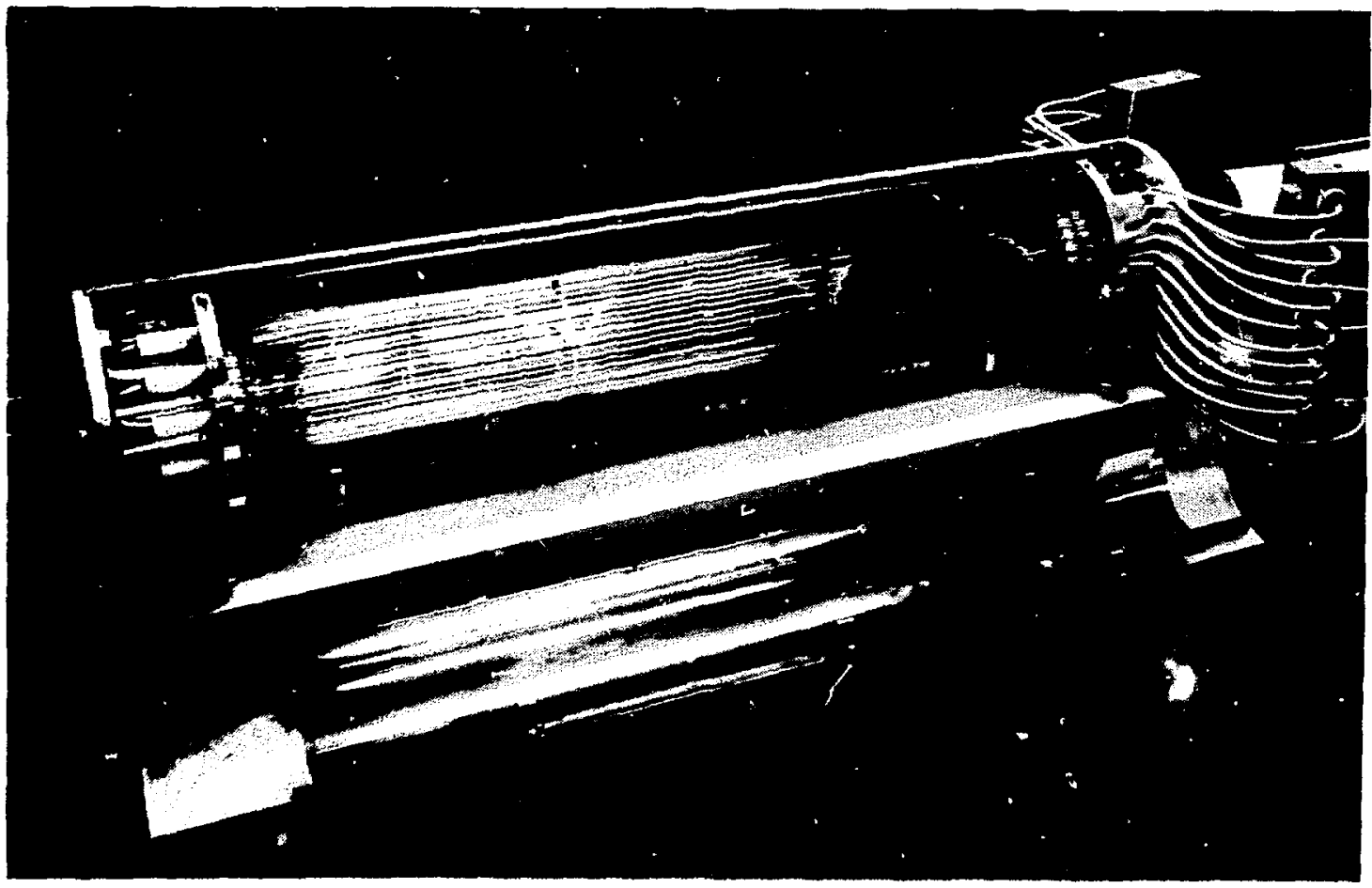

Fig. 22. The $B$ amplifier with one reflector module removed. The three pairs of disis are visible under the flashlamps. 
and an arc length of 44 in. Use of the same flashlamp for the $B, C$, and $D$ amplifiers reduces the number of spares required and allows standardization of the energy storage banks, reducing co:t and complexity. Again two flashlamps are connected in series to reduce the number of circuits required and to reduce the magnetic forces on the lamps. The $C$ module is designed for 32 lamps, the $D$ module for 40 .
The flashlamp modules for the $C$ and $D$ amplifiers are srranged in quarters instead of half shells, for ease in maintenance. In addition the disk holders are no longer machined out of a solid piece but are formed from a heavy band. The cooling flow will be directed through nozzles in the rails onto the faces of the disks instead of passing through the disk holder itself. Initial testing of the $C$ module is anticipated :: February 1974.

\section{PERFORMANCE OF THE PROTOTYPE SYSTEM (CYCLOPS)}

Development of the prototype laser chain for use in the $10-\mathrm{kJ}$ irradiation facility is a many-faceted effort involving detailed component characterization, system integration, and beam propagation studies. A broad range of laser and laser-related technologies existing both at Livermore and other industrial and governınent laboratories will be called upon to perform these tasks.

From the system standpoint, the effort to date has been largely directed toward the development and installation of energy storage and its concomitant delivery control instrumentation. In addition, detailed component integration of the oscillator and preamplifier stages is nearing completion and preliminary integration of the $A$ and $B$ power amplifiers is well

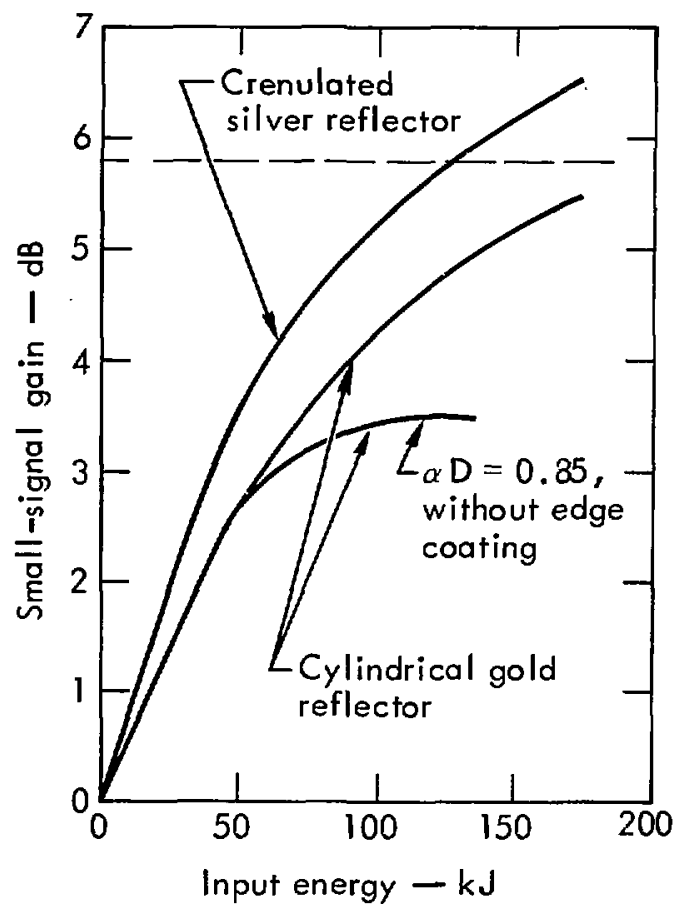

Fig. 23. Measured performance characteristies of the $B$ amplifier with $10-\mathrm{mm}$-bore flashlamps and $2 \%$-Nd-doped ED-2 disks. under way. Some beam diagnosics and power extraction measurements have been performed out through the glass rod preamplifiers. Preliminary results have also been obtained through the first $B$ amplifier stage.

Beam propagation studies will develop in complexity and sophistication as the laser system evolves in time. Much is known about the physical principles that limit the propagation of in:ense pulses in glass. How far we can go in approaching the limit will be critically dependent upon the attention that is given to such details as beam shape, spatial and temporal frequency content, and wavefront distortions. A variety of experimental, analytical, and computational tools already exist for this task, and others will be developed. To date, for example, we have used a multipleexposure camera to photograph our beam at the output of the last glass rod preamplifier. The photographs are then scanned with a microdensitometer and the resulting data are numerically reduced to yield both contour and three-dimensional beam intensity profiles. These data are in turn used as input to computer codes designed to investigate the effects of whole-beam seiffocusing and beam stability.

Detailed characterization of the component parts of the amplifier chain must be made both from the point of view of beam propagation and system reliability. Active distortions in the rod and disk amplifiers, for example, may determine when, in the pump cycle, the optical pulse can propagate without suffering phase front degradation and depolarization. Also, long-term thermal effects will limit the frequency of operation. We are presently pursuing a program to examine such effects through the use of holography and interferometry.

\section{System Integration}

The developmental program for the prototype 1-kJ laser amplifier chain, as outlined in the previous semiannual report. ${ }^{4}$ has progressed through preliminary fabrication and assembly of all components up to and including the first $B$-disk module. In the couse of this work. subsystem development and integration has proceeded apace, the intent being (1) to bring the 


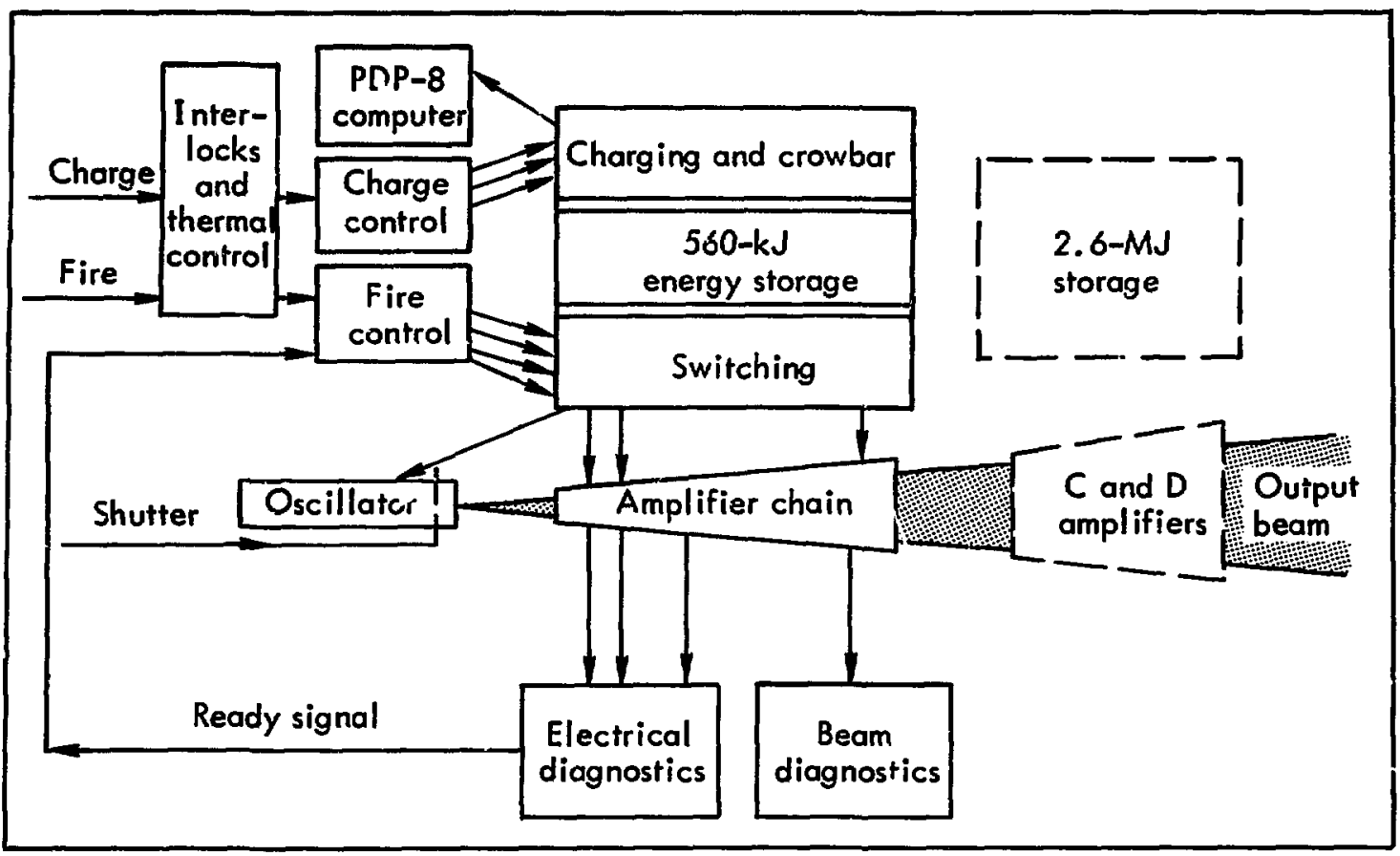

Fig. 24. Block diagrim of the 1-kJ laser chain.

entire chain on-line, and (2) to begin performing meaningful large-beam propagation experiments with this chain by the end of FY 1974. This section of the report is intended to document overall system status as of December 1973, and to report on system performance as of the same date.

Figure 24 is a block diagram of the 1-kJ laser system, which illustrates the interaction among the principsl subsystems: the oscillator, its optical train (with associated beam diagnostics), energy storage, charge control, fire control, oscillator thermal and mechanical support, and safety interlock subsystems. The status of each will be discussed in brief. In this and all subsequent diagrams in this section, dashed lines correspond to hardware to be implemented ciuring the first six months of 1974 , while solid lines indicate working status as of December 1973.

Oscillator and Associated Optical Train. The oscillator is a dye-mode-locked Nd:YAG laser, whose nominal output is a single, 2.5-mJ, 150-psec-duration pulse of $1.06-\mu \mathrm{m}$-wavelength light. A more detailed description of the oscillator and its associated singlepulse-selection gear is given in the previous section of this report. A small sampling of on-line performance statistics was gathered late in 1973, and can be summarized as follows:
- Output pulse train energy: $16.0 \pm 1.0 \mathrm{~mJ}(98$ shots).

- Switchout pulse energy: $2.5 \pm 0.5 \mathrm{~mJ}$ (105 shots).

- Pulse train failure: $4 / 109$.

- Multiple pulse train occurrence: 5/105.

- Switchout malfunction: $17 / 105$.

- Effective usefulness: $83 / 109$ (or 76\%).

These $d^{n}$ ta were gathered over a period of days, at a repetition rate similar to that expected in normal system use (one shot every few minutes). Care was exercised to set flashlamp voltage properly for the dye concentration of the day, ${ }^{*}$ and spark gap cabling was optimized. These data differ from statistical data obtained earlier, in October 1973, during testing of the oscillator sans switchout. Individual inspection of all oscillator components has failed to uncover the source of the discrepancy. Examination of the above results revealed that our principal operational problem is the poor reproducibility and repeatability of the spark gap and laser pulse switchout. A major task to be addressed during the next six months is to improve this switching element of the oscillator.

*The procedure is to determine the threshold voltage for mode locking, and then to operate either 20 or $30 \mathrm{~V}$ higher for the remainder of that day. 


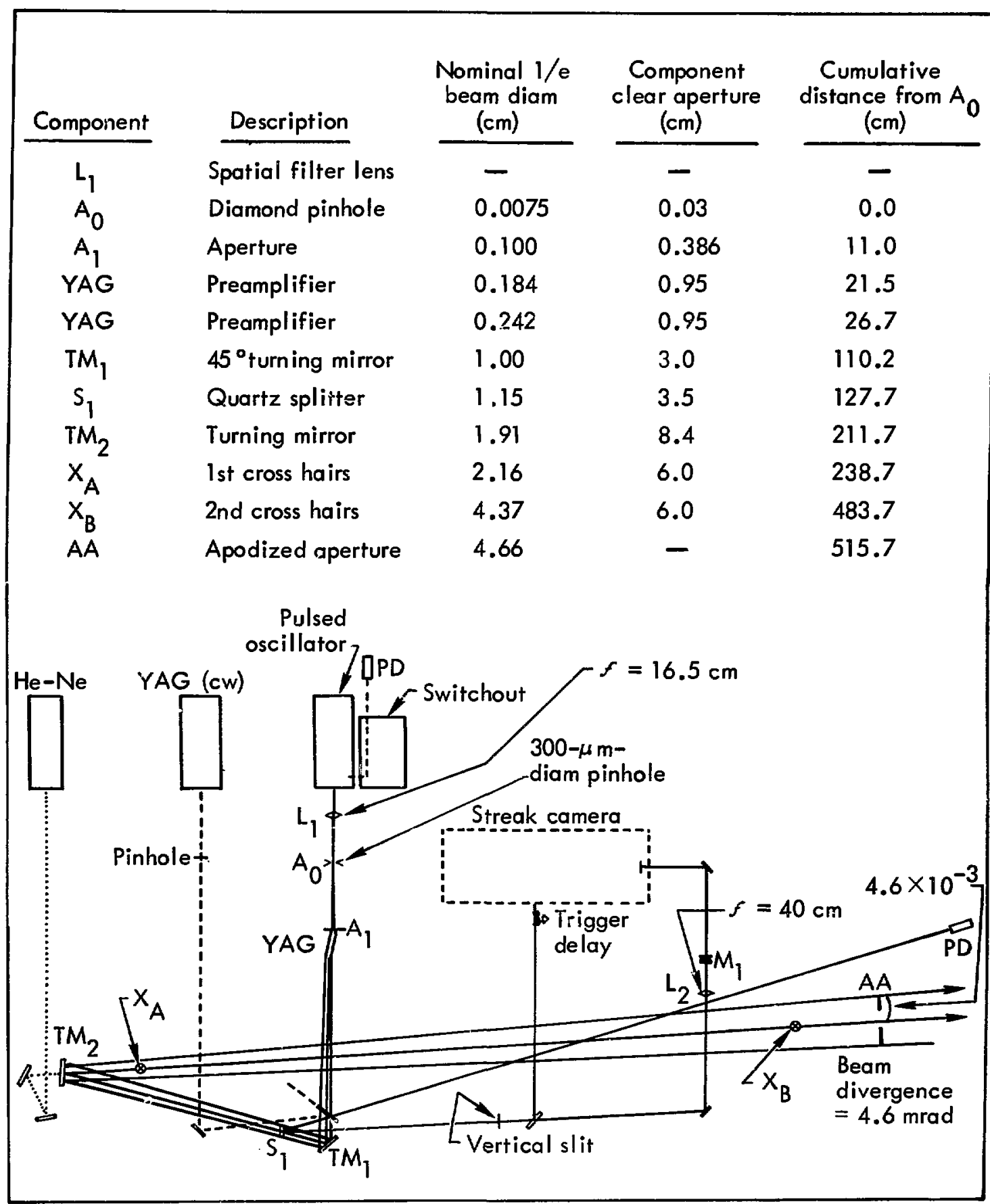

Fig. 25. Beam shaping and early preamplification portion of the optical train for the $1-\mathbf{k J}$ system.

The amplifier train and associated beam diagnostics are shown schematically in Figs. 25 and 26. Figure 25 represents the beam shaping and early preamplification portions of the optical train. The output beam from the oscillator passes through a lens/pinhole combination which serves to filter ${ }^{5}$ high-spatial-frequency noise arising from the switchout Pockels cell and 


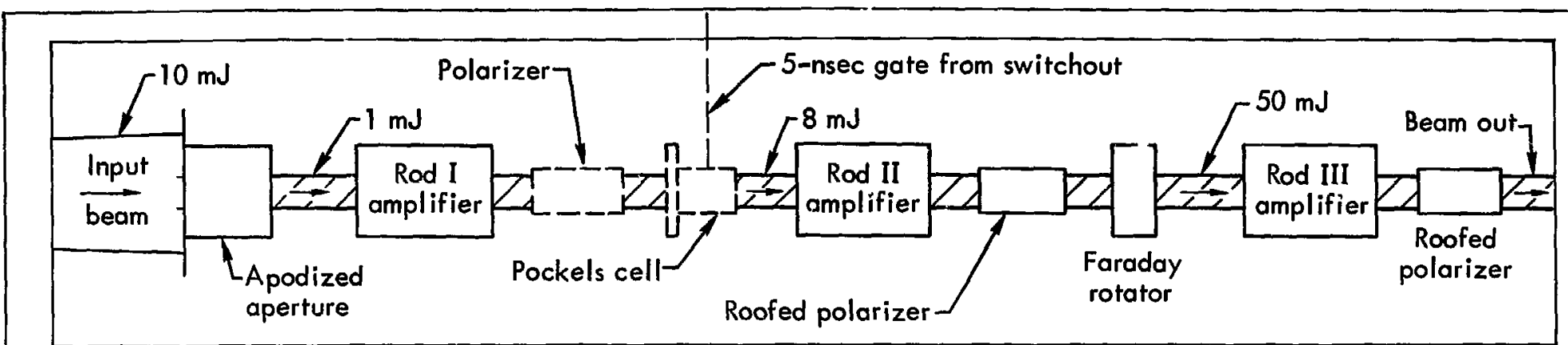

(a) Beam divergence $=1.44 \mathrm{mrad}$.

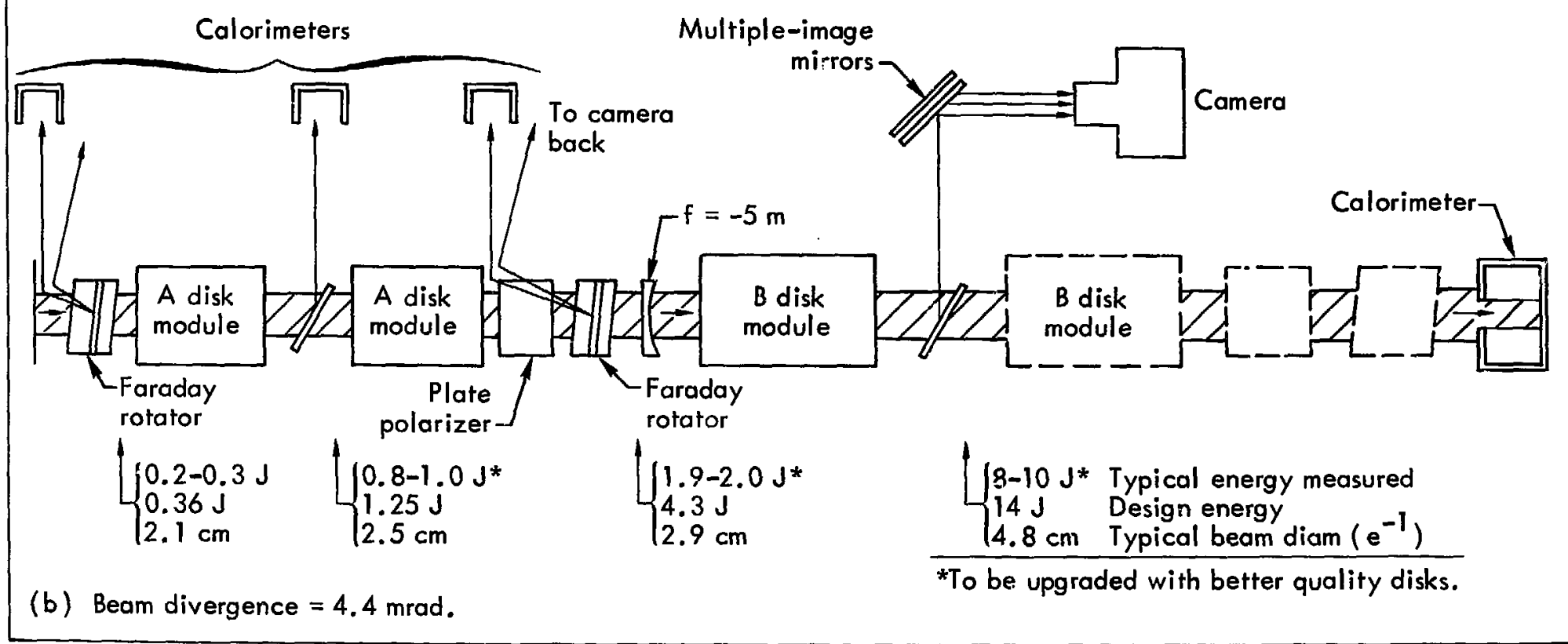

Fig. 26. Schematics of component anrangement in early part of $1-k J$ chain (dashed-line components are not yet in operation). (a) Preamplifier chassis, illustrating present and planned (dashed lines) component placement. (b) Disk module portion of chain; also shown are calorimeter and beam-profile diagnostic units, and typical pulse ensigy and beam diameter at the several intermediate stages. Performance levels are conservative (relative to design goals), and will be upgraded as soon as finished disks are available. 
associated Glan polarizer. The spatial filter also produces, without additional focusing components, ${ }^{*}$ a far-field beam with diameter and divergence compatible with the apodized aperture and 1-in.-clearaperture rod preamplifier described below. (A nominal optical design, ${ }^{6}$ carried out with the aid of the computer code BOBG, ${ }^{7}$ calls for 5-mrad (half-angle) beam divergence from a point $500 \mathrm{~cm}$ in front of the apodized aperture.) The limiting beam aperture for this configuration occurs at the output face of the YAG amplifier, whose location is determined by the tradeoff between pulse gain saturation (too close to pinhole) and beam "barreling," or total internal reflection (too far from pinhole). The location shown ( $21 \mathrm{~cm}$ from the pinhole) was found experimentally to yield a beam completely free of barreling effects; however, the pulse gain of 7.5 (compared to a small-signal gain of 30) represents a heavy price to pay. This amplifier may profitably be replaced during the next half-year by an equivalent-small-signal-gain glass rod amplifier of larger (0.5-in.) clear aperture. The remaining elements in the optical beam are two turning mirrors, which serve for beam alignment with res-ect to the long optical chain following the oscillator and preamplifier optics, and also to admit $\mathrm{He}-\mathrm{Ne} 6328 . \AA$ and $\mathrm{cw}$ YAG $1.06-\mu \mathrm{m}$ auxiliary alignment beams, as shown.

The wedged fused-silica splitter $S_{1}$ provides $3 \%$ samplings of the beam, for streak camera ${ }^{8}$ measurements of the temporal beam characteristics (Fig. 27) and for beam energy measurements by the integrating photodiode (as shown). Not shown is our most recent diagnostic aid, a Tektronix Transient Digitizer, which we have used for real-time display of oscillator train and output pulse characteristics. We estimate that oscillator alignment time is reduced by a factor of 2 to 3 through the use of this instrument.

Figure 28 shows a photograph of the $1-\mathrm{kJ}$ chain's front end. Major boxes enclose the oscillator and switchout assemblies. The YAG preamplifier (in cylindrical housing) is also shown downstream from the oscillator. The front of the preamp chassis, containing the apodized aperture, appears at the far right of the photo. Also shown are the two $\mathrm{cw}$ alignment lasers, He-Ne and Nd:YAG. Turning mirrors and peripheral optics occupy the foreground table space.

Figure 26a represents the component arrangement for the beam preamplifier. The apodized aperture $(A A)$ serves to spatially shape the beam for minimal edge diffraction and maximal filling of the amplifier's clear

*This feature is important, not only to minimize the optical path in glass, but also to avoid inevitable interference fringes stiperposed on the beam by thin lenses. In addition, simplicity of the beam-forming optics is an aid to system alignment.
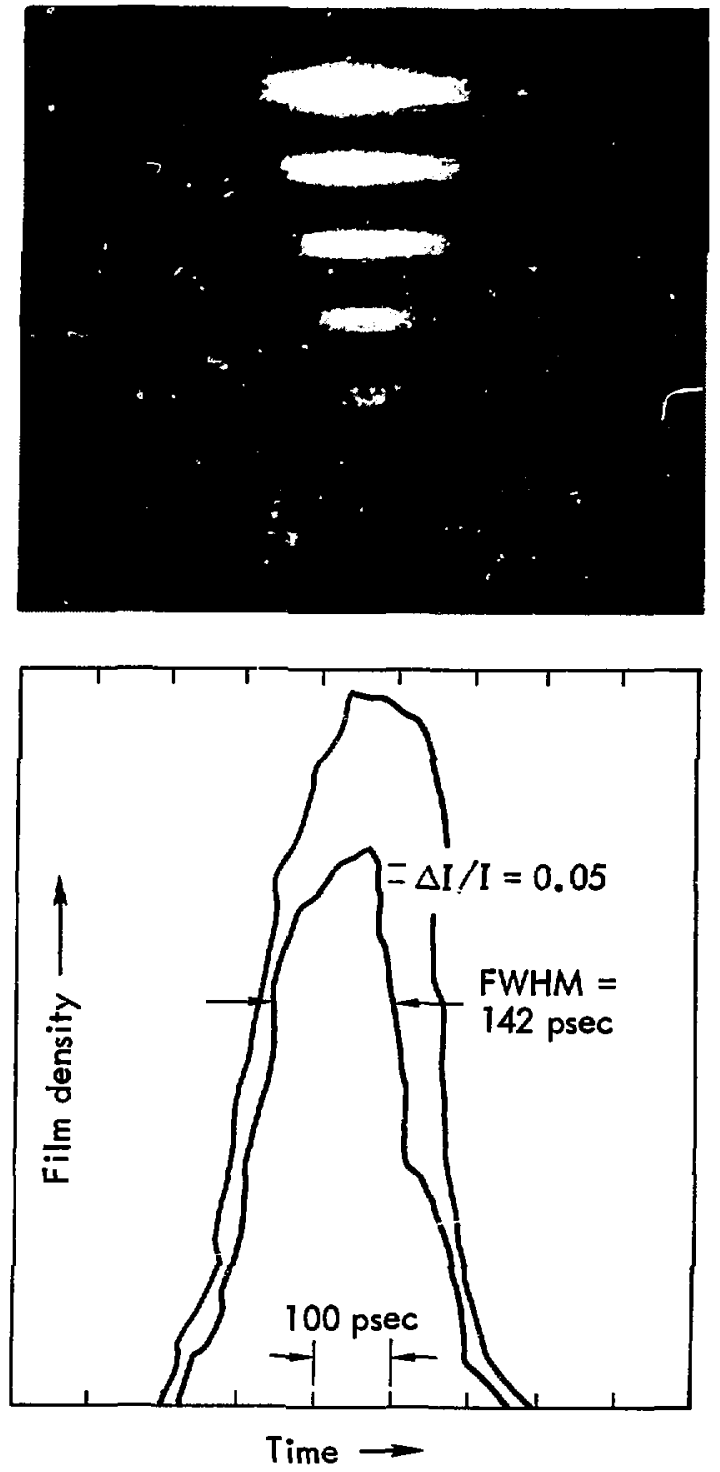

Fig. 27. Typical temporal pulse shape from beam preamplifier section of the chain, as determined with the LLL 10-psec-resolution streak camera. The streak photograph shows exposure intensity vs time, left to right, with a multiple-image mirror pair producing successively wea'ier streaks top to botfom, thus facilitating scanning densitometer analysis, whose results are shown in the graph.

aperture. More specifically, the ideal $A .4$ possesses a transmission function given by $\exp \left[-\left(r / r_{a}\right)^{N}\right]$, where $r_{a}$ is a radius chosen for convenience and $N$, the weighting (WT) of the $A A$, determines the sharpness of beam truncation. The prototype film emulsions we have used to date are all approximately $N=5 W T$ 


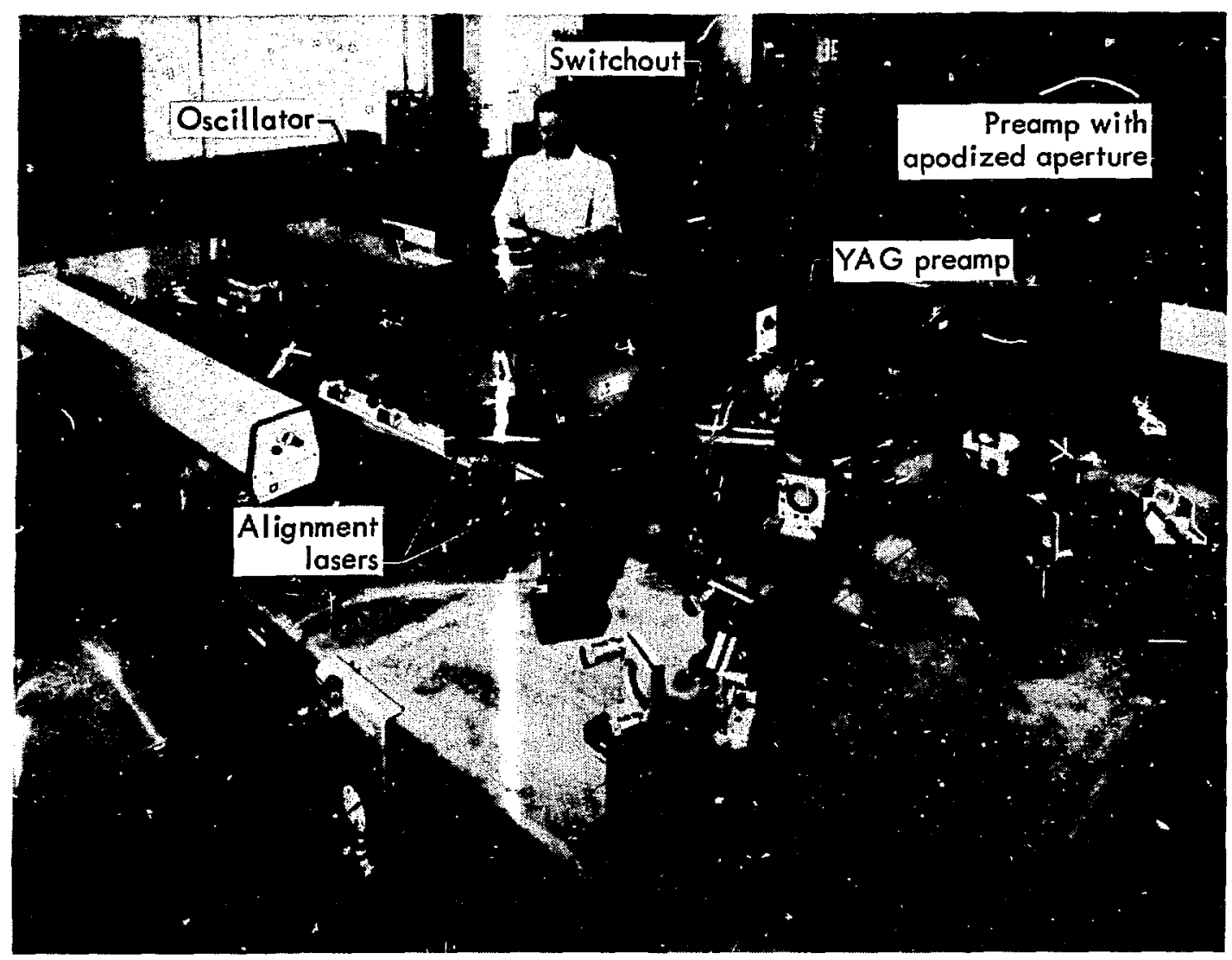

Fig. 28. Components of the early stages of the 1-kJ chain.

apertures, with a cutoff radius of $0.875 \mathrm{~cm}$. Th ey perform qualitatively as expected in operation, although their transmission ( $66 \%$ on center line) is sor lewhat lower than expected. Although they do not ap jear to be affected by the transmitted pulse, they re destroyed by preamp prelasing. Details of the A $\mathrm{A}$ compc ient fabrication are discussed in the next section. Ore of the important tasks to be addressed during the nerit six months is to further characterize these ap rtures in terms of downstream beam diffraction, esf ecially for apertures with $N \geqslant 10 W T$. A second task is o evaluate the performance of metal film and/or crystalline quartz $A A$ 's in on-line system application.

Maximum beam divergence is determined by the limiting clear apertures of the $A A(1.74 \mathrm{~cm})$ and the third amplifier rod's output face $(2.45 \mathrm{~cm}$ diam), together with their $200 \mathrm{~cm}$ relative displacements; this div rgence angle $(1.75 \mathrm{mrad})$ in turn defines the focal len ${ }^{\text {th }}$ of $L_{1}$ and the beam travel distance from dia nond pinhole to $A A$ (Fig. 25).

The 1-by-10-in. rod amplifiers, each with nominal gais. of $9 \mathrm{~dB}$, amplify the shaped beam to approxi- mately $0.5 \mathrm{~J}$ at the preamp output. The polarizer and Faraday rotator combination form the first of several one-way bear isulators for protection against prelasing and amplification of backreflected light. The component arrangement that gives maximum isolation, i.e., amplifier-polarizer-rotator in that order, ${ }^{10}$ has been followed throughout the whole chain. Shown in dashed lines is a Pockels-cell/polarizer combination intended to serve as a short-time-duration (5-nsec) optical gate for two-way beam isolation. The Pockels cell ${ }^{11}$ is driven by the voltage waveform that was applied 25 nsec earlier to the switchout cell at the oscillator. Lack of availability of components with unifornı transmission in the desired clear aperture has delayed installation of this unit.

Figure 26b shows component arrangements and diagnostics for the $A$ and $B$ module amplifiers. The 5-m-focal-length diverging lens inserted between $A 2$ and $B 1$ modules serves to match beam diameters into the $B$ stage (and subsequently $C$ and $D$ stages) in a reasonable chain length. As of the end of 1973, the chain had been operated through the first $B$ amplifier 


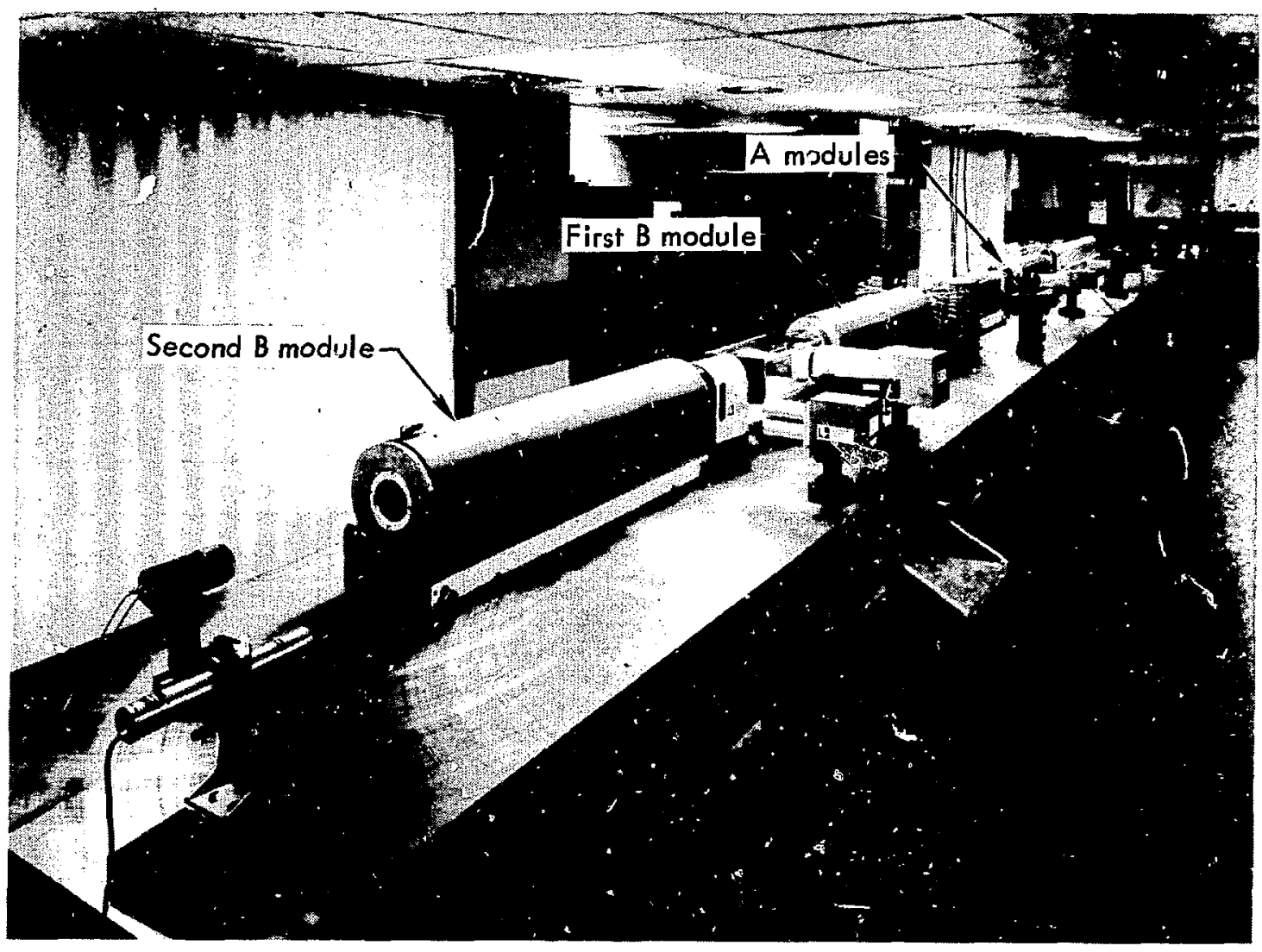

Fig. 29. The major amplifier components operated to date in the 1-kJ chain.

at nominal energy levels as shown in the figure. Disks used in the $A$ and $B$ modules were preliminary production (we are still awaiting delivery of fine-finished and properly edge-coated disks) and beam quality was accordingly suboptimal. Figure 29 is a r'hotograph of the existing system, looking up the chain from the output end of the second $B$ module.

Control Subsystem. Some effort has been devoted to component protection in the event of failure of a part of the system during operation. The following discussion of the firing and charging schemes incorporated into the system during the second half of 1973 emphasizes this asnect of the electrical systems. Their operation is, for the most part, evident from the diagrams.

Figure 30 illustrates the firing logic of the system. The rate generator periodically drives the oscillator flashlamps (after a 10-msec delay) with a 2-sec repetition period, thus ensuring that reasonably steady-state thermal conditions prevail ir the oscillator head. When operation of the laser is aesired, and all capacitor banks are fully charged, the mechanical shutter (solenoid-driven and located inside the oscillator head) is cpened. The oscillator then produces output pulses, but no further activity occurs until the FIRE button is activated. When this is done the next rate generator pulse passes the gate and activates the pulse distribution network. Figure 31 ilustrates the order in which flashlamps and rotator banks are fired by this network. If all Faraday-rotator banks work satisfactorily (we have not yet had an operational failure), a summing circuit generates a READY signal from the individual rotator ready signais generated by current loops at each rotator magnet. This READY signal allows the oscillator flashlamp trigger to bypass the $10-\mathrm{msec}$ delay and fire on schedule $(t=-100 \mu \mathrm{sec}$ in Fig. 31). The optical pulse is thus launched down the chain in near synchronism with maximum fluorescence gain at each amplifier stage. At the close of 1973, amplifier flashlamp current monitors were installed and their outputs were recorded on oscilloscopes. However, no means of aborting the shot in the event of flashlamp failure was incorporated. 


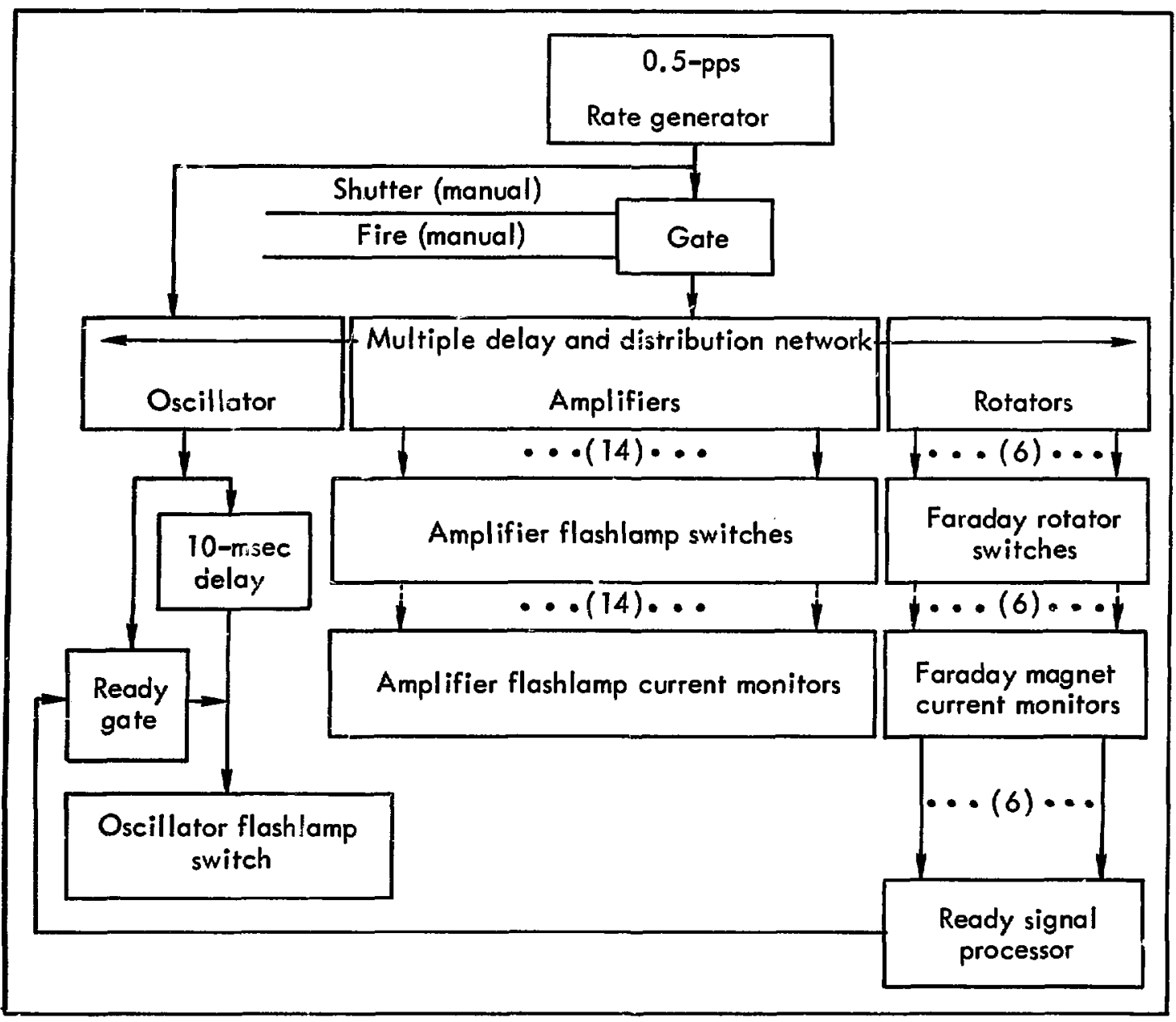

Fig. 30. Block diagram of firing control system for the 1-kJ chain.

Figure 32 illustrates the implementation of the PDP-8 coinputer in controlling capacitor bank voltages during the charging cycle. At the end of 1973, the computer (1) sets bank voltages, (2, monitors for interlock OK, (3) charges capacitors for amplifier flashlamps and rotators, (4) watches for negative slope on all charging curves (and crowbars all banks if such a slope occurs), (5) monitors for "at voltage" signal from all banks (presently this takes approximately a minute), and (6) activates the FIRE bution. The computer can also be cross-coanected out of operation, in which case many red/green lights at the operator console indicate the state of charge readiness of the banks. During the first six months of 1974, the computer will take over much of the record keeping that is presently done by hand.
Total energy storage as of December 1973 was sufficient to operate the syst $\mathrm{Em}$ through the second $B$ module.

\section{Beam Propagation}

Because of the essential need for a spatially wellcharacterized beam our efforts to date have been largely directed at the problem of providing a spatially clean beam for input to the disk power amplifiers. Modulation resulting from diffraction and multiple beam interference effects has been substantially eliminated in the preamplifier stages through the use of a spatial filter and simplified optical geometry. The impetus for this effort is the hope that a significant increase in the threshold for the onset of small-scale beam breakup can be attained. 


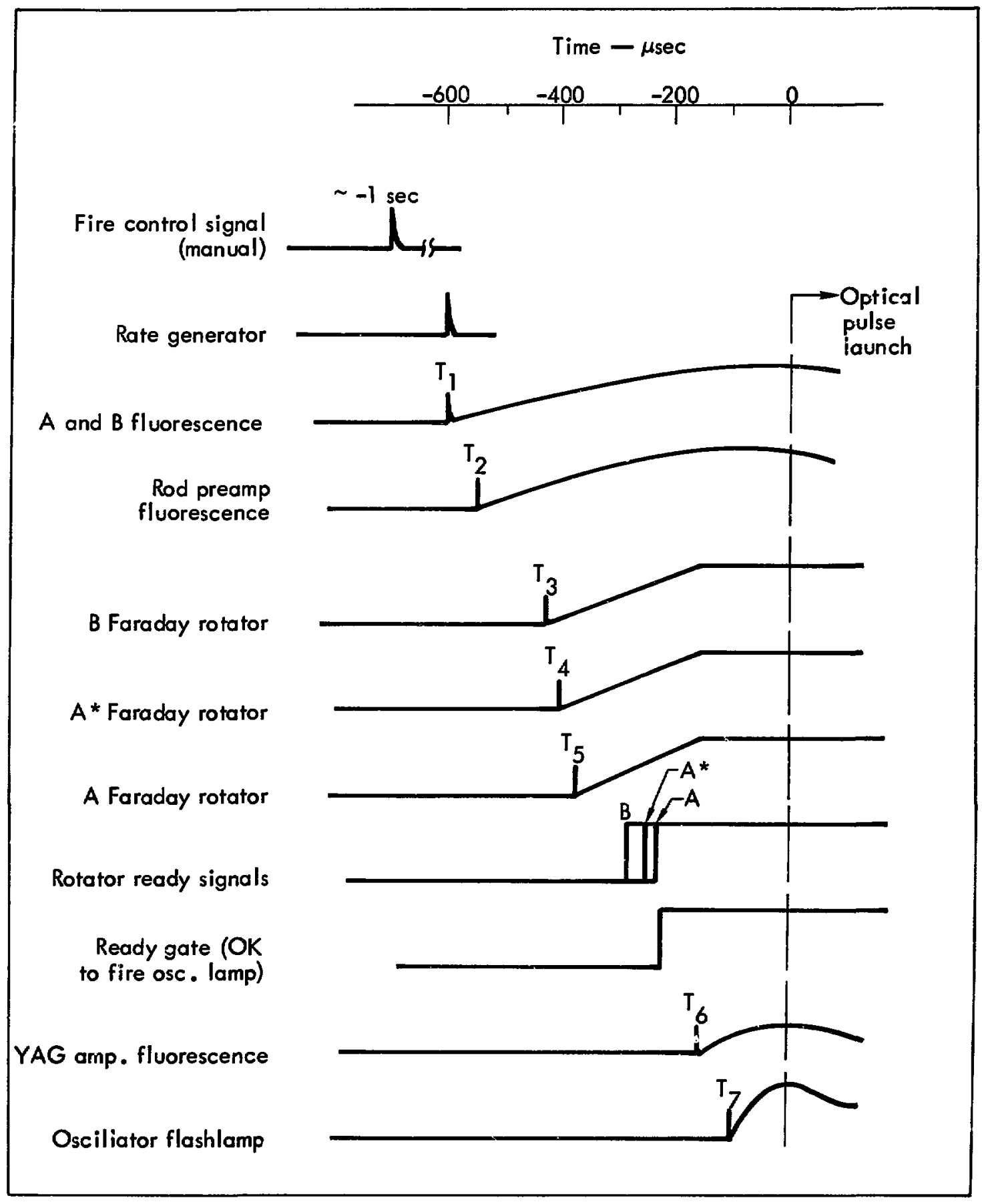

Fig. 31. Firing sequence for the 1-kJ chain. Trigger signals $T_{1}-T_{3}, T_{5}$, and $T_{7}$ initiate flashlamp currents, and signals $T_{3}-T_{5}$ initinte current in the Faraday rotator magnets. Rotator ready signals are generated by pickup loops and integrating networks at each magnet, and the ready gate is generated by scanning individual rotator ready signals. 


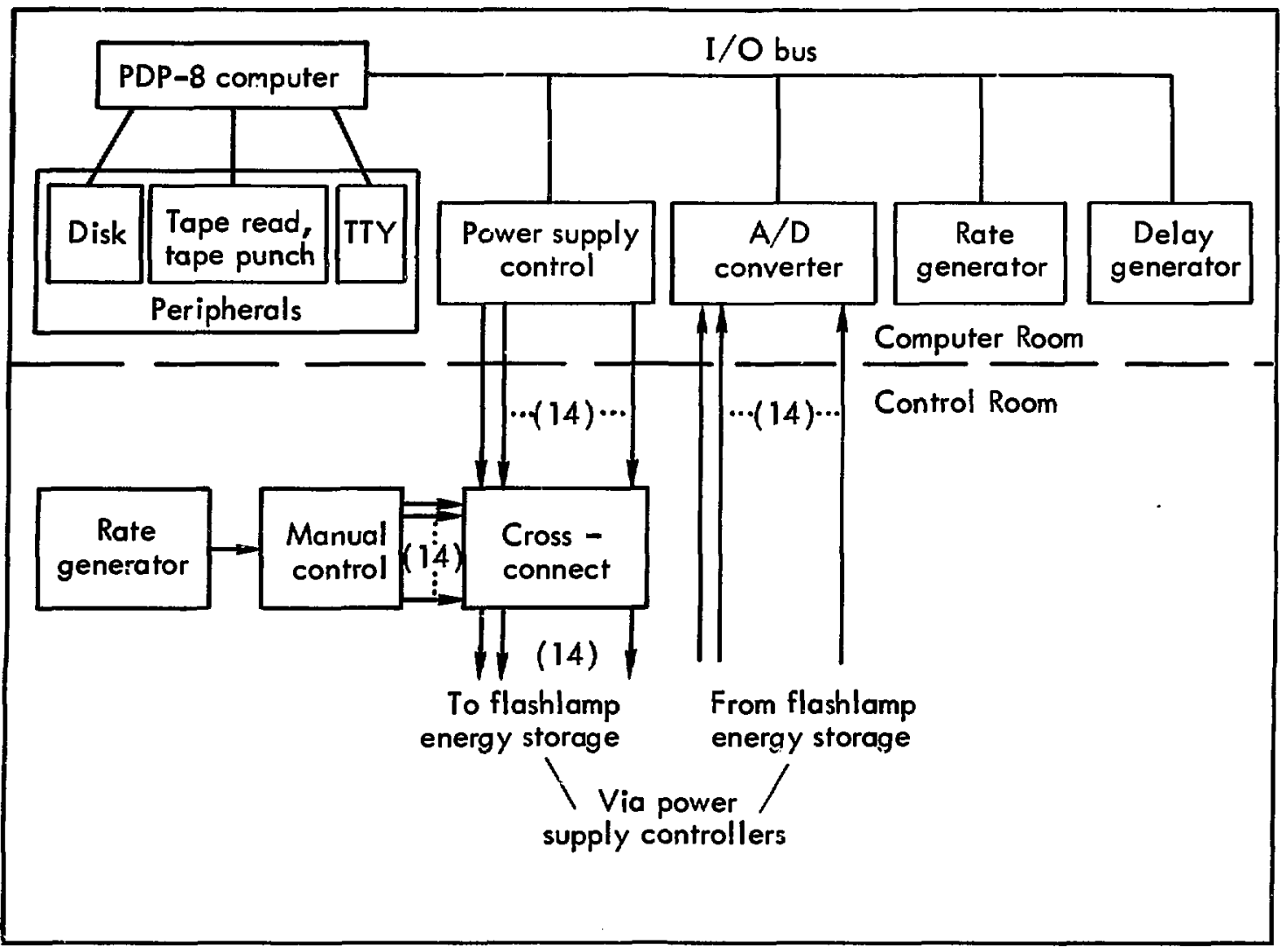

Fig. 32. PDP-8 compuier control of capacitor bank voltages during the charging cycle for the 1-kJ chain.

Figure 33 is a schematic of the propagation path from the oscillator through the apodized aperture. In brief, the near-Gaussian output from the oscillator is spatially filtered with a pinhole aperture positioned in the focal plane of a $17-\mathrm{cm}$ lens. The beam expands from the aperture with a haif-angle divergence of $5 \mathrm{mrad}$ as measured to the $e^{-2}$ intensity point. At the apodized aperture the beam is attenuated and the central portion propagates with a half-angle divergence of $1.7 \mathrm{mrad}$. This divergence angle is fixed by the position of the pinhole filter and the point on the aperture where the transmission drops to about $10^{-3}$ of its on-axis value. Also shown in Fig. 33 are nominal values for the spacing of the elements, beam energy, and waist size.

The spatial character of the switched-out pulse from the oscillator is best described as a perturbed Gaussian. The sources of the perturbations have been identified:

1. A ring surrounding the central spot (this is a farfield diffraction pattern arising from truncation of the beam by the intracavity mode-limiting aperture), which is down from the central spot by $e^{-5}$ in intensity.
2. A two-sided flattening of the otherwise circularly symmetric bean profile by the Pockels cell.

3. A one-dimensional set of interference fringes arising from the Glan polarizer immediately following the Pockels cell.

A Polaroid photo of the oscillator output is shown in Fig. 34a. The aforementioned perturbations are clearly in evidence.

The spatial filter in current use has an aperture diameter of $300 \mu \mathrm{m}$. The beam waist, $w_{1}$, at the aperture is about $70 \mu \mathrm{m}$. This filter transmits about $80 \%$ of the incident energy and is efficient in removing spatial frequencies as low as $0.1 w_{1}$.

Work on beam diagnostics is also progressing. Efforts to date, however, have been limited to the use of photographic techniques to examine near-field intensity distributions in the preamplifier stages. Figure 34b is a Polaroid photo of the beam taken at the entrance to the apodized aperture. Figures $34 \mathrm{c}$ and $34 d$ show intensity contours and profiles of this same beam. These were obtained by numerically reducing microdensitometer traces of IZ photographic plates; 


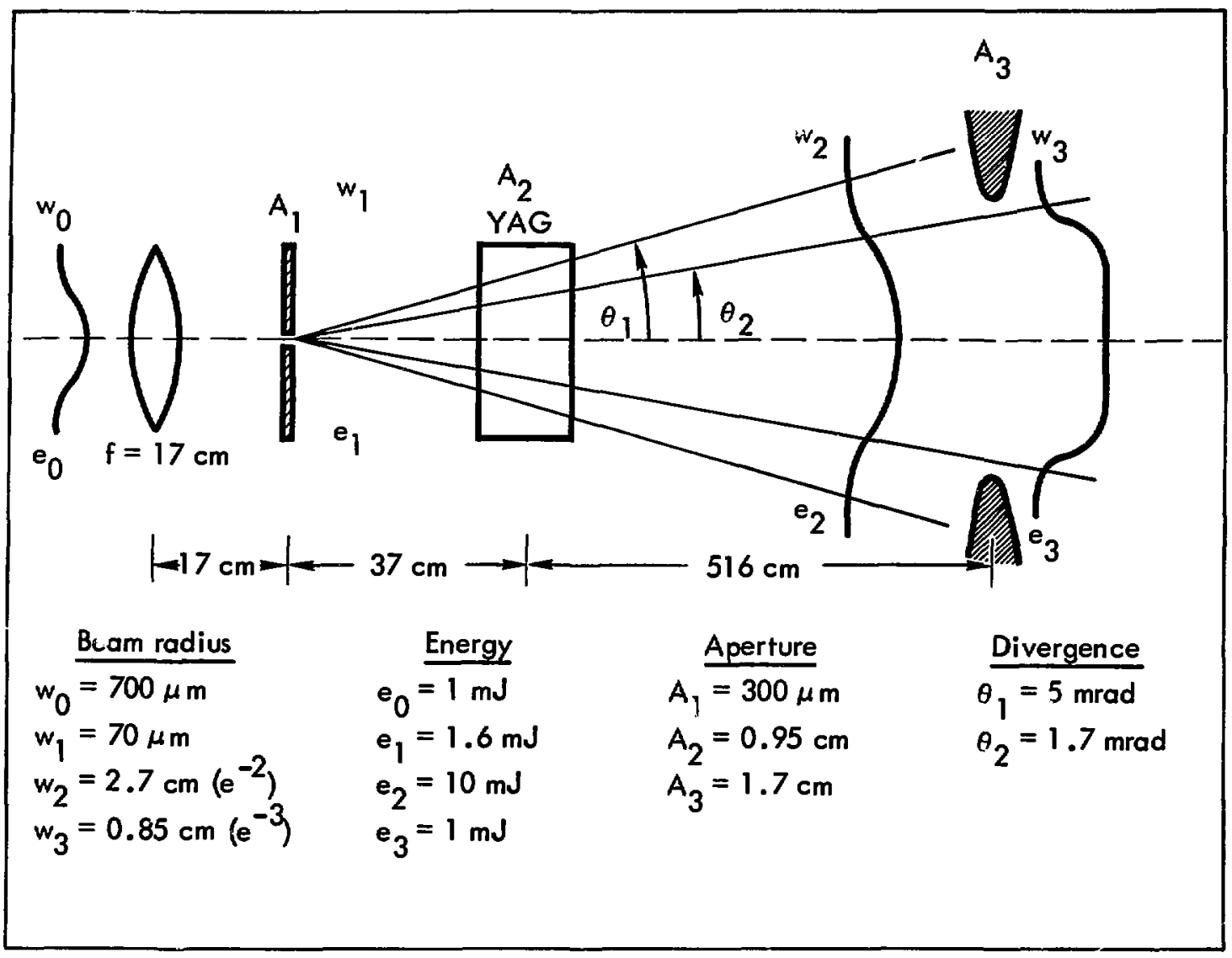

Fig. 33. Beam-shaping optics for the 1-kJ chain.

details of this technique appear below. From these traces it is seen that the beam has retained its Gaussian character. The small asymmetry that is in evidence results from distortion by the Pockels cell, as noted above. For reference, the theoretical transmission function of the apodized aperture $(N=5$, see below $)$ is given. For the optical train geometry presented in Fig. 36 , approximately $11 \%$ of the incident beam passes the aperture.

Figure 35a shows a multiple exposure of the amplified beam at the output of the glass rod preamplifiers. From this photograph it is apparent that the beam has appioximately the desired shape and is essentially free of spatial noise arising from interference effects. The small rings that occasionally appear arise from imperfectioss and dust particles on the surface of the rods.

Figures 35b through 35d again show microdensitometer profiles of the apodized beam. A notable feature of these traces is the appeaiance of spatial intensity modulation. Examination of this modulation reveals low frequency variations of about $14 \%$ along the top of the beam. High frequency noise of 3-5\% variation is also in evidence.

To date we have not identified the sources of these spatial variations. The $3.5 \%$ modulation may represent instrument noise. Larger variations, for example, may arise from mottling of the photographic plate that forms the apodized aperture. Calibration procedures designed to delineate the sources of noise are currently in progress.

Apodized Apertures. In order to efficiently fill the amplifier apertures without introducing strong diffraction effects, the spatial profile of the input beam must be carefully controlled. ${ }^{12}$ This shaping can be accomplished by an apodized aperture having the following characteristics: (a) a radial transmission pro. file which will convert the input intensity profile to the optimum form for transmission through the rest of the system, (b) a peak-t ransmission-tominimum-transmission ratio of at least $10^{3}$, (c) random 


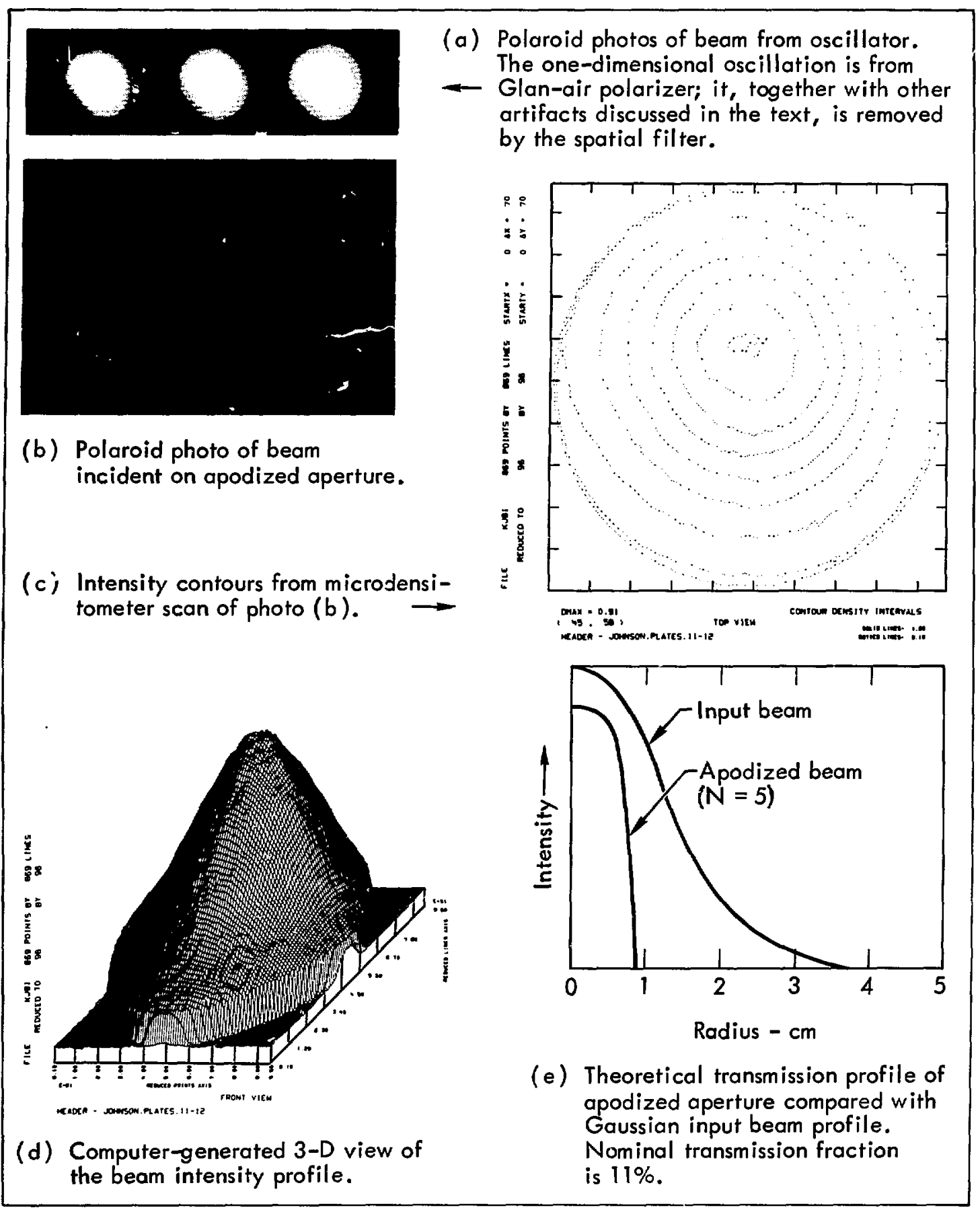

Fig. 34. Beam profiles at several points in the beam-shaping portion of the optical train for the 1-kJ chain.

transmission fluctuations less than the random intensity fluctuations on the input beam, (d) optical quality equal to or better than other system com- ponents, (e) long life at the laser intensity and energy density levels to which it will be exposed.

Various types of apodized apertures being con- 


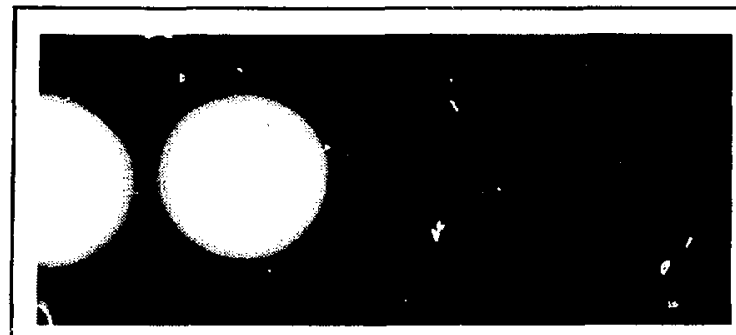

(a) Multiple-image photograph. Each successive spot is reduced by $37 \%$ in intensity.

\section{(b) Computer-generated 3-D view of beam intensity-back view. $\rightarrow$}

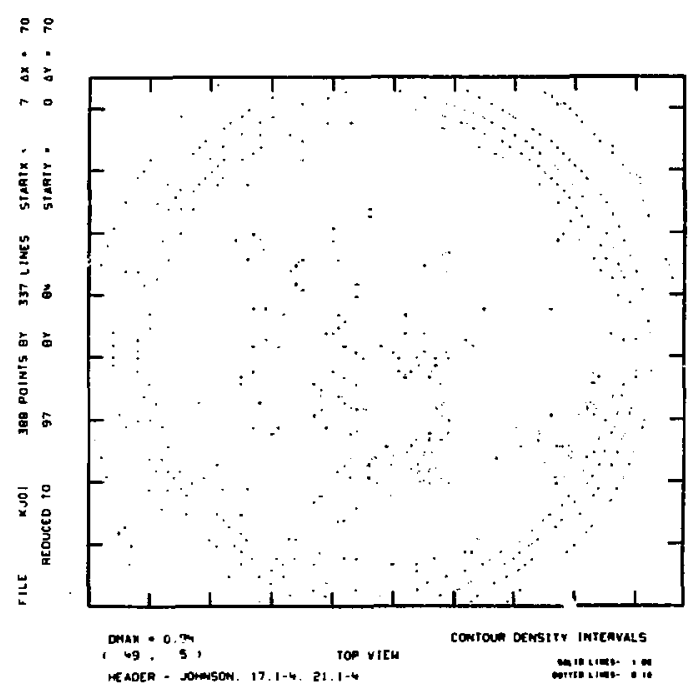

\section{(c) Intensity contours from micro- densitometer scan of beam photo.}
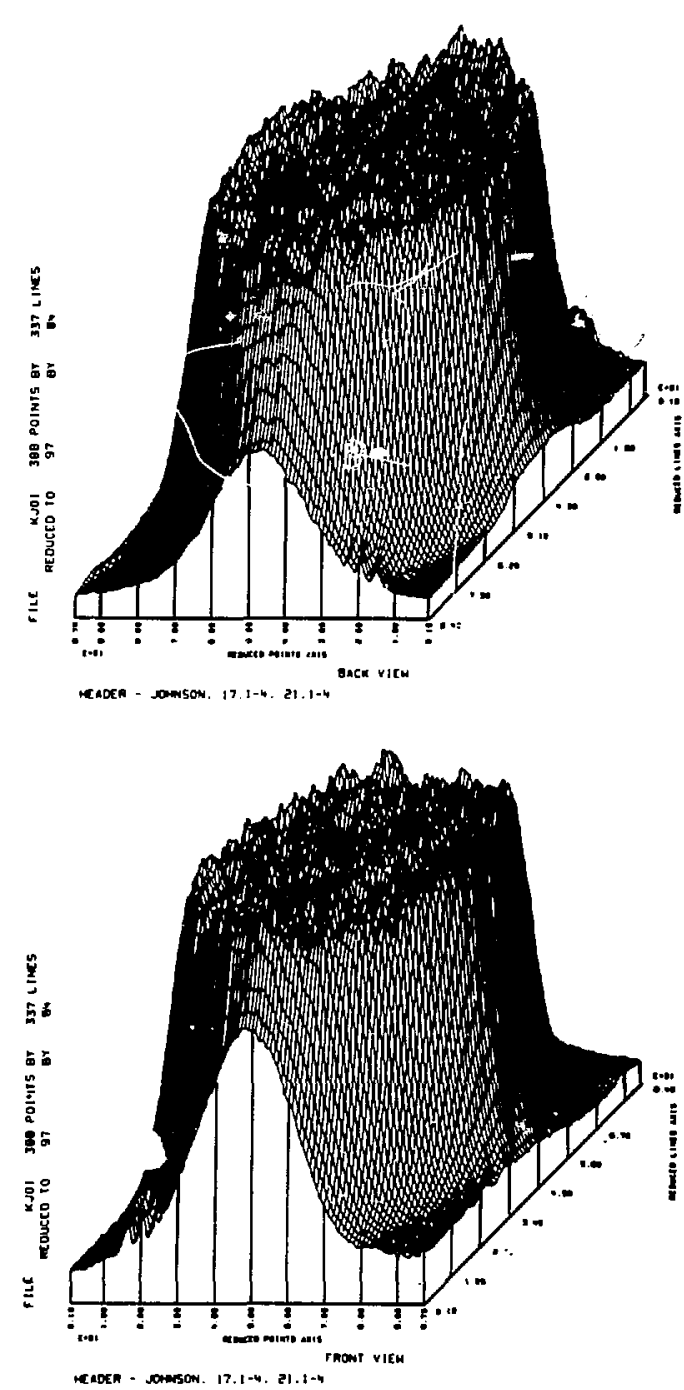

(d) Computer-generated 3-D view of beam intensity-front view.

Fig. 35. Beam intensity profiles at the output end of the glass preamplifiers in the 1-kJ chain.

sideren for this purpose include shaped dye cells, ${ }^{13}$ shaped solid absorbers, fringing-field Pockels cells, ${ }^{14}$ shaped-field Faraday rotators, ${ }^{15}$ metal film coatings, ${ }^{16}$ multilayer dielectric coatings, and photographic plates. Photographic plate and vapor-deposited metal film apertures are presently preferred since they provide considerable flexibility in the choice of transmission profile and can be obtained with reasonably short turnaround times. The photographic plates can withstand energy densities up to $25 \mathrm{~mJ} / \mathrm{cm}^{2}$ without damage.
Both types of aperture are produced by means of a "rotating mask" technique, in which an opaque mask having an angular aperture that varies with distance from the center is placed between a source (i.e., light or metal evaporator) and a rotating plate. The rotating plate, which may be either a photographic plate or a flat glass substrate, receives a varying amount of exposure, depending on the opening of the mask, thus resulting in a graded transmission function that depends on radius only. 


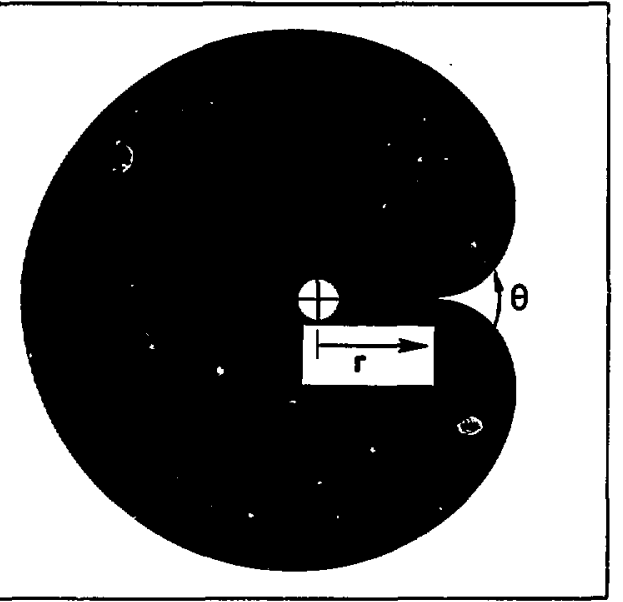

Fig. 36. Opaque mask used for genesating apodizing aperture. Fiducial crosshairs in center are for alignment, and are covered during exposure.

For a given set of conditions, it is possible to generate a mask that will produce arbitrary transmission-vs-radius functions. The fabrication process is calibrated by starting with a mask that has a known $\theta$-vs- $r$ characteristic. The purpose of this calibration is to obtain a specific functional relationship $\theta(T)$ between $T$, the transmission of the final device, and $\theta$, the angle opening of the mask. This relationship is sufficient to characterize the entire fabrication process, provided that the process is exactly duplicated on future runs. For a final mask, the combination of a specified transmission-vs-radius characteristic $T(r)$ and $\theta(T)$ from the calibration determines $\theta(r)$ for the mask.

One example of such a mask is shown in Fig. 36. Note that the angle opening of the mask is a smoothly varying function of radius, starting from zero near the center and gradually increasing to $360^{\circ}$ at a specified maximum radius. The small circle with fiducial crosshair in the center is used for alignment purposes and is covered during exposure.

A typical photographic aperture is shown in Fig. 37. Also shown in the figure is the optical density distribution across the plate diameter. The transmission of the plate is given by

$$
T=10^{-D} ;
$$

for the plate shown, $T(r) \approx \exp \left[-\left(r / r_{0}\right)^{5}\right]$, where $r_{0} \approx$ $7 \mathrm{~mm}$. Spatial distributions of this general form have been demonstrated to be an optimum shape for propagation in laser amplifier chains. ${ }^{12}$ The optical ciensity of $\sim 5$ achieved at the edg $\epsilon$ of the plate reduces

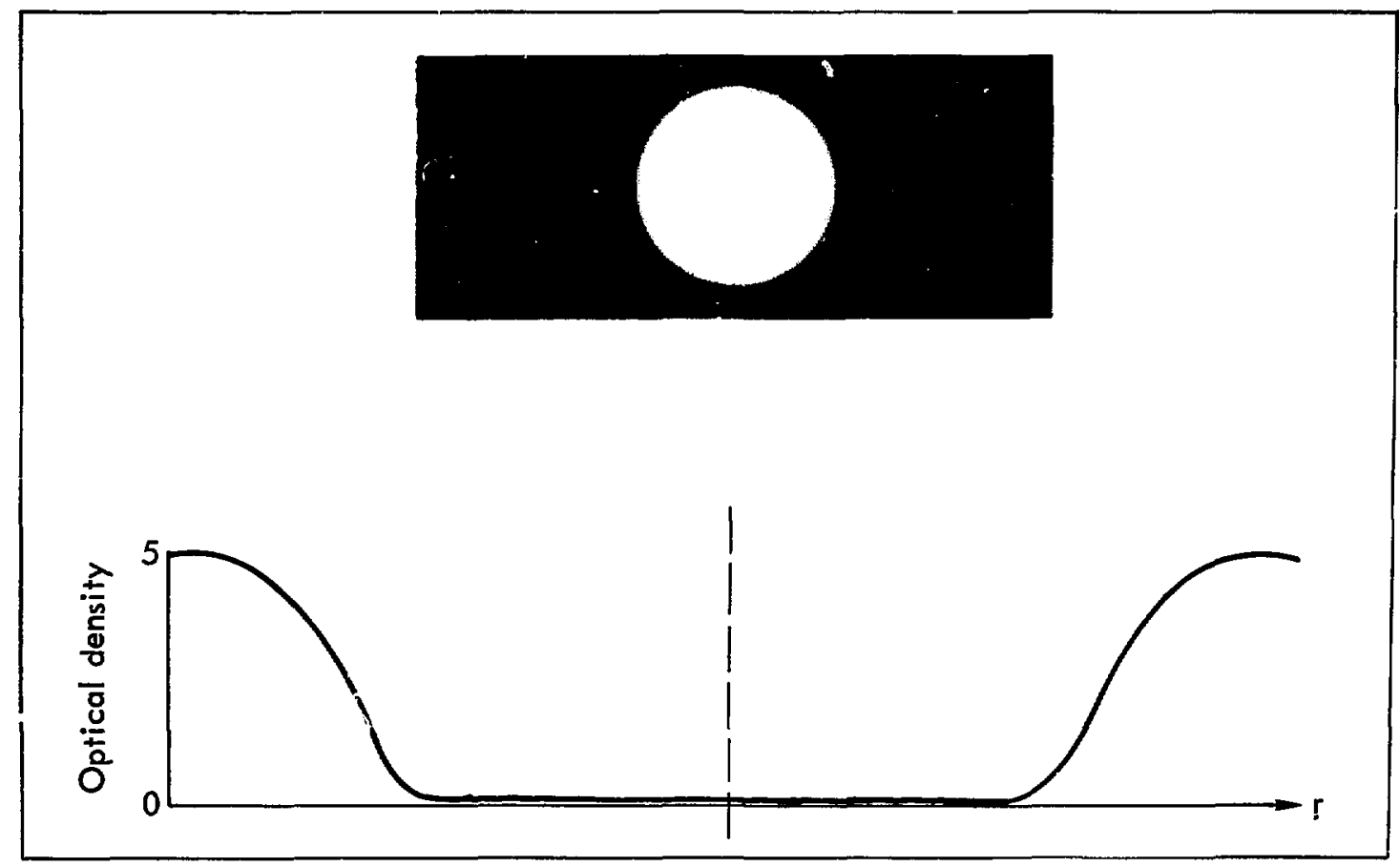

Fig. 37. Example of photographic-plate apodizing aperture. Upper tigure is a plioto of a sample plate, and below is an enlarged microdensitomet $\mathrm{g}$ scan taken across a diameter of the plate. 
the intensity at the edge of the beam by about five orders of magnitude compared to the center.

We have fabricated a number of apodized aperture plates using the "rotating mask" technique described above. Photographic plates, such as the example shown in Fig. 37, are mounted in a cell containing inciexmatching fluid. Figure 38 shows a schematic of a cell that is currently in use. The transmitted beam profiles indicate that the photographic version of the apodized aperture is performing as expected.

We have also made several apertures with vapordeposited metal film. The vapor deposition process is currently being calibrated in order to generate new masks with the desired $T(r)$ characteristics. These, along with the photographic plates, should satisfy the principal requirement for beam shaping in the $1-\mathrm{kJ}$ laser chain.

Numerical Reduction of Photographic Data. To aid investigation of beam intensity distributions, the computer program INTENSITY developed for target intensity measurements with the Long Path Laser was modified. Like the original program, the new version, INTENSITK (where the $K$ is for kilojoule), produces relative and absolute intensity distribution date from photographic density values provided by a PDS microdensitometer. The resulting data can be used to provide contour and three-dimensional representations of the intensity distribution across the laser beam. The flow of data from the photograph to the final displayed laster intensity is shown in Fig. 39.

The main feature found in INTENSITK which differs from the original program is the replacement of a film self-calibration technique by a standard calibration. From previous film calibrations, a photographicdensity-to-relative-intensity conversion curve was constructed by fitting a cubic spline curve to 44 calibration points. This standard conversion curve, shown in Fig. 40, is built into the program.

As density data from an image is processed, the peak density is found and converted to the frak relative intensity through the standard conversion curve. The peak value thus obtained may be less than 1.0 since the peak image density is, in general, less than the maximum density of the standard conversion curve. The peak value is thus used to rescale subsequent relative-intensity values to provide a 0-to-l scale.

Techniques used in the program INTENSITY to provide an absolute intensity scale and relationships of intensity and energy on an area basis have not yet been implemerted for INTENSITK.

For licpresentation of intensity as a function of position (rather than area), the intensity data produced by the program may be processed by other programs to produce the contour, three-dimensional, and crosssectional views shown in Figs. 34 and 35.

Future diagnostics of the beam quality will involve self-film calibration technique to avoid the use of a standard conversion curve and the discriminator.

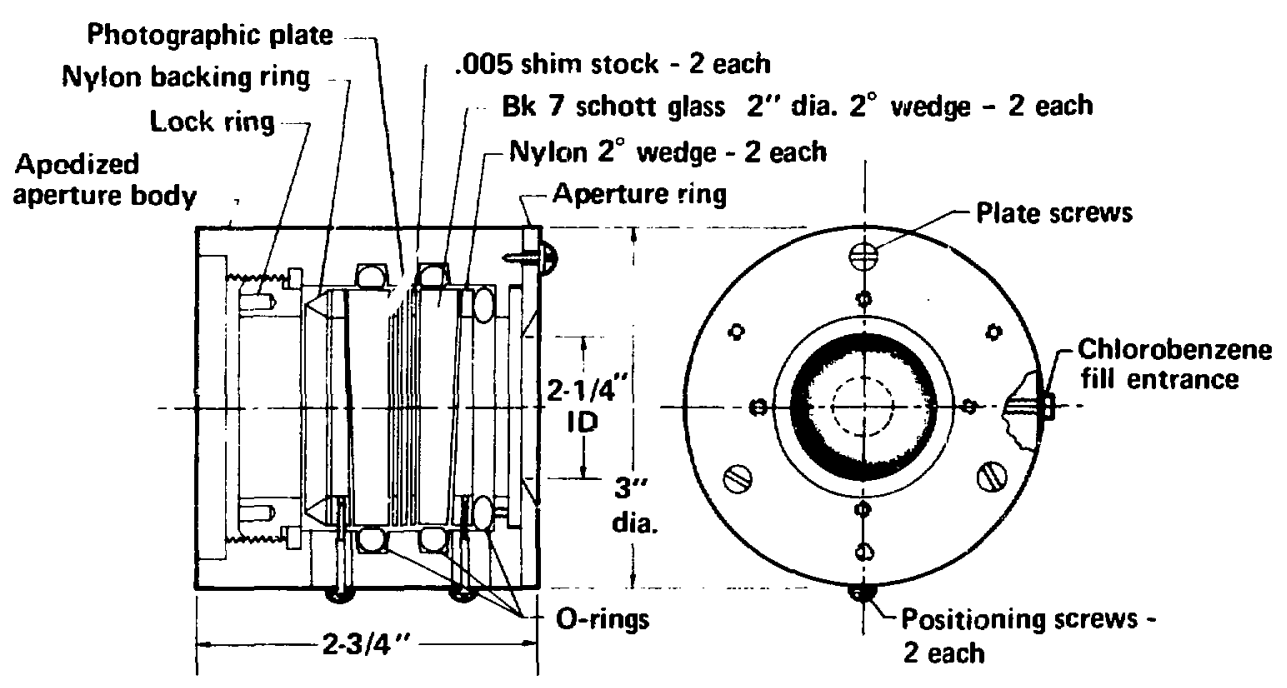

Fig. 38. An apodized aperture assembly currently in use, incorporating a photographic plate similar to the one in Fig. 37 mounted in a cell containing index-matching fluid. 


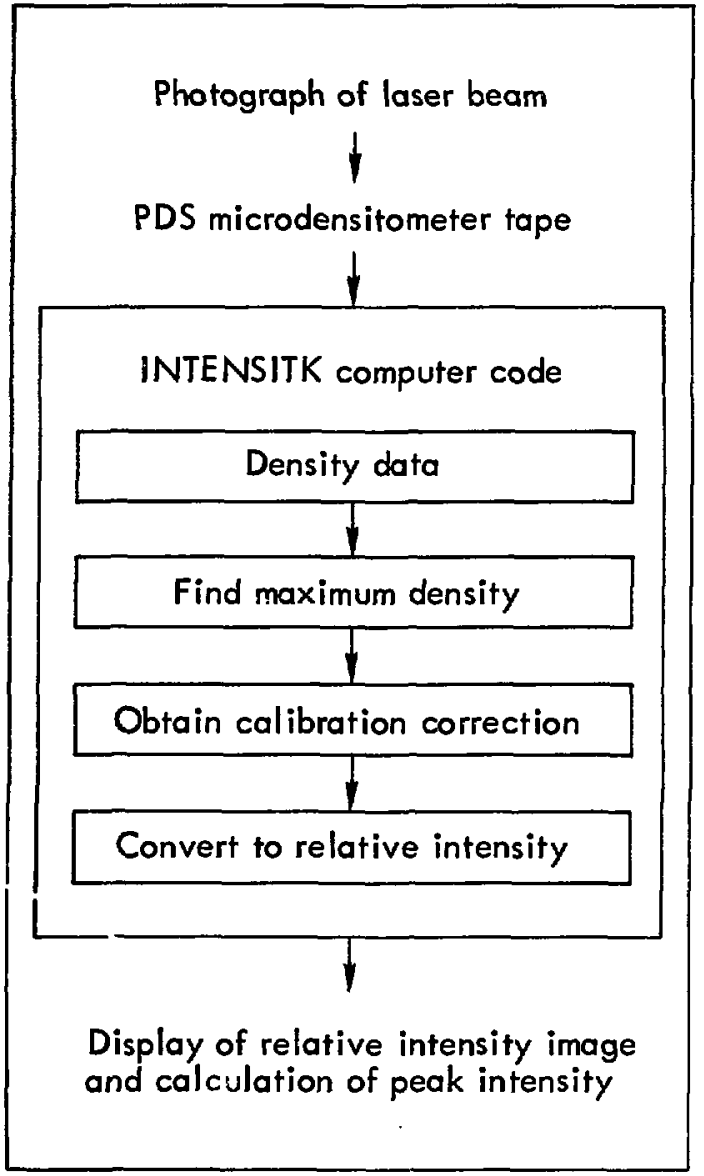

Fig. 39. Flow diagram for the numerical reduction of $1 Z$ photographic plates with the INTENSITK computer code.

\section{Supportive Technological Efforts}

Glass Laser Materials Development. One of the most important properties of $\mathrm{Nd}$-doped laser glass for the development of multikilojoule, subnanosecond lasers is the stimulated-emission cross section, $\sigma_{p}$. A large $\sigma_{p}$ minimizes the amount of glass required for a given small-signal gain, thereby minimizing nonlinear optical effects on the propagating beam. It also provides a lower saturation flux, resulting in increased energy extraction efficiency.

The $\sigma_{p}$ of a laser glass is determined by the chemical composition of the base glass to which $\mathrm{Nd}$ ions are added. It is pertinent to note that the chemical compositions of commerciaily available $\mathrm{Nd}$ laser glasses have not been optimized with respect to producing a high $\sigma_{p}$ while maintaining other desirable optical properties. During the reporting period we have developed an experimental/computational technique to determine all of the relevant radiative properties of Nd laser glass in terms of a few easily measured parameters. Using this model, we have fully characterized the radiative transition probabilities of two important coinmercial Nd glasses (ED-2 and LSG-91H) and several other glasses. This work has been fully reported in the literature ${ }^{17}$ and only a synopsis is given here. Using this computational model, we have begun to explore the effects of compositional variations on $\sigma_{p}$ within the lithium/aluminum-silicate system used in present commercial glasses. This work will be extended to other glass forming systems of potential utility for our application: borates, phosphates, germanates, etc. Studies compieted in the past few months on lithium/ aluminun-silicate glasses have already identified a new glass composition with a $\sigma_{p} 10 \%$ greater than the highest $\tau_{p}$ available in commercial glasses. Moreover, this superior glass is expected to be available in large s.ces at high quality.

The Judd-Ofelt model ${ }^{18,19}$ of ligand-field-induced electric dipole transitions in rare-earth-doped solids has been applied to Nd:glass systems. ${ }^{17}$ According to this model, the squared expectation value of the induced dipole moment between initial level $|(S, L) /\rangle$ and terminal level $\left|\left(S^{\prime}, L^{\prime}\right) Y^{\prime}\right\rangle$ can be expressed as

$$
S\left(J ; J^{\prime}\right)=\sum_{t} \Omega_{t}\left|\left\langle(S, L) J\left\|U^{(t)}\right\|\left(S^{\prime}, L^{\prime}\right) J^{\prime}\right\rangle\right|^{2}
$$

where the $\left|\left\langle\left\|U^{(t)}\right\|\right\rangle\right|^{2}$ quantities are doubly reduced unit tensor operators evaluated in intermediate coupling appropriate to the trivalent rare-earth ions. 18,19 These quantities are independent of the properties of the host material and have been calculated for the $\mathrm{Nd}^{3+}$ ion. ${ }^{20,21}$ The $\Omega_{t}$ parameters $(t=$ $2,4,6)$ may be viewed as phenomenological parameters characterizing to ion-lattice interaction which produces observed optical spectra. The three $\Omega_{t}$ parameters for a given $\mathrm{Nd}$ glass are determined by the following procedure.

The integrated absorbances $\int k(\lambda) d \lambda$ of a number of bands originating on the ground ${ }^{4} I_{9 / 2}$ electronic level are measured (on a dual beam Cary spectrophotometer, for example). These data are converted to dipole strengths, $S_{m}$ :

$$
\int k(\lambda) d \lambda=\frac{8 \pi^{3} e^{2} \bar{\lambda}}{3 \operatorname{ch}(2 J+1)} \frac{1}{n} \rho\left\{\frac{\left(n^{2}+2\right)^{2}}{9}\right\} S_{m}
$$

where $\bar{\lambda}$ is the mean band wavelength, $n=n(\bar{\lambda})$ is the bulk index of refraction at $\bar{\lambda}$, and $\rho$ is the Nd ion 


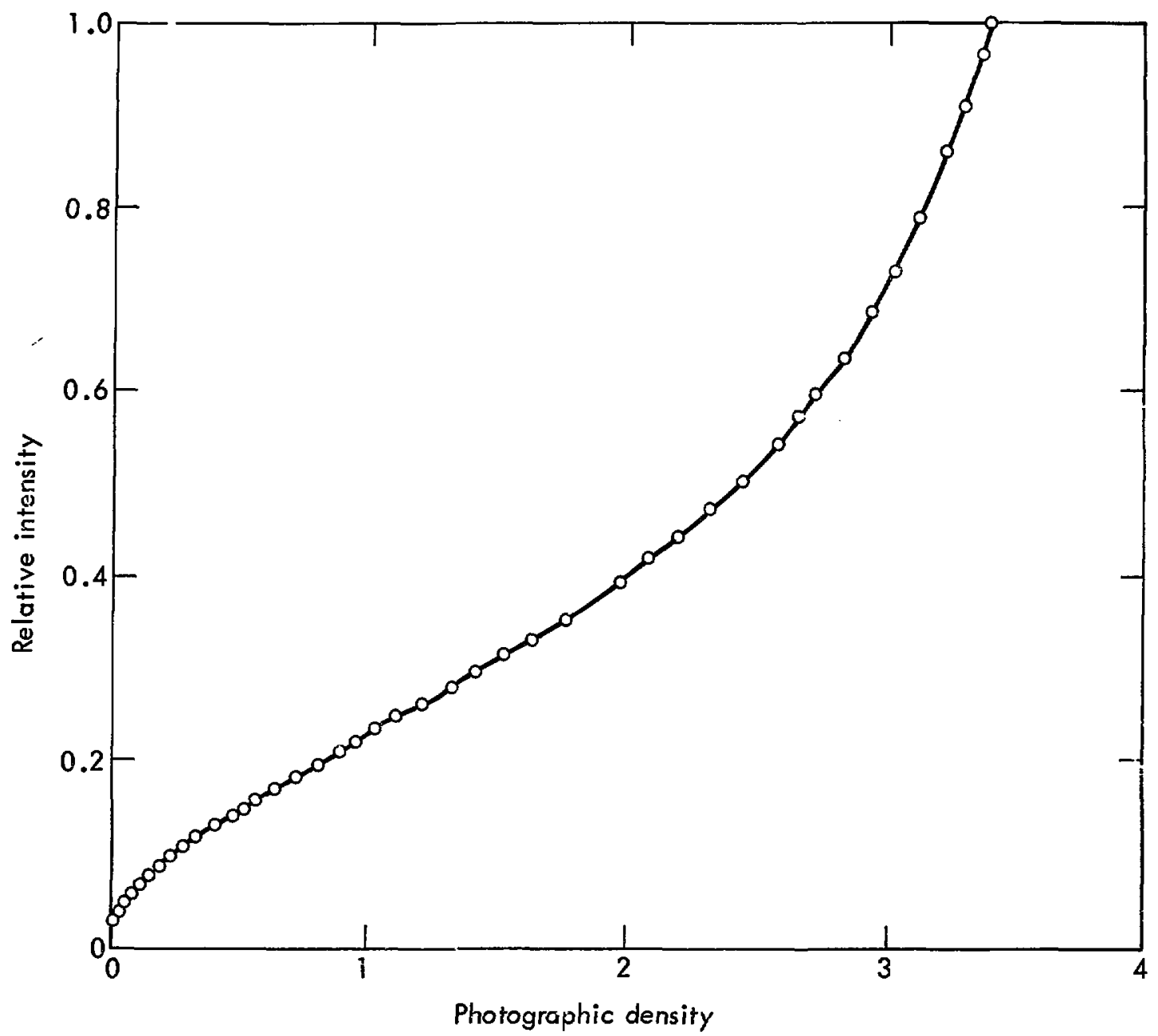

Fig. 40. This standard conversion curve relating photographic density to relative beam intensity is built into the INTENSITK code.

concentration. An equation of the form (1) is written for each of the transitions (bands) measured. The values of the three $\Omega_{t}$ parameters are varied in a leastsquares fitting procedure, to minimize the difference between the sum of squares of measured and calculated dipole strengths. Typically, the root-mean-square error in predicting intensities is about $5 \%$. Once the $\Omega_{t}$ parameters have been determined, all possible radiative transition probabilities between excited levels can be calculated using the expression ${ }^{22}$

$$
\begin{aligned}
A(J ; J)=\frac{64 \pi^{4} e^{2}}{3 h\left(\overline{2} J^{\prime}+1\right)} \frac{n\left\{\left(n^{2}+2\right)^{2}\right\}}{(\bar{\lambda})^{3} 9} \\
\quad \times \sum_{t} \Omega_{t}\left|\left\langle\left(S^{\prime}, L^{\prime}\right) J^{\prime}\left\|U^{(t)}\right\|\left\langle S^{\prime}, L^{\prime}\right) J^{\prime}\right\rangle\right| .
\end{aligned}
$$

The stimulated-emission cross section at $1.06 \mu \mathrm{m}$ for the ${ }^{4} F_{3 / 2} \rightarrow{ }^{4} I_{11 / 2}$ transition follows imrrediately from

$$
\sigma_{p}=\frac{\bar{\lambda})^{2}}{8 \pi c n \Delta \nu} A\left(\left[{ }^{4} F_{3 / 2}\right] ;\left[{ }^{4} I_{11 / 2}\right]\right) .
$$

To evaluate Eq. (4), the fluorescence line shape must be measured (not the absclute fluorescence intensityj to establish the value of $\Delta \nu$ :

$$
\Delta v \equiv \frac{1}{\sigma_{p}} \int \sigma(\nu) d \nu .
$$

The analysis has been carried through for ED-2, LSG-91H, AOLUX3669, and S33 (experimental) laser glasses. The latter two glasses were studied because 
"able 7. Measured and calculated absorption line strengths $\left(S_{m}\right.$ and $\left.S_{c}\right)$ for 3669A, S33, ED-2, LSG-91H, and ED-H laser glasses

Line strengths $\left(10^{-20} \mathrm{~cm}^{2}\right)$ by glass type

\begin{tabular}{|c|c|c|c|c|c|c|c|c|c|c|c|}
\hline \multirow[b]{2}{*}{$\begin{array}{c}\text { Band } \\
\text { o. }\end{array}$} & \multirow[b]{2}{*}{$\bar{\lambda}(\mu)$} & \multicolumn{2}{|c|}{$3669 \mathrm{~A}$} & \multicolumn{2}{|c|}{$\mathbf{s 3 3}$} & \multicolumn{2}{|c|}{ ED-2 } & \multicolumn{2}{|c|}{ LSG-91H } & \multicolumn{2}{|c|}{ ED-H } \\
\hline & & $s_{m}$ & $s_{c}$ & $s_{m}$ & $s_{c}$ & $s_{m}$ & $s_{c}$ & $s_{m}$ & $s_{c}$ & $s_{m}$ & $s_{i}$ \\
\hline 1 & 1.62 & $-{ }^{a}$ & 0.08 & $--^{a}$ & 0.22 & 0.26 & 0.23 & $-{ }^{a}$ & 0.21 & $-{ }^{a}$ & 0.25 \\
\hline 2 & 0.88 & 0.52 & 0.78 & 1.15 & 1.30 & 1.23 & 1.37 & 1.20 & 1.36 & 1.32 & 1.50 \\
\hline 3 & 0.81 & 1.62 & 1.67 & 3.73 & 3.59 & $4.0^{\prime \prime}$ & 3.84 & 3.69 & 3.56 & 4.36 & 4.18 \\
\hline 4 & 0.75 & 1.32 & 1.30 & 3.26 & 3.36 & 3.50 & 3.62 & 3.14 & 3.23 & 3.70 & 3.84 \\
\hline 5 & 0.68 & 0.10 & 0.10 & 0.22 & 0.24 & 0.31 & 0.25 & 0.18 & 0.23 & 0.24 & 0.27 \\
\hline 6 & 0.63 & $-{ }^{a}$ & 0.03 & 0.06 & 0.06 & 0.068 & 0.066 & 0.04 & 0.06 & 0.06 & 0.07 \\
\hline 7 & 0.58 & 5.61 & 5.62 & 6.20 & 6.20 & 6.31 & 6.31 & 6.17 & 6.17 & 6.41 & 6.42 \\
\hline 8 & 0.53 & 1.22 & 1.12 & 1.83 & 1.83 & 1.90 & 1.92 & 1.90 & 1.86 & 2.21 & 2.12 \\
\hline 9 & 0.47 & 0.25 & 0.18 & 0.42 & 0.35 & 0.45 & 0.37 & 0.46 & 0.36 & 0.62 & 0.43 \\
\hline 10 & 0.43 & 0.12 & 0.11 & 0.15 & 0.19 & 0.13 & 0.20 & 0.15 & 0.20 & 0.16 & 0.23 \\
\hline 11 & 0.35 & 1.81 & 1.73 & 2.83 & 2.82 & $-^{b}$ & 2.97 & $--^{c}$ & 2.95 & -0 & 3.24 \\
\hline
\end{tabular}

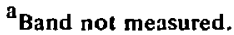

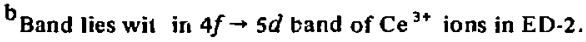

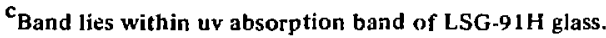

their radiative fluorescence properties have been measured and could be used to check the accuracy of the JO model. Calculated absolute radiative properties, including stimulated-emission cross sections, radia've quantum efficiencies, ${ }^{4} F_{3 / 2}$ radiative lifetime, and fluorescence branching ratios, proved to be accurate to $10 \%$ or better. The results for the four glasses cited are presented in Tables 7-10. Absolute transition probabilities for all 820 possible transitions in ED-2 and LSG-91H glasses have been calculated ${ }^{23}$ but will not be presented here.

Having verified the validity of the model, we examined new glass compositions within the lithium/ aluminum-silicate system. This was a cooperative effort with Owens-Illinois. Five glasses were melted whose chemical compositions were modest variations from the standard ED-2 formulation. Relative fluorescence intensity measurements ${ }^{24}$ suggested that of the five glasses, the glass designated ED-H (to be relabeled ED- 8 for commercial purposes) exhibits a $10 \%$ higher $\sigma_{p}$ than that of ED-2. We have applied the JO model to ED-H and have confirmed the higher cross section. The properties of this glass are included in Tables 7-10.

We exanined the differential spectral absorbance of ED-H and ED-2.3 glass sample materials. The spectral absorbance of a sample $G(\lambda)$ is proportional to $\operatorname{lo} \sigma(\lambda)$ where $\ell$ is the sample thickness, $\rho$ is the $\mathrm{Nd}$ ion concentration, and $\sigma(\lambda)$ is the transition cross section at wavelength $\lambda$. The ED-2.3 sample we tested was
$0.500 \mathrm{~cm}$ thick with $\rho=2.85 \times 10^{20}$ ions $/ \mathrm{cm}^{3}$, and the ED-H sample was $0.434 \mathrm{~cm}$ thick with $3.01 \times 10^{20}$ ions $/ \mathrm{cm}^{3}$. For equal transition cross sections, the spectral absorbance of the ED-2 sample would exceed that of the ED-H sample by a factor $\left.(0.5)^{r} 2.85\right) /(0.434)(3.01)=1.09$ at all wavelengths. In actuality, the differential absorbance is virtually zero at all wavelengths, indicating that ED-H transition c: sections are about 1.09 times larger than those of ED-2.

We have procured $A$-size disks of ED-H glass and made preliminary gain measurements confirming the higher $\sigma_{p}$ for this glass. Casting of larger disks of ED.H for evaluation in the prototype chain is in progress. The yield of this modest effort has been sufficiently productive to warrant extension of the technique to other glass systems in an effort to obtain materially improved glass material for the $10-\mathrm{kJ}$ laser system.

Tahle 8. Intensity parameters for 366yA, S33, ED-2, LSG-91H, and ED-H laser glasses -

Glass type

3669A S33 ED-2 LSG-9HII ED-H

Intensity parameters

$\left(10^{-20} \mathrm{~cm}^{2}\right)$ :

\begin{tabular}{rlllll}
$\Omega_{2}$ & 3.84 & 3.35 & 3.30 & 3.14 & 2.88 \\
$\Omega_{4}$ & 2.95 & 4.47 & 4.68 & 4.79 & 5.53 \\
$\Omega_{h}$ & 1.76 & 4.79 & 5.18 & 4.59 & 5.46 \\
Error (rms, \%) & 5.8 & 3.5 & 3.9 & 4.4 & 5.7 \\
\hline
\end{tabular}


Table 9. The ${ }^{4} F_{3 / 2}$ fluorescence intensities $(A)$ and branching ratios $(\beta)$ for 3669A, S33, ED-2, LSG-91H, and ED-H laser glasses. Subscript $c=$ calculated, $m=$ measured

\begin{tabular}{|c|c|c|c|c|c|c|c|c|c|c|c|c|c|c|c|}
\hline \multirow[b]{2}{*}{$\left(S^{\prime}, L^{\prime}\right) \mathcal{J}^{\prime}$} & \multirow[b]{2}{*}{$\bar{\lambda}(\mu \mathrm{m})$} & \multirow[b]{2}{*}{$\left\langle\mu^{4}\right\rangle$} & \multirow[b]{2}{*}{$\left\langle\mu^{6}\right)$} & \multicolumn{3}{|c|}{$3669 \mathrm{~A}$} & \multicolumn{3}{|c|}{ s33 } & \multicolumn{2}{|l|}{ ED-2 } & \multicolumn{2}{|c|}{ LSG-91H } & \multicolumn{2}{|l|}{ ED-H } \\
\hline & & & & $A\left(\sec ^{-1}\right)$ & $\beta_{c}$ & $\beta_{m}$ & $A\left(\sec ^{-1}\right)$ & $\beta_{c}$ & $\beta_{m}$ & $A\left(\sec ^{-1}\right)$ & ${ }^{\beta}{ }_{C}$ & $A\left(\sec ^{-1}\right)$ & $\beta_{c}$ & $A\left(\sec ^{-1}\right)$ & $\beta_{c}$ \\
\hline${ }^{4} I_{9 / 2}$ & 0.88 & 0.230 & 0.056 & 406 & 0.41 & 0.37 & 963 & 0.38 & 0.36 & 1030 & 0.38 & 1070 & 0.41 & 1170 & 0.38 \\
\hline${ }^{4} I_{11 / 2}$ & 1.06 & 0.142 & 0.407 & 508 & 0.51 & 0.51 & 1289 & 0.51 & 0.53 & 1380 & 0.51 & 1290 & 0.50 & 1600 & 0.52 \\
\hline $4_{1 / 3 / 2}$ & 1.35 & 0.0 & 0.212 & 82 & 0.08 & 0.12 & 250 & 0.10 & 0.11 & 272 & 0.10 & 235 & 0.09 & 300 & 0.10 \\
\hline $4_{I_{13 / 2}}$ & 1.88 & 0.0 & 0.028 & 4 & 0.004 & - & 12 & 0.005 & -- & 12 & 0.004 & 12 & 0.005 & 15 & 0.005 \\
\hline
\end{tabular}

Note: $\left\langle\mu^{t}\right\rangle=\left|\left(\left(^{4} F_{3 / 2}\right)\left\|U^{(t)}\right\|\left(S^{\prime}, L^{\prime}\right) S^{\prime}\right\rangle\right|^{2}$.

Propagation Studies: Small Scale Instabilities. The instability of intense laser beams in the presence of a refractive index nonlinearity is well established and is a fundamental consideration in the design of high-peakpower laser systems. ${ }^{25-27}$ For beams of large aperture with power far above the critical power for selffocusing, this instability leads to the growth of smallscale intensity and phase variations in the beam. The growth rate for such structure has been measured in ED-2 laser glass as a function of intensity and the spatial scale the variations. Calculations based on linearized small-scale instability theory compare favorably with the measured rates. This work represents part of a continuing effort to experimentally check the accuracy of the theories and computational techniques that will be used to optimize the propagation properties of large laser systems for laser fusion research.

The experimental arrangement for measuring the growth of small-scale intensity variations in an unpumped glass sample is shown in Fig. 41. A spatially and temporally smooth input beam enters from the left after its spatial and temporal intensity profiles have been recorded by a multiple-image camera ${ }^{28}$ and a 10-psec-resoiution sireak camera 8,29 respectively. Typical pulses contain 0.25 to $0.75 \mathrm{~J}$ in $150 \mathrm{psec}$ (FWIM) with a $1 / e$ beam diameter of $7 \mathrm{ml}$ 1. The shear plate impresses a sinusoidal intensity $m$ sulation on the beam before it reaches the sample of unpumped ED-2 laser glass, and the intensity profiles of the beam at the sample input and output are imaged ontc the film of the two multiple-image cameras shown. Slightly wedged mirror pairs consisting of one partially reflecting mirror and one totally reflecting mirror gerierate a linear array of spots of successively reduced intensity in each camera. Since the relative intensities of the spots are known, each photograph contains all the information needed for accurately deterinining the response characteristics of the film ${ }^{30}$ and thus for reliably converting from modulations in film density to modulations in intensity.

Table 10. Radiative properties of 3669A, S33, ED-2, LSG-91H, and ED.H laser glasses

\begin{tabular}{|c|c|c|c|c|c|}
\hline & $3669 A$ & $\mathbf{S 3 3}$ & ED-2 & LSG-91H & ED-H \\
\hline Calc. radiative lifetime, $\tau_{\mathrm{rad}}(\mu \mathrm{sec})$ & 1000 & 400 & 372 & 384 & 326 \\
\hline Meas. fluorescence lifetime, $\tau_{f}$ ( $\mu \mathrm{sec}$ ) & 520 & 244 & 310 & 300 & 254 \\
\hline Calc. radiative quantum effic., $n_{\mathcal{c}}$ & 0.52 & 0.61 & 0.83 & 0.78 & 0.78 \\
\hline Measured line wid th $\Delta \tilde{\nu}_{\text {eff }}\left(\mathrm{cm}^{-\mathcal{C}}\right)$ & 296 & 300 & 300 & 310 & 305 \\
\hline $\begin{array}{l}\text { Calc. induced-emission cross section, } \sigma_{p},\left(10^{-20} \mathrm{~cm}^{2}\right) \\
\text { Measured stimulated-emission cro } s: \text { section, }\end{array}$ & 1.2 & 2.8 & 2.9 & 2.6 & 3.2 \\
\hline $\begin{array}{l}a_{p}\left(10^{-30} \mathrm{~cm}^{2}\right) \\
\text { Calc. induced-emission cross section at } \lambda=1.064 \mu \mathrm{m} \text {, }\end{array}$ & - & 3.2 & 3.1 & 2.5 & - \\
\hline$\sigma_{p}(1.064)\left(1 \mathrm{~J}^{-20} \mathrm{~cm}^{2}\right)$ & 1.05 & 2.62 & 2.71 & 2.45 & 3.0 \\
\hline Small-signal gain coefficient at $1.064 \mu \mathrm{m}, \gamma\left(\mathrm{cm}^{2} / \mathrm{J}\right)$ & 0.056 & 0.139 & 0.145 & 0.130 & 0.160 \\
\hline
\end{tabular}




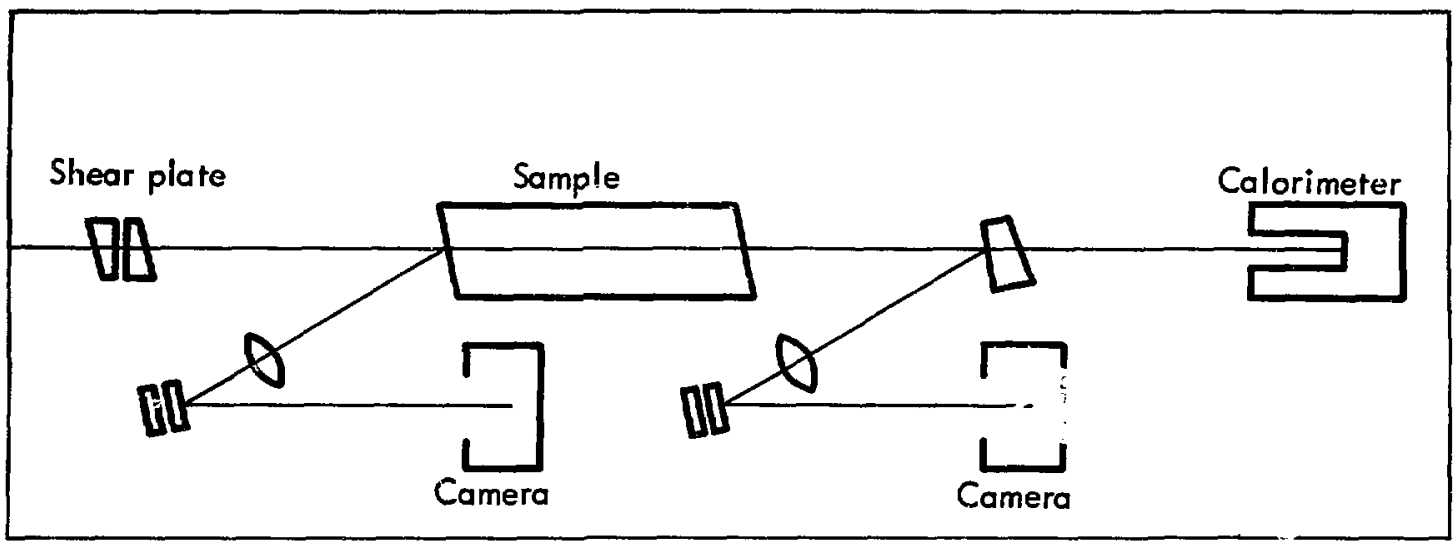

Fig. 41. Experimental arrangement for measuring the growth of small-scale intensity variations in a beam passing through an unpumped glass sample.

For some spatial frequencies dramatic growth of the impressed modulation is observed, as seen in Fig. 42. Linearized instability theory predicts that such modulations superimposed on a uniform-intensity background undergo exponential growth with a gain coefficient $g$ given by 25

$$
g=K\left(\frac{n_{2} E^{2}}{n}-\frac{K^{2}}{4 k^{2}}\right)^{1 / 2},
$$

where $K$ is the wave number of the intensity modulation, $n_{2}$ is the nonlinear refractive index coefficient, $n$

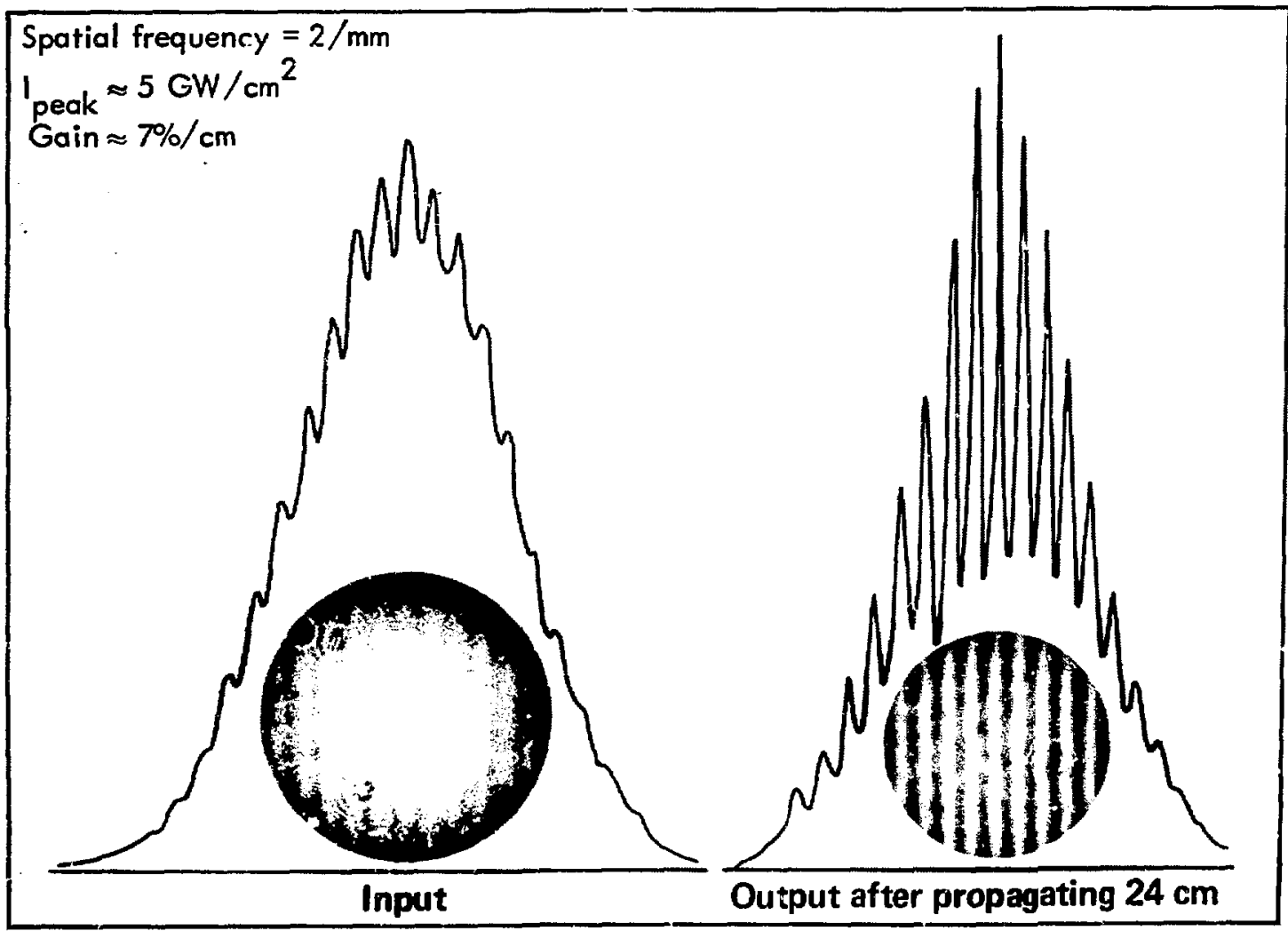

Fig. 42. Modulation growth in a nonlinear medium as determined with the experimental arrangement of Fig. 41. 
is the normal refractive index, $E$ is the rms value of the laser field, and $k$ is the wave number of the laser light.

To make a quantitative comparison between the measured growth of the sinusoidal intensity modulation and the growth predicted by linearized instability theory, the input intensity modulation is multiplied by the calculated gain and integrated over the measured temporal profile of the input pulse to obtain the timeintegrated energy-density modulation expected at the rod output. A time-averaged gain coefficient $\bar{g}$ is then defined by setting $e^{\bar{g} L}$ equal to the ratio of the output and input energy-density modulations. Experimental and theoretical values of $\bar{g}$ are plotted over a range of intensity for fixed spatial frequency in Fig. 43 and over a range of spatial frequency for fixed intensity in Fig. 44.

Considering that the calculation of $\bar{g}$ described above ignores the effects of gain saturation and fringe distortion and the fringe smearing resulting from a dependence of fringe position on intensity, the agreement is quite good. Computer calculations accounting for all of these effects are expected to result in even better agreement between theory and experiment.

In the last semiannual report ${ }^{31}$ we described preliminary measurements of the nonlinear refractive index coefficient, $n_{2}$, of some important optical glasses and crystals, using the time-resolved Mach-Zehnder interferometer. Final $n_{2}$ values obtained from careful data reduction are given here in Table 11 .

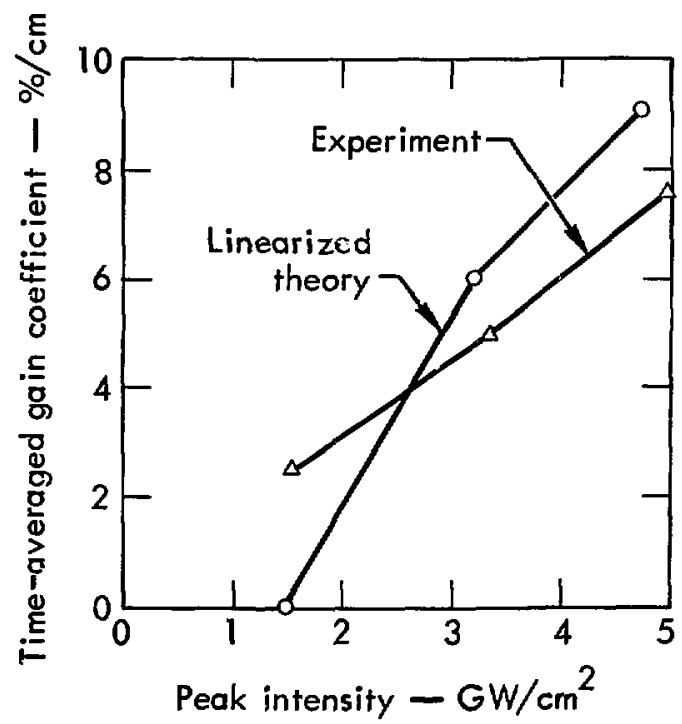

Fig. 43. Modulation growth as a function of peak intensity for fused wave number of the intensity modulation $\left(K=119 \mathrm{~cm}^{-1}\right)$.
Characteristic dimension - $\mathrm{mm}$

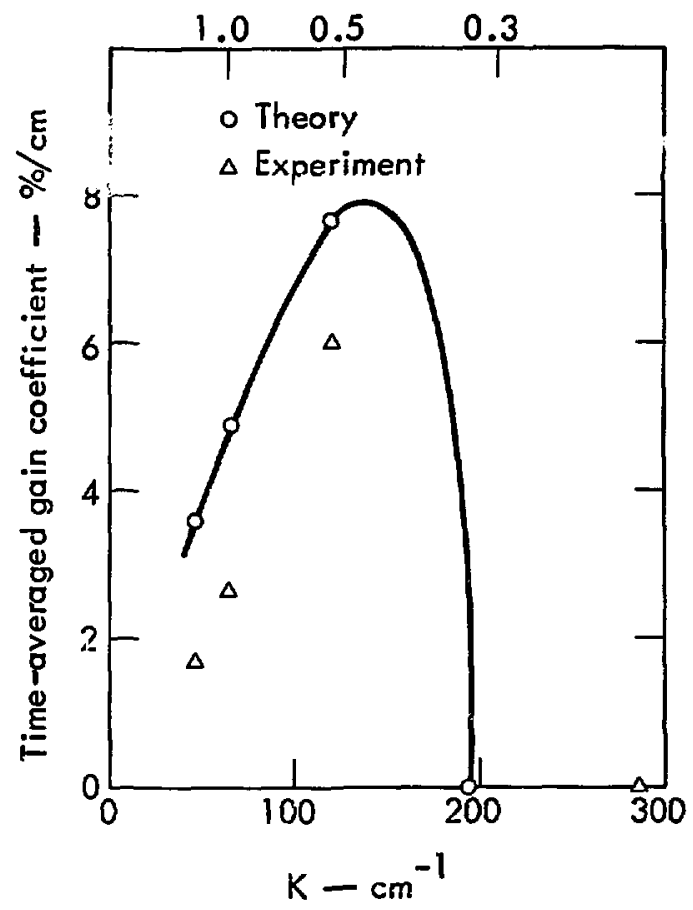

Fig. 44. Modulaticn growth as a function of wave number of the intensity modulation, for feak intensity of 4 $\mathrm{GW} / \mathrm{cm}^{2}$.

Flashlainp Pumping of ED-2 Laser Glass. Since the excitation energy of the laser glass is derived from flashiamps, it is important to have a detailed understanding of both the properties and operation of these lamps in laser configurations. The primary purpose of this work is to normalize the xenon flashlamp models which we have been using to estimate laser energy requirements. We have used a $1-\mathrm{cm}$-bore, $127-\mathrm{cm}-\mathrm{long}$, 450-Torr xenon flashlamp to measure the following aspects of flashlamp pumping as applied to disk iasers:

1. The absorption of visible flashlamp radiation as a function of Nd concentration in ED-2 laser glass.

Table 11. Measured $n_{2}$ values for four Nd-doped laser materials

Material

Measured $n_{2}$ value (esu)

\begin{tabular}{ll} 
ED-2 glass & $1.52 \pm 0.3 \times 10^{-13}$ \\
EY-1 glass & $1.77 \pm 0.3 \times 10^{-13}$ \\
FR-4 glass & $1.95 \pm 0.3 \times 10^{-13}$ \\
YAG(111) & $3.16 \pm 0.6 \times 10^{-13}$ \\
\hline
\end{tabular}


2. The absorption of uv flashlamp radiation by $\mathrm{Ce}$ in ED-2 laser glass.

3. The pumping efficiency of ED-2.3 laser glass as a function of pulse duration for constant energy loading.

4. The absolute electrical-to-optical conversion efficiency as a function of energy loading and current density.

The following sections of this article discuss each of these aspects.

\section{Absorption in ED-2 Glass}

A series of absorption experiments was performed in which the output from a 1-cm-bore, 450-Torr xenon flashlamp was attenuated by ED-2 laser glass. We used glass plates with different Nd concentrations and obtained the attenuation of xenon flashlamp radiation as a function of absorption length.

The flashlamp was connected to a single-mesh discharge circuit which delivered $53 \mathrm{~J}$ per centimeter of lamp length ( $6.73 \mathrm{~kJ}$ total). The peak current density was $3.8 \mathrm{kA} / \mathrm{cm}^{2}$ and the pulse length was $500 \mu \mathrm{sec}$, slightly overdamped ( $\alpha=0.95$ ). The pulse-to-pulse reproducibility of the flashlamp output, as measured by the calorimeter, was $\pm 5 \%$. This variation includes the calorimeter fluctuations. For low concentrations of $\mathrm{Nd}$ in the glass samples, in which case there was very little absorption, the attenuation is masked by the lamp output fluctuations which, when averaged, are about $\pm 3 \%$.

Two glass plates of constant thickness were used in order to maintain a constant zeometry and to provide a wide variation in optical desısity. ED-4 glass plates (ED-2 with no Nd) were used to normalize out the effects of Fresnel reflection and the uv absorption from Ce. The transmitted signal through the ED-2 plate was simply compared to the signal through the ED-4 plate to find the attenuation due to the Nd ions.

The transmitted radiation was detected with a ballistic calorimeter designed by Stuart Gunn of LLL as a laser energy monitor. It was modified to accept a broadbank black coating (Nextel Black) which has been estimated to be $97 \pm 2 \%$ absorbing from $0.2 \mu \mathrm{m}$ to $>2.0 \mu \mathrm{m}$. The calibration is $120 \mu \mathrm{V} / \mathrm{J}$, and typical signal levels were $2.0 \mu \mathrm{V}$.

The attenuation of xenon flashlump radiation vs optical thickness in ED-2 glass is plotted in Fig. 45.

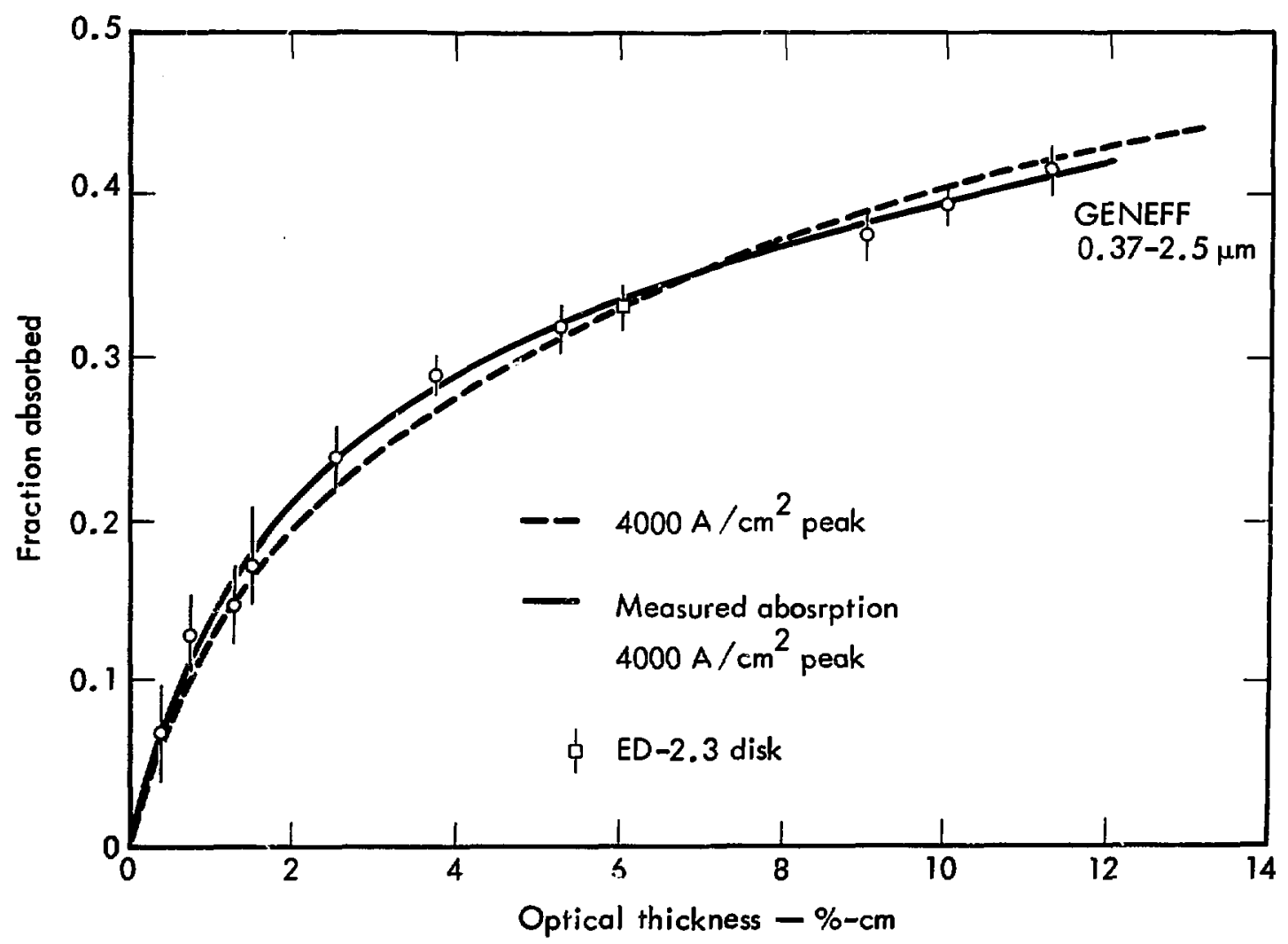

Fig. 45. Absorption of xenon flashlomp radiation as a function of optical thickness in ED-2 glass. 
Contributions to the attenuation due to Fresnel reflections and to $\mathrm{Ce}$ absorption have been normalized out. The dotted line is the absorption vs optical thickness" generated by GENEFF, the Trenholme-Emmett computer-simulated flashlamp model. This model predicts very accurately the absorption characteristics of xenon flashlamp radiation in ED-2 laser glass.

The derivative of Fig. 45 yields the specific absorption of flashlamp energy per $\mathrm{cm}^{3}$ at a specified optical thickness. This is shown on the lower curve in Fig. 46. This curve is the profile of energy deposition into a disk of ED-2 laser erass irradiated from one side. Distributions for laser disks of different optical thickness which are irradiated from two sides are also shown in Fig. 46.

Figure 47 shows the percent of total light that is absorbed by the Ce in ED-2 as a function of peak current density. Ce starts absorbing very strongly below $3800 \AA$. Notice that up to $30 \%$ of the total

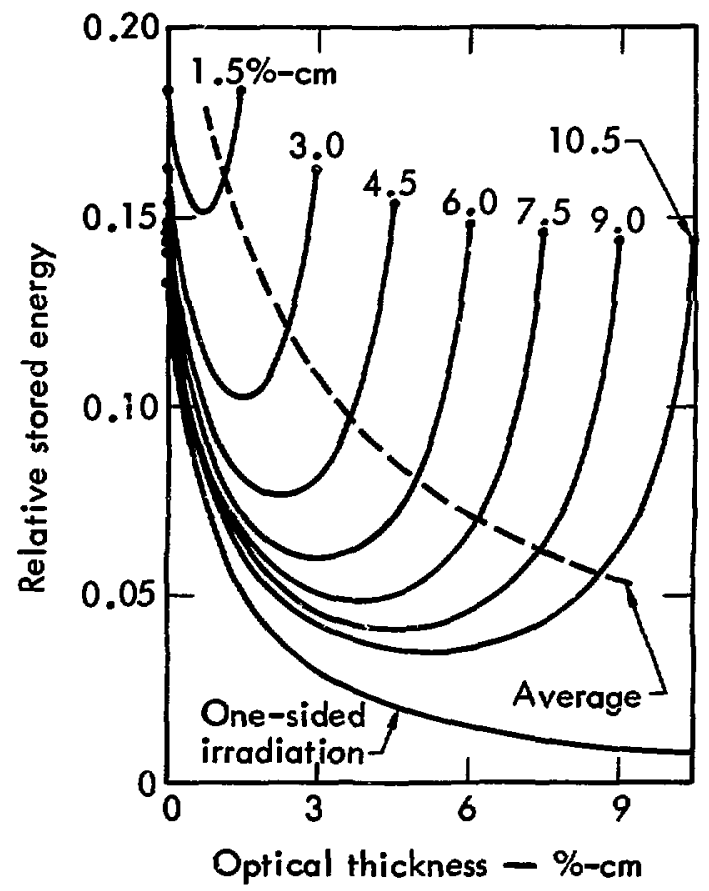

Fig. 46. Gain profiles in ED-2 laser disiss as functions of disk optical thickness. Dashed curve is average relative stored energy in disk.

"Optical thickness is defined to be the distance into the absorbing material in $\mathrm{cm}$ times the \% doping of $\mathrm{Nd}_{2} \mathrm{O}_{3}$ by weight. If the spectral absorption strength per $\mathrm{Nd}^{2}$ ion is independent of Nd concentration, use of the optical thickness unit allows one to parametrize the absorption properties for disks of all concentrations and thicknesses.

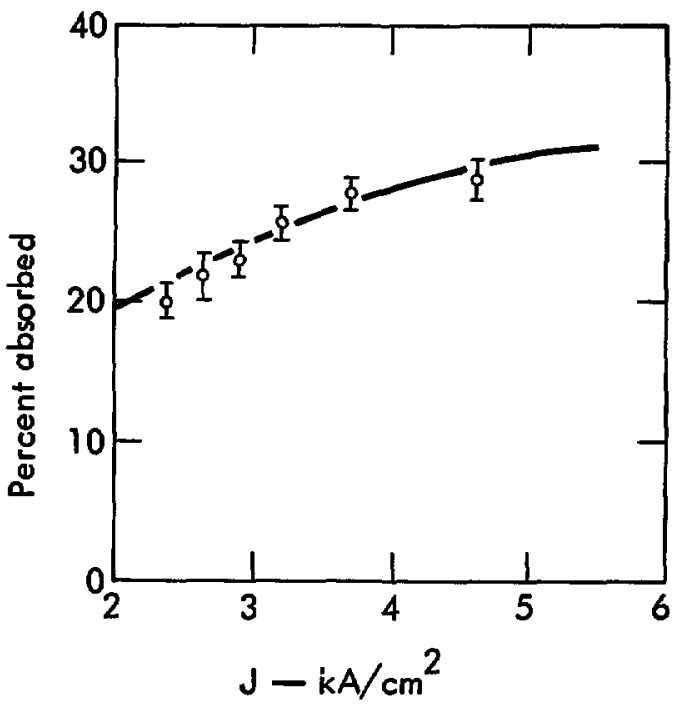

Fig. 47. Absorption of xenon flashlamp light in ED-2 glass as a function of current density, with lanup loading constant at $70 \mathrm{~J} / \mathrm{cm}$.

flashlamp energy is absorbed by the Ce. The GENEFF code predictions for $\mathrm{Ce}$ absorption are about $32 \%$ at $4 \mathrm{kA} / \mathrm{cm}^{2}$ compared to a measured $28 \%$. At $>4$ $\mathrm{cm}^{2}$ the code predicts about $23 \%$ absorption. we measure $22 \%$ absurption.

Pumping of ED-2 vs Pulse Duration at Constant Energy Input

A series of experiments was conducted in order to determine whether flashlamps operating under a long pulse condition ( $\left.\tau_{\text {flashlamp }} \gg \tau_{\text {fluorescence }}\right)$ pump ED-2 laser glass in a way predicted by, the flashlamp model. This question was important since the $A$ and $B$ disk laser modules were designed to operate with an $800-\mu \mathrm{sec}$ pulse duration in order to increase flashlamp life expectancy.

The output from a 450-Torr xenon flashlamp was directed onto a slab of ED-2.3 laser glass $\left(3 \% \mathrm{Nd}_{2} \mathrm{O}_{3}\right.$ by weight). The fluorescence from the initial laser levels (the ${ }^{4} \mathrm{~F}_{3 / 2}$ levels) of the Nd ion is detected with a photodiode. A $1.06-\mu \mathrm{m}$ interference filter $(\sim 200-\AA$ passband) rejects scattered pump radiation, and the geometry of the glass detector system is chosen to minimize $1.06-\mu \mathrm{m}$ radiation generated by the flashlamp. No scattered flashlamp light is detected with this system. A similar technique was used by ILC Inc. several years ago to optimize small lamps which were used in YAG rangefinder lasers. ${ }^{32}$

The peak of the fluorescence signal, as measured by the photodiode, is proportional to the peak stored energy in the laser glass. By measuring the change in 
diode signal as a function of flashlamp energy or pulse duration, we can test the flashlamp pumping model.

The flashlamp circuits were carefully diagnosed in order to determine the actual energy delivered to the flashlamp. This was done by measuring $V(t)$ and $I(t)$ and by determining $\int V \cdot I d t$, the energy dissipated in the lamp, and $1 / 2 C V_{0}^{2}-R \int I^{2} d t$, the stored energy minus the transmission loss. These two comparisons were self-consistent and agreed with the circuit loss estimates presented by Markiewicz and Emmett ${ }^{33}$ on flashlamp circuit design to within $\pm 2 \%$. Once the characterictics of the bank were established, the data were reduced by measuring the series resistance of the bank with a four-point resistance probe and using the results of Markiewicz and Emmett to estimate the transmission loss. The current and often the voltage signals were monitored on each shot to determine whether or not the bank was changing its characteristics.

The experimental fluorescence data (see Fig. 48) have been normalized to the theoretical curves produced by GENEFF* by placing the $330-\mu \mathrm{sec}, 50-\mathrm{J} / \mathrm{cm}$ point on the theoretical curve. The other points are

* GENEFF is a computer code which incorporates experimentally measured xenon plasma emissivities into a flashlamp radiation model. The model is then normalized to experimentally measure total radiative efficiencies.

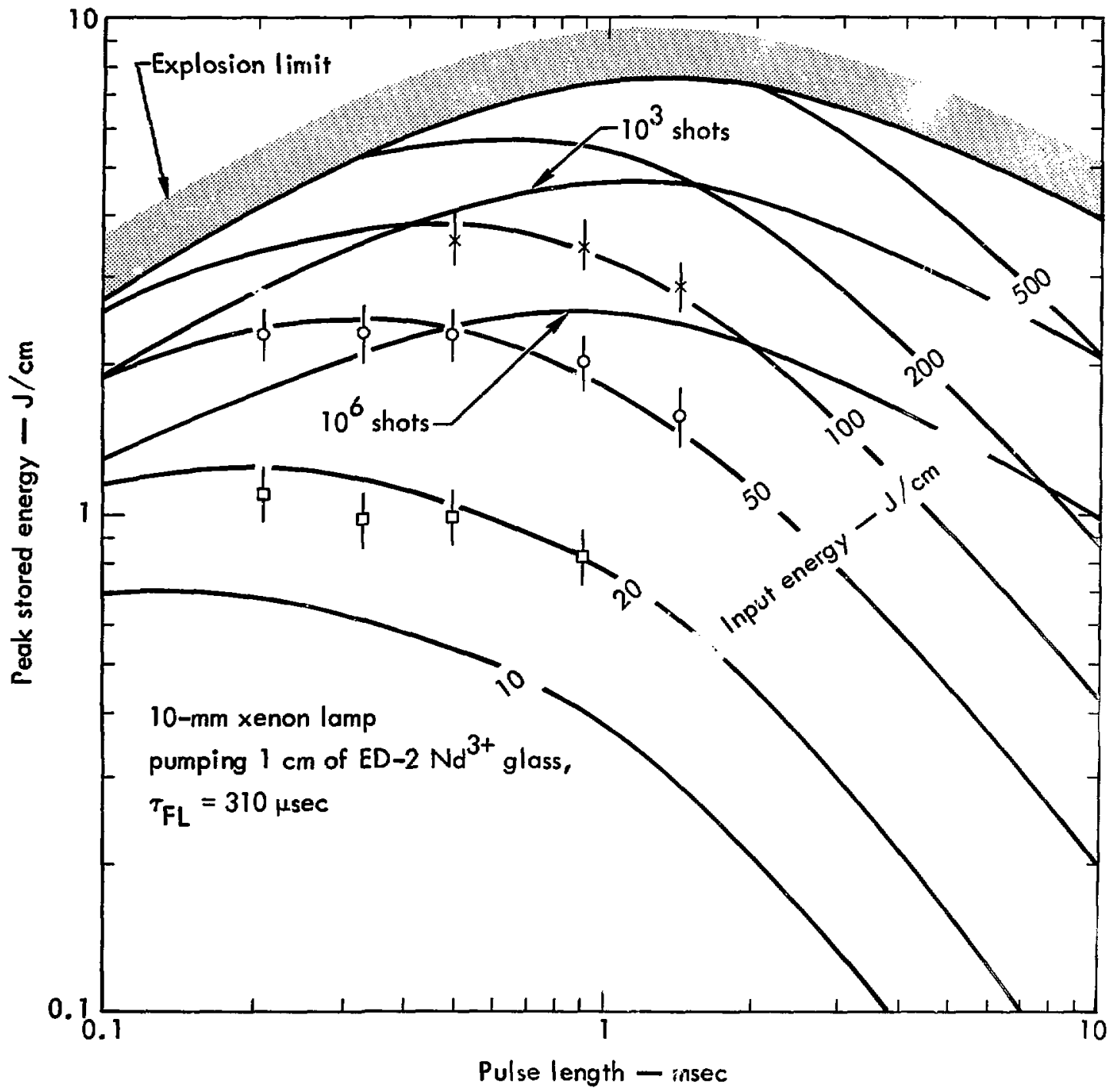

Fig. 48. Peak stored energy it: ED-2.3 lass: glass as a function of fashlamp pulse length. Experimental nuorescence data are normalized to theorctical curves calculated by GENEFF code. 
fixed, experimentally, relative to the one normalized point and thus show the relative fit to the theoretical curves. As can be seen, the fit is very good. A more detailed examination shows that the lamps should be about $10 \%$ more efficient in pumping ED-2 with an $800-\mu$ sec pulse duration than with a $350-\mu \mathrm{sec}$ pulse duration.

\section{Flashlamp Electrical-to-Optical Conversion \\ Efficiencies}

The calorimeter we used to measure the flashlamp light absorption in ED-2 glass can be used to measure the absolute flashlamp radiative output. Figure 49 shows two views of the experimental arrangement we used to measure total radiative efficiencies. Vignetting effects around masks and apertures were very small, typically $3 \%$.

Many experiments were performed to make certain that no scattered light entered the calorimeter. These in:luded tests for low angle scattering, re-reflection off the flashlamp walls, and reflections off walls, tables, etc. The calorimeter signal dropped as $1 / r^{2}$ as the calorimeter was moved from 40 , to 50 , and to $60 \mathrm{~cm}$ from the lamp center. In addition, quartz filters were placed between the lamp and the calorimeter to test for effects due to hot gas or to ir radiation from the warm $\left(10^{\circ} \mathrm{C}\right.$ above ambient $)$ lamp envelope.
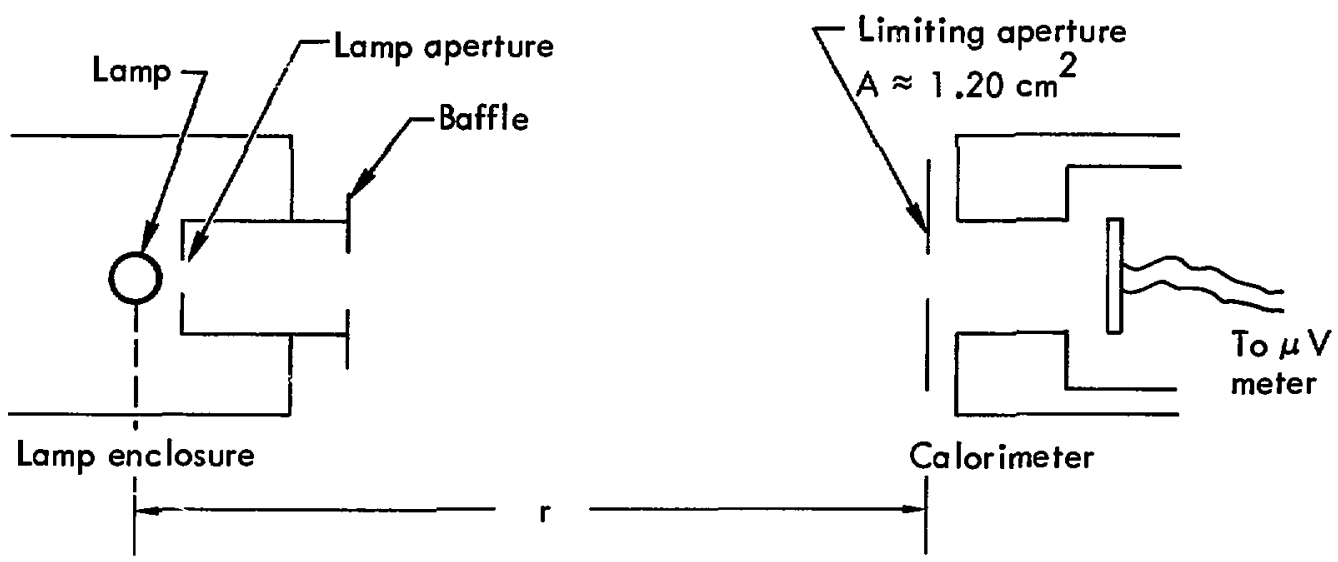

(a) End view
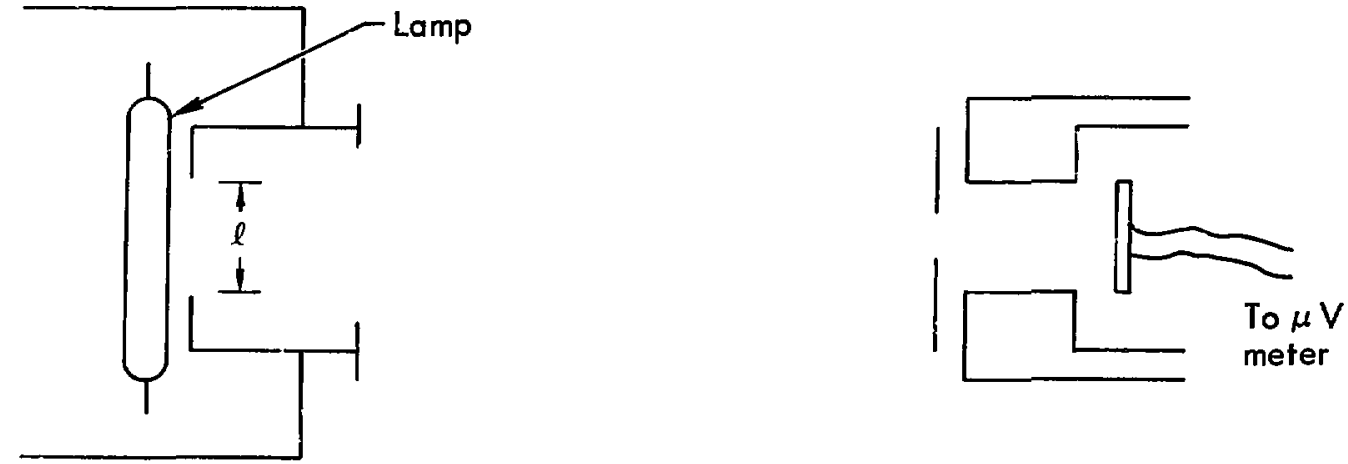

Lamp enclosure

Calorimeter

(b) Top view

Fig. 49. Experimental arrangement for measuring total radiative efficiency of flashlamps. 
The formula for converting the calorimeter signal into lamp radiative efficiency is

$$
E_{\text {rad }} / \mathrm{cm}=k \frac{\pi^{2}}{d \Omega} \frac{1}{\ell^{\prime}} E_{m},
$$

where $d \Omega=A / r^{2}$ is the solid angle subtended by the limited calorimeter aperture, $\ell$ is the effective lamp length corrected for end effects $\left(\ell^{\prime} \approx 1.034 \ell\right.$ for our geometries), $E_{m}$ is the energy measured by the calorimeter (usually averaged over a series of 5-10 shots), $\pi^{2}$ is the effective total solid angle of a Lambertiar linear radiator when viewed normal to the axis, and $k$ is a correction term which accounts for the lamp not being a true Lambertian radiator, as the lamp radiates more strongly at low angles than the $\cos \theta$ dependence of a Lambertian source. If the lamp were a blackbody, it would radiate like a Lambertian source (excluding envelope effects), and $k \approx 1$. J. Trenholme has estimated $k \approx 103$ from the present flashlamp model using $\mathrm{ZAP}^{34}$ to compare the on-axis radiation intensity to the total radiated intensity.

For a pulse width of $500 \mu \mathrm{sec}$, a peak current density of $4.5 \mathrm{kA} / \mathrm{cm}^{2}$, and an energy of $72 \mathrm{~J} / \mathrm{cm}$ delivered to the lamp, we get

$$
\begin{aligned}
\ell^{\prime} & =3 \times(1.034) \mathrm{cm}, \\
r & =50 \mathrm{~cm}, \\
A & =1.20 \mathrm{~cm}^{2}, \\
k & =1.03, \\
E_{m} & =1.101 \mu \mathrm{V} \div 120 \mu \mathrm{V} / \mathrm{J}=8.42 \mathrm{~mJ}, \\
E_{\text {rad }} \mathrm{cm} & =k \frac{\pi^{2}}{\Omega} \frac{1}{\ell^{\prime}} E_{m}=57.5 \mathrm{~J} / \mathrm{cm} . \\
\text { Efficiency } & =\frac{57.5}{72}=0.798 .
\end{aligned}
$$

$A$ cross check on the radiative efficiency was made by measuring the residual thermal energy left in the lamp. The lamp we had used to make the radiative efficiency measurements was placed in a quartz vacuum jacket, and a Chromel-Alumel thermocouple was tied to the wall with a thin nickel wire. The temperature rise of the wall was measured over a period of about $2 \mathrm{~min}$, a time long enough for all local nonequilibrium effects to have died away. The temperature-vs-time decay curves were then extrapolated back to zero time to find the temperature rise corresponding to the thermal energy being lost uniformly in the walls.

After the experiment, the lamp was cut apart to determine the tube's average inner diameter and wall thickness. The energy required to heat a $1 \cdot \mathrm{cm}$-long section of this lamp was $1.09 \mathrm{~J} /{ }^{\circ} \mathrm{C}$. The typical temperature rise was about $10^{\circ} \mathrm{C}$. The data shown in Table 12 are for a lamp operated with $500-\mu$ sec pulse duration from the same circuit described earlier in this section.

These thermal measurements together with the radiative calorimeter data show that this $1-\mathrm{cm}$-bore lamp is more efficient than one would have estimated from the data taken on smaller lamps ( $50 \%)$. The radiative measurements are consistent with measurements made by Gonez and Newell, ${ }^{35}$ who measured $65 \%$ efficiency from 0.35 to $1.1 \mu \mathrm{m}$. If one includes the additional uv output detected by the calorimeter, $0.17 \mu \mathrm{m}<\lambda_{\text {det }}\lceil 5 \mu \mathrm{m}, 80 \%$ radiative efficiency is a very reasonable total radiative value.

\section{Conclusions}

1. The absorption of flashlamp light in ED-2 laser glass is accurately predicted by the present lamp model.

2. The dynamic pumping of ED-2 laser glass by xenon flashlamps, under conditions of interest to laser builders, is accurately predicted by the model to within $10 \%$. For long lamp pulses, the model is about $10 \%$ pessimistic. For short, low-energy pulses the model is about $25 \%$ optimistic. We are not concerned with this latter operating region.

3. The overall e!ectrical-to-radiative efficiency of these lamps is about $80 \%$. This is higher than the model estimate of $62 \%$. These values are consistent with measurements made on large-bore lamps. This new measurement is being included in the lamp model. The consequences of these new measurements on energy transport calculations are being examined.

Table 12. Radiative efficiency data for $1 \cdot \mathrm{cm}$-bore, $127 \cdot \mathrm{cm}$-long, 450-Torr xenon flashlamp operated with 500- $\mu$ sec pulse duration

\begin{tabular}{cccccc}
$\begin{array}{c}\text { Energy per unit } \\
\text { length into lamp } \\
(\mathrm{J} / \mathrm{cm})\end{array}$ & $\begin{array}{c}\text { Peak current } \\
\text { density } \\
\left(\mathrm{kA} / \mathrm{cm}^{2}\right)\end{array}$ & $\begin{array}{c}\text { Envelope } \\
\text { temp rise } \\
(\mathrm{C})\end{array}$ & $\begin{array}{c}\text { Residual thermal } \\
\text { energy } \\
(\mathrm{J} / \mathrm{cm})\end{array}$ & $\begin{array}{c}\text { Implied liative } \\
\text { efficiency } \\
(\mathbf{( \% )}\end{array}$ & $\begin{array}{c}\text { Measured radiative } \\
\text { efficiency } \\
(\%)\end{array}$ \\
\hline 65 & 4.2 & 11.5 & 11.1 & $80.6 \pm 5$ & $80 \pm 5$ \\
51.7 & 3.7 & 9.5 & 9.2 & $79.9 \pm 5$ & $77 \pm 5$ \\
39.7 & 3.06 & 8.1 & 7.8 & $77.7 \pm 5$ & $76 \pm 5$ \\
\hline
\end{tabular}


During this semiannual ieporting period, the principal activity of the Design Analysis Group was in the area of detailed simulation of the temporal behavior of pumping in disk laser anplifiers. The motivation of this effort was the necessity of developing a method of selecting disk thickness and doping which was more accurate than the approximate method outlined in the last semiannual report. The effort culminated in the GAINPK package, which has satisfied the need for accurate temporal simulation of laser pumping.

Experiment and theory have made it clear that the factor which limits the power output of large fusion lasers is small-scale beam breakup rather than whiolebeam self-focus or surface damage. Therefore, the implications of the theory of this effect in the presence of disk boundaries and amplifier gain were investigated with an eye toward using the results to guide system optimization calculations in the presence of small-scale breakup. The whole-beam ray trace effort outlined in the last report has continued, but numerical problems associated with small-scale growth have prevented successful conclusion of the project.

The 10-kJ system optimization and layout effort has continued. A number of individual chain layouts have been studied, as have various combinations of chains to form the whole array. Simplified programs for rapid study of various alternative chain layouts have been produced. An intensive stidy of various methods of postoscillator spatial noise suppression was carried out.

In addition to the above activities, programming assistance was supplied to the $\mathrm{CO}_{2}$ system and new laser activities.

\section{TEMPORAL PUMPING SIMULATION}

A package of programs has been developed around a set of subroutines (GAINPK) which finds the peak gain in a laser inplifier for one shot. The programs allow investigation of laser behavior as a function of bank energy (PUMPER), of the effect of varying the drive pulse width (TIMVAR), and of changing the doping in the disks (DOPING). The programs are designed with self-explanatory input file format for easy use by casual computer users.

The basic input to the programs is a description of the energy storage bank, the disk parameters, and the geometry of the laser head. Also required is a curve of transfer efficiency of laser lamp power to inversion rate, either as two coefficients of an analytic expression or as a set of ZAP points. If points are supplied, a curve is least-squares-fitted to them. To find the laser gain, the equations of the flashlamp discharge circuit are then integrated to find the lamp power as a function of time. The lamp power is then multiplied by the transfer efficiency (which is a function of current) to find the rate of inversion generation. The inversion decay rate is then calculated from the inversion decay level, and the equation for inversion is integrated in time to find the inversion behavior. The decay is due to fuorescence, superfluorescence, and parasitiz oscillation. The fluorescence is modeled by a single exponen. tial decay. The superfluorescence (amplified spontaneous emission) is simulated by multipiying the fluorescent rate by the factor $s$ (found by ZAP calculations of superfluorescence),

$$
s=\exp \left(0.18 \beta+0.018 \beta^{2}+0.002 \beta^{3}\right)
$$

where $\beta$ is the product of the gain coefficient and the disk's long axis. In fact, since only a fraction $\gamma$ of the inversion decays at $1.06 \mu \mathrm{m}$ and is amplified, we multiply by

$$
q=(1-\gamma)+\gamma s
$$

Parasitic oscillation is approximated by multiplying the decay rate by

$$
p=1+\exp \left[50\left(\beta-\beta_{0}-0.03\right)\right],
$$

where $\beta_{0}$ is the parasitic threshold.

The PUM?ER code repeatedly goes through the above process while varying the bank energy. The result is a plot of peak gain versus bank energy, as shown in Fig. 50. If experimental gain points are supplied to the program, it will adjust a factor for best fit to the points and report back the required value. In addition, information on lamp lifetime, peak wn -ents, bank damping, and the effect of inductor changes is supplied.

The TIMVAR code calculates peak gain as a function of the drive pulse width. It does this for a nui ,er of bank energies and then summarizes the maximum gain as a function of bank energy, as illustrated in Fig. 51. This code allows tradeoffs between 
peak gain and lamp lifetime, or between gain and capacitor value.

The DOPING code (which is still under development) varies the doping as well as the drive pulse

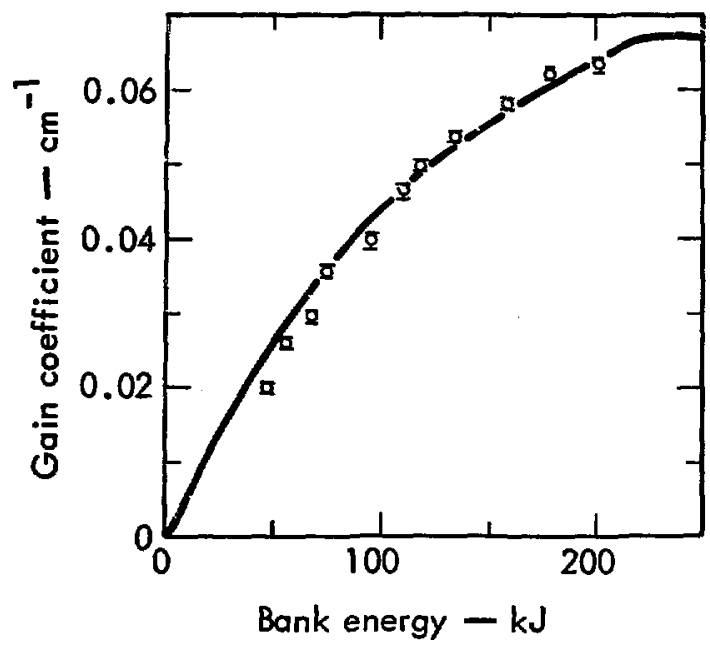

Fig. 50. Laser gain coefficient as a function of flashlamp bank energy. PUMPER code rms-fits the curve to the data points by adjusting one parameter.

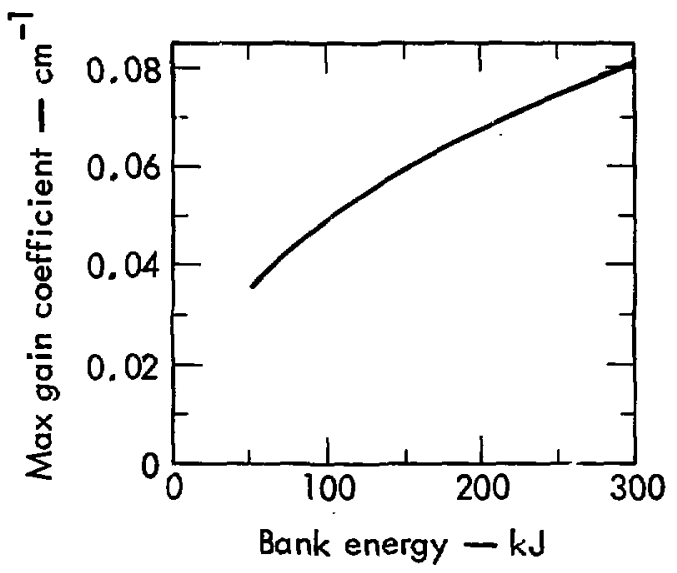

Fig. 51. Maximum gain coefficient vs bank energy as calculated by TIMVAR code.

vidth. The doping change modifies both the fluorescent lifetime and the efficiency of optical absorption. The main output is a contour plot of disk gain as a function of doping and drive pulse width. An example is shown in Fig. 52.
The emphasis of the reflector optimization project has shifted to investigation of automatic multidimensional optimization in the presence of noise, since hand optimization (even with extensive use of computer graphics) has proven inadequate. Several methods have been investigated; the most promising is Nelder and Mead's creeping simplex method. Various modifications to improve its behavior in noise are being tried. A program which tests the minimizer against a large sumber of representative test functions has been written. It systematically varies the parameters of the method and produces copious graphic diagnostic information. Once the most effective approach has been found, it will be applied to the reflector optimization problem. As an added beriefit, the resulting meth 1 should be applicable to numerous other problems _. the laser fusion area.

While automatic search methods are being investi- gated, a large amount of experimental data on various reflectors has become available from the vigorous laser development area, and this information is being used to refine the parameters of the 2-D Monte Carlo reflector simulation program.

\section{ZAP Progress}

The ZAP program has remained essentially unchanged, and is now a production "workhorse" rather than a developing code. The major ZAP effort has therefore switched to documentation and userawareness seminars. The automatic disk-laser input generator has been extended to all presently used types of LLL disk lasers, which makes job setup a simple task. This support program for ZAP has now grown to over 2000 lines of Fortran. 


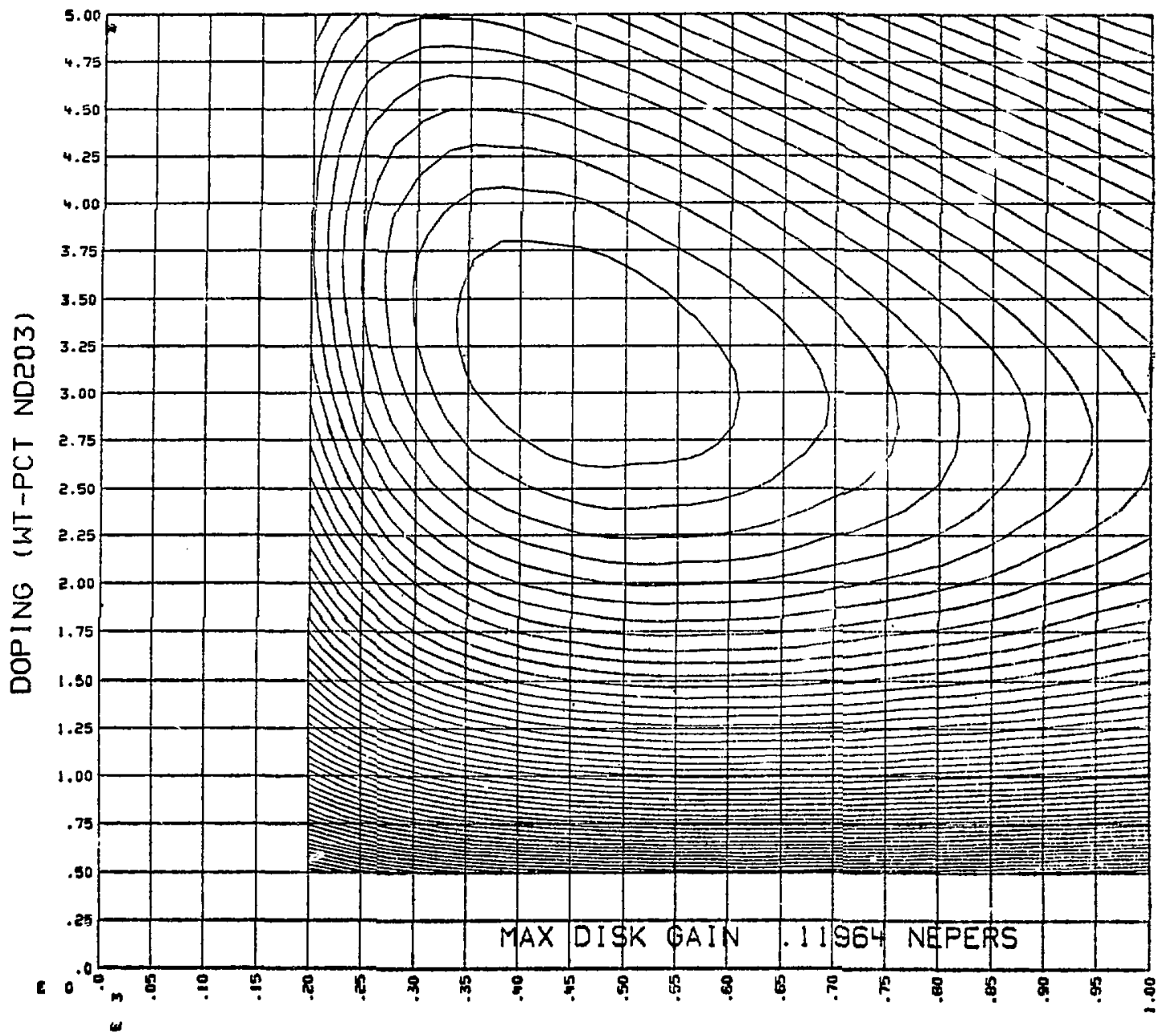

FULL PULSE WIDTH (MICROSECONDS)

Fig. 52. Cortour plot of disk gain as a function of disk doping and flashlamp drive pulse width, as produced by the DOPING code.

\section{SMALL-SCALE GROWTH}

When an intense laser beam travels through a solid material, the electric field distorts the electron configuration in the solid in such a way that the refractive index is raised by a small amount (a few parts per million in typical fusion lasers). This change tends to concentrate more light in the intense regions, raising their intensity even higher. This self-focus effect can raise the light flux to a level high enough to damage the solid.(For experimental results, see the previous section.)
At any specific intensity, the growth rate for a pattern of evenly spaced intensity ripples depends on the ripple spacing. Slow (widely spaced) ripple patterns have low growth because the distance over which the intensity changes is large, so the equivalent induced lens is weak. Fast patterns have low (or no) growth because diffraction of the ripples is stronger than the self-fucus effect. The maximum growth rate occurs between these two limits. 
As the intensity in the beam rises, the size of the maximum-gain ripples becomes smaller and smaller (Fig. 53). When the maximum-gain ripple size becomes smaller than the size of the laser beam, the beam tends to break up into a number of filaments rather than collapsing as a whole. At the extreme intensities encountered in fusion lasers, this small-scale beam breakup is the factor which limits the output power from the laser system.

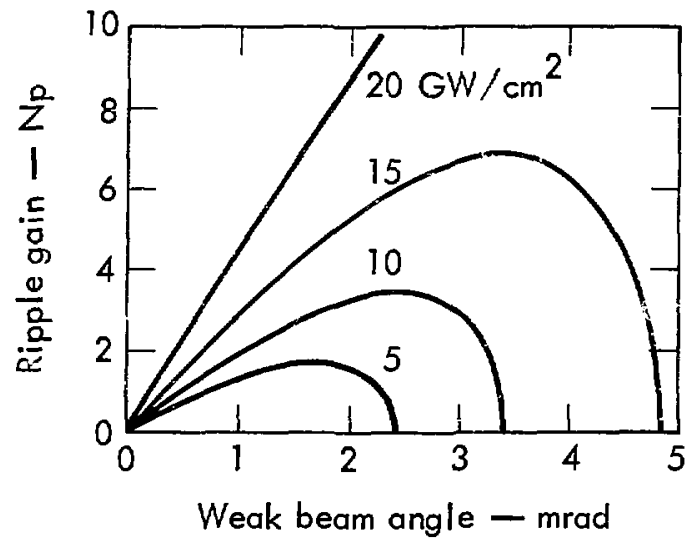

Fig. 53. Ripple gain as a function of tipple scale for a beam passing through $20 \mathrm{~cm}$ of ED-2 las. glass, for various beam intensities. The weak beam angle is the angle between the strong main beam and the weak interfering beam that produces the ripples. As this angle increases, the ripples become more closely spaced.

We have studied two implications of the standard theory of small-scale growth. One is the effect of passing through separated slabs of material rather than a uniform solid. The other is the effect of changing intensity of the beam due to amplification.

When a ripple pattern passes through a slab of material, the gain is affected by the boundary conditions at the slab interfaces. When ihere are only two interfering plane waves causing the ripples (a strong background beam and a weak interfering beam), the ripple gain is the same as the bulk result and the ripples grow as $\exp (g x)$. The gain coefficient $g$ is given by

$$
g=k \theta \sqrt{\mu-\left(\frac{\theta}{2}\right)^{2}},
$$

where $k$ is the radian wave number.

$$
k=\frac{2 \pi}{\lambda},
$$

the angle $\theta$ is the angle between the strong and weak beans (the ripple pattern has wave number $k \theta$ ), and $\mu$ is the relative index ciange caused by the strong beam:

$$
\mu=\frac{\delta n}{n} .
$$

When a beam encounters an absorbing region or an index inhomogeneity, the pattern produced is not the simple one caused by the strong beam and one weak beam. Instead, the pattern is that due to a strong beam with two weak beams, one at angle $\theta$ and one at angle $\rightarrow$ to the strong beam. When such a pattern strikes a slab, the gain depends on the phase relation between the weak beams and the strong beam at ine slab entrance and exit faces. Since beam imperfections occur at random, we concentrated on the worst-case phase which gives highest gain. We found that the gain under these circumstances was higher at low spatial frequencies than the two-beam case, so that the breakup features tend to be larger in a series of slabs than in a solid rod (Fig. 54).

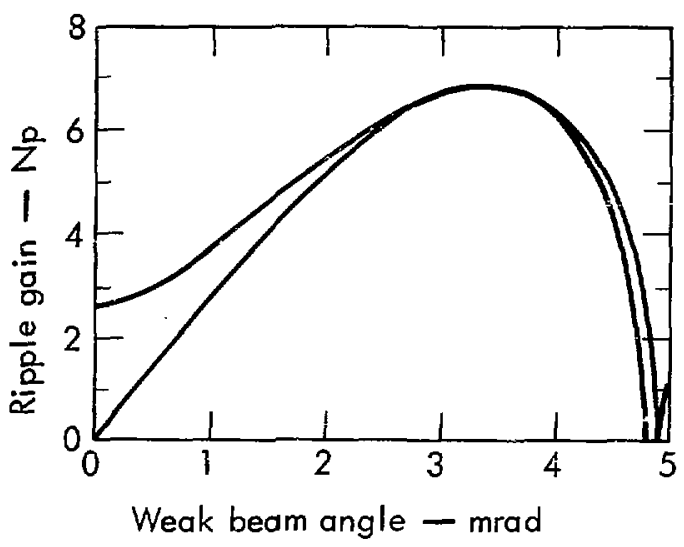

Fig. 54. Comparison of ripple gain with and without the imposition of boundary conditions at slab surfaces. The actual gain is raised by the effects of the boundaries, both at low angles (large ripples) and high angles (small ripples). The peak gain is unchanged.

When a ripple pattern passes through an amplinier. the entire shape of the gain-vs-spatial-frequency curve is changed. This is because as the intensity rises through the amplifier, ripples of smaller and smaller spacing begin growing (recall Fig. 53). in the two-beam case, calculation of the gain is straightforward and yields results like those of Fig. 55. In the limit of large gain in the amplifier, the pe.dk-gain ripples have about twice the size of those in the unpumped case. The gain is sharply reduced for fixed outpul flux as the gain is raised, because the length of material over which the beam has high intensity is reduced. This result emphasizes the need for the highest possible gain coefficient in amplifiers which are limited by smallscale growth. 
The calculation of optimum system design described in the previous semiannual report is now being reciune using a small-scale growth limit instead of the previously assumed flux limit. The optimization is more difficult because two variables (gain and growth) can be adjusted in each stage. Results will be reported in the next semiannual.

Fig. 55. Effect ${ }^{\wedge}$ gain in the material on the ripple growth factor. 1 this example, the output intensity is kept constant at $20 \mathrm{GW} / \mathrm{cm}^{2}$ while the input is decreased as the gain is raised. The rapid decrease of ripple grow th argues strongly for the highest possible gain coefficient in large laser systems.

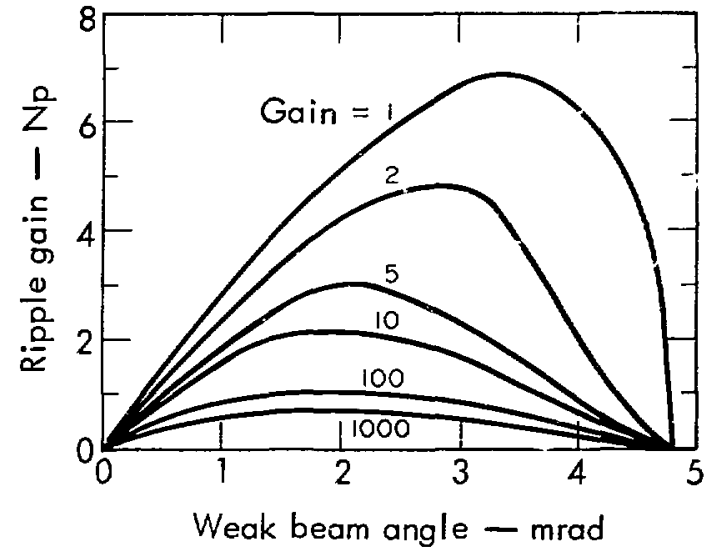

Once a system has been designed so that small-scale growth is tolerable (see above), we still want to know the effects of nonlinear effects on the beam shape as a whole. In addition, we need to know the effect of linear distortions due to tilted plates and surface irregularities. Development is therefore continuing on the diffractionless ray-trace code described in the previous semiannual. This code, called SNORT, has encountered great difficulties due to the unstable nature of the equations being solved. Because of the absence of diffraction, the irregularities lave a growth rate which goes inversely with their size. Thus nuise on the scale of the mesh has very high gair, and irregularities at mesh changes grow rapidly and destroy the calculation. Numerous methods of uniform gradient calculation and intensity smoothing have been attempted without success. After consultation with the theoretical group at LASL, we have tried a PIC method, but find that a very large number of rays is necessary for successful computation, leading to excessive computation time. A method in which $r_{i} f$ deviations are accumulated through several disks before they are applied is now the most promising approach.

Although the nonlinear part of the code is still not satisfactory, the linear part is quite accurate. Analysis shows that the axial offset of the tangential focus due to nassage through a slab of thickness $\ell$, index $n$, and tilt angle $\theta$ is

$$
T=\ell \cos \theta\left(1-\frac{\cos \theta}{\sqrt{n^{2}-\sin ^{2} \theta}}\right),
$$

and the sagittal offset is

$$
\begin{array}{r}
S=\ell\left[\cos \theta+\frac{\sin ^{2} \theta-\cos ^{2} \theta}{\sqrt{n^{2}-\sin ^{2} \theta}}\right. \\
\left.-\frac{\sin ^{2} \theta \cos ^{2} \theta}{\sqrt{\left(n^{2}-\sin ^{2} \theta\right)^{3 / 2}}}\right] .
\end{array}
$$

The SNORT code correctly reproduces these astigmatic foci. In addition, beam steering due to the change in index passing through a disk is also correctly reproduced. We expect th t the entire code wili soon be operating correctly. 


\section{COMPONENT DEVELOPMENT}

The development and refinement of reliable system components is an important aspect of the implementation of the LLL solid-state system. Component development in this period has been directed primarily at producing hardware for the solid state program. In particular, this section addresses work on Faraday isolators, multilayer dielectric-coated polarizers, calorimeters, and Pockels cells. Figure 56 shows a typical laser chain, with the components discussed in this section noted. The Faraday rotator and polarizers act in corijunction as isoldtors against light traveling backward through the chain. Thus protection is provided against damage due to reflection as well as oscillation in the high-gain amplifier stages. The Pockels cells are used to switch out a single pulse from a modelocked irain, and aiso to provide isolation in both directions. The calorimeters are used both for wholebeam energy measurements and for routine performance monitoring, the latter done by collecting the $5 \%$ reflection from the face of the rotators. In addition, evaluation of detectors at $10.6 \mu \mathrm{m}$ and progress in development of apodized apertures is reported.

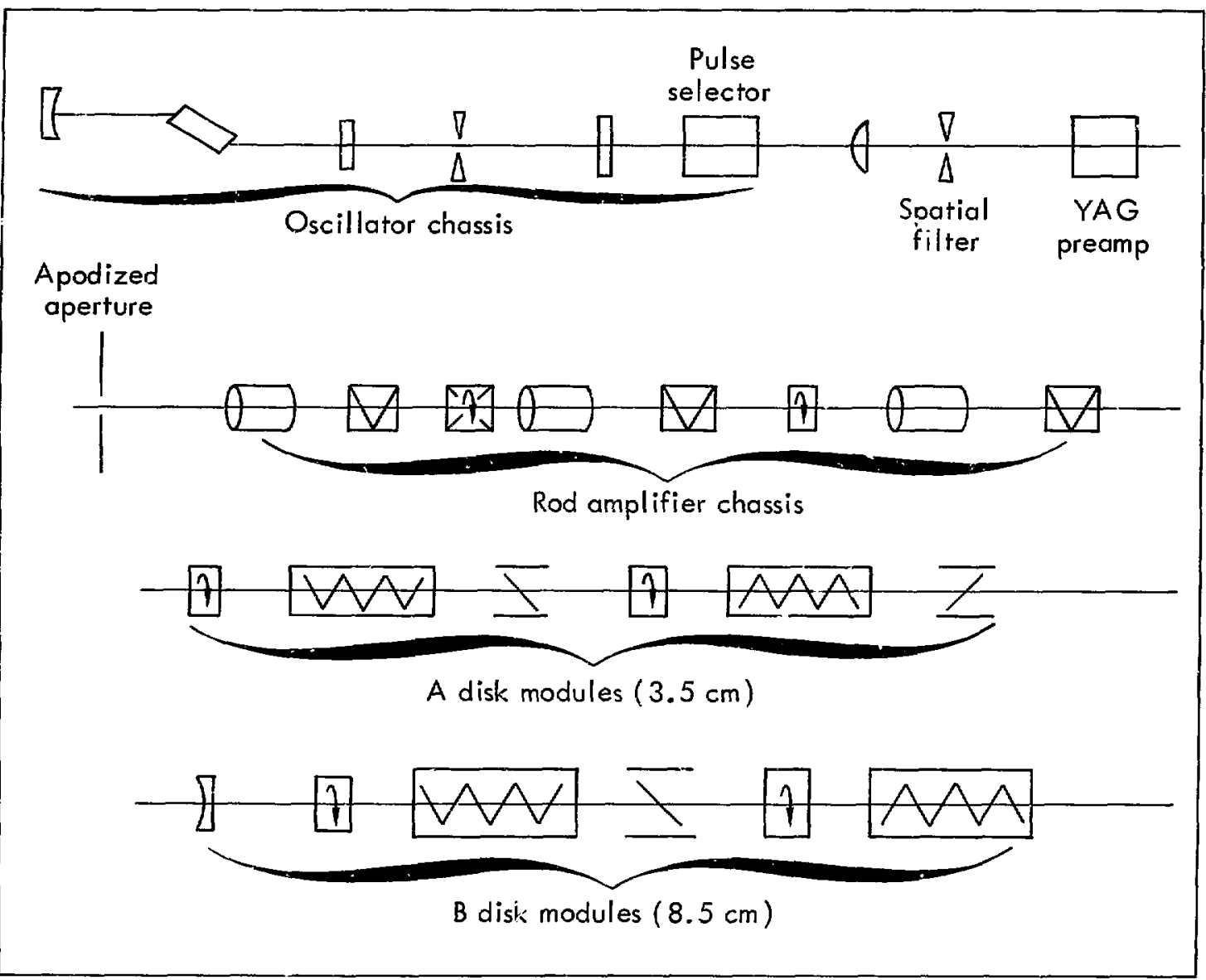

Fig. 56. Typical laser chain (part of the $1-\mathrm{kJ}$ system) showing the components involved.

\section{FARADAY ISOLATORS}

Table 13 contains a summary of the parameters for the six sizes of Faraday rotators we have built, or are building, with clear apertures ranging from $22.6 \mathrm{~mm}$ for the $A^{*}$ module to $334 \mathrm{~mm}$ for the $D$ module. Required stored energy ranges from 2 to $170 \mathrm{~kJ}$. Superconducting magnets were also considered: for 
Table 13. Summary of Faraday rotator parameters

\begin{tabular}{|c|c|c|c|c|c|c|}
\hline Module & $A^{*}$ & $A$ & $B$ & $C_{1}$ & $C_{2}$ & $D$ \\
\hline Clear aperture ${ }^{a}(\mathrm{~mm})$ & 22.6 & 41.1 & 98 & 166 & 225 & 334 \\
\hline Overail length (mm) & 101.6 & 152.4 & 241.3 & 294 & 370 & 507 \\
\hline Peak magnetic field (kOe) & 55 & 55 & 27.5 & 27.5 & 27.5 & 22.2 \\
\hline Bank voltage $(\mathrm{kV})$ & 3.5 & 4.1 & 5.0 & 19.5 & 19.4 & 19.8 \\
\hline Bank capacitance $(\mu F)$ & 240 & 480 & 720 & 182 & 430 & 868 \\
\hline Bank energy $(\mathrm{kJ})$ & 1.5 & 4.0 & 9.0 & 35 & 81 & 171 \\
\hline
\end{tabular}

${ }^{a}$ At $5^{\circ}$ angle of incidence.

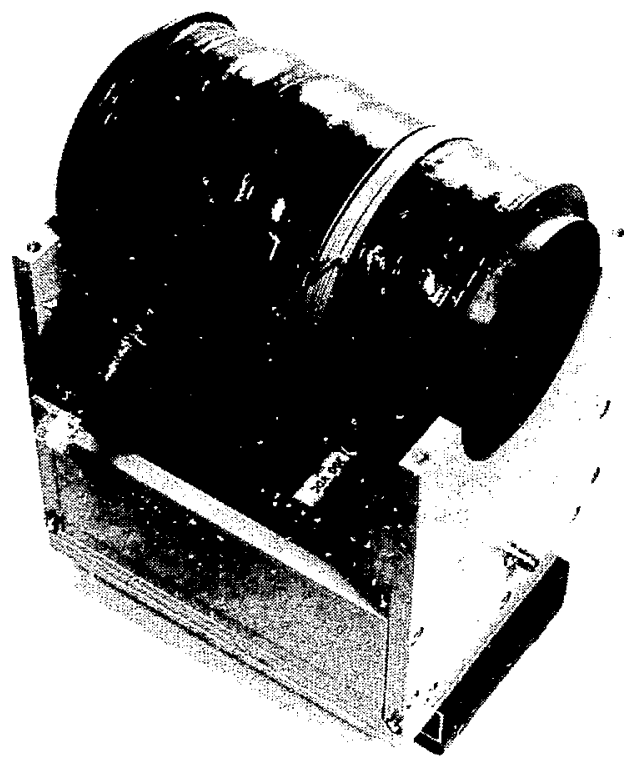

Fig. 57. Faraday rotator used in $B$ module of the $1 \cdot \mathrm{kJ}$ system, with cover removed. Aperture is $100 \mathrm{~mm}$. Secondary coil monitors magnetic field.

apertures greater than about $220 \mathrm{~mm}$ the acquisition cost of a superconducting magnet is less than the corresponding pulsed magnet with power supply and capacitor bank, but the cost of supplying liquid helium, either in bulk or as a closed refrigeration loop, makes the superconducting system more expensive even at the largest aperture.

The $A^{*}, A$, and $B$ (shown in Fig. 57) rotators have been replicated in quantities of six and have been integrated with various LLL systems. They are powered by a common design power-supply/ capacitor-bank, which differs from one rotator to another only in the installed capacitance. The $C_{1}, C_{2}$, and $D$ magnets are presently under construction, as are their power-supply/capacitor-bank units, which again are common to all three types.

Evaluation of rotator glass has continued. Both Ho $\rightarrow$ FR-4 and Owens-lllinois EY-1 have proved acc table in terms of damage threshold, nonlinear

index of refraction, and freedom from absorption, striae, inclusions, and birefringence. In the previous report the Verdet constant for FR-4 was erroneously represented as $-0.034 \mathrm{~min} / \mathrm{Oe}-\mathrm{cm}$; the correct value is $-0.031 \mathrm{~min} / \mathrm{Oe}-\mathrm{cm}$.

The actual isolation obtained is limited by the polarizer extinction ratio, the magnetic field uniformity, and the birefringence in the glass. The last can be due to residual strains or it can be thermally induced. Figure 58 shows the transmission through a Faraday isolator after the edges of the glass have been warmed by repeated pulsing of the magnet. The transmission of the brightest spot was measured to be $-23 \mathrm{~dB}$.

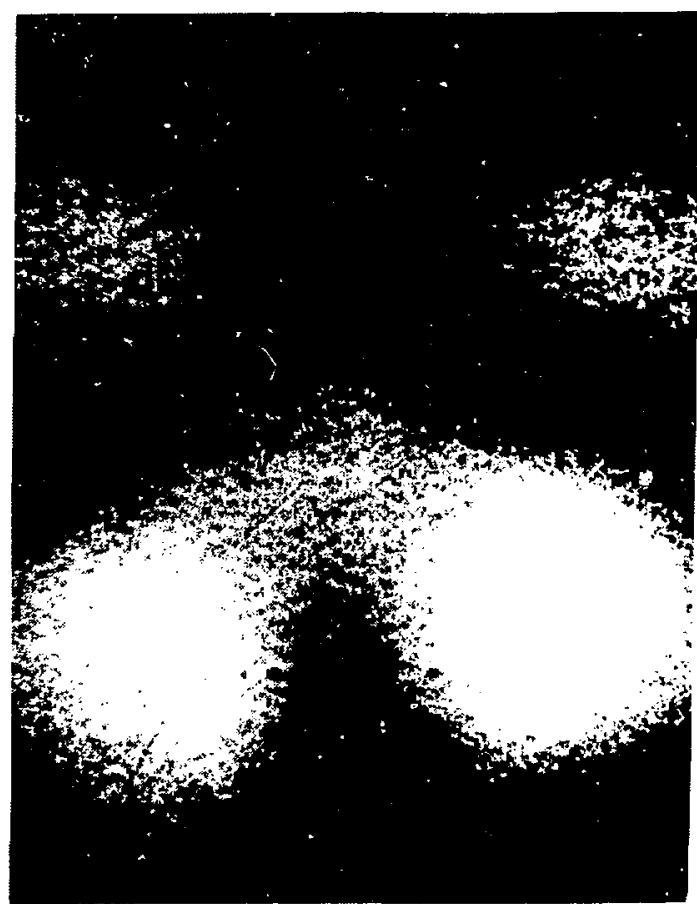

Fig. 58. Backward transmission through a Faraday rotator isolator whose glass-rod edges have heen warmed by repeated pulsing of the magnet, showing pattern characteristic of radial stress birefringence. Maximum transmission is $-23 \mathrm{~dB}$; minimum transmission is limited by polarizers to $-30 \mathrm{~dB}$. 
Multilayer-dielectric-coated polarizer samples have been acquired from some eight munufacturers. The polarizers are stop-band filters, generally designed for operation at an angle of incidence of $60^{\circ}$, so that the low-wavelength edge (reflection-to-transmission) occurs for a $4-6 \%$ shorter wavelength for $P$-polarization than for $S$-polarization. At our behest, several of the manufucturers have successfully moved their design from 60 to $56.5^{\circ}$, which is Brewster's angle for the substrate, thus avoiding interference from sesond-surface reflection. Considerable decrease of insertion loss has also been obtained, so that we now have polarizers with less than 5\% inser: 'on losis and an extinction ratio of better than $100: 1$, which do not damage at $3.4 \mathrm{~J} / \mathrm{cm}^{2}$ for 200-psec pulses.

Damage testing wili resume when the Internediate Laser System (ILS) is alvailable again. Generally we have found that the zirconium dioxide coatings have withstood damage better than titanium dioxide. Howe ever, in the light of recent work showing that damage in thin fiber dielectrics initiates at defects, our results may still be more characteristic of the individual coat. ing process than of the materials.

\section{APODIZING APERTURES}

The development of both the electrooptic and magnetooptic devices for using accentuated fringing fields to provide soft aperturing of a beam, described in the previous semiannual report, has been completed and is reported in UCRL-75272. The purpose of soft aperturing is to prevent the beam from developing diffraction fringes, which would self-focus and cause damage further down the chain. Figure 59 slows the theoretical transmission plus measured experimental points for a $10-\mathrm{mm}$-diam Pockels cell with the usual polarizer and analyzer, while Fig. 60 shows the theoretical transmission for a counterwound solerioid as well as photographs of the corresponding actual transmitted and rejected beams. The Pockels cell technique is limited by the availability of high quality crystals to apertures less than about $25.35 \mathrm{~mm}$, and the Fariday effect technique is limited in practice 10 apertures larger than about $50 \mathrm{~mm}$ by the fact that high radial inhomogeneity requires thin solenoid magnets: smaller apertures then require excessively lighi current destsities. The results illustrated in Fig. 59 were obtained at $5353 \AA$ with an EY-1 glass rod $50 \mathrm{~mm}$ in diameter by $25 \mathrm{~mm}$ long: the magnet was driven by $5.4 \mathrm{~kJ}$ of stored energy.
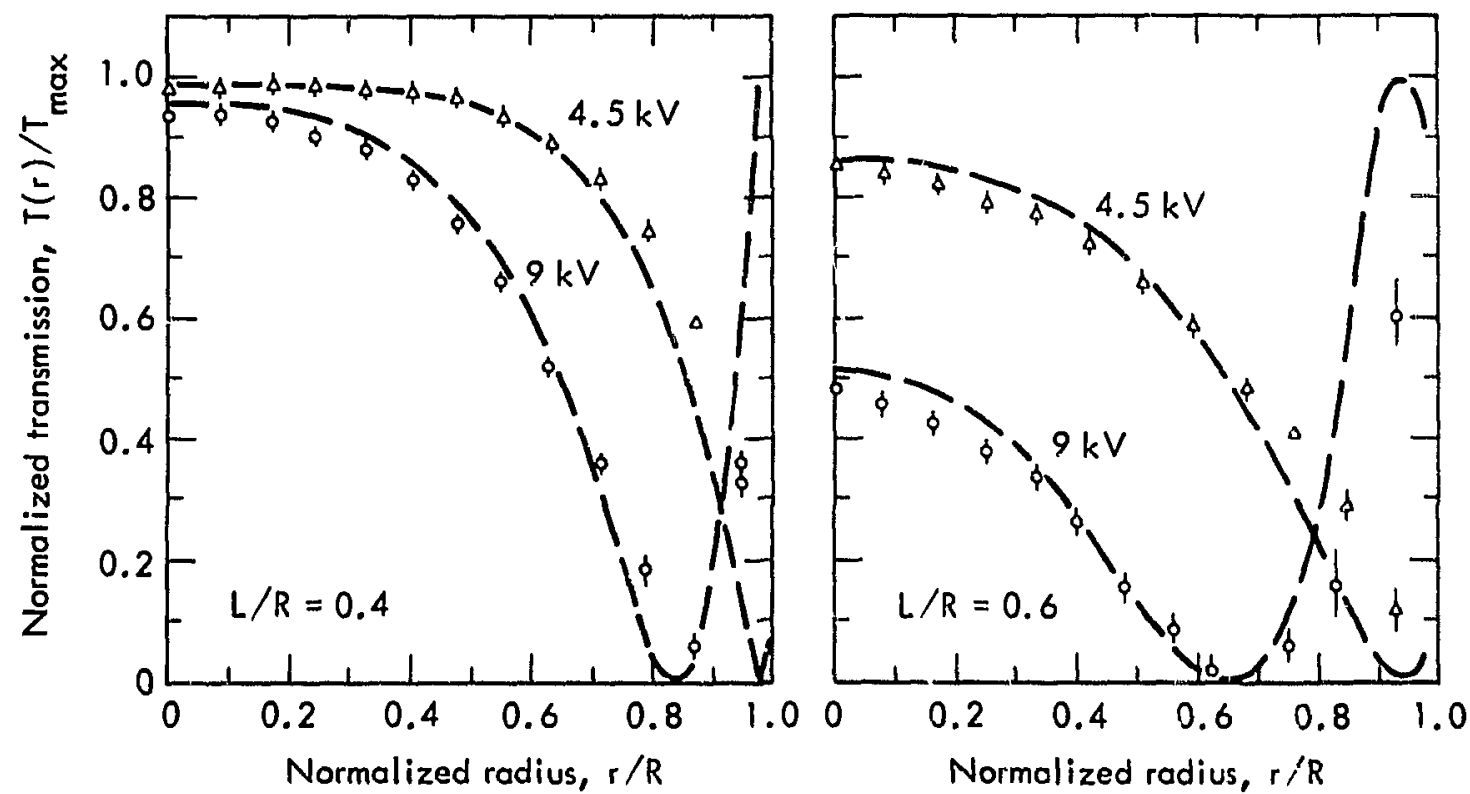

Fig. 59. Theoretical and messurcd transmission of a soft-aperturing $10-\mathrm{mm}$-diam Pockels cell (apedized aperture) as a function of radial distance from beam ecnter, with usual polarizer and analyzer. 


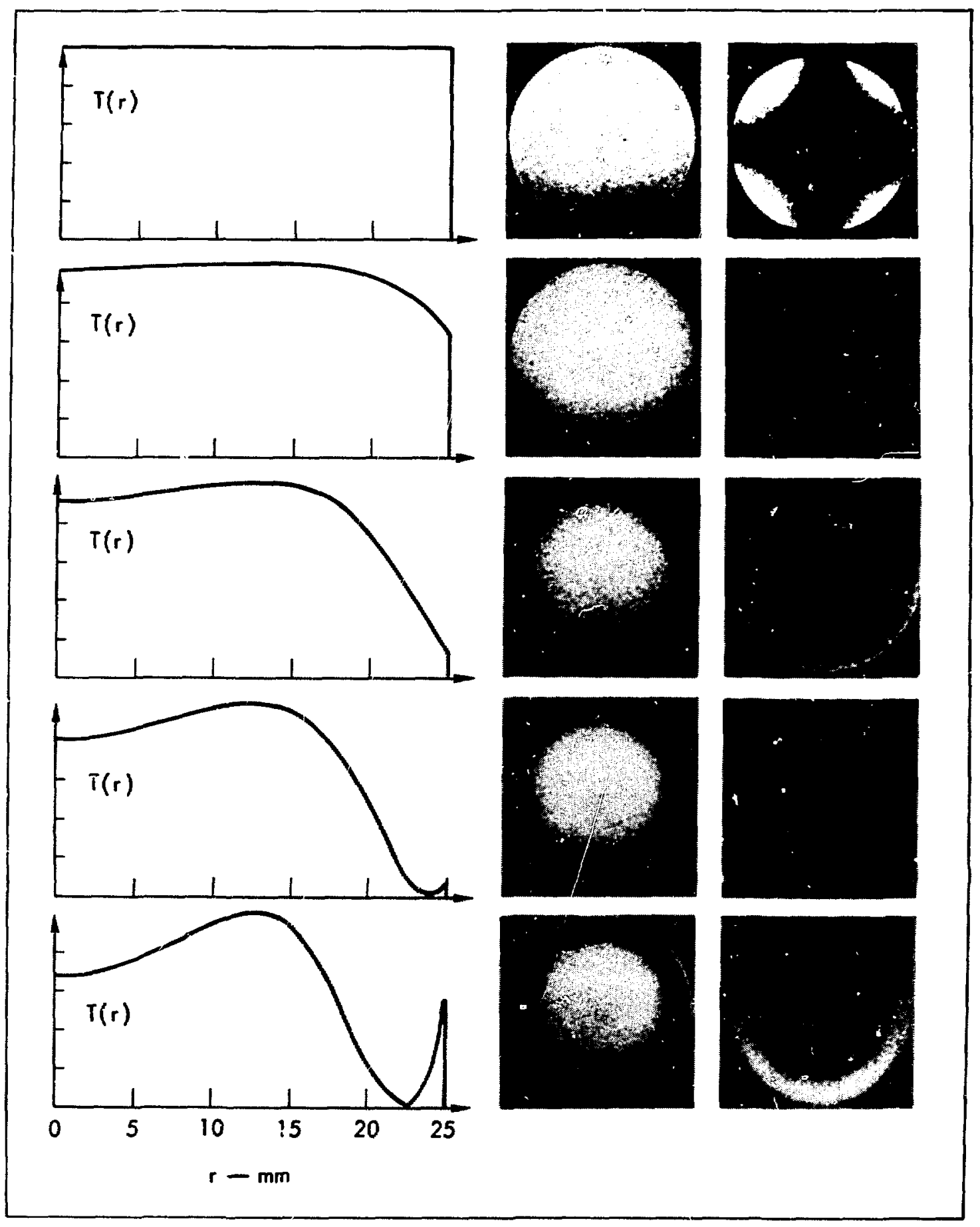

Fig. 60. Theoretical transmisxion for a counterwound-sotenoid apodized aperture, together with photos of the transmitted and rejected besms. 
As reported previously, evaluation of various calorimeter geometries has led to our adoption of 1-mm-thick BG-18 glass absorbers mounted on aluminum disks, one signal absorber and one reference or "dummy" absorber per calorimeter. The calorimeters were developed primarily for whole-beam energy measurement and system monitoring under the conditions of very high power. In practice the calorimeters have proven to be accurate and repeatable to a few tenths of a percent - even less under special circumstances - and hence are used in a wide variety of experiments including flashlamp studies, study of both linear and nonlinear absorption, measurement of nonlinear index of refraction, and harmonic generation. In the LC-3 calorimeter the absorbers are mounted over/ under, complete with entry ports. Although this arrangement is in part responsible for the low noise - less than $0.01 \mu \mathrm{V}$ witli sensitivity as high as $700 \mu \mathrm{V} / \mathrm{J}$ for the 12-mm-bore units - it is bulky. For apertures $50 \mathrm{~mm}$ and larger, the LC-10 type has been developed, wherein the absorbers are back-to-back with the dummy in a blind cavity. Suprisingly, this lias not increased the noise noticeably. The two types are illustrated in Fig. 61.

An extensive series of comparisons of various calorimeters was performed at the ILS, using a splitconverging beam arrangement. The calorimeters were of the LC-3 and LC-10 types with BG-18, VG-14, and
NG-1 absorbing glasses and of the LC-1 type with aqueous copper-sulfate solution. The response of the glass absorbers at various positions in the converging beam was constant up to the energy density where surface damage occurred, about 20 to $50 \mathrm{~J} / \mathrm{cm}^{2}$ for 200-psec pulses. The reflected and reradiated energy was measured with a reflectance calorimeter. Computer codes were also developed to estimate systematic errors due to differences in the heat losses for laser pulses and for electrical heating calibrations. After application of the measured loss corrections, the results for calorimeters with the four absorbing materials agree within a range of $0.5 \%$ and the absolute accuracy is estimated to be within $1 \%$. In use, the repeatability-i.e., the standard deviation of the ratio of two calorimeter readings in a series of shots-varies between 0.2 and $0.5 \%$.

Twenty-nine calorimeters of the LC-3 and LC-10 types, all using BG-18 glass as the absorber, have been built. with apertures from 13 to $127 \mathrm{~mm}$. A second series, all LC. 10 type, with apertures ranging from 100 to $300 \mathrm{~mm}$ are presently under construction. A number of these are fitted with Irtran as the absorber to be used at $10.6 \mu \mathrm{m}$. Two calorimeters of the LC-10 type without jackets have been fitted with sapphire absorbers and are being used for measurements of vacuum-ultraviolet laser systems.
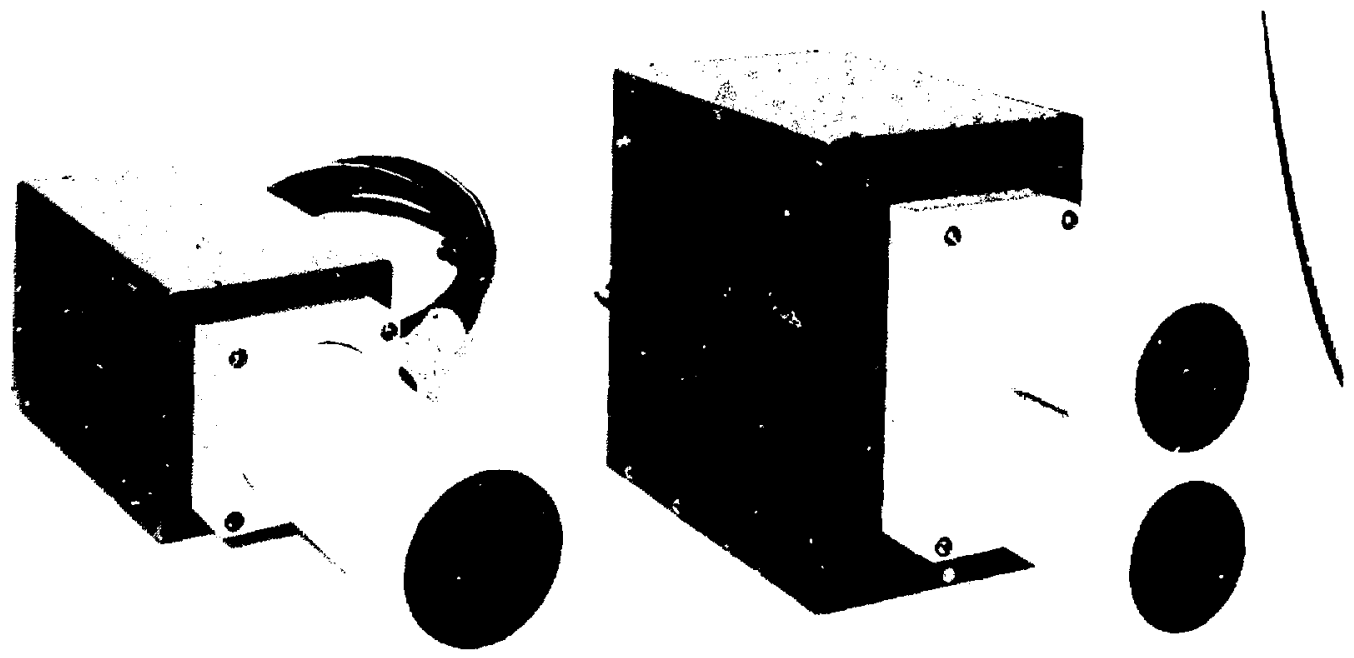

Fig. 61. Back-to-back LC-10 calorimeter with $26 \cdot \mathrm{mm}$ aperture (left), and side-by-side LC-3 ealorimeter with 13 -mm aperture (right). 
We evaluated series of Ge crystals, variously doped with $\mathrm{Hg}, \mathrm{Cu}, \mathrm{Au}$, and $\mathrm{Cu} / \mathrm{Sb}$, for sensi'ivity and rise time in a number of different mounts. The purpose of these tests was to evaluate different sources, determine variability, and evaluate the Dewars available for mounting the detectors (which must be cooled, usually to liquid helium temperature). Table 14 is a summary of the resuits. ${ }^{36}$

Clearly the response of the $\mathrm{Ge}: \mathrm{Hg}$ detector is the fastest, while the copper-doped and copper-tin-doped crystals were somewhat more sensitive.
Table 14. Results of tests of variously doped $\mathrm{Ge}$ detector crystals

\begin{tabular}{|c|c|c|c|}
\hline $\begin{array}{l}\text { Detector } \\
\text { crystal }\end{array}$ & $\begin{array}{c}\text { Sensitivity } \\
\text { (mifos) }\end{array}$ & $\begin{array}{c}\text { Rise time, } \\
10 \%-90^{\circ} \\
\text { (nsec) }^{\circ}\end{array}$ & $\begin{array}{c}\text { Fall time, } \\
90^{\circ}-10 \% \\
\text { (nsec) }\end{array}$ \\
\hline Ge:Hg ${ }^{\mathrm{a}}$ & $2-7 \times 10^{-3}$ & $<0.5$ & $1.4-2.0$ \\
\hline Ge:Cu & $2 \times 10^{-7}$ to $3 \times 10^{-4}$ & $1-2$ & $3.5-6$ \\
\hline $\mathrm{Ge}: \mathrm{Cu} / \mathrm{Sb}^{b}$ & $10^{-6}-10^{-4}$ & $1-3$ & $1-3$ \\
\hline Ge:Au & $10^{-2}$ & - & - \\
\hline
\end{tabular}

a One Ge:Hg sample had rise and fall times of 35 nsec, but a sensitivity of $3.5 \times 10^{-3} \mathrm{mho}$.

$b_{\text {Three of four samples were from the same boule and }}$ differed in sensitivity only by a factor of two. 


\section{DIAGNOSTICS}

Several discussions in this report have explicitly registered the need for detailed characterizations of the experimental variables (e.g., laser pulse length and spatial profile) involved in the exploration of the laserfusion concept. In most cases the required characterizations and measurements must be derived from carefully instrumented, sensitive, and highly sprecific diagnostics. Since comparison of theory and experiment is !imited by the quality and range of experimental information, considerable effort has been devoted to the development of the appropriate diagnostic packages. The Diagnostics Group has carried on a variety of projects concerned with developing high-resolution techniques for measuring the characteristics of laser pulses and laser-produced plasmas. Our development work on ultrafast streak cameras, making them applicable to a variety of wavelengths from the infrared to the $x$-ray region, has been very successful. Now we are investigating data acquisition techniques that can replace the film in streak cameras. We have worked out methods for dignosing optical distortion in active lasing media, and these methods will soon be applied to the study of the Nd:glass amplifier modules. Developments relevant to target/plasma diagnostics have proceeded in the areas of highresolution interferometry and charged-particle diagnostics. Details of these activities are presented in the following sections.

\section{LASER-PLASMA FACILITY}

An important advance in our capabilities was provided by the opening of our Laser-Plasma Diagnostics Laboratory. A modest Nd laser produces nominally 1-J, 130-psec-FWHM pulses. The laser output has been used for testing and calibrating laser pulse diagnostics as well as for target irradiations in order to pursue the development of plasma diagnostic techniques. A schematic of the target chamber and some of the current instrumentation that is being used is shown in Fig. 62 .
Figure 63 is a photograph of the target chamber; the diagnostic instrumentation can be identified by comparison with Fig. 62. We have irradiated slab targets of plast:c and metal with beams having focal spot diameters of about $200 \mu \mathrm{m}$. Results of work using this facility are shown in some of the following sections. The facility has provided an important and reliable tool for our diagnostics development.
The basic concept and design of the 10-psecresolution ultrafast compact streak cameras have been described previously. ${ }^{8}$ Figure 64 is a photograph of one of the cameras which incorporates a videu data acquisition system (an SSRI optical multichannel analyzer) in place of photographic film. Five compact cemeras have been constructed and are in use in the laser program.

We have obtained additional data relating to the detailed characteristics and operation of the camera. The film exposure threshold for the camera using a 30-psec Nd:YAG oscillator pulse is between $10^{-9}$ and $10^{-11} \mathrm{~J}$ at the camera slit. The differences are due to variations in the $S-1$ cathode sensitivities in the imageconverter (streak) tubes. This input sensitivity is roughly $10^{2} \mathrm{~W} / \mathrm{cm}^{2}$ on the photocathode. These values are for cathodes with sufficient sensitivity to insure linear operation of the camera. We have had problems with some cameras showing a nonlinear intensity response. The source of this problem was found to be the badly degraded sensitivity of the S-1 photocathodes in some of the tubes. Figure 65 shows an example of our measurements of the streak tube response functions using 30-psec, $1.06-\mu \mathrm{m}$ pulses. As can be seen, sensitive cathodes (those acceptable for streak camera use) exhibit a linear response, that is, the curve has a slope of 1 on the log-log plot. Degraded cathodes have a large loss in sensitivity at $1.06 \mu \mathrm{m}$ and show a highly nonlinear response (slopes varying from about 2 to 6 in our experience). This degradation appears to occur randomly with time. Some cathodes lose sensitivity (this loss occurring predominantly at the long- 


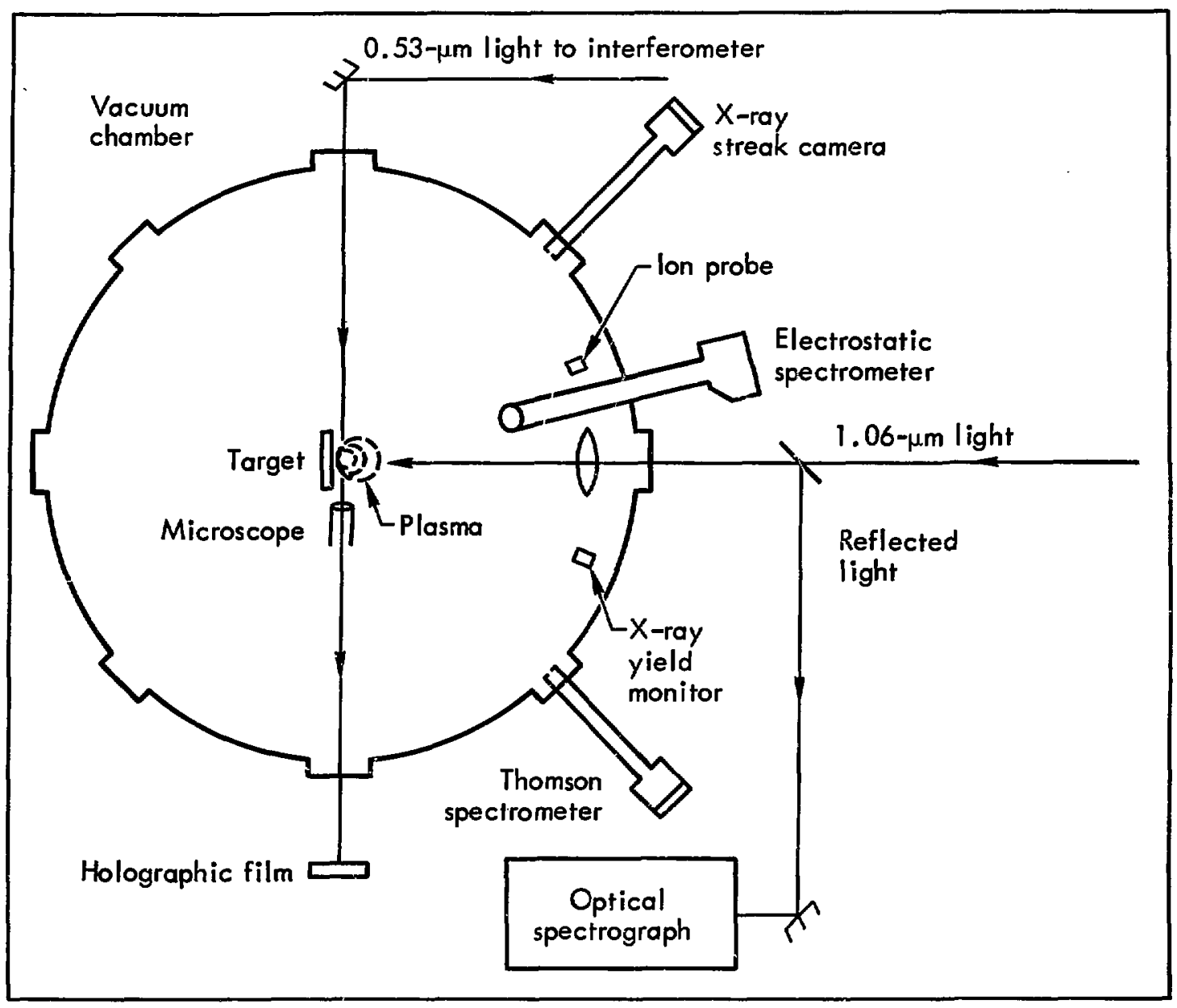

Fig. 62. Target chamber and some of the associated diagnostic equipment for laser-induced plasma studies.

wavelength region of their response characteristic) within two v'eeks of manufacture, yet others have retained ingh sensitivity for over three years. The high slope exhibited by degraded cathodes severely limits the dynamic range of the camera and compromises its overall capabilities. We are beginning efforts to support study and solution of this cathode stability problem.

Figure 66 is a curve of the intensity response at $1.06 \mathrm{\mu m}$ of a compact camera containing a sensitive S-1 photocathode. The da: a were recorded on film, but the film response has been normalized out in order to show the response of the camera. As can be seen, the response is linear and the usable dyanamic range exceeds $100: 1$, the highest yet reported for ultrafast streak cameras.

Figure 67 is a detailed sweep linearity curve we have obtained. The sweep speed is constant to $3 \%$ over $80 \%$ of the $40-\mathrm{mm}$ output aperture.

The reliability and operating convenience of these cameras continues to be good as they provide valuable diagnostic capability in our program.

\section{X-RAY STREAK CAMERA}

The basic concept and the design modification of a standard image-converter tube to make an ultrafast $x$-ray-sensitive streak camera were discussed in the previous progress report. ${ }^{37}$ During this reporting period we have addressed the questions raised in that initial discussion. For our first rather crude camera the 


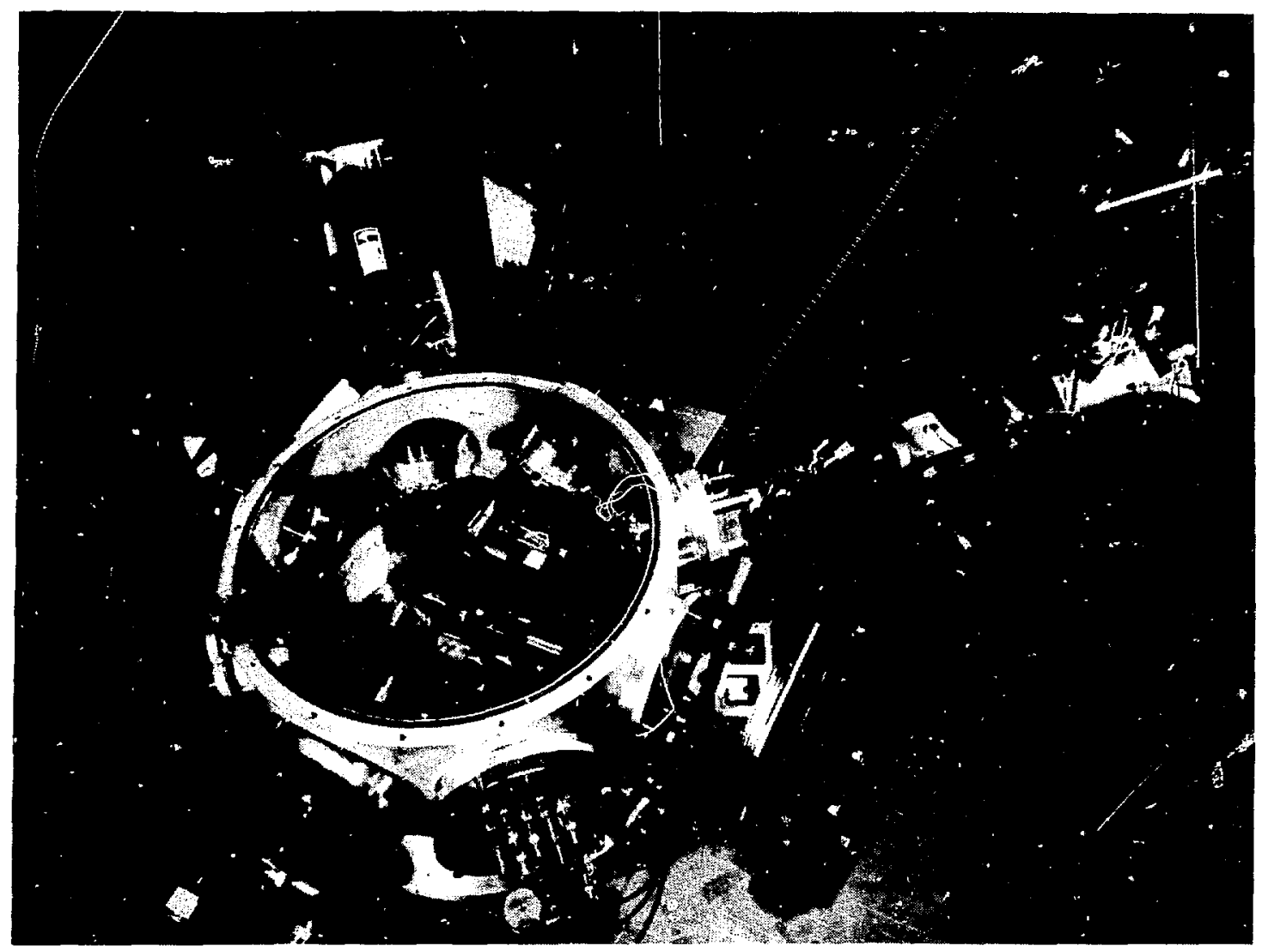

Fig. 63. Photugraph of the laser-plasma target chamber.

sensitivity has been found to be quite high, an estimate of the streak speed has been obtained, and the photocathode electron energy spread has been determined not to be a severe limitation on time resolution for $x$ rays in the few-kilovolt range.

The $x$-ray-sensitive streak tube was made by installing an $x$-ray-sensitive photocathode and a new extraction grid in a standard commercially available image-converter streak tube (RCA C73435 series). ${ }^{40}$ The cathode is a $100-\AA$-thick gold layer supported on a 0.33 -mil Be vacuum window. 37

In our initial experiments with the x-ray streak camera, which is shown schematically in Fig. 68, a 13-mil-diam aperture was placed in front of the $x$-ray photocathode. This aperture fixes the spatial resolution: by limiting the usable photocathode area. The resultant streak is a straight line of varying intensity. The camera depicted in Fig. 68 uses components that were easily available. Because of the lens coupling and the characteristics of the image intensifier, the overall optical gain of the system is low, about 20 . Nevertheless, the sensitivity of the camera is quite good. X-ray streaks have been obtained with the camera 10 in. from an iron target irradiated with a 1-J, 130-psec Nd:glass laser pulse. The exposure on the apertured cathode was about $30 \mathrm{~nJ}$ of $1-\mathrm{keV}$-average $\mathrm{x}$ rays.

The streak speed of the $x$-ray camera has been determined by observing the $x$-ray emission from an iron target during irradiation by two 0.5-J, 130-psec laser pulses separated by 300 psec. Figure 69 is the streak photograph obtained, and Fig. 70 is a densitometer scan of the streaks. The ripples in the densitometer scan are noise due to low incident flux and low film exposure. The resolution determined from Fig. 70 is consistent with our original estimate of 50 psec for the camera's time resolution. ${ }^{37}$

A new x-ray streak camera is presently under construction which will have a $1-\mathrm{cm}-$ by-150- $\mu \mathrm{m}$ slit photocathode. The tube has a fiber-optic output window and will be contact-coupled to a high-gain, micrchannel-plate image intensifier as used in our optical streak cameras. This will provide higher light gain and better usable range on the recording film. The slit cathode on the new camera will provide one degree 
of spatial resolution, naking possible time-resolved measurements of x-ray spectra and spatial intensity (imaging) variations which will substantially enhance our diagnostics capabilities for laser-produced plasmas, which in turr will provide fundamental information necessary for effective target design.

\section{OPTICAL KERR EFFECT DETECTION SYSTEM FOR 10.6- $\mu \mathrm{m}$ PULSFS}

A subnanosecond detection system for use with high-power 10.6- $\mu \mathrm{m}$ pulses has been built around an ultrafast streak camera and an optically driven Kerr cell. ${ }^{38}$ Previous reports have described this system, its operation, and its characterjstics. ${ }^{39}$ This report describes recent improvements in the system.

Until recently, the major obstacle to perfecting this system was the unavailability of a probe beam which was temporally smooth on the subnanosecond time scale. This problem has been overcome recently by using a $\mathrm{cw}$ argon-ion laser stabilized on a single longitudinal mode by an intracavity etalon. Good results have been obtained with both the Spectra-Physics model 165 and the Coherent Radiation model 52.

An LLL ultrafast streaking camera with an S-20 photocathode and a time resolution of $10 \mathrm{piec}$ has been used for high-time-resolution streak photography of 10.6- $\mu \mathrm{m}$ pulses from a mode-locked $\mathrm{CO}_{2}$ TEA oscillator. The experimental arrangement is shown in Fig. 71. The $\mathrm{CO}_{2}$ and argon lasers are linearly polarized $45^{\circ}$ relative to each other. Salt beam splitters are used for supplying a small fraction of the $10.6-\mu \mathrm{m}$ energy to nanosecond-response helium-cooled detectors. One of these detectors supplies a timing signal for the streak camera and the other is used for observing the pulse shape. A screen room provides electrical isolation for the streak camera and oscilloscope.

Representative streak photographs of $10-\mu \mathrm{m}$ pulses are shown in Fig. 72. The total streaking time for the camera is less than the pulse duration in these cases. The streaking speed is $33 \mathrm{psec} / \mathrm{mm}$, yiclding a frame duration of $1.3 \mathrm{nsec}$. A variable time delay allows one to change the streak camera timing relative to the $\mathrm{CO}_{2}$ pulse. Figures $72 a$ and $72 b$ show the front and rear portions of a pulse. These data are the first measurements made of $10.6-\mu \mathrm{m}$ laser pulses with 10-psec time resolution. The pulses are smooth and there is good contrast between the background and the peak of the pulse.

In the near future, this system is scheduled io be used for diagnosing a laser which is expected to produce a much shorter $10.6-\mu \mathrm{m}$ pulse. At that time, turther experimental verification of its high-timeresolution capability will be demonstrated.

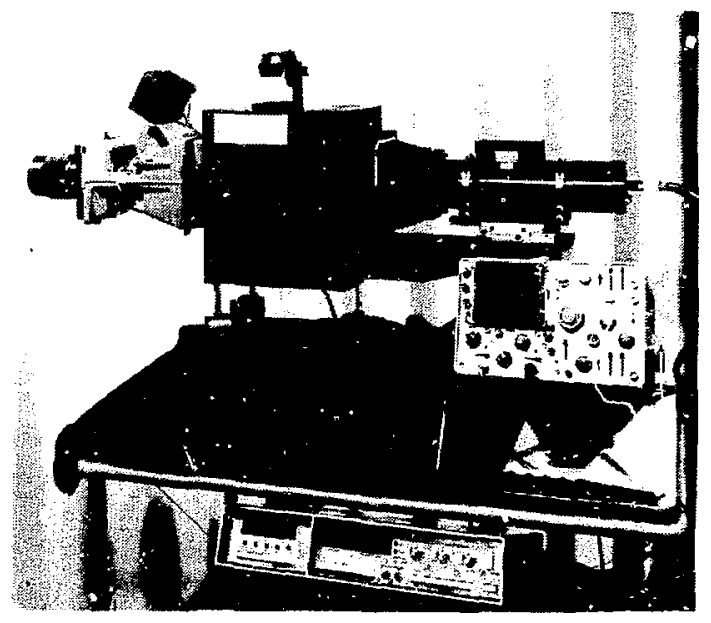

Fig. 64. This compact 10-pse، esolution streak camera uses a video data acquisitioli system instead of photographic film.

\section{HOLOCAMERA FOR DIAGNOSTICS OF WAVEFRONT DISTORTION IN LASING MEDIA}

fin the previous semiannual report we discussed the use of holographic interferometry for the investigation of pump-induced dynamic wavefrent distortion in Nd:glass disk amplifiers. A "holocamera" based on the configuration of a Mach-Zehnder interferometer has been assembled with 10-cm-aperture optics. The capabilities of the system have been fully evaluated with a single-pulse ruby laser. These test. results give us confidence that this simple "holocamera" is capable of producing high-quality holographic interferograms, even when operating with a single-pulse ruby laser. We plan to first use the device to examine dynamic wavefront distortion in the $A$-amplifier $\mathrm{Nd}$ :glass disk module. which has a clear aperture $3.5 \mathrm{~cm}$ in dianeter and a holising length of approximately $1 \mathrm{~m}$. The actual holographic testing of the $A$-module is scheduled for 


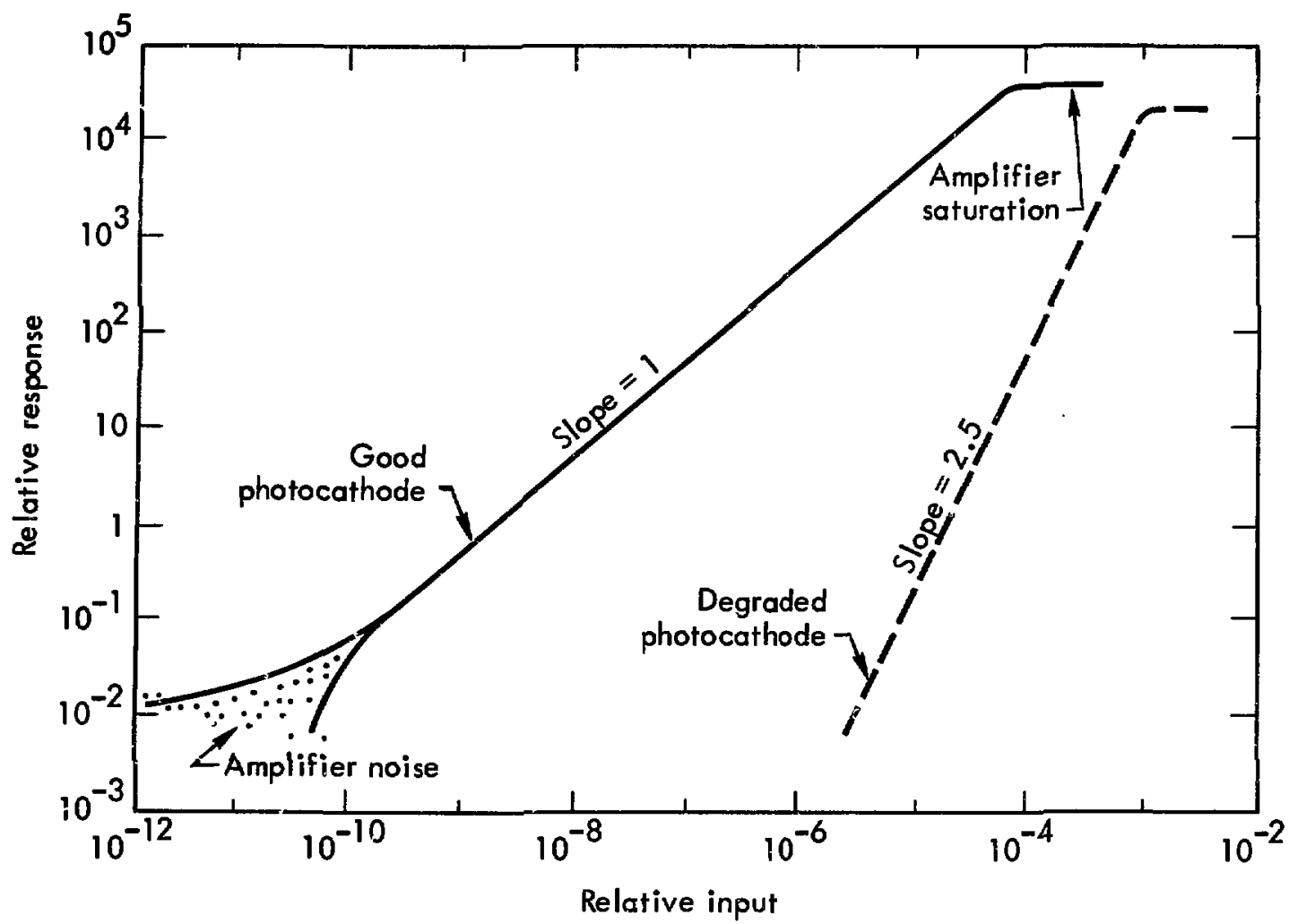

Fig. 65. Typical measure 1 response of S-1 streak tube with good photocathode and degraded photocathode to 30-psec, 1.06- $\mu \mathrm{m}$ input pulse.

Late January 1974.

As mentioned in the previous report, short coherence lengths may be experienced with double-pulse operation of a ruby laser, thus requiring the use of a path-matched holographic interferometer when the aperture of the tested module becomes large (e.g., modul: $D$ will have a $30-\mathrm{cm}$ clear aperture). A reflective turning plate has thus been proposed for insertion into the holographic interferometer in order to tilt the phasefront of the "reference" beam. Such piecewise turning elements have until now been used only in conjunction with a nearby ground-glass diffuser and imaging optics in the "scene" beam. 40 In that scheme, the optical quality of the turning elements is immaterial because of the diffusing effect of the nearby ground glass. However, because of the small aspect angles in the amplifier modules, we were unable to efficiently use the ground glass. In our case, where the turning elements are used in the "reference" beam without a ground-glass diffuser, high optical quality is essential. Our first efforts at making a suitable reflective turning plate were not successful; holograms werc made but the reference beam quality was poor. Further efforts are now under way to improve the optical quality of this unusual optical element. The

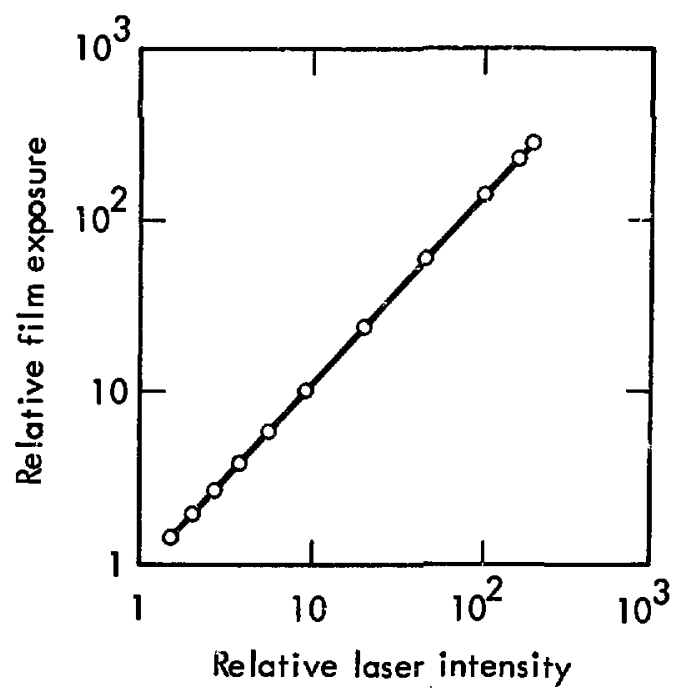

Fig. 66. Measured response of a compact streak camera with sensitic S-1 photocathode to a 150-psec, $1.06-\mu \mathrm{m}$ pulse. 
coherence length problem may also be minimized by operating the laser in single-pulsed mode, and by decreasing the angle subtended by the "scene" and "reference" beams in the interferometer configuration. Each of these techniques will be used to full advantage as this program develops.

The short pulse ( $30 \mathrm{nsec}$ ) will allow us to take a series of "stop action" interferograms during the much longer (several hundred microseconds) pump cycle of the Nd:glass disk amplifier modules under test. In this way, the development of optical distortions may be traced and operating points at which these effects are minimized can be found. The possibility also exists of making holograms instead of phase interferograms in order to help localize the causes of severe disturbances. These techniques will provide experimental data to guide iteration of the amplifier designs to minimize optical distortions and inhomogeneities.

\section{HOLOGRAPHIC MICROINTERFEROMETRY OF LASER-?RODUCED PLASMAS}

During the last reporting period, we described a holographic microscope $e^{41,42}$ capable of $2-\mu \mathrm{m}$ resolution when viewing stationary objects. In the past six months we have extended the use of this holographic scheme to obtain microinterferograms of laserproduced plasmas with a temporal resclution of approximately 100 psec. A single laser is used for both production and diagnostics of the plasma, as shown in Fig. 73. A sample of the mair heating pulse is split off and frequency-doubled to $5320 \AA$ before entering the interferometer. The holographic interferometer is shown in Fig. 74. The snly unusual element is the microscope objective which permits us to obtain high spatial resolution. The absence of diffuse surfaces between the laser and holographic plate eliminates the diffractive effects of coherent speckle.

Two results obtained with this technique are shown in Fig. 75. They were each obtained with a half-joule of $1.06-\mu \mathrm{m}$ radiation focused to a $200-\mu \mathrm{m}$ spot on the surface of an aluminum slab. The duration of the heating pulse was approximately $130 \mathrm{psec}$ FWHM. The result shown in Fig. 75a was obtained at a delay time of 1 nsec after the arrival of the 1.06- $\mu \mathrm{m}$ heating pulse. Six fringes are evident in the original reconstruction, indicating an electron density of $10^{20} \mathrm{e} / \mathrm{cm}^{3}$ at a point $40 \mu \mathrm{m}$ from the slab surface. The spatial resolution evident here is approximately $30 \mu \mathrm{m}$, a value determined by the $0.1-n s e c$ duration of the diagnostic pulse and the observed fringe velocit; of $300 \mu \mathrm{m} / \mathrm{nsec}$. The result shown in Fig. 75b, which has three fringes, was obtained with a delay of 0.5 nsec. In this case, the main laser pulse was not focused on the surface of the target; hence the flattened appearance.

Future work will concentrate on development of shorter diagnostic pulses, which will improve spatial resolution as well, and the use of shorter-wavelength probing radiation. The use of $3547-\AA$ or $2660-\AA$ radiation will reduce refractive effects within the plasma, and reduce this number of fringes in higher energy laser systems. The ultimate goal of this program is to determine the spatial distribution of electron density in the vicinity of the critical $\left(10^{21} \mathrm{e} / \mathrm{cm}^{3}\right)$ surface associated with the heating $1.06-\mu \mathrm{m}$ pulse. The determination of density gradients in this vicinity will be an important aid in assessing the importance of competing absorption and reflection processes in the laser-proriuced plasma.

\section{ION SPECTROSCOPY OF LASER-PRODUCED PLASMAS}

Two diagnostic devices for ion spectroscopy of laser-produced plasmas have been built. We have constructed a $127^{\circ}$ curved-plate electrostatic energy analyzer and a Thomson mass spectrometer from basic designs provided by Sandia Laboratories, Albuquerque. Both devices have been used to observe plasmas produced by subnanosecond $1.06-\mu \mathrm{m}$ laser pulses striking planar targets in our diagnostics facility.

The curved-plate electrostatic energy analyzer which is shown in Fig. 76 has been used to measure the energy and charge distributions of ions emanating from laser-produced plasmas. This is basically a time-of-flight device in which only ions of a preselected energy-tocharge ratio pass through the spectroreter and reach the detector at times determined by the masses of the ions and their kinetic energies. $A$ representative analyzer output is shown in Fig. 77. This device requires many repeatable laser firings in order to determine average energy and charge distributions of the ions; however, it is well suited for studies at particular energies. 
The Thomson spectrometer, which is shown in Fig. 78 , is capable of measuring a wide range of ion energy and charge distributions on one shot. It uses parallel magnetic and electric fields to spatially separate ions of different energies and charge-to-mass ratios. Ions with the same charge-to-mass ratio form a parabola at the output; position along the parabola is directly related to ion energy. A separate parabola is obtained for each individual ion species, so that the output appears photographically as a series of parabolas. The density of the photograph at any point on a parabola is related to the number of ions of that charge-to-mass ratio with that energy. A novel feature of this instrument is the use of a channel-electron-multiplier array to amplify the signal while retaining spatial information by converting ions to electrons, increasing the number of electrons by secondary emission along a continuous dynode, and finally accelerating the electrons to a phosphor-coated sreen that can be photographed. Figure 79 is is photograpl taken with the instrument after irradiation of a $\mathrm{CH}_{2}$ target. The parabolas corresponding to ionized hydrogen and all six carbon species are clearly visible.

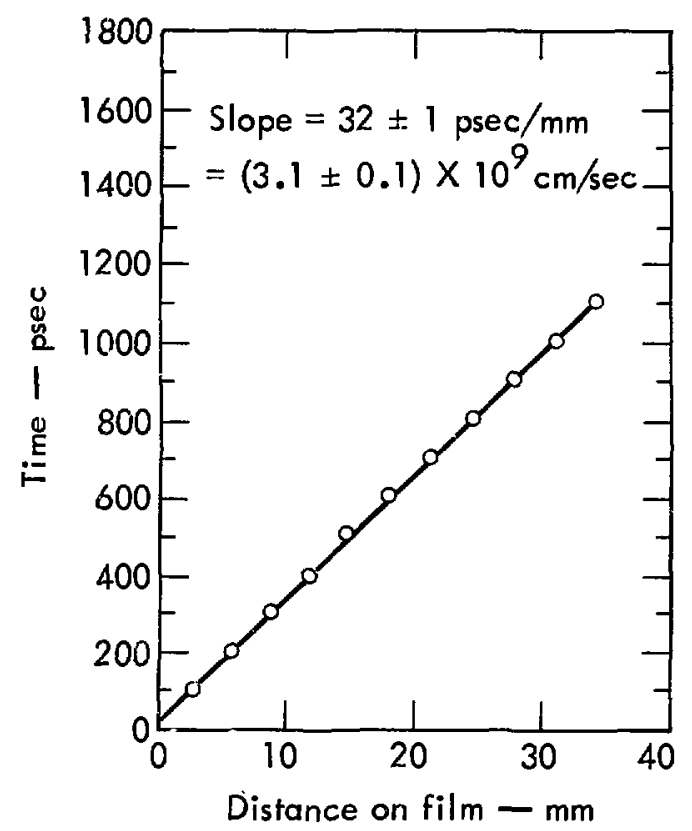

Fig. 67. Sweep linearity curve for compact streak camera.

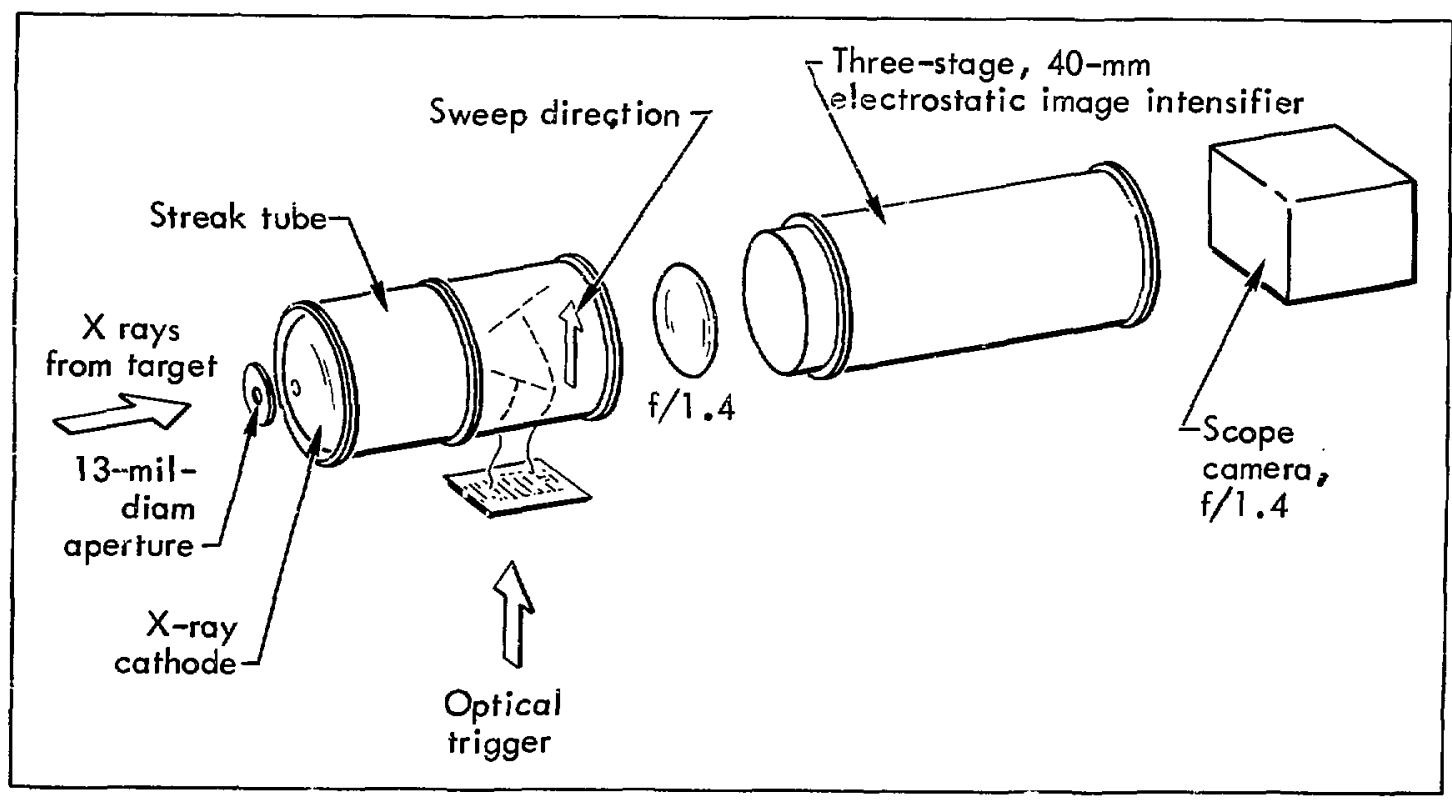

Fig. 68. Schematic of LLL's x-ray streak camera. 


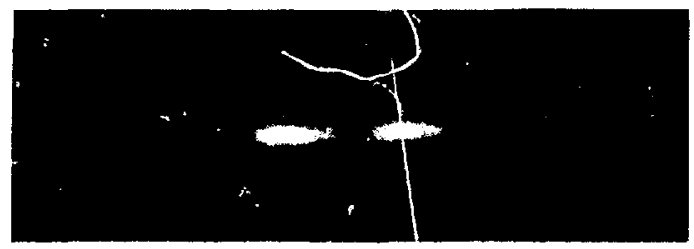

Fig. 69. Photograph obtained with the $\mathrm{x}$-ray streak camera.

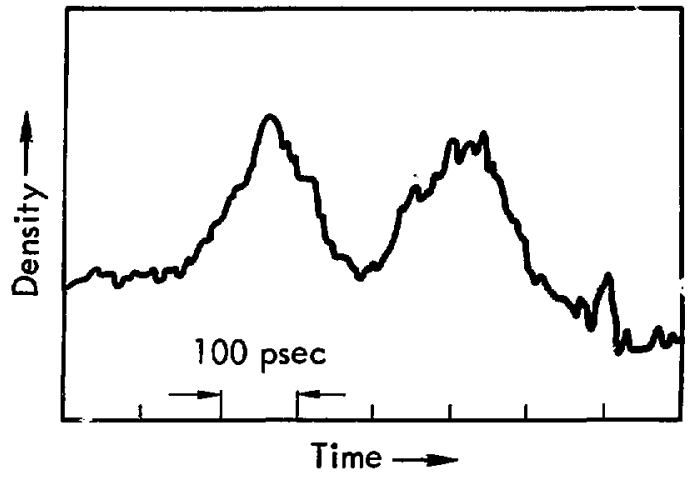

Fig. 70. Densitometer scan of the struaks in Fig. 69.

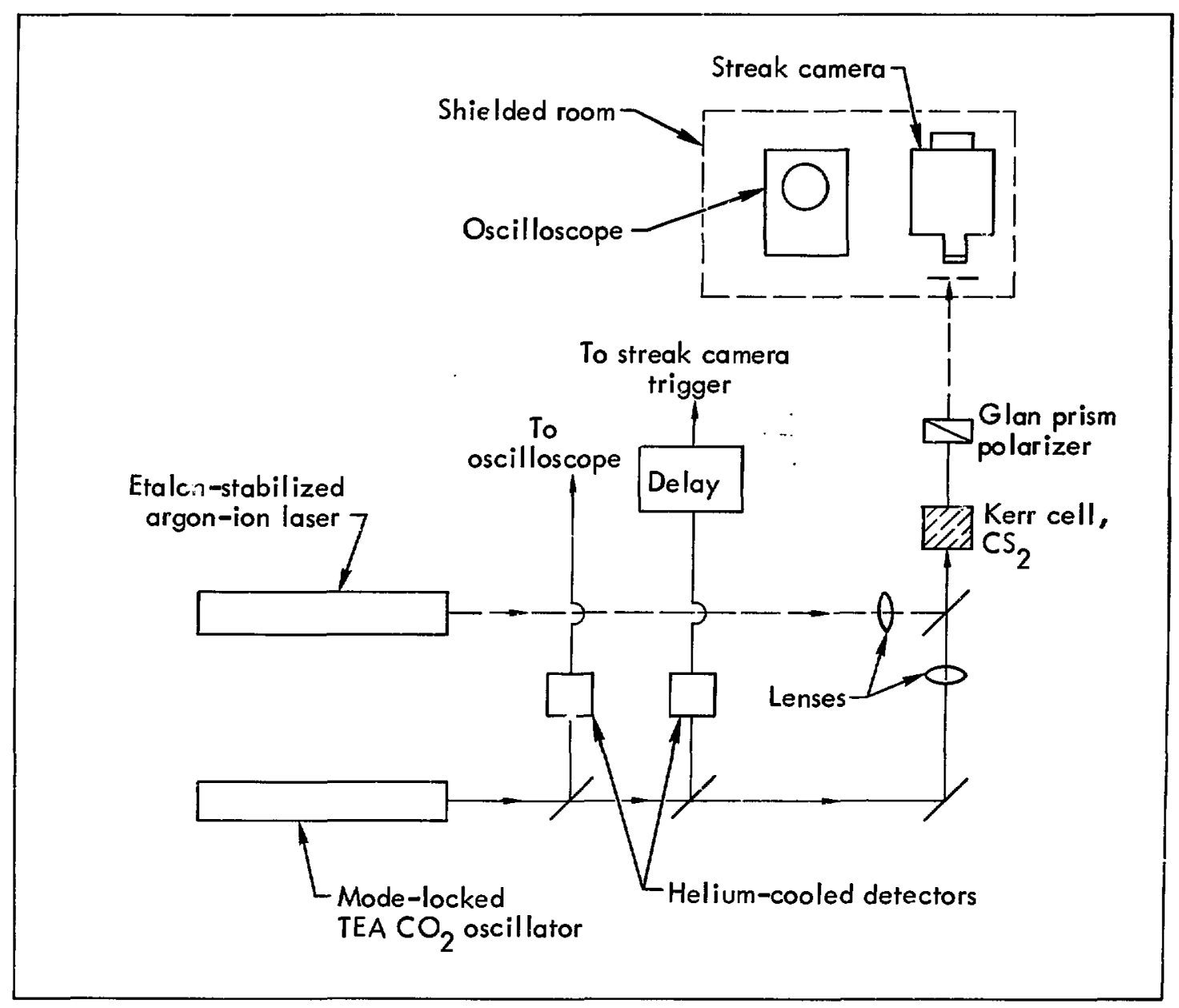

Fig. 71. Schematic of subnanosecond detector system for $10.6-\mu \mathrm{m}$ pulses based on an ultrafast streak camera and the optical Kerr effect. 

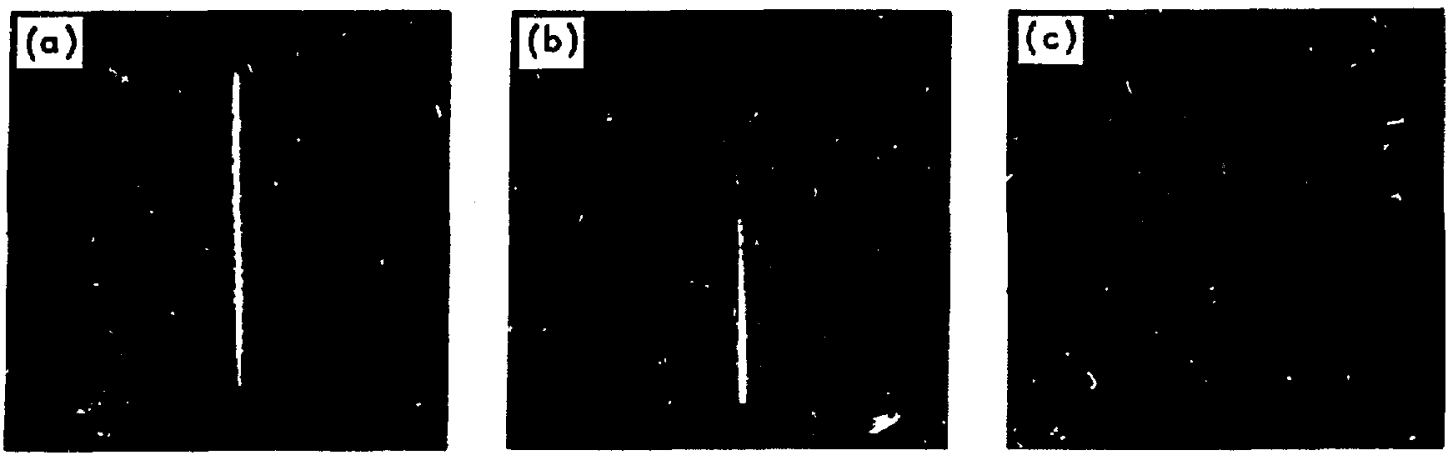

Fig. 72. Typical streak photos mede of $10.6 \cdot \mu \mathrm{m}$ pulses with the detector system of Fig. 71.

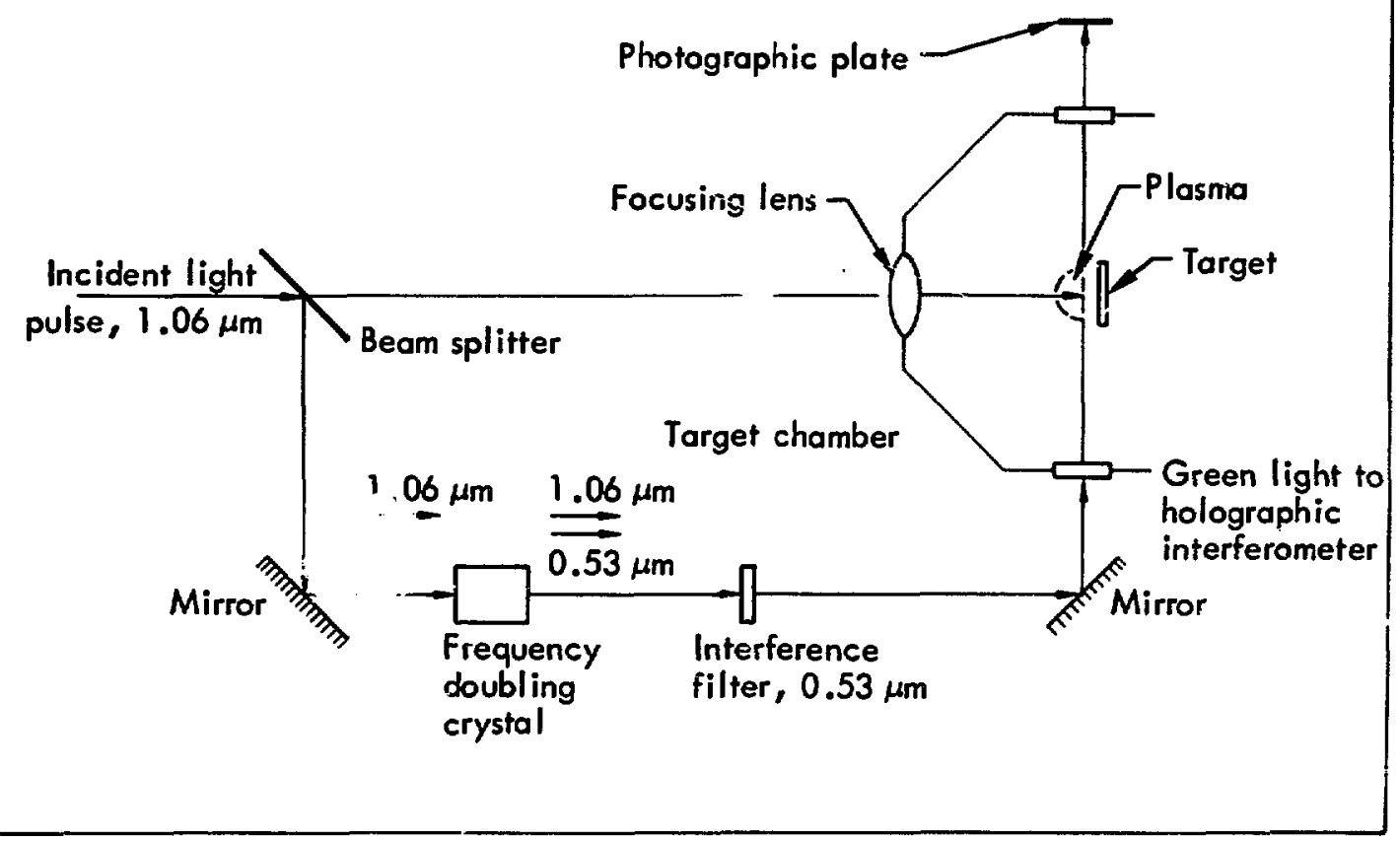

Fig. 73. A single laser is used both to produce and diagnose the plasma in this LLL holographic interferometer experiment. 


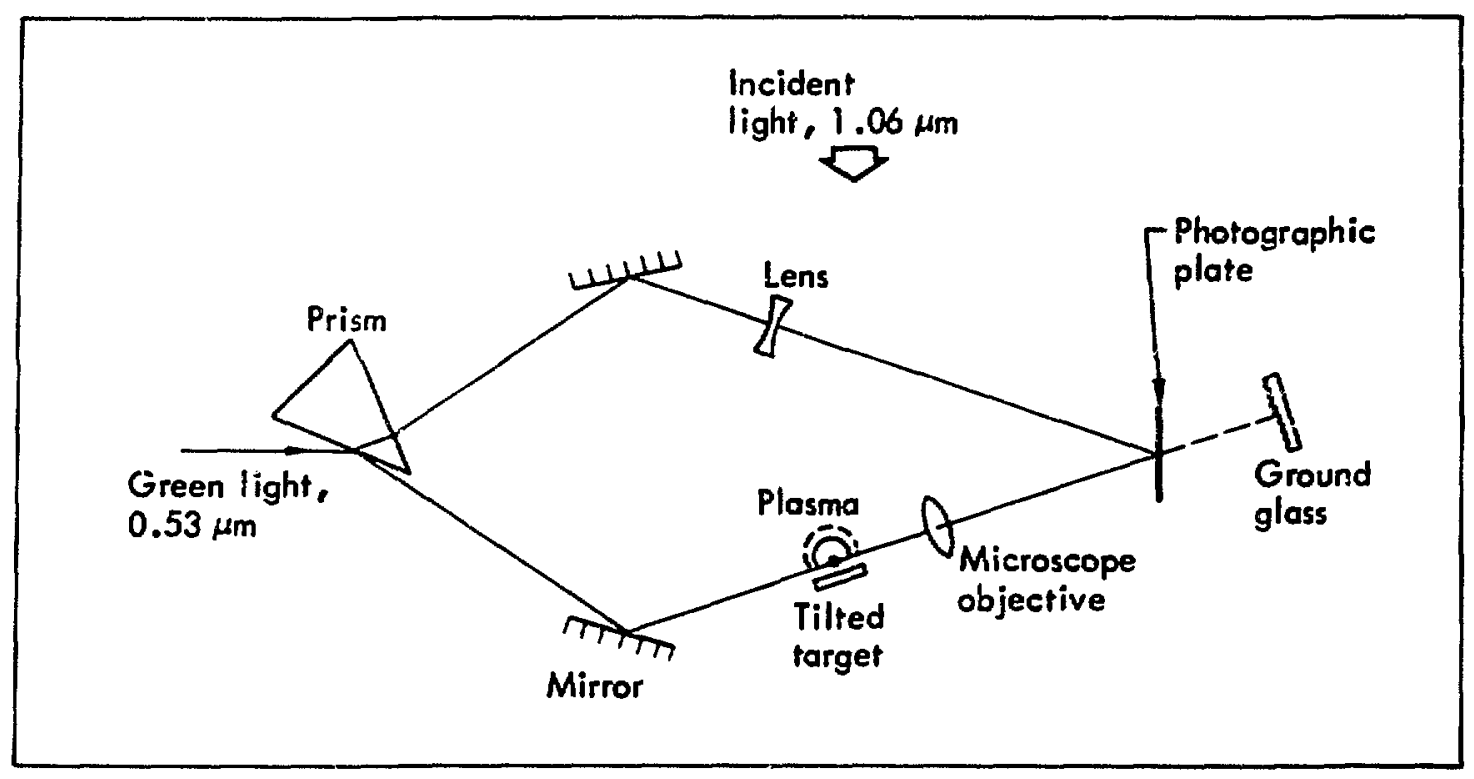

Fig. 74. Optical interterometer uxed in experiment of Fig. 73 employs hologrsphic microscopy.
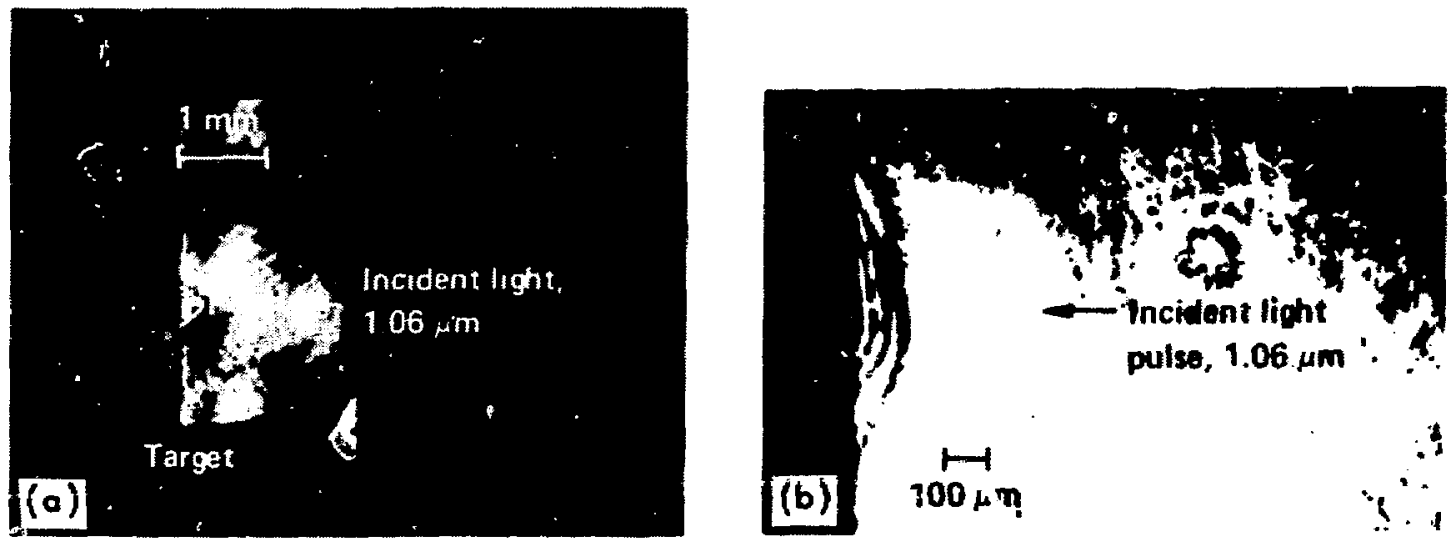

Fig. 75. Interterogrems of laser-produced plasms obtained using hologaphic microceopy. (a) Delay time 1 noec after irrediation. (b) Delay time 0.5 nuec after irrediation. 


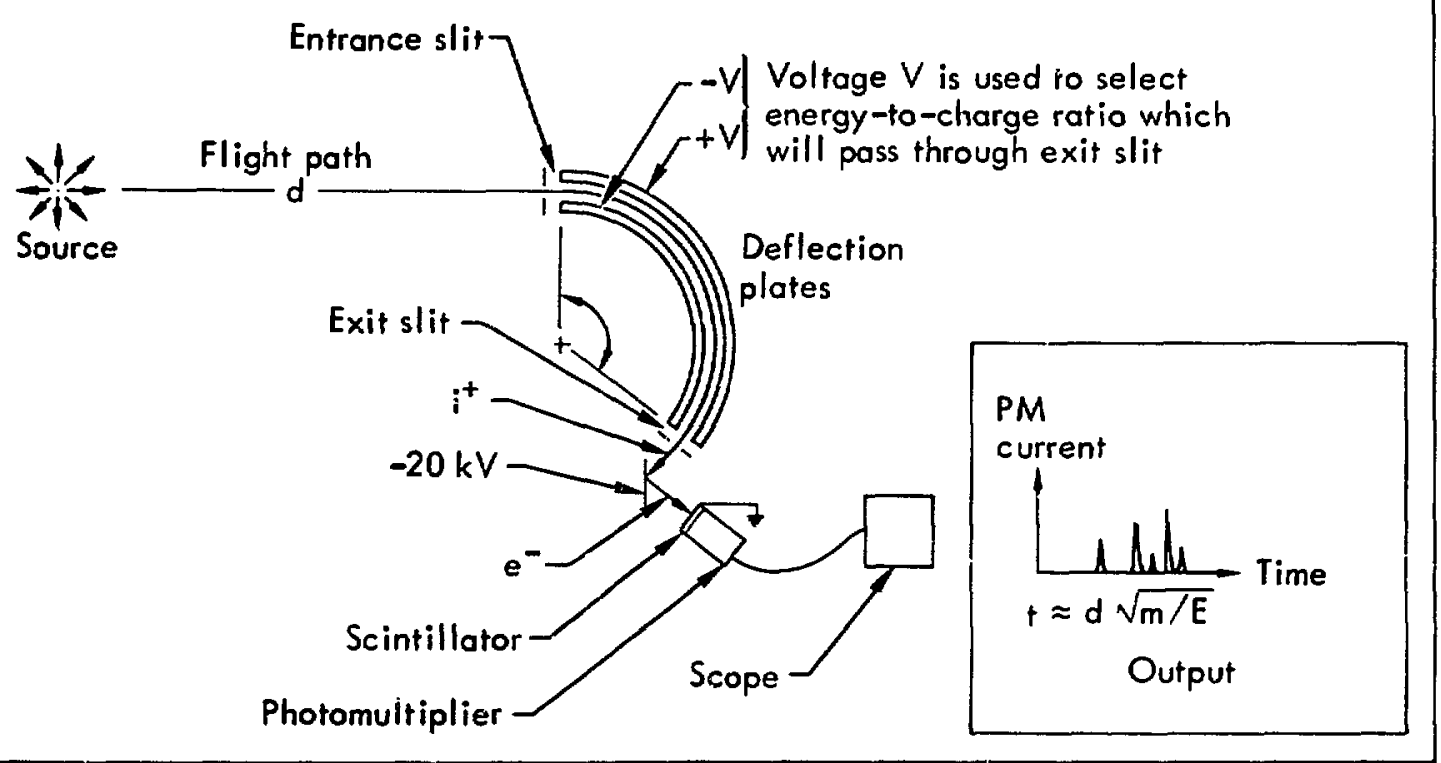

Fig. 76. Curved-plate electrostatic energy analyzez for ion spectroscopy of laser-produced plasmas.

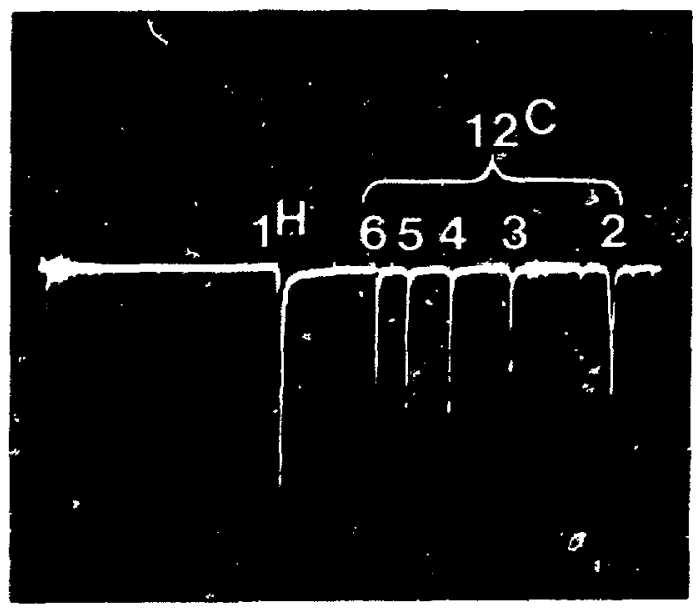

Fig. 77. Typical output of Fig. 76 energy analyzer, showing ion currents from a Cll, larget. Peaks are from $\mathrm{H}^{* 1}(1.05 \mathrm{keV}), \mathrm{C}^{* 2}(2.10 \mathrm{keV}), \mathrm{C}^{* 1}(3.15 \mathrm{keV})$, $C^{*}(4.20 \mathrm{kcV}), C^{-3}(6.30 \mathrm{kcV})$, and $\mathrm{C}^{* \circ}(6.30 \mathrm{kcV})$. Scope sensitivities are $0.5 \mathrm{~V} /$ division and $1 \mu \mathrm{sec} /$ division. Numbers show ion charge. 


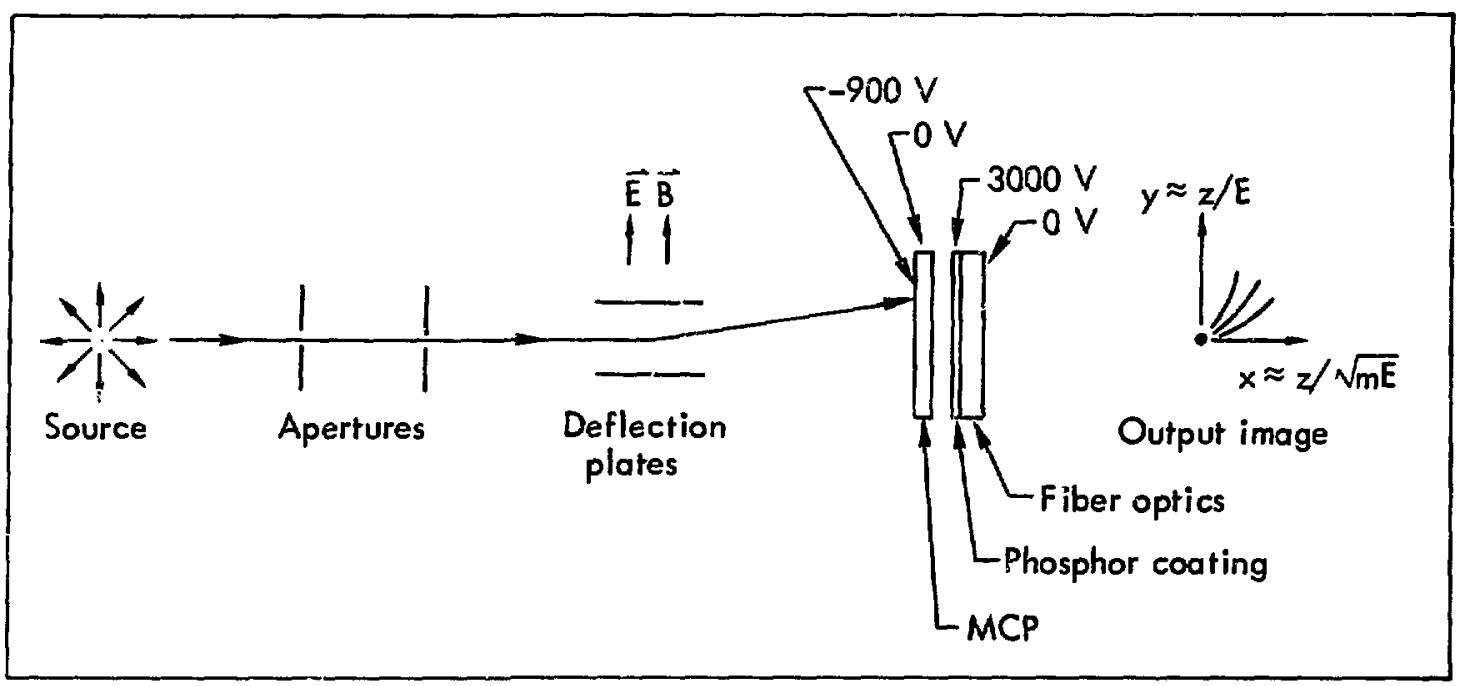

Fig. 78. Thomson spectrometer uses parallel magnetic and clectric fields to sparially scparate ions of different cnergics and charge-to-mass ratios. 


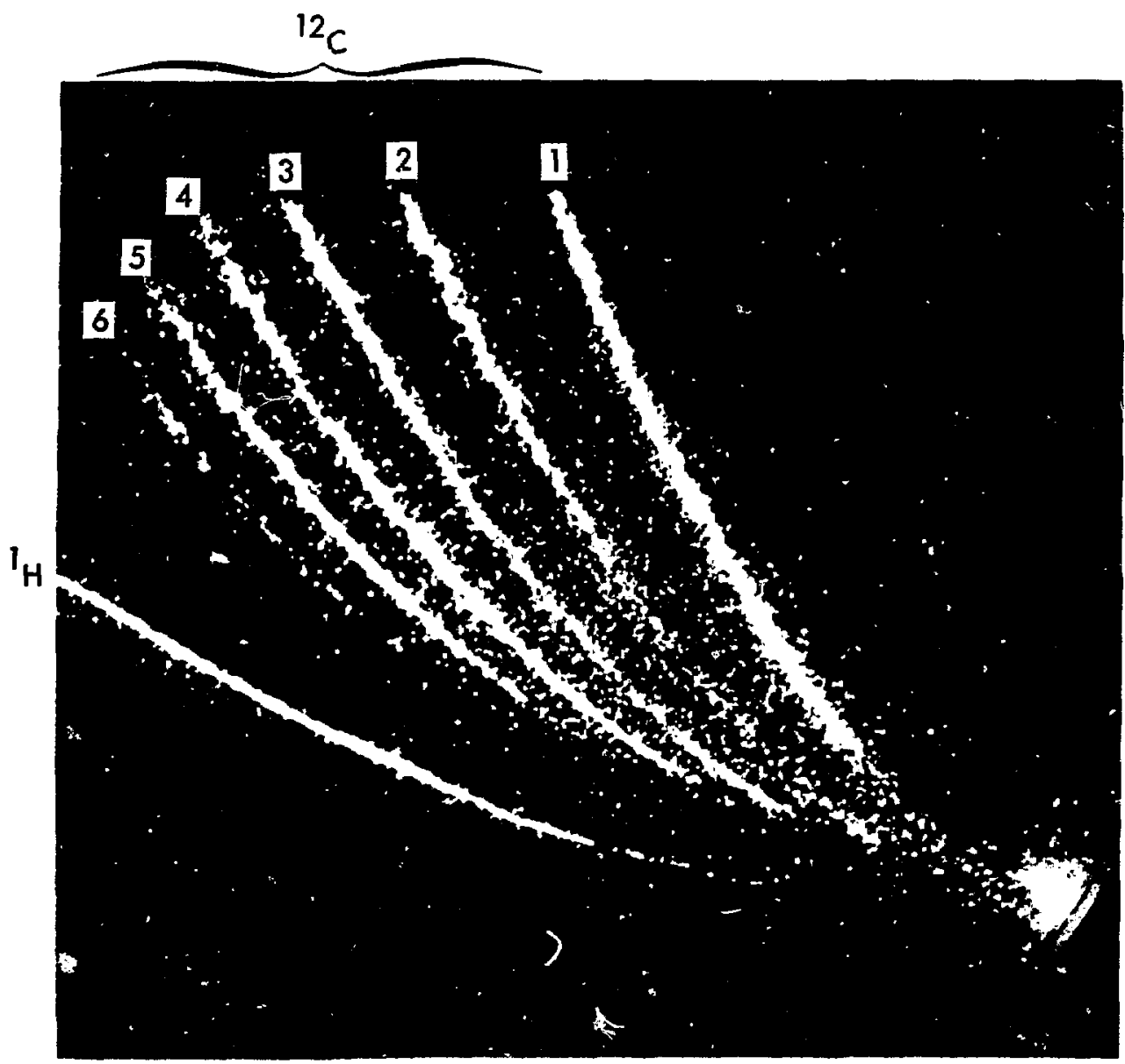

Fig. 79. Photo taker with Thomson spectrometer after irradiation of a $\mathrm{CH}_{2}$ target shows all six carbon species. 
The role of the laser-target design group is to collaborate with the laser design and laser-plasma experiment groups to design the optimal targeis for the lasers which are constructed, provide target designs and requirements for the laser-target fabrication group, and participate in interpreting the experiments. Central to the successful execution of these tasks is the LASNEX implosion-fusion computer program.

The behavior of laser-produced plasmas hinges directly on microscopic electron transport and interaction processes. Consequently, experimental verification of the LASNEX approximations to these processes is important. The validity of the prescription used to describe the velocity distribution of electrons heated by the laser, particularly those heated via plasma instabilities, and their subsequent transport within the plasma has been corroborated in part by comparison with experiments conducted both at LLL and at LASL. However, there are as yet unexplained discrepancies between the calculation and at least one experiment.

Progress has continued on refining our calculations of laser-driven implosions. The following issues have been addressed:

1) Is the implosion sufficiently symmetric and stable despite the nonuniform laser intensity and the imperfect pellet? Should the implosion depart sufficiently from spherical symmetry, then the subsequent thermonuclear yield will be significantly degraded. Results of preliminary series of calculations of solid pellets suggest that, even with laser intensity variations as great as $\pm 10 \%$ over $18^{\circ}$ laser beams, the implosion is highly symmetric provided two conditions are satisfied: first, that the critical radius is maintained at least twice as large as the pellet redius-in part by varying the laser wavelength from long $(4 \mu \mathrm{m})$ to short $(0.5 \mu \mathrm{m})$ over the duration of the laser irradiation; and second, that the underdense atmosphere is sufficiently heated with a prepulse to insure supersonic energy transport to the pellet surface. It is anticipated that hollow pellets will be much more sensitive to asymmetries and instabilities.

2) Does the strong spontaneous magnetic field generated because of spatial irregularities in the laser beam adversely affect the implosion? For spatial variations of $10 \%$ over $18^{\circ}$-wide laser beams, calculations predict the generation of magnetic fields as large as several megagauss. For long-wavelength laser light, magnetic fields this large inhibit electron thermal conduction and reduce, thereby, the ablation pressure attainabie with any given laser intensity. In addition, the inhomogeneous magnetic fields may generate asymmetries in the implosion. It appears that for laser wavelengths much less than $10 \mu \mathrm{m}$ the ablation can be corrected by changing the pulse shape and that the asymmetries are not serious. However, more calculations are needed to confirm these preliminary results.

3) How severely will suprathermal electrons degrade the performance of these implosions? LASNEX calculations indicate the fusion yield ratio is diminished by a factor of 2 if $20 \%$ of the laser energy is absorbed via plasma instabilities. The gain is reduced more than tenfold if $50 \%$ of the light is absorbed via instabilities.

4) Can the suprathermal electron problem be bypassed? Targets have been designed which absorb the laser light by classical processes, and in which the peak laser intensity does not exceed the threshold for anomalous absorption. This is accomplished by several means: short-wavelength, finite-bandwidth lasers; seeding th, pellet with materials having $Z>1$; and use of hollow pellets. The use of hollow pellets also minimizes the required peak laser power. LASNEX calculatior \& indicate that an approach combining hollow pellets and frequency-doubled Nd:glass lase: light $(\sim 1 ; 2, \mathrm{~m})$ might work.

5) Whai is the utility of $10-\mu \mathrm{m}$-wavelength light for laser fusion? With $10-\mu \mathrm{m}$ light it is apparently not possible to avoid exci dtion of plasma instabilities and generation of suprathermal electrons in laser implosions of potentially high enough gain to be suitable for power pioduction. These electrons cause failure of the implosion. Our conclusion is that implosions with $10-\mu \mathrm{m}$ light may be successful only if high- $Z$ materials are used in the pellet, and that this possibility should be carefully calculated.

\section{DEVELOPMENT OF LASNEX COMPUTER CODE}

The LASNEX code has been developed to study the many physical processes important in the effort to achieve laser-initiated fusion. These processes and the models LASNEX uses to calculate them are outlined below.

\section{Physical Processes and Models}

Figure 80 is a schematic representation of the physical processes important to laser fusion. The circles in this figure represent torms in whicli energy can exist 


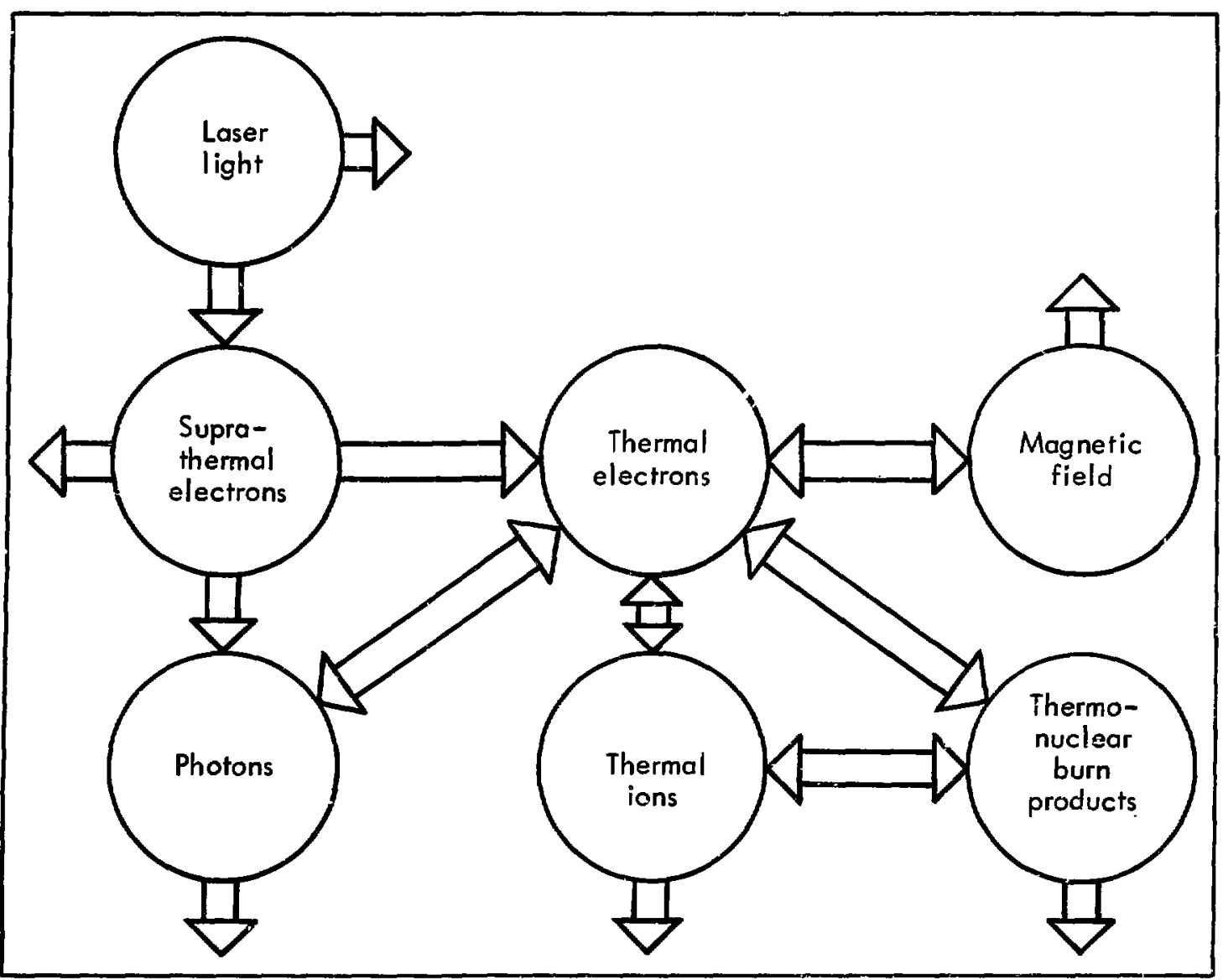

Fig. 80. The plasma components (circles) and interactions (arrows) important to laser fusion are modcled in LASNEX calculations.

within a laser-heated plasma while the arrows represent interaction mechanisms and generally show the direction of energy flow. Possible plasma diagnostics are depicted by the arrows that do not enter another circle.

One can also consider this figure as a description of the equations solved by the LASNEX code. In this sense each circle represents a set of the unknown functions that are subject to initial and boundary conditions while the arrows correspond to sets of integral and partial differential equations. Let us consider a typical flow of energy through its different forms in a laser-heated plasma and at the same time discuss the models and approximations LASNEX employs.

Laser light enters the plasma from vacuum. The boundary conditions on the laser light are both temporal (the pulse shape) and spatial (the spot shape), but since LASNEX can only consider two space dimensions we must restrict the spot shape to one with axial symmetry.
Of course, combinations of laser beams with different frequency, temporal, and spatial distributions can be considered. As the laser light travels through the zones of the LASNEX spatial mesh it deposits energy according to classical inverse bremsstrahlling ab-orption. If the light is sufficiently intense as it nears the critical density (where the laser light frequency equals the plasma frequency), then plasma instabilities will develop and cause increased absorption of the light. The rate of change of the local electron distribution caused by light absorption is given by

$$
\begin{aligned}
\dot{n}(v)=C_{I B} v \exp & \left(-\frac{1}{2} m v^{2} / K T\right) \\
& +C_{P I} v^{2} \exp \left(-\frac{1}{2} m v^{2} / \alpha K T\right),
\end{aligned}
$$

where the first term is due to inverse bremsstrahlung and the second to plasma instability absorption. The parameters $C_{P I}$ and $\alpha$ are determined from detailed 
plasma-simulation calculations ${ }^{43}$ of plasmas under somewhat idealized conditions and thus are not universally accepted For the most critical parameter $(\alpha)$, LASNEX uses the value 12 , thereby producing a high-energy tail to the electron distribution. It has been shown ${ }^{44}$ that this value is consistent with experimental measurements of hard $\mathrm{x}$ rays produced by bremsstrahling suprathermal electrons. If not all of the laser light is absorbed by these processes, then the light is reflected at the critical density and suffers further attenuation on its way back out to vacuum.

Since the distribution of electrons he ated by laser light absorption may not be close to a Maxwellian, it is necessary to treat the nonequilibrium aspects of the transport and thermalization of these electrons in some detail. LASNEX accomplishes this by allowing the electron distribution to be described in terms of a speed coordinate as wall as a temporal and two spatial coordinates. This model is actually quite a simple one compared to a full transport model that would allow two angular coordinates as weil. The validity of the simpler model rests upon the assertion that the electrons will always be nearly isotropic even if they are far from Maxwellian in their energy distribution. In high- $Z$ plasmas this situation can arise simply from classical Coulomb collisions since the isotropization rate is proportional to $Z^{2}$ while the thermalization rate is proportional to $Z$. In low- $Z$ plasmas the electrons are rendered isotropic by magnetic fields which always seem to be produced in laser plasmas ${ }^{45}$ and by infinite mass charge fluctuations caused by collective effects in the laser absorption and other regions of the plasma. Given that the suprathermal electrons are nearly isotropic their transport and thermalization can be handled by a combined multigroup flux-limited diffusion and simplified Fokker-Planck treatment. In this modei a self-consistent electric field is set up by using a generalized Ohm's law and the condition of no net current. $^{46}$

Therinal electrons receive energy from Coulomb collisions with the suprathermal elecurons and from Ohmic heating caused by the previously mentioned electric field, the so-called $\vec{J} \cdot \vec{E}$ heating. The thermal electrons are assumed to be described by a Maxwellian distribution whici simplifies the calculation of their interactions with the other plasma components. The transport of the thermal electron ene gy is accomplished by using iensorial plasma conductivities in a magnetic field, ${ }^{47,4 \varepsilon}$ somewhat modified by a flux limiter and variable degree of ionization.

The hydzodynamic pressire and $s_{1}$ :cific heat of the thermal electrons are taken from a Thomas-FermiDirac model which includes both partial ionization and electron degeneracy.

The thermal ions are heated by Coulomb collisions with thermal electrons and by hydrodynamic compression. The ions' intertial structure is assumed to be given by an average atom mor ' alculated in local thermodynamic equilibrium with . .e thermal electron temperature. From this model are calculated frequency-dependent radiative opacities due to boundbound, bound-free, aná free-free processes. ${ }^{49}$ At ion temperatures above $1 \mathrm{keV}$ thermonuclear reactions begin. LASNEX uses Maxwellian-averaged cross sections for most interesting ihermonuclear reactiuns, and for the $\mathrm{D}+\mathrm{T} \rightarrow \mathrm{n}+\alpha$ reaction it transports the neutron and alpha particle separately with single-group flux-limited diffusion methods. ${ }^{46}$ The use of a singlegroup representation of neutron transport is valid for laser fusion because even the most highly compressed laser-fusion pellets are relatively thin to $14-\mathrm{MeV}$ neutrons. The thermonuclear burn products either escape from the plasma providing a diagnostic possibility or deposit their energy back into the plasma electrons and ions. The increased ion temperature causes more thermonuclear reactions, leading to a runaway condition that is slowed only by depletion of reacting ions and by cooling caused by hydrodynamic expansion and radiative anc conductive losses.

The photons in the plasma are handled by a multigroup flux-limited diffusion model ${ }^{50}$ similar to that used for suprathermal electrons. As previously mentioned, frequercy-dependent opacities are taken from an LTE average atom model. The redistribution of photon energy due to Compton scattering is treated in the Fokker-Planck approximation. ${ }^{51}$ In addition, the frequency distribution of photons produced by bremsstrahlung from suprathermal electrons is calculated for diagnostic purposes.

In an axially symmetric laser-produced plasma $(r, z$ coordinates in LASNEX), magnetic fields perpendicula- to the plane of calculation can be generated spoutaneously by nonparallel electron pressure and density gradients:

$$
\dot{\vec{D}}=\operatorname{curl}\left(\frac{\iota}{c n_{e}} \nabla P_{e}\right) .
$$

Such a magnetic field then influences the plasma hydrodynamically through the $\vec{J} \times \vec{B}$ force :1, most importantly, by altering transport coefficients for all charged particles. The transport coefficients used in LASNEX are essentially those derived by Braginskii ${ }^{48}$; however, because of plasma turbulence effects these

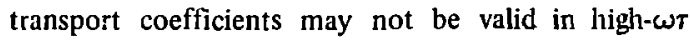
situations. It has been shown, at least in some magnetic confinement geometries, ${ }^{32}$ that the theory of Bohm ${ }^{53}$ may approximately describe the transport of part: les across magnetic field lines in these situations. We have so far considered the range between Braginskii and Bohm diffusion as an uncertainty in our calculations. 
The hydrodynamics in LASNEX is two-dimensional Lagrangian with axial symmetry. Quadrilateral zones are used to perform the calculation in a manner similar to that described by Schulz. ${ }^{54}$ Partial pressures due to all of the plasma components represented by circles in Fig. $\mathbf{8 0}$ are included. Since photons and thermonuclear burn products are not electrostatically confined to the plasma, their partial pressures are reduced in a manner that yields the correct momentum deposition rate in streaming situations.

\section{Diffusion Theory}

Except for the hydrodynamic and laser deposition methods, most of the transport models used in LASNEX involve diffusion theory. In many cases arguments can be made in favor of using a diffusion model, but ather than detail these here it is better to consider a particular example in which diffusion theory breaks down whereas multigroup flux-limited diffusion produces an adequate result.

Consiat: the problem of calculating the steady state radiation from the sun at the radius of the earth's orbit.

1) By diffusing the radiation temperature:

$$
0=\operatorname{div}\left(\frac{a c}{3 \times \rho} \nabla T^{4}\right),
$$

which yields

$$
T^{4} \propto 1 / r \text { or } T=1500^{\circ} \mathrm{K} .
$$

2) By thux-limited diffusion of the radiation temperature:

$$
0=\operatorname{div}\left(\frac{a c}{3 \kappa \rho+2 / T^{4}\left|\nabla T^{4}\right|} \nabla T^{4}\right)
$$

which yields

$$
T^{4} \propto 1 / r^{2} \text { or } T=400^{\circ} \mathrm{K} .
$$

3) By muitigroup flux-limited diffusion:

$$
0=\operatorname{div}\left(\frac{c}{3 k_{\nu} \rho+2 / U_{\nu}\left|\nabla U_{\nu}\right|} \nabla U_{\nu}\right),
$$

which yields

$$
U_{\nu}(r)=U_{\nu}\left(r_{\odot}\right)\left(\frac{r_{Q}}{r}\right)^{2} .
$$

The straight diffusion of the radiation temperature produced a completely erroneous result, but with only the addition of a flux-limiter the temperature of a body at the earth's orbital radius is very close to the correct value. If in addition there is a need to know the frequency distribution of photons, then multigroup flux-limited diffusion retains that information as well. Both flux-limited models also contain an estimate of the direction of the radiative flux in the terms

$$
-\frac{\nabla T^{4}}{\left|\nabla T^{4}\right|} \text { or }-\frac{\nabla U_{\nu}}{\left|\nabla U_{\nu}\right|}
$$

but detailed information about the angular distribution of photons cannot be obtained with any diffusion model.

\section{EXPERIMENTAL CHECK OF LASNEX CODE}

Discussed below are two comparisons of LASNEX predictions with experiments conducted recently.

\section{Hard X-Ray Spectra and Suprathermal}

\section{Electron Distributions}

One of the central issues for the design of laser fusion pellets is the question of the velocity distribution of electrons heated by the absorption of laser light. Several experiments conducted recently here at LLL by Slivinsky and Kornblum ${ }^{55}$ and at LASL by $\mathrm{McCall}^{56}$ address this matter directly. In these experiments, one measures the spectra of hard $x$ rays $(\sim 5.150 \mathrm{keV}$ at LLL and $\sim 5.50 \mathrm{keV}$ at LASL) and infers the electron velocity distribution. At LLL the experiment was conducted with the Long Path Laser

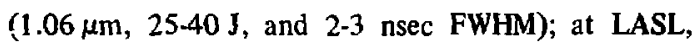
with both a $\mathrm{CO}_{2}$ laser $(10.6 \mu \mathrm{m}, 10 \mathrm{~J}$, and $1.5 \mu \mathrm{sec}$ FWHM) and Nd:glass laser $(1.06 \mu \mathrm{m}, 10 \mathrm{~J}$ and $\sim 30$ psec FWHM). The reduced data are displayed in Figs. 81-83.

Using electron distributions characteristic of both inverse bremsstrahlung absorption, $f(v) \propto v \exp \left[-v^{2} /\right.$ $\left.\left(v_{\text {thermal }}\right)^{2}\right]$, and anomalous absorption, $f(v) \propto v^{2}$ $\exp \left[-v 2 / \alpha(v \text { thermal })^{2}\right]$, the pellet design code LASNEX has predicted $X$-ray spectra for these experiments. Katz et al. ${ }^{43}$ have conducted numerical simulations of the plasma instabilities responsible for anomalous absorption and predict $6 \leqslant \alpha \leqslant 12$, that is, that the distribution of heated elecirons has significantly more energy in the high energy tail than does a Maxwellian distribution. The LASNEX calculations indicate that, 


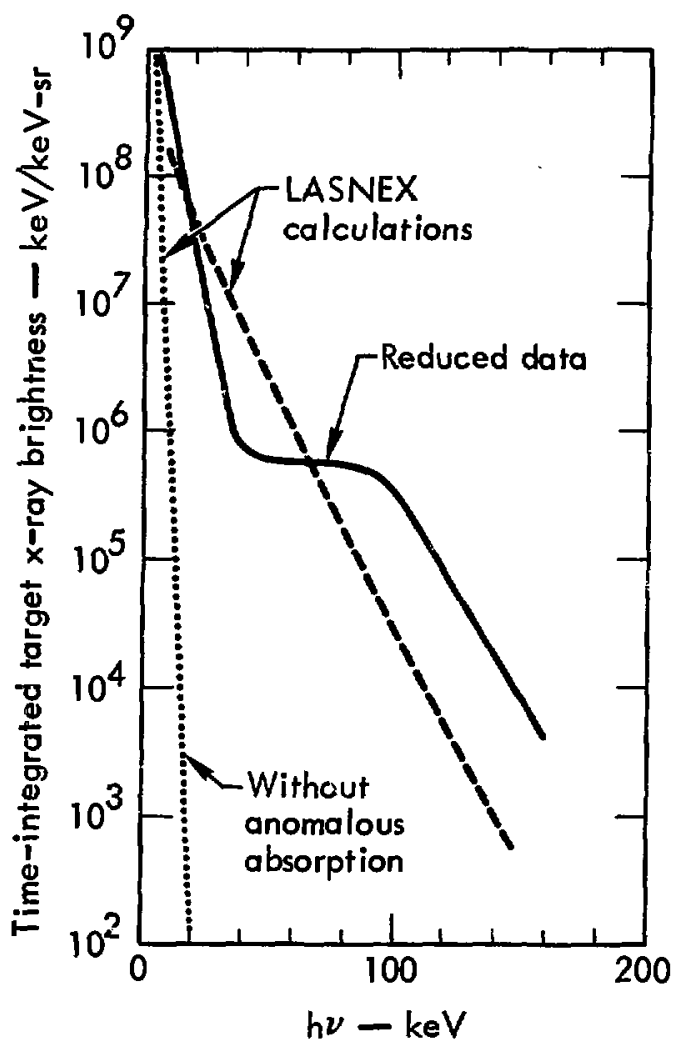

Fig. 81. Data and LASNEX predictions for experiments conducted with the LLL Long Path Laser (30 J, 2 nsec, 100- $\mathrm{un}$-diam focal spot, $\mathrm{CD}_{2}$ target). Dotted line assumes absorption exclusively by inverse bremsstrahlung, dashed line assumes both inverse bremsstrahlung and anomalous absorption.

were the absorption of laser light exclusively by inverse bremsstrahlung, the resulting $x$-ray spectra would be far too soft, typically about 14 orders of magnitude too low at $h v \approx 50 \mathrm{keV}$ (see Figs. 81 and 82). By contrast, the $x$-ray spectra predicted by LASNEX calculations in which absorption occurs via the competing mechanisms of inverse bremsstrahlung and anomalous absorption (in these calculations $\alpha$ is 12) agree rather well with the experimental measurements except for McCall's Nd experiment. This agreement tends to corroborate the theoretical electron distribittion and serves as a check on LASNEX itself.

Several imponderables remain. The pronounced plateau observed in Siivinsky's experiment implies an electron distribution more highly structured than that predicted by Katz et al. ${ }^{43}$ In simulating McCall's 30-psec Nd experiment, LASNEX has been less successful: regardless of the contribution from the suprathermal electrons, the $x$-radiation from the thermal

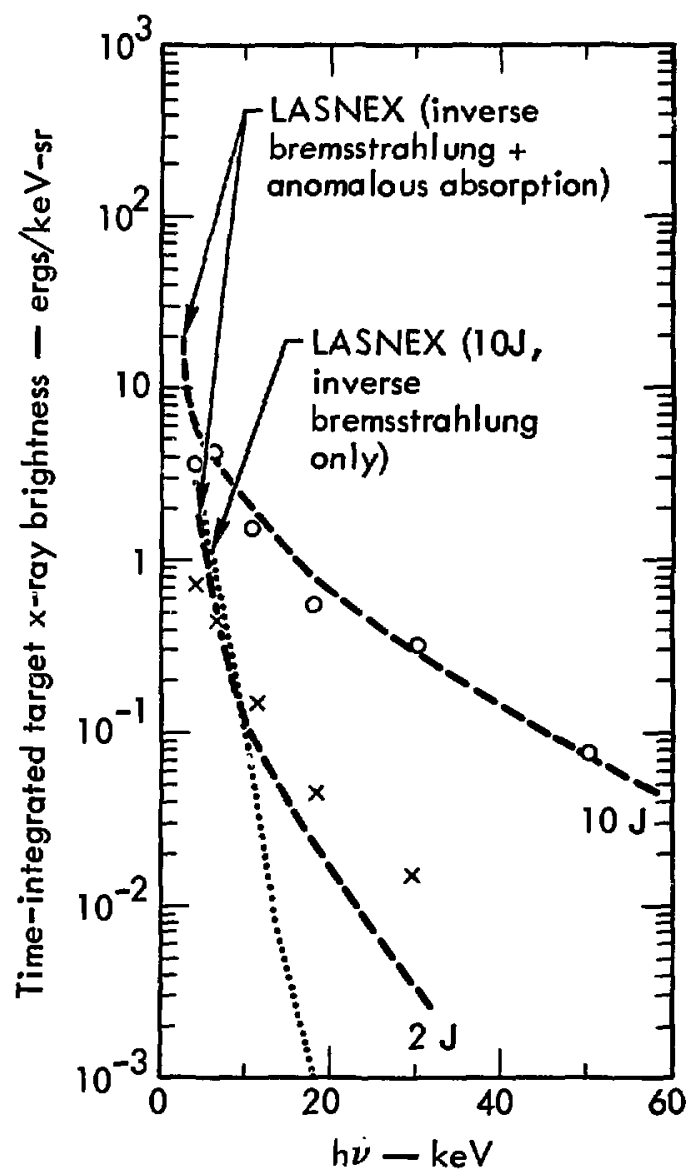

Fig. 82. McCall's $\mathrm{CO}_{2}$ laser experiment at $\mathrm{LASL}^{\mathrm{s}}$ (2 and $10 \mathrm{~J}, 1.5 \mathrm{nsec}, 100-\mu$ m-diam focal spot, $\mathrm{CH}_{2}$ target). Dots are 10-J data, X's 2-J data. Dashed lines are LASNEX predictions for 2- and 10-J cases assuming both inverse bremsstrahlung and anomalous absorption. Dotted line is LASNEX calculation for 10-J case assuming absorption exclusively by inverse bremsstrahlung.

background is itself too intense by one order of magnitude at the lower end of the spectrum, $4-6 \mathrm{keV}$, as depicted by the dotted line in Fig. 83. LASNEX calculations agree with this experiment when a large fraction of laser energy is not absorbed and/or transported-the dashed line indicates LASNEX predictions when only $10 \%$ of the $10 \mathrm{~J}$ is absorbed by the electrons. This hypothesis apparently contradicts both reflection measurements taken only at a limited number of angles and ion kinetic energy measurements which account for all the laser energy. It does not, however, exclude the possibility of an anomalous ion acceleration tapping energy from the electrons. Alternatively, LASNEX predictions match the experimental results if 


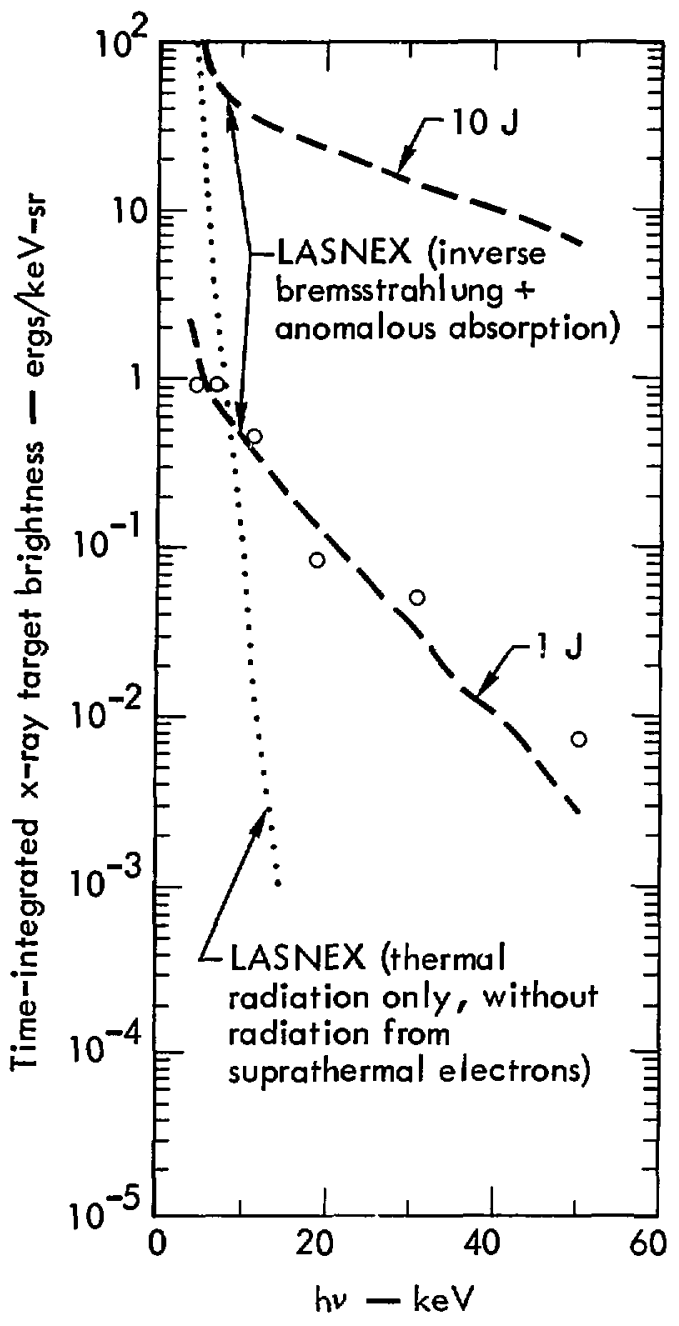

Fig. 83. McCall's Nd:glass laser experiment at $\mathrm{LASL}^{56}(10 \mathrm{~J}$, 30 psec, 50- $\mu$ m-diam focal spot, $\mathrm{CH}_{2}$ target). Dots are data. Dasied lines show LASNEX predictions assuming both inverse bremsstrahlung and anomalous absorption, calculated both for $10 \mathrm{~J}$ and for $1 \mathrm{~J}$ of beam energy (the latter assumes only $10 \%$ of the 10- $\mathrm{J}$ beam energy is absorbed by the plasma). Dotted line shows the LASNEX-predicted spectrum assuming no contribution from the suprathermal electrons.

the assumed laser intensity is wel! below the nominal experimental values (assuming greater spot size and pulse width). Because of the possible importance of this discrepancy, this experiment will probably be repeated with the new 50-J, 100-psec Nd:glass laser here at LLL; particular emphasis will be placed on a complete accounting of the laser energy and on the careful measurement of the thermal $x$ rays as well.

\section{Spatial Distribution of the Region}

\section{Emitting Thermal $X$ Rays}

In a number of proposed laser-fusion experiments, an understanding of the spatial distribution of x-ray emission may prove to be extremely useful. Seward et al. have completed the construction of a prototype $x$-ray microscope.$^{60}$ To predict the images obtained with such a device, a postprocessor code (TDG) has been written for the hydrodynamics code LASNEX. At prescribed intervals during the calculation of a pellet implosion, LASNEX writes the value of the relevant variables (position, density, electron temperature, and the multigroup opacities) for each zone on an output buffer. As indicated in Fig. 84, the 2-D Lagrangian mesh fully describes the radiative properties of a solid of revolution. After the LASNEX calculation, TDG reads this information, and, by integrating the transport equations (one for each frequency group) along rays parallel to the direction of observation, determines the instantaneous $x$-ray snapshot for each frequency group. By folding in the spectral response of the detector, TDG then computes an instantaneous, spectrally integrated $\mathrm{x}$-ray snapshot. For comparison with measurements which are not time-resolved, such as the micrographs taken with Seward's instrument, TDG integrates all of the snapshots.

Seward has exposed $x$-ray micrographs with iron targets at both the Long Path Laser and the Plasma-X laser. The left side of Fig. 85 show isoflux contours (in relative units) for a micrograph taken at the Plasma-X with an iron slab target and with the microscope at an angle of $45^{\circ}$ to the plane of the target. In this shot the microscope is sensitive only to photons of energies $h v$ $\leqslant 1.7 \mathrm{keV}$. The right-hand side of Fig. 85 depicts the LASNEX-TDG prediction. LASNEX indicates that these soft $X$ rays are emitted by a very narrow region near the target surface-hence the $x$-ray image appears as a disk. The data appear to substantiate this hypothesis.

Future experiments witl plasma targets promise to tiucidate the emission of $x$-radiation over a range of photon energies $(\leqslant 10 \mathrm{keV})$, and, moresver, this instrument should prove quite useful in diagnosing fusion-pellet irradiations. TDG will play a key role in interp:eting these results. 


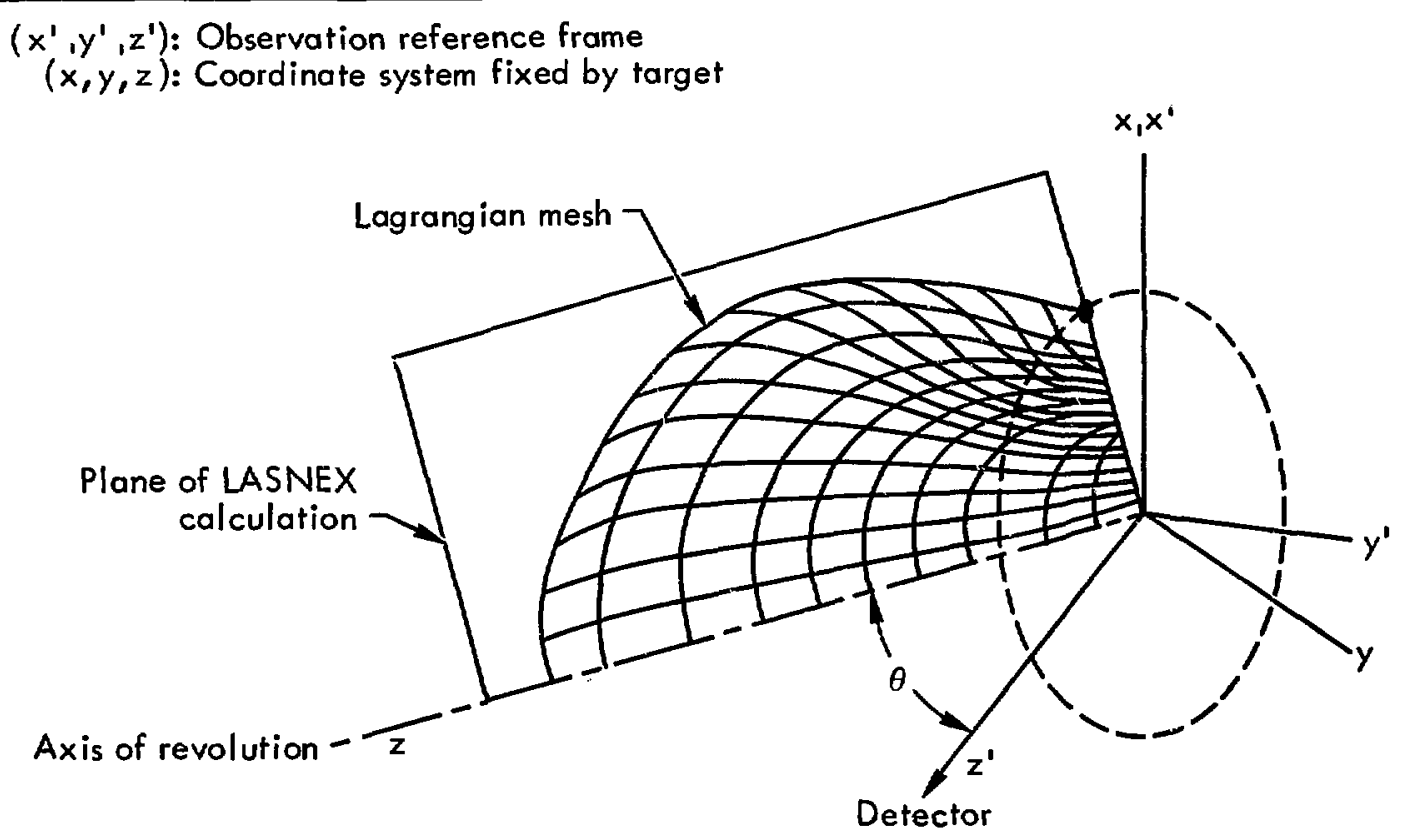

Fig. 84. The 2-D Lagrangian mesh of LASNEX can fully describe the radiative properties of a solid of revolution, as shown here.

\section{2-D CALCULATIONS TO STUDY SYMMETRY AND STABII.ITY OF SOLID SPHERICAL DT TARGETS}

Symmetry and Stability of Solid DT Pellet Implosion

A two-dimensional LASNEX calculation was made of the implosion and burn of a $60-\mu \mathrm{g}$ pellet of deuterium-tritium. The initial geometry (Fig. 86) consists of the $400-\mu \mathrm{m}$-radius drop surrounded by a $1400-\mu \mathrm{m}$-radius low-density atmosphere created by a laser prepulse. Counters at the bottom of the figures show the maximum electron and ion temperatures in $\mathrm{keV}$, the maximum; density in $\mathrm{g} / \mathrm{cm}^{3}$, the total laser energy input and thermonuclear energy generated in $\mathrm{kJ}$, the time in nsec, and the size of the picture in $\mu \mathrm{m}$.

The two-dimensional analog of an actual multibeam irradiation consists of two regular beams-one at each pole-and nine annular beams equally spaced between them. The beams join in such a way as to result in a sinusoidal intensity variation of $\pm 10 \%$. Because of the symmetry about the equatorial plane we show only half of the drop and thus consider only five full wavelengths of the intensity variation.

At the start of the calculation a 100-J, 10-psec laser pulse flash-heats the electrons in the low density atmosphere to a temperature of several hundred electron volts. This is done to insure that electron transport in the atmosphere is always supersonic. A supersonic atmosphere guarantees that the intial motion of the surface of the drop is caused by ablation-a hydrodynamically stable situation. LASNEX calculations of a similar impiosion using a subsonic atmosphere show that the initial motion of the droplet surface is caused by pressure forces within the atmosphere itself-the classical unstable situation. In these calculations the implosion symmetry was seriously degraded (e.g., see Fig. 87).

The successful calculation makes use of four different laser wavelengths $(4,2,1$, and $0.5 \mu \mathrm{m})$. The longer-wavelength light is used during the first part of the implosion when symmetry requirements are most severe. The short-wavelength light is used near the end of the laser pulse in order to minimize electron decoupling effects. ${ }^{58}$ Because of the steeply rising power history of the optimal pulst shape ${ }^{59}$ the $4-\mu \mathrm{m}$ laser is employed for almost $90 \%$ of the implosion time but the $0.5 . \mu \mathrm{m}$ laser delivers more than $80 \%$ of the total 


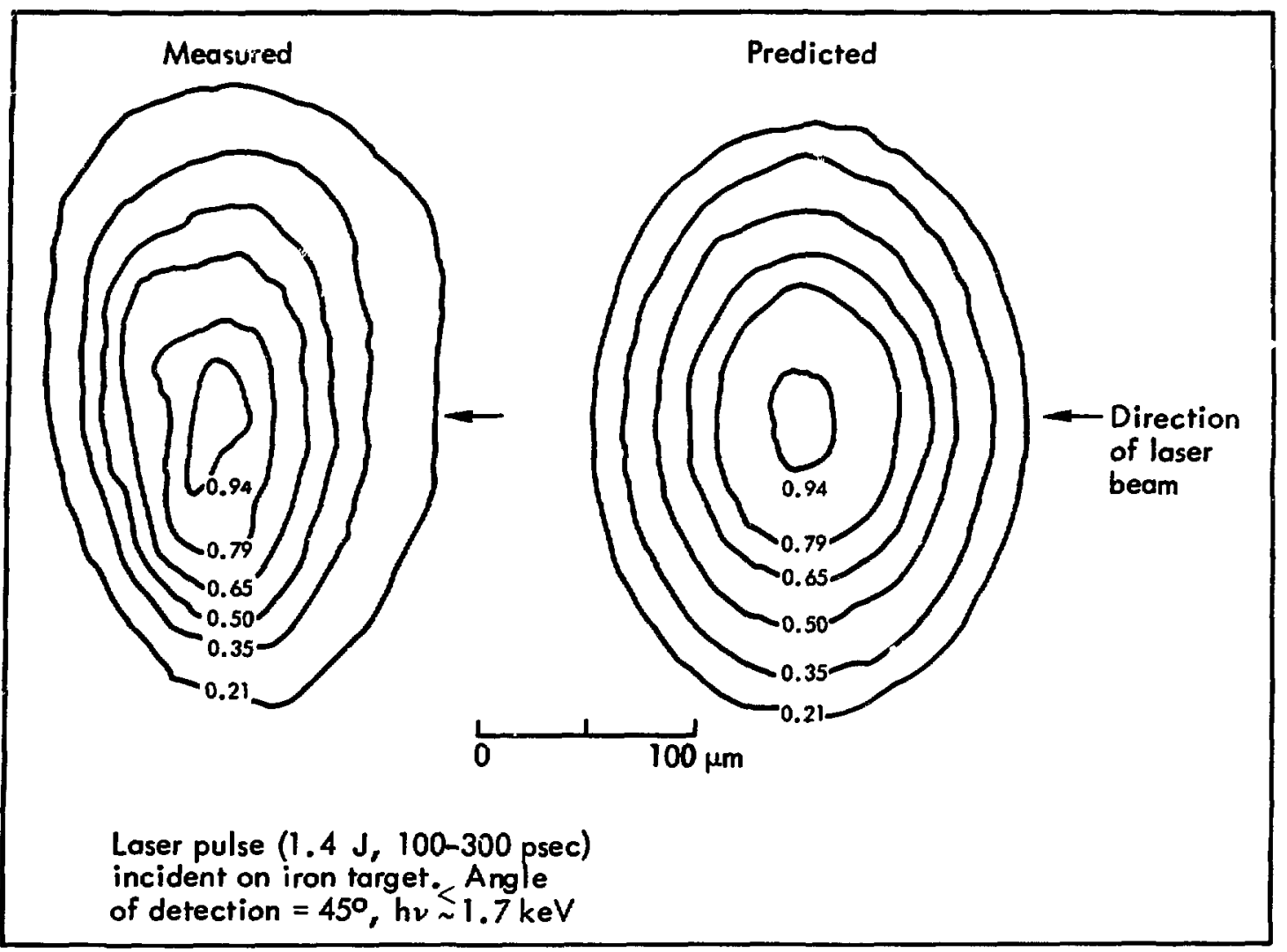

Fig. 85. Comparivon of experimental and calculated x-ray micrographs.

energy. The pellet implosion depicted in Fig. 86 is carried out nearly isentropically and results in a compression of more than $10^{4}$-fold.

Figure 88 shows the 10-psec-long thermonuclear bum phase of the calculation. Only a small fraction of the imploded drop $\left(\sim 0.3 \mathrm{~g} / \mathrm{cm}^{2}\right)$ has been heated hydrodynamically to ion temperatures $\gtrsim 10 \mathrm{keV}$. The alpha particles produced in this region heat the surrounding matter while hydrodynamic, radiative, and electron conduction losses try to cool it. Eventually the alpha particle heating wins out, resulting in an outward-propagating thermonuclear burn front. The ions quickly achieve temperatures above $50 \mathrm{keV}$. The rising pressure, however, causes a violent hydrodynamic expansion which cools the fuel and quenches thermonuclear reactions. In this short period $1.7 \mathrm{MJ}$ of thermonuclear energy was released-more than 30 times the laser energy required to bring it about and only slightly less than the $1.8 \mathrm{MJ}$ produced in a onedimensional simulation of the same pellet.

Although this calculation appears to satisfy the two-dimensional requirements imposed by symmetry and stability, several effects, such as the possibility of numerical diffusion and instability, may weaken such a statement. We are currently studying the limitations of LASNEX in this respect and then will proceed with similar two-dimensional calculations of hollow (shell) targets.

\section{Effects of Self-Generated Magnetic Fields} on the Implosion of Solid DT Spheres

Self-generated magnetic fields have been observed in experimental laser-produced plasmas ${ }^{60}$ and are believed to be produced primarily by nonzero cross products in the thermoelectric gradients of the plasma that are present when a laser target is nonuniformly irradiated. Other sources for self-generated fields that have been investigated recently are direct radiation production $^{61}$ of the magnetic field, and unstable growth of magnetic fields ${ }^{62}$ in a uniformly irradiated plasma.

LASNEX calculations have elucidated the impact of these fields on the implosion of DT drops. The 

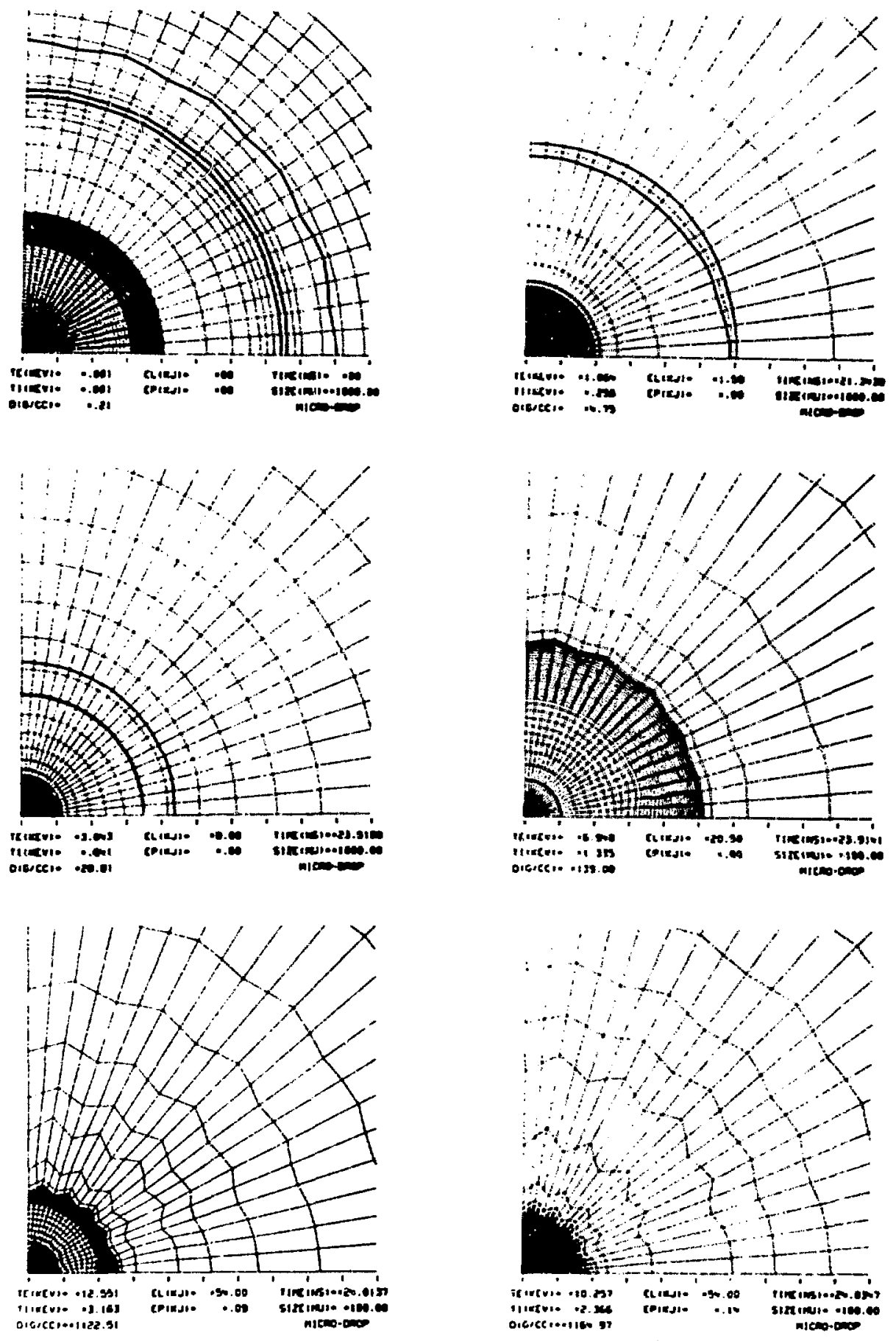

Fig. 86. Two-dimensional implocip:a sit a laser fusion pellet showing the LASNEX mesh and etectron temperature isotherms (dark ares at $0.1,0.3,1,3$, and $10 \mathrm{kcV}$ al various times. 


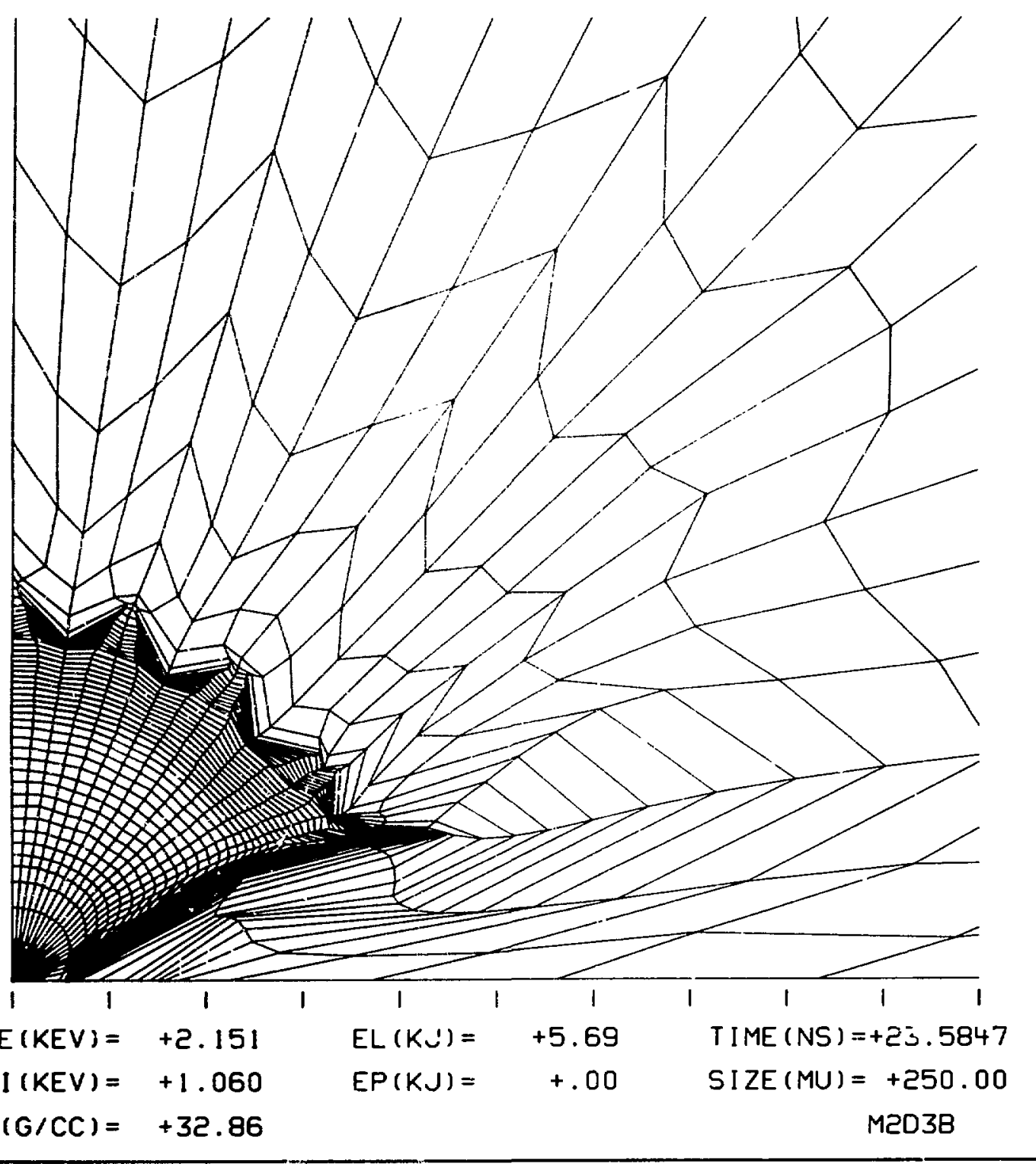

Fig. 87. Asymmetrical implosion of a lascr-fusion pellet showing imprini of nonunifoim laser irradiation and destructive axis jet. Pellet and laser uniformity are same as in Fig. 86, but this calculation was unsuccessiul because it employed only a short-wavelength laser $(1-\mu \mathrm{m})$ and did not fash-heat the pellet atmosphere to achieve adequate eiectron conductivity.

system investigated here is illustrated in Fig. 89. In particular, an $18^{\circ}$ cone was taken that represented $1 / 50$ th of a sphere and one cycle of variation in the intensity of the laser radiation. The 2-D, radially directed magnetic fields were required to be zero on the conical boundary.
The magnetic field can affect a hydrodynamic implosion in two basic ways. It inhibits the transport of energy across the field lines, thereby elevaing the temperature of the electrons in the region of the sritical density and diminishing electron temperature (and pressure) in the region of peak ablation pressure. 


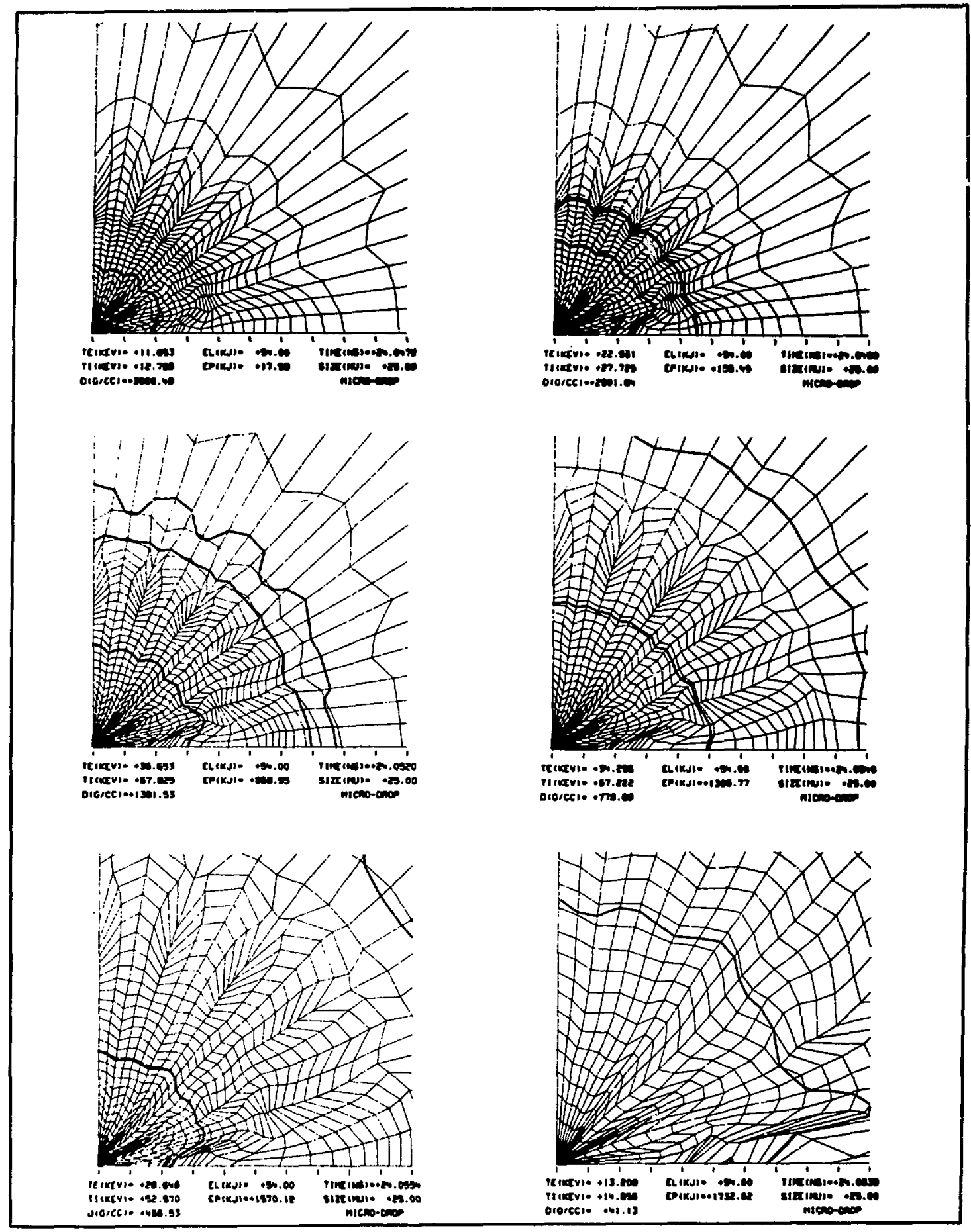

Fig. 88. Two-dimensional thermonuclear explosion of pellet imploded in Fig. 86 showing ion temperature isotherms of 10, 20, and $50 \mathrm{keV}$. 


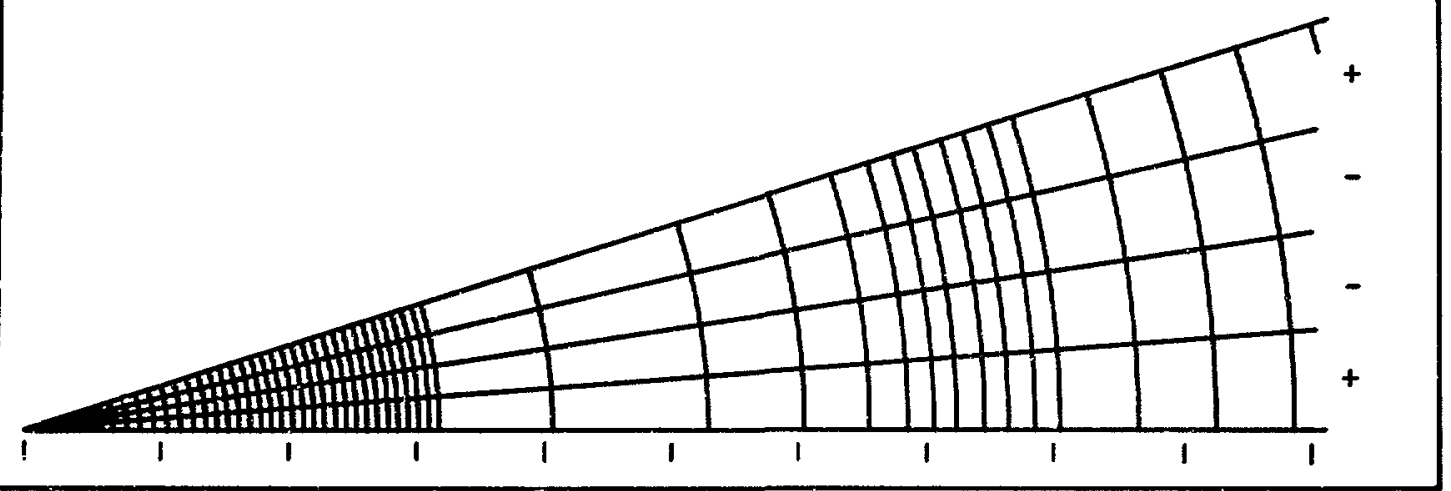

Fin. 89. Initin peometry and zoning used in calculating the effect of self-generated magnetic fields. The +'s and - 's indicate the hypotherized varitions in laser intensity.

In addition, the magnetic fields exaggerate the varia. tion in the ablation pressures at various points on the pellet surface. The second effect directly degrades the symmetry of the implosion.

As discussed above, LASNEX calculates the eration of axially symmetric magnetic fields arising from thermoelectric gradients and inhibits electron thermal cor duction by either Braginskiit's or Bohm's prescription!. These two approximations to thermal conduction iepresent opposite extremes. Since correct calculation : hrould be intermediate to both, LASNEX magnetir field calculations are typically conducted twice, once with each approximation. The effects of adding the magnetic field physics with and without the anomalous diffusion term (Braginskii or Bohm conduction, respectively) are illustrated in Table 15. In the calculation without magnetic fields the average ablation pressure at the very end of the laser pulse is about 100 Gbar (gigabars) over the entire ablation surface. Because of spherical convergence, he $10 \%$ variation in laser intensity is amplified to a $17 \%$ variation in the ablation pressure.

The second model ("standard fields") represents a calc:lation conducted with the classical magnetic inhibition to thermal conduction. The order-ofmagnitude decrease in the ablation pressures is the most obvious effect of the self-generated magnetic fields. Initial magnetic fields of kilogauss magnitude lead to $\omega \tau$ on the order of $10^{2}$. These initial fields diffuse into the center of the pellet while the lengthy ramp on the laser pulse slowly rises. Near the end of the laser pulse the magnetic fields in the region of the critical density increase to megagauss values when the DT is compressed $10^{4}$ times. The field in the compressed DT (again in the megagauss range) has little effect on the problem since it is not large enough to change the trajectory of alpha particles produced in the thermonuclear burn. In addition to changes in the magnitude of the ablation pressure, the asymmetry in the ablation pressure increases from 17 to $25 \%$ because of spatial nonuniformities of the magnetic field.

The anomalous diffusion coefficient changes the effective collision time in the plasma quite drastically. For the large wr values that are observed near the critical density the effective collision time changes from $10^{-8}$ to $10^{-12} \mathrm{sec}$. This results in an effective $\omega \tau$ of about 16. The final column illustrates that the ablation pressure computed within the context of Bohm diffusion is about $70 \%$ of that present without magnetic fields. There is as much overall symmetry in this calculation, but the modification of the coefficients of the

Table 15. Spatial variation of pressure at the pellet ablation surface, as calculated for three different models. The first column under each model gives the ablation pressure for each of four angles at the time corresponding to the end of the laser pulse; the second column shows relative ablation pressures for the four angles, normalized to the peak ablation pressure for that calculation. The spatial variation of laser intensity, $10 \%$, is indicated by the +'s and -'s on the right side

Calculated absolute and reiative pressures ${ }^{a}$

\begin{tabular}{|c|c|c|c|c|c|c|}
\hline \multirow{2}{*}{$\begin{array}{l}\text { Angular } \\
\text { Iocation }\end{array}$} & \multicolumn{2}{|c|}{ No fields } & \multicolumn{2}{|c|}{ Standard fields } & \multicolumn{2}{|c|}{ Anomalous diffusion } \\
\hline & Pressure & $\%$ & Pressure & $\%$ & Pressure & $\%$ \\
\hline $18^{\circ}$ & 92.0 & 84 & 11.0 & 100 & 60.0 & $75(+)$ \\
\hline $13.5^{\circ}$ & 97.0 & 88 & 9.4 & 86 & 71.0 & $90(\rightarrow)$ \\
\hline $9^{\circ}$ & 100.0 & 91 & 8.4 & 77 & 79.0 & $100(-)$ \\
\hline $4.5^{\circ}$ & 110.0 & 100 & 8.3 & 76 & 73.0 & $92(+)$ \\
\hline
\end{tabular}

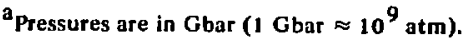


transport equation allowed signiffcant ablation pressure to develop.

The combined consequences of these effects can be illustrated graphically by plotting the path (Fig. 90) of a typical fuel zone in the $(P, \rho)$ plane. If the fuel is compressed along the Fermi-degenerate adiabat, then $P$ $\propto \rho^{5 / 3}$. This represents the most efficient compression of a system. Curve 1 is the path followed without a magnetic field. The fuel reaches densities of $1100 \mathrm{~g} / \mathrm{cm}^{3}$ and pressures of about $8 \times 10^{3}$ Gbar. Curve 2 represents the path followed by the problem without anomalous diffusion. The naximum compression and pressure are both orders of magnitude too low for ignition. Curve 3 represents the path for the problem with anomalous diffusion. In this case the fuel becomes dense enough but the decreased ablation pressure does not provide enough kinetic energy during the implosion to raise the fuel to ignition temperature. Slight modifications in. the laser pulse shape can easily increase this temperature.

Table 16 illustrates the effect on the yield ratios of these calculations for the two different models for 10 , 5 , and $1 \%$ variations in the intensity. These results indicate that a decrease in the amplitude of the variation by about a factor of 2 will almost eliminate the effects of the magnetic field regardless of which model is chosen for the transport. If, however, Bohm

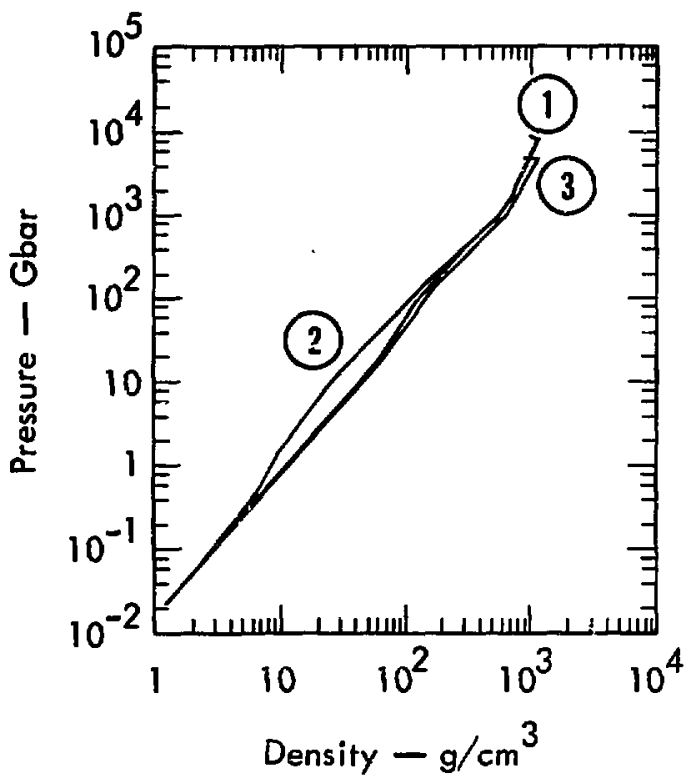

Fig. 90. Calculated adiabat for the DT fucl for three different models: (1) Without self-generated magnetic fields. (2) With magnetic ficlds and Braginskii thermal conduction. (3) With magnetic fields and Bohm diffusion. diffusion pertains then variation in the intensity up to $10 \%$ may be permitted.

The effects of the magnetic field on the implosion can also be reduced by starting the implosion with

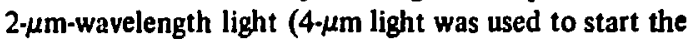
calculations reported here), by seeding the pellet with materials having $Z \gg 1$ to increase the collision frequency, and by optinizing the pulse shape to compensate for decoupling. Consequently, we tentatively conclude that self-generated magnetic fields will not seriously degrade laser implosions where the wavelength is much smaller than $10 \mu \mathrm{m}$. However, further calculations are required.

\section{Effect of Suprathermal Electron Tail on the Thermonuclear Yield of DT Pellets}

As related above, when laser light is absorbed by a hot plasma, energy is transferred to electrons by a number of processes:

1. Inverse bremsstrahlung.

2. Plasma decay instabilities.

3. Resonant absorption.

Of these, inverse bremsstrahlung results in bulk heating of the electrons and a near-Maxwellian distribution, while theory indicates that the other processes will produce an energetic suprathermal component of electrons.

The parametric decay instability, which is excited at densities from about 0.8 to 1.0 times the critical density (where $\omega=\omega_{p e}$ ) for sufficient intensity, is an efficient absorber of electromagnetic radiation, and will completely absorb the energy of a wave in about 10 free-space wavelengths. In one dimension and in the limit that an electron makes an infinite number of passes through the instability region, the electron distribution becomes $F(v)=A \exp \left[1 / 2\left(1 / 2 m v^{2} / K T_{e}\right)^{1 / 2}\right]$. In the limit that an electron makes but a single pass through the absorption region, the distribution is given by $F(v)=B \exp \left(-1 / 2 m v^{2} / \alpha K T_{e}\right)$. The parameter $\alpha$ depends on the size, $L$, and temperature of the interaction region and scales as $\left(L / \lambda_{D}\right)^{2 / 3}\left(\lambda_{D}\right.$ is the Debye length). At $2 \mathrm{keV}$, and an interaction length of 10

Table 16. Calculated vield ratios for two magnetic field models as a function of percent of variation of incident laser insensity

Calculated yield ratios for:

\begin{tabular}{rcc}
$\begin{array}{c}\text { Intensity } \\
\text { variation }\end{array}$ & $\begin{array}{c}\text { Standard } \\
\text { fields }\end{array}$ & $\begin{array}{c}\text { Anomalous } \\
\text { diffusion }\end{array}$ \\
\hline $1 \%$ & 34 & 35 \\
$5 \%$ & 2.3 & 33 \\
$10 \%$ & $6 \times 10^{-4}$ & 0.31 \\
\hline
\end{tabular}


wavelengths, $\alpha \approx 12$. An $\alpha \approx 12$ is often quoted, but it is by no means a universal constant. At higher temperatures, $\alpha$ becomes somewhat less than 12 , but then the electrons are more likely to return to the heating region before losing their energy and the distribution starts to go over to the infinite pass model. This occurs because, at high energies, electrons can completely cross the low density atmosphere without significant energy loss and are reflected back into the center of the pellet by the electrostatic sheath at the outer surface of the atmosphere. Results presented below have been obtained using the single-pass distribution.

The current state of knowledge about the electron spectrum produced by the parametric decay instability can be summarized as follows:

1. The energy will be absorbed with an effect:-m absorption length of about 10 free-space wavelengths.

2. Some fraction $f$ of the absorbed energy is absorbed into a suprathermal tail characterized by the distribution $F(v)=B \exp \left(-1 / 2 m v^{2} / \alpha K T_{e}\right)$.

3. Some fraction $1-f$ of the energy is absorbed by the bulk of the electrons in something like a Max. wellian distribution.

Only suprathermal electrons produced by the parametric decay instability have been included in the results below. When the nonlinear states of the Raman and $2 \omega_{p e}$ instability and resonant absorption are better understood, their electron spectrum can be readily included.

Before beginning a quantitative discussion of the effect of the suprathermal electrons on a laser-driven implosion, it is useful to review their effect qualitatively.

During an ideal implosion history, an initial weak shock is sent through the target. This shock heats the electrons and ions to temperatures of about $1 \mathrm{eV}$, compresses severalfold, and starts the matter moving inward at about $1 \mathrm{~cm} / \mu \mathrm{sec}$. The target material is then adiabatically compressed using a shaped laser pulse. ${ }^{59}$ Adiabatic compression is desired so that the pressure required for compression can be kept as low as possible and as near the Fermi-degenerate pressure as practical. If nearly adiabatic compression is tu be achieved, then the supersonic transport of energy into the center of the target must be small compared to the Fermi energy. This means there must be strong coupling between the electrons heated by the laser and those electrons inside the critical radius. Very energetic electrons do not satisfy this criterion and can deposit their energy deep inside the pellet. This energy deposition results in a continually rising "adiabat" and is called preheat. A higher adiabat means that a larger driving pressure is required to achieve a given compression and also results in a somewhat more rapid decompression of the pellet during burn.
A second eff ' $t$ of suprathermal electrons is a lower ablation pressure for a given laser power, i.e., decoupling. This occurs because the energy coupling rate between hot and cold electrons decreases as the energy of the hot electrons goes up. Above a certain energy, the energy an electron loses to background electrons is less than the energy it gains in each pass through the heating region and these electrons run away from the rest of the distribution. When only a fraction of the laser energy goes into a suprathermal tail, one possible cure for decoupling is to increase the laser power, but this also increases the preheat. One gains only if the increase in driving pressure exceeds the increase in the pressure required for compression. If the intensity is raised to a high enough value, the background electrons in the absorption region as well as the suprathermal tail will decouple. When this condition is reached, increasing the intensity will not result in a higher ablation pressure. 58

The quantitative effects of preheat and decoupling will vary from one target design to another, but the above qualitative effects are the same. The quantitative results presented below are for a single target design, shown in Fig. 91. This target has a $58-\mu \mathrm{g}$, $400-\mu$ m-radius solid DT core surrounded by a low density atmosphere which might be produced by a laser prepulse. The atmosphere proceeds in two steps to a density slightly greater than the critical density for

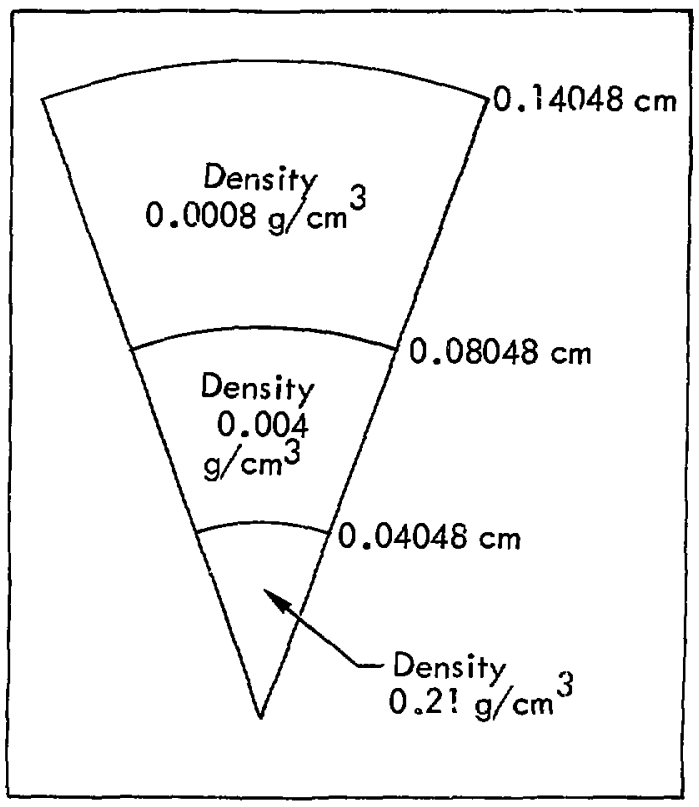

Fig. 91. Section of the DT target whose implosion and thermonuclear burn was tested for sensitivity to suprathermal electrons. 
$2-\mu \mathrm{m}$ light. This is a crude approximation to the density gradient that would exist when the atmosphere is generated. The laser pulse used to drive ihis target was chosen so that thermal conduction could smooth $\pm 10 \%$ variations in intensity from conical laser beams whose peak intensities were separated by $18^{\circ}$ across the surface of the drop, while at the same time giving high yields. The laser pulse is shaped in frequency as well as in intensity and proceeds in steps from $2 \mu \mathrm{m}$ to $1 \mu \mathrm{m}$ to $1 / 2 \mu \mathrm{m}$. The $2-\mu \mathrm{m}$ laser is necessary to achieve the required $1 \%$ uniformity in ablation pressure, and the $1 / 2-\mu \mathrm{m}$ laser is used to minimize decoupling during the high-power end of the pulse. The $1 / 2-\mu \mathrm{m}$ laser can be replaced with a $1-\mu \mathrm{m}$ laser with only about a $10 \%$ drop in the fusion gain. This target gives a yield of $2.2 \times 10^{6}$ $\mathrm{J}$ when imploded with $4.6 \times 10^{4} \mathrm{~J}$ if all the energy is deposited in an inverse bremsstrahlung spectrum. The laser power profile used to drive the peilet is listed in Table 17.

The concomitant effects of preheat and decoupling on this target are shown in Fig. 92. The curves shown are for implosions of the same target with varying fractions of energy into a suprathermal spectrum and the rest into an inverse bremsstrahlung spectrum. In each case, the laser power is readjusted to optimize the yield with a given fraction of energy in a suprathermal tail. The indicated fraction of the energy is put into the suprathermal tail only when the instability threshold is exceeded and only between 18 and 1.0 times the critical density. The results presented are remarkably insensitive to the estimate of the threshold. This insensitivity occurs because early in time most of the laser light is absorbed by inverse bremsstrahlung. By the time inverse bremsstrahlung has become ineffective, the laser intensity is rising very rapidly to a value far above the threshoid. Varying the threshold between

Table 17. Laser power profile used to drive the pellet in Fig. 91

\begin{tabular}{ccc} 
Time (nsec) & Power $\left(\mathrm{W} / \mathrm{cm}^{2}\right)$ & $\frac{M^{\mathrm{a}}}{100}$ \\
\hline 0 & $2.4 \times 10^{\mathrm{B}}$ & 100 \\
11.0 & $2.6 \times 10^{11}$ & 100 \\
12.3 & $1.5 \times 10^{12}$ & Constant \\
12.65 & $1.5 \times 10^{12}$ & power \\
\hline
\end{tabular}

${ }^{a} M$ is from the equation

$$
c(l)=c(0)\left[\frac{1}{1-t \frac{1-R}{t}-\underline{t}}\right]^{M}, R=\left[\frac{\epsilon(0)}{\varepsilon_{f}}\right]^{1 / M} .
$$

for energy in a typicill laser pulse, where $c(0)$ and $c$ are intial and final energies. The effect of $M$ is discussed a few pages further on.
0 and $10^{14} \mathrm{~W} / \mathrm{cm}^{2}$ for $1-\mu \mathrm{m}$ light at $1 \mathrm{keV}$ only shifts the time of onset for strong instability absorption by a small incren:er. In fact, the difference in yield between the assumption of a zero threshold and $10^{14}$ $W / \mathrm{cm}^{2}$ is only a couple of percent.

The upper set of curves in Fig. 92 is a set of pressure-vs-lensity curves for calculations in which the fraction of energy absorbed into a suprathermal tail is varied from 0 to $40 \%$ for $\alpha=12$. The pressure-vsdensity curve for D' at $10^{-5} \mathrm{keV}$ is shown for reference. The implosion with all the energy going into an inverse bremsstrahlung spectrum lies close to this and is a nearly ideal implosion. On the othcr hand, the implosion with $40 \%$ of the energy in a supratiermal tail was veiy badly preheated, and it barely ignited.

The lower set of curves in Fig. 92 shows the ablation pressure developed as a function of laser power for the various cases. The large degradation as the suprathermal energy increased is evident, and the peak power required to ignite the $40 \%$ case was a factor of 5 higher than for thic $0 \%$ case.

In all the cases shown in Fig. 92, decoupling of electrons in the suprathermal tail could be jvercome by an increase in the laser pcwer. Figurc 93 shows a case for which no power is high enough to get ignition. These runs were done with a slightly different target and laser pulse. The upper curves are again pressure-vsdensity plots. The lowest of these had a peak power of $1.35 \times 10^{15} \mathrm{~W}$. It achieved significant compression but did not ignite. The other curves are for the same target and pulse with the power increased over the last 400 psec. The lower plot in Fig. 93 shows that the ablation pressure continued to rise as the power increased, but the pressure required to drive the pellet increased even faster, as seen by the sharp kink in the pressure-vsdensity curves.

The effects of a suprathermal tail are a strong function of the hardness of the electron spectrum. Figure 94 shows the gain $\eta$ as a function of the fraction $f$ of the energy going into a suprathermal tail for $\alpha=6$ and $\alpha=12$. Also snown is the laser energy required to achieve ignition as a function of $f$. With $\alpha=6$, gains of better than 20 are outained with $50 \%$ in supiathermal tails. At $\alpha=12$, however, $40 \%$ of the energy in a suprathermal tail causes severe degradation, and gains of 20 are achieved with no more than $20 \%$ of the energy in the tails.

The fraction of energy in suprathermals that can be tolerated levels off as $\alpha$ is increased much beyond 12, as seen in Fig. 95 for $f$-values of 0.1 and 3. The gain reaches a minimum at $\alpha \approx 24$ and rises slightly thereafter. The gain at very large $\alpha$ 's occurs because the very energetic electrons produced cannot couple to the fuel in the time it takes for the implosion to occur, and so less preheat results. In the limit of infinite $\alpha$, there 

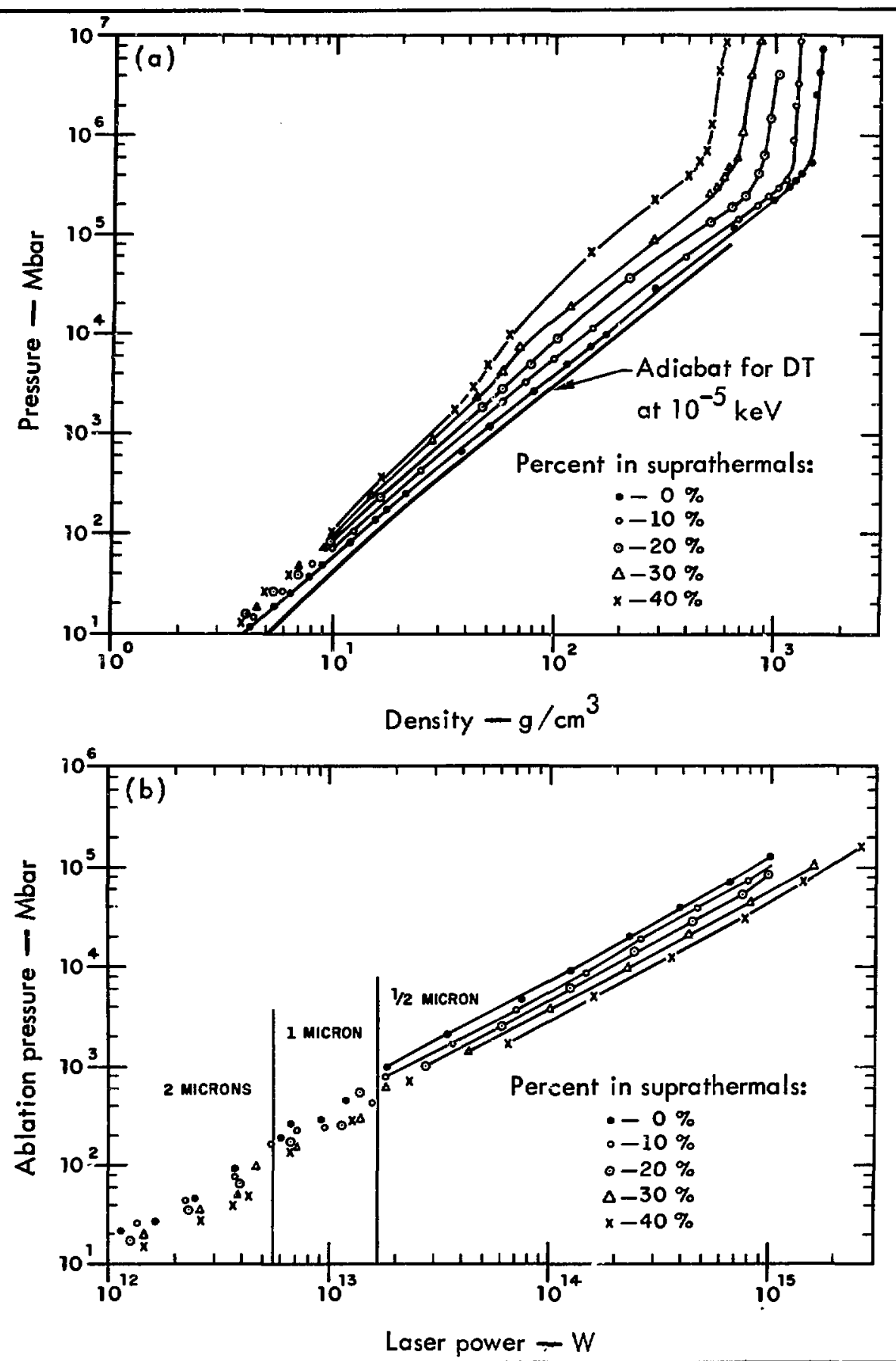

Fig. 92. Pressure vs density in a central fuel zone (a), and ablation pressure vs laser power (b), calculated for various fractions of the laser energy absorbed in a suprathermal electron tail of the form $F(v)=B v^{2} \exp \left(-1 / 2 m v^{2} / 12 K T_{e}\right)$ and the remainder of the laser energy absorbed in an inverse bremsstrahlung spectrum. 

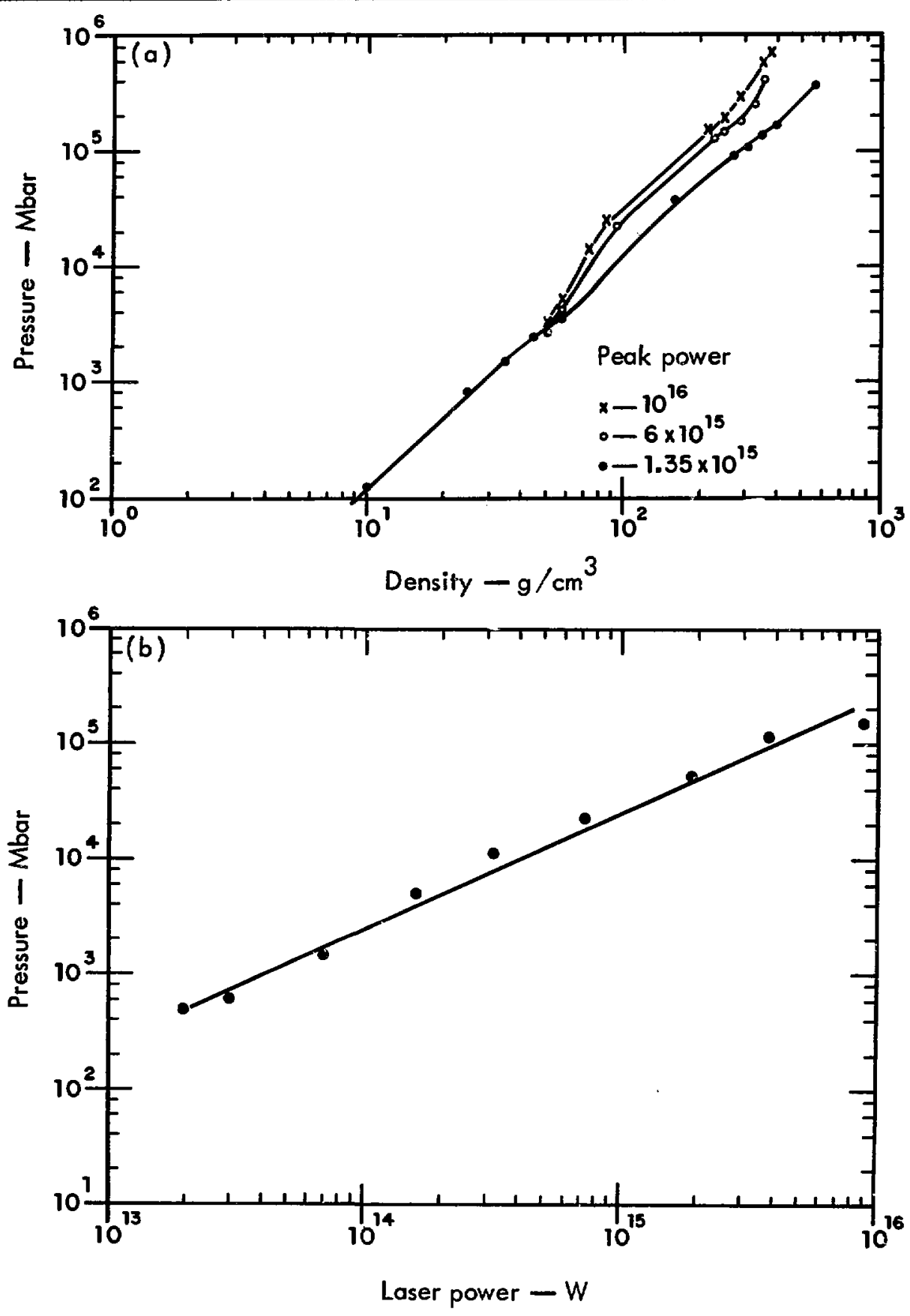

Fig. 93. Pressure vs density in a central fuel zone (a), and ablation pressure vs laser power (b), calculated for a problem in which laser power was incicased to overcome decoupling during the final $\mathbf{4 0 0}$ psec. Note that the pressure required to drive the pellet increased even faster than the ablation jressure, thus precluding ignition. 


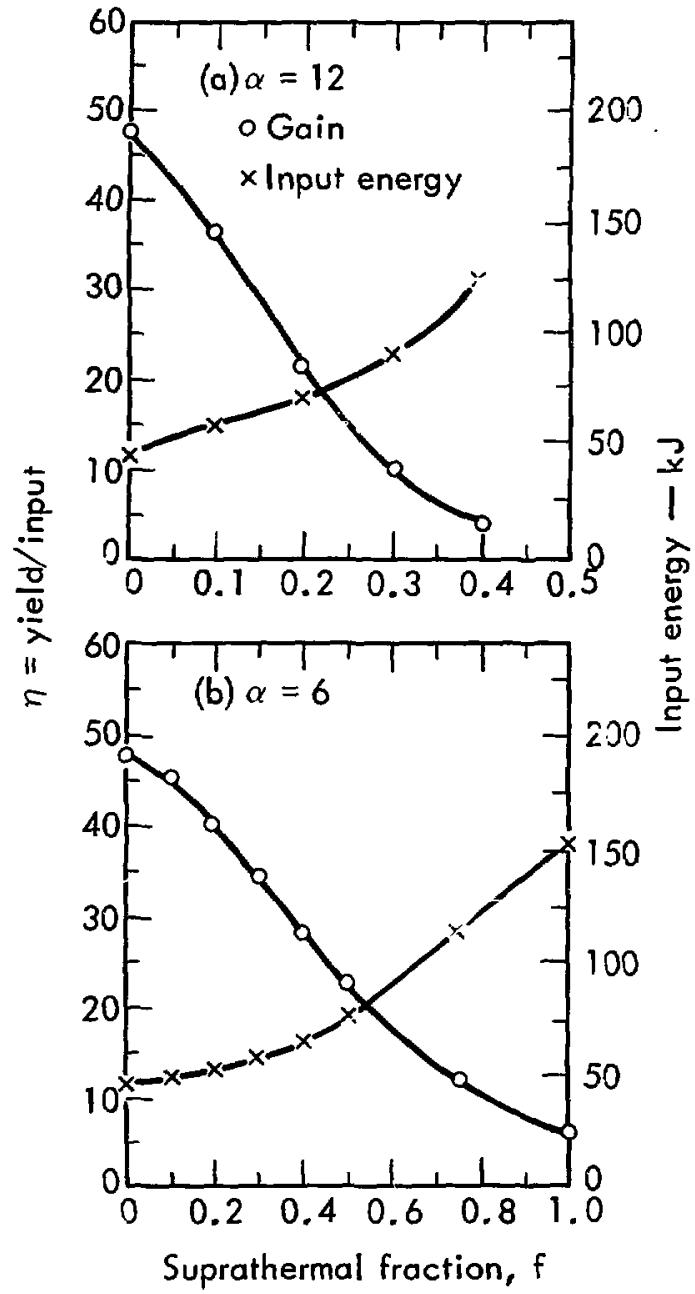

Fig. 94. Gain and input energy as functions of the fraction $f$ of energy deposited in electrons with a suprathermal tail, for two different values of $\alpha$ in the suprathermal distribution function $F(v)=B v^{2} \exp \left(-1 / 2 m v^{2}\right.$ / $\alpha K T_{e}$.

would be no preheat and one would only have to contend with decoupling. Frot: Fig. 95 one sees that with the most pessimistic choice of $\alpha$ one can achieve break-even at about $100 \mathrm{~kJ}$ with $30 \%$ of the energy in a suprathermal tail.

The performance of solid spheres of DT is sensitive to the production of suprathemil electrons when the laser light is absorbed above the threshold of the parametric decay instability. However, the targets can be successfully imploded to give better than a breakeven yield of thermonuclear energy with a significant fraction of the laser energy in such a tail. These tails

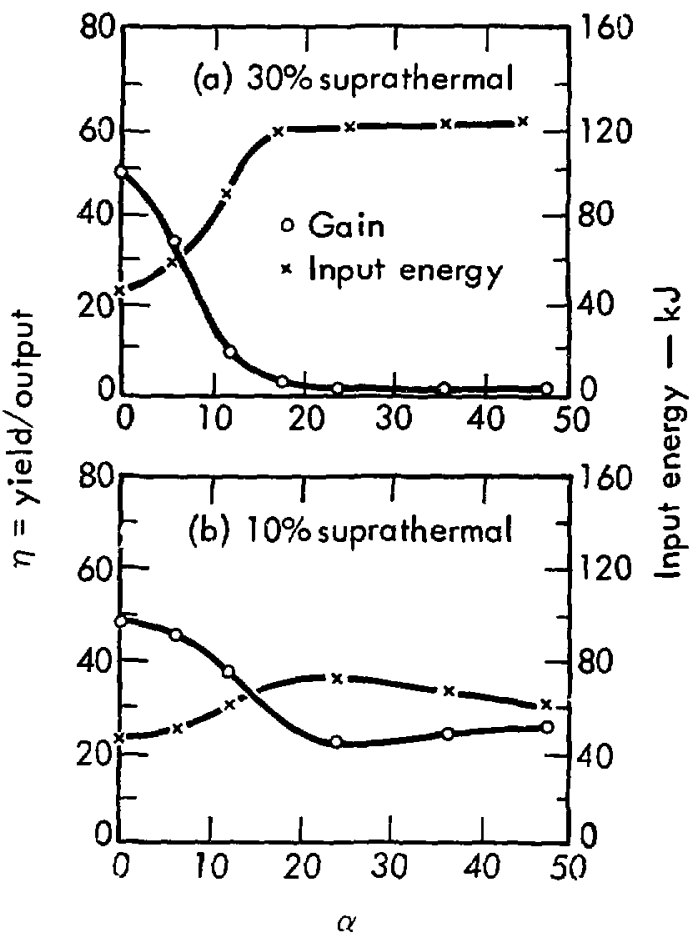

Fig. 95. Gain and input energy as functions of the slope $\alpha$ of the suprathermal distribution $F(v)=B v^{2} \exp$ $\left(-1 / 2 m r^{2} / \alpha K T_{e}\right)$ for (a) $30 \% \%$ of absorbed laser energy in suprathermal electrons, and (b) $1 r_{i c}$ of absorbed energy in suprathermals.

are characterized by a distribution of the form $F(v)=$ $B v^{2} \exp \left(-1 / 2 m v^{2} / \alpha K T_{e}\right)$. If $\alpha=6$, then five times break-even can be achieved at $150 \mathrm{~kJ}$ with all of the energy being dumped into a suprathermal tail, and gains of better than 20 achieved at $70 \mathrm{~kJ}$ with $50 \%$ of the energy in tails. In the worst case, with $\alpha \approx 24$. break-even at $100 \mathrm{~kJ}$ is possible with $30 \%$ of the enersy in suprathermal tails.

No attempt has been made to stay below threshold in these calculations, and indeed, the peak intensities of about $10^{17} \mathrm{~W} / \mathrm{cm}^{2}$ are several orders of magnitude above the threshold for the parametric decay instability. If it turns out to be impossible to limit $\alpha$ to 6 or $f$ to $0.3-0.4$. then one still has the choice of modifying the target and laser pulse to stay below threshold. This can be done by seeding the pellet 10 incleisc the effective $Z$, by decreasing the laser wave length, and by making various optimistic estimates about the threshold intensity at which significant energy is dumped into suprathermal tails. Our calculations indicate that one call inplode a target below threshold by seeding to get an effective $\bar{Z} \approx 10$, using 
$0.2-\mu \mathrm{m}$ light and assuming that one can go a factor of 10 above the linear threshold before producing energetic tails. In addition. these calculations assumed the effective threshold intensity could be raised a factor of
10 by about $1 \%$ frequency modulation of the laser beam. One can also greatly reduce the required intensities by use of hollow pellets. However, stability and symmetry requirements are then more severe.

\section{LASER TARGETS REQUIRING MINIMUM LASER POWER}

\section{Hollow DT Shells Imploded with}

\subsection{6- $\mu \mathrm{m}$ Light}

As discussed above, one would like to develop a target that can be imploded with power levels below those which would excite instabilities. Moreover, this target should exhibit efficient absorption by inverse bremsstrahlung. One-dimensional studies with the LASNEX code indicate that there exists a parameter regime in which highly levitated DT shells fill these requirements. The target shown in Fig. 96 gives a yield of $4.3 \mathrm{~kJ}$ when imploded with $1.8 \mathrm{~kJ}$ of $1 / 2-\mu \mathrm{m}$ laser light. This target has a DT mass of $21.5 \mu \mathrm{g}$ which is levilated $50: 1,{ }^{*}$ and the peak laser power is $1.5 \times$ $10^{12} \mathrm{~W}$.

The work done on a target is given by $\int P d V(P=$ pressure, $V=$ volume). The levitation of a target increases the volume and permits the same work to be done with a smaller driving press'ıre. Stability considerations place a limit on the allowed levitation. The growth rate for perturbations on an ablatively accelerated material is given by

$$
\gamma^{2}=k a-\frac{P a}{\rho} k^{2} \text {. }
$$

where $k$ is the wave number of the perturbation, $P_{a}$ is the ablation pressure. and $\rho$ is the density. The approximation $P_{a} / \rho \approx a \Delta r(\Delta r=$ shell thickness) gives the result that perturbations with $k>1 / \Delta r$ are stable. The most unstable mode is for $k=l_{1}^{\prime} 2 \Delta r$ with growth rate $\gamma=\sqrt{a / \Delta r}$. An initial pertusbation $x_{0}$ at this wavelength will grow to $x=x_{0} \exp \left(\sigma^{0} d t\right)=x_{0}$ $\exp \left(\int^{3} / 2 \sqrt{a / \Delta r} d t\right)$. For accelerations that vary as $a t^{2 m}$, the interral give

$$
x=x_{0} \exp \left(\sqrt{\frac{(2 m+1)(2 m+2)}{4(m+1)} \frac{r}{\Delta r}}\right) .
$$

To prevent penetration of the shell with $r / \Delta r=50$, we require $x_{0} / \Delta r<0.0062$ for $m=0$, and $x_{0} / \Delta r<0.00085$ for $m \rightarrow \infty$. These simple calculations indicate that a highly levitated shell would have to be constructed very carefully if it is to be imploded intact. The

* The levitalion ratio is the ratto ol the radius of tise hollow splierical pellet to the thickness of ils DT shell-je.., r/Lr. numbers given here are only a crude estimate of the required surface finish, and there is some theoretical uncertainty about the applicability of Eq. (22). Detailed two-dimensional hydrodynamic calculations are required for an accurate estimate, and these are being carried out.

To take advantage of the large volume attained by levitation, one must put the laser energy into the target earlier in the pulse than would be optimal for a solid target. When imploding solid spheres of DT, a typical laser pulse is given by

$$
\begin{gathered}
\epsilon(t)=\epsilon(0)\left[\frac{1}{1-t \frac{1-R}{t_{f}}}\right]^{M} . \\
R=\left(\frac{\epsilon(0)}{\epsilon_{j}}\right)^{1 / M},
\end{gathered}
$$

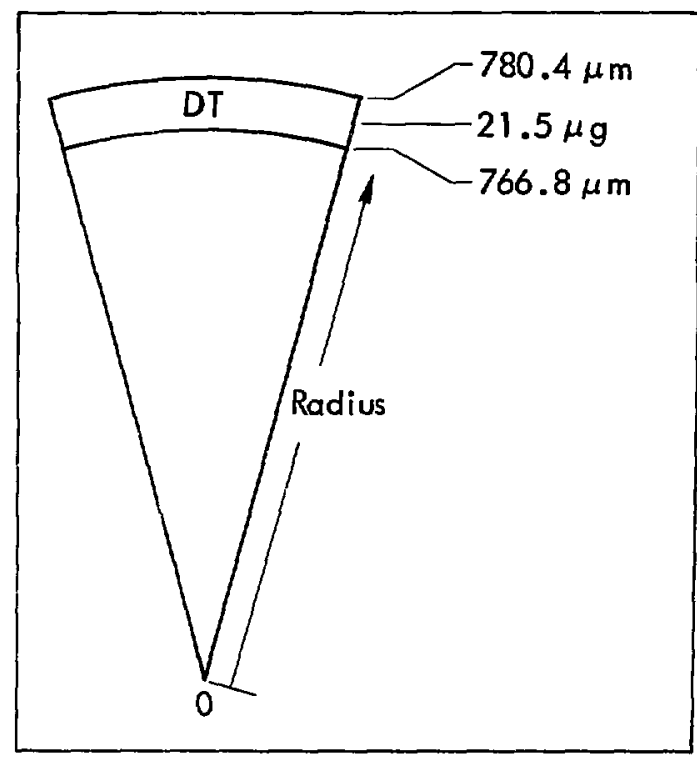

Fig. 96. Levitated DT shell (i.e., hollow, with thin walls) allows implosion at power levels below those which would excite instabilities. 
where $\epsilon(0)$ and $\epsilon_{f}$ are initial and final energies. As $M \rightarrow$ $\infty$ this function approaches an exponential, and it is more rapidly rising than an exponential for all $M>1$. The smaller $M$ is, the more rapidly is energy put into the target at the end of the pulse. For a solid sphere of $D T, M$ is about 2 , and half of the energy is put into the target in the final $0.2 \%$ of the laser pulse. The levitated target in Fig. 96 was imploded with $M=100$, essentially an exponential power profile. For this pulse, given in Table 17, half of the energy goes into the target in the last $6 \%$ of the pulse. Expressed another way, in the solid sphere target, $r / r_{0}$ has decreased a factor of 10 before half of the energy is deposited, while in the hollow target, $r / r_{0}$ has only decreased a factor of 2 when half the energy is deposited. As a consequence of this earlier energy deposition, a much larger fraction of the target mass is ablated. In typical solid-sphere targets with $50 \mathrm{~kJ}$ input energy, between $1 / 4$ and $1 / 3$ of the target mass is compressed and bur: In the levitated target of Fig. 96, more than 99\% of $\mathrm{t}_{\mathrm{l}}$ - target is ablated away. Because of the much greater ablation, the initial target mass relative to the input energy is much larger for a levitated target than for a solid sphere. For example, a solid sphere imploded with $50 \mathrm{~kJ}$ would have a mass of about 60 $\mu \mathrm{g}$ while the target of Fig. 96 has $21.5 \mu \mathrm{g}$ with a driving energy of only $1.8 \mathrm{~kJ}$.

In order to get efficient absorption by inverse bremsstrahlung and stay below threshold for instabilities, one must use a laser of sufficiently short wavelength. When the target in Fig. 96 is imploded with a $1 / 2-\mu \mathrm{m}$ laser, $99 \%$ of the energy is absorbed by inverse bremsstrahlung. The threshold for the parametric decay instability is given by

$\eta^{2}=\frac{E^{2}}{4 \pi n_{c} K T_{e}}<4\left(1+\frac{T_{i}}{T_{e}}\right) \frac{\nu_{e i}}{\omega}\left(\frac{1+f}{s}\right)$,

where $E^{2} / 4 \pi$ is the electric field's density, $n_{c}$ is the critical density, $T_{e}$ is the electron temperature, $T_{i}$ is the ion temperature, $\nu_{e i}$ is the electron-ion collision frequency, and $\omega$ is the laser frequency. The factor $f$ accounts for the increased threshold because of frequency modulation of the laser; $s$ is a factor decreasing the threshold because of swelling of the intensity in the density gradient. For conditions appropriate to the levitated shell $\left(T_{f} \approx 1 \mathrm{keV}, T_{i} / T_{e} \approx 1 / 3, I_{\max } \approx 7 \times\right.$ $\left.10^{13} \mathrm{~W} / \mathrm{cm}^{2}\right), \eta^{2} \approx 0.0075$ and we get $(1+f) / s \geq 1.5$ for stability. The value for $s$ is expected to be small because the laser light is heavily damped by inverse bremsstrahlung. For $f \approx 2$, which is typical of neodymium, the proposed pulse should be below threshold. To prevent significant backscatter due to the Brillouin instability, we require

$$
\eta^{2} \frac{L}{\lambda} \leq 10(1+f)
$$

or for conditions here $L / \lambda \leqslant 4000$ where $L$ is the density scale length and $\lambda$ is the laser wavelength. This condition is easily satisfied.

The pulse shape, target mass, and dimensions presented here are not crucial. We have also considered a similar target, levitated 50:1, but have used an $M=4$ laser pulse and a target with about $h_{\llcorner}$'? the mass of that in Fig. 96. In that case, a smaller fraction of the mass is ablated and about the same mass of material is left to burn. Using such a pulse, a slightly smaller fraction of the energy is absorbed by inverse bremsstrahlung and peak intensities are about a factor of 2 higher, but the yield is about the same.

These calculations imply that successful laser-driven implosions are possible with much lower total energy and smaller peak laser intensities than previously thought feasible. The results obtained so far have been one-dimensional. Before this type of target can be considered viable, we must determine the symmetry of the laser power required to get a spherical implosion. We are proceeding with two-dimensional calculations designed to answer this question.

\section{Utility of 10.6- $\mu \mathrm{m}$ Laser Light for}

\section{High-Density Laser-Induced Fusion with} Levitated DT Shells

It has been suggested that long-wavelength $\mathrm{CO}_{2}$ light may not be as effective as shorter-wavelength laser light for high-density laser-induced fusion. ${ }^{58}$ Within the last six months, we have considered the implosion of hollow shells of DT produced by shaped pulses of $10-\mu \mathrm{m} \mathrm{CO}$ laser light. Since the implosion of levitated shells requires so much lower laser intensities than the implosion of solid spheres, one might hope that, while $10-\mu \mathrm{m} \mathrm{CO}_{2}$ light is not useful with solid spheres, it might be with levitated shells. Unfortunately, our calculations do not substantiate this hope. In these LASNEX calculations, we investigated the effects of suprathermal electron spectra, electron decoupling, preheat, flux limiting, and classical $\vec{J} \cdot \vec{E}$ coupling. These predictions are compared with earlier results ${ }^{63}$ and with results based on the more restricted three-temperature approximation. ${ }^{64}$ Successful $\mathrm{CO}_{2}$ implosions were only achieved when unduly optimistic assumptions were made about the electron spectra. Unfavorable effects arising from self-generated magnetic fields or frum anomalous reflection of the laser light have not been included in this analysis. The option of seeding the pellet with high- $Z$ material ${ }^{45}$ has not, however, been fully investigated. The feasibility of $\mathrm{CO}_{2}$ implosions may depend on the success of this option.

The calculations assumed laser energies of about 5 $\mathrm{kJ}$ and $500 \mathrm{~kJ}$ in order to investigate pellet performance near break-even as well as at high gain. 
The larger $500 \cdot \mathrm{kJ}$ pellet is less affected by electron decoupling and prehea: because it implodes in a longer time and contains many more electron mean free paths. Figure 97 shows the dimensions of the pellets and the laser pulse shapes. The pellets are hollow shells of DT weighing 7.5 and about $1000 \mu \mathrm{g}$, respectively. The ratio of pellet radius to shell thickness is about 60 to 1 in each case. As pointed out above, any greater ratio is highly susceptible to Taylor instability. Our LASL co-workers have reported a 5-kJ calculation of the same $7.5-\mu \mathrm{g}$ pellet. We may, thus, compare our results with those computed independently. Most of the $5-\mathrm{kJ}$ laser pulse shape is determined by the following equation:

$$
\dot{E}(t)=\dot{E}_{0}\left(1-\frac{t}{t_{0}}\right)^{-m} \text {, }
$$

with

$$
\begin{aligned}
\dot{E}_{0} & =5.7 \times 10^{8} \mathrm{~W}, \\
t & =30 \mathrm{nsec}, \\
m & =1.875 .
\end{aligned}
$$

This equation was followed to a peak power of $3.9 \times$ $10^{13} \mathrm{~W}$, and the total source energy was $5.3 \mathrm{~kJ}$.

Two different sources were employed in the calculations of the $1000-\mu \mathrm{g}$ pellet. One source, shown by the dashed curve, is merely the $5 \cdot \mathrm{kJ}$ pulse shape scaled to 1 MJ. The other source, the solid curve, was used in an attempt to reduce the peak power required to implode the shell and, thereby, the electron energy, decoupling, and preheat. In addition to a contraction of time scale and reduced peak power, there is a departure from the "ideal pulse shape" given by the above equation for $\dot{E}$ This pulse has a total energy of $500 \mathrm{~kJ}$.

Table 18 summarizes the results obtained at $5 \mathrm{~kJ}$. Problem $A$ was run with the 3-T approximation (three temperatures, including one temperature for an assumed Maxwellian electron spectrum). Problem $B$ shows that in this case use of a flux limiter makes little difference. In problems $C$ through $F$, multigroup diffusion . of electrons was used so that the effects of electron decoupling and preheat could be calculated. In problem $C$, a Maxwellian electron spectrum was assumed and the $\vec{J} \cdot \vec{E}$ coupling between the suprathermal and thermal electrons was assumed to be negated by electron runaway and other effects. With these assumptions, preheat and decoupling severely degrade implosion.

In calculating $D$, the $\vec{J} \cdot \vec{E}$ coupling was assumed to be fully effective. This reduces the decoupling and preheat effects sufficiently so that a substantial thermonuclear yield is achieved. Since an inverse bremsstrahlung distribution of electrons is probably an
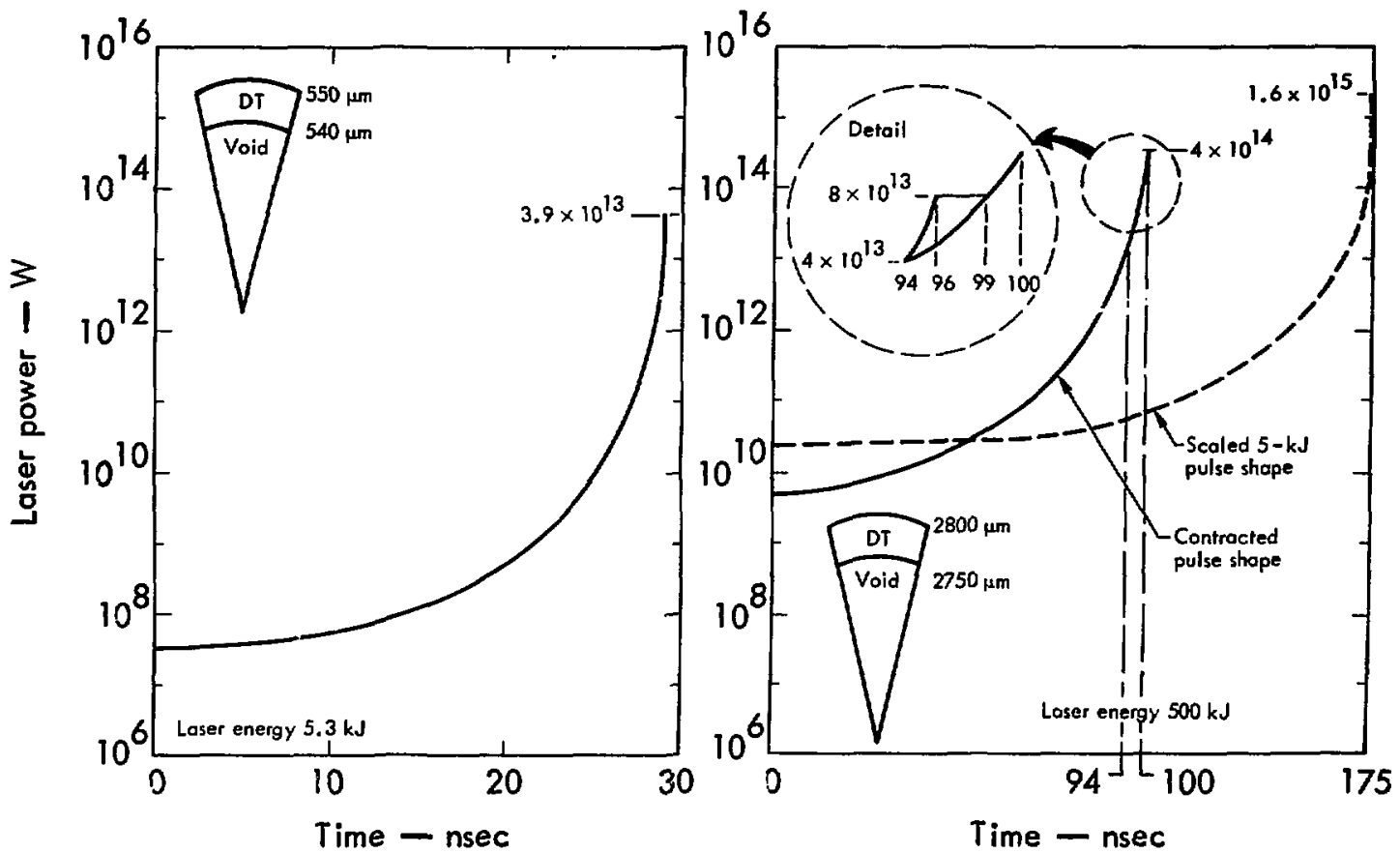

Fig. 97. Pellet dimensions and pulse shapes used in calculations of implosion of levitated pellets of DT by $10.6-\mu \mathrm{m} \mathrm{CO}_{2}$ laser light at energies of 5 and $500 \mathrm{~kJ}$. 
Table 18. Calculational assumptions and results of six problems run for the case of the 7.5- $\mu \mathrm{g}$ pellet irradiated by the $5-\mathrm{kJ}$ laser pulse

\begin{tabular}{|c|c|c|c|c|c|}
\hline Problem & $\begin{array}{l}\text { Multigroup } \\
\text { clectron } \\
\text { diffusion? }\end{array}$ & $\begin{array}{l}\text { Hot electron } \\
\text { distribution }\end{array}$ & $\begin{array}{l}\text { Flux-limited } \\
\text { electrons? }\end{array}$ & $\begin{array}{c}\overrightarrow{\mathbf{J}} \cdot \overrightarrow{\mathbf{E}} \\
\text { couplin_? }\end{array}$ & $\begin{array}{c}\text { Calculated } \\
\text { yield } \\
(\mathrm{kJ})\end{array}$ \\
\hline$A$ & No & - & Yes & - & 70 \\
\hline$B$ & No & - & No & - & 60 \\
\hline C & Yes & $\begin{array}{l}\text { Inverse brems- } \\
\text { strallung }(I B)\end{array}$ & Yes & No & $\mathbf{0}$ \\
\hline$D$ & Yes & $\begin{array}{l}\text { Inverse brems- } \\
\text { strahlung }(1 B)\end{array}$ & Yes & Yes & 30 \\
\hline$E$ & Yes & $97 \%\left(B+3 \% F(v)^{i}\right.$ & Yes & Yes & 20 \\
\hline$F$ & Yes & $90 \% 1 B+10 \% F(v)^{3}$ & Yes & Yes & 0 \\
\hline
\end{tabular}

${ }^{a} F(v) \propto \exp \left[\frac{-v^{2}}{12\left(v_{t h}\right)^{2}}\right]$.

optimistic assumption, a more realistic non-Maxwellian distribution function ${ }^{59}$ was used in calculations $E$ and $F$. In $E$, with $3 \%$ of the electron energy in a nonMaxwellian distribution (and $97 \%$ in a near-Maxwellian

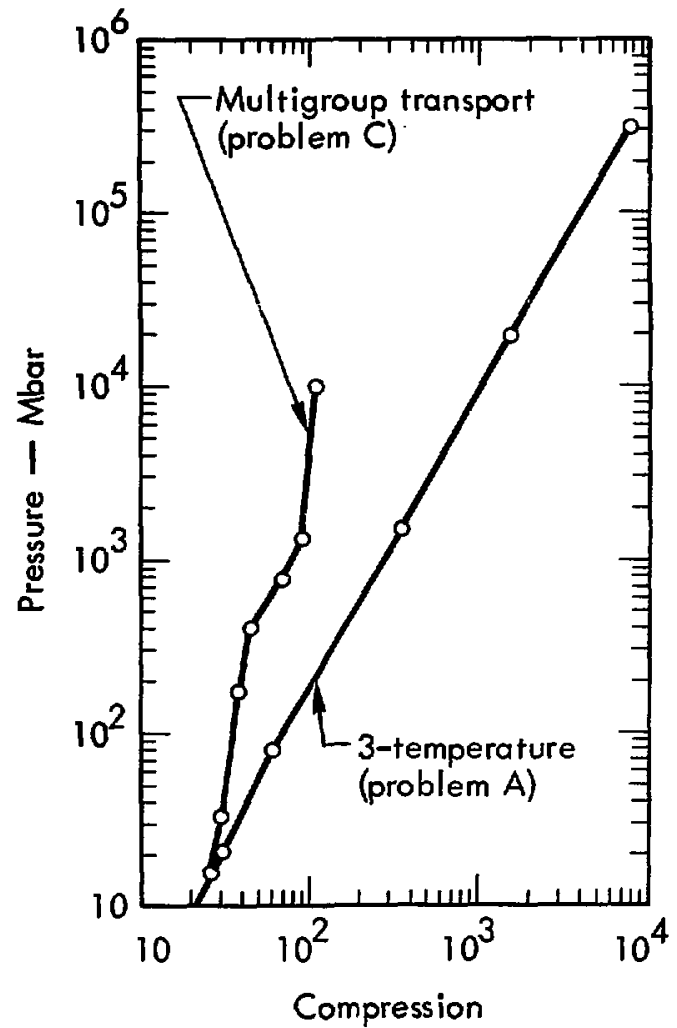

Fig. 98. Comparison of adiabats of typical fucl zones as calculated for problems $A$ and $C$ (sec Table 18). inverse bremsstrahlung distribution) the fusion yield is moderately reduced. With only $10 \%$ of the electron energy in a hot component, the implosion is degraded despite the assumption of $\vec{J} \cdot \vec{E}$ coupling (calculation $F$ ).

The $\vec{J} \cdot \vec{E}$ coupling term is treated classically by LASNEX. The possibility of anomalous coupling of hot electron energy into thermal electrons exists and could improve the pellet performance.

Figure 98 illustrates the effects of decoupling and preheat in the calculations of Table 18. The adiabats of typical fuel zones in problems $A$ and $C$ are compared. As is seen, not only is the shell severely preheated (a much higher pressure is required to attain a given compression), but also the hot electrons are decoupled from the pellet. In problem $D$, the $\vec{J} \cdot \vec{E}$ heating effectively couples most of the inverse bremsstrahlung tail into the thermal electrons with the result that its adiabat is quite close to the 3-T curve shown in Fig. 98 (problem $A$ ).

Figure 99 shows the suprathermal electron spectra generated in problem $C$, both in the corona and in the core (dense imploding fuel). In contrast to the 10-to-20-keV electrons which are generated in the 3-T calculation, we see that the electrons have energies in excess of $50 \mathrm{keV}$ when multigroup electron diffusion is used. Heuristic calculations with the flux limiter and range equations show that such electrons are generated, that they will decouple from the thermal electrons, and that they severely preheat the imploding shell.

Table 19 summarizes the results obtained for the larger pellet. We note two interesting effects. The $\vec{J} \cdot \vec{E}$ coupling term has virtually no effect on the pellet performance $\left(A^{\prime}\right.$ and $\left.B^{\prime}\right)$, nor do the decoupling and preheat effects, provided absorption proceeds via inverse bremsstrahlung distribution $\left(A^{\prime}\right.$ and $C^{\prime}$ ). 
Table 19. Calculational assumptions and results of five probiems sun for the case of the $1000-\mu \mathrm{g}$ pellet irradiated by a $500-\mathrm{kJ}$ or $1-\mathrm{MJ}$ laser pulse

\begin{tabular}{|c|c|c|c|c|c|}
\hline Problem & $\begin{array}{c}\text { Multignoup } \\
\text { electron } \\
\text { diffusion? }\end{array}$ & Hot electron distribution & $\begin{array}{c}\quad \overrightarrow{\mathbf{J}} \cdot \overrightarrow{\mathbf{E}} \\
\text { coupling? }\end{array}$ & $\begin{array}{l}\text { Laser pulse } \\
\text { energy } \\
\text { (kJ) }\end{array}$ & $\begin{array}{l}\text { Calculated } \\
\text { yield } \\
\text { (MJ) }\end{array}$ \\
\hline$A^{\prime}$ & Yes & Inverse bremsstrahlung (IB) & Yes & 500 & 30 \\
\hline$B^{\prime}$ & Yes & Inverse bremsstrahlung ( $I B$ ) & No & 500 & 30 \\
\hline$C^{\prime}$ & Yes & Inverse bremsstrahlung (IB) & Yes & 1000 & 60 \\
\hline$D^{\prime}$ & Yes & $\begin{array}{l}90 \% I B+10 \% F(v)^{a} \text { for power } \geqslant 10^{12} \mathrm{~W} / \mathrm{cm}^{2} \\
100 \% I B \text { for power } \leqslant 10^{12} \mathrm{~W} / \mathrm{cm}^{2}\end{array}$ & Yes & 500 & $\mathbf{0}$ \\
\hline$E^{\prime}$ & Yes & Same as $D^{\prime}$ & Yes & 1000 & $\mathbf{0}$ \\
\hline
\end{tabular}

${ }^{a} F(v) \propto \exp \left[\frac{-\nu^{2}}{12\left(v_{t h}\right)^{2}}\right]$.

Calculation $D^{\prime}$ assumes that $10 \%$ of the laser energy is deposited via plasma instabilities and the remainder of the energy via inverse bremsstrahlung. In addition, the threshold for the anomalous absorption (instability threshold) was raised to $10^{12} \mathrm{~W} / \mathrm{cm}^{2}$ to mock up very crudely the effect on the instability thresholds of seeding the pellet with high $-Z$ material. Problem $E^{\prime}$ was a similar recalculation of problem $C^{\prime}$. In both cases, the

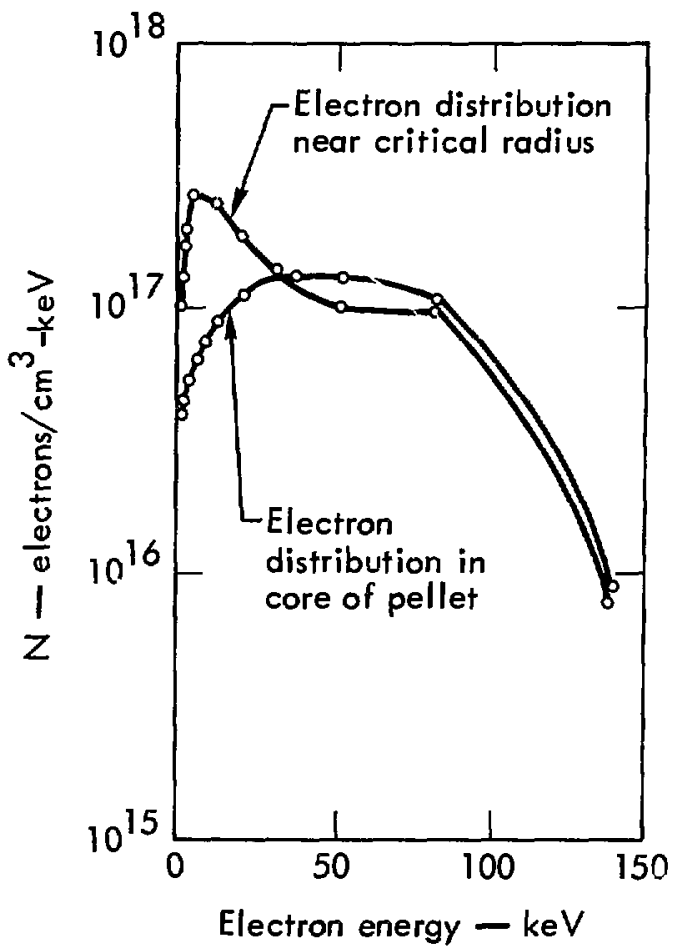

Fig. 99. Suprathermal electron spectra generated in problem $C$ (see Table 18) in the corona and the core.

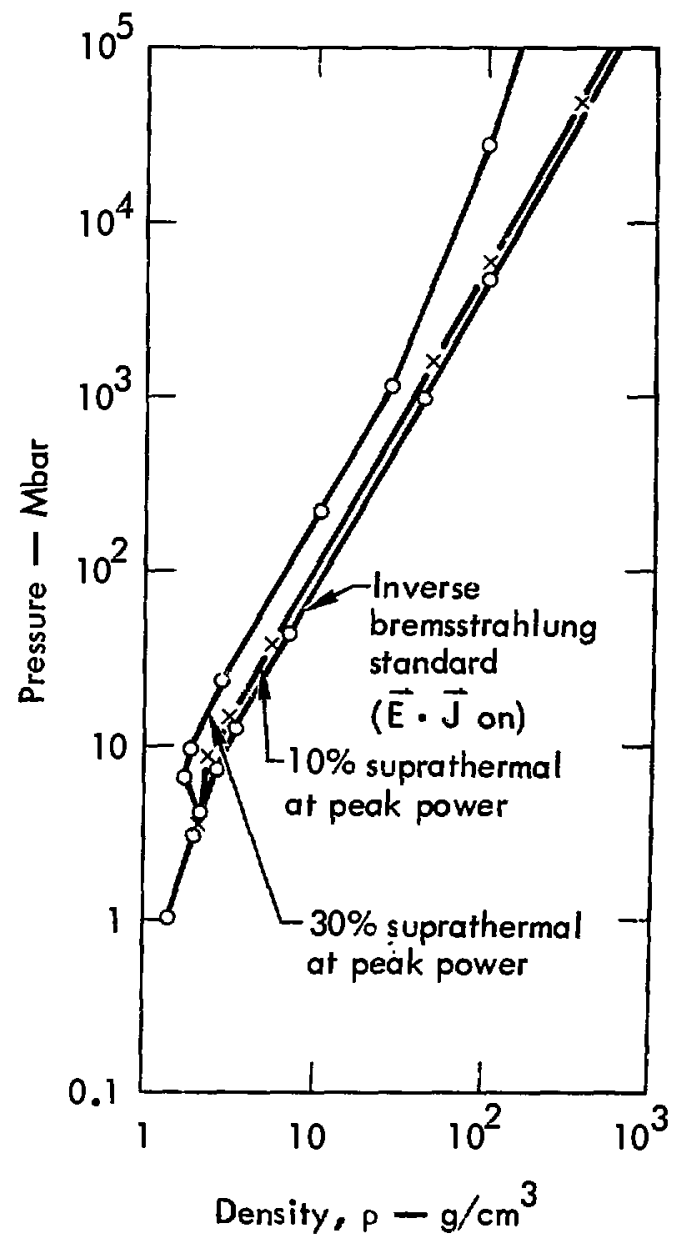

Fig. 100. Effect of electron preheat on pellet compression. Increasing percent of non-Maxwellian electrons degrades pellet performance. 
implosion is degraded despite the fact that only $10 \%$ of the electron energy has appeared in the non-Maxwellian tail!

To explore the sensitivity of a hollow pellet to the instability threshold, a number of calculations were carried out allowing the hot electrons to be generated only after the laser pulse reached peak power. The use of shorter-wavelength light is a known means by which the threshold could be increased to such levels. Figure 100 gives a com. parison of the adiabats of these calculations. If $\mathbf{3 0 \%}$ of the laser energy supplied at peak power appears as non-Maxwellian electrons, severe degradation of the pellet performance results because of preheat. With $10 \%$ of the energy in non-Maxwellian electrons, we predict a slightly degraded pellet performance.

Unless optimistic electron spectra were assumed, successful implosion of hollow DT pellets was not achieved with $10-\mu \mathrm{m} \mathrm{CO}$ light. The implosion of pellets seeded with. high- $Z$ materials has not yet been fully explored. 


\section{LASER-PLASMA EXPERIMENTAL}

The laser-fusion experimental prograin has close and active relationships with the work on laser-fusion target design (Sec. 6), laser-plasma theory (Sec. 8), and diagnostics (Sec. 5). This association is best described as symbiotic and the reader is encouraged to regard Secs. $5-8$ as a unified entity.

The investigations of laser-produced plasmas continue to be guided by the need for an understanding of laser/target interaction mechanisms and of those plasma characteristics, such as anomalous electron distributions, that are especially significant for laser fusion. We have enhanced our ability to explore spectral and geometrical features of laser-produced plasmas with the completion of two new instruments. One of these-a simple $x$-ray microscope-has been used to examine the spatial characteristics of the $x$-rayemitting plasmas produced with our laser systems. The LASNEX computer program has successfully reproduced characteristic features of the micrographs obtained with the $\mathrm{x}$-ray microscope. A second instrument has resulted from our ability to bend KAP crystals to a small radius of curvature, leading to an $x$-ray spectrograph that permits the examination of a broad spectral range with a single exposure. The spectral data are converted to absolute values with the aid of a computer program.
Our studies of the very high energy $x$ rays (above $\sim 10 \mathrm{keV}$ ) emitted from laser-produced plasmas have been augmented by simultaneous measurements of the low energy spectrum, and by investigations of the angular distribution of the high energy $x$ rays. These experiments, in conjunction with the corresponding computational studies, are providing information on laser/plasma coupling mechanisms of special significance for laser fusion. Since a knowledge of focused laser beam intensity is essential to the study of laser/target interactions, we have developed an experimenta' and computational technique that provides detailed information concerning the intensity pattern of the focused beam. Other developments include improvements is the accuracy with which we measure the $x$-ray yield from laserproduced plasma and, consequently, an increased knowledge of the efficiency with which laser light is converted to soft $x$ rays by plasma production. We have also developed a new method of measuring the total laser light reflected from the target, and some preliminary results have been obtained. Many of these diagnostic techniques are increasingly used in parallel, which provides data that are particularly helpful in constructing a more complete picture of laser-plasma characteristics.

\section{X-RAY MICROSCOPY}

Since $\mathrm{x}$ rays can be reflected through small angles from highly polished surfaces, it is possible to construct an $\mathrm{x}$-ray imaging arrangement, or lens, using this reflection property. We have produced a simple twoelement grazing-incidence $x$-ray lens having a focal length of about $10 \mathrm{in}$. When this lens is placed $14 \mathrm{in}$. from the $x$-ray source, it forms an image with a magnification of 3 at a distance of about 42 in. from the lens. It therefore functions as an $\mathrm{X}$-ray microscope suitable for examining the geometrical features of a laserproduced plasma viewed as an $\mathrm{x}$-ray source.

The $x$-ray lens consists of two cylindrically ground glass surfaces. Each cylindrical surface produces a line focus in the image plane. The two suriaces are oriented at $90^{\circ}$ with respect to one another and are placed so that a small fraction of the incident $x$-ray beam is reflected from both surfaces. The combination of the two perpendicular line foci produces a true image as indicated in Fig. 101.

The present version of the $\mathrm{x}$-ray microscope has a resolution of less than $25 \mu \mathrm{m}$. The $\mathrm{x}$-ray reflection properties of the glass surfaces produce a high-energy cutoff at about $2 \mathrm{keV}$. Figure 102 illustrates th 3 transmission properties of the $\mathrm{x}$-ray lens, including the use of a filter to provide additional spectral selectivity.

The $x$-ray micioscope has been used to examine the spatial characteristics of plasmas produced with both the Plasma-X and the Long Path Laser systems. Figure 103 shows a schematic representation of a laserproduced plasma and a photograph of a Jong Path Laser plasma obtained with the $\mathrm{x}$-ray microscope. We have successfully reproduced such photographs using the LASNEX code (see Sec. 6). More advanced $\mathrm{X}$-ray microscopes with improved resolution and extended spectral range have been planned for future measurements. 


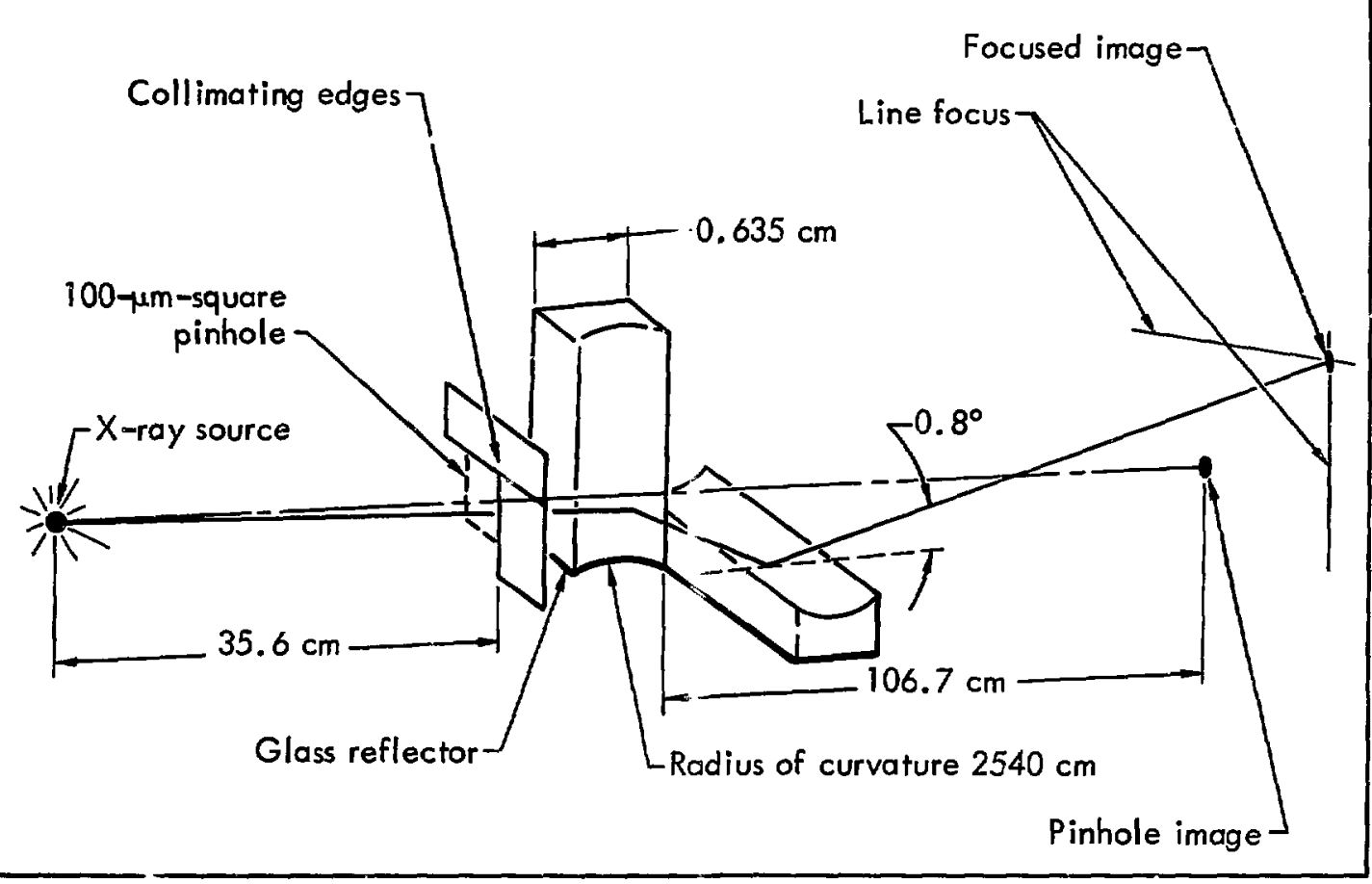

Fig. 101. Schematic representation of the x-ray microscope used to study laser-produced plasmas.

\section{X-RAY SPECTROSCOPY}

We have augmented our spectroscopic capabilities with the development of a bent KAP crystal spectrograph. The crystals are bent to a radius of only $2.3 \mathrm{~cm}$, thereby providing a broad range of spectral covercge from approximately $600 \mathrm{eV}$ to 10 $\mathrm{keV}$. The $n \in w$ spectrograph has been used to examine the $\mathrm{x}$-ray emission from various targets irradiated with the Long Path Laser. An example of these spectra, converted to absolute values with the aid of a computer program, is shown in Fig. 104. Interpretation of these spectra is currently in progress.

\section{THE HIGH-ENERGY X-RAY SPECTRUM}

The high-en ergy $x$-ray spectrum (above $\sim 10 \mathrm{keV}$ ) from laser-procuced plasmas is of special interest because of its relationship to plasma instabilities excited by the intense laser light. High-energy $x$ rays may therefore $f$ rovide an inmportant key to the understanding of th. physics of laser/plasma coupling mechainisms. $O$ particular interest is the fact that instability heating may produce a suprathermal electron spectrum, otentially detrimental for some laserfusion schernes.
The x-ray spectrum for a laser-heated $\mathrm{CD}_{2}$ target from about $10 \mathrm{keV}$ to above $110 \mathrm{keV}^{65}$. has been redetermined with improved accuracy. The improvements resulted in part from auxiliary measurements in which we obtained the $\mathrm{x}$-ray angular distribution as a function of x-ray energy. The new spectrum, Fig. 105, shows a more pronounced shelf (the nearly horizontal region) between about 50 and $100 \mathrm{keV}$. A comparison with computational results is discussed in the previous section (Sec. 6). 


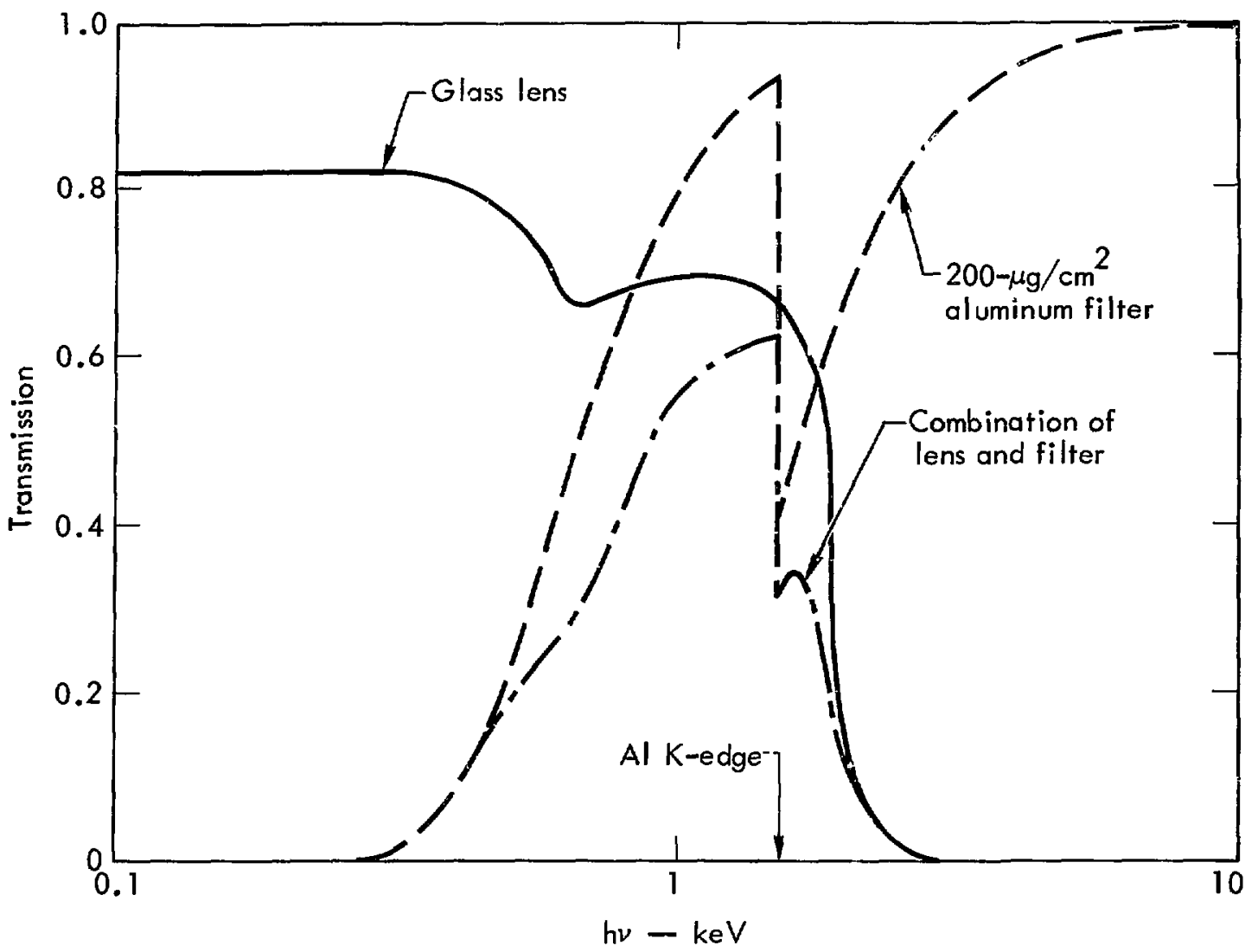

Fig. 102. X-ray transmission characteristics of the X-ray microscope.

In addition to the measurements using $\mathrm{CD}_{2}$ targets, we have explored the effect of target material on the angular distribution of the high-energy $x$ rays. High- $Z$ targets (e.g., $T$ a) give a much lower forward-to-back asymmetry than does $\mathrm{CD}_{2}$. This Z-dependent asymmetry can be used as a diagnostic tool to infer the penetration and hence the approximate energy of the high energy electrons. Some preliminary results were obtained using layered targets consisting of a sandwich of $\mathrm{CD}_{2}$ backed with Ta. If the high energy electror . formed in the $\mathrm{CD}_{2}$ plasma penetrate to the tantalum, they produce a nearly isotropic $x$-ray spectrum; otherwise, the angular distribution will be characteristic of $\mathrm{CD}_{2}$. Additional measurements using a variety of $\mathrm{CD}_{2}$ thicknesses will provide further evidence concerning the electron distribution.

Recent experimental determinations have extended the spectral measurements to the most prominent portion of the spectrum around $1 \mathrm{keV}$. A battery of six detectors provided simultaneous spectral data from 0.5 to $50 \mathrm{keV}$-an intensity range of 11 orders of magnitude. When analyzed and combined with our eailier results, the data wil! provide a spectrum from 0.5 to $100 \mathrm{keV}$, and hence a determination of the relative intensity of the high-energy $x$ rays and a broad range of spectral data for code normalization. The low energy data are a particularly important aid in interpreting the significance of the high-energy $x$ rays. In conjunction with the spectral measurements, we made simultaneous determinations of the $\mathrm{x}$-ray yield above about $1 \mathrm{keV}$ and, as discussed below, we obtained detailed intensity profiles of the laser's far-field pattern. The intensity profiles are essential to a complete computational treatment of the $x$-ray experiments. 


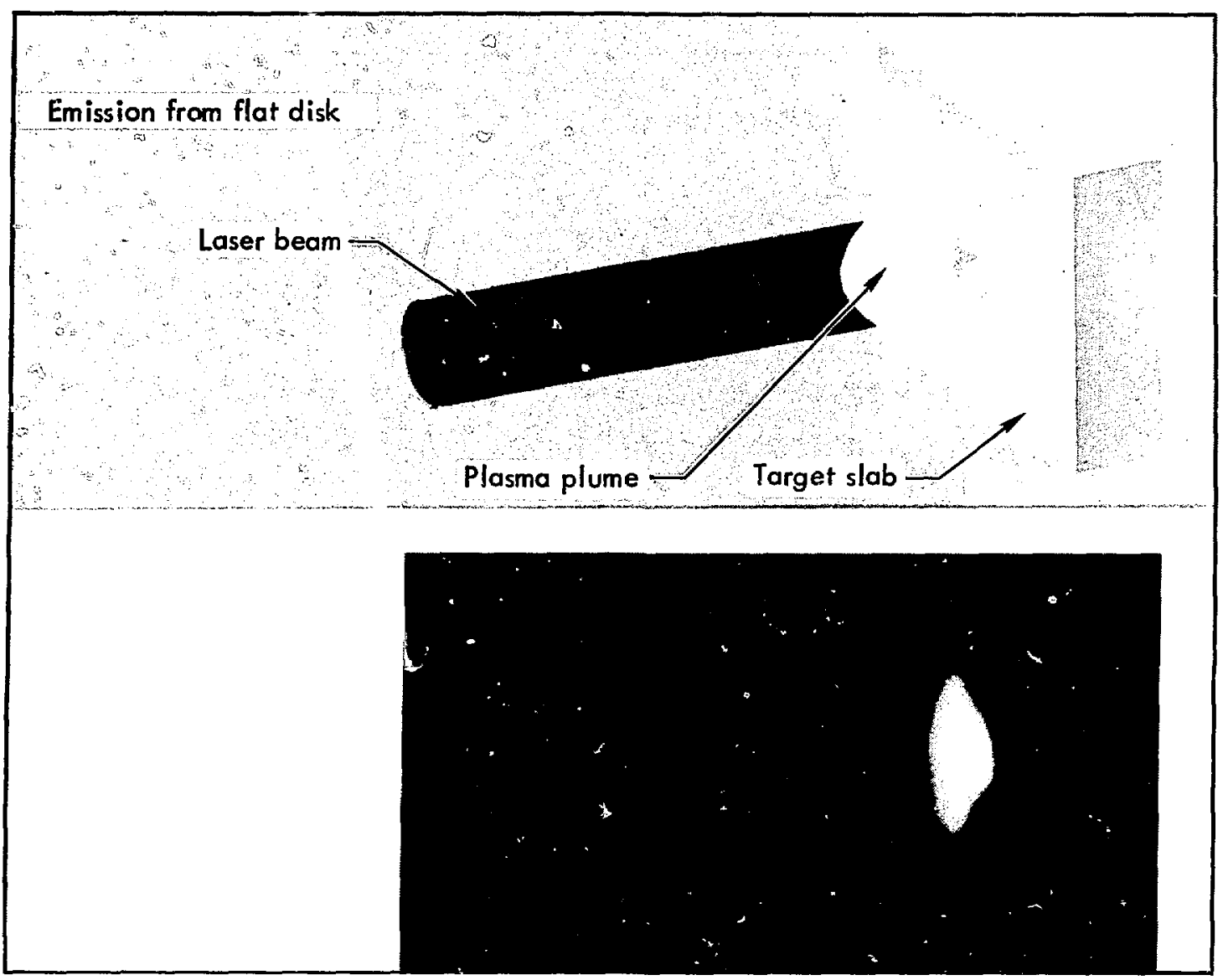

Fig. 103. Schematic representation of a laser-prodused plasma, and a photograph mzde with the x-ray microscope of a plasma produced with the Long Path Laser. The X-ray-emitting region of the plasma has a size of roughly $400 \mu \mathrm{m}$.

\section{LASER BEAM INTENSITY PROFILES}

A determination of the far-field pattern of a laser's output bean can in general provide information concerning the intensity profile of the focused beam at the target. Such data is of key importance in any attempt to analyze laser/target interactions. The method we have developed for far-field exploration involves the calorimetric determination of total laser energy, photodiode measurement of laser intensity as a function of time, and densitometer measurements of multipleimage far-field patterns.
The optical systems used for beam analysis of the Long Path Laser are shown schematically in Fig. 106. Our far-field camera uses a grating, constructed photo. graphically, to produce multiple images whose relative intensities are controlled by the grating's characteristics. The several images provide data for a calibration curve of film density as a function of relative exposure. An important advantage of this system is its capability for self-calibration in a manner that eliminates concern over reciprocity failure. 


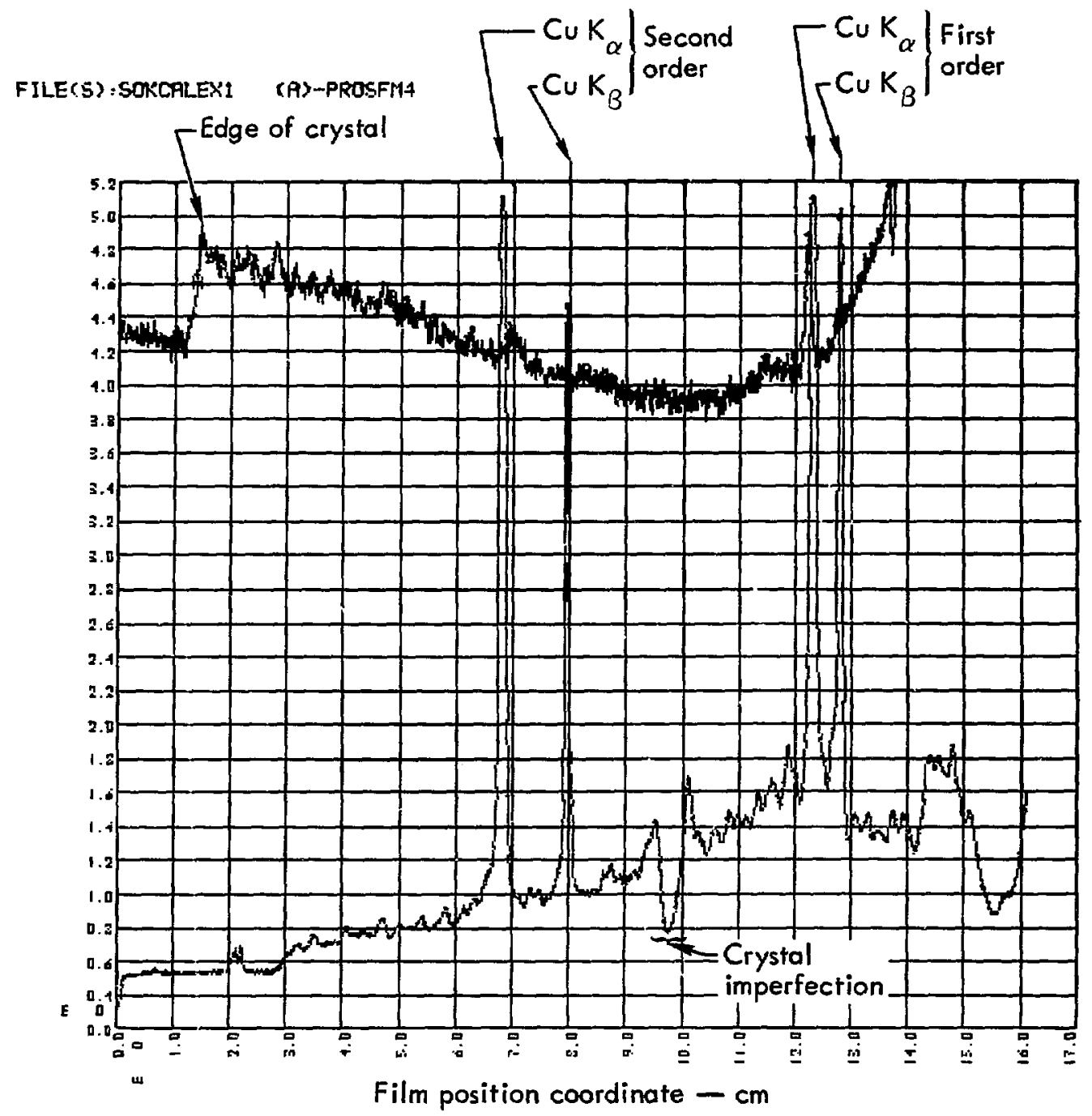

Fig. 104. Film from $x$-ray crystal spectrograph of laser plasma produced at a copper target. Curved pyrolytic carbor crystal with radius of curvature $3.8 \mathrm{~cm}$, crystat-to-film distance $10.2 \mathrm{~cm}$. Upper curve, film density $\times 10$. Lower curve, x-ray machine calibration (copper anode). Curves are displaced in $x$ position to match $\mathrm{Cu} K_{\alpha}$ line.

The multiple film images produced by the far-field camera are scanned by a computer-operated microdensitometer that records the photographic density, as a function of position, on magnetic tape. A computer program has been written that converts the film density data and laser pulse characteristics to the following information, interpreted at the target:

- Intensity contours.

- Three-dimensional intensity profile.
- Peak intensity.

- Distribution of intensity and energy.

Ar example of a three-dimensional intensity distribution and intensity contours for a particular firing of the Long Path Laser is shown in Fig. 107.

Our technique for beam analysis will also be applicable to the new laser systems to be employed in future experiments. A more detailed description of the new method is in preparation. 
Efforts have continued to develop and evaluate $\mathrm{x}$-ray calorimeters and to improve the accuracy of $\mathrm{x}$-ray fluence measurements and laser-light-to- $x$-ray conversion efficiencies. Among the detectors that we have tested extensively are two that are well suited to laserplasma $x$-ray yield measurements and appear to provide very reliable results. These detectors-the silicon surface barrier detector (SiSB) and a special thin-film thermopile matrix (TPM)-give absolute energy readings that are in excellent agreement $( \pm 10 \%)$ when used simultaneously to measure laser-plasma $x$-ray yields. We conclude that we are now able to measure $\mathrm{x}$-ray fluences from laser-produced plasmas to an accuracy of $\pm 10 \%$. The TPM seems particularly

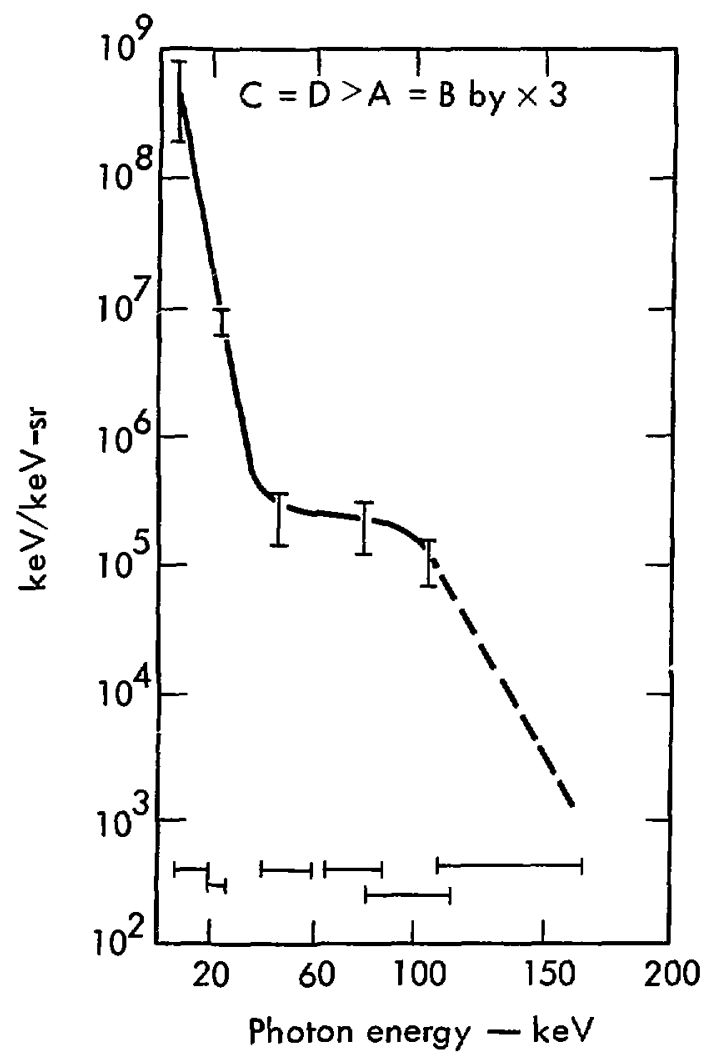

Fig. 105. The high-energy $x$-ray spectrum emitted by a plasma from a $\mathrm{CD}_{2}$ target irradiated by a Long Path Laser pulse of about $30 \mathrm{~J}$ in 3 nsec. appropriate for the higher $\mathrm{x}$-ray fluences anticipated in future experiments with higher energy lasers. Both detectors require some special care under certain circumstances; the performance of the SiSB detector may be degraded by exposure to excessively high $x$-ray fluxes, and careful shielding from electrical noise is an important requirement for satisfactory operation of the TPM.

Among the other detectors we have investigated is a nickel bolometer, which has rather low sensitivity but may be satisfactory for future high-flux experiments. Film calorimetry is an important technique, and a film calibration project is in progress. A pyroelectric detector will be evaluated in the near future.

Our most recent determinations of the laser-lightto-X-ray conversion efficiencies $\left(\epsilon_{x}\right)$, obtained using the Plasma-X and Long Path lasers, are given in Tables 20 and 21. Thic conversion efficiency $\epsilon_{x}$ is the percentage of the incident laser energy that is reemitted as $x$ rays from the target plasma into the backward $2 \pi$ steradians and transmitted by a specified absorber. The measurements were made at an azimuthal angle of $135^{\circ}$ from the laser beam direction; $\epsilon_{x}$ is determined from the measurements on the assumption of isotropic $x$-ray emission into the backward hemisphere.* The random fluctuation in $\epsilon_{x}$ for these lasers is about $\pm 50 \%$. The accuracy of the average value of $\epsilon_{x}$ is about $50 \%$ for the thinnest absorber (1850- $\AA$ aluminum) and $25 \%$ for the thicker absorbers. The errors involved in these efficiency measurements include errors in measuring the incident laser light and in determining the $x$-ray transmission losses in the imperfectly evacuated target chamber. It is of interest to note that the average conversion efficiencies for the iron target and both the 1850- $\AA$ aluminum and the $1 / 2$-mil beryllium absorbers are, perhaps fortuitously, about the same for both lasers. We also note that the measured conversion efficiencies obtained with the Plasma-X laser show a variation with $Z$ that is consistent with similar measurements reported by NRL ${ }^{66}$ and Lockleed. ${ }^{67}$

\footnotetext{
* Recent laser-plasma x-ray measurements by a group at Locklieed Palo Alto Research Laboratory show some anisotropy for $\mathrm{x}$-ray enargies above about $1 \mathrm{keV}$. However, their data indicate that the $x-$-ray fluence at $135^{\circ}$ from the laser beam direction (the position of our detectors) is nearly the sume as the value obtained by averaging over tlic backward hemisphere. Therefore, the issumption of isotropy in interpreting our data is a reasonable approximation.
} 


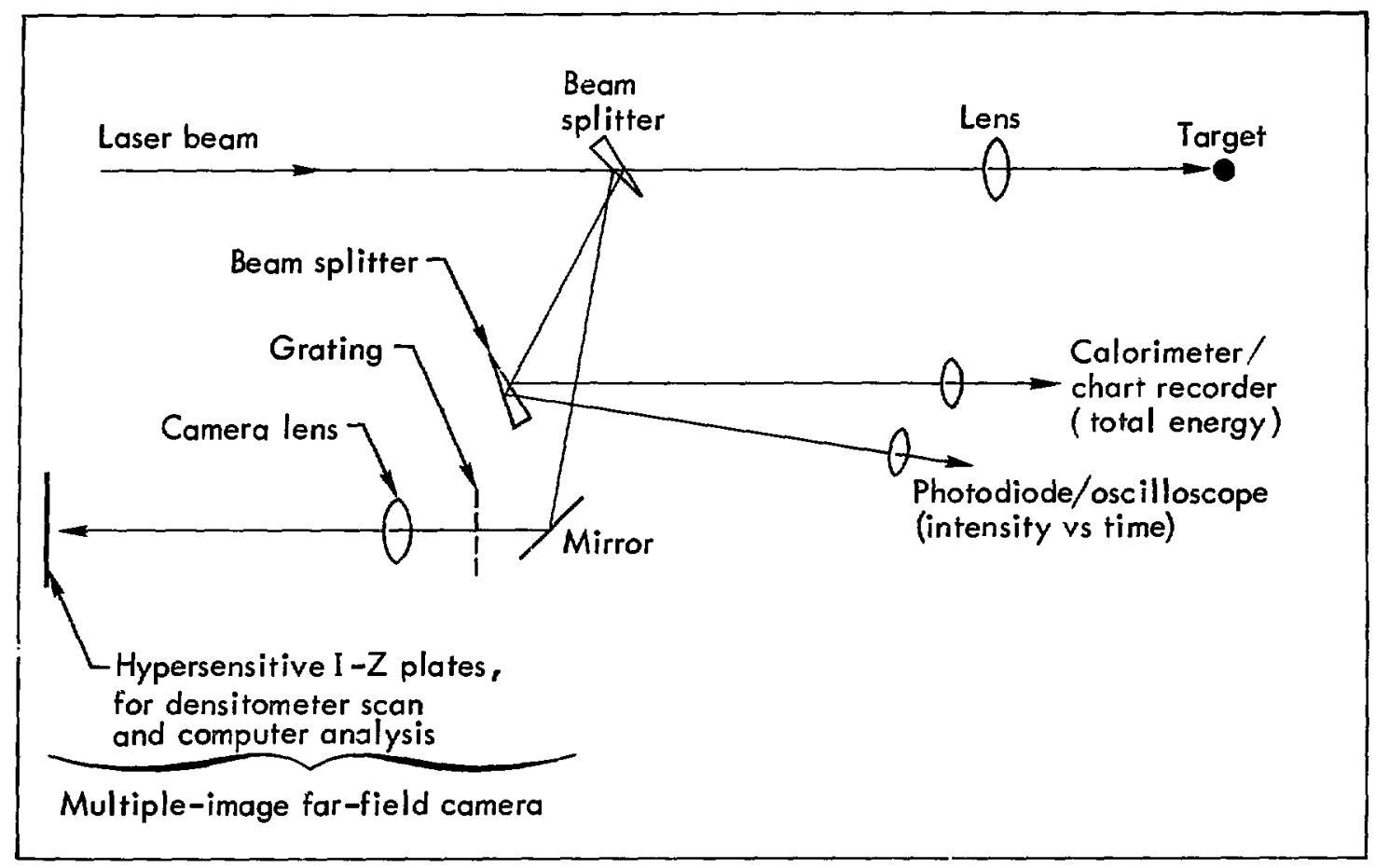

Fig. 106. Schematic representation of optical systems used for beam analysis of the Long Path Laser.

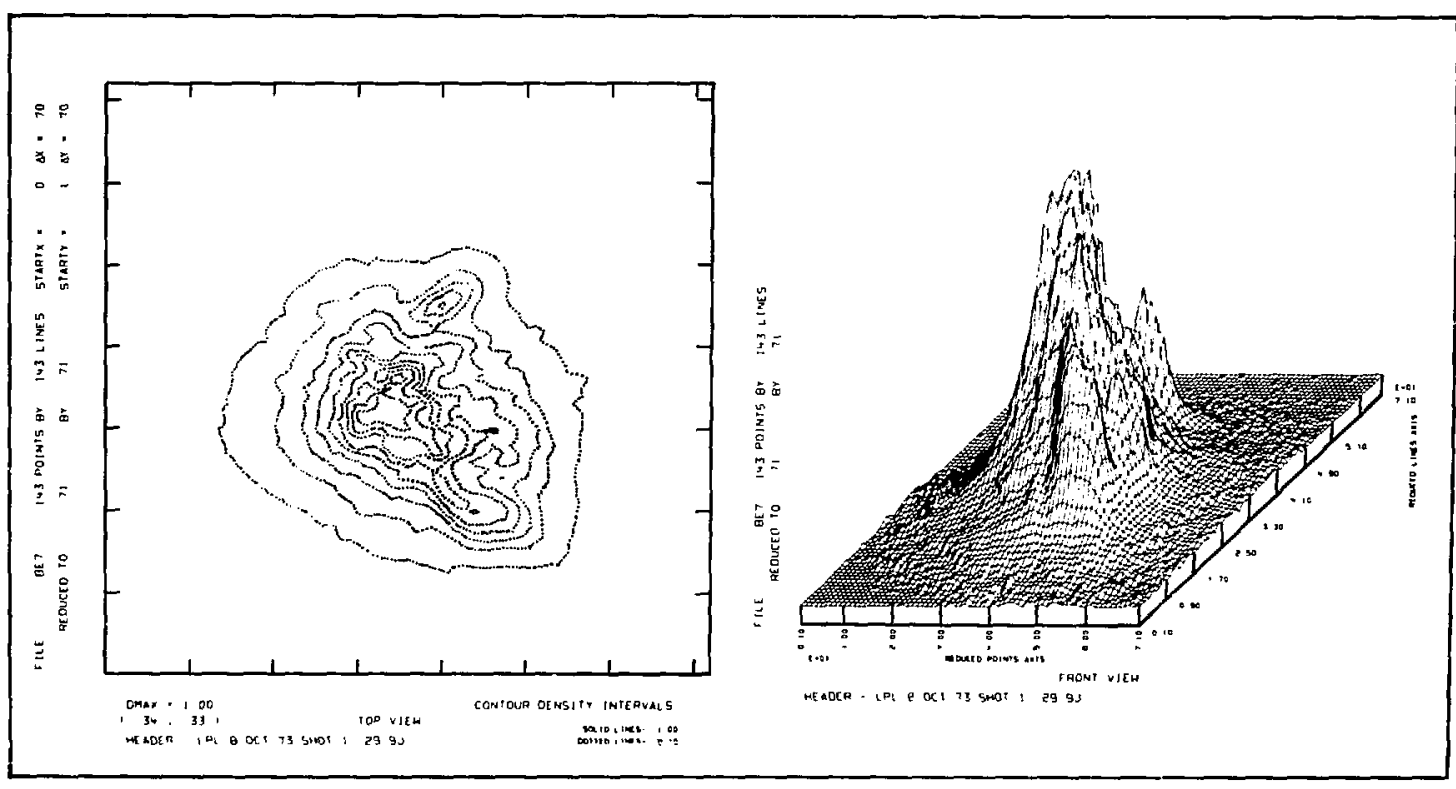

Fig. 107. Computer-generated reproductions of the far-field intensity profile of a Long Path Laser pulse. Contour plot at left, 3-D representation at right. The density provided to the computer was from a multiple-image far-field photograph. Shelf at base of 3-D representation is a result of our method of processing low-density data. 
Table 20. X-ray conversion efficiencies $\left(\epsilon_{x}\right)$ for the Plasma-X laser. Values are average efficiencies for isotropic emission into $2 \pi$ steradians, for laser energy of $1.5 \mathrm{~J}$ in about $\mathbf{3 0 0}$ psec

Absorber

\begin{tabular}{lccc} 
& \multicolumn{2}{c}{ Absorber } & \\
\cline { 2 - 3 } Target & Material & Thickness & Efficiency, $\epsilon_{x}(\%)^{\mathrm{a}}$ \\
\hline $\mathrm{CH}_{2}$ & $\mathrm{Be}$ & $1 / 2 \mathrm{mil}$ & 0.005 \\
$\mathrm{C}$ & $\mathrm{Be}$ & $1 / 2 \mathrm{mil}$ & 0.02 \\
$\mathrm{Al}$ & $\mathrm{Be}$ & $1 / 2 \mathrm{mil}$ & 0.27 \\
$\mathrm{Zr}$ & $\mathrm{Be}$ & $1 / 2 \mathrm{mil}$ & 0.35 \\
$\mathrm{Ta}$ & $\mathrm{Be}$ & $1 / 2 \mathrm{mil}$ & 0.60 \\
$\mathrm{~Pb}$ & $\mathrm{Be}$ & $1 / 2 \mathrm{mil}$ & 0.35 \\
$\mathrm{Fe}$ & $\mathrm{Al}$ & $1850 \AA$ & 18.0 \\
$\mathrm{Fe}$ & $\mathrm{Be}$ & $1 / 2 \mathrm{mil}$ & 1.5 \\
$\mathrm{Fe}$ & $\mathrm{Be}$ & $1 \mathrm{mil}$ & 0.30 \\
\hline
\end{tabular}

\footnotetext{
${ }^{\text {a }}$ Measurement accuracy is about $25 \%$ for all cases except the thin aluminum absorber, for which the accuracy is about $50 \%$.
}

Tabie 21. $X$-ray conversion efficiencies $\left(\epsilon_{x}\right)$ for the Long Path Laser. Values are average efficiencies for isotropic emission into $2 \pi$ steradians, for laser energy of 20-50 J in about 3 nsec

\begin{tabular}{cccc} 
& \multicolumn{2}{c}{ Absorber } & \\
\cline { 2 - 3 } Target & Material & Thickness & Efficiency, $\epsilon_{x}(\%)^{\mathrm{a}}$ \\
\hline $\mathrm{Fe}$ & $\mathrm{Al}$ & $1850 \AA$ & 18.0 \\
$\mathrm{Fe}$ & $\mathrm{Be}$ & $1 / 3 \mathrm{mil}$ & 3 \\
$\mathrm{Fe}$ & $\mathrm{Be}$ & $1 / 2 \mathrm{mil}$ & 2 \\
$\mathrm{Fe}$ & $\mathrm{Be}$ & $5.33 \mathrm{mils}$ & 0.05 \\
\hline
\end{tabular}

\footnotetext{
${ }^{\text {a }}$ Measurement accuracy is about $25 \%$ for all cases except the thin aluminum absorber, for which the accuracy is about $50 \%$.
}

\section{LASER LIGHT REFLECTED FROM THE TARGET}

A knowledge of the intensity and directional characteristics of the laser light reflected from the target is an essential ingredient in the study of laser/target interactions. We have developed a new technique that permits a determination of the total laser light reflected from the target during plasma production. The inethod can also be used to provide a photographic record of the spatial intensity pattern of the reflected laser light. The rentral feature of the method is the use of a parabolic reflector located so as to collect the target-reflected light and direct it through a window at the rear of the target chamber, as shown in Fig. 108. The light is then photographed or focused into a calorimeter. The incident laser light reaches the target by passing through a hole in the center of the parabolic reflector. The light reflected back through this hole passes through the focusing lens and is monitored with the aid of a beam splitter. Preliminary results using an $f / 7$ focusing lens with the Long Path Laser system indicate a total reflection equal to less than $10 \%$ of the incident laser light. Additional measurements with improved reflectors are in progress. 


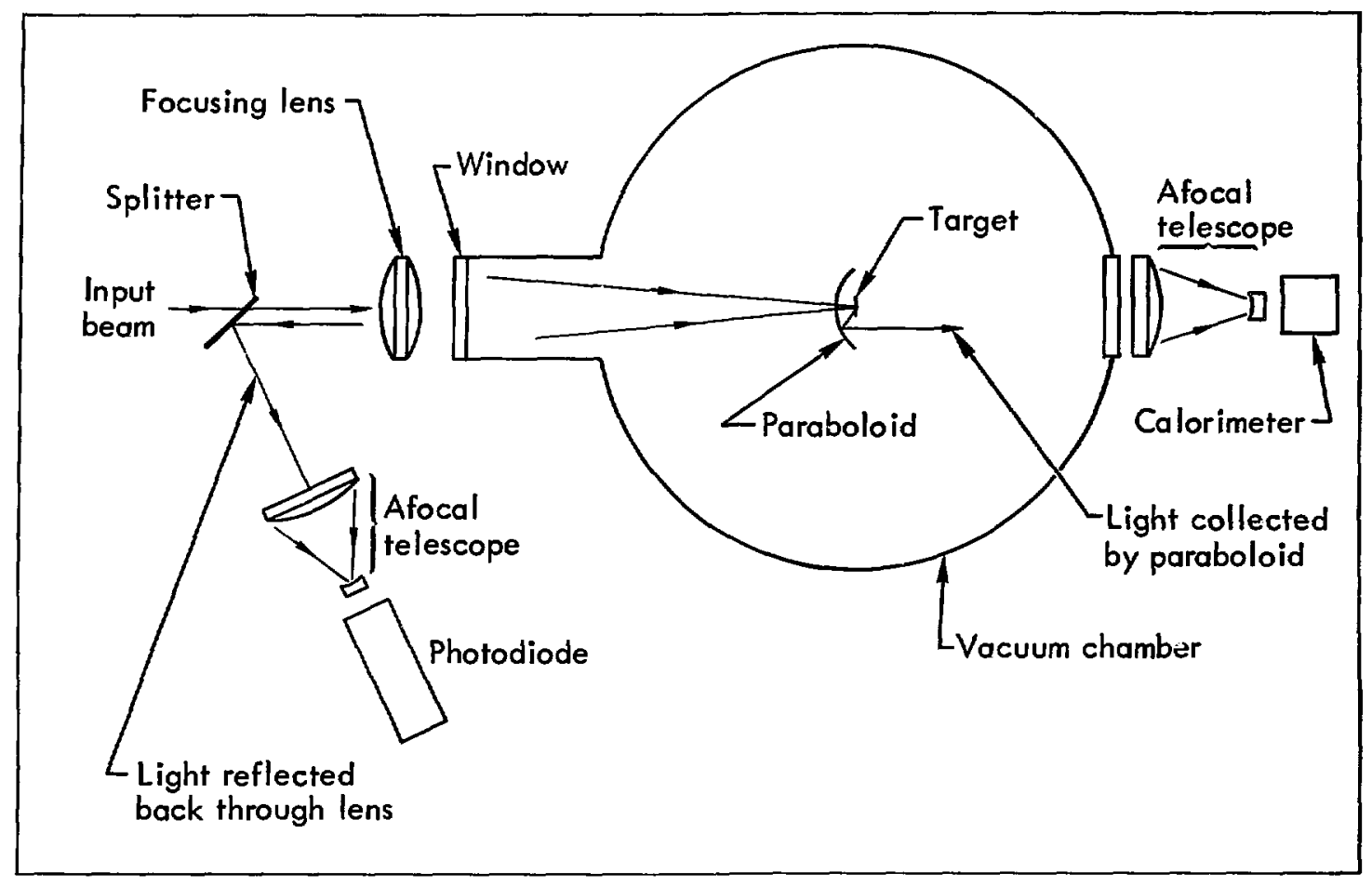

Fig. 108. Principal components of the target reflection experiments done with the Long Path Laser system. 


\section{LASER-PLASMA THEORY}

The design of pellets requires an understanding of how plasina processes affect the absorption of laser light and the transport of electron energy. We have continued to examine these questions in both theory and computer simulation. Let us first present an overview of our principal achievements during the past six months and then discuss these results in more detail.

Our first emphasis has been on understanding the processes which lead to light absorption. The heating of an inhomogeneous plasma both near the critical density and near $1 / 4$ the critical density has been calculated using our 2-D electromsznetic, relativistic particle-simulation code. The results of these calculations extend our previous estimates of instability absorption lengths from idealized 1-D electrostatic codes. In addition, we have simulated resonant absorption, which occurs when the laser light is incident at an angle to the density gradient. Resonant excitation of plasma waves is shown to also occur due to the finite diameter of the laser light beam. NonMaxwellian heated distributions are observed for both instability heating and resonant absorption. The simulations also demonstrate the importance of density steepening due to the light wave pressure.

Since non-Maxwellian heated distributions are undesirable for pellet design, we have continued to study how to minimize them by operating near instability threshold. This is feasible if one maximizes collisional heating by using a sliort-wavelength laser. We have included electron-ion collisions in our 1-D simula- tions and find that the production of suprathermal tails sets in strongly for intensities abou! 5 times instability threshold. Hence it is often desirable to increase the threshold intensity by bandwidth. We have extended the finite bandwidth theory to determine the threshold as a function of bandwidth for both the ion acoustic decay and oscillating two-stream instabilities. The simulation codes with collisions have also been applied to study how hot electron transport is impeded because the cold electrons which supply the return current are collisional. The calculations show that this effect is often reduced by the development of runaways.

Finally, we have further examined the reflection and self-focusing of laser light in the underdense plasma before the critical density. To study the reflection a code has been written which solves nonlinear differential equations for the coupling between the light waves and the plasma waves. This code gives good agreement with our particle simulations for the onset of reflection and has the advantage that very long density gradients can be considered. In addition to delineating where the reflection is important, we have continued to stidy ways to reduce the reflection. Both bandwidth and gradients in the blowoff velocity are demonstrated to be effective. We have also observed the formation of filaments of light in underdense plasn:tas. These filaments give rise to new heating mechanisms and are shown to be unstable to kink rerturbations.

\section{ABSORPTIVE PROCESSES NEAR THE CRITICAL DENSITY}

Previous calculations have predicted that light is efficiently absorbed via the generation of plasma waves near the critical density and that non-Maxwellian velocity distributions are produced, ${ }^{68}$ as observed experimentally. These predictions were made on the basis of model calculations, most of which considered unirorm plasmas and spatially homogeneous pump fields. To extend these results, we have recently focused on examining light absorption with a 2-D, electromagnetic, relativistic particle-simulation code. We can then propagate laser light into a plasma with a density gradient and calculate the plasma wave generation and the light absorption.

The light can be incident at an arbitrary angle to the density gradient. Let us first consider normal incidence of the laser light in order to isolate the parametric instability effects from resonant absorption. ${ }^{69}$ Figure 109 shows a typical case we have simulated. We consider a slab of plasma with an initial density which rises from $0.8 n_{c r}$ to $1.15 n_{c r}$ in five light wavelengths $\left(\lambda_{0}\right)$, where $n_{c r}$ is the critical density. The incident light (Nd) has an intensity of $10^{16} \mathrm{~W} / \mathrm{cm}^{2}$, and the plasma has an initial electron temperature of about $4 \mathrm{keV}$. We observe strong plasma wave generation near the critical density, where the plasma wave energy becomes about equal to the laser light energy. The absorption is efficient, about $70 \%$ even for this relatively small slab of plasma. (By varying the number of simulation particles, we find that less than $20 \%$ of this absorption is due to collisional effects.) If these results with an 


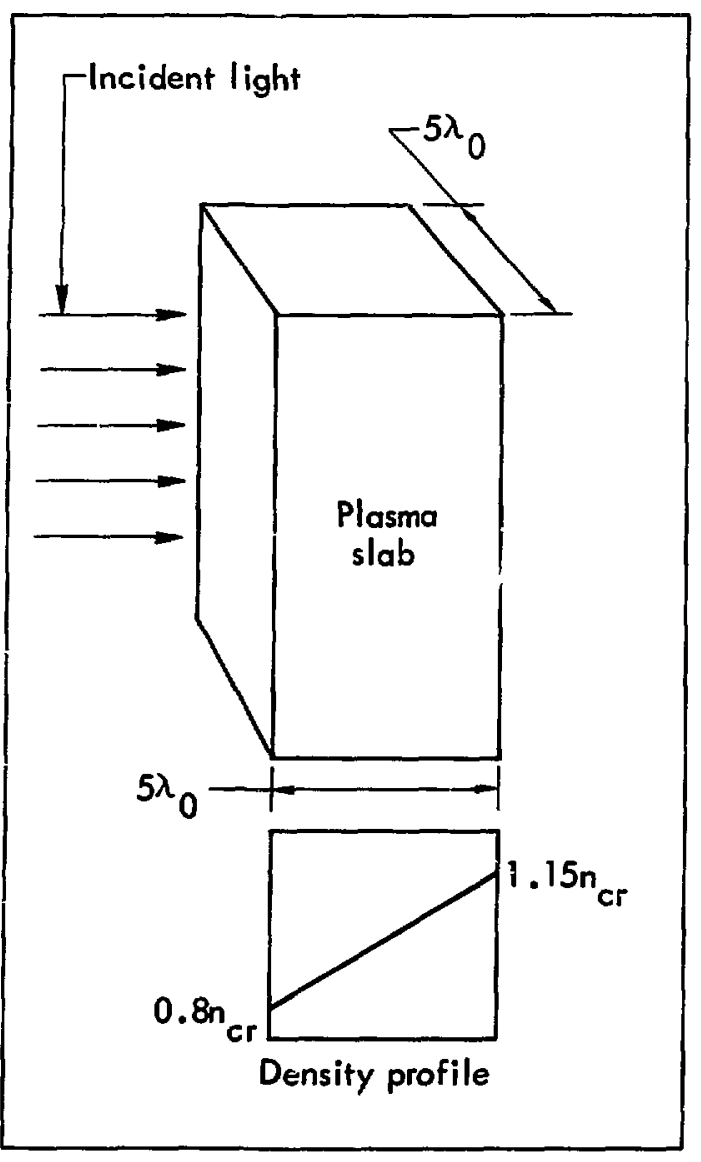

Fig. 109. Schematic of a typical 2-D plasma simulation study of tight absorption near the critical density.

electron/ion mass ratio of 100 are scaled to the pellet case, they show that the laser-light abscrption length by instabilities is about $10 \lambda_{0}$. This is in reasonable agreement with predictions of our model calculations.

The heated distribution function is shown in Fig. 110. Here we plot $f_{0}(v)$ vs $\gamma v / c$, where $\gamma=\left(1-v^{2} / c^{2}\right)^{-1 / 2}$. Note the long suprathermal tails as predicted. NonMaxwellian distributions are generated even allowing for appreciable density gradients.

The electromagnetic simulations both here and at Los Alamos ${ }^{70}$ show another potentially very important effect. The density profile alters due to the electromagnetic wave pressure. In particular, there is a steepening near the critical density, which on a long time scale can lead to a sharp step in density. This steepening has indeed been observed in LASNEX calculations $^{71}$ and can substantially affect the light :bsorption by limiting the region of plasma accessible to instabilities.

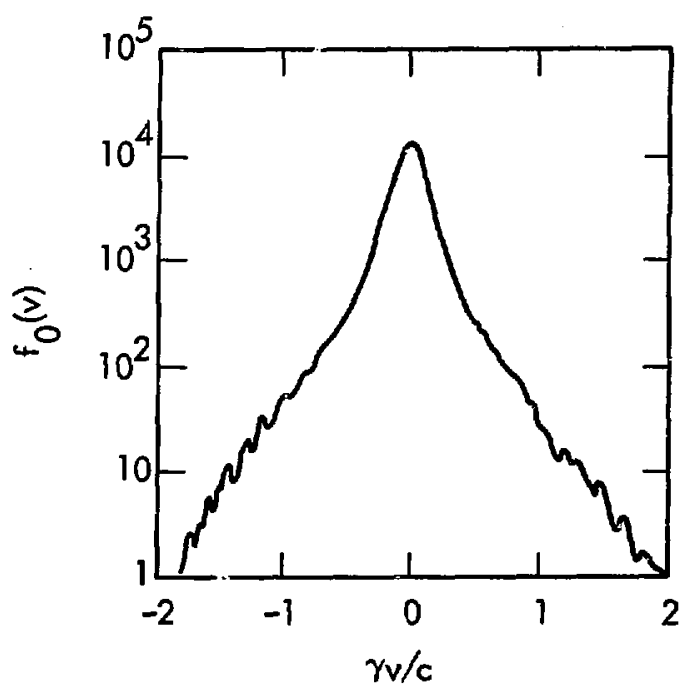

Fig. 110. Typical turbulently heated electron distribution function calculated with the 2-D electromagnetic code.

This nonlinear modification of the density gradient also plays a role in resonant absorption. ${ }^{72}$ In this case, the ponderomotive stress due to the gradient in the energy density of the resonantly driven plasma wave and the temperature gradient resulting from its heating combine to create a step in the density. ${ }^{73}$ Because of this density jump, the critical density point is accessible to electromagnetic radiation with a wide range of angles of incidence. Consequently, resonant absorption becomes an efficient process over a broad range of angles of incidence.

Figure 111 shows the density gradient from a computer simulation of resonant absorption. The light is incident at an angle of $24^{\circ}$ to the gradient with an intensity of $5 \times 10^{15} \mathrm{~W} / \mathrm{cm}^{2}$ (Nd). The plasma has an initial temperature of $4 \mathrm{keV}$ and an initial density which rises from 0 to $1.5 n_{c r}$ in $2 \lambda_{0}$. Note the sharp gradient near the critical density. The depressions it: lower density are caused by spatial variations in the time-averaged electromagnetic field energy due to the standing wave pattern set up by reflection at the critical density.

Another important feature is the observed production of very fast electrons. These hot electrons are of two classes. The first appear concomitantly with the formation of the density depressions discussed above. As shown in Fig. 112, they appear in characteristic streamers in phase space, primarily with velocities directed toward lower density. Such electrons are apparently due to coherent acceleration by the electro. 


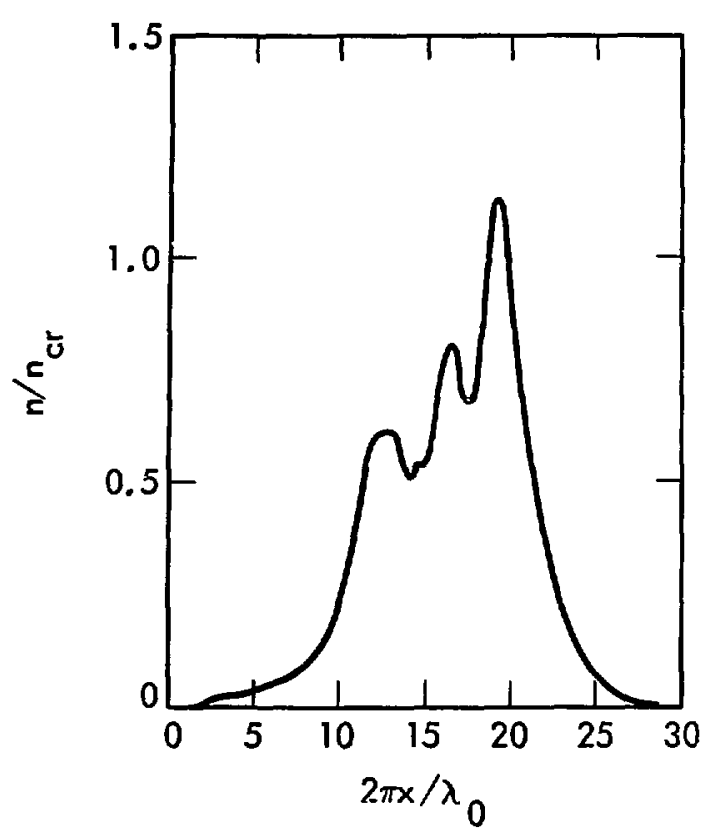

Fig. 111. Plasma density profile observed in a 2-D simulation of resonant absorption.

static plasma wave resonantly excited near the critical density. Potential plots show this long-wavelength structure with a maximum amplitude of $e \phi / m c^{2} \approx 1 / 4$, sufficient to generate the observed fast electrons. This class of very fast electrons apparently disappears only at very low light intensities. The second class is due to parametric instabilities as previously discussed. The principal difference is that the heating is done preferentially in the direction of the density gradient.

We have also examined resonant absorption in plasmas with long density gradients. For these calculations the process was modeled by including a density gradient in the 1-D electrostatic code. In the case of long density scale length the resonantly excited plasma wave is weakly damped. It is driven to very large amplitudes and directly traps the electrons. ${ }^{69}$ The heated distributions are quite similar to those shown in Fig. 110. We have also tried to model the finite laser spot size by including a spatially dependent pump in the 1.D code. The spatial dependence of the pump field resonantly excites plasma waves even in the absence of density gradients. These plasma waver have very long wavelength (of the order of the laser-light spot size). Hence they are again driven to large amplitude. Since their phase velocity is very high, they can now decay by parametric instabilities rather than by trapping. The simulations indicate improved heated distributions, since the effective pump field driving the instabilities has a large amplitude. We are now considering more realistic, 2-D calculations of this promising effect.

Finally, we have continued to examine light absorption via instabilities ncai $1 / 4$ the critical density. In particular, we have extended our previous 2-D electromagnetic simulations ${ }^{74}$ of this heating to include nonuniform plasmas. First we verified the theoretical prediction ${ }^{75}$ that the density gradient threshold for the two-plasmon decay is lower than the threshold for the Raman backscatter. Further simulations were carried out well above this threshold, where both instabilities are absolutely unstable near $1 / 4 n_{c r}$. Typicilly the light density was $10^{16} \mathrm{~W} / \mathrm{cm}^{2}$, the electron temperature was $1 \mathrm{keV}$, and the effective density gradient length was $K_{0} L=40$, where $L^{-1}=n^{-1} \partial n / \partial x$. In these runs the two-plasmon decay was always dominant. We observed laser light absorptions of $10-20 \%$. This indicated an absorption length near $1 / a n_{c r}$, which is several times longer than that observed in uniform plasma simulations. The longer absorption length is plausible, since the density gradient reduces the instability growth rate. Strongly non-Maxwellian distributions are again produced. The two-dimensional character of the electrostatic potential is clearly shown in Fig. 113. In order to further establish that the absorption was a multidimensional process, onedimensional simulations were carried out with identical parameters. The instability was then weak and little heating was observed.

\section{CONTROL OF NON-MAXWELLIAN HEATED DISTRIBUTIONS}

Since non-Maxwellian distributions are undesirable for pellet fusion, we want to determine how to minimize their generation. One option is to use a shortwavelength laser and heat at high density in order to remain near the instability threshold, while maximizing collisional heating (see Sec. 6 of this report). Hence, it is important to determine the transition from thermal particle heating below threshold to suprathermal tail formation well above threshold.

To determine how far above threshold this transition occurs, we have included electron-ion collisions in our 1-D simulations of instability heating. Two 


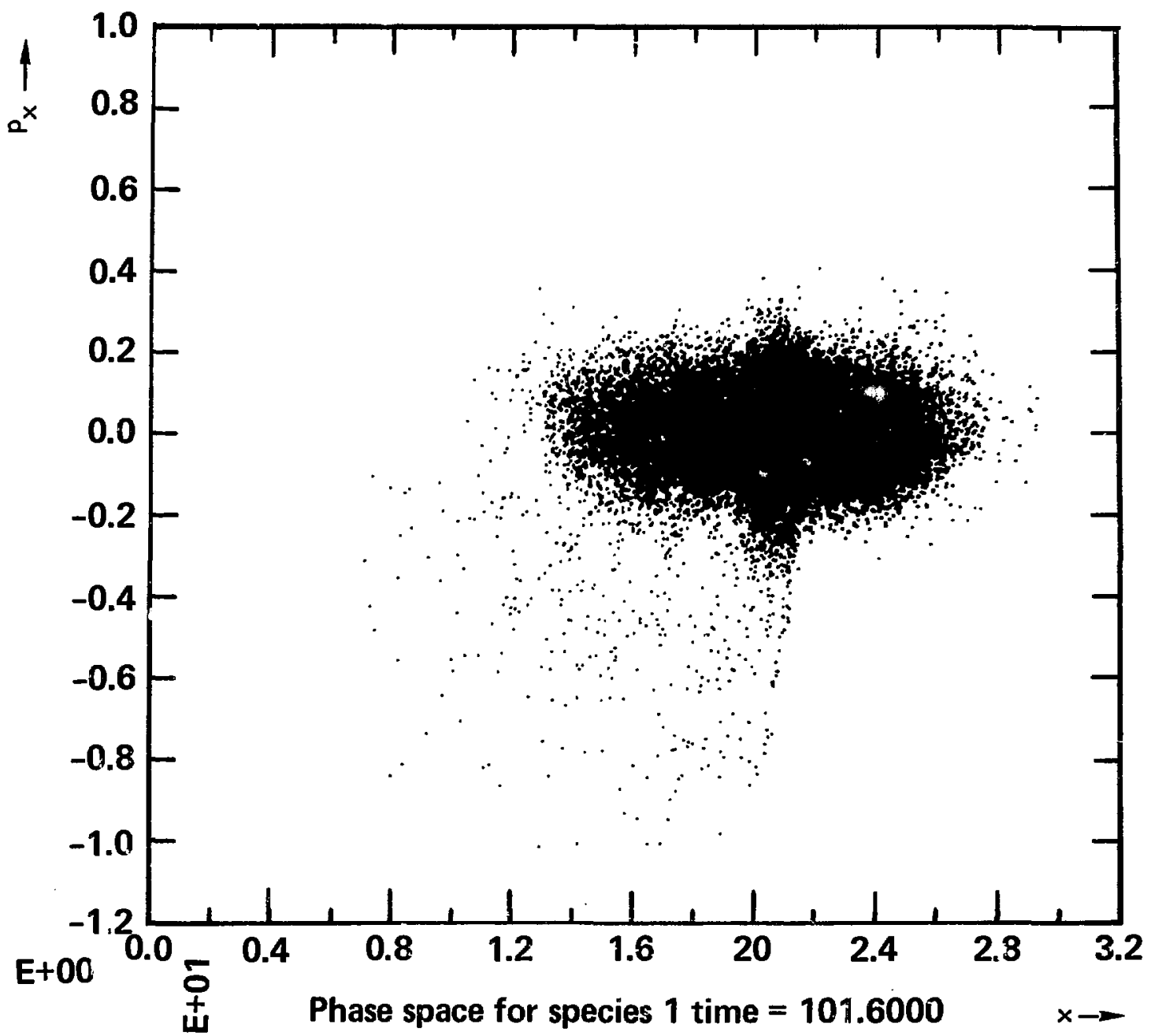
Fig. 112. Phase space plot from a simulation of resonant absorption; $p_{x}$ is plotted vs $x$, which is along the direction of the density
gradient.

separate approaches have been used. The first method is to apply a simplified Fokker-Planck operator to the electron distribution function, $f$ :

$$
\left(\frac{\partial f}{\partial t}\right)_{C}=\frac{\partial}{\partial v}\left[\nu v f+a^{2} \frac{\partial}{\partial \nu}(\nu f)\right],
$$

where $\nu(v)$ is the velocity-dependent collision frequency, and $a^{2}$ is specified by energy considerations. This approach has been incorporated into a 1-D code which uses periodic smoothing in phase space. ${ }^{76}$ The second method is to use a standard PIC simulation code and model collisions by randomly rotating the velocity vectors of the particles. Results from the two approaches are in reasonable agreement.
Figure 114 shows the heated electron distribution functions observed in a series of simulations for parameters characteristic of laser fusion. The pump field frequency is equal to $\omega_{p e}$, and its intensity was varied from the threshold value to many times threshold. At threshold, we indeed observe a heating of slow electrons as predicted classically. When the threshold is exceeded, tail formation begins. At about 5 times threshold, this tail heating begins to dominate the thermal particle heating. Finally, for about 20 times threshold intensity, the limiting case collisionless heated distributions are produced. These calculations did not use a velocity-dependent collision frequency, but further simulations have shown that the results are 


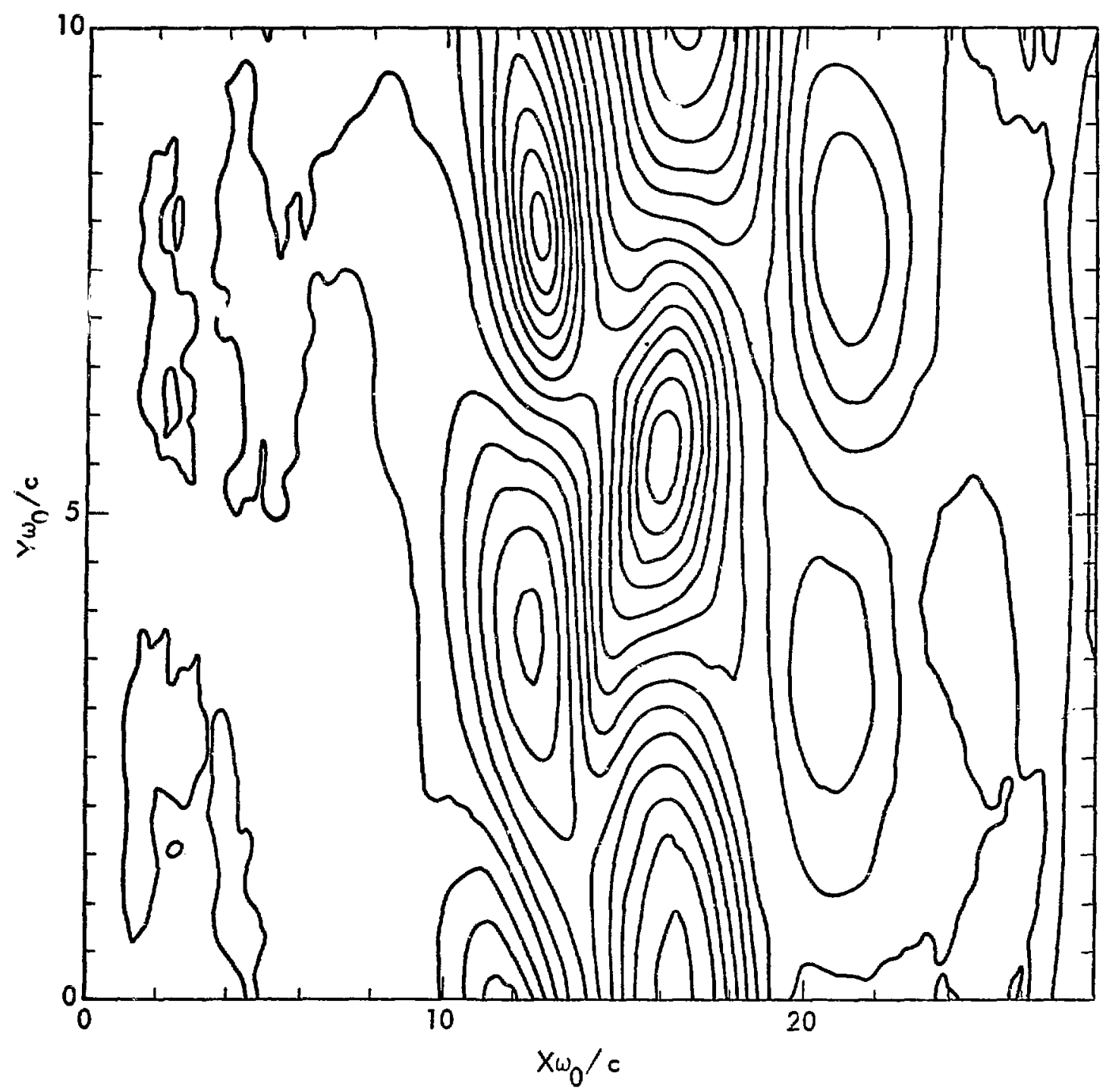

Fig. 113. Contour plot of the electrostatic potential observed in a 2-D simulation of instability heating near $1 / 4 n_{c r^{*}}$

not su'bstantially changed. The important point is that fo: the high intensities used in laser fusion, the supra. thermal tails appear rather quickly once instability threshold is passed. This is in contrast to the weak intensity cases examined in ionospheric studies.

Since non-Maxwellian distributions appear so near to threshold, it is often desirable to increase the threshold intensity by the use of bandwidth. We have previously shown 77 that the threshold for the ionacoustic-decay instability is increased by the factor $\Delta \omega / \nu$ in the special case in which the electron and ion wave dampings are equal ( $\nu)$. This theory has been extended to include unequal damping on the plasma waves and also the oscillating two-stream instability.

The most important new result is that the threshold for the oscillating two-stream instability is less sensitive to bandwidth than is the ion acoustic decay. Let us give a heuristic derivation. With a weak monochromatic pump the growth rate for this instability is $\gamma \approx 1 / 4$ $\left(v_{o s} / v_{t e}\right)^{2} \omega_{p e}$, where $v_{o s} / v_{t e}$ is the ratio of an electron's oscillatory velocity to its thermal velocity. Bandwidth decreases the growth by a factor estimated as $\gamma_{0} / \Delta \omega$, which is the fraction of the time a given plasina wave sees a coherent pump field. The condition that 


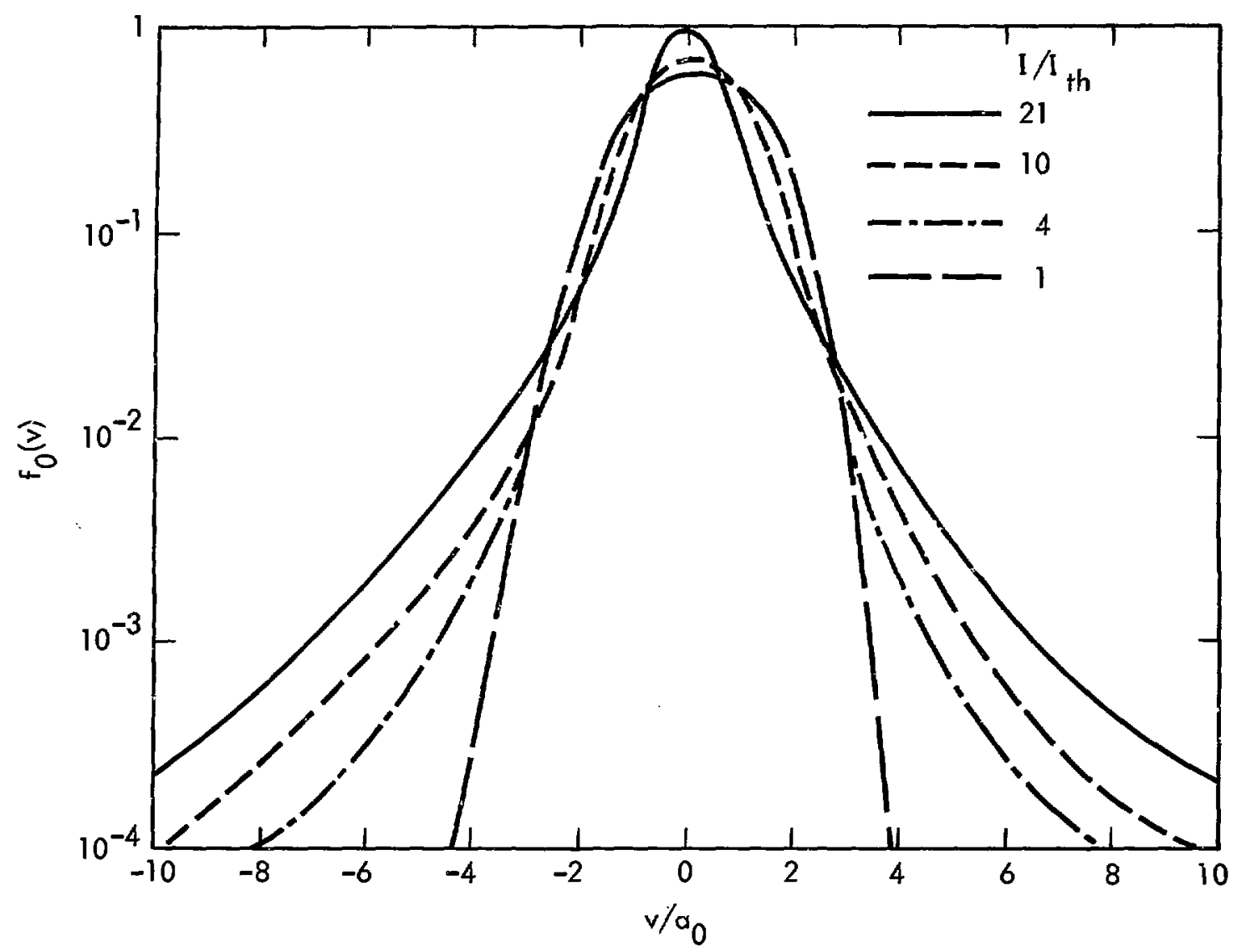

Fig. 114. Series of heated electron distribution functions calculated with a 1-D simulation code which incorporates electron-ion collisions.

this reduced growth rate exceed collisions is $\gamma_{0}{ }^{2} / \Delta \omega>$ $\nu_{e} / 2$. Hence the threshold becomes $\left(\nu_{O S} / \nu_{t e}\right)^{2} \gtrsim$ $4 \sqrt{\Delta \omega \nu} / \omega_{0}^{2}$. Note that bandwidth only appears as a square root. This is plausible since the oscillating twostream instability is less resonant in character than the ion acoustic decay, which excites both an electron plasma wave and an ion acoustic wave. This weaker dependence of the threshold on bandwidth indicates that in practice on'y about one order of magnitude increase in thresholu can be obtained using reasonable bandwidths.

There are important restrictions to the theory which are being further explored. In particulai, we have neglected the double resonance effect, ${ }^{78}$ which occurs when the bandwidth exceeds the ion acoustic frequency. This effect is basically due to different frequencies present in the pump field resonating with ion waves.
Finally, we have continued to search for plasma effects which play a role in equilibrating hot electrons once they are prodiced. A well-known example is the $\vec{J} \cdot \vec{E}$ effect. Laser-heated electrons strean: into and heat the dense plasma beyond the critical density. Charge neutrality requires a return current. During the early stages of pellet compression, this reiurn current is composed of relatively cold electrons, which are much more collisional than the laser-heated ones. The dissipation caused by the cold return current reduccs the mean free path of the hot electrons.

We are calculating how runaway electrons modify this $\vec{J} \cdot \vec{E}$ effect. An electric field is set up in the plasma to draw into the absorption region the required return current. Since the collision frequency is a strong function of velocity, some of the cold electrons run away in this field. This allows the return current to be supplied by fast electrons rather than by slow collisional ones. 
We have observed these runaways in simulations with our collisional codes. The effect is especially important when the density of the cold plasma is comparable to that of the hot. Then most of the cold electrons are found to run away. The laser-heated electron energy is reduced by approximately $1 / 2$, but it is transferred to energetic electrons rather than to cold ones. In the other limit in which the density of cold electrons is much larger than that of the hot ones, the runaway effect is small.

\section{REFLECTION AND SELF.FOCUSING IN THE UNDERDENSE PLASMA}

We have further examined the reflection and selffocusing of laser light due to instabilities which occur in the underdense plasma before the critical density. It is especially important to delineate where this reflection is large. The reflection was previously examined in computer simulations, ${ }^{79,80}$ but these simulations are in general limited to a study of relatively short density gradient lengths. Hence it is worthwhile to obtain a reduced description to supplement the simulations.

The simplest approach is to directly solve coupled nonlinear differential equations for the evolution of the light waves and the plasma waves. For the light waves it is convenient to work in terms of the vector potential $A$. Specializing to one dimension to first study backscatter, we then have

$$
\left(\frac{\partial^{2}}{\partial t^{2}}-c^{2} \frac{\partial^{2}}{\partial x^{2}}+\omega_{p e}^{2}\right) A=-\omega_{p e}^{2} \frac{\delta n}{n_{0}} A \text {, }
$$

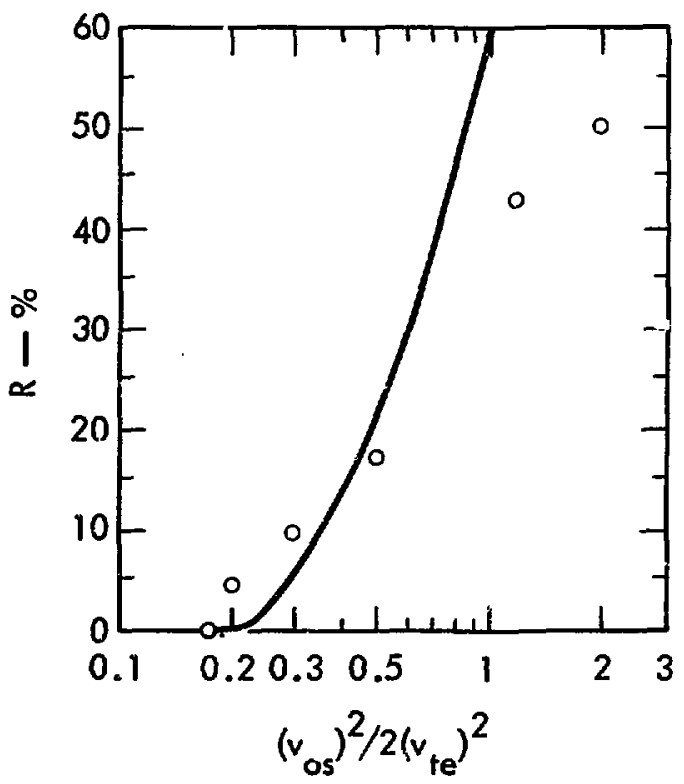

Fig. 115. Comparison of laser light roffection as calculated by the particle simulations (circles) and by coupled equations for the light waves and plasma waves (the curve). where $\omega_{p e}$ is the electron plasma frequency, $c$ is the velocity of light, and $\delta n$ is the density fluctuation associated with the electrostatic plasma waves. Since ion waves are most efficient (and hence dangerous) in reflecting the light, we have concentrated on the Brillouin instability. For the ion density fluctuations we have

$\left(\frac{\partial^{2}}{\partial t^{2}}-v_{a c}^{2} \frac{\partial^{2}}{\partial x^{2}}+\nu \frac{\partial}{\partial t}\right) \frac{\delta H}{n_{0}}=\frac{-e^{2}}{m M c^{2}} \frac{\partial^{2}}{\partial x^{2}} A^{2}$,

where $\nu_{a c}$ is the ion acoustic velocity, $\nu$ the damping rate, and $M$ the ion mass. These coupled equations can be simply derived by treating the plasma as a fluid. ${ }^{81}$

Linearized versions of these equations have been widely studied for both homogeneous and inhomogeneous plasmas. ${ }^{82}$ We have numerically examined the nonlinear behavior. Our solutions allow for pump depletion, nonlinear frcquency shifts, and re-backscattering of the light. Nonlinear damping of the ion waves is not included, which is reasonable until the reflection becomes sizable. Figure 115 shows a comparison of these calculations with our previously reported particle-simulation results. ${ }^{80}$ For these resuits the plasma density rises linearly to $0.7 n_{c r}$ in $10 \lambda_{0}$. The electron/ion temperature ratio is 5 . Note that there is good agreement until the reflection becomes larger than about $20 \%$. Then the particle simulations show smaller reflection as expected, since trapping cccurs to limit the ion wave amplitudes.

The results from the nonlinear differential equations are more illuminating if we plot the transmitted versus incident intensity, as in Fig. 116 . Note that as the incident intensity increases beyond a critical value, the reflection builds up to keep the transmitted intensity a constant. In other words, in the absence of nonlinear damping there is a maximum intensity which can be transmitted through a given region of plasma. We also find multiple frequency structure in the reflected light due to repeated re-backscattering, and a broadening in frequency of the transmitted light.

We have calculated how the light reflection depends on the plasma properties, such as density gradient, blowoff velocity gradient, electron and ion temperatures, and plasma wave damping. In agreement with 


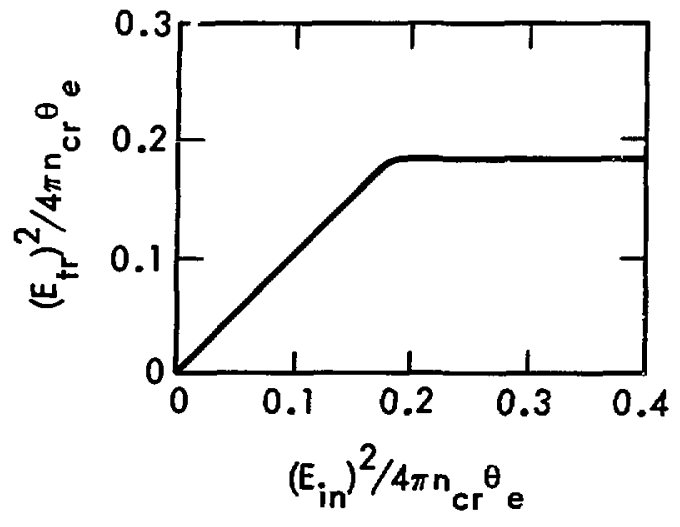

Fig. 116. Transmitted intensity vs incident intensity as calculated from the coupled equations. The plasma density rises linearly to $0.7 n_{c r}$ in $55 \lambda_{0}$.

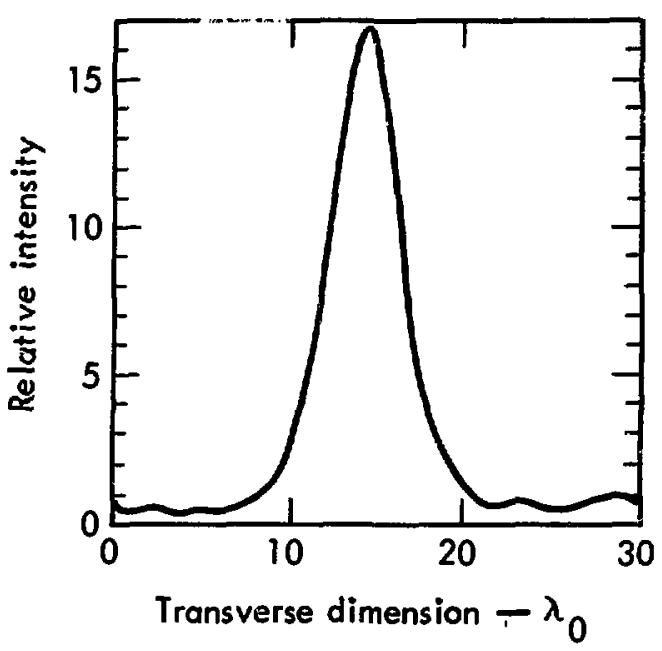

Fig. 117. Laser light intensity profile observed in a 2-D simulation of self-focusing.

filament significantly extend the regions o: $2 x$ which frequency matching for parametric instabilities can occur. For example, consider the " $2 \omega_{p e}$ " instability. When a filament forms in a higher density region, the density depression establishes the frequency matching necessary for the $2 \omega_{p e}$ instability to occur in the channel. The wave coupling is provided by the strong spatial variation of both the lasur wave field and the plasma density. Since the frequency of the decay waves is below the electron plasma frequency of the surrounding medium, they cannot propagate away from the hannel. Instead large-amplitude oscillations grow whicn cause strong plasma heatir $\bar{s}_{\bar{B}}$ as observed.

We have also investigated the stability of the filaments. ${ }^{86}$ Our calculation shows that they are unstable to perturbations which bend the channel periodically along the direction of radiation propagation (see Fig. 118.) The mechanism for this instability is the greater electromagnetic wave pressure on the outer wall of the channel than on the inner. This pressure imbalance leads to motion which increases the curvature and thus the pressur: differential. A purely growing mode results. Let $\ell$ be the wave vector of the perturbation along the channel, $K$ that of the radiation, and $c_{s}$ the sound speed. Then the growth rate is $\gamma \propto \ell c_{s}$ for $\ell / K$ $<1$. The final consequences of this instability remain to be explored. For example, the filament may be completely destroyed or it may simpl'; relax into a more complicated configuration. 


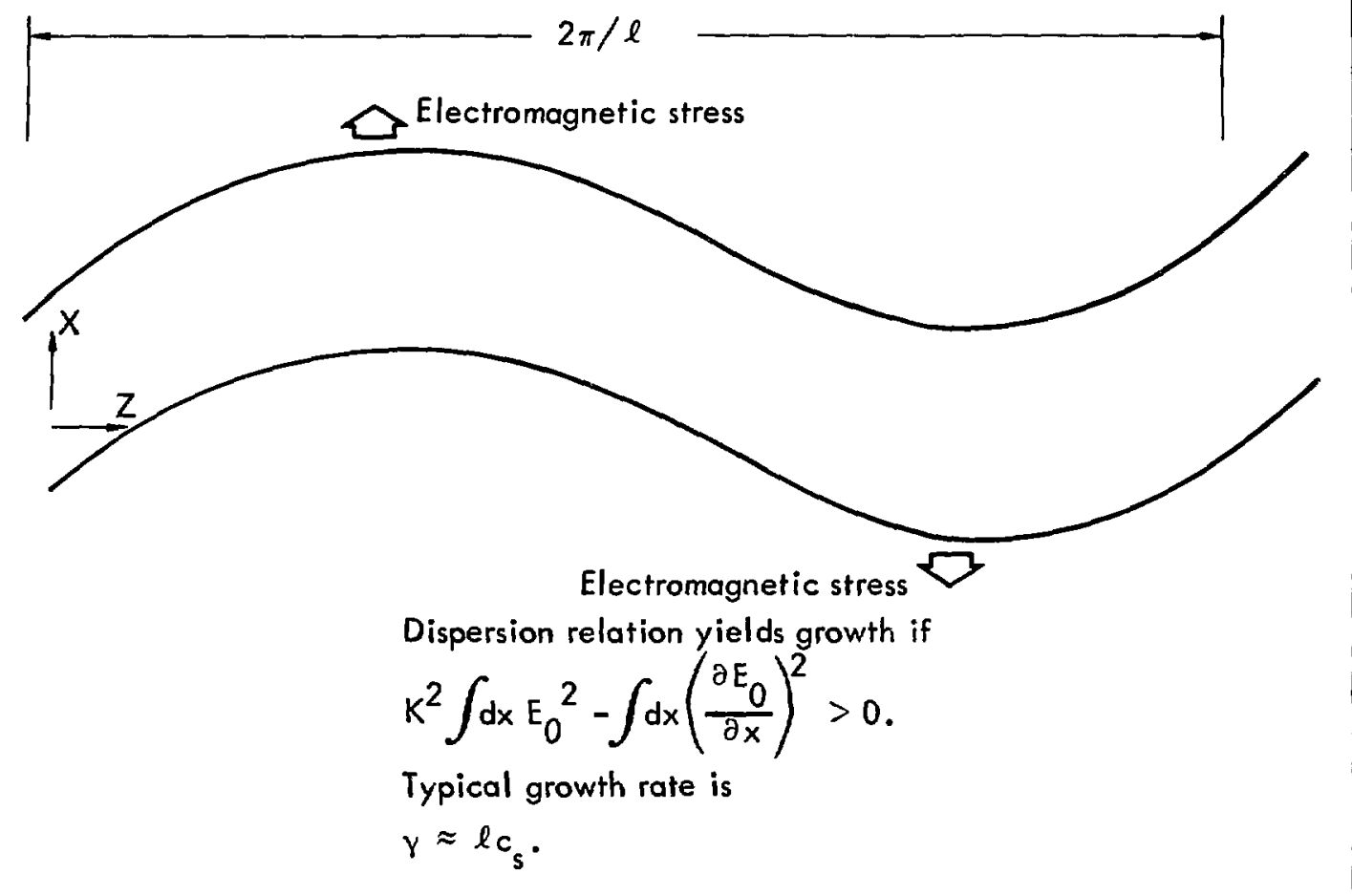

Fig. 118. Schematic diagram of a filament of light ui.uergoing a kink instability.

\section{SUMMARY REMARKS}

Let us conclude with a very brief summary of our present understanding of laser.plasina theory.

1. Absorption: Light is efficiently absorbed through the generation of plasma waves. Suprathermal electrons are produced both via instabilities and resonant absorption. Particular attention is now being given to elucidating the consequences of the observed nonlinear steepening of the densicy profile near the critical density.

2. Control of energetic electrons: Suprathermal electrons due to instabilities can be controlled by enhancing the electron/ion collision frequency and by using finite-bandwidth laser light. Fast electrons due to resonant absorpition appear to be the most difficult to avoid, but may be tolerable if the local density profile hecomes very steep. The role of ion turbulence in thermalizing hot electrons is under active investigation.

3. Reflection: Instability-generated reflection continues to be a potentaal problem for pellet fusion. This reflection can be avoided by the use of shells and shortwavelength lasers or by the use of finite-bandwidth light. The competition between the many instabilities taking place in the underdense plasma necessitates further study. 
Basic investigations in support of the Laser Program activities fall generally into two broad categories: fundamentai material properties, and concepts for programming pulses in frequency and time. During the period covered by this report, emphasis was placed on investigations of the magnitude and origin of the index nonlinearity in transparent dielectrics, stimulated Raman scattering in gases, pulse compression via self-phase modulation, and fundamental properties of self-focusing. Experiments were also initiated to obtain a more detailed understanding of the spectral properties of laser glasses, to study fluorescence sensitization, and to investigate new lasing media. In addition, three studies were performed under contract: one at the University of Southern California on the relative contributions of electronic and nuclear motions to index nonlinearity; another at the University of Southern California on spectroscopy of neodymium in laser glasses; and a third, at Stanford University, on the efficient generation of optical harmonics in alkali metal vapors.

\section{INDEX NONLINEARITY AND SELF-FOCUSING}

The fundamental limitation on the design of a large-aperture laser for fusion research arises from the phenomenon of self-focusing. Self-focusing of light is a consequence of the dependence of the refractive index of the optical medium on the light intensity. It is customary to characterize this dependence by the index nonlinearity parameter, $n_{2}$, where the refractive index is given by

$$
n=n_{0}+n_{2}\left\langle E^{2}\right\rangle
$$

where $\left\langle E^{2}\right\rangle$ is the mean squared field amplitude in the medium. It is well known that for most transparent dielectrics, $n_{2}$ is of the order of $10^{-13}$ esu, so that at a light intensity of $10^{10} \mathrm{~W} / \mathrm{cm}^{2}$, the nonlinear refractive index change is of the order of $10^{-5}$ to $10^{-6}$. Although this value is locally very small over a path $1 \mathrm{~m}$ long, several wavelengths of optical path change can occur. More important, it can be shown that in the presence of index nonlinearity, a uniform plane wave $i$, unstable against perturbations of the phase front. This effect is discussed elsewhere in this report.

The principal questions concerning index nonlinearity are then, how large is $n_{2}$, how does it arise, can it be altered, and what are the consequences for propagation? To measure $n_{2}$ accurately, She, Moran, and Carman ${ }^{87}$ have measured $n_{2}$ in several materials reiative to $\mathrm{CS}_{2}$. The heart of the measurement technique is a Mach-Zehnder interferometer ${ }^{88}$ shown in Fig. 119. One arm contains the sample of unknown nonlınearity, while the reference arm contains a $\mathrm{CS}_{2}$ cell with known nonlinear response. The output beams of the interferometer are then carefully overiapped spatially at the slit of an LLL ultrafast streak camera (resolution $\sim 10 \mathrm{psec}$ ). Furthermore, the two interferometer-arm path lengths are adjusted to be identical to within $3 \mathrm{~mm}$ using a low-intensity, 20-psec ruby laser pulse in conjunction with the streak camera. The interferometer beam splitters were uncost-1 and wedged glass substrates, while the reflectors $k \quad ; 0 \%$ mirrors, thus necessitating the above technique for linear path length adjustment. Since the $n_{2}$ of $\mathrm{CS}_{2}$ was typically 10-100 times that of the samples, the nonlinear phase delay was also approximately the same in both arms. By varying the relative intensity between the two arms (using identical filters before one sample and after the other), it was possible to equate the nonlinear path lengths in the two arms. This was established using the streak camera to determine the interferometer fringe distortion as a function of intensity (or time). When the fringe pattern is independent of time (intensity) the increases in nonlinear path length are the same in both arms. From this null condition, the relative intensities in the two arms establish the ratio of the $n_{2}$ of the two samples. The measure of the relative intensities is further reduced to a measurement of the relative pulse energies (good to $\pm 1 \%$ ), since the pulses in both arms are of equal duration.

Self-focusing iri either the sample or the $\mathrm{CS}_{2}$ leads to a complete washing out of the interferometer fringes and in practice imposed a limitation on the maximum allowable path-length change. By employing our veryhigh-brightness Nd:YAG-Nd:glass !aser system, the excellent quality of our beam when telescoped to $2-3$ $\mathrm{mm}$ diameter aliowed up to $\lambda / 2$ increase in nonlinear path length without self-focusing.

The largest errors in this experiment come from the minimum detectable fringe distortion vs time. The 


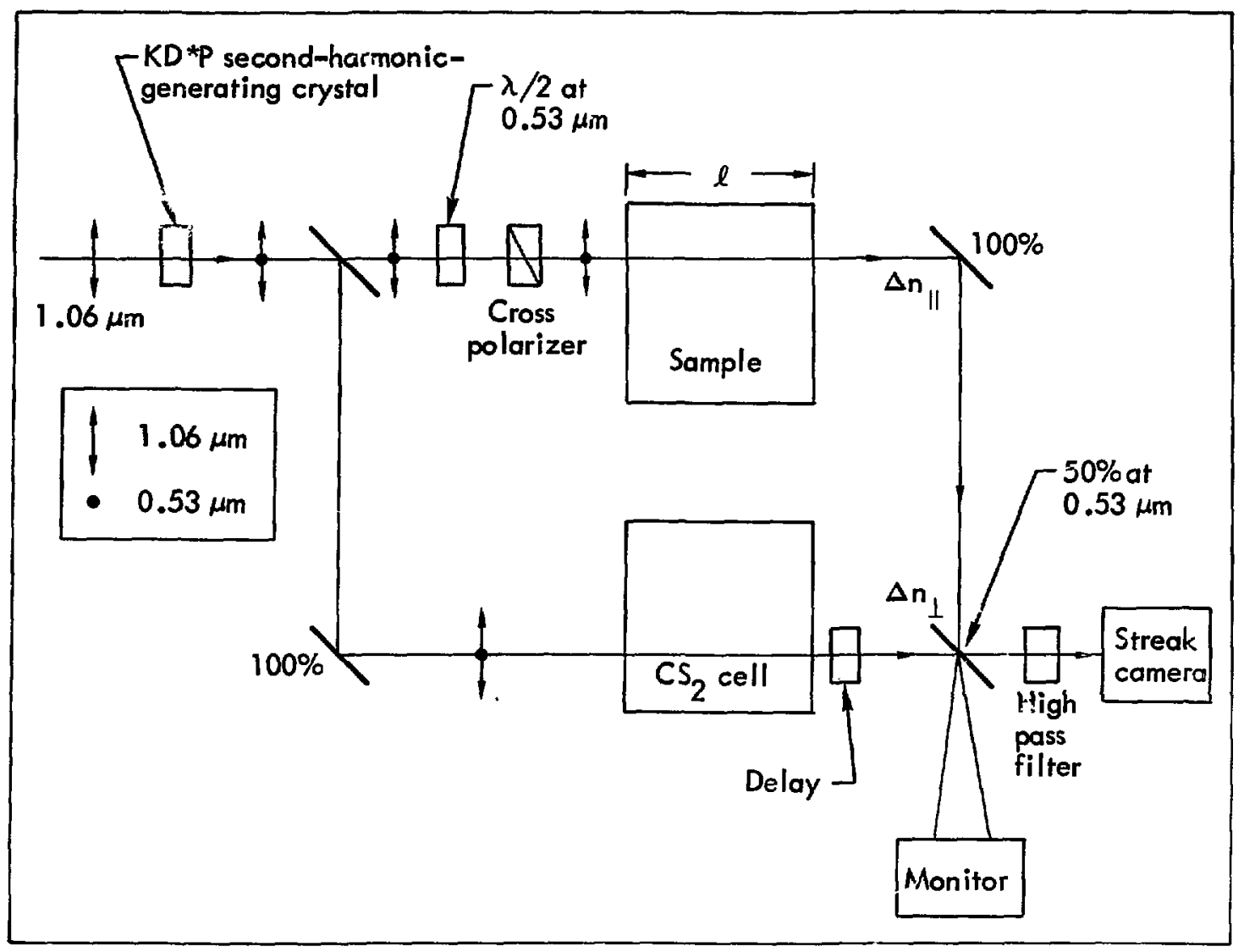

Fig. 119. Experimental setup showing Mach-Zehnder interferometer.

spatial resolution of the streak samera is $\geqslant 5$ lines $/ \mathrm{mm}$, and the slit is about $2.5 \mathrm{~cm}$. With six fringes on the slit, we are limited to about 25 resolution points between fringes. Using microdensitometry it was possible to see 1/30 of a fringe by enhancing the statistics through scanning several positions and using fringe symmetry. A second source of error was the absolute calibration of our filters. Both of these errors could be reduced by performing a least-squares fit to a plot of relative fringe distortion vs relative intensity as a means of establishing the null condition. However, the relative fringe distortion had to be normalized to the same incident puise intensity, which was established using a Tektronix 519 scope and an ultrafast diode (good to about 10\%). After applying all of these techniques plus corrections for linear refractive indices, beam crosssectional uhanges, Fresnel reflection losses, etc., we believe the $n_{2}$ values are good to about $10 \%$. The results are generally in agreement with previous work, 89 although some discrepancies have been noted. For several compositions of AO (American Optical) laser glass, no variation in $n_{2}$ as a function of $\mathrm{Nd}$ concentration was noted, which rules out $\mathrm{Nd}$ as the source of the nonlinearity. A definite dependence of $n_{2}$ on glass composition was observed. Our preliminary values for several materials are quoted in Table 22.

The correlation of observed values of $n_{2}$ with linear dispersion data was examined by Boling 90 and by Glass and Owyoung. ${ }^{91}$ It had earlier been proposed by Wang ${ }^{92}$ on the basis of a perturbation theory argument that the third-order polarizability of an atomic system should be relatad to the first-order polarizability by a relation of the form

$$
\alpha^{(3)}=g\left(\alpha^{(1)}\right)^{2} / \hbar \omega_{0},
$$

where $\omega_{3}$ is the uv resonance frequency, and $g$ is an empirical factor of the order of unity. $N$ and $\omega_{0}$ are obtained by fitting the refractive index data to a dispersion curve for a single uv resonator. Unforunately, the local field corrections were not treated correctly and systematically in the literature, leading to some 
Table 22. Values of $n_{2}$ measured with reference to $\mathrm{CS}_{2}$

\begin{tabular}{|c|c|c|c|}
\hline Material & $n_{0}$ & $10^{13} n_{2}$ (esu) & Polarization \\
\hline $\mathrm{CS}_{2}{ }^{\mathrm{a}}$ & 1.63 & $110 \pm 11$ & Linear \\
\hline ED-2 & 1.56 & $1.53 \pm 0.18$ & Linear \\
\hline $\mathrm{AO} C 835^{\mathrm{b}}(3 \% \mathrm{Nd})$ & 1.51 & $1.83 \pm 0.18$ & Linear \\
\hline $\mathrm{AO}$ C1020 (5\% Nd) & 1.51 & $1.83 \pm 0.18$ & Linear \\
\hline AO C2828 (3\% NG) & 1.51 & $2.02 \pm 0.20$ & Linear \\
\hline Ruby $^{c}(0.05 \%$ Cr) & 1.76 & $1.47 \pm 0.15$ & Linear \\
\hline SF-6 & 1.77 & $9.02 \pm 0.99$ & Linear \\
\hline SF-6 & 1.77 & $5.61 \pm 0.56$ & Circular \\
\hline $\mathbf{Y A G}^{\mathrm{d}}$ & 1.83 & $3.47 \div 0.35$ & Linear \\
\hline YAG & 1.83 & $2.78 \pm 0.28$ & Circular \\
\hline Lucite & 1.49 & $2.75 \pm 0.50$ & Linear \\
\hline
\end{tabular}

${ }^{\mathrm{a}} \mathrm{CS}_{2}$ value normalized in $n_{2}(\mathrm{ED}-2)=1.53$ at $1.06 \mu \mathrm{m}$. All values given here were actually measured with reference to $\mathrm{CS}_{\mathbf{2}}$.

$b_{\text {We are grateful to Dr. Eli Snitzer of the American Optical }}$ Company for supplying these samples.

CE-field parallel to c-axis, crystal axis at $60^{\circ}$ to c-axis.

Beam propagated in 111 direction, $(1,-1,0)$ axis at $45^{\circ}$ to plane of polarization.

onfusion. Furthermore, the relation of expressions ke that given above to the generalized Miller's rule ${ }^{93}$ was not clearly established.

In the present research, the nonlinear response of the meäium was modeled by a nonlinear oscillator satisfying Duffing's equation. It was shown that the model yields both the third-order nonlinear polarizability and the optical Stark shift. The nonlinearity was related to observable parameters, such as ionization potential and uv resonance. Systematic treatment of the local field corrections for cubic or isotropic media led to the result that the third-order susceptibility is given by

$\chi^{(3)}=\frac{K}{4}\left(\frac{n^{2}-1}{4 \pi}\right)^{2}\left(\frac{n^{2}+2}{3}\right)^{2} \frac{\omega_{0}}{N h\left(\omega_{0}^{2}-\omega^{2}\right)}$,

where $N$ and $\omega_{0}$ are obtai. ed from fiting the refractive data to a Lorentz-Lorenz relation,

$$
\left(\frac{n^{2}+2}{3}\right)\left(\frac{4 \pi}{n^{2}-1}\right)=\frac{\omega_{0}^{2}-\omega^{2}}{N}\left(\frac{m}{e^{2}}\right) .
$$

Owyoung had previously measured ellipse rotation in a number of materials. An effective value of the index nonlinearity $n_{2}$ can be obtained from the value of $\chi_{1221}^{(3)}$ through the relation $n_{2}=36 \pi \chi_{! 221}^{(3)} / n$. We would thus expect that the quantity

$$
9 \pi\left(\frac{n^{2}-1}{4 \pi}\right)^{2}\left(\frac{n^{2}+2}{3}\right)^{2} / n n_{2}
$$

would be numerically equal to $N \omega_{0}$. In Fig. 120 the quantity given in Eq. (35) determined from experimental measurement is compared to $N \hbar \omega_{0}$ for a number of transparent dielectrics. It is seen that the agreement is generally good. This analysis has been applied to the laser crystal GGG, for which it predicts an $n_{2}$ value of approximately $8.7 \times 10^{-13}$ esu. To

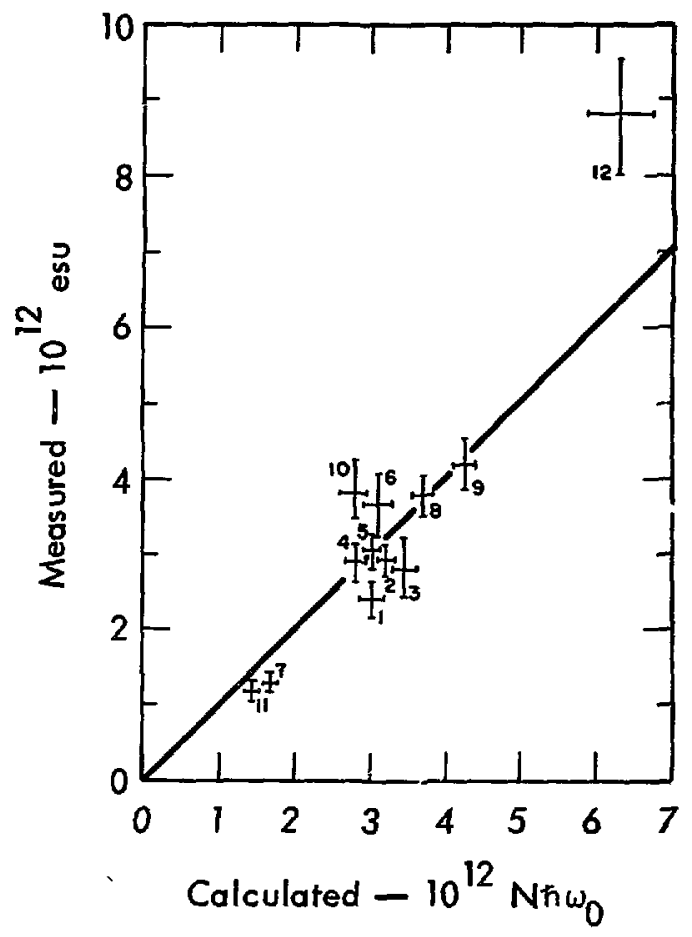

$\begin{array}{clccc}\text { Point } & \text { Material } & n & \text { Measured } & \text { Calcd. } \\ 1 & \text { FK-6 } & 1.446 & 1.13 \pm 0.08 & 0.90 \\ 2 & \text { Fused silica } & 1.456 & 0.99 \pm 0.07 & 0.91 \\ 3 & \text { BK-7 } & 1.517 & 1.46 \pm 0.14 & 1.18 \\ 4 & \text { LSO } & 1.519 & 1.43 \pm 0.10 & 1.45 \\ 5 & \text { ED-4 } & 1.567 & 1.73 \pm 0.12 & 1.75 \\ 6 & \text { EY-1 } & 1.635 & 2.03 \pm 0.20 & 2.35 \\ 7 & \text { SF-7 } & 1.640 & 5.83 \pm 0.40 & 4.36 \\ 8 & \text { LAK-3 } & 1.694 & 2.57 \pm 0.18 & 2.58 \\ 9 & \text { YAG } & 1.829 & 4.09 \pm 0.28 & 4.08 \\ 10 & \text { LASF-7 } & 1.914 & 6.20 \pm 0.60 & 8.39 \\ 11 & \text { CCI } & 1.460 & 2.50 \pm 0.25 & 2.03 \\ 12 & \text { Ruby } & 1.75-1.76 & 1.4 \pm 0.14 & 2.11\end{array}$

Fig. 120. Plot of the quantity shown in exprescion (35). determined from experimental measurements, $v$ the quantity $N$ w w, determined by allculation, for 12 different transparent dielectrica. 
date, no experimental measurement of this material has been made.

All indications point to the conclusion that we are unlikely to find any laser host with an index nonlinearity much smaller than that of present laser glass. By careful selection of the material, it might be possible to find a glass with an $n_{2}$ value as low as $1 \mathrm{X}$ $10^{-13}$ esu, but probably not much lower. It is apparent that electronic nonlinearities which exist in any transparent dielectric give rise to this lower limit on $n_{2}$. It is also apparent that higher index values generally lead to higher nonlinearity, although the actual dispersion properties of the material must be taken into account. Sapphire, for example, with an index of 1.76 would be expected to exhibit a high $n_{2}$; but measured values range from 1.4-to- $2.4 \times 10^{-13}$ esu. Our computed value is $2.1 \times 10^{-13}$ esu.

It is important to understand the other factors contributing to the index nonlinearity. In a contractsupported effort at the University of Southern California, Prof. R. Hellwarth has been investigating the relative contributions of nuclear and electronic motion to the nonlinearity.

It can be demonstrated theoretically that the desired nonlinear susceptibility of a material can be closely determined from its differential Raman scattering cross section (as a function of frequency), and its coefficient for intensity-induced polarization changes (IIPC). Absolute measurements of the necessary Raman cross sections were made for fused quartz; American Optical LSO laser glass and its Nd-doped form, C1020; Owens-Illinois ED-4 laser glass and its Nd-doped form, ED-2; and American Optical Nd-doped laser glass, C2828. Published measurements of Owyoung and Hellwarth and George were used for the IIPC coefficients. ${ }^{89,94}$

Preliminary results indicate that in the three glasses for which complete data exists, the nonlinear index $n_{2}$ for static self-focusing arises mainly (70-80\%) from electronic nonlinearities. Therefore $n_{2}$ arises mainly from the same physical source as the linear refractive index $n$ and should correlate more strongly with $n$ than with the Raman scattering strength. The peak values of the Raman scattering cross sections were also used to obtain the maximum stimulated gain. The value obtained for these glasses was about $1.5 \times 10^{-11} \mathrm{~cm} / W$. These gains are just below what might be troublesome in a large disk amplifier, but may be increased by cousoling anong stimulated Raman waves.

The propagation of light in nonlinear media has been extensively investigated in the past. In the analy. sis of self-focusing, use is often made of the "constant shape" approximation, in which the beam is assumed to maintain a Gaussian prefile,

$$
I=I_{0} e^{-r^{2} / f^{2}(2)} \text {. }
$$

and the variation of the Gaussian parameter $f^{2}(z)$ is calculated. Recently, A. Glass of LLL has investigated the existence of self-similar solutions for stationary self-focusing. Thus, the question has been posed, do solutions exist for the problems which indeed satisfy the constant-shape assumption? If so, under what conditions, and what shape (radial profile) do they assume?

It has been shown that self-similar solutions do indeed exist for this problem, but that they are not necessarily of the simple form generally assumed. For a phase surface of constant curvature, a complete set of self-similar solutions exists. In the absence of the nonlinearity, these reduce to the well-known GaussLaguerre modes of the spherical resonator. ${ }^{95}$

The light field is conveniently expressed in terms of a real amplitude $A$ and phase $\phi$. We introduce a similarly variable $\rho=r / f(z)$, where $f(z)$, the radial scale function, satisfies the simple differential equation $f^{\prime \prime} f^{3}(z)=b$, and where $b$ represents the radius of curvature of the phase front. The phase and amplitude of the wave are given by ${ }^{96}$

$$
\phi=\phi_{0}(z)+\frac{1}{2} k \rho^{2} \int f^{\prime}
$$

and

$$
A=F_{n}^{(m)}(p) / f(z) .
$$

The explicit forms of $\phi_{0}(z)$ and $F_{n}^{(m)}(\rho)$ are given as functions of the ratio of the total power in the mode to the Talanov critical power. ${ }^{97}$ In the case where $b=$ 0 , the scale function $f$ is constant, and the solutions reduce to the "self-trapped" modes of Chiao et al.," Haus, ${ }^{99}$ and Yankansuas. ${ }^{100}$ Since the parameter $b>$ 0 , these modes do not exhibit self-focusing, but always diffract according to the equation $f^{\prime \prime} f^{3}=b$, independent of the value of the nonlinearity. The corresponding radial distribution is given as a solution of the nonlinear eigenvalue problem,

$$
\begin{aligned}
& \frac{1}{\rho} \frac{\partial}{\partial \rho}\left(\rho \frac{\partial F_{n}^{(m)}}{\partial \rho}\right)-\frac{n^{2}}{\rho^{2}} F_{n}^{(m)} \\
& \quad+2 k^{2}\left(F_{n}^{(m)^{2}}-2 k^{2} a+b \rho^{2} / 2\right) F_{n}^{(m)}=0 .
\end{aligned}
$$

where $a$ is the eigenvalue of the problem. For $b>0$, the problem reduces in the motion of an oscillator in an at tractive potential well. In the absence of the nonlinearity, the solutior are the well-known GaussLaguerre modes of the spherical resonalor. The effect of the nonlinearity is to deepen the well in regions of high intensity. For $b>0$, the parameter $a=-E \sqrt{b} / k$. The eigenvalue $E$, which reduces to $(1+n+2 m)$ for 
the linear case, governs the variation of the phase on-axis. In Fig. 121 the dependence of $E$ on total power is given for the lowest order modes with $n=0$ (cylindrically symmetric). It is seen that there is a maximum power allowable in each mode, corresponding to the "self-trapped" modes. As the power increases above this level, the radial function $F_{n .}^{(m)}(\rho)$ is drawn in closer to the axis, necessitating an additional zero crossing, and thereby raising the mode index $m$. The radial dependence of the lowest order mode for various powers is seen in Fig. 122.

These propagation modes play an important role in describing the field variation in high power oscillators, especially in crystalline materials like YAG or GGG in which the index nonlinearity is high. The interface between the linear and nonlinear medium in the cavity can be approximated by a surface of constant phase. On this surface the field distribution can be expressed both as a single mode of the nonlinear medium and as a superposition of modes of the linear medium. For weak nonlinearity, the expansion coefficients can be found analytically. From this analysis one san obtain the power dependence of the output beam, divergence, for a spherical resonator containing a nonlinear element, and the influence of rod positioning.

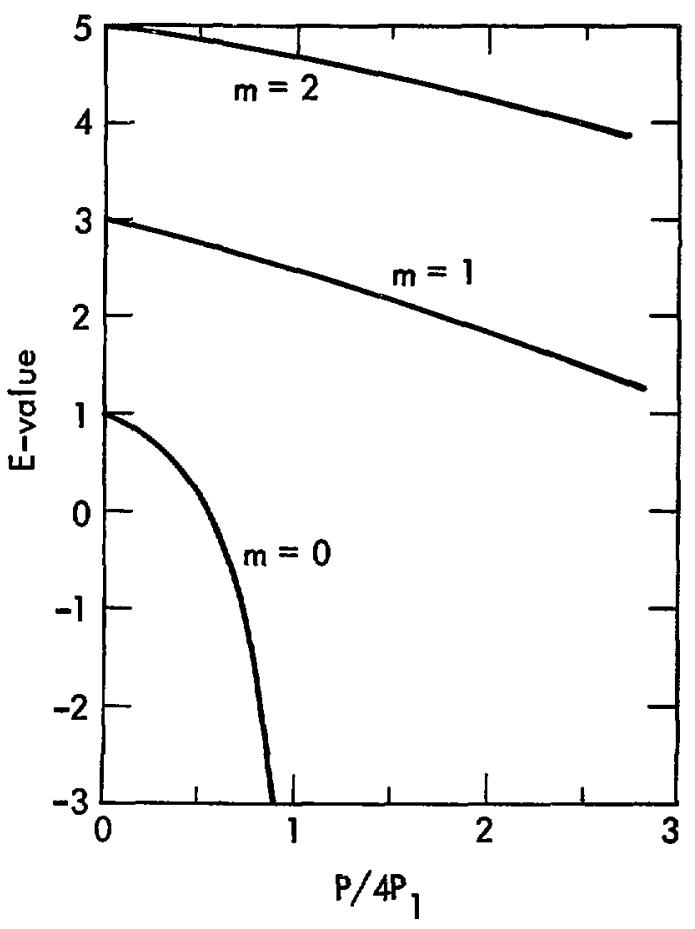

Fig. 121. Dependence of the eigenvalue $E$ on total power.

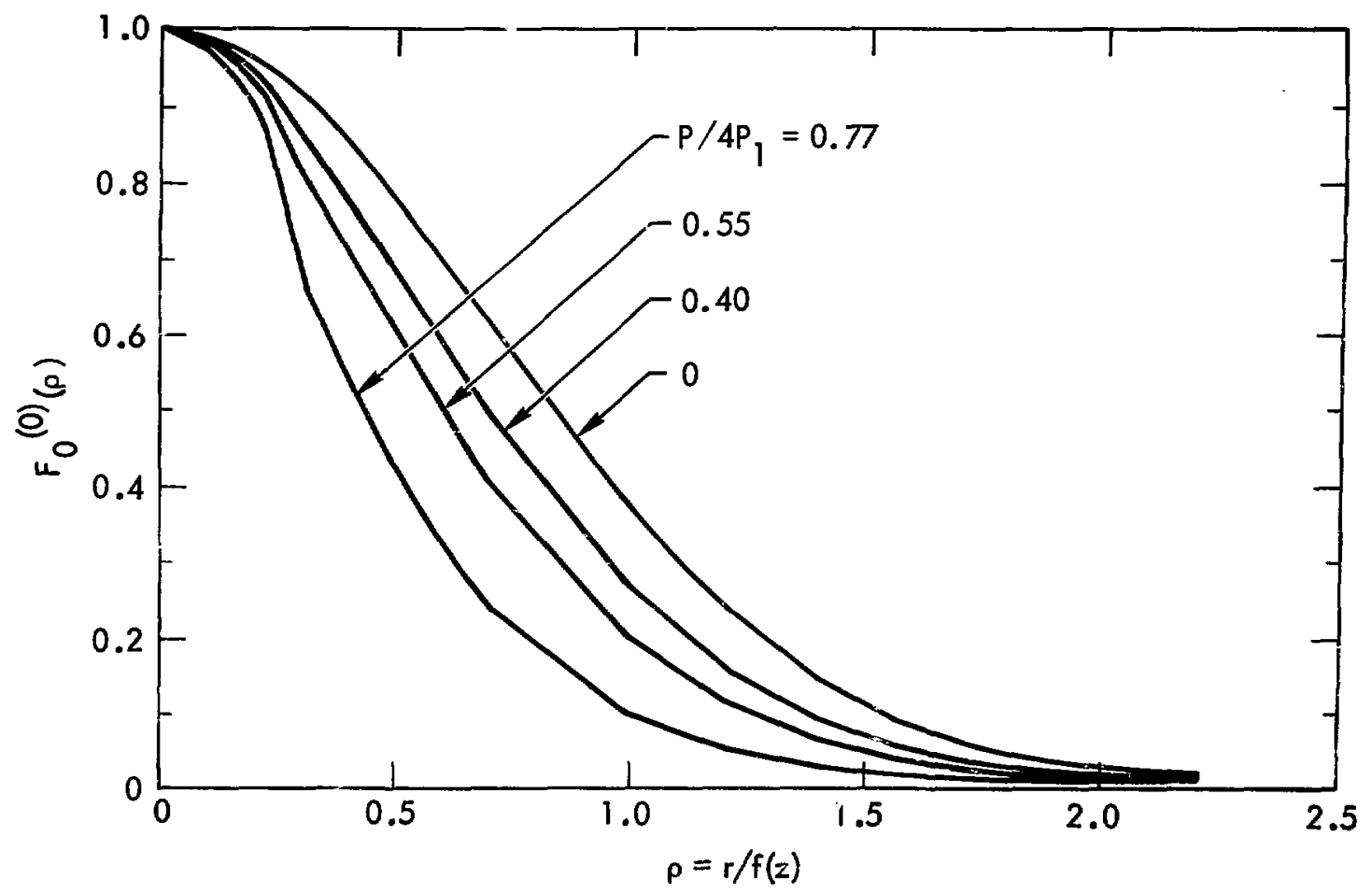

Fig. 122. Radial dc andence of lowest order mode for four power levels. 
The constant-shape approximation in self-focusing corresponds to the case $b<0$. In this case, no true eigenmodes exist. The effective potential well is a parabolic repulsive well, with an attractive minimum in the center due to the self-action of the intense beam. The constant-shape approximation treats the problem locally, in the beam center, neglecting the boundary clndition at large $r$. It is clear, however, that no bound state, corresponding to a real eigenvalue, can exist in such a well. The self-focusing solutions of the constantshape approximation are therefore "quasi-modes" of the problem, corresponding to complex eigenvalues, since they are decaying solutions of the potential problem. If the intensity is great at the beam center, the amplitude of the self-focused solution outside the central minimum of the well will be small, and most of the beam will follow convergent trajectories of the shape

$$
f(z)=\sqrt{1-z / z_{0}}
$$

Clearly, however, it is impossible to trap the entire beam inside a self-induced well. The amplitude of the field in the repulsive region will be small but nonzero, therefore corresponding to infinite power in a steadystate solution. Self-focused modes thus correspond to decaying states in a repulsive well, with a solf-induced minimum at the center.

There is an abundant literature regarding the development of propagation instabilities in nonlinear media at high power. The modes described in this paper represent stable solutions, and exist at all powers. However, these modes cannot be superposed. Mixed modes will develop many zero crossings and are not similarity solutions. In very large beams with weak phase curvature, the beam axis is not locally defined, so that power concentration can develop at any region of high intensity. Thus the existence of stable modes and the appearance of the instability at high power are not inconsistent.

\section{FREQUENCY CONVERSION AND PULSE COMPRESSION}

The process of stimulated Raran scattering (SRS), the inelastic scattering of light from an atomic or molecular excitation, is significant for a number of reasons. Raman converters have been proposed as a means of isolating a high power laser from target reflec. tions. Pulse shortening has been observed in backward, traveling-wave Raman amplifiers. Inverted Raman systems offer the possibility of amplification of large signais in the absence of small-signal gain. Thus, they would not be subject to the problem of parasitic oscillation, which limits the size of laser elements. Additionally, the Raman process is a prototype two-photon process, and its study is relevant to all multiphoton parametric eptical processes.

The singly stimulated two-photon emission and stimulated anti-stokes Raman decay modes of excited metastable atomic systems have been previousiy examined by numerous authors. A laser amplifier based on one of these iwo-photon decay modes has wellknown, at active properties. Such an amplifier operating on the $S^{2} P_{1 / 2} \rightarrow 5^{2} P_{3 / 2}(1.315-\mu \mathrm{m})$ transition of atomic iodine has recently been proposed by Vinogradov and Yukov. ${ }^{101}$ R. Carm and H. Lowdermilk of LLL have experimentally investigated this possibility and reported the first observation of stimulated electronic anti-Stokes Raman gain in a nonresonant inverted medium, an accomplishment that reciuired careful optimization of the available experimental parameters.
In the steady-state regime, the intensity in the stimulated Raman wave grows exponentially in space with a small-signal gain coefficient $g_{s}$ given by

$$
g_{s}=4 \lambda_{s}^{2}\left(\frac{\partial \sigma}{\partial \Omega}\right) I_{L} N / h \omega_{L} \Gamma,
$$

where $\lambda_{s}$ is the wavelength of the scattered radiation, $\partial \sigma / \partial \Omega$ is the differential scattering cross section, $I_{L}$ is the incident laser intensity, $N$ is the inversion density, $\omega_{L}$ is the laser frequency, and $\Gamma$ is the Raman linewidth. The laser pump pulse at $1.06 \mu \mathrm{m}$ had a duration of $1 \mathrm{nsec}$ and bandwidth of $0.015 \mathrm{~cm}^{-1}$. Since the Raman linewidth of the iodine transition is $\Gamma \geqslant 0.1$ $\mathrm{cm}^{-1}$, the steady-state expression for $g_{s}$ is applicable. The maximum laser intensity $I_{L}$ is limited to about $10^{10} \mathrm{~W} / \mathrm{cm}^{2}$, beyond which damage to the quartz window of the iodine cell occurs. It should also be noted that, at this flux level, the Stark shift of the atomic levels is about one-third the linewidth. A diagram of the experiment is shown in Fig. 123.

The atomic iodine inversion was obtained by flashlamp pumping of $\mathrm{CF}_{3} \mathrm{I}$ at a pressure of 100 Torr. It was verified that neither $\mathrm{CF}_{3} I$ nor anv of the photolysis reaction products produced $s^{\prime}$ : $\boldsymbol{r}_{i} u l a t e d$ vibrational Raman scatfering at the power levels of interest. To establish initially the existence and timing of the inversion, the iodine cell was operated as a laser. On this basis it was determined anat $I^{*}$ densities of $N \geqslant 3 \times$ $10^{16} \mathrm{~cm}^{-3}$ were achieved. With this excited-state de.t- 


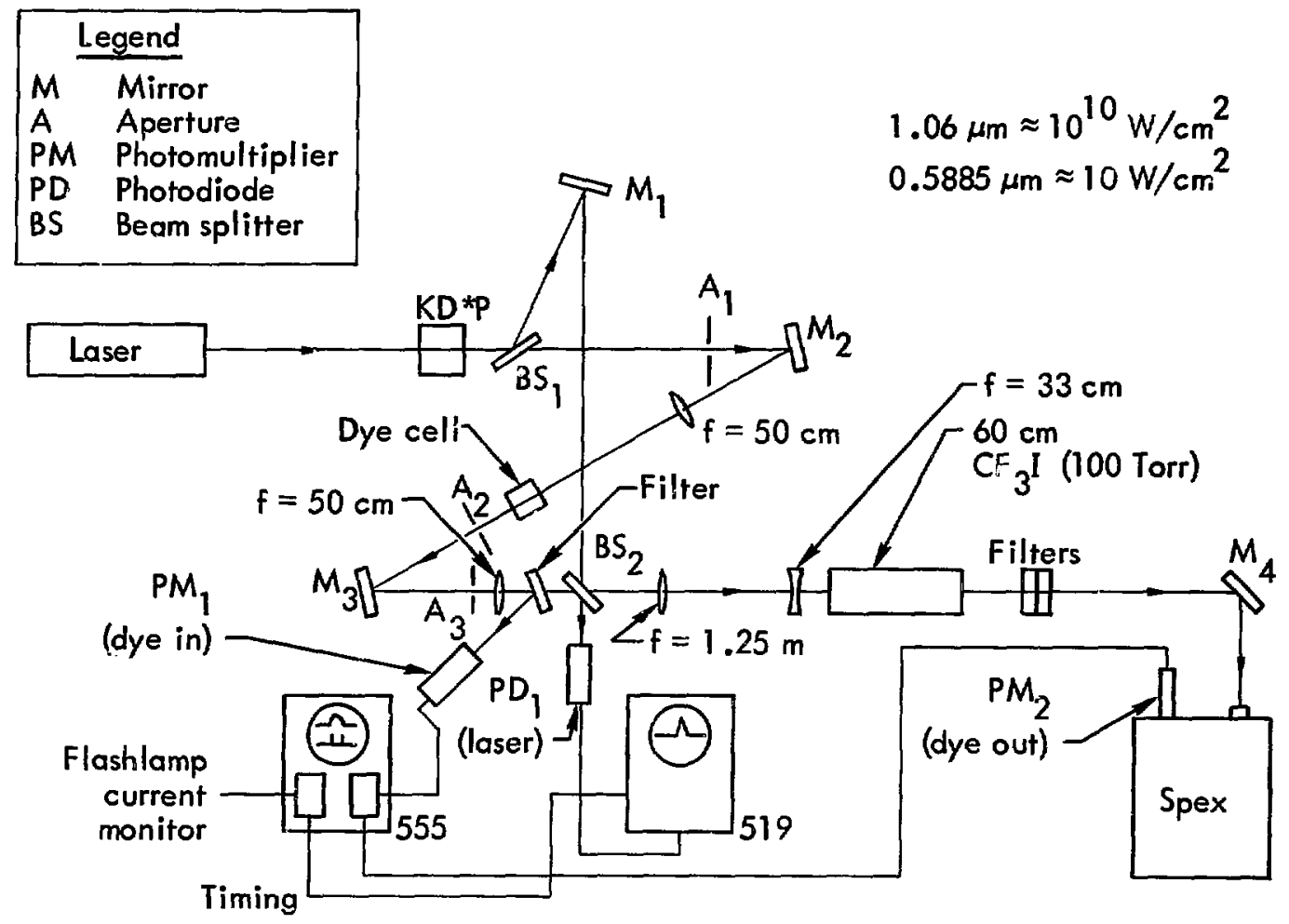

Fig. 123. Anti-Stokes Raman experimental setup, in which atomic iodine inversion was observed.

sity and a cell length of $60 \mathrm{~cm}$, the single-pass gain at $1.3 \mu \mathrm{m}$ with a cross section of $2 \times 10^{-18} \mathrm{~cm}^{2}$ is 18 $\mathrm{dB}$, thus avoiding superfluorescence depumping of the excited state. By observing the transmission of a $\mathrm{cw}$ He-Ne laser beam through the iodine cell, Carman and Lowdermilk found, as others have, that the flashlamp pumping of the $\mathrm{CF}_{3} \mathrm{I}$ produces severe optical distortion of the medium due to propagation of shock waves, caused by heating, which originate at the cell wall. Thus it was required that the pump pulse pass through the center of the iodine ceil befure the arrival of the shock wave, which travels at a sonic velocity of about $10^{5} \mathrm{~cm} / \mathrm{sec}$. Due to strong absorption of flashlamp light by the $\mathrm{CF}_{3} \mathrm{I}$, a 12 -mm-diam iodine cell was used to achicve uniform pumping. Under these conditions the laser pump pulse, with its 2 -min beam diameter, passing through the iodine cell at the end of the $8-\mu \mathrm{sec}$ flashlamp puise experienced no observable distortion.

After these considerations, the expected Raman gain was found from Eq. (41) to be $e^{7.2}$, assuming $\partial \sigma / \partial \Omega \approx 10^{-28} \mathrm{~cm}^{2}$. With such a low gain it would not be possible to observe the anti-Stokes signal built up from noise. Consequently, an input signal derived from a traveling-wave rhodamine $B$ dye laser pumped by the second harmonic of the 1.06- $\mu \mathrm{m}$ laser pulse was provided. It was then necessary to consider the fact that, since the energy stored in the volume traversed by the pump light is only $8 \mathrm{~mJ}$, the expected gain of $e^{7.2}$ requires that the input anti-Stokes signal not significantly exceed $10 \mathrm{~W}$ in the Raman bandwidth to avoid amplifier saturation and nonexponential growth.

The traveling-wave dye laser's power thus was attenuated to $100 \mathrm{~W}$ in the entire $100-\mathrm{cm}^{-1}$ bandwidth of the dye emission. The anti-Stokes gain of $e^{3}$ was then observed by passing the amplified dye-laser pulse through a monochromator with resolution of $0.3 \mathrm{~cm}^{-1}$ and comparing the signals from photomultipliers monitoring the input and output energy as the monochromıator was tuned through the anti-Stokes line at 5884 A.

In conclusion, a stimulated anti-Stokes Raman gain in inverted atomic iodine has been observed. The measured gain of $e^{3}$ is well within the limits of experimental uncertainty. 
It has been previously recognized that a laser pulse may be converted into a pulse of significantly shorter duration and higher peak power by a backward, traveling-wave Raman amplifier. ${ }^{102}$ In view of the rapidly increasing interest in the application of very intense laser pulses of subnanosecond duration and the extreme difficulties involved in their direct generation by solid state lasers, G. Kachen and H. Lowdermilk of LLL have undertaken a detailed examination of the temporal and spatial characteristics of Raman-Stokes pulses under well-controlled experimental conditions. Observation has been made, with 10-psec resolution, of pulsations in time occurring in both the forward and backward scattered waves. These pulsations, due to laser depletion, had been predicted to occur in stimulated Rarian scattering ${ }^{103}$ A diagram of the experiment is shown in Fig. 124.

The laser pump source was the second harmonic at $5324 \AA$ from an LLL NA:YAG-glass laser system which produces a 1-nsec pulse with a bandwidth of 0.01 : $\mathrm{cm}^{-1}$ and divergence angle twice the diffraction limit.
The spatial and temporal intensity distribution showed no detectable fluctuations. Competition from stimulated Brillouin scattering and interference from ordinary transient effects, which were related to the damping of the material excitation, were both avoided by the choice of a pump pulse duration of 1 nsec. To avoid the effects of self-focusing, our observations were confined to gases $\left(\mathrm{SF}_{6}, \mathrm{CO}_{2}, \mathrm{~N}_{2} \mathrm{O}, \mathrm{N}_{2}, \mathrm{CH}_{4}, \mathrm{H}_{2}\right)$. Lenses of focal length 60,40 , or $25 \mathrm{~cm}$ focused the pump pulse into the gases contained in a cell which was $1 \mathrm{~m}$ long to avoid feedback due to reflections from cell windows. The time sequence of the forward and backward emission was obseryed simultaneously using a streak camera of 10-psec resolution. A photodiode was used to measure Raman conversion efficiencies. The number of Stokes orders scattered was detected by a spectrograph and image-converter camera, and finally the spatial distribution of the Stokes scattering was photographed. The temporal and spatial characteristics of the Raman scattering were studied in each case as a function of the laser pump power.

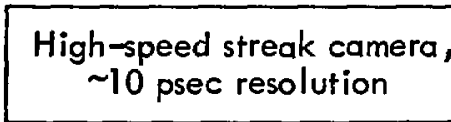
Synchronously
driven Nd:YAG oscillator

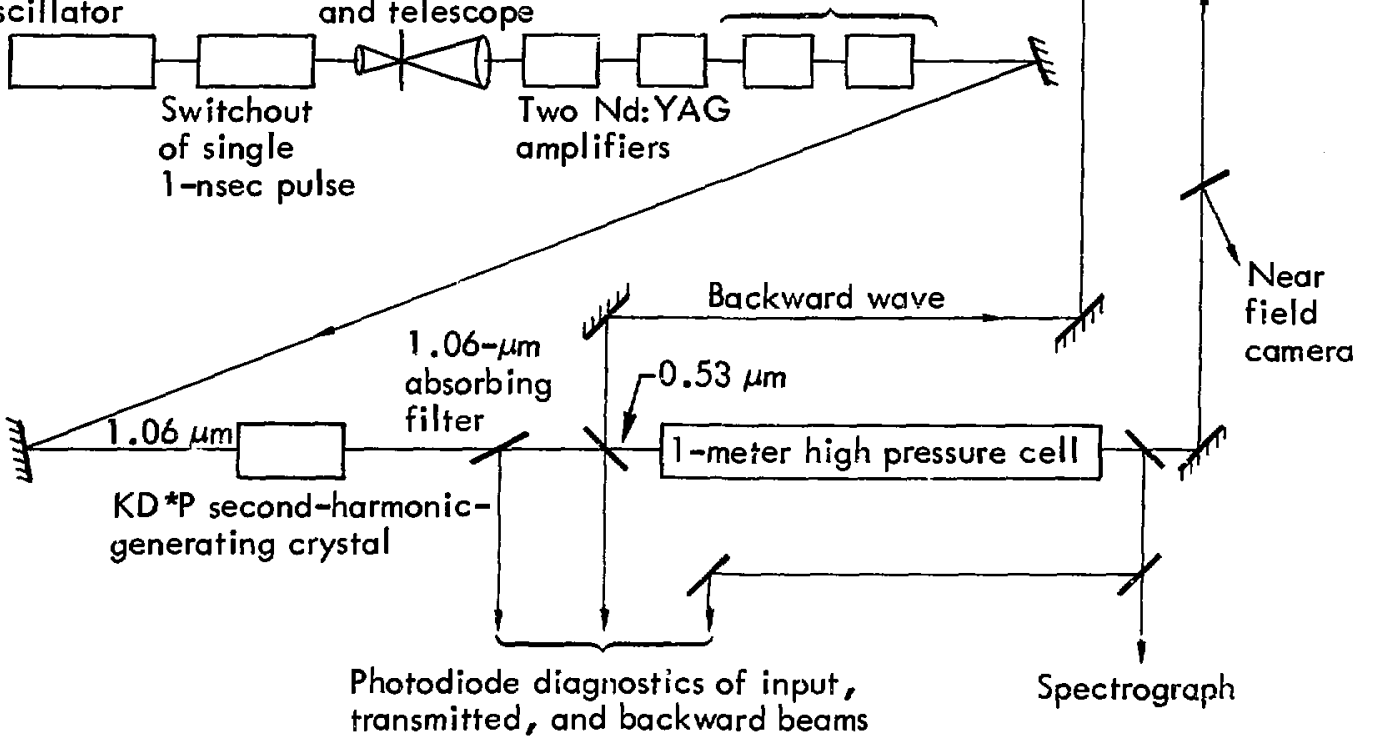

Fig. 124. Experimental setup for studying stimulated Raman scattering in high-pressure gases. 
The same qualitative behavior was observed for all the gases studied. For laser powers just above the first Stokes threshold, the spatial intensity distribution of the Stokes radiation is Gaussian with fluctuations less than $4 \%$. When the pump power is increased substantially, the spatial intensity distribution of the Rasıan pulses contains severe small-scale fluctuation. This behavior may be understood by consideration of the intensity distribution of the laser pulses in the region in which the Raman conversion occurs. Conversion efficiencies of pump to Raman radiation of up to $90 \%$ have been observed with significant variation of the ratio of forward to backward intensity among the geses studied. With the pump power at the first Stokes threshold, a single backward Stokes pulse is generated with a duration of $100 \mathrm{psec}$. As the pump power is increased, several pulses are observed in both the forward and backward direction as shown in Fig. 125. The spacing and duration of the pulses are reproducible in each case. The pulses are shorter and more closely spaced for increased pump power, for shorter focal length of the focusing lens, and for gases with larger Raman cross section, as expected.

In work carried out at Stanford by Prof. S. Harris and co-workers under contract with Lawrence Livermore Laboratory, experiments have been under way to improve the conversion efficiency for tripling $1.06-\mu \mathrm{m}$ laser radiation to $0.354 \mu \mathrm{m}$ in alkali metal vapors. Present conversion efficiencies of $2.7 \%$ have been demonstrated in $\mathrm{Na}$. This result was obtained at $\mathrm{Na}$ vapor pressure of 5 Torr phase-matched with $\mathrm{Xe}$ in a $40-\mathrm{cm}$ zone. The laser beam was focused confocally to the zone length, and the input energy was varied from 0.3 to $10 \mathrm{~mJ}$. The conversion efficiency varied

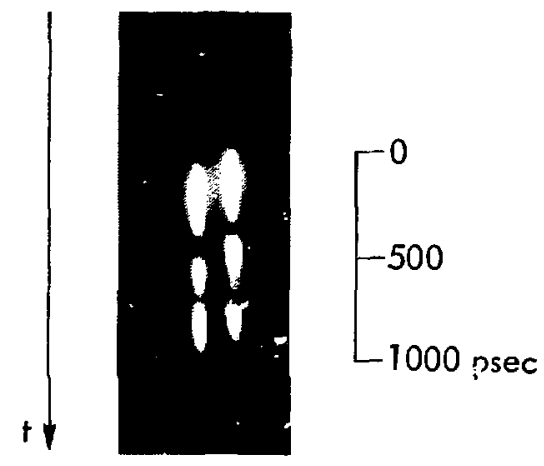

Fig. 125. Pulsations in forward (left trace) and backward (riglit trace) stimulated Raman scattering in Ioti-atm $\mathrm{CH}_{4}$. quadratically with the input energy up to an input energy of about $5 \mathrm{~mJ}$; for inputs above that level the conversion efficiency rose much less sharply, until for input energies of above $10 \mathrm{~mJ}$, increasing the input energy caused a decrease in output. This effect is believed to be due to multiphoton ionization of the $\mathrm{Na}$. The pover density at which this effect was observed was 2-to-4 $\times 10^{11} \mathrm{~W} / \mathrm{cm}^{2}$, which corresponds closely to theoretical calculations of the breakdown density in Na. Difficulties with condensation prevented carrying out experiments at higher pressures. However, it is expected ce this problem is eliminated, the conversion effic $\ldots y$ ' should rise as the square of the pressure until saturation caused by absorption of the $1.06-\mu \mathrm{m}$ light occurs.

Construction of an $\mathrm{Rb}$ cell almost identical in design to the $\mathrm{Na}$ cell is almost completed. This work was undertaken since $\mathrm{Rb}$ has a ncnlinearity almost 30 times that of $\mathrm{Na}$ and shou:a allow efíicient tripling at lower power densities.

An extensive analysis of the compression of light pulses via self-phase modulation has been carried out by R. Fisher and W. Bischel of LLL. In particular, they have examined the feasibility of constructing a pulse compression scheme based on the interferometer of Gires and Tournois, and they have calculated the effects of self-phase modulation in a long amplifier chain on subsequent compression. They conclude tha: there is sufficient self-phase modulation induced in the laser glass itself to generate a bandwidth wide enough to accommodaic tenfold pulse compression, from $1 \mathrm{nsec}$ to $100 \mathrm{psec}$.

The calculation of phase modulation in a lorg amplifier chain was carried out using a split-step algorithm described elsewhere. ${ }^{104}$ The linear dispersion of the mateial, the nonlinear index $\left(n_{2}\right)$, and the anomalous dispersion and gain of the active medium were all takeil into account. In order to simulate staging and other 'umped losses in this plane-wave calculation, a distributed loss was assumed, such that the intensity in the system was kept at very nearly a constant value.

The resultant self-phase modulation was calculated for average intensities of 1,2 , and $5 \mathrm{GW} / \mathrm{cm}^{2}$, in $1-\mathrm{nsec}$ pulses, propagating in a $2-\mathrm{m}$ chain. In each case, there is very little pulse distortion in the amplifier, but significant self-phase modulation. The effect of dispersion, which tends to reduce self-phase modulation, was found to be nonnegligible, amounting to roughly $30 \%$ on the final frequency profile. For each case, the optimal compression was calculated and the optimally compressed pulse envelope was examined. Results are shown in Fig. 126.

The stability of the compression scheme against noise was examined. It was found that a white nowe 

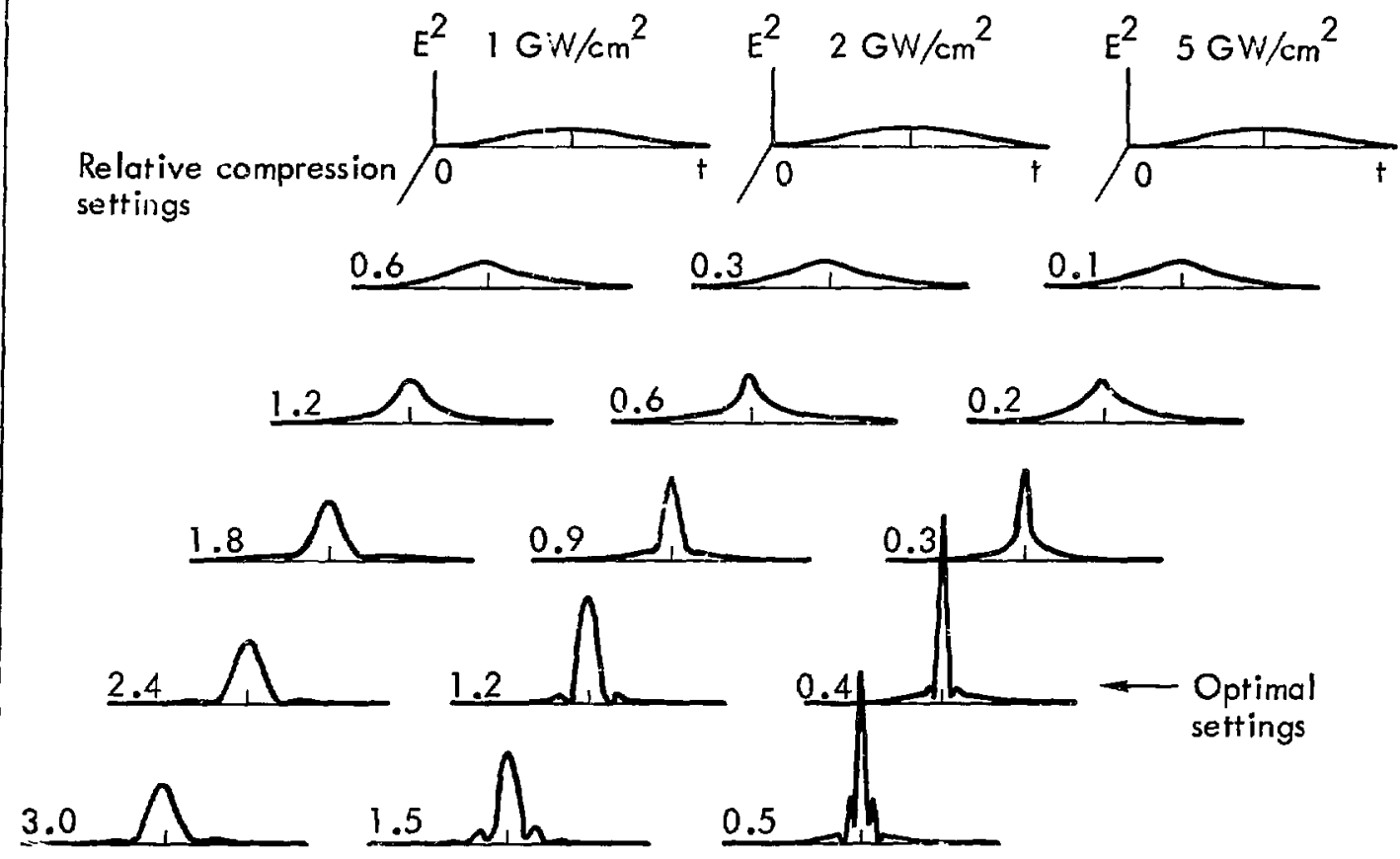

Fig. 126. Calculated dispersive compressibility of pulses from a $2-\mathrm{m}$-long Nd:glass laser chain for input peak intensities of 1 , 2 , and $5 \mathrm{GW} / \mathrm{cm}^{2}$. Spatial effects due to the radial intensity profile are not considered. In each case, the initial full $1 / e$ intensity duration is 1 nsec.

amplitude modulation had very little effest on the compression scheme, while the presence of a single frequency component of significant power, i.e., a sinusoidal amplitude modulation, was profoundly deleterious in the achievement of efficient compression.

In a beam of Gaussian profile, the beam center will be more effectively charged than the edges, due to its higher intensity, and subsequently will be more effectively compressed. This effect was simulated by averaging the resultant self-phase modulation over a Gaussian distribution. It was found that the efficiency of pulse compression for a Gaussian beam was 10 to $20 \%$ lower than that of a plane wave of comparable peak intensity.
In summary, it appears possible to compress 1-nsec pulses of powe $e_{i}$ densities of $10^{9} \mathrm{GW} / \mathrm{cm}^{2}$ on the basis of self-phase modulation alone. It is unlikely that any practical system will rely solely on self-phase modilation in the amplifier chain. The ability to compress pulses implies the ability to shape pulses, given a prescribed frequency ciirp. In any system of pulse compression using an imposed modulation, self-phase modulation in the amplifier must be taken into account in a realistic manner.

A detailed investigation of the optimal design for a Gires-Tournois interferometer is presently being carried out to assess the feasibility of constructing such a device.

\section{LASER MATERIALS STUDIES}

A key element in the solid-state laser system under development is the lasing medium-a neodymiumdoped glass. In addition to the significant cost of the lasing medium, its properties dictate the design of many of the major components of the laser, such as the flashlamps, power supplies, and arnplifier configura- tions. The output characteristics of the final laser system are a principal function of the lasing medium selected.

An extensive investigation of the properties of various solid-state laser media has been initiated in an effort to optimize the performance of existing 
materials and to develop new and alternative materials. This includes:

- New Nd-doped glasses. Changes in glass composition are correlated with the effects upon spectral properties and transition probabilities of Nd.

- Nd glasses co-doped with fluorescence-sensitizing ions to increase optical pumping efficiency. Iron group, rare earth, and post-transition metal ions having absorption bands which complement those of $\mathrm{Nd}$ and which effectively transfer excitation to the ${ }^{4} F_{3 / 2}$ state of Nd are being surveyed.

- Other amorphous and crystalline Nd host materials that can be prepared in the size and quality required for fusion lasers. Among materials being considered are fused silica and polycrystalline $\mathrm{CaF}_{2}$. Bot. of these materials have small nonlinear refractive indices. Techniques exist for preparing pure materials in large sizes and of good optical quality. Introduction of $\mathrm{Nd}$ dopants and detailed measurements and evaluation of spectral properties are required.

- New opticaily pumped lasing ions and schemes for operation at greater efficiency and/or at new wavelengths. For example, $f d$ transitions of several rareearth ions in crystals and glasses offer the potential for tunable, optically pumped lasing in the visible/nearultraviolet region. Given the spectroscopic properties of the lasing medium, it is possible to predict, using existing computer codes, the design criteria and output characteristics of high-energy Nd laser systems. Input data required include absorption spectra and cross sections. stimulated-emission cross sections or specific gain coefficients, and the fluorescence lifetime and its variation with $\mathrm{Nd}$ concentration. To obtain such data rapidly for a large number of materials, two systems have been designed: one for absorption and emission spectroscopy, and one for transient fluorescence studies. Both systems have digital data acquisition and processing capabilities.

To complete the spectroscopic characterization of materials, additional measuraments of excitation spectra and fluorescence kinetics are planned, including determination of fluorescence conversion efficiency, radiative quantum efficiency, terminal laser-level lifetime, excited state absorption, two-photon absorption, homogeneous and inhom:ogeneous linewidths, and ionion interaction and cross-reiaxation rates. Such measurements, however, will be mady only on laser material selected for actua' or potential use.

The extraction of the stored energy in Nd glass by short, intense narrow-band optical pulses can be affected by hole-burning in the spectral gain profile and by bottlenecking due to slow terminal-level relaxa. tion. Experiments are being set up to measure these properties. They involve optically pumping a sample to invert the ${ }^{4} F_{3 / 2} \rightarrow{ }^{4} I_{11 / 2}, 1.06-\mu \mathrm{m}$ laser transition and then applying a short $(\leqslant 1 \mathrm{nsec})$, narrow-band saturating pulse. A series of weak $1.06-\mu \mathrm{m}$ pulse: of varying time delays will follow to probe the gain recovery as the ${ }^{4} I_{11 / 2}$ manifold relaxes to the ground state. Alternatively the transient $660-\mathrm{nm}$ excited-state absorption can be monitored as described in the preceding Semiannual Report. ${ }^{105}$ The ${ }^{4} I_{11 / 2}$ lifetime in ED-2 glass at $300^{\circ} \mathrm{K}$ is reported to be 11 nsec. 106

Laser-induced fluorescence line narrowing has been reported in Nd-doped glass. ${ }^{107}$ The presence of spectral hole burning will be studied in the above arrangement using a second tunable laser pulse to probe the gain profile at various times after saturation. Timeresolved spectra of the fluorescence will also be recoruled.

In addition to the ${ }^{4} I_{11 / 2} \rightarrow{ }^{4} I_{9 / 2}$ relaxation, two other relaxation rates are pertinent to the above experiments. One is the population equilibration rate among the Stark levels of the ${ }^{4} I_{11 / 2}$ manifold. These relaxation times are expected to be very fast at room temperature-picoseconds or less. The second is the cross relaxation rate due to ion-ion energy transfer. The rates of energy transfer involving electric dipole and electric quadrupole transitions can be calculated, given the spectral overlap of the transitions for the two ions and the ion-ion separations. Although these quantities are not well known at present, estimates made using Judd-Ofelt intensity parameters ${ }^{17}$ yield predicted ${ }^{4} F_{3 / 2} \rightarrow{ }^{4} I_{9 / 2}:{ }^{4} I_{9 / 2} \rightarrow{ }^{4} F_{3 / 2}$ transfer rates of $\lesssim 10^{8}$ sec $^{-1}$ for nearest neighbor ions $(\sim 3 \AA$ separation). Average cross relaxation times at $\mathrm{Nd}$ doping levels of $3 w t \%$ should be in the range of microseconds. ${ }^{4} I_{11 / 2} \rightleftharpoons{ }^{4} I_{9 / 2}$ transfer rates are predicted to be of a similar order of magnitude.

Optical transitions between individual Stark levels of rare earths are usually not clearly resolved in glasses due to inhomogeneous line broadening. The Stark splitting of $\mathrm{Nd}$ in glass has been unraveled by Prof. L. G. DeShazer and co-workers at USC from careful analysis of absorption and emission spectra at various temperatures. ${ }^{108}$ By the application of these techniques, the energy levels, linewidths, and radiative transition probabilities associated with the ${ }^{4} F_{3 / 2}$ and ${ }^{4} I$ states of $\mathrm{Nd}$ in Ed-2 glass have been determined under contract with LLL. Additional studies of LSG-91H glass are in progress. These results will be of value in analyzing and predicting lasing properties. 
We anticipate continuing research activities in the areas outlined above during the next six months. We are continuing olir investigations into the origins of index nonlinearity, in conjunction with Professor Hellwarth at USC. Special emphasis will be placed on assessing the iesonant effects on $n_{2}$ due to the inverted population of $\mathrm{Nd}^{3+}$ ions. The consequences of index nonlinearity on laser system design and performance are under continuing investigation both in the Theoretical Studies and Design Analysis groups, as well as in $T$ Division. The effort is closely coordinated with experimental studies in the Solid State Laser group.

In the realm of frequency conversion, the Raman studies will concentrate on assessing the feasibility of developing a $1.9-\mu \mathrm{m}$ source for the early part of the plasma heating pulse. Special attention will be concentrated on the problems of suppressing instabilities in the spatial and temporal development of the pulse. As regards up-conversion, we will continue to work with Professor Harris at Stanford toward the development of alkali-metal-vapor frequency tripling.
Emplasis will be placed on understanding the limits of the process at very high power levels.

We will assess the engineering difficulties associated with realizing the Gires-Tournois pulse compression scheme. Damage phenomena in the compressor structure will be examined, and inproved compression designs will be formulated.

The investigation of new solid-state laser media will be getting under way. These studies will include both compositional studies on laser glasses-in an effort to improve spectral properties and reduce index non. linearity-and investigation of new crystalline hosts, as indicated above. Finetic studies on lifetimes and energy transfer processes will be initiated.

Over all. the goal of the Basic Studies activities is to provide the underlying analytical and computational base. and to assess new approaches for laser design, new materials for lasers, and basic phenomena of importance in the construction and implementation of high power lasers for fusion research. 
The use of lasers for the photochemical separation of isotopes is at present a topic of considerable national interest. A number of processes have been proposed and are reviewed in the literature. ${ }^{109.110}$ The separation of isotopes using laser-induced photochemical processes has been reported by several groups. $111-113$ In most such isotope-separation processes a laser is tuned to resonance with an atomic or molecular absorptiun line and is used to excite one isotope in a mixture of atoms or isotopic molecules. The excitation is isotopically selective only if the isotopic absorption lines are resolved and if the laser linewidth is less than the isotope shift. The excited atoms or molecules produced when the laser light is absorbed can subsequently undergo chemical reaction or ionization to form a separable entity. Details of several specific processes are given in Refs. 109.113.

The Luser Isotope Separation Program at Livermore has as its primary objective the development and evaluation of laser-induced photochenical processes for the separation of isotopes. Although the prinlary focus of the program is on the enrichment of uranium because of the critical need faced by the nation for new enrichment capacity during :he coming decades, processes for the separation of other isotopes will be considered as well.

In achieving the primary objective, it is intended that the program will produce a broad technical data base which will be useful for the assessment of many laser isotope separation processes. Accordingly, much of the effort during the past six months has been devoted to establishing the facilities for developing this broad approach. The major activities, which are reviewed separately below, are:

- Establishment of facilities for atomic and molecular spectroscopy.

- Construction of apparatus for uranium kinetic studies using the crossed-beam technique.

- Development of stable, reliable sources of uranium vapor for the spectroscopic and kinetic studies.

- Development oĩ a high-power, stable, tunable dye laser as a tool for separation studies.

- Photochemical separation of ${ }^{202} \mathrm{Hg}$ from natural mercury.

\section{SPECTROSCOPIC FACILITIES AND EXPERIMENTS}

A knowledge of absorption cross sections, excitedstate lifetimes, and photoionization cross sections is required for the evaluation of isctope separation processe' based upon atomic vapors. A facitity for the measurement of these parameters has been set up and is now operating.

Approximately 30.000 spectral lines of the uranium spectrum have been tabulated. ${ }^{1 / 4}$ Although many assignments have been made. oscillator strengths and radiative lifetines are available for only a few of these lines. Using the most rigorous criteria in the choice of lines for photochemical isotope-separation processes. one finds that there remain at least several dozen transitions which must be investigated with respect to the parameters mentioned above.

The atomic spectroscopy facility consists of two tunable $\mathrm{cw}$ dye lasers for excitation of the atomic vapor. a uranium-vapor oven source, and several monochromators for excitation and spectral analysis. The experimental Irrangernent is shown in Fig. 127.

The $\mathrm{cw}$ dyc laser to be used in the measurement of absorption cross sections is a Spectra-Physics Model
470 dye laser excited by a Spectra-Physics Model 170 argon ion laser. The dye laser incorporates a piezoelectrically driven intracavity etalor to provide continuous tuning, without mode hopping, of the dye laser over a spectral range of about $\pm 1 \mathrm{~cm}^{-1}$ around any chosen center frequency in the spectral range from 5000 to $6500 \AA\left(20.000 \mathrm{~cm}^{-1}\right.$ to $\left.15,400 \mathrm{~cm}^{-1}\right)$. The continuous tuning range is sufficient to cover the expected isotope effects of the spectral lines to be studied. The linewidth of the $\mathrm{cw}$ dye laser is approximately $10 \mathrm{M} ! \mathrm{iz}$, mucl smaller than the $1.5 \cdot \mathrm{GHz}$ Doppler width of the uranium lines.

The capability of the tunable laser is illustrated in Fig. 128 where the output of the dye laser is split to pass through two cells containing iodine vapor. One of the cells contains isotopically pure ${ }^{129} \mathrm{I}_{2}$ while the other contains isotopically pure ${ }^{127} l_{2}$. The isotope splitting of the rotational lines is about $3 \mathbf{G H z}$ while the Doppler width is about $360 \mathrm{MHz}$. In the plotograph the laser is tuned to a resonance line of the ${ }^{2} \mathrm{I}_{2}$ cell. The absorption is indicated by the resonance fluorescence of the ${ }^{127} \mathrm{I}_{2}$ sample. At the 


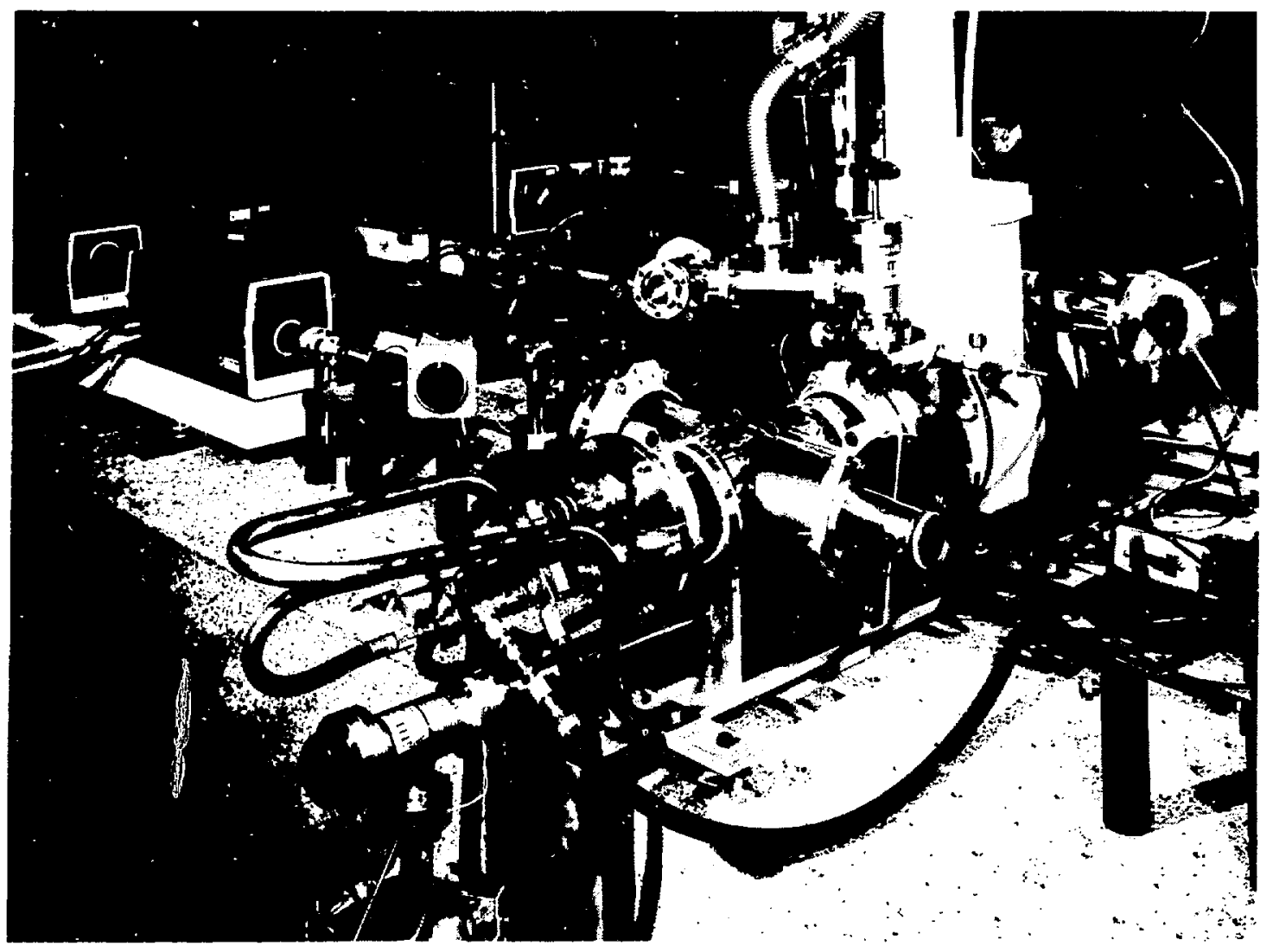

Fig. 127. Experimental arrangement for atomic spectroscopy studies.

same time the ${ }^{129} 1_{2}$ sample is unexcited and remains dark.

The apparatus is now being used to measure absorption cross secticns for selected transitions in the UI spectrum. Some 15 resonance transitions have been chosen on the basis of a large isotope shift, expected small hyperfine splitting of the ${ }^{235} \mathrm{U}$ transitions, and estimated lifetimes. The lines were chosen by a computer search of the UI line list. ${ }^{114}$ The decay channels Ior these lines were also taken from the line list, and excited-state lifetimes were estimated from line intensities and oscillator strengths where available. 115

In operation, the laser is tuned to a chosen uranium line by monitoring a portion of the laser output with the $1.5-\mathrm{m}$ Jubin-Yvon THRP spectrometer. This instrument, with a resolution of better than $1: 300.000$ in first order, is used to set the laser wavelength close to the chosen line. The uranium line is then found by scanning the dye laser wavelength over its $\pm 1-\mathrm{cm}$ l range at a $50-\mathrm{Hz}$ rate while the center frequency is changed slowly and absorption of the sample cell is monitored on an oscilloscope. The absorption is measured by taking the ratio of signals obtained from photodiodes $A$ and $B$ in Fig. 127. When the desired line is found, the laser wavelength is scanned slowly over the linewidtl and the ratio of the $A$ and $B$ photocell signals is plotted on a chart recorder.

The sample cell is in the form of a small oven having a path length of about $1 \mathrm{~cm}$. The vapor density in the oven is known from the characterization of the source, which permits the absorption cross section to be determined. Calculations indicate that absorption cross sections for the lines of interest should be in the range from $10^{-12}$ to $10^{-14} \mathrm{~cm}^{2}$, a range well within the capability of the existing setup.

Measurement of excited-state lifetimes will use a slightly different arrangement. The uranium source will produce a beam of atoms, and the lifetimes will be measured by monitoring fluorescence decay times after pulse excitation of the atomic beam. The beam geometry provides a more favorable arrangement for optically collecting the huorescence and imaging it on 


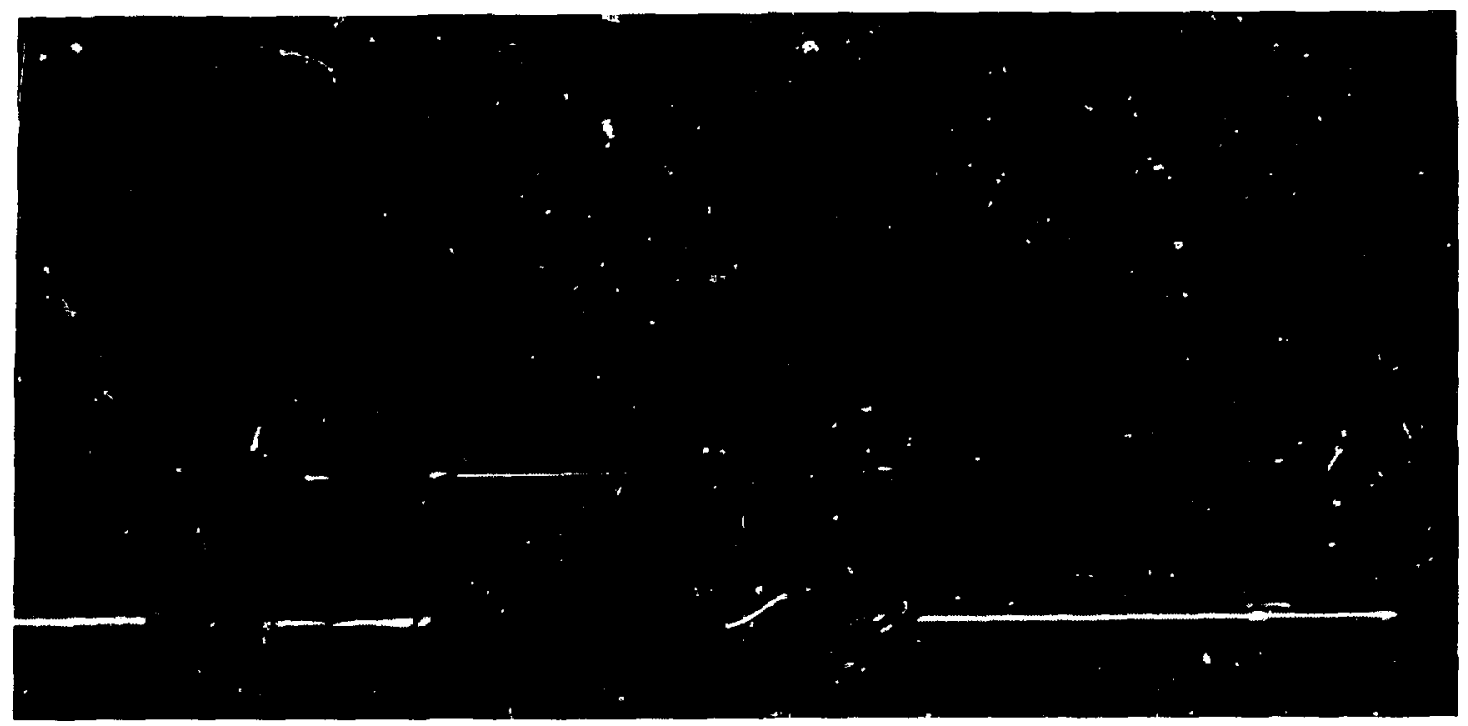

Fig. 128. Jllustration of tunable dye laser's operation. When laser is tuned to a resonance line of ${ }^{127} I_{1}$, upper cell which contains ${ }^{127} I_{2}$ fluoresces while lower cell which contains ${ }^{129} I_{2}$ remains dark.

the entrance slit of a monochromator than does the oven geometry used in the cross section measurements.

Fluorescence detection and signal processing will be done by a conventional photomultiplies-tube/boxcar integrator arrangement. The excitation pulses will be produced by a Pockels cell electrooptical shutter operating on the $\mathrm{cw}$ dye-laser output. Lifetimes in the 1-to-5 $\times 10^{-7}$-sec range are anticipated and should be measurable with the apparatus described. The optical and mechanical systems for this experiment are under construction and expected to be operating in the last quarter of FY 74.

Very little information has been published on the vapor phase spectroscopy of the uranium compounds. Since this information is essential for the development of laser isotope separation processes, we have established a molecular spectroscopy facility to bridge this gap.

In molecular isotope separation processes the spectral region from 0.25 to $20 \mu \mathrm{m}$ is of interest. Since the absorption cross sections of promising materials are expected to be much smaller in the ir than in the uv and visible regions, it is convenient to break the total spectral range into two parts which are studied with different experimental arrangements. Special facilities for sample handling are also required because of the low vapor pressure of the samples, their radioactivity, and their toxicity.

For investigations in the range from 0.25 to $2.5 \mu \mathrm{m}$ a $0.75-\mathrm{m}$ Spex spectrometer is being used. The absorp- tion cell is a $1.25-\mathrm{m}$-long stainless steel tube, $1 \mathrm{~m}$ of which is heated in a tube furnace. A dry-box system has been fabricated which allows the introduction of samples into the absorption cell under a dry, inert atmosphere. A vacuum system is attached to the absorption cell so that the atmosphere in the cell can be changed. The lamp used in the initial studies is a $1000-W$ xenon arc.

For investigations in the spectral range from 2.5 to $20 \mu \mathrm{m}$ a stainless steel multiple-reflection absorption cell has been designed for use with a Beckman IR-9 recording spectrophotometer. The cell, with provision for 24 optical passes, has an effective length of $5 \mathrm{~m}$. It can be heated to about $1000^{\circ} \mathrm{C}$.

Compounds for initial study in the molecular spectroscopic effort include the uranium tetrahalides $\left(\mathrm{U} X_{4}\right)$, mixed tetrahalides of the form $\mathrm{U} X_{3} Y$ where $X$ is one halogen and $Y$ is another, and the uranyl halides $\mathrm{UO}_{2} X_{2}$. Other compounds are of interest as well.

There are several reasons for selecting these compounds. First, several of these materials have reasonable vapor pressure ( 0.1 to 1.0 Torr) at temperatures below $500^{\circ} \mathrm{C}$ and appear to be stable at these temperatures. Additionally, the $\mathrm{U} X_{4}$ and $\mathrm{U} X_{3} Y$ molecules have high molecular symmetry, either $T_{a^{2}}$ or $C_{3 y}$, which leads to rather simple spectroscopic properties. These include a sizable predicted vibrational isotope shift in one of the degenerate vibrational modes. Also, from estimated rotational constants it appears that the rotational line structure should be well spaced. This is important if the isotope shift is to be resolved. 
Some initial spectroscopic studies of the uranyl halides have yielded results which are not very promising. These compounds tend to decompose rather than to vaporize congruently.

The molecular spectroscopic studies arc predicate.: upon the availability of well-characterized samples of the materials of interest. Since many of the compcunds are not commercially available, it is necessary to have a synthetic chemistry capability associated with the program. This capability is provided by personnel within the Laser Isotope Separation Program in cooperation with the Chemistry Department at LLL. Facilities for synthesizing and purifying uranium compounds have been set up. A complete range of analytical capabilities and expertise is available for support within the Chemistry Department. This talent has been drawn upon in the initial studies on the materials listed above.

\section{KINETICS OF URANIUM REACTIONS}

Another area of critical importance to the development of laser photochemical processes for isotope separation is the understanding of the kinetics of uranium and uranium-compound reactions. As in the case of vapor phase spectroscopy, the relevant literature is, at best, sparse. To overcome this difficulty, a strong effort in chemical kinetics is being established. The focus of this effort will be the measurement and theoretical interpretation of the reaction cross sections, chemi-ionization cross sections, and charge and energy exchange rates for the reactions of uranium and uranium compounds.

Three types of reactions to be considered, together with examples of each, are given below:

\section{Normal chemical reactions}

$$
\begin{aligned}
& \mathrm{U}^{*}+\mathrm{CO}_{2} \rightarrow \mathrm{UO}+\mathrm{CO}, \\
& \mathrm{U}^{*}+\mathrm{O}_{2} \rightarrow \mathrm{UO}+\mathrm{O}, \\
& \mathrm{U}^{*}+\mathrm{N}_{2}+M \rightarrow \mathrm{UN}_{2}+M(M \equiv \text { third body }) .
\end{aligned}
$$

\section{Chemi-ionization reactions}

$$
\begin{aligned}
& \mathrm{U}^{*}+\mathrm{O}_{2} \rightarrow \mathrm{UO}_{2}^{+}+\mathrm{e}^{-}, \\
& \mathrm{U}^{*}+\mathrm{N}_{2} \mathrm{O} \rightarrow \mathrm{UO}^{+}+\mathrm{N}_{2}+\mathrm{e}^{-} .
\end{aligned}
$$

Ion-molecule reactic'ss

$$
\mathrm{U}^{+}+\mathrm{O}_{2} \rightarrow \mathrm{UO}^{+}+\mathrm{O} \text {. }
$$

Different techniques are used to study different types of reactions. Reactions which yield a charged product are generally easier to st dy than those which do not.

Our initial experiments will be concerned with the investigation of chemi-ionization reactions by the technique illustrated in Fig. 129, which shows the chemi-ionization reaction of uranium with molecular oxygen ${ }^{116}$ as an example. In this experiment, beams of uranium and $\mathrm{O}_{2}$ intersect at right angles. The oxygen beam, produced by a capillary-array effusion source located in a separately pumped vacuum system, is collimated into a narrow molecular beam before entering the main scattering chamber. There it intersects an atomic uranium beam that is directed into the plane of the paper and normal to the $\mathrm{O}_{2}$ beam. The uranium beam is produced by a source of the type described in the following section. In the beam intersection region the uranium ian be excited by a laser. lons produced by a chemi-ionization reaction are extracted by an electric field and mass-analyzed with a quadrupole mass filter.

The production of molecular ions is monitored as a function of the excitation wavelength. The results will be used to determine the reaction cross section as a function of atomic excitation. It is expected that in at least some chemi-ionization reactions the cross section will increase strongly with the excitation of the uranium atoms. After suitable modification, ionmolecule and charge exchange studies can be conducted in the apparatus using standard techniques. 117

Reactions such as these could serve as the basis for a process to remove the enriched product after laser excitation. Such a chemical extraction process offers distinct advantages with respect to physical extraction. First of all, loss of product by charge exchange is minimized since the charge exchange process between atoms and molecular ions is not symmetric. Also, i' requirements upon magnetic and electric fields used iu. physical extraction are not so severe if the enriched product is extracted as a molecular ion.

The crossed-beam apparatus illustrated schemalically in Fig. 129 is best adapted to the study of reactions which yield a charged product. For normal reactions. such as those in the examples, the product is uncharged and detection is more difficult. For such reactions, we plan? to use a modified "flowing afterglow" technique as shown schematically in Fig. 130. In this arrangement the uranium atoms are intunduced into a flowing helium stream. They drift into a region which contains a reagent gas where they are excited by 


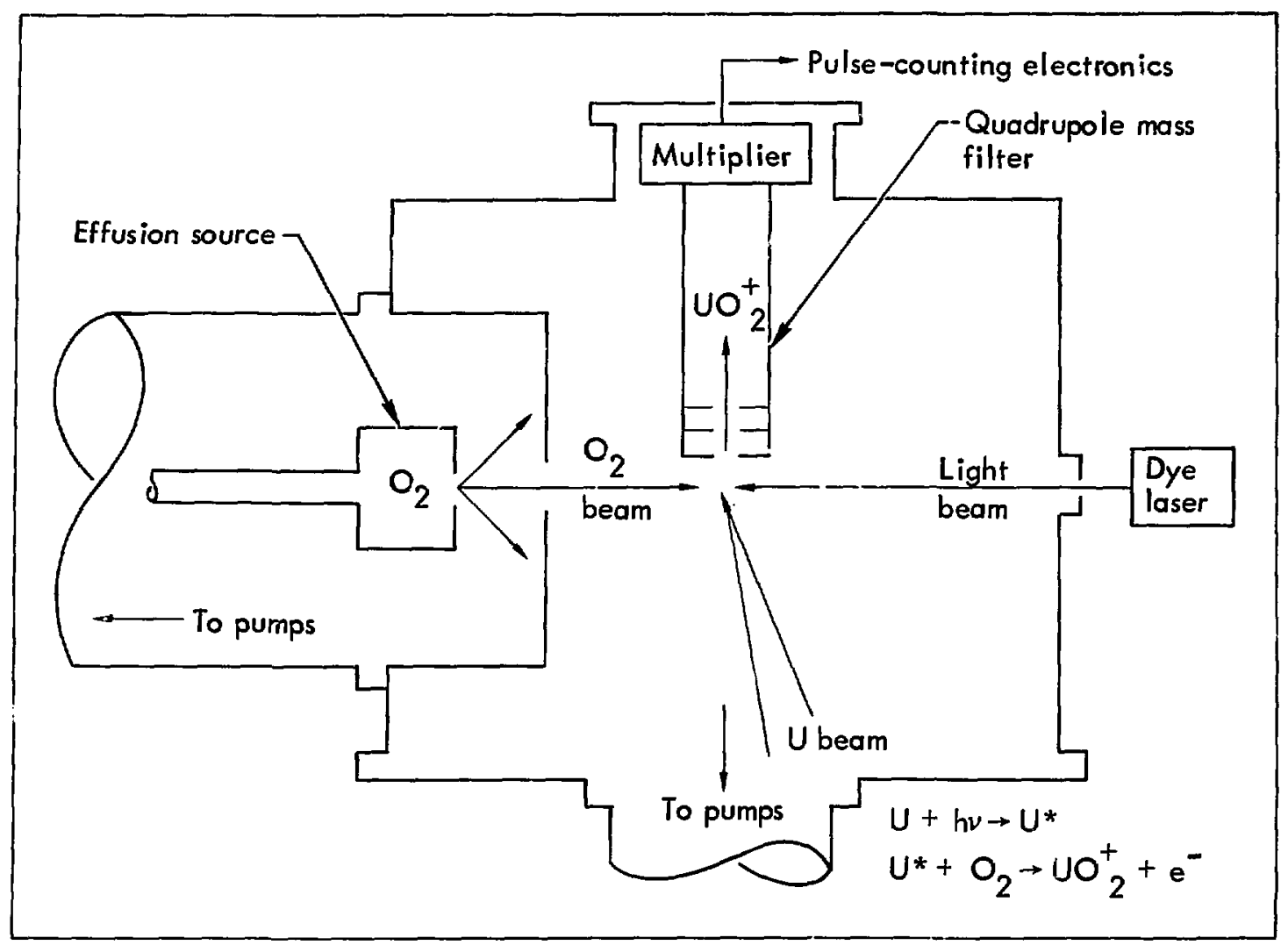

Fig. 129. Experimental setup for studying chemi-ionization reaction of uranium with molecular oxygen.

a tunable laser. The products formed in the excited-state reactions with the reagent gas are to be analyzed optically. A quadrupole mass filter with an electron gun ionizer is also included in the system to aid in the analysis of reaction products.

\section{URANIUM VAPOR SOURCES}

Any isotope separation process based upon uranium vapor requires a source for the vapor. That this is a difficult problem can be appreciated from consideration of the simplest case: a source in which uranium metal is heated in a hot crucible to produce a vapor. To produce a vapor pressure of 0.1 to 1.0 Torr, a convenient pressure range for separation processes, the uranium must be heated to approximately $2100^{\circ} \mathrm{C}$. Uranium is extremely reactive, and no suitable material has been developed for containing molten uranium at this temperature for long periods. This is a problem which has plagued experimenters concerned with the spectroscopic and kinetic properties of uranium.
Some techniques used to produce rapid evaporation of refractory materials-plasma and electron-gun sources, for instance-are unsuitable because of the large number of ions they produce. Since the first step of the isotope separation process creates isotopically specific jons or excited atoms, ions and excited neutrals produced by the source would tend to reduce, or even destroy, the enrichment.

In connection with the Laser Isotope Separation Program, this problem seems to have been solved for laboratory-scale experiments. ${ }^{118}$ Novel uranium-vapor sources have been developed for atomic beam and spectroscopic studies. In these sources, a high-melting 


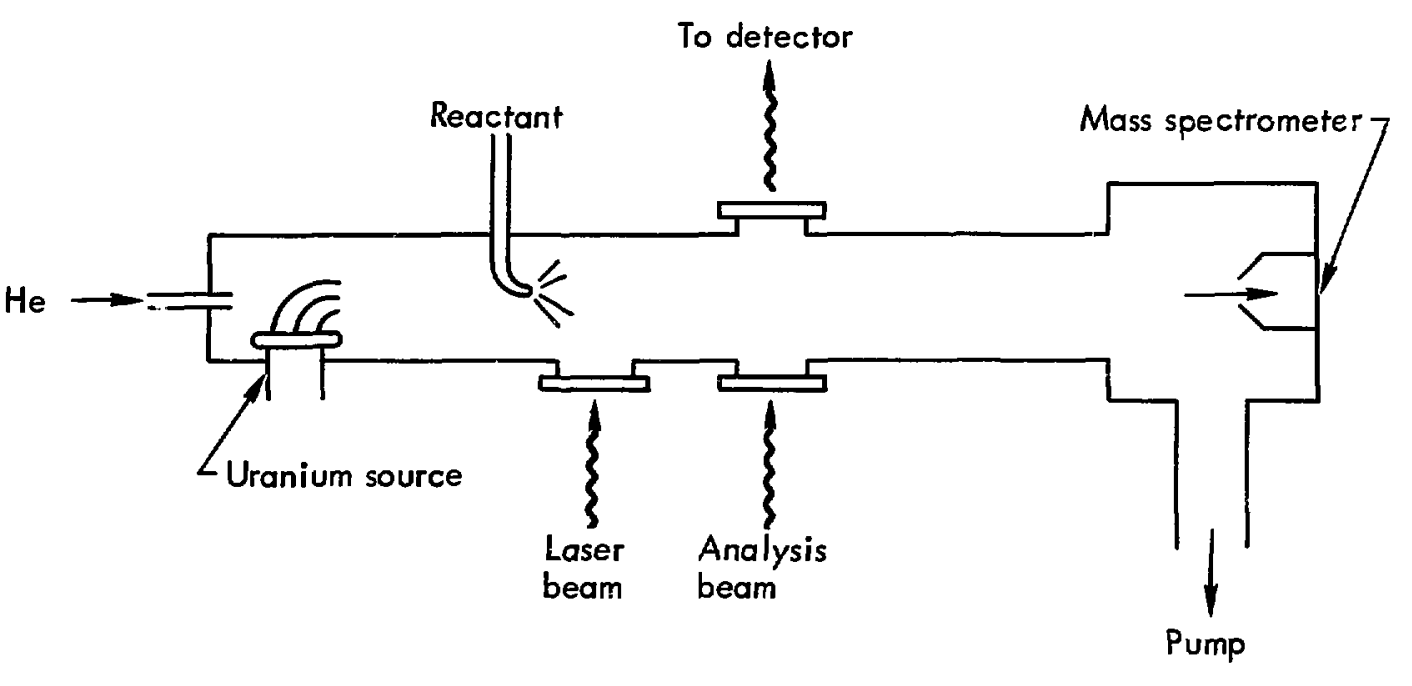

Fig. 130. Modified "flowing afterglow" technique for identifying uranium reactions and reaction products.

compound, $\mathrm{URe}_{2}$, is heated on tungsten or rhenium filaments. The uranium vaporizes, leaving behind a nonvolatile rhenium residue. These sources will provide uranium vapor densities greater than $10^{13}$ atoms $/ \mathrm{cm}^{3}$ for a number of hours.

Molten $\mathrm{URe}_{2}$ is found to attack rhenium heaters rapidly but, surprisingly, appears to be compatible with tungsten heaters for a few hours. This oehavior contrasts markedly with the severe attack experienced in containing pure liquid uranium in tungsten, where the liquid $U$ rapidly penetrates through $1-2 \mathrm{~mm}$ of tungsten in a few minutes at $1600^{\circ} \mathrm{C}$.

The uranium vapor source used in the atomic spectroscopy experiments is shown in Fig. 131. The vapor source is contained in the Pyrex tee. The optical probe beams enter the source through the arm of the tee which points toward the lower left corner of the figure. The system is pumped by an ion pump mounted on the rear arm of the tee. This source is capable of stable operation for periocis greater than $5 \mathrm{hr}$ at uranium pressures in the active region of 0.01 to 0.1 Torr.
Experiments with high-temperature uranium sources are being continued to develop highly reliabie souices for high-throughput vapor systems.

Concurrent with the investigation of high temperature sources, some effort is being devoted to the development of "low-temperature" vianium sources. These sources would produce uranium vapor by the thermal, or photolytic, decomposition of volatile compounds which contain uranium. Although these systems would produce atomic vapor as a transient species, the atorns nucleate quickly and condense, and a relatively short time is required for the several steps of the isotope separation process. Thus, the separation system can be operated at temperatures much below the temperature of a molten uranium source.

The process presently under investigation is based upon the thermal decomposition of $\mathrm{UI}_{4}$ to produce $\mathrm{U}$ $+4 \mathrm{I}$. There are several quasi-stable, volatile uranium co:npounds which will be investigated for use in produring nonequilibrium sources of uranium and simple uranium-containing molecules.

\section{DEVELOPMTENT OF A HIGH-POWER TUNABLE LASER}

The scalability, and economic feasibility, of largescale laser isotope separation systems is dependent upon the scaling behavior of tunable lasers. The development of large tunable lasers is being undertaken at an early stage in the Isotope Separation Program.
Several of the investigations planned will require a high-repetition-rate pulsed tunable laser operating in the visible region of the spectrum. The first application of such a laser is in the separation of mercury isotopes as described in the following section. To meet these 


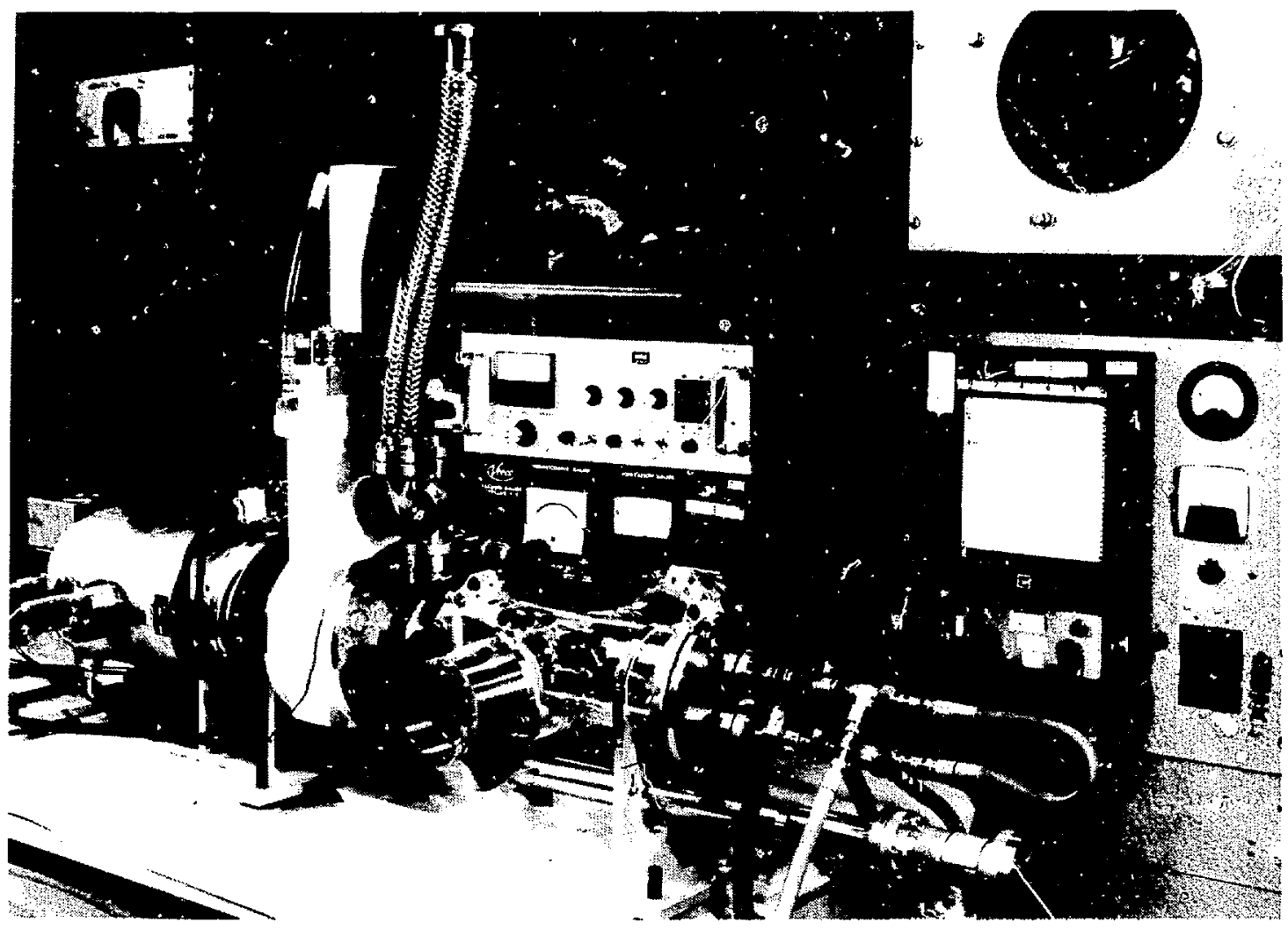

Fig. 131. Uranium vapor source for atomic spectroscopic studies.

needs, a dye laser with the target specifications given in Table 23 is being developed. Some of the design criteria for this laser will be discussed.

The active length of the laser is kept shou $t$ to minimize the effects of thermal schlieren and refractive index variations upon performance. An active length of $7.5 \mathrm{~cm}$ was chosen as the minimum possible length consistent with the design power goal of $10 \mathrm{~W}$. To achieve sufficient excitation power input with this length, and maintain the spatial uniformity of excitation necessary for a fundamental mode output, a twolobe elliptical cavity is used with two linear xenon flashlamps.

The ZAP computer code was used to optimize the geometry of the elliptical pump cavities. With the ZAP code it was possible to design a system which mininizes preheating of the dye solution by pump light that falls outside of the active region. The calculations also showed that filling the entire flashlamp cavity with water improves the pumping efficiency greatly by improving the index match between the pump cavity volume and the dye cell. This reduces the aberrations introduced by the flat walls of the dye cell and improves the excitation uniformity.
The water in the laser head is continuously circulated to maintain temperature uniformity throughout the head. Particular care has been taken to insure that the dye solution and pumping head will be at the same temperature to prevent any thermally induced refractive index variations from occurring within the active volume.

The maximum permissible average power input to each of the lamps is $2 \mathrm{~kW}$. The lamps have an inside diameter of $4 \mathrm{~mm}$ The electrodes are especially large to maximize the lainp life under high average pcwer conditions. The design goal is a lamp lifetime greater than $10^{6}$ shots.

Table 23. Design goals for the high-power tunable dye laser being developed at LLL

Average power ........... 10 w

Wavelength $\ldots \ldots \ldots \ldots \ldots \ldots 50 \ldots$

Pulse duration ............. $10^{-6} \mathrm{sec}$

Pulse repetition rate. ........ 300-500 $\mathrm{Hz}$

Linewidth .............. $10^{-2} \times\left(\sim 10^{9} \mathrm{~Hz}\right)$

Average power at second harmonic . . . . 0.I W 
To design the pulse-forming networks for driving the flasilamps, use was made of the MINI-SCEPTRE network design code. Unfurtunately, design experience for lamps sperating in the time/snergy regime of the present systejn is somewhat sparse. However, on the basis of available information, a lamp impedance of about $0.5 \Omega$ was anticipated and the pulse-forming networks were designed to operate into the 1.0- $\Omega$ impedance of the two lamps in series.

To meet the design-goal repetition rate and power, thyratrons were chosen as the switching elements foi driving the lamps. The voltage and current requirements of the system were met by using four EG\&G thyratrons mounted in low-inductance chassis. The energy is stored in four pulse-forming networks mounted on the thyratron chassis to form a lowimpedance, lumped-constant transmission line. Each of the four networks has a characteristic impedance of $4 \Omega$. The four are operated in parallel to give the desired 1- $\Omega$ impedanca. Tests of the discharge circuits into a dummy resistance gave a pulse width of $0.7 \mu \mathrm{sec}$ (FWHM), which compares favorably with the predicted value of $0.6 \mu \mathrm{sec}$.

Initial $t \in s t s$ with the flashlamps have given a pulse width of $1.7 \mu \mathrm{sec}$. Impedance measurements have revealed tha: the lamp turn-on time is longer than expected so that the lamp impedance is higher than the $1-\Omega$ design value. Driving the lamps separately, each from two pulse-forming networks in parallel, has reducer the pulse width to $1.0 \mu \mathrm{sec}$.

The plasma in the lamps is maintained by a dc simmer circuit during the time between pulses. The magnitude of the simmer current affects the turn-on time and plasma growth rate when the lamps are fired. Further investigations are being carried out to optimize this current and approach for the design goal of $0.5-\mu$ sec pulse width.

The optical design of the laser resonator was subject to several limitations imposed by the intended use of the laser. In order to obtain the $0.005-\AA$ linewidth required for several isotope-separation experiments, a number of possible schemes for narrowing the 20-to-30- $\AA$ linewidth of the dye laser were evaluated. Based on considerations of stability, simplicity of utilization, and minimal insertion losses, a two-element transmitting filter system was selected. The first filter has a pair of low-reflectivity Fabry-Perot etalons. The first of these has a free spectral range of $2.0 \AA$ while the second has a free spectral range of $0.1 \AA$. The elements of the etalons are coated to have a reflectivity of $20 \%$ at the operating wavelength. This system was chosen in preference to a single high-reflectivity etalon because it was feared that the coatings of the etalon would be damaged by the high average intracavity power.

The combination of the Lyot filter and two etalons should yield the required linewidth. Wavelength should $L$ : readily settable to $\pm 0.5 \AA$. The fine-tuning of the wavelength will be done by a piezoelectric drive on the output mirror of the laser cavity.

The optical cavity is hemispherical with the curvature radius of the high-reflectance mirror chosen to give a mode diameter in the active region slightly smaller than the dye channel thickness. The nearly flat wavefront near the flat output mirror provides a convenient place in the cavity to place the tuning elements. Figure 132 shows the geometry of the laser and placement of the tuning elements.

The laser is presently undergoing its initial tests. These tests have revealed some aspects of the system which need to be modified. Modifications to the dye cell and to the electronics are being made as the tests are being carried out. After the system is proven, which should be sometime during the next few months, it will be reengineered to simplify construction and mainienance. The knowledge gained from this system will be applied to the design of considerably larger systems.

\section{PHOTOCHEMICAL SEPARATION OF ${ }^{202} \mathrm{Hg}$ FROM NATURAL MERCURY}

The primary interest in the Laser Isotope Separation Program is in the development of processes for enrich. ing uranium. However, during the initial phase of the program, when it is necessary to develop uranium sources suitable for separation experiments, it seems expedient to examine other laser photoseparation systems in order to gain insight into some of the pioblems cornmon to all laser photoseparation processes. Some of these problems are the stabilization of large tunable lasers on isotopic absorption lines, the separation of an enriched product from a rapidly flowing gas stream with high efficiency and without isotope scrambling, and the assessment of the influence upon separation processes of laser-specific effects such as bleaching and coherence phenomena, to name a few.

As an initial demonstration of laser isotope separation, a photochemical process similar in those developed by Gunning and co-workers ${ }^{119-121}$ has 


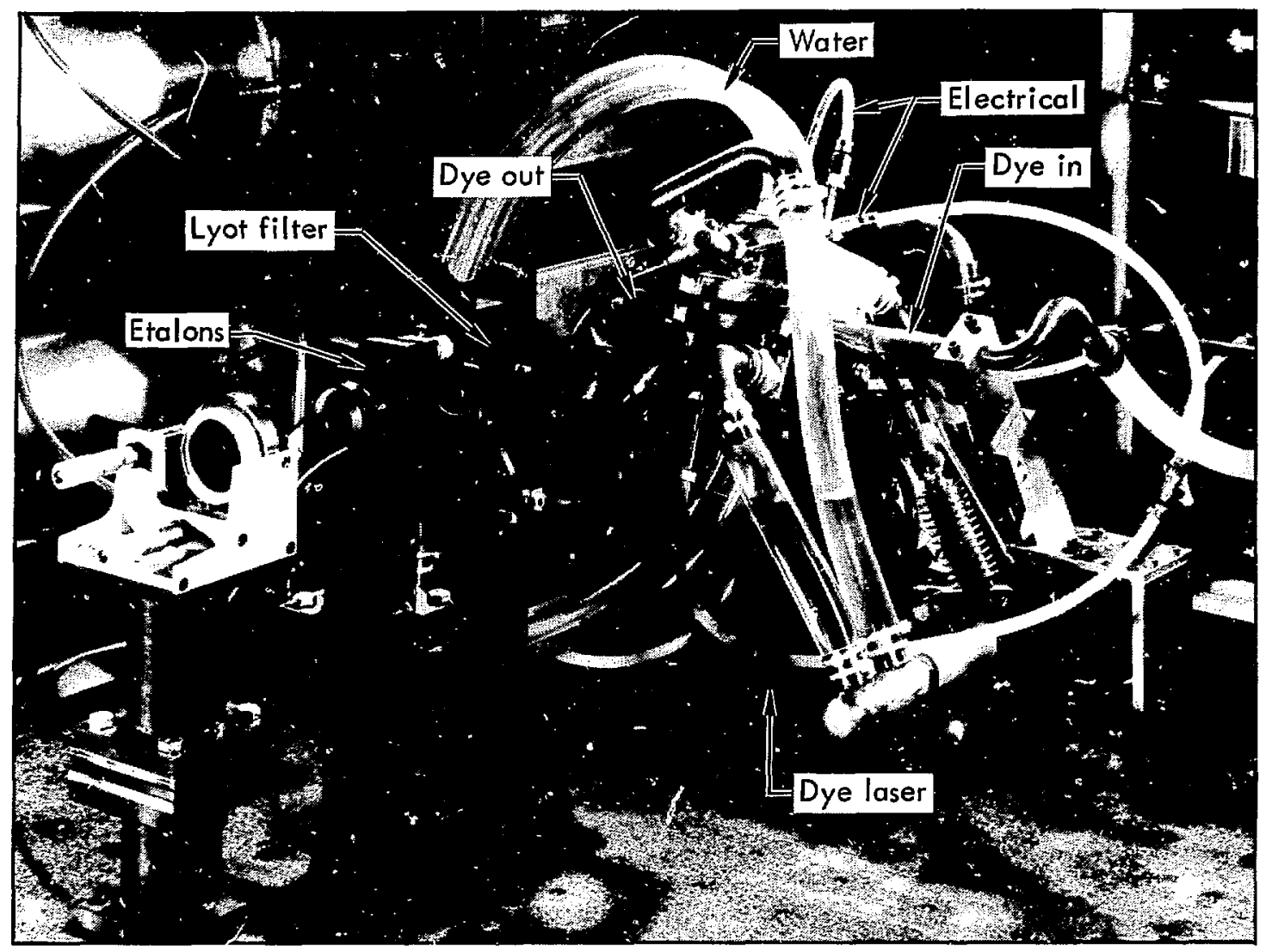

Fig. 132. Basic elements of the high-power, high-repetition-rate pulsed dye laser which will be used in studies of the scalability of tunable lasers to sizes appropriate for large-scaie isotope separation systems.

been chosen. This photochemical process is thought to involve the following reactions:

$$
\begin{aligned}
{ }^{N} \mathrm{Hg}\left(6^{1} S_{0}\right)+h \nu \rightarrow{ }^{202} \mathrm{Hg}\left(6^{3} P_{1}\right) \\
+{ }^{(N-202)} \mathrm{Hg}\left(6^{1} S_{0}\right),
\end{aligned}
$$

${ }^{202} \mathrm{Hg}\left(6^{3} P_{1}\right)+\mathrm{CH}_{3} \mathrm{Cl} \rightarrow{ }^{202} \mathrm{HgCl}+\mathrm{CH}_{3}$,

$$
{ }^{202} \mathrm{Hg}\left(6^{3} P_{1}\right)+\mathrm{CH}_{3} \mathrm{Cl} \rightarrow{ }^{202} \mathrm{Hg}\left(6^{1} S_{0}\right)+\mathrm{CH}_{3}+\mathrm{Cl},(50)
$$

$\mathrm{Cl}+{ }^{N} \mathrm{Hg}+M \rightarrow{ }^{N} \mathrm{HgCl}+M$,

in addition to other reactions involving $\mathrm{CH}_{3}$ and $\mathrm{Cl}$. In this series, step (49) provides enriched ${ }^{202} \mathrm{HgCl}$ while (50) and (51) tend to dilute the enriched product. As a result, complete enrichment by this process is not possible. This process is favored, however, with respect to other processes which yield greater enrichment because the quantum yield for the process is very high.

An alternative photochemical separation process, also developed by Gunning, involves the reaction of mercury with water vapor to produce mercuric oxide enriched in ${ }^{202} \mathrm{Hg}$. The steps of this process are believed to be:

$$
\begin{aligned}
& { }^{202} \mathrm{Hg}\left(6^{3} P_{1}\right)+\mathrm{H}_{2} \mathrm{O} \rightarrow{ }^{202} \mathrm{HgOH}+\mathrm{H}, \\
& { }^{202} \mathrm{Hg}\left(6^{3} P_{1}\right)+\mathrm{H}_{2} \mathrm{O} \rightarrow{ }^{202} \mathrm{Hg}\left(6^{1} S_{0}\right)+\mathrm{H}+\mathrm{OH}, \\
& N_{\mathrm{ilg}}+\mathrm{OH}+M \rightarrow{ }^{N} \mathrm{HgOH},
\end{aligned}
$$

where the initial excitation of ${ }^{202} \mathrm{Hg}$, as expressed by Eq. (48), is assumed to have taken place. In this series of reactions, equations (53) and (54) lead to isotope scrambling. This is minimized in Gunning's experiments by the mixture of butadiene with the reactants to scavenge the $\mathrm{H}$ and $\mathrm{OH}$ radicals from the gas phase, thereby decreasing the rate of reaction (52). In practice, very little enrichment is obtained unless substantial quantities of butadiene are present with $\mathrm{H}_{2} \mathrm{O}$ in the reaction mixture. 
The water vapor process is found to yield a higher enrichment than the calomel process of reactions (49) to (51). However, the quantum yield for the calomel process is found to be about ten times greater than the quantum yield for the water vapor process.

The apparatus used for carrying out the photochemical enrichment process using water vapor is shown in Fig. 133. It is generally similar to that described by Gunning et al. but with a number of modifications for better controi of pressures and flows and with a substantially different irradiation cell.

Although the intent is to carry out the enrichment experiment using the laser described in the previous section, preliminary experiments have been carried out with a microwave-powered resonance lamp. The total lamp power incident upon the active region of the cell at $2537 \AA$ was $23 \mathrm{MW}$ as determined by hydrogen iodide actinometry. For the laser source, the highrepetition-rate laser will be operated at $5074 \AA$ and frequency-doubled to obtain the required $2537-\AA$ wavelength. The design goal for power output at $2537 \AA$ is $100 \mathrm{MW}$.

The choice of crystals for critical $\left(90^{\circ}\right)$ phase matching at $2537 \AA$ is limited. Of the standard nonlinear optical materials, only ADP can be phasematched for $2537-\AA$ generation. Phase matching with

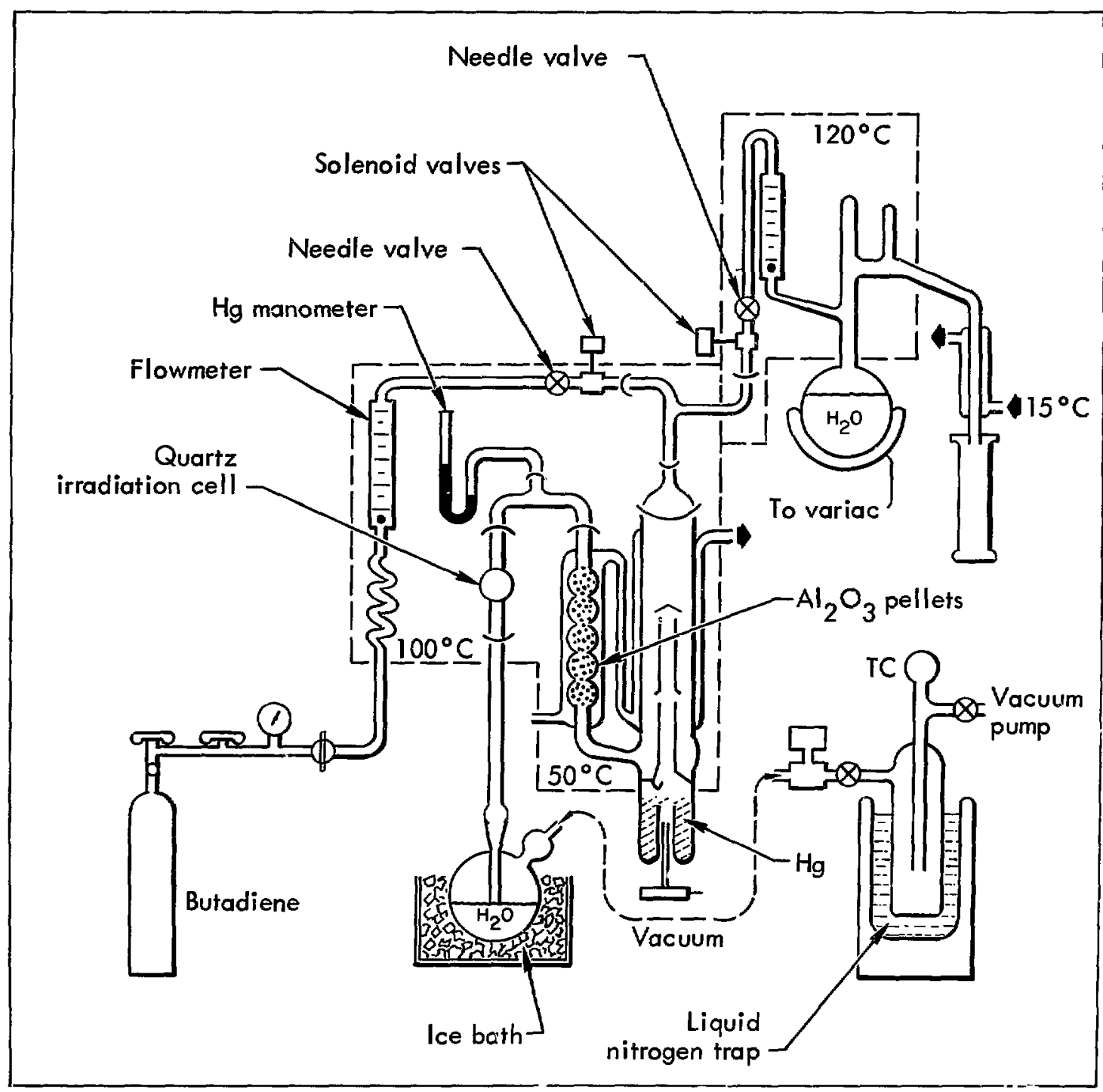

Fig. 133. Apparatus for carrying out the photochemical enrichment process for 20 a $\mathrm{Hg}$ using water vapor. 
$A D P$ at this wavelength requires a temperature of -40 to $-60^{\circ} \mathrm{C}$. A frequency doubler using ADF has been constructed but not yet tested, since the highrepetition-rate laser is not completed.

The reaction cell in the separation setup is cylindrical with a $5.0-\mathrm{cm}$ diameter and a thickness of $2.0 \mathrm{~cm}$. The gas mixture enters at the top of the cell, flows through the active region and exits at the bottom. In order to equilibrate the $\mathrm{Hg}$ vapor temperaivic with the temperature of the water jacket at the flow rates used, it was found necessary to pack the condenser icgion of the diffusion pump transpiration saturator with a heattransfer material. $\mathrm{Al}_{2} \mathrm{O}_{3}$ chips were used for this purpose.

In the apparatus of Fig. 133, the enriched $\mathrm{HgO}$ is captured from the gas stream by a cold water trap through which the reacted gases are bubbled. Preliminary experiments have indicated that some isotopic scrambling is taking place in the water trap; apparently the slight water solubility and amphoteric behavior of $\mathrm{HgO}$ coupled with relatively rapid exchange between $\mathrm{Hg}$ and $\mathrm{HgH}$ in a water-mercury slurry makes an aqueous acid recovery of ${ }^{202} \mathrm{Hg}$ from ${ }^{N} \mathrm{Hg}$ very difficult.

The results of a photoseparation experiment using water vapor are shown in Fig. 134. In this experiment, the $\mathrm{Hg}$ pressure was 0.022 Torr while the $\mathrm{H}_{2} \mathrm{O}$ and $\mathrm{C}_{4} \mathrm{H}_{6}$ pressures were 18.5 and 16.5 Torr respectively. The total flow rate (all components) was $0.12 \mathrm{liter} / \mathrm{sec}$. The ${ }^{202} \mathrm{Hg}$ abundance after one pass was $55.6 \%$, as compared with $29.6 \%{ }^{202} \mathrm{Hg}$ in the starting material.

The completion of this experiment awaits the completion of the high-power, high-repetition-rate laser. After the initial proof-of-principle experiments with the laser $F$-1otoseparation, and the investigation of several techniques for separating the aggregated photoproduct from the gas stream, we plan to exanine the effects of absorption bleaching on the photoseparation

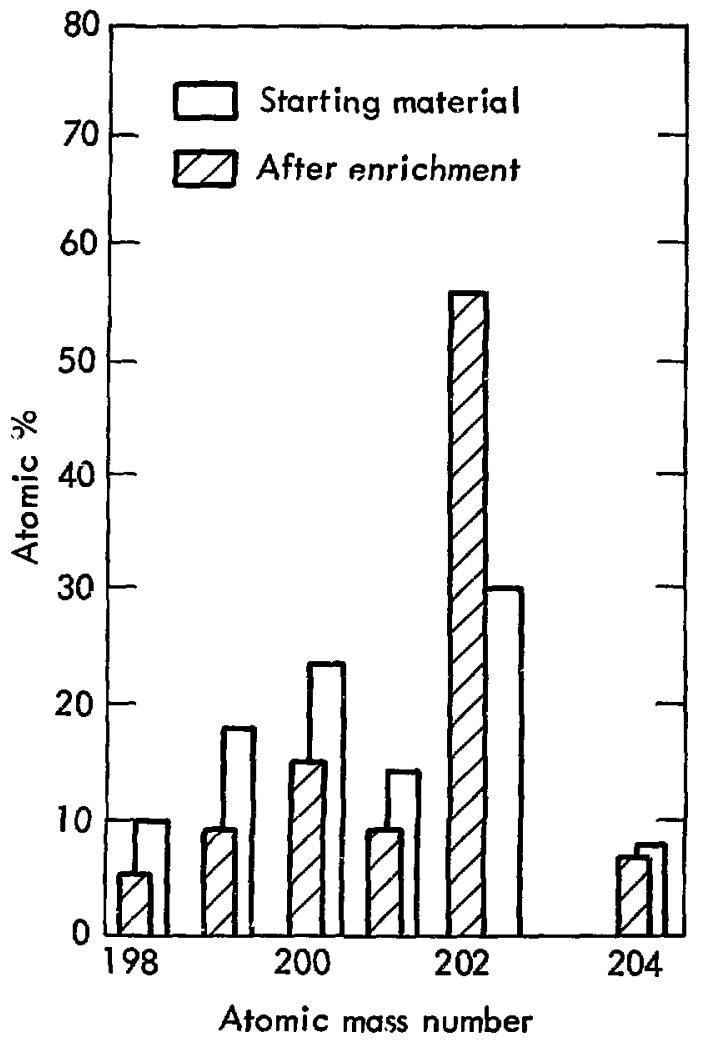

Fig. 134. Results of one pass through the apparatus of Fig 133, as determined by mass spectrometric anatyses of the starting material and the enriched material.

yield. This will be done by monitoring the rate at which the enriched product is formed as a function of laser power. Anomalies related to wall effects should be revealed, as well as effects due to excited-state quenching by stimulated emission. 


\section{PROPAGATION}

The properties of electromagnetic propagation are fundamental issues concerning both laser design and the laser/plasma interaction. This general area of research has been continued during this reporting period with specilic emphasis on the disk laser system, nonlinear ef- feets in the I-kJ laser. and the self-focusing of elliptical beams. These altivities are described below. From considerable experience it is clear that a detailed understanding of the plyysics governing propagation is essential for optimum design of a reliable high-power laser system.

\section{PROPAGATION CODE FOR DISK LASER SYSTEMS}

A new nonlinear propagation code has been developed which is based on the fast Fourier Iransform algorithm. This code solves the basic parabolic wave equation in $x, y, z$ geometry,

$2 i k \frac{\partial E}{\partial z}+\frac{\partial^{2} E}{\partial x^{2}}+\frac{\partial^{2} E}{\partial y^{2}}+\left(\gamma|E|^{2}+i k \sigma\right) E=0$.

and is capable of being applied to complicated disk systems. The solution is advanced by alternately upgrading the nonlinear phase front and then linearly propagating the altered phase front using a two. dimensional fast Fourier transform. This operation is accomplished by means of a symmetrized splitting of the transverse Laplacian and nonlinear operators in Eq. (55).

The advantage of this method is that it allows a problem to be done with a minimal number of axial space increments and with the maximum accuracy possible for a given mesh spacing. In particular, if one is dealing with an air gap or a linear medium, the integration can be carried out in a single step and without truncation error in the $z$-direction. In the transverse direction the method is equivalent to a spline method in which the basic functions are passed through all points in th: mish. thus guaranteeing the maximum possible a - allowed by the given mesh spacing.

The treatment o1 disks is based on the fact that the projection of a circle on a tilted disk is an cllipse. Let $\theta$ be the angle between the incoming propagation direction and the direction of propagation alter refrac. tion within the tilted disk. Then the intensity of the refracted beam is rescaled according to

$$
I_{r}=I_{i} \cos \theta .
$$

The mesh within the disk is transformed according to the rule

$$
\begin{aligned}
& x_{r}=x_{i}, \\
& y_{r}=y_{i} / \cos \theta .
\end{aligned}
$$

where for convenience we have assumed here that the tilt of the disk is with respect to the $y$-direction. The beam is then propagated the required distance using the nonlinear equation (55) and the conditions (56)-(58). As the beam emerges from the disk the transformation (56)-(58) is reversed and the beam is propagated in the old mesh to the new disk. The above method accounts for the main nonlinear effects of the disk system on the laser field. Individual disks can create asymmetric refractive and propagation effects not accounted for by the above treatment, but such effects will tend to be compensated for in any pair of disks.

The code is capable of treating either large-scale or small-scale self-focusing effects. To examine the latter, one simply propagates a sinall (in spatial extent) periodic window of the main beam containing an appropriate noise signal through the disk system.

Nonline ir solutions $f: r$ the fast Fourier transform code have been tested against solutions by other codes and have been found to possess greater accuracy for a smaller number of axial space increments. An example of the self-focusing of a beam with elliptical symmetry is shown in Fig. 135. The code is constructed to allow a simulation of realistic disk-amplifier systems, allowing for an arbitrary number of elements such as apertures, !enses. rotators. disk modules. and rods.

\section{NONLINEAR EFFECTS AND THE 1-kJ LASER DESIGN}

Efícient use of high-power glass laser amplificers requires an input pulse which is near its peak intensity over as much of the amplifier's aperture as is possible and which has as high an intensity as is allowed by self-focusing and damage mechanisms. In the absence of self-focusing and diffraction effects, the ideal intensity profile would be a radial step function or "flattopped" pulse. Although distortion of the pulse shape due to linear diffraction also limits the pulse shape. 2 the dominant limitation at the $5-10-20-\mathrm{GW} / \mathrm{cm}^{2}$ 

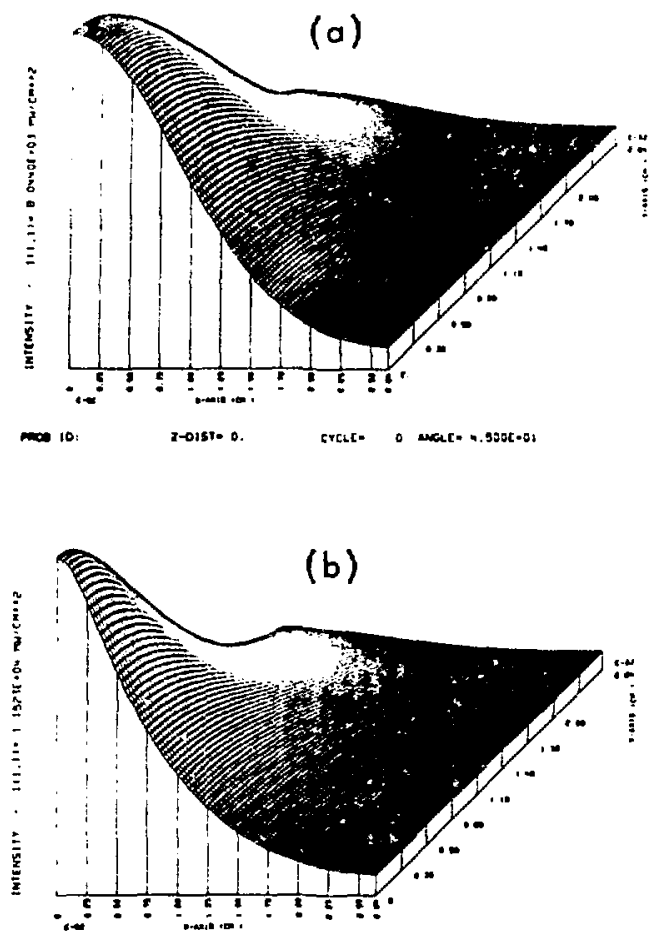

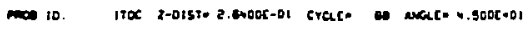

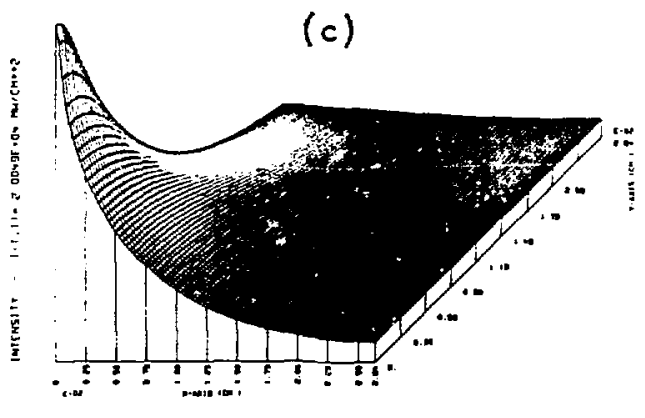

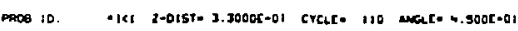

Fig. 135. Calculations of self-focusing of a laser beam with elliptical symmetry made with cole based on fast Fourier transform. Time progression is from top to bottom.

amplifier output intensities contemplated for the $1-\mathrm{kJ}$ laser appears to be pulse shape distortion due to the self-focusing nonlinearity. For pulses with the profile $I_{N}(r)=I_{0} \exp \left[-\left(r / r_{0}\right)^{N}\right]$, the safe value of $N$ based on linear diffraction was estimated to be 10 (Ref. 12), while large profile distortions occur for $N$ greater than 5 or 6 when nonlinear effects are taken into ascount.

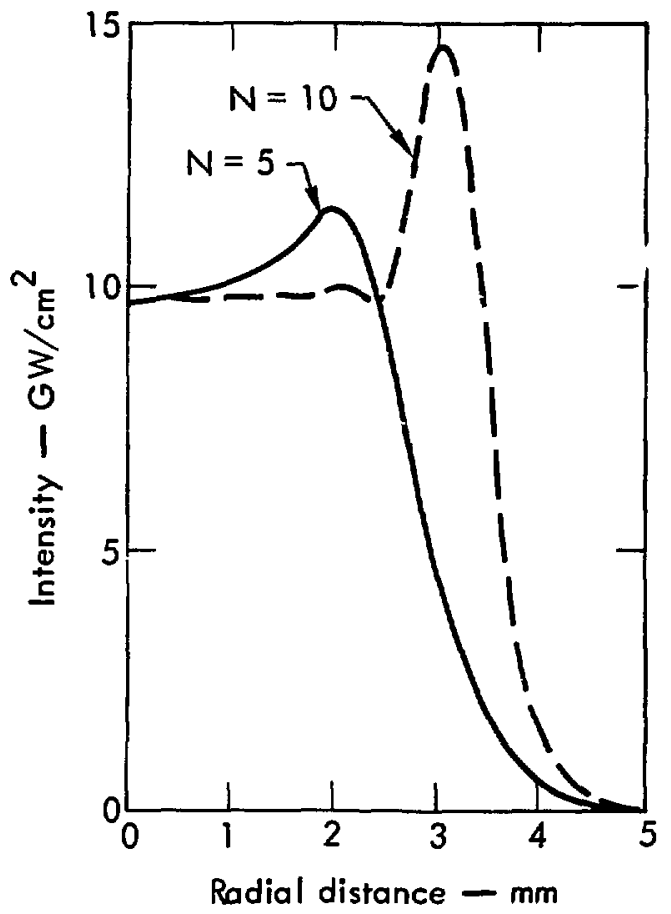

Fig. 136. Calculation of pulse shape distortion induced by self-focusing in a glass laser amplifier for two $N$-values.

The self-focusing-induced shape distortion is illustrated by the simple calculation of Fig. 136. The nonlinear coefficient $n_{2}$ causes the glass elements to act like a lens with a radially dependent focal length and to alter the constant plase surfaces of the laser pulse. As the pulse propagates across air-spaces between the glass elements a high-intensity num forms on it. For the cases shown in Fig. 136, $I_{0}$ was chosen to be $10 \mathrm{GW} / \mathrm{cm}^{2}$ (Ref. 122) and the constant $r_{0}$ was chosen so that $/(r=0.5 \mathrm{~cm})=10^{-3} I_{0}{ }^{*}$ (Hereafter this criterion will be used as the definition of the bean radius.) The pulses were numerically propagated through $\ell=10 \mathrm{~cm}$ of glass $\left(n_{2}=1.6 \times 10^{-13}\right.$ esu, $n=$ 1.556 ) and $l=117.5 \mathrm{~cm}$ of air using a cylindrically symmetric version of the parabolic wave equation ${ }^{123}$ :

$\operatorname{2in} \frac{\omega}{c} \frac{\partial E}{\partial z}+\frac{1}{r} \frac{\partial}{\partial r}\left(r \frac{\partial}{\partial r} r\right)$

$$
+\frac{\omega^{2}}{\because c^{2}} n n_{2}|E| P^{2} E=0
$$

* A fractional intensity cutoff at a lard aperture must be imposed (see Ref. 12) due to small-icale self-focusing of diffracion rings. 
where $E$ is the peak electric field in the pulse at the point $(r, z)$, and $n$ is the linear refractive index. We also ran the corresponding linear diffraction problerns $\left(n_{2}=\right.$ 0 ) and found that the diffraction contribution to the rim is less than $0.005 I_{0}$.

The parameters used to simulate the pulse propagation in the disk amplifiers of the complete $1-\mathrm{kJ}$ laser chain are summarized ${ }^{124}$ in Table 24 ; the overall net of gain over losses used here is approximately 2000. taking into account the losses of the post-D2 module isolator. Each disk module was simulated by a single slab of glass centered at the location of the disk module center and having a thickness equal to the optical path of the beam through the glass portion of the module. Input pulses with a $1.53-\mathrm{cm}$ beam radius and $I_{0}=1.02 \mathrm{GW} / \mathrm{cm}^{2}$ were propagaied through this system. (The intensity actually propagated was reduced by $1 / n=1 / 1.556$ to account for the Brewster's angle of incidence of the disks and the subsequent refraction inside the disk.) With the failure point arbitrarily defined to occur when the self-focused rim in:ensity is twice the on-axis intensity, the following results were obtained:

$$
\begin{array}{lcccccc}
N & 5 & 6 & 7 & 8 & 9 & 10 \\
\begin{array}{l}
\text { Failure point } \\
\text { (module) }
\end{array} & >D 2 & >D 2 & \begin{array}{l}
\sim D 2 \\
\text { exit }
\end{array} & <D 2 & <D 1 & <D 1
\end{array}
$$

In the above table the symbol $<$ means failure occurred before the disk module in question, while $>$ means that propagation would be safe beyond the disk module in question. The conclusion to be drawn from these computations is that a value of $N$ exceeding 5 is clearly to be avoided if the system is to survive largescale self-focusing damage.

The question of small-scale self-focusing has of course not been addressed here. It is interesting to note that there may be some conflict in using diffraction in air-spaces between disks to avoid the two kinds of seiffocusing. Large air-spaces may reduce the importance of small-scale self-focusing, but may enhance the iniportance of large-scale effects.

Table 24. Amplifier chain parameters for the $1-k J$ laser simulations ${ }^{124}$

\begin{tabular}{lcccccccc} 
Air length (m) & 0.15 & 2.5 & 4.5 & 3.0 & 6.0 & 7.0 & 9.5 & 9.6 \\
Module number & $A 1$ & $A 2$ & $B 1$ & $B 2$ & $C 1$ & $C 2$ & $D 1$ & 02 \\
Glass tength $(\mathrm{cm})$ & 11 & 11 & 17 & 17 & 18 & 18 & 29 & 29 \\
Small-signal gain $\left(\mathrm{cm}^{-1}\right)$ & 0.128 & 0.128 & 0.094 & 0.084 & 0.058 & 0.058 & 0.035 & 0.035 \\
Telescope power & - & - & 2.77 & - & 2.41 & - & 1.5 & - \\
\hline
\end{tabular}

Note: Glass parameters are $n=1 . n_{2}=1.7 \times 10^{-13}$ esu: interstage isolato: losses are $20 \%$.

\section{NONLINEAR SELF-FOCUSING OF ELLIPTICAL BEAMS}

A code which solves the parabolic wave cquation in cylindrical polar coordinates $(r, 0, z)$ is now opera. tional. Since many nonsymnetric beams of interest have fairly smooth angular distributions liat require only a coarse angular resolution, the use of polar coordinates allows bo:h flexibility and economy of zoning. The transverse discretization in this cole is based on cubic spline interpolation. In this technique a piecewise cubic polynomial is passed through the nicsh points in such a way that both first and second derivatives of the solution are continuous at these points. The solution of the resulting difference equations is implicit and is based on a symmetrized splitting of the noncommuting transverse-discretizing operators which is second-order accurate.

A theory of the self-focusing of elliptical beams based on the paraxial ray appronimation has been
Jeveloped by Marburger. ${ }^{125}$ In this theory it is assumed that the beam always retains its elliptical symunetry. However. Marburger's fornula for the selffocusing length differs from the well-known Kelly formula 123 for beams of circular symusetry by a factor of $\sqrt{2}$ in the limit when both minor and major axes are equal. Our calculations show that the Marburger formula is accurnte if reduced by the factor of $\sqrt{2}$ in qutestion. Figure 137 illustrates self-focusing of an elliptical beam in a distance of $0.7 \mathrm{~cm}$ when the Mar. burger formula would give a distance of $1.0 \mathrm{~cm}$. Note that as the self-focus is approached the elliptical symmetry is eventually lost.

The analysis of the self-focusing of circularly symmetric beams using the paraxial ray approximation has demonstrated the existence of a critied power $P_{\text {, }}$ stuch that the initial slope of the un-ixis intensity as a 


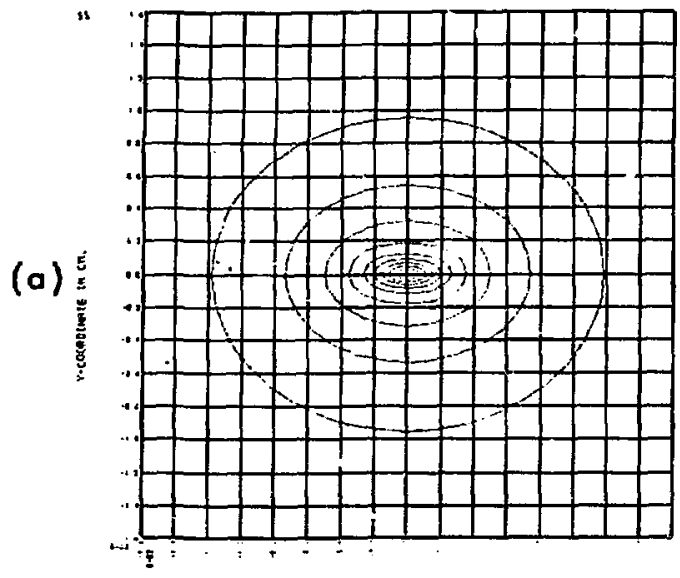

(a)

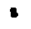

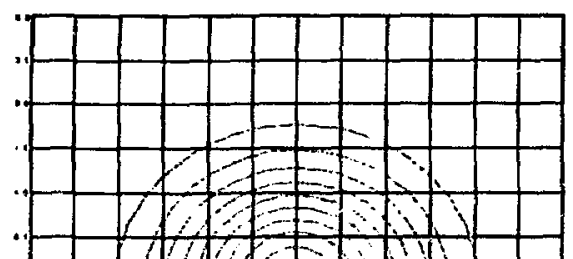

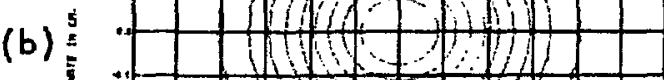
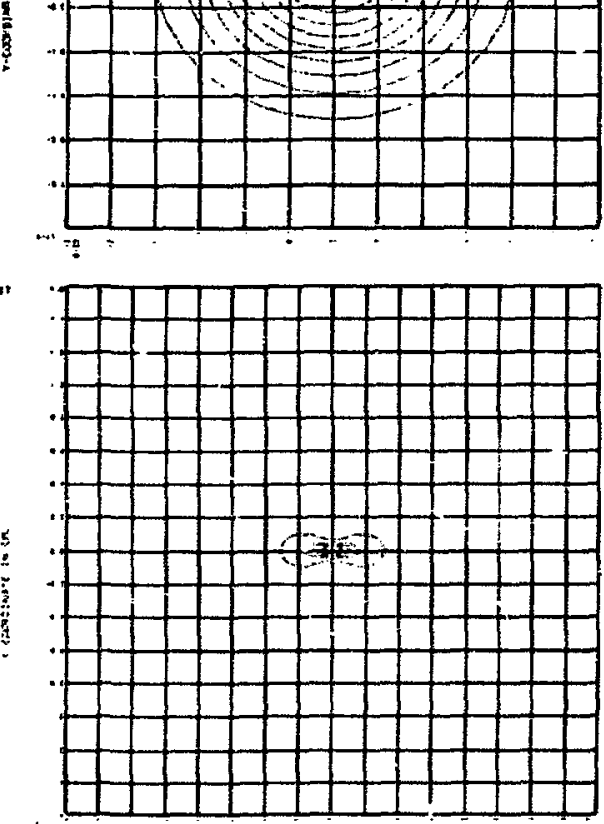

(b)

$$
\because
$$
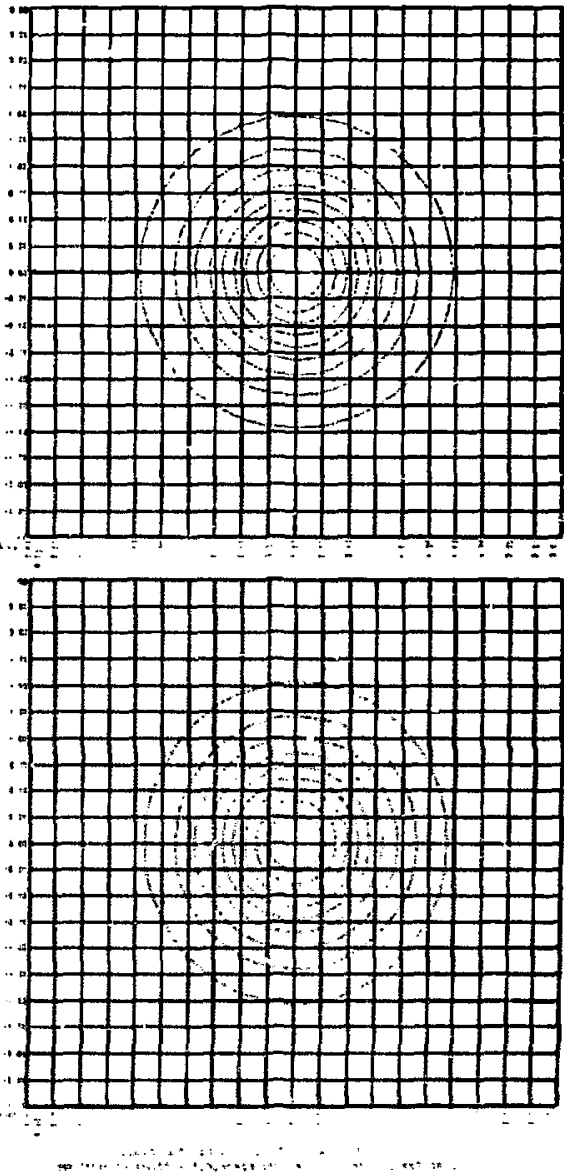

Fig. 138. Catculated self-focusing behavior of an elliptical beam with $\tilde{P}_{1} \leqslant \tilde{P}_{5} \tilde{P}_{2}$ shows that beam's crass section undergoes complicated cyclic variations in shape: (a) initial intendity contours $a t=0$, maximum intensity $2.3 \times 10^{7} \mathrm{~W} / \mathrm{cm}^{2}:$ (b) $a t==$ $14 \mathrm{~cm}$, maxinum intensity $3.9 \times 10^{\circ} \mathrm{W} / \mathrm{cm}^{\circ}$; (c) at : = $26 \mathrm{~cm}$, maximum intensily is $3.9 \times 10^{7}$ $W / \mathrm{cm}^{2}$ and major and minor axes of beam's efliptical eross section have reversed. 
function of axial displacement vanishes, and :. critical power $P_{2}=P_{1} / 0.273$ corresponding to a self-focus at $\infty$. If the beam power satisfies the inequality $P_{1}<P<$ $P_{2}$, the on-axis intensity increases to a maximum and then decreases as if the beam were diffracting.

We have found in the case of elliptical-cross-section beams a complete analog to $P_{\mathcal{L}}$ and $P_{2}$, namely a complete $\widetilde{P}_{2}=\widetilde{P}_{1} / 0.273$, where $\widetilde{P}_{1}$ is predicted by Marburger's theory. Thus, an elliptical beam with power $\widetilde{P}$ satisfying the inequality $\widetilde{P}_{1}<\widetilde{P}<\widetilde{P}_{2}$ will initially focus through a maximum on-axis intensity before it diffracts away. However, the in:ensity contours undergo a complicated cycling behavior. Figure 138 illustrates the behavior of an elliptical beam with power $\widetilde{P}_{2}$. At $z=0 \mathrm{~cm}$ (Fig. 138a) the contours are ellipses with eccentricity 0.5 and the on-axis intensity is 23.8 $\mathrm{MW} / \mathrm{cm}^{2}$. The contours evolve into circles at about $z=$ $14 \mathrm{~cm}$ (Fig. 138b) and the on-axis intensity increases to $39.7 \mathrm{MW} / \mathrm{cm}^{2}$. At $z=26 \mathrm{~cm}$ (Fig. 138c) the elliptical axes are interchanged. The on-axis intensity of $39.2 \mathrm{MW} / \mathrm{cm}^{2}$ is considerably larger than the initial value, and the axes of this ellipse are not as large as those in Fig. 138a. The subsequent evolution of the bean indicates a "cycling" of the mean radius of the contours. and the largest on-axis intensities generally correspond to circular contours except for a certain amount of hysteresis. This cycling is more intricate than simple harmonic motion. The amplitude of oscillation of the mean radius is about $25 \%$ of the initial mean radius up to an axial distance of $200 \mathrm{~cm}$. 


\section{GAS LASER RESEARCH AND TECHNOLOGY}

To produce a net energy gain from thermonuclear fusion of a small, inertially confined DT pellet requires a laser energy of some $10^{4}-10^{6} \mathrm{~J}$ delivered to the pellet within $10^{-9}-10^{-10} \mathrm{sec}$ with an efficiency approaching $10 \%$. Such a laser system is not currently in existence. New lasers are needed, and a substantial fraction of the effort has been in this direction, particularly with regard to the recently realized bound-free systems. It is the main goal of the Gas Laser Research and Technology effort to develop both the detailed concepts and the technologies required to produce a large and sufficiently scalable system applicable in the laser fusion context. This encompasses an enormots range of issues including spectroscopic studies, the physics of atomic collisional processes, electromagnetic propagation, the development of the pulsed power technology apropos to laser requirements, and materials studies pertinent to both electron and optical windows.
In order to effectively screen potential candidate systems, it is useful to refer to criteria established by both the pellet requirements and the considerations governing scalability and operation of laser systems. Table 25 presents a summary of system requirements and limitations based on our current knowledge of the relevant criteria. It is interesting to observe that the implications follow quite readily and provide considerable insight into the physical properties of the desired laser medium.

The discussion that follows will report on the combined activities of the Gas Laser Research and Technology areas. Prominent in this analysis are the topics of (1) bound-free systems, (2) pulsed power development and electron transport studies, and (3) theoretical studies. These are discussed in detail below.

\section{BOUND-FREE SYSTEMS}

During the perisd covered by this report our data base concerning the interesting $\mathrm{Xe}_{2}^{*}$ and $\mathrm{Kr}_{2}^{*}$ molecular bound-free ultraviolet systems has been considerably expanded. In addition to the behavior of the singlecomponent xenon and krypton sytems, a detailed experimental analysis of rare-gas mixtures has heen made. In this latter case, there is strong evidence for the existence of rapid and efficient electronic energy transfer processes among the participating atoms and molecules. ${ }^{126}$ Since analogous processes are common to a large variety of systems, we believe that energy transfer mechanisms of tlis genre represent an important class of phenomena directly applicable to the laser fusion needs.

The experimental apparatus used in the rare-gas mixture studies is illustrated in Fig. 139. Under typical experimental conditions, a high-pressure gas cell with internal mirrors and mode-limiting optical apertures was altached to a Febetron 705 relativisticelectron-beam generator which emits a 40-nsec pulse in a $10-\mathrm{kA}$ beam with a noninal cnergy of $1.5 \mathrm{MeV}$ over a 2-cm-diam aperture. The electron-beam current density penetrating the diapliragm and incident on the gas was $\sim 300 \mathrm{~A} / \mathrm{cm}^{2}$ as measured by a calibrated Faraday cup. The optical cavity consisted of two 1-in-radius-ofcurvature mirrors separated by $5 \mathrm{~cm}$. The mirrors were fabricated from highly polished $\mathrm{MgF}_{2}$ substrates which were Al-coated and $\mathrm{MgF}_{2}$-overcoated. The reflectance and transmittance of the mirrors were measured to be 80 and $8 \%$, respectively. Thu $2 \cdot \mathrm{mm}$-diam intracavity apertures limited the field of view of the diagnostic systems essentially to the volume contained in the cylinder defined by the apertures. The spectral composition of the radiation along the optical axis was detected by a $75-\mathrm{cm}$ Seya-Namioka spectrograph. The temporal characteristics were observed using an ITT 4115 photodiode with a 2-nsec rise time. Spatial propagotion stud: $s$ were performed by replacing the photodiode with SWR film mounted on a variablelength line-of-sight pipe, and attenuation in this pipe was accomf:is .ed with calibrated sapphire attenuators. The spontaneolis radiation emitted perpendicular to the cavity axis was monitored using an $82-\mathrm{cm}$ CzernyTurner spectroneter with a typical bandpass of $5 \AA$.

The observed behavior of the stimulated emission of the $\mathrm{Xe}_{2}^{*}$ and $\mathrm{Kr}_{2}^{*}$ systens as a function of gas density is shown in Figs. 140 and 141. Both exhibit a maximum output at an intermediate density; that is, at sufficiently high pressure the stimulated output rapidly deteriorates. In contrast to this behavior, the spontaneous emission intensities do not decrease at the ligher densities. These spontaneous emission data are illustrated in Figs. 142 and 143. These results strongly indicate the presence of an optical loss associated with 
Table 25. Criteria for evaluating new laser-systein candidates for use in laser fusion

Reason for the requirements

Implications for the laser

A. Requirements for pellet coupling:

1. Short wavelength $(\leqslant 6000 \AA)$.

2. Pulse output of $10^{6} \mathrm{~J}$ in $10^{-10}$ sec.
To provide optimal coupling of energy to the thermonuclear pellet.

To establish conditions for efficient thermonuclear burn.
Laser should operate on an electronic transition.

Entire pulse energy should be stored in laser upper level, since pumping or relaxation from other levels is insignificant in $10^{-10} \mathrm{sec}$.
B. Requirements for practical applications:

1. High efficiency $(\geqslant 5 \%)$.

2. Pulse repetition rate about 100 per sec.

3. Low cost, low maintenance.

\section{Same as B.1.}

4. Smallest possibie size. consistent with other require-

4. Inversion lifetime (energy storage time) as long as possible and $>10^{-6}$ sec.
2. Energy flux in pulse $<1-10 \mathrm{~J} / \mathrm{cm}^{2}$.

3. Lowest small-signal gain ments (see C.2).

C. Requirements due to limitations imposed by properties of matter:

1. Wavelength $\gtrsim 3000 \AA$.
Needed for any practical applications such as commercjal power generation.

Same as B.1 above.

To reduce cost of optical and other components: also, because of difficulty of transferring large laser pump energies over long distances in very short times.
Energy should be transferret selectively from electrical storage to laser upper level. No losses such as absorption of laser radiation from this level to higher level. Efficient energy extraction (saturated amplifier).

Gas laser with convective cooling; large solid-state lasers cannot be cooled quickly.

Infrequent need to replace expensive components; tends to favor gas lasers. High energy storage density is desirable. Collisions of excited states should not lead to deexcitation.
At shor ter wavelengths there is severe two-photon absorption in all optical materials at the power levels $\left(10^{1}-10^{12}\right.$ $W / \mathrm{cm}^{2}$ ) the laser will generate.

To prevent damage to optical components. Fluxes in solid state lasers where the beam travels long distances in high density material are limited to near $1 \mathrm{~J} / \mathrm{cm}^{2}$ to avoid self-focusing and damage. Low-density gas lasers will pass fluxes nearer the limit at $\sim 10 \mathrm{~J} / \mathrm{cm}^{2}$ imposed by window and optics surface damage.

To suppress parasitic oscillations and superfluorescence which deplete the energy stored in the laser.
Because of engineering complexity required to transfer $\sim 10^{7} \mathrm{~J}$ of energy into the laser medium.
$\mathrm{Xe}_{2}$ and $\mathrm{Kr}_{2}^{*}$, though potentially efficient, are not suitable.

For efficient energy extraction (see B.1 above), laser must saturate at fluxes $<1-10 \mathrm{~J} / \mathrm{cm}^{2}$. Since saturation flux $\approx h \nu / \sigma$, this implies that stimulated-emission cross section o for a nominal 5000- $\AA$ laser should be $\geqslant 10^{-19}$ to $10^{-20} \mathrm{~cm}^{2}$.

Stimulated-emission cross section as small as possible consistent with C.2 above $\left(-10^{-20} \mathrm{~cm}^{2}\right.$ for $\left.5000 \AA\right)$. This implies that laser transition will be a "forbidden" optical transition, since allowed transitions typically have $\sigma \approx 10^{-16} \mathrm{~cm}^{2}$.

Laser npper level resistant to deactivation and free of other loss mechanisms. 


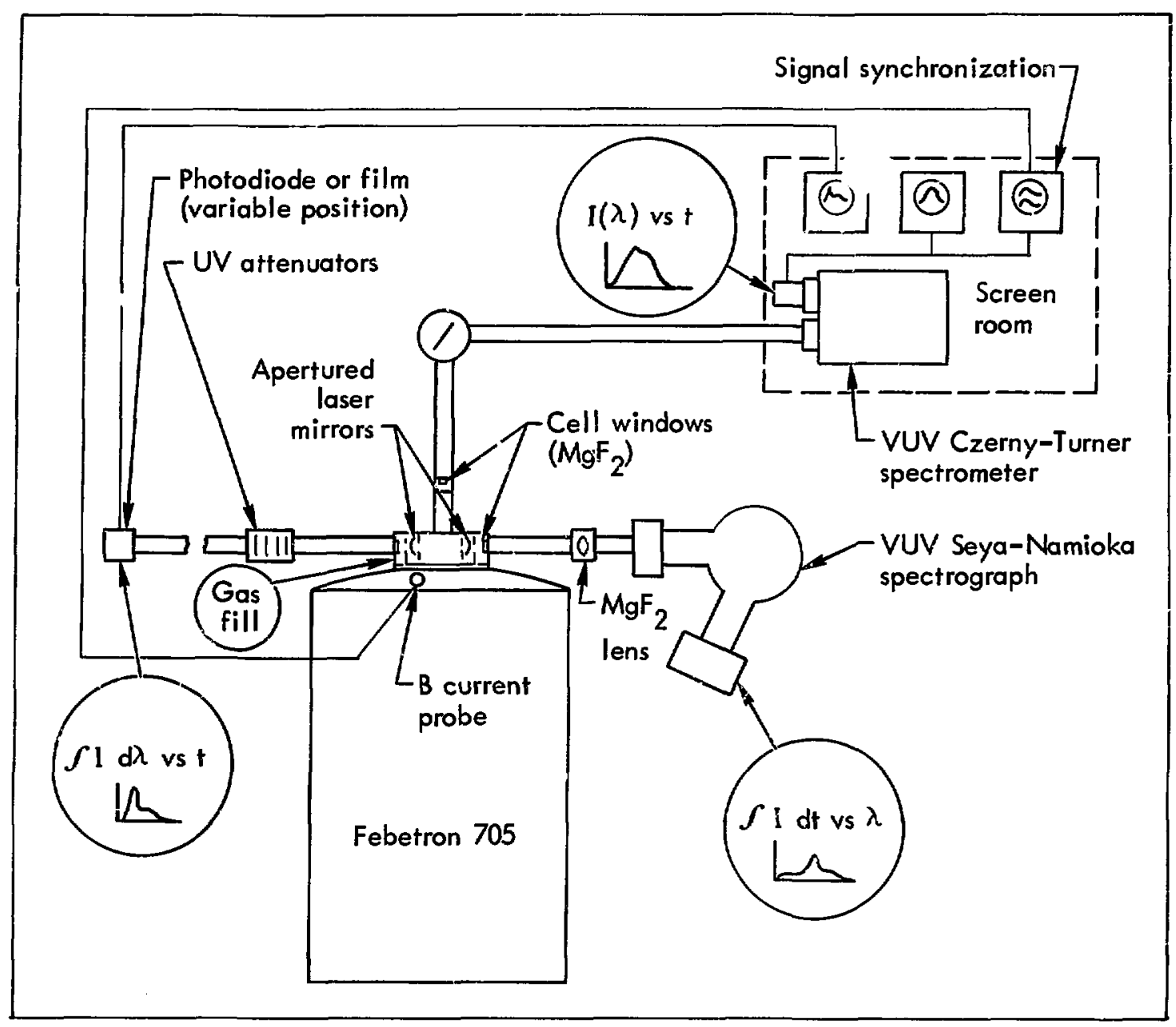

Fig. 139. Schematic of the experimental apparatus showing the three diagnostic systems: Seya-Namioka spectrograph, photodiode, and Czerny-Turner spectroneter viewing the sidelight.

the gas density, causing a reduction in the medium's gain. This clearly imposes a limit on the density scaling of these systems. Such scaline mndirinns, as well as other factors (see Table 25), make it dubious that these laser systems are directly appropriate to the fusion problem.

The data observed with mixed gas media (e.g., $\mathrm{Ar} / \mathrm{Xe}$ ) are qualitatively different. Figure 144 shows the results for the stimulated output in argon/xenon mixtures. Not only does the oscil ation continue to occur at considerably higher total pressures, but also the stimulated energy output of the system was observed to $b$. . iter than the maximum attainable with pure xenon. This effect is attributable to the combined action of the energy transfer process 127

$$
\mathrm{Ar}_{2}^{*}+\mathrm{Xe} \rightarrow \mathrm{Ar}+\mathrm{Ar}+\mathrm{Xe}^{*}
$$

and collisional coupling ${ }^{128}$ of the ${ }^{1} \Sigma_{u}^{+}$and ${ }^{3} \Sigma_{u}^{+}$manifolds of the $\mathrm{Xe}_{2}^{*}$ molecule. 
Source technology efferts have concentrated on the development of electron beam sources specifically tailored for laser excitation and the associated studies required to understand electron beam transport to and coupling with the medium.

All gas laser systems under present consideration require electron-beam pump sources with pulse widths ranging from $30 \mathrm{nsec}$ to $30 \mu \mathrm{sec}$. With the aid of industry, we have interactively developed two sources in this pulsewidth regime which are specifically designed for iaser research and development. Table 26 summarizes the source characteristics.

MEG I, shown in Fig. 145, arrived at LLL at the beginning of FY 74. This source produces a trapezoidal pulse with. $<5$ nsec jitter. It is configured to be a versatile tool for a wide range of laser studies.

A cold electron beam (one with mininal transverse encrgy) is required because it propagates more efficiently, passes more easily through a foil and support structure into the laser cell, and deposits more uniformly and over a greater range. We have utilized our previously reported cathode technology to provide a cold, uniform e-beam with $<10 \%$ spatial variation and 95\% reproducibility.

The EB-300 gun, shown in Fig. 146, has recently been installed. It has the flexibility of operating in the

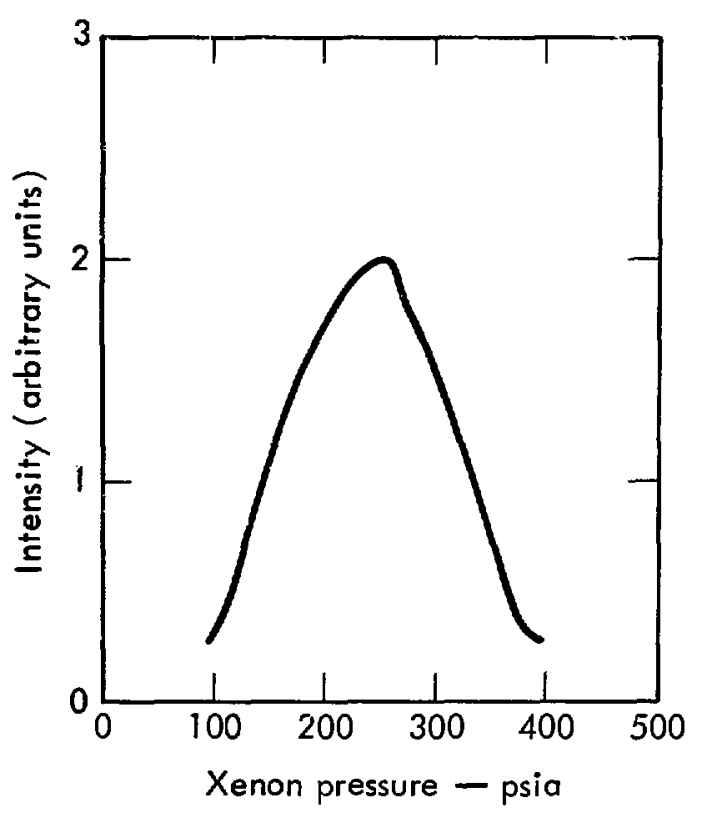

Fig. 140. Stimulated emission of $\mathrm{Xe}_{2}^{*}$ as a function of gas pressure. direct pump mode, as in MEG I, or in the sustainer mode, wherein an additional voltage is applied across the laser cell.

In anticipation of this gun, we characterized blade cathodes, the blades providing the field enhancement necessary for cathode turn-on. The ECUN code, developed by Hermerfeld at SLAC. was utilized to determine electron trajectories and current densities at the anode plane. These calculations were experimentally correlated with our high-resolution diagnostics with excellent agreement. Typical resulting data are shown in Fig. 147 for several interblade spacings and sinall $(\sim 1-\mathrm{cm})$ gap widths. We learned that although one can obtain a uniform beam with widely spaced blades, closely spaced blades are required to generate the desired cold e-beam. These results will be extended to the EB-300 and wider gap spacings.

We have developed some novel e-beam injection systems applicable to MEG I to transmit the beam from the low-pressure drift regime into the high-

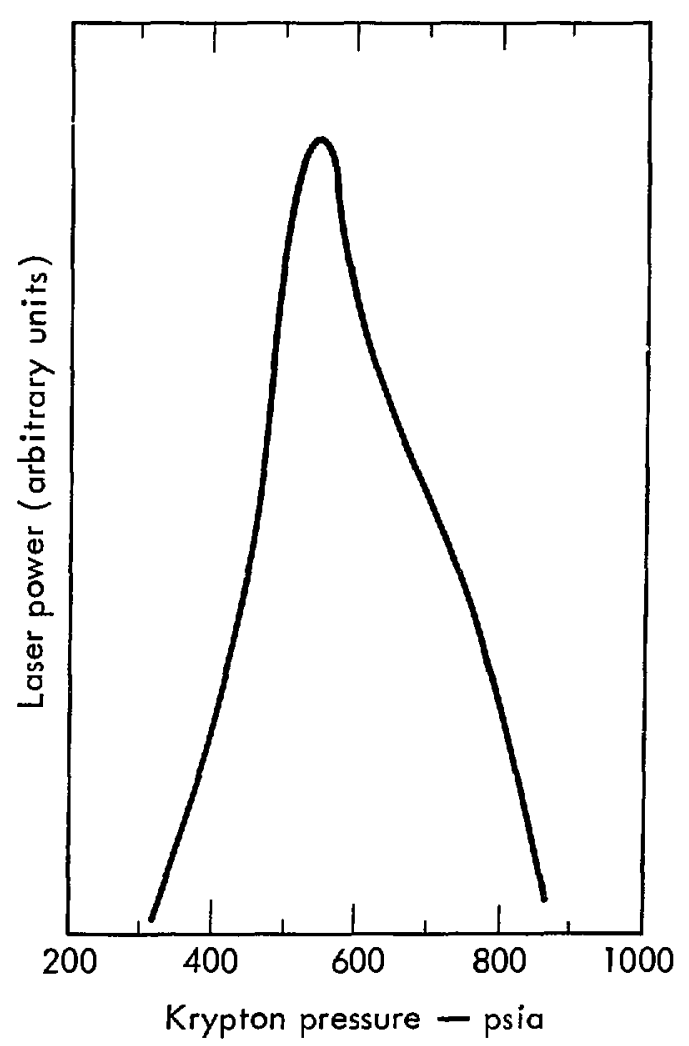

Fig. 141. Stimulated emission of $\mathrm{Kr}_{2}^{*}$ as a function of gas pressure. 


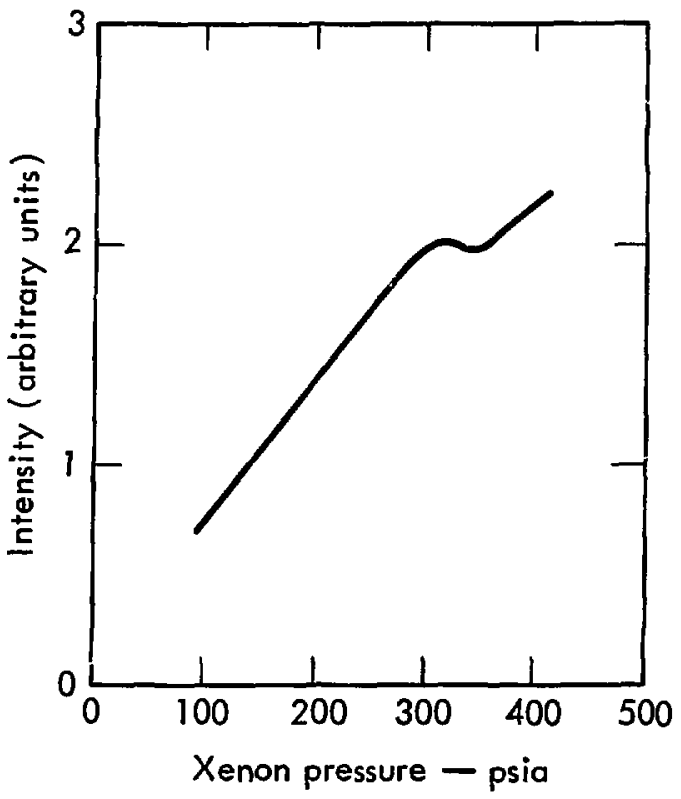

Fig. 142. Spontaneous emission of $\mathrm{Xe}_{2}$ as a function of gas pressure.

pressure laser cell. These are shown in Fig. 148. In the past, a standard "hibachi configuration" has been used to support the ihin electron-transmission window. One improvement has been to taper the support segments to better guide the electrons into the cell. A second modification has been to use a single slit $\sim 0.5 \mathrm{~cm}$ wide into the cell. This supports the window and permits all

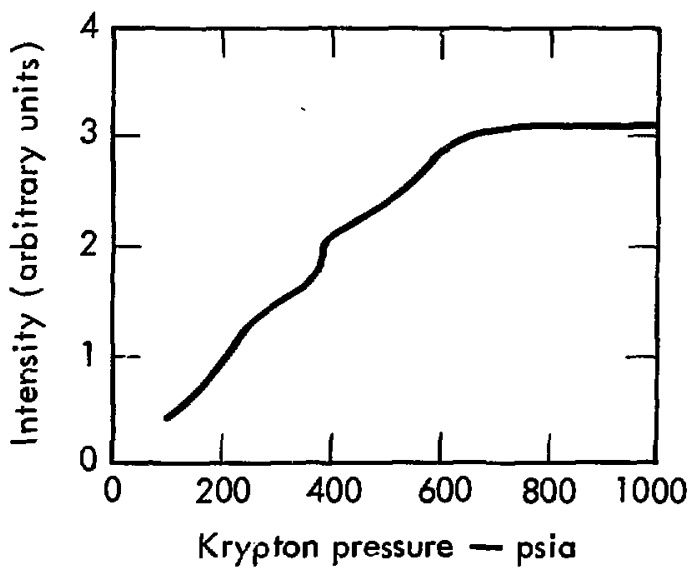

Fig. 143. Spontaneous emission of $\mathrm{Kr}_{2}^{*}$ as a function of gas pressure.

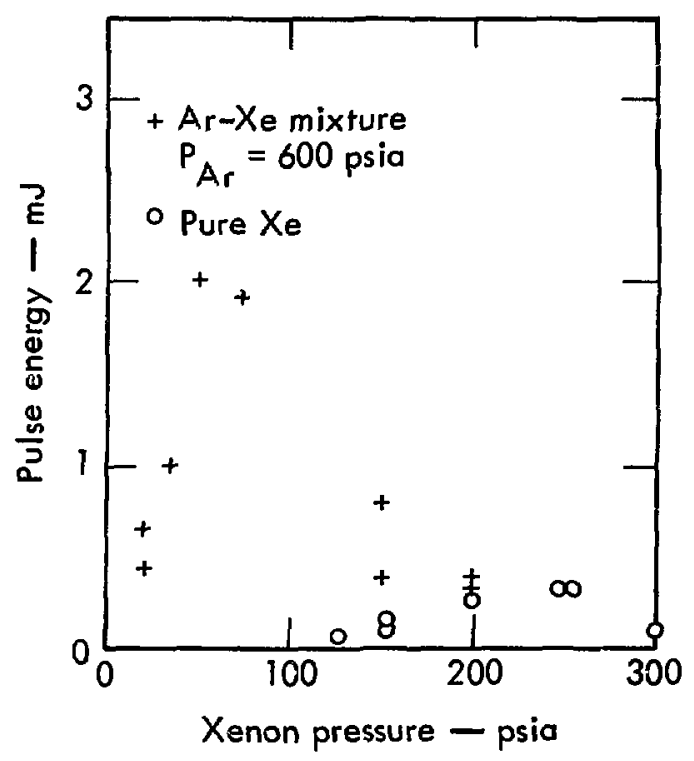

Fig. 144. Stimulated emission of a mixture of Ar and Xe as a function of pressure.

of the e-beam to be injected. Subsequent electron scattering in the high pressure gas fills the optical cavity.

Previously, to reduce the injected current density, one changed the cathode-anode gap. This required a change in source switch pressures and voltages. We have since evolved simple attenuators which attenuate the e-beam with no change in source parameters. As is also shown in Fig. 148, these attenuators are variable carbon apertures contiguous to the laser cell.

Evaluation of electron energy deposition in the high pressure gas of the laser cell has required the use of SANDYL, an electron-photon Monte Carlo code originally developed by Sandia Laboratories, Livermore, for weapon effects analysis. We have been the first to employ such a code for laser applications. It is required for cases of interest in which cell dimensions are less than the mean range of electrons. In this case

Table 26. Characteristics of the MEG I and EB-300 electron beam sources developed for laser excitation

Source

Voltage (MeV)

MEG 1

Current density $\left(A / \mathrm{cm}^{2}\right)$

Sustainer voltage $(\mathrm{kV})$

5000

None

Beam cross section $(\mathrm{cm})$
EB-300

0.3

50

100

$10 \times 100$ 


\section{MeV-100 KA MEG EIECTRON BEAM GENERATOR}

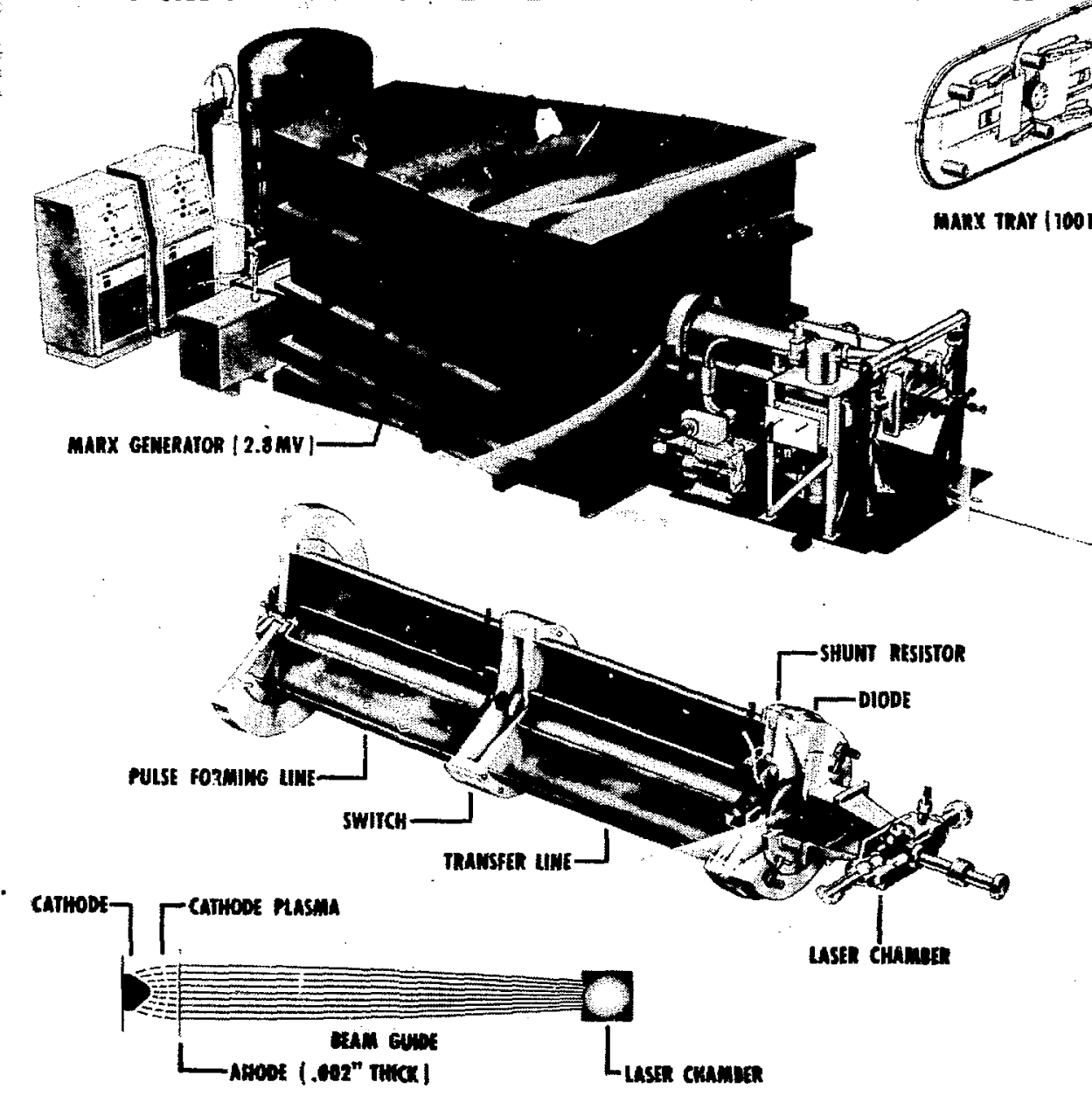

Fig. 145. The MEG I electron beam generator is designed to transversely pump a gas laser cavity.

multiple scattering of electrons is important, particularly with higher $Z$ gases such as xenon. Failure to use the code, or use of only a one-dimensional code, leads to errors of approximately a factor of 4 in the value of deposited energy. Calorimetry, commonly used with e-beam machines, overestimates deposition by a factor of 4 in the high-pressure laser gas unless it is corrected with the aid of the three-dimensional analysis. These are important considerations in the evaluation of laser efficiency, one of the most critical requirements for laser fusion devices.

The importance of using this calculation is illustrated by the case of a $1-\mathrm{MeV}$ pencil beam of electrons axially incident on a $2-\mathrm{cm}$-diam cylindrical cell filled with $10 \mathrm{~atm}$ of Xe. Twenty-five percent of the energy is backscattered out of the cell, $55 \%$ is scattered to the walls, and only $20 \%$ of the incident energy remains in the cell. With a $2-\mathrm{cm}$-wide beam into the same $2-\mathrm{cm}$ cell, the losses are even greater.

Since electron beams and the associated source technology are becoming increasingly important to the development of new gas lasers, we have developed a strong in-house capability in this area. We are working interactively with inclustry, thereby expanding our capabilities to develop these sources and to advance this important technology.

Finally, we are currently extending the technology to acquire more compact sources with longer gain lengths. Toward this end, industry will provide some 


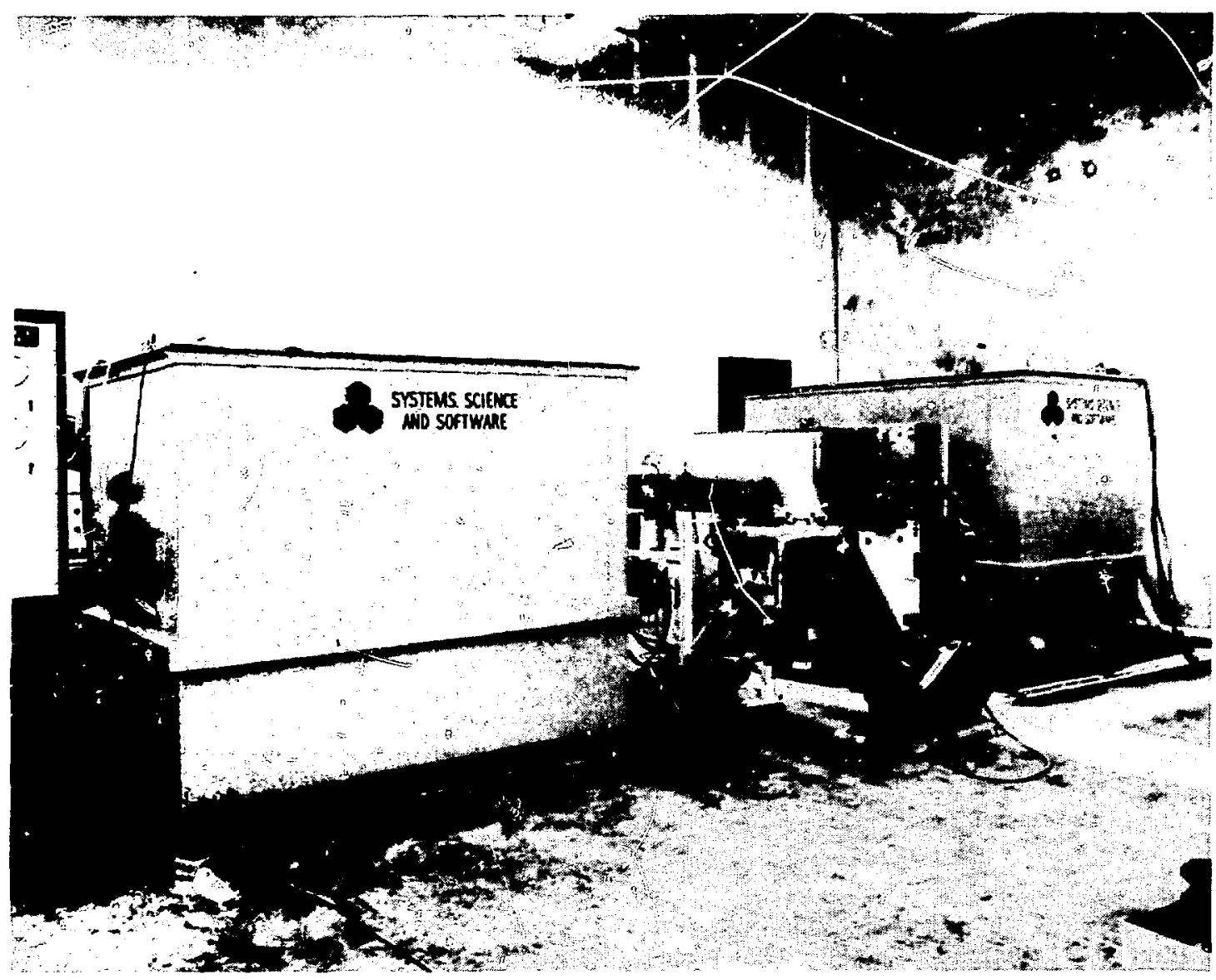

Fig. 146. The EB-300 electron beam generator is a flexible souzce that can produce a $300-k e V$ electron beam $S$ by $100 \mathrm{~cm}$ in cross section and with intensity of $10 \mathrm{~A} / \mathrm{cm}^{2}$. This generator is used cither in the direct ebeam mode or 28 an auxiliary sustainer.

sources. We are concentrating on the technology of switching, PFL dielectrics, and e-beam generation as being the most essential areas in this development. Thus we are developing novel, low-jitter pressurized gas switches. We are beginning to investigate liquid dielectrics, aiming at obtaining a more optimum dielectric than the water or oil currently used. We are also investigating the manipulaticis of e-beams to fan out from a source of given dimensions to a wider laser cavity. Such drifting e-beams would allow greater flexibility in the laser experiment and provide lasersource isolation.

\section{THEORETICAL STUDIES}

Theoretical studies have concentrated in two areas: (1) ab initio calculations of molecular properties, and (2) kinetic modeling of laser systems. The former provides us with independent data that bears on the parameters used in the latter.
The molecular calculations have centered on the properties of the argon dimer, since this system is light enough to be accurately studied with existing com. putational facilities, represents a prototype of the heavier $\mathrm{Xe}_{2}^{*}$ and $\mathrm{Kr}_{2}^{*}$ systems, and can be compared 

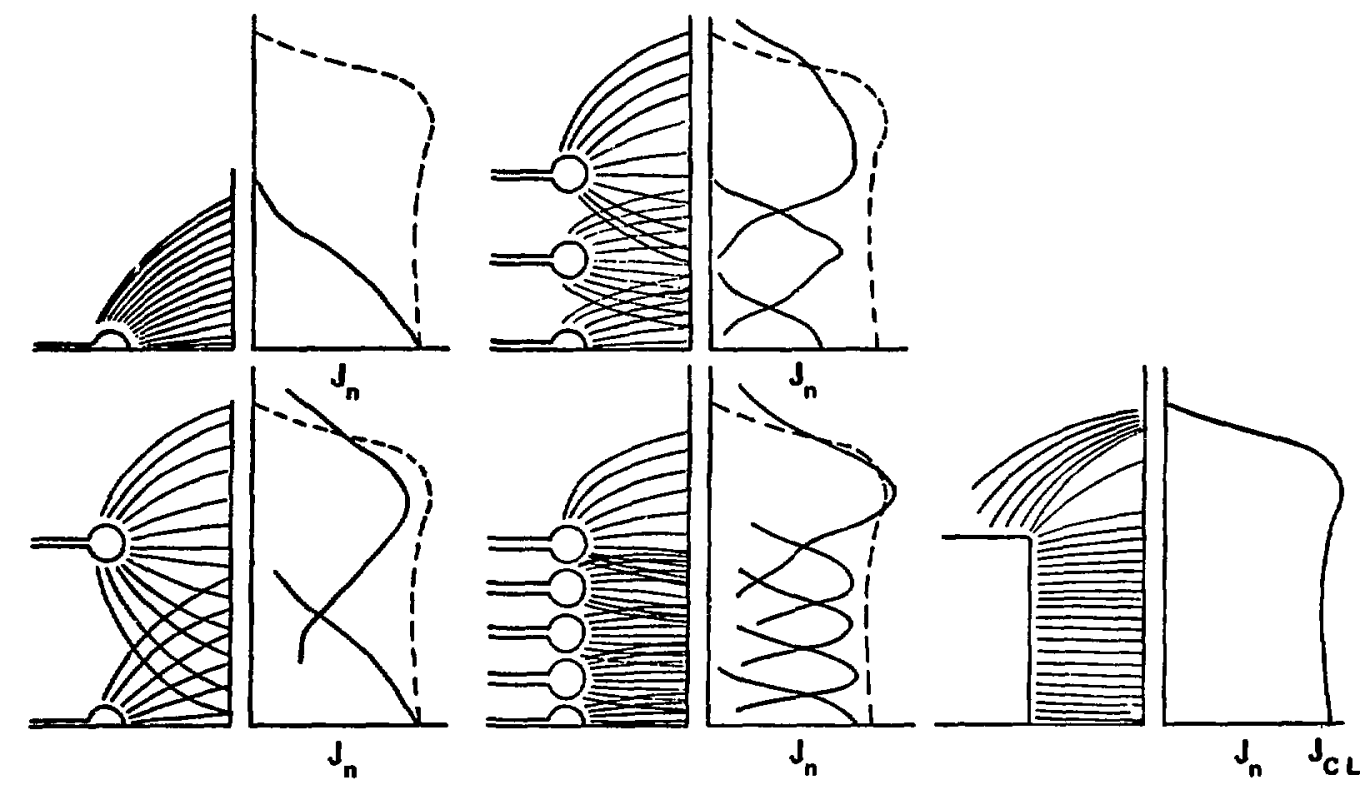

Fig. 147. Electron trajectories and current densities for blade cathodes, ranging from a single blade cathode to a continuum. Cathode plasma expanding about the blade edge is accounted for.

with experiment. In comparison with experiment excellent agreement is found for the $1300-\AA$ continuum originating from $\mathrm{Ar}_{2}^{*}$. This has enabled us to establish the values of $r_{e}$ (equilibrium internuclear separation), $\omega_{e}$ (vibrational quantum of the excimer), and $D^{0}$ (excimer binding energy). Extension of this work by analogy has provided useful estimates of the corresponding quantities for $\mathrm{Kr}_{2}^{*}$ and $\mathrm{Xe}_{2}^{*}$, which comprise essential input data for the modeling of these laser systems.

The modeling efforts have dealt with two major arcas: the rare-gas excimer systems, and photolytically driven systems generating excited atoms with $n p^{2}$ and $n p^{4}$ configurations.

In the modeling of the rare-gas media it has been found that the spin-changing collisional processes

$$
\mathrm{Xe}_{2}\left({ }^{1} \Sigma_{u}^{+}\right)+\mathrm{Xe}=\mathrm{Xe}_{2}\left({ }^{3} \Sigma_{u}^{+}\right)+\mathrm{Xe}
$$

and

$$
X e_{2}\left({ }^{l} \Sigma_{u}^{+}\right)+\mathrm{e}^{-}-X e_{2}\left({ }^{3} \Sigma_{u}^{+}\right)+\mathrm{e}^{-}
$$

are essential for a quantitative understanding of these systems. The inclusion of these considerations has produced a refinement in our previously constructed analysis. 129
Examination of Table 25 and comparison of the properties of atomic systems with $n p^{2}$ and $n p^{4}$ electronic configurations suggests their general suitability. Recalling some of the requirements we observe that:

1) The stimulated-emission cross section must be small enough that superfluorescence and other parasitic processes do not depopulate the upper state in a large amplifier, yet large enough that the saturation flux for a subnanosecond pulse is less than the optical breakdown threshold of the active medium. This condition requires that the stimulated-emission cross section be near $10^{-20} \mathrm{~cm}^{2}$.

2) The wavelength of the laser should be short, yet not so short that two-photon absorption to the intrinsic conduction band will be possible in con. venient optical materials.

3) Storage of energy at high excited-state densities (a few hundred or thousand joules per liter) is desirable to minimize the bulk of the laser.

In this context the 5577. $\AA$ auroral line of atomic oxygen is an interesting candidate ${ }^{130}$ for such a laser. (Other systems such as sulfur also seem applicable.) The energy level structure of oxygen is shown in Fig. 149. The stimulated-emission cross section for this transition, assuming a $300^{\circ} \mathrm{K}$ Doppler-i roadened line, is $\sim 9 \times 10^{-20} \mathrm{~cm}^{2}$. This leads to a saturation energy 


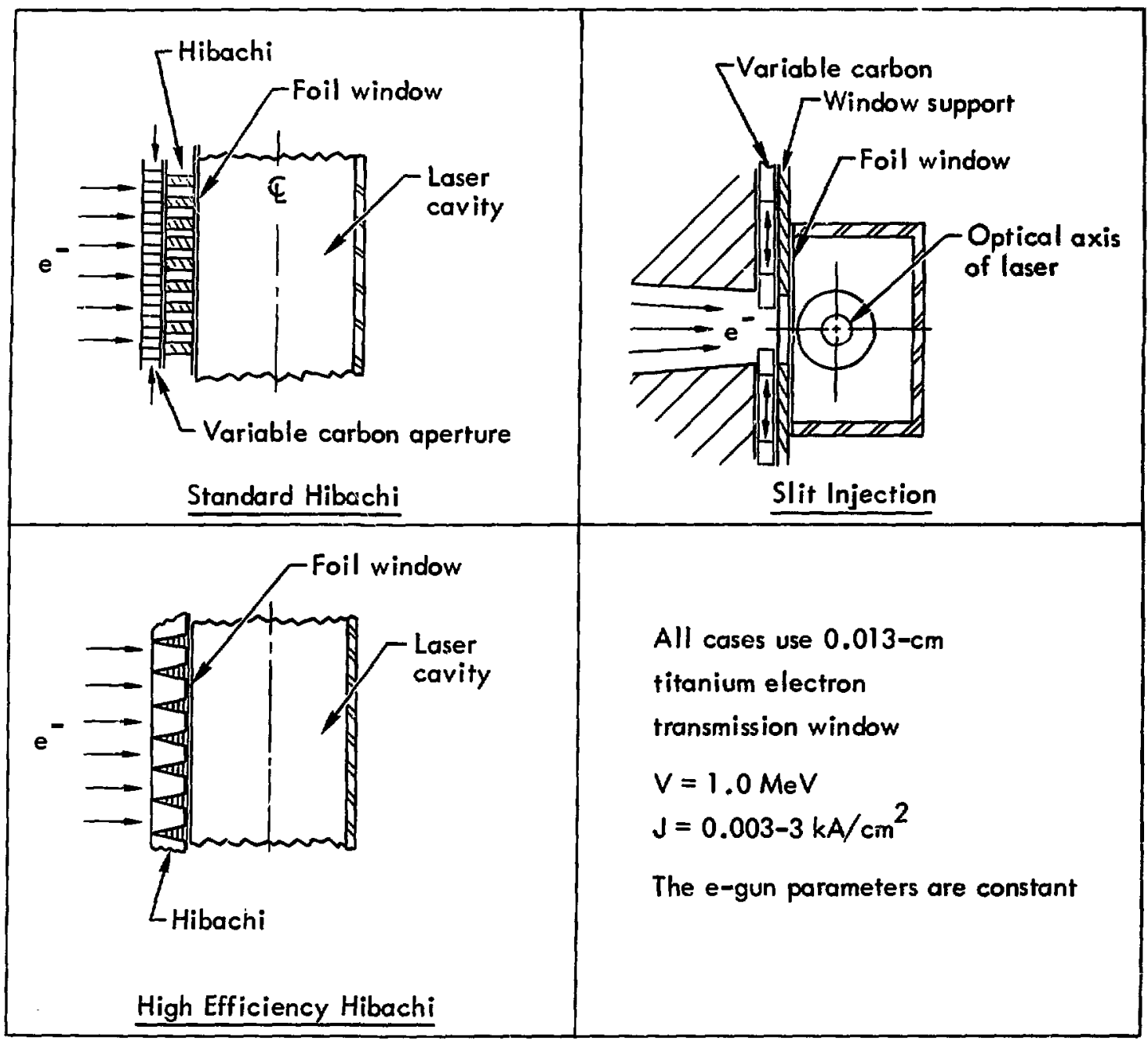

Fig. 148. Several e-beam injection systems applicable to MEG I, using various window support and current control schemes

flux of $4 \mathrm{~J} / \mathrm{cm}^{2}$, a value within the limits of technology for mirrors and windows for a subnanosecond pulse. The oxygen ${ }^{1} S_{0}$ state also has a low probability of deactivation on collision with a variety of collision partners, including the important case of a second excited $O\left({ }^{1} S_{0}\right)$ atom, leading to a prediction of highdensity energy storage on a microsecond time scale or longer. Furthermore, since this energy is stored in a single atomic state, it is available for efficient picosecond pulse extraction.

It remains to be demonstrated that these states can be efficiently generated in sufficient density. Preliminary modeling studies ${ }^{130}$ indicate that this is feasible by both photolytic and direct collisional energy transfer with triatomic donors. Experiments are currently being prepared to test these concepts. 


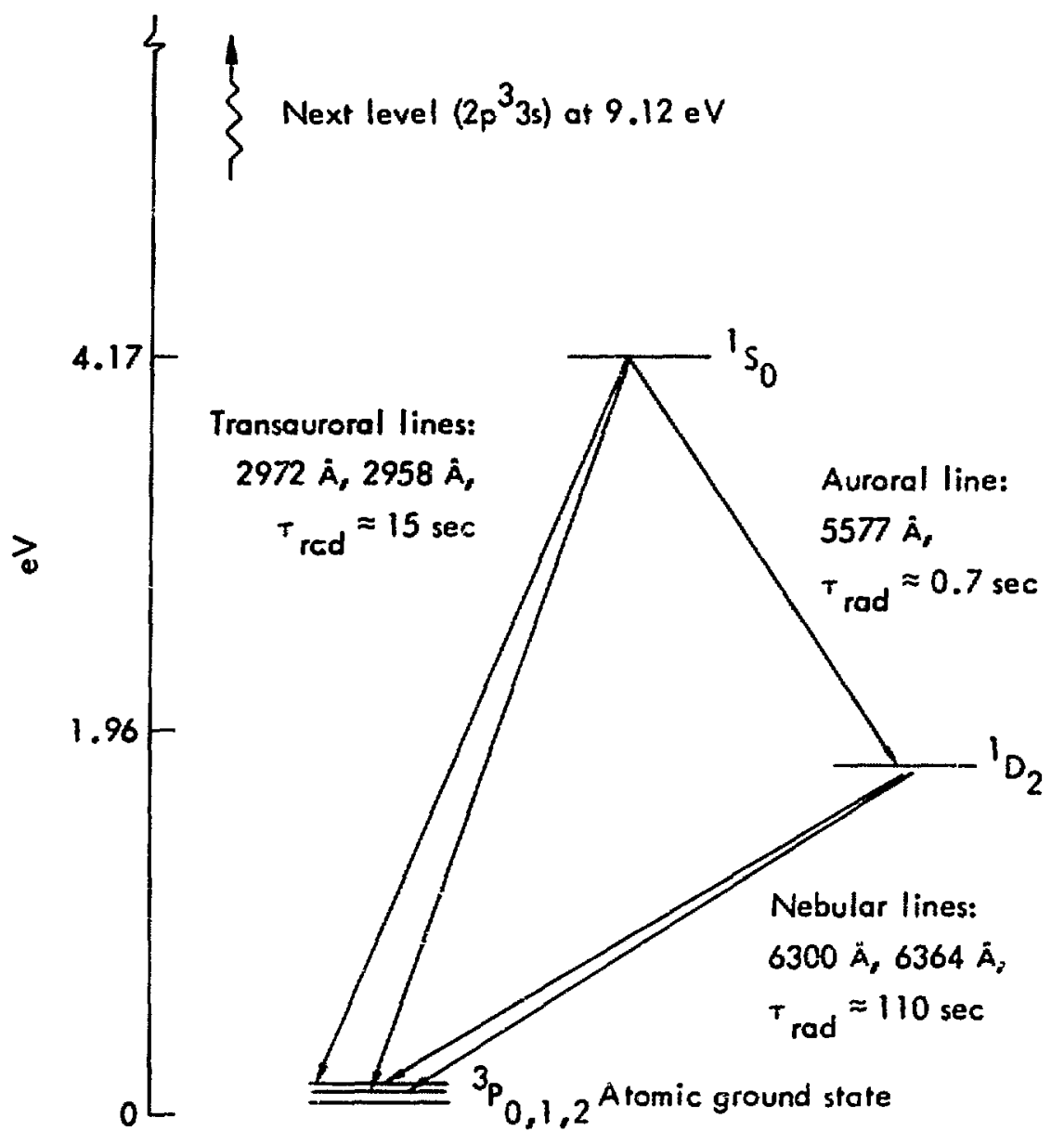

Fig. 149. Energy levels of the $2 p^{n}$ configuration of atomic oxygen.

\section{CONCLUSIONS}

1. Intelligent use of energy transfer processes can significantly improve laser performance. and these pro. cesses are generally applicable.

2. The scaling laws and intrinsic properties (see Table 25) of the vacuum-ultraviolet xenon and krypion excimer systems suggest that these systems do not apply directly to the fusion problem. Their study. however, has pointed a clear path toward the examina. tion of cettain energy-transfor processes in which these excimer states may play an important role.

3. Pulsed-power electron beam machines are directly applicable to the laser fusion problem, but considerable enginecring refinement is needed to optimize lheir use.

4. The generation of $n p^{2}$ and $n p^{4}$ systems by both photolytic and heavy-body collisional processes appeare is an altractive candidate scheme on which a largt scaluble laser systen mighı be based. 
Broadly stated, the abjectives of the $\mathrm{CO}_{2}$ Luser System group at Livermore have been to construct an intermediate-size $\mathrm{CO}_{2}$ laser system in order to examine system scaling laws and to perform plasma plıysics experiments (e.g., see Secs. 6 and 8 for discussions related to the wavelength dependence of the plasma heating). Because our laser system was dedicated from its inception as a facility for target interaction studies, we have imposed several requirements on its design. (1) Reliability of the laser system must be high. (2) Pulse repetition rate should be at least one pulse per minute, so that data can be acquired in a reasonable tine span. (3) Preventive maintenance to insure reliability should not be required more often than every 1000 shots. (4) Electrical noise must be minimized within reasonable economic limits.

The Livermore $\mathrm{CO}_{2}$ system consists of devices which we closely specified and then purchased from vendors who normally sell similar units. Beyond our modelocked TEA oscillator and GaAs $\lambda / 2$ switchout unit, we have assembled an amplifier chain consisting of ( 1 ) a uv-preiunized TEA preamplifier, (2) a beam expander with a magnification of three, and (3) a 9.cm-aperture uv-preionized TEA amplifier followed by 1 wo $11 \cdot \mathrm{cm}$ aperture cold-cathode e-beam-sustained amplifiers operated at $2 \mathrm{~atm}$. Our system is designed to produce spatially smooth $10.6-\mu \mathrm{m}$ pulses of 1.5 -nsec duration containing $\sim 50 \mathrm{~J}$. It should be possible to extract more than $50 \mathrm{~J}$, but only at the expense of beam quality.

During the past six months our experimental studies have continued to provide improved input for com. puter programs designed to simulate the energy extraction efficiency and pulse shapes from the highpower $\mathrm{CO}_{2}$ lasers being assembled for laser fusion experiments. Generally these experimental and theoretical findings have supported our confidence in the validity of our initial design, and consequently the largest part of our effort has been directed toward the realization of this system. The following sections contain an account of experimental and theoretical work completed during this reporting period as well as a report on the status of our $\mathrm{CO}_{2}$ laser system.

\section{STUDIES OF RAPID RELAXATION IN $\mathrm{CO}_{2}$}

Since the last semiannual report, we have conducted iwo experimental investigations into certain rapid relaxational processes which are important in characterizing the extraction of energy from $\mathrm{CO}_{2}$ gain media on a short time scale. The first of these studies consisted of the redetermination of the rotational relaxation rate constants, $k_{\text {rot }}$, for pure $\mathrm{CO}_{2}$ and $\mathrm{CO}_{2}$-He and $\mathrm{CO}_{2}-\mathrm{N}_{2}$ mixtures. The second study has yielded a p:eliminary determination of the rate constants describing the thermalization of the $\mathrm{CO}_{2} \mathrm{O2}^{\circ} \mathrm{O}$ and $10^{\circ} 0$ levels in pure $\mathrm{CO}_{2}, \mathrm{CO}_{2} \cdot \mathrm{He}$, and $\mathrm{CO}_{2} \cdot \mathrm{N}_{2}$ mixtures.

The rotational relaxation rate constants, $k_{\text {rot }}$, were measured for the $\mathrm{CO}_{2} \mathrm{O0}^{0} 1$ level in three kinds of low pressure amplifiers: one using $\mathrm{CO}_{2}$ alone, and two others using mixtures of $\mathrm{CO}_{2} \cdot \mathrm{Hl}$ and $\mathrm{CO}_{2}-\mathrm{N}_{2}$. The experiment consists of perturbing the $\mathrm{CO}_{2} \mathrm{OO}^{\mathrm{O}_{1}^{2}}$ level with a $\sim 2$-nsec saturating pulse at $P(20)$ in the $10.4-\mu \mathrm{m}$ band and monitoring the subsequent repopulation of the $J=19$ rotational state witl a probe beam set at $P(20)$ in the 9.4- $\mu \mathrm{m}$ band (see Fig. 150). This approach is similar to earlier work of Cheo and Abrams that measured the same rate constants. ${ }^{131}$ Because the influence of these rate constants substantially affects the performance of the short-pulse ( $\$ 1-\mathrm{nsec}) \mathrm{CO}_{2}$ amplifiers, we undertook to redetermine them and thereby establish the accuracy of their numerical values. The experimental technique is described in detail in the previous semiannual report. The experimental oscillograms, showing the recovery of th: $J=$ 19 level after perturbation by the $10.6-\mu \mathrm{m}$ pulse, were digitized by tracing the signal shape with a manual scanner and then fitted by a single exponential function utilizing a computerized least-squares program to elicit the time constant $1 / \tau$ toral Such data were taken as a function of pressure for three amplifier cases: (1) $\mathrm{CO}_{2}$ alone, (2) fixed $\mathrm{CO}_{2}$ pressure and varied $\mathrm{He}$ pressure, and (3) fixed $\mathrm{CO}_{2}$ pressure and varied $\mathrm{N}_{2}$ pressure. For the three cases, the total amplifier pressure was measured by a Wallace-Tiernan gauge at one output of the tube. In the latter two instances, calibrated Fischer \& Porter flow meters (one for each gas) were used to maintain a fixed $\mathrm{CO}_{2}$ pressure while the He and $\mathrm{N}_{2}$ were varied in known partial pressures. The partial pressures in the latter cases were equal to the product of the ratio of flow rate for a particular gas to the sum of flow rates for the two gases times the total pressure. As a check to insure the accuracy of this 


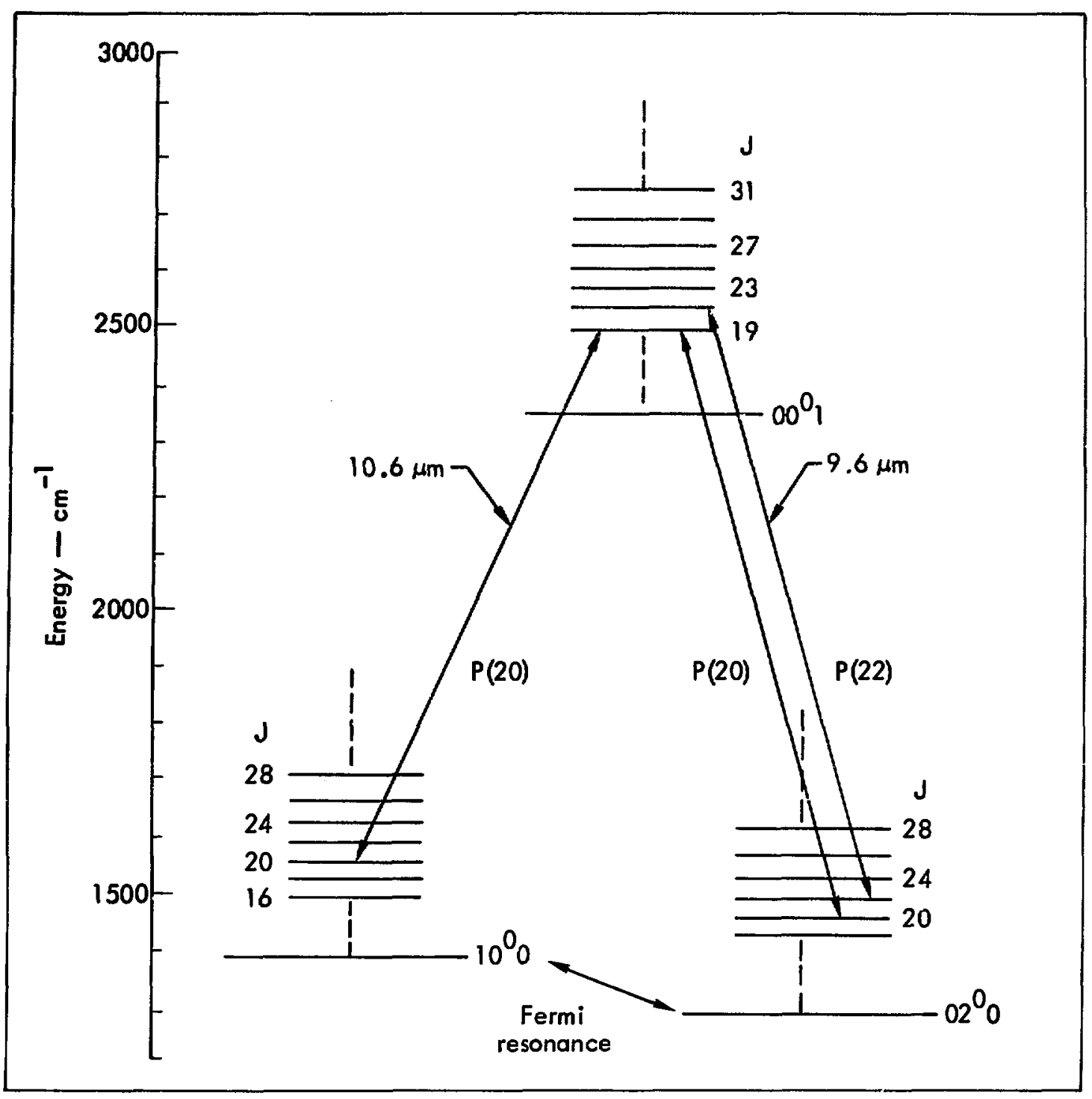

Fig. 150. Energy level diagram of certain low-lying vibrational levels in $\mathrm{CO}_{2}$ indicating the rotational substructure. Not shown are the lower lying $02^{2} 0,01^{\prime} \mathrm{O}$, and $00^{\circ} \mathrm{O}$ vibrational states.

technique, sample bottles were taken of all the mixed gas data and the subsequent mass-analyzed partial pressures agreed to within a few percent of the flow meter determinations. For the mixed gas situations the probe signal recovery time, $\tau_{\text {total }}$, is given by the expression

$$
\begin{aligned}
1 / \tau_{\text {total }}=k\left(\mathrm{CO}_{2}-\mathrm{CO}_{2}\right) P\left(\mathrm{CO}_{2}\right) \\
+k\left(\mathrm{CO}_{2}-x\right) P(x),
\end{aligned}
$$

where $x=\mathrm{He}$ or $\mathrm{N}_{2}$.
In Fig. 151 we plot $1 / \tau_{\text {total }}$ vs $P(x)$ for the three amplifier cases. The data were well fitted by a single exponential function with better than $1 \%$ agreement. As a protection against the data being influenced by coherent propagation effects, which would be dependent upon the particular intensity of the $10.6-\mu \mathrm{m}$ pulse, the oscillograms were analyzed starting at a point several nsec beyond the initial gain decrease caused by the saturating pulse. For all the data, at least one $e$-folding time of the signal was analyzed. The results for the rotational rate constants from this analy- 


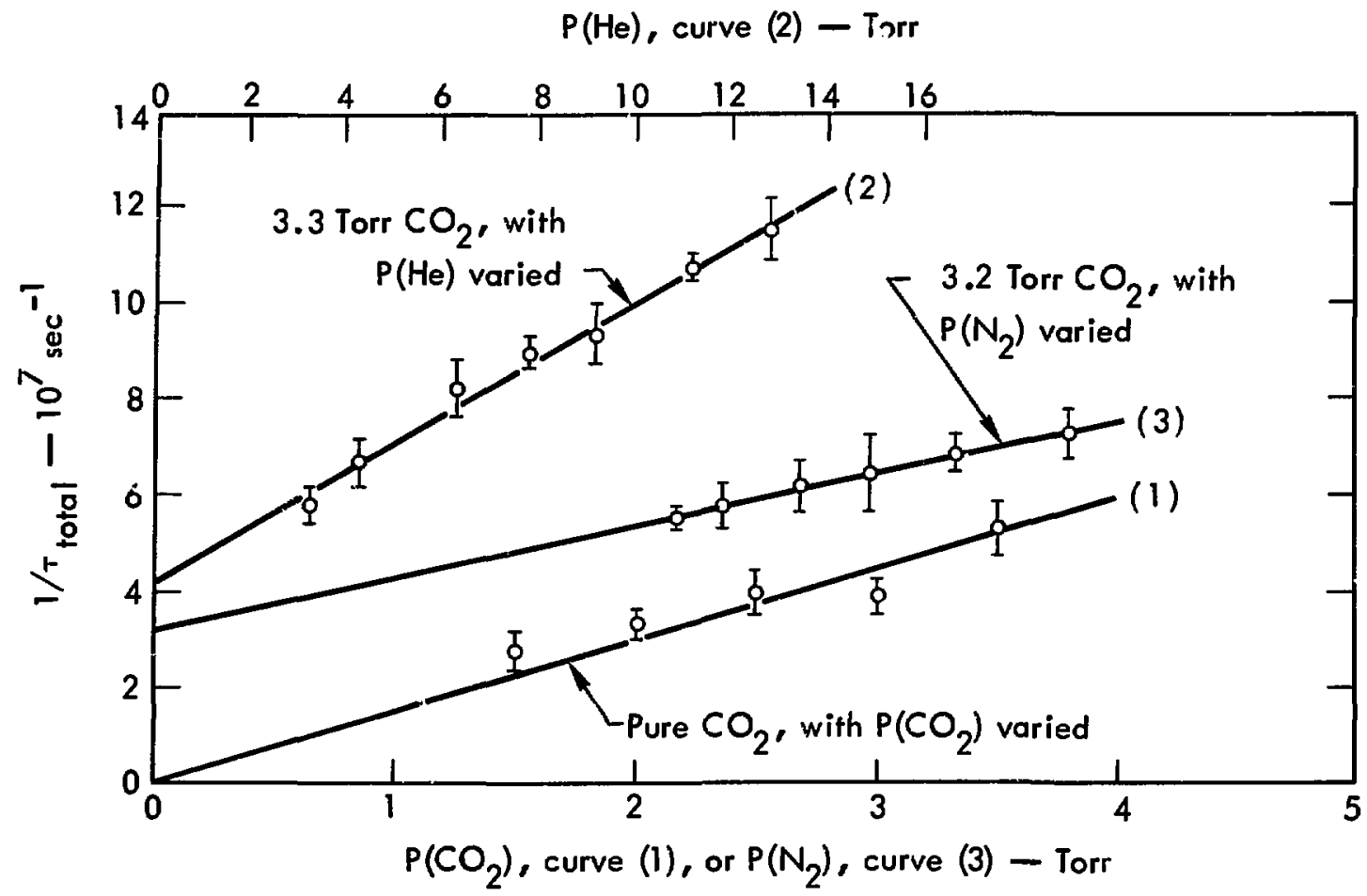

Fig. 151. Linear least-squares computer fits to the $1 / \tau_{\text {total }}$ data vs pressure for three amplifier cases: (1) pure $\mathrm{CO}_{2},(2) \mathrm{CO}_{2}$ fixed at 3.3 Torr with $P(\mathrm{He})$ varied as indicated, and (3) $\mathrm{CO}_{2}$ fixed at 3.2 Torr and $P\left(\mathrm{~N}_{2}\right)$ varied. Individual error flags express \pm 1 standard deviation for repeated measurements taken under fixed experimental conditions.

sis are summarized in Table 27. The error bars were selected to encompass the spread of results for the $k\left(\mathrm{CO}_{2}-x\right)$ values determined from repeated data runs.

The second area of investigation yielded a preliminary value for the equilibration rate between the $\mathrm{CO}_{2}$ $02^{\circ} 0$ and $10^{\circ} 0$ lower laser levels in pure $\mathrm{CO}_{2}$ of $\sim 4 X$ $10^{5} \mathrm{sec}^{-1}$ Torr $^{-1}$. This is the elusive "Fermiresonance time" for which literature values range over a factor of 400 and which could influence the satur?. tion conditions of a short-pulse amplifier. This experiment is similar to the previous investigation except that now a $\mathrm{CO}_{2}$ absorption cell is used instead of the $\mathrm{CO}_{2}$ amplifier. 132 Approximateiy 2-nsec-long pulses containing several rotational-vibrational frequencies in the $10.4 \mu \mathrm{m}$ band are absorbed in a heated $\mathrm{CO}_{2}$ zell ( pressure 10-200 Torr) causing a nonequilibrium situation to exist between the population in th. $02^{\circ} 0$ and $10^{\circ} 0$ levels. A weak probe be im set at the $P(24)$ line in the 9.4- $\mu \mathrm{m}$ band spatially and temporally overlaps the saturating pulse to monitor the recovery of the $02^{0} 0$ state. The signal shape for the transmitted probe beam first rises in time, then exhibits a decrease suggesting transfer to the $02^{0} 0$ state from either or both the $02^{2} 0$ and the $01{ }^{1} 0$ levels, and then shows the slow $00^{\circ}$ 1 decay. A single exponential function was used to analyze the initial probe-signal increase. The inverse of the fitted time constant, $1 / \tau$, was plotted as a function of pressure and the rate constant was obtained from the slope of this curve. Rate constant data for this process were also taken for two binary gas mixtures using a fixed $\mathrm{CO}_{2}$ pressure and varied pressures of $\mathrm{He}$ and $\mathrm{N}_{2}$ and are presently being reduced. Finally, the rapidly decreasing components of the probe signal shape are under investigation in an attempt to elicit rate constants for the $V-V$ processes $02^{2} 0 \leftrightarrow 02^{0} 0$ and $(2 x) 01^{1} 0 \leftrightarrow 02^{0} 0$.

Table 27. Measured rotational rate constants (units of $10^{7} \mathrm{sec}^{-1} \mathrm{Torr}^{-1}$ ) for low-pressure amplifiers cortaining $\mathrm{CO}_{2}$ alone, $\mathrm{CO}_{2}$ mixed with $\mathrm{He}$, and $\mathrm{CO}_{2}$ mixed with $\mathrm{N}_{2}$

\begin{tabular}{lcc} 
& Present work & Cheo and Abrams ${ }^{131}$ \\
\hline$k\left(\mathrm{CO}_{2}-\mathrm{CO}_{2}\right)$ & $1.3 \pm 0.2$ & $1.1 \pm 0.2$ \\
$k\left(\mathrm{CO}_{2}-\mathrm{Hc}\right)$ & $0.6 \pm 0.1$ & $0.7 \pm 0.1$ \\
$k\left(\mathrm{CO}_{2}-\mathrm{N}_{2}\right)$ & $1.2 \pm 0.2$ & $1.0 \pm 0.2$ \\
\hline
\end{tabular}


In the last semiannual report, observations were presented on the $J$-dependence of rotational relaxation in the $\mathrm{CO}_{2} \mathrm{OO}^{\circ} 1$ vibrational level for mixtures of $\mathrm{CO}_{2}$, $\mathrm{He}$, and $\mathrm{N}_{2}$. The experiment consisted of injecting a saturating $\sim 2$-nsec pulse at the $P(20)$ line in the $10.4 \mu \mathrm{m} \mathrm{CO}$ band into a low-pressure $\mathrm{CO}_{2}$ laser amplifier while simultaneously monitoring the transient gain response of an overlapping weak probe beam in the $9.4-\mu \mathrm{m}$ band restricted to operate on any of the transitions $P(10)$ through $P(34)$. A partial energy diagram depicting some of these states is given in Fig. 150. The data showed that the decay times of the various $J$-states in the $\mathrm{CO}_{2} \mathrm{OO}^{0} \mathrm{I}$ level got progressively longer for increasing or decreasing $J$-values centered about the perturbed $J=19$ state. Such behavior may be expected to have a significant effect on the efficiency of energy extraction and pulse shapes in $\mathrm{CO}_{2}$ amplifiers for nanosecond and especially subnanosecond laser pulses and is therefore important for fusion applications. In an attempt to analyze this stepwise equilibration we utilized a recent approach which proved very successful in describing the dynamics of an ensemble of perturbed simple-harmonic oscillators. ${ }^{133}$ This method was modified for the present case of a rigid rotator. The analysis considers only $\Delta J= \pm 2$ transitions and expresses the time rate of change for the occupation probability $\dot{P}_{J}$ of a perturbed system of rigid rotator states in contact with a thermal reservoir as

$$
\dot{P}_{J}=-\left(a_{J}+b_{J}\right) P_{J}+a_{J+2} P_{J-2}+b_{J-2} P_{J-2} .
$$

In Eq. (64) the first term denotes population transfer from $J$ states, via collisions, into $J-2$ and $J+2$ states. The second term expresses population transfer from the $J+2$ states into the $J$ states, the third term population transfer from the $J-2$ states into the $J$ states. A coupled set of 35 such equations for $\dot{P}_{1}$ through $\ddot{P}_{69}$ was computer-integrated. The analysis contained the requirement of detaiiad balance. The various $\boldsymbol{P}_{J}$ 's at time-zero were described by a Boltzmann distribution at $\Gamma=400^{\circ} \mathrm{K}$ except that the $P_{19}$ probability was half its normal value which is consistent with full saturation. The results of this analysis were as follows: (1) states lying closer in $J$-value on either side of the perturbed $J=19$ level decayed faster than farther-lying $J$ states, (2) levels adjacent to the perturbed $J=19$ state exhibited a behavior similar to, but more pronounced than, that seen in the waveforms for the $P(18)$ and $P(22)$ gain signals, i.e., an initial overshoot of the steady-state value, (3) increasing time delays were observed for appreciable population changes in states successively farther removed in $J$ value on either side of the $J=19$ level (this effect was not looked for experimentally), (4) the approach to steady state for the various $P_{J}$ values could not, except for a few levels on each side of $J=19$, be well approximated by a linear temporal function, and (5) the total decay rate ratio for the $J=33$ to the $J=21$ states was predicted to be about a factor of 10 , whereas the observed ratio was about 2.5 . Thus, within the context of this model, we conclude that the various $J$-state decays are influenced by interactions in which $|\Delta|$ changes by two and more than two units or angular momentum. One can straightforwardly extend Eq. (64) to include such extra contributions, but only by making an independent calculation which determines the relative amplitudes of interactions involving $|\Delta J|=2$ changes and those in which $|\Delta J|$ changes by more than two units. Such calculations are under way.

In a related effort, these recently measured rotational cross-relaxations and vibrational intranode relaxation ${ }^{134}$ processes have been included in a fivetemperature model of an electrically pumped $\mathrm{CO}_{2}$ amplifier. This model assumes that the $\mathrm{CO}_{2}-\mathrm{N}_{2}$ system may be described by four Landau-Teller energy-de.1sity equations which specify the dynamics of the $\mathrm{CO}_{2}$ and $\mathrm{N}_{2}$ vibrational modes. The distinguishing feature of this kind of description is its ability to exploit the wide disparity in the rates of the many energy-transfer processes that occur as the laser medium interacts with pumping electrons and stimulating photons. The requirement that the vibrational modes and rotational manifolds be separately in dynamic euculibrium has been lifted by the inclusion of several adcitional rate equations. The addition of these rapid rate equations has further improved the previously reported good agreement between experimentally observed and theoretically predicted oscillator pulse shapes.

By incorporating our model into an optical pulse propagation routine, we have begun to simulate the performance of a chain of high-pressure electrically pumped $\mathrm{CO}_{2}$ amplifiers. The inputs to this chain that we have considered so far consist of spatially and temporally Gaussian pulses of variable duration containing up to six rotational lines in the $10.6-\mu \mathrm{m}$ band. Our single mode-locked, switched-out $\mathrm{CO}_{2}$ oscillator pulses have been found to contain up to nine rotational lines with a reproducible spectrum. In predicting the performance of large $\mathrm{CO}_{2}$ systems we have confined ourselves to such experimentally achievable pulses. Output temporal pulse shape, spatial pulse shape, spectral character, energy extraction, and overall electrical 
efficiency have been predicted. We find that intense pulses propagating through saturating amplifiers will steepen slightly in front, broaden spatially, grow a tail, and generally take on the appearance of an ocean wave. The intelligent choice of optical anerture dimensions in large $\mathrm{CO}_{2}$ systems depends on this information.

Because our model includes $\mathrm{CO}_{2}$ vibrational as well as rotational kinetics, it is able to make realistic calculations of the dependence of extracted energy on pulse duration. Figure 152 is a comparison plot of experimental data and theory. The experimental data in Fig. 152 was reported by Figueira et al., ${ }^{136}$ and because it was presented in terms of total energy rather than energy density, we have scaled the data to fit our 20-nsec pulse duration prediction. It can be seen from Fig. 152 that such a procedure yields a reasonably good fit to the 5-nsec pulse duration and 70-nsec pulse duration data also. Although our relative fit is superior to that of Figueira et al., ${ }^{36}$ lack of information has prevented an absolute comparison. An estimate of lasersystem electrical efficiency for vazicus pulse lengths can be made by recalling that 200 to $250 \mathrm{~J} /$ liter-atm must be rieposited in gas mixtures ranging from $3: 1 / 4: 1$ to $3: 1: 2\left(\mathrm{He}: \mathrm{N}_{2}: \mathrm{CO}_{2}\right)$ in order to obtain small-signal gains in excess of $4 \% / \mathrm{cm}$. Figure 153 is a compilation of many computer runs at 750 Torr, and the shaded areas are meant to indicate variations in gas mixture and pump energy. In calculating electrical efficiencies we have assumed that by the use of pulse-forming lines $100 \%$ of the electrical energy is delivered to the gas.

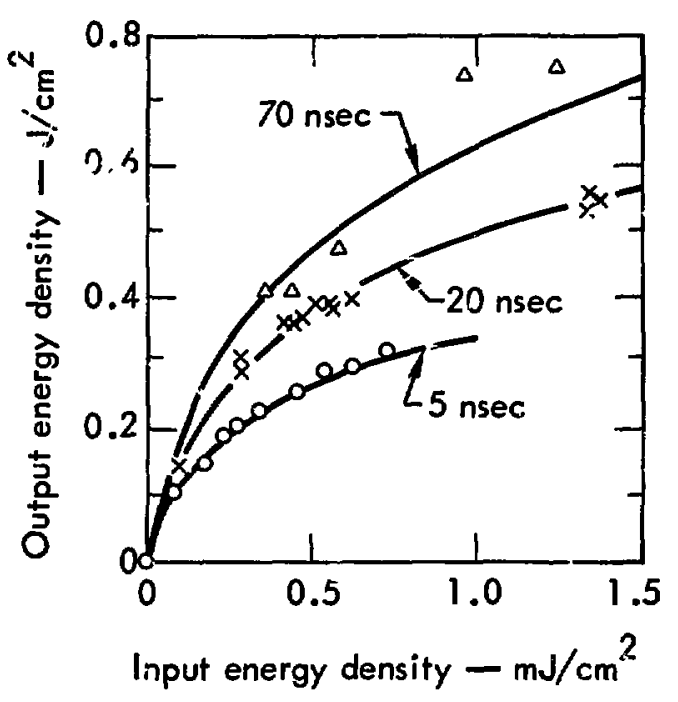

Fig. 152. Compa ison of experimental data obtained by Figueira et al. ${ }^{136}$ with theory.

Further we have assumed that aporizing apertures will permit $100 \%$ beam filling factors in the implifiers. Subject to these qualifications, we conciude that it should be possible to obtain overall electrical efficiencies higher than $2 \%$ with present technology in large systems designed to produce 1-nsec-duration pulses containing several rotational lines in the $10.4-\mu \mathrm{m}$ band.

\section{$\mathrm{CO}_{2}$ SYSTEM STATUS}

Construction of the $\mathrm{CO}_{2}$ Laser System described in the January-June 1973 Semiannual Report is on schedule, and the system is expected to be fully operational in March 1974. Figure 154 is a photograph of the system and associated control room. The oscillator system and first large-aperture amplifier are operational and characterized, and the two e-beam-sustained amplifiers are installed and under test. The facilities are essentially complete, with the control and diagnostic components in the final phase of completion.

\section{Oscillator System}

The oscillator system, similar to those used elsewhere, is shown schematically in Fig. 155. The oscillator portion of the system is now operational and displays the following characteristics:

Peak power

Pulse duration $3 \mathrm{MW}$ (photon drag) $1.3 \mathrm{nsec}$

Prepulse extinction ratio
Postpulse extinction ratio
Amplitude jit ter
Beam divergence
Reliability

$400: 1$

$100: 1$

$\pm 12 \%$

$\theta_{1 / 2} \approx 1 \mathrm{mrad}$

$0.96 @ 50 \%$ confidence

Amplifiers

The first large-aperture amplifier, a Lumonics 602, has been installed and fully characterized:
No. of shots
216
Repetition rate
$1 \mathrm{ppm}$
Gas mix
$\mathrm{He} / \mathrm{N}_{2} / \mathrm{CO}_{2}=70 / 10 / 20$
Gain uniformity $\quad \bar{\alpha}=3.8 \% / \mathrm{cm}, \sigma_{\alpha}=0.1 \% / \mathrm{cm}$
(total pressure 760 Torr)
Repeatability $\quad \pm 5 \%$
Reliability $\quad 0.98$ (a) $50 \%$ confidence 


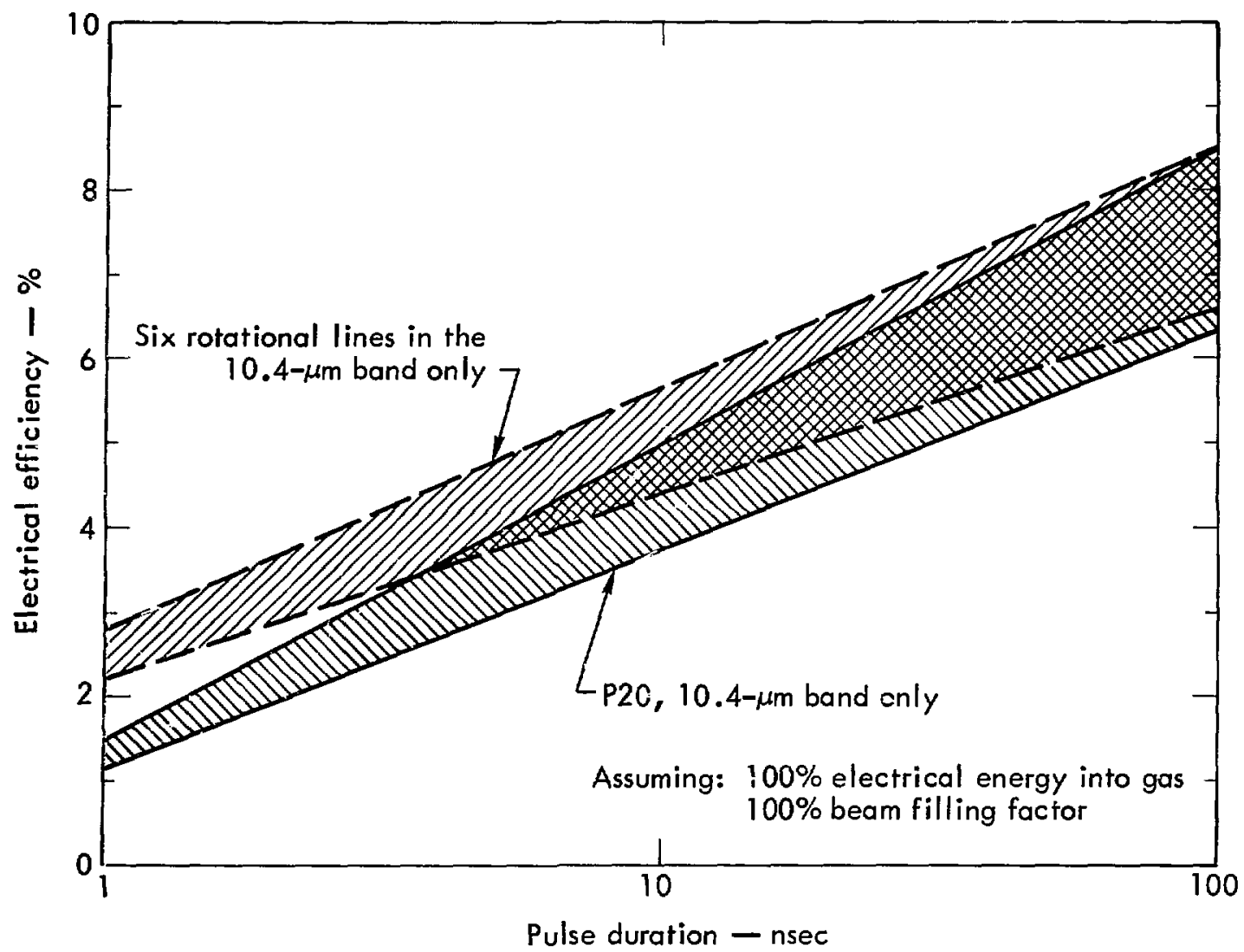

Fig. 153. Estimate of overall $\mathrm{CO}_{2}$ Laser System's electrical efficiency at 760 Torr. Shaded areas indicate variations in gas mixture in the range $3: 1 / 4: 1$ to $3: 1: 2\left(\mathrm{He}: \mathrm{N}_{2}: \mathrm{CO}_{2}\right.$ ) and pump energy in the range $200 \mathrm{~J} /$ liter-atm.

The second and third amplifiers. Systems Science and Software e-beam-sustained, have been installed. The preliminary test results follow ( $\sigma \equiv$ standard deviation):

$$
\begin{aligned}
& \text { E-gun repeatability } \sigma_{J}=0.002 \mathrm{~A} / \mathrm{cm}^{2} \cdot \bar{J} \\
& =0.68 \mathrm{~A} / \mathrm{cm}^{2} \\
& \text { E-gun uniformity } \quad \begin{aligned}
\sigma_{J} & =0.11 \mathrm{~A} / \mathrm{cm}^{2}-\mathrm{J} \\
& =0.68 \mathrm{~A} / \mathrm{cm}^{2}
\end{aligned} \\
& \text { (over } 10 \times 100-\mathrm{cm} \text { area) } \\
& \sigma_{J}=0.08 \mathrm{~A} / \mathrm{cm}^{2}-\bar{J} \\
& =0.68 \mathrm{~A} / \mathrm{cm}^{2} \\
& \text { (over } 8 \times 100-\mathrm{cm} \text { area) }
\end{aligned}
$$

$\begin{array}{ll}\text { No. of shots } & 190 \\ \text { Repetition rate } & 1 \mathrm{ppn} \\ \text { Electron energy } & \sim 190 \mathrm{keV} \\ \text { Gas mix } & \mathrm{He}: \mathrm{N}_{2}: \mathrm{CO}_{2}=3: 1: 2 \\ \text { E/P } & 4 \mathrm{kV} / \mathrm{cm}-\mathrm{atm} \\ \text { Pressure } & 760 \mathrm{Torr}\end{array}$

$$
\begin{array}{ll}
\text { Peak discharge } & I_{p k}=35 \mathrm{kA} \\
\text { current } & \sigma_{I}=1.2 \mathrm{kA} \\
\text { Pulse width } & \overline{\Delta t}=2.04 \mu \mathrm{sec} \\
& \sigma_{\Delta t}=0.067 \mu \mathrm{sec} \\
\text { Energy deposited } & \bar{E}=203 \mathrm{~J} / \text { liter } \\
& \begin{array}{l}
\sigma_{E}=9 \mathrm{~J} / \mathrm{liter} \\
\sigma_{t}=0.027 \mu \mathrm{sec}
\end{array} \\
& \text { (from trigger to stant of } \\
& \text { discharge current) } \\
\text { Reliability } & 0.92(a 50 \% \text { confidence } \\
\text { Gain iniformity } & \bar{\alpha}=4.72 \% / \mathrm{cm} \\
& \sigma_{a}=0.12 \% / \mathrm{cm} \\
& \text { (measured on a } 35 \text {-shot }
\end{array}
$$
sequence at five locations over the $10-\mathrm{cm}$ aperture)

Final acceptance tests, currently in progress, will include a full-gain scan of each amplifier separately. The tests are being performed with a gas mix of He: $\mathrm{N}_{2}: \mathrm{CO}_{2}=3: 1: 2$ at a total pressure of $2 \mathrm{~atm}$. 

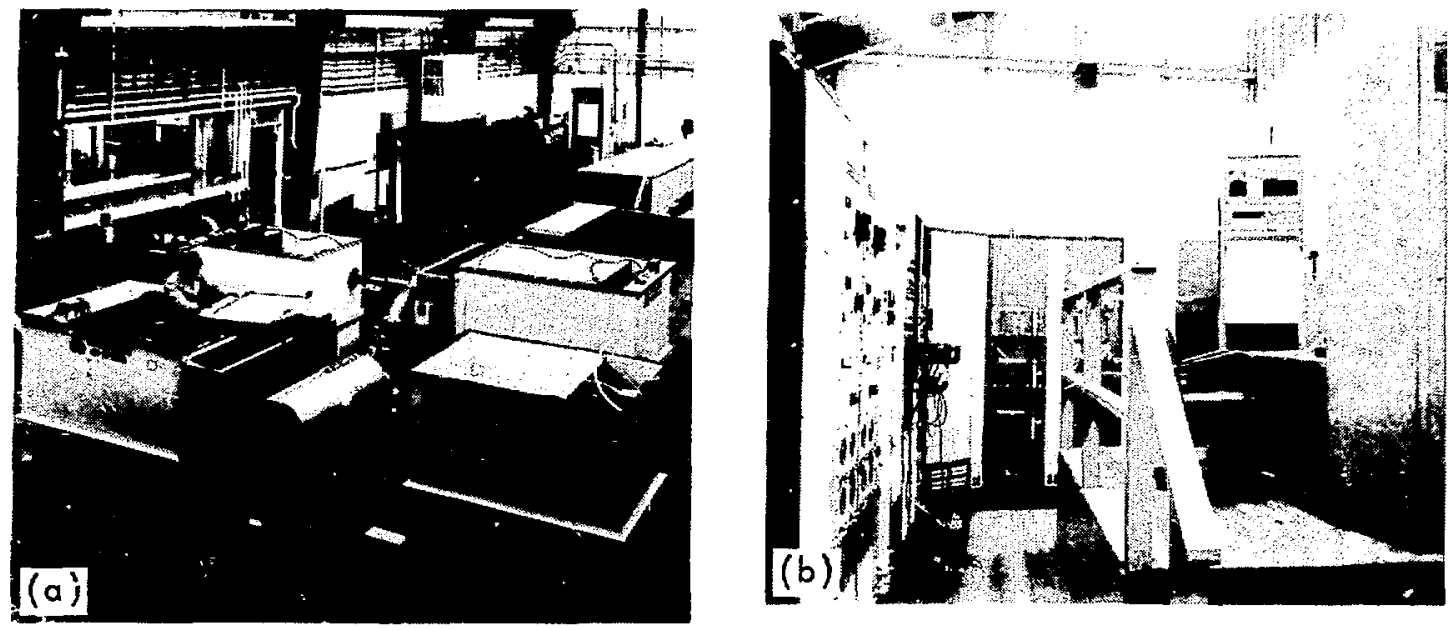

Fig. 154. (a) The $\mathrm{CO}_{2}$ Laser System. In the foreground are the two cold-cathode, electron-beam-controlled amplifiers. (b) Control room for the $\mathrm{CO}_{2}$ Laser System. The amplifier control chassis are in the racks on the left, and the elevated control console is on the right.

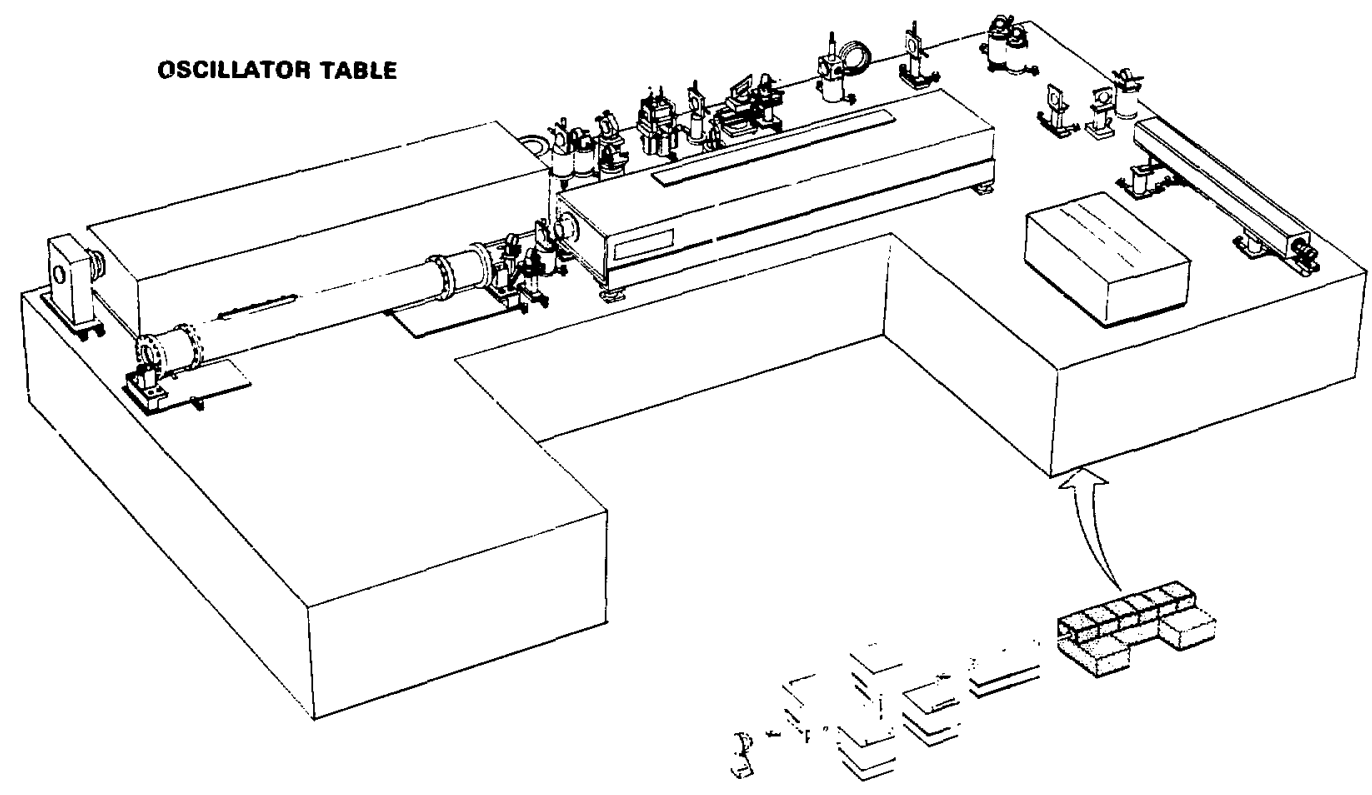

Fig. 155. The oscillator for the $\mathrm{CO}_{2}$ Laser System. 
The system control and diagnostics are located in a separate control room. Control is maintained from an elevated control console from which the entire system may be viewed through a large window. Laser and facilities functions are displayed and interlocked at the control console. System interconnections and power are rouied in floor trenches with enclosed junction boxes and patch panels located where appropriate.
Control chassis, diagnostics, trigger systems, and laser and spark-gap gas and oil system control are located in a contiguous set of racks directly behind the eicvated control console.

The system control and diagnostics are essentially complete with the exception of high-speed iaser diagnostics and a master sequencing chassis.

\section{FACILITIES}

The facilities are complete and in operation. All gases are stored in a fenced gas farm which includes a liquid- $\mathrm{N}_{2}$ storage tank, trailer-mounted He tanks, and banks of $\mathrm{CO}_{2}, \mathrm{~N}_{2}$, and $\mathrm{SF}_{6}$ bottles. The gases are distributed through a common rack in the control room. Electrically operated valves and pressure sensors have been employed for ease of operation. All used gases are vented to the outside of the building via a gas exhaust system. The dielectric oil used in the amplifiers is provided from a 2000 -gallon storage tank with pump and filter. All oil is transferred via permanent plumbing located in the floor trenches.

In summary, system assembly is progressing satisfactorily and as originally planned. Initial experimentation should begin in March 1974.

\section{CONCLUSIONS}

The construction of a $\mathrm{CO}_{2}$ laser system capable of producing 10.6 $\mu \mathrm{m}$ light pulses of about 50-J energy and 1-nsec duration in a spatially well-characterized beam is on schedule. This facility will enable the performance of vital plasma-physics experiments examining the properties of the light/plasma coupling. By means of visible-wavelength pulsed holographic and interferometric techniques, the heating of a target plasma by a $10.6-\mu \mathrm{m}$ pulse can be studied in detail. Such information is necessary for the testing and normalization of computer models for laser heating of high-density target plasmas. Early experimental studies using this $\mathrm{CO}_{2}$ laser zystem will be attempts to elucidate this light/plasma coupling phenomenon. 


\section{PROGRAM RESOURCES}

\section{MANPOWER}

The LLL laser program averaged 257 people directly assigned to it during this reporting period, the firse half of FY 1974. Of the total $35 \%$ are scientific stafl and the remaining $65 \%$ are engineers, technicians, and other support personnel. During the second half of FY 1974 the total manpower will increase to a June end-point of about 340 full-time-equivalent employees.

Following is a manpower breakdown by major task during this six-month reporting period:

\begin{tabular}{lr} 
& Man-years \\
\cline { 2 - 2 } Solid state lasers & 131 \\
Targel development & 39 \\
Gas lasers & 68 \\
Isotope senoxation & 19 \\
\multicolumn{1}{r}{ Total } & 257
\end{tabular}

\section{OPERATING costS}

Operating costs for this reporting period totaled $\$ 7.2$ million. Manpower costs (wage expenses, suppor: burden, and overhead) totaled $\$ 4.4$ million. Procurements, supplies, and travel accounted for $\$ 2.2$ million, and computer recharges amounted to $\$ 0.6$ million. The breakdown by major task is as follows:

\begin{tabular}{lr} 
& \multicolumn{1}{c}{ S Million } \\
\cline { 2 - 2 } Solid state lasers & 3.4 \\
Target development & 1.2 \\
Gas lasers & $\mathbf{2 . 1}$ \\
Isotope separation & $\underline{0.5}$ \\
\multicolumn{1}{r}{ Total } & $\mathbf{7 . 2}$
\end{tabular}

pumps, optical tables, and alignment lasers. Following is the six-month summary by major tasks:

\begin{tabular}{lr} 
& S Thousand \\
\cline { 2 - 2 } Solid state lasers & 620 \\
Target development & 20 \\
Gas lasers & 175 \\
Isotope separation & $\underline{85}$ \\
\multicolumn{1}{c}{ Total } & $\mathbf{9 0 0}$
\end{tabular}

\section{FACILITIES}

In May of 1973 it was recognized that the design of the 1-kJ Nd:glass laser would require more length than was available in Building 174. An addition of approximately 0 by $30 \mathrm{ft}$ would be required just to house the laser and target systems. Energy storage space amounting to approximately $2000 \mathrm{sq} f \mathrm{ft}$ to house the banks for the $C$ and $D$ amplifiers was also needed, but it had been assumed this would be outside as were previous banks. 


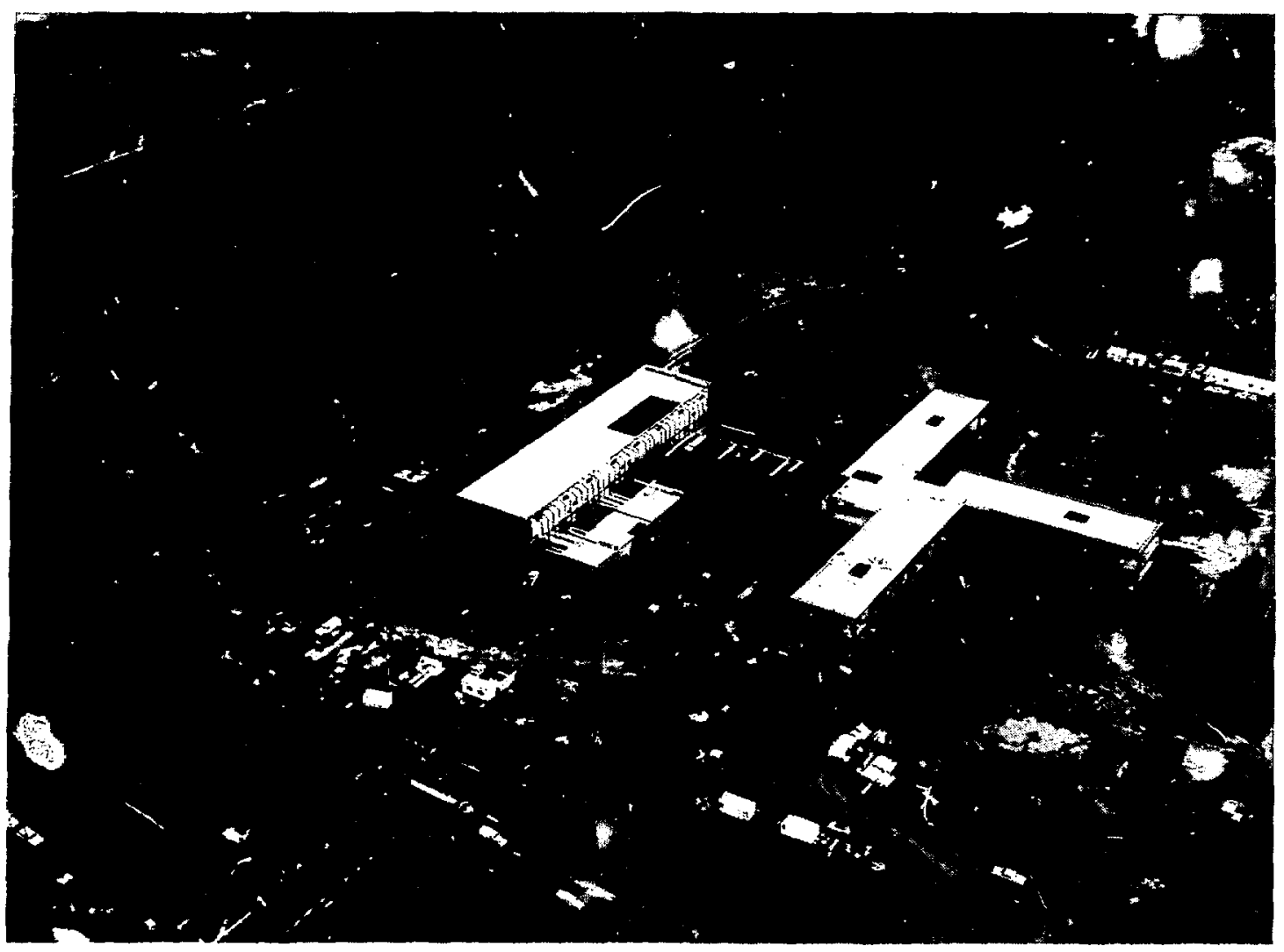

Fig. 156. The LLL Laser Fusion Laboratory had reached this stage of construction by December 1973.

However, cost analysis showed that a "bare bones"designed energy storage area could be built as part of the addition at a price competitive with outside storage systems. Locating the energy storage system inside had the added advantage of making it virtually mairtenance-free because of better environmental control (dust, rain, and heat). With LLL Plant Engineering acting as the architect-engineer and prime contractor, construction began in July. By December the building was essentially complete and occupancy began shortly thereafter. The total area addition was $4000 \mathrm{sq} \mathrm{ft}$, at a total project cost, including engineering, of $\$ 185,000$.

Construction of the Laser Fusion Laboratory, shown in Fig. 156, is progressing on schedule toward an initial occupancy date of June 26, 1974. The provision of a construction manager, Norman Engineering Company, has proved to be an effective means for modifying subcontract schedules as required by material shortages or delayed deliveries so as to still maintain the overall schedule.

Design concepts for the High-Energy Laser Facility
(HELF) are being looked at as part of a laser-programfunded pre-Title I contract with Norman Engineering Company. This preliminary design effort is directed toward early recognition of the relationships between the physical plant and the experiment to be housed. Interface problems can then be identified and resolved prior to the actual design of the buildings. Several suggested design geometries will be studied to define how the basic elements such as the laser bay, control and diagnostics room, target area, entrgy storage, support labs, etc., can be optimally configured. Other studies included in pre-Title I will investigate methods for providing a controlled environment for the laser and target system. In addition to the technical aspects of the design, two further studies will provide current estimates for total construction costs and an evaluation of the time scales associated with various construction options. The use of a pre-Title I study for the HELF is an effective way to accelerate the construction of the building so as to be :ompatible with program goals in solid-state laser research. 


\section{REFERENCES}

1. Semiannual Report January-June 1973, UCRL-50021-73-1. pp. 31-33.*

2. Semiannual Report July-December 1972, UCRL-50021-72-2, pp. 16-17.

3. Semiannual Report January-June 1973, UCRL-50021-73-1, pp. 19-20.

4. Ibid., pp. 4-7.

5. J. Weaver, Kilojoule Laser System Spatial Filter Analysis, LLL internal memorandum, SSL-73-338 (Oct. 29, 1973). Readers outside the Laboratcry who desire further information on LLL internal documents should address their inquiries to the Technical Information Department, Lawrence Livermore Laboratory, Livermore, California 94550.

6. J. Weaver, BOBG Code Runs 5KJS Through B Disk and 10KJS Through B Disk, LLL internal memorandum (Nov. 7, 1973).

7. Semiannual Report January-June 1973, UCRL-50021-73-1, pp. 71-74.

8. Ibid., pp. 88-89.

9. Ibid., pp. 48-58.

10. G. Leppelmeier and W. Simmons, "Application of Faraday Rotation to Isolation in High-Power, Large Aper* ture Nd:Glass Laser Systems," to be published.

11. Semiannual Report January-June 1973, UCRL-50021-73-1, p. 87.

12. Ibid., p. 48.

13. Semiannual Report July-December 1972, UCRL-50021-72-2, p. 19.

14. Semiannual Report January-June 1973, UCRL-50021-73-1, p. 29.

15. W. W. Simmons, G. W. Leppelmeier, and B. C. Johnson, to be published.

16. P. Jacquinot and B. Roizen-Lossier, in Progress in Optics, edited by E. Wolf (North-Holland Publ. Co., Amsterdam, 1964), vol. III, p. 130.

17. W. F. Krupke, "Induced-Emission Cross Sections in Neodymium Laser Glasses," IEEE J. Quant. Electron. QE-10, 450 (1974).

18. B. R. Judd, "Optical Absorption Intensities of Rare Earth Ions," Phys. Rev. 127, 750 (1962).

19. G. S. Ofelt, "Intensities of Crystal Spectra of Rare Earth lons," J. Chem. Phys.37, 511 (1962).

20. W. T. Carnall, P. R. Fields, and K. Rajnak, "Electronic Energy Levels in Trivalent Lanthanide Aquo Ions," $J$. Chem. Phys. 49, 4424 (1968).

21. J. Caird, Rare Earth Laser Engineering Program, Final Report, Air Force Contract F33615-72-C-2038, Rept. No. AFAL-TR-73-323 (1973).

22. W. F. Krupke, "Optical Absorption and Fluorescence Intensities in Several Rare-Earth Doped $\mathrm{Y}_{2} \mathrm{O}_{3}$ and LaF $_{3}$ Single Crystals," Phys. Rev. 145, 325 (1966).

23. W. F. Krupke, Optical Properties of ED-2 Laser Glass, LLL Rept. UCIR-741 (Dec. 1973).

24. C. Rapp, Characterization and Optimization of ED-2 Laser Glass Composition, Owens-Illinois, documentation in response to LLL Purchase Order No. 292015 (Sept. 1973).

25. V. I. Bespalov and V. I. Talanov, "Filamentary Structure of Light Beams in Nonlinear Liquids," JETP Lett. 3, 307 (1966).

26. Semiannual Report January-June 1973, UCRL-50021-73-1, pp. 26-28 and 145-148.

27. A. J. Campillo, S. L. Shapiro, and B. R. Suydam, "Periodic Breakup of Optical Beams Due to Self-Focusing," Appl. Phys. Lett. 23, 628 (1973).

28. D. C. Burnham, "Laser Beam Photography by a Multiple Beam Technique," Appl. Opt. 9, 1482 (1970).

29. Semiannual Report July-December 1972, UCRL-50021-72-2, pp. 51-52, and January-June 1973, UCRL-50021-73-1, pp. 88-89.

30. I. M. Winer, "A Self-Callibrating Technique for Measuring Laser Beam Intensity Distributions," Appl. Opt. 5, 1437 (1966).

31. Semiannual Report January-June 1973, UCRL-50021-73-1, pp. 22-27.

32. Optical Pumps for Laser Lamps, Final Report, ECOM Contract No. DAAB-07-70-C-0035 (May 1971).

33. J. P. Markiewicz and J. L. Emmett, "Design of Flashlamp Driving Circuits," IEEE J. Quant. Electron. QE-2, 707 (1966).

"The full title of this report is Laser-Fusion Program Semiannual Report-January-June 1973, but we abbreviate it here to save space because of the frequent references made to past Semiannuals. 
34. ZAP is a Monte Carlo ray-tracing code which allows one to incorporate the flashlamp model into arbitrarily complicated mechanical environments. See J. H. Alexander, M. Troost, and J. E. Welch, The ZAP Laser Analysis Program, Systems, Science and Software Inc., available from the Defense Documentation Center as AD-884-920 (1971).

35. J. H. Gonez and P. B. Newell, J. Opt. Soc. Amer. 56, 87 (1966).

36. For the full report see LLL Internal Document LER-73-103701 (1973).

37. Semiannual Report January-June 1973, UCRL-50021-73-1, pp. 92-95.

38. T. C. Owen, L. W. Coleman, and T. J. Burgess, Appl. Phys. Lett. 22, 272 (1973).

39. Semiannual Report January-June 1973, UCRL-50021-73-1, p. 98.

40. R. E. Brooks, L. O. Heflinger, and R. F. Wuerker, IEEE J. Quant Electron. QE-2, 275 (1966).

41. R. R. van Ligten and H. Ostenberg, Nature 21 1. 282 (1966).

42. G. W. Ellis, Science 154, 195 (1966).

43. J. Katz, J. Weinstock, W. Kruer, J. DeGroot, and R. Faehl, Turbulently Heated Distribution Functions and Perturbed Orbit Theory, LLL Rept. UCRL-74334 Preprint (Nov. 1972). Accepted for publication by Phys. Fluids.

44. H. D. Shay, LLL Rept. UCRL-7\$185 Preprint (Oct. 26, 1973).

45. J. Stamper, K. Popadopoulos, R. Sudan, S. Dean, E. McLean, and J. Dawson, Phys. Rev. Lett. 26,1012 (1971).

46. Semiannual Repor, January-June 1972, UCRL-50021-72-1, p. 107.

47. Semiannual Report July-December 1972, UCRL-50021-72-2, p. 131.

48. S. Braginskii, in Reviews of Plasma Physics (Consultants Bureau, New York, 1965), vol. 1, p. 205.

49. W. Lokke and J. Ramus, LLL, private communication (1971).

50. P. Brown, D. Post, and J. Wilson, LLL, private communication (1973).

51. J. Chang and G. Cooper, "A Practical Difference Scheme for Fokker-Planck Equations," J. Comp. Phys. 6, 1 (1970).

52. L. Spitzer, "Particle Diffusion Across a Magnetic Field," Phys. Fluids 3, 659 (1960).

53. D. Bohm, in The Characteristics of Electrical Discharges in Magnetic Fields (McGraw-Hill, New York, 1949), ch. 2 , sec. 5 .

54. W. Schulz, "Two-Dimensional Lagrangian Hydrodynamic Difference Equations," in Methods in Computational Physics, edited by B. Alder, S. Fernbach, and M. Rotenberg (Academic Press, New York, 1964), vol. 3, p. 1 .

55. V. W. Slivinsky and Harry N. Kornblum, Bull. Amer. Phys. Soc. 18, 1256 (1973).

56. G. H. McCall, Bull. Amer. Phys. Soc. 18, 1255 (1973).

57. F. Seward and T. Palmieri, LLL Internal Rept. UOPT-73-23 (1973); F. Seward, LLL Internal Rept. UOPT-73-39 (1973); H. D. Shay, F. Seward, T. Palmieri, G. B. Zimmerman, and J. Nuckolls, LLL Rept. UCRL-74925 Preprint (1973).

58. R. Kidder and J. Zink, "Decoupling of Corona and Core of Laser-Heated Pellets," Nucl. Fusion 12, 325 (1972).

59. J. Nuckolls, L. Wood, A. Thiessen, and G. Zimmerman, "Laser Compression of Matter to Super-High Densities: Thermonuclear (CTR) Applications," Nature 239, 139 (1972).

60. See Ref. 45.

61. J. A. Stamper, Magnetic Field Generation Due to Radiation Pressure in a Laser-Produced Plasma, Naval Research Laboratory, NRL Memorandum Rept. 2492 (1973).

62. D. A. Tídman and R. A. Shanny, "Field-Generating Thermal Instability in Laser-Heated Plasmas," communication to Phys. Fluids (1973).

63. A. R. Thiessen, G. Zimmerman, L. Wood, and J. Nuckolls, LLL Rept. UCRL-741 14 Preprint Rev. 1 (1972).

64. J. S. Clark, H. N. Fisher, and R. J. Mason, Phys. Rev. Lett. 30, 89 (1973).

65. Semiannual Report January-June 1973, UCRL-50021-73-1, pp. 171-174.

66. D. Nagle, Naval Research Laboratory, private communication (1973).

67. L. Chase, Lockheed Palo Alto Research Laboratory, private communication (1973).

68. J. J. Thomson, R. J. Faehl, and W. L. Kruer, Phys. Rev. Lett. 31, 918 (1973), and many references therein.

69. J. P. Freidberg, R. W. Mitchell, R. L. Morse, and L. I. Rudsinski, Prys. Rev. Lett. 28, 795 (1972).

70. R. L. Morse et al., Bull. Amer. Phys. Soc. 18, 1274 (1973).

71. G. Zimmerman, LLL, private communication (1973).

72. N. G. Denisov, Sov. Phys. JETP 4, 544 (1957). 
73. R. E. Kidder, Interaction of Photon Beams with Plasmas (II), LLL Rept. UCRL-74040 Preprint (1972).

74. A. B. Langdon, B. F. Lasinski, and W. L. Kruer, LLL Rept. UCRL-75018 Preprint (1973).

75. M. N. Rosenbluth, Phys. Rev. Lett. 9, 565 (1972).

76. J. Denavit, J. Comp. Phys. 9, 75 (1972).

77. J. J. Thomson, W. L. Kruer, S. E. Bodner, and J. DeGroot, LLL Rept. UCRL-74637 Preprint (1973), to be published in Phys. Fluids.

78. K. Nishikawa, private communication (1973).

79. D. W. Forslund, J. M. Kindel, and E. L. Lindman, Phys. Rev. Lett. 30, 739 (1973).

80. W. L. Kruer, K. G. Estabrook, and K. H. Sinz, Nucl. Fusion 13 (1973).

81. S. Bodner, LLL Rept. UCRL 74074 Preprint (1972).

82. D. Forslund and A. Galeev, International Center for Theoretical Physics, Preprint IC/73/125 (1973), and references therein.

83. J. Shearer and J. Eddleman, Phys. Fluids 16, 1753 (1973).

84. P. Kaw, G. Schmidt, and T. Wilcox, Phys. Fluids 16, 1522 (1973).

85. A. B. Langdon and B. F. Lasinski, LLL Rept. UCRL-75029 (1973).

86. E. Valeo, LLL Rept. UCRL-75226 Preprint (1973).

87. C. Y. She, M. J. Moran, and R. L. Carman, LLL Rept. UCID-16423 (Nov. 1973).

88. E. A. Jenkins and H. E. White, Fundamentals of Optics (McGraw-Hill, New York, 1957), ch. 13.

89. A. Owyoung, IEEE J. Quant. Electron. QE-9, 1964 (1973).

90. N. Boling, Owens-Illinsis Technical Center, Toledo, Ohio, private communication.

91. A. Glass and A. Owyoung, Sandia Laboratories, Albuquerque, N. Mex., private communication.

92. C. C. Wang, Phys. Rev. B2, 2045 (1970).

93. J. J. Wynne, Phys. Rev. 178, 1295 (1969).

94. A. Owyoung, R. W. Hellwarth, and N. George, Phys. Rev. B5, 628 (1972).

95. Handbook of Lasers, R. J. Pressley, editor (Chemical Rubber Publishing Co., Cleveland, Ohio, 1971), ch. 14.

96. S. A. Akhmanov, A. P. Sukhorokov, and R. V. Khokhlov, Sov. Phys. Usp. 93, 609 (1968).

97. V. I. Talanov, JETP Lett. 2, 138 (1965).

98. R. Y. Chiao, E. Garmire, and C. Townes, Phys. Rev. Lett. 20, 479 (1964).

99. H. A. Haus, Appl. Phys. Lett. 8, 128 (1966).

100. Z. K. Yankansuas, Izv. Vyssh. Uchebn. Zaved. Radiofiz. 9, 417 (1966).

101. A. V. Vinogradov and E. A. Yukov, JETP Lett. 16, 447 (1972).

102. R. W. Minck, E. E. Hagenlocker, and W. G. Rado, J. Appl. Phys. 38, 2254 (1967); W. H. Culver, J. T. A. Vanderslice, and V. W. T. Townsend, Appl. Phys. Lett. 12, 189 (1968); M. Maier, W. Kaiser, and J. A. Giordmaine, Phys. Rev. 177, 580 (1969).

103. R. V. Johnson and J. H. Marburger, Phys. Rev. 4, 1175 (1971).

104. R. A. Fisher and W. K. Bischel, Appl. Phys. Lett. 23, 661 (1973).

105. Semiannual Report January-June 1973, UCRL-50021-73-1, pp. 160-162.

106. D. K. Duston, Ph.D. thesis, Rensselaer Polytechnic Institute, Troy, New York (1969).

107. L. A. Riseberg, Phys. Rev. A7 671 (1973).

108. M. M. Mann and L. G. DeShazer, J. Appl. Phys, 41, 2951 (1970).

109. C. B. Moore, Accounts Chem. Res. 6, 323 (1973).

110. V.S. Letokhov, Science 180, 451 (1973).

111. S. W. Mayer, M. A. Kwok, R. W. F. Gross, and D. J. Spencer, Appl. Phys. Lett. 17, 516 (1970).

112. E. S. Yeung and C. B. Moore, Appl. Phys. Lett. 21, 109 (1972).

113. R. U. Ambartzumian, V.S. Letokhov, C N. Makarso, and A. A. Paretzki, JETP Lett. 18, 91 (1973).

114. D. W. Steinhaus et al., Los Alamos Scientific Laboratory Rept. LA-4501 (1971).

115. C. H. Corliss and W. R. Bozman, NBS Monograph 53 (1962).

116. W. L. Fite and P. lrving, J. Chem. Phys. 56, 4227 (1972).

117. J. Dubrin and M. Henchman, Chemical Kinetics, J. C. Polanyi, editor (Butterworths, University Park, 1972), vol. 9 , ch. 7.

118. O. H. Krikorian, LLL Invention Case No. IL-5809.

119. K. R. Osborn, C. C. McDonald, and H. E. Gunning, J. Chem. Phys. 26, 125 (1957).

120. J. R. McDowell, C. C. McDonald, and H. E. Gunning, Can. J. Chem. 37, 1432 (1959).

121. R. Pertel and H. E. Gunning, Can. J. Chem. 37, 35 (1959). 
122. For this intensity the most rapidly varying plane-wave perturbation would have an $e$-folding distance of $4 \mathrm{~cm}$. See, for example, V. I. Bespalov and V. I. Talanov, JETP Lest. 3, 307 (1966).

123. P. L. Kelley, Phys. Rev. Lett. 15, 1005 (1965).

124. John Trenholme, LLL, private communication. These numbers are from a preliminary design of about August 1973. The small-signal gains were inferred from expected energy output values after the A, B. C, and D module pairs.

125. J. Marburger, "Self-Focusing with Elliptical Beams," in Damage in Laser Materials, NBS Special Publication No. 356 (U.S. Govt. Printing Office, Washington, D. C., 1972), p. 84.

126. Charles K. Rhodes, IEEE J. Quant. Electron. QE-10, 153 (1974).

127. P. W. Hoff, J. C. Swingle, and C. K. Rhodes, Appl. Phys. Lett. 23, 245 (1973).

128. C. W. Werner, E. V. George, P. W. Hoff, and C. K. Rhodes, to be published.

129. E. V. George and C. K. Rhodes, Appl. Phys. Lett. 23, 139 (1973).

130. J. R. Murray and C. K. Rhodes, The Possibility of a High-Energy-Storage Visible Laser on the Auroral Line of Oxygen, LLL Rept. UCRL-51455 (Sept. 1973).

131. P. K. Cheo and R. L. Abrams, Appl. Phys. Lett. 14, 47 (1969); R. L. Abrams and P. K. Cheo, Appl. Phys. Lett. 15, 177 (1969).

132. A similar approach was utilized in C. K. Rhodes, M. J. Kelley, and A. Javan, J. Chem. Phys. 48, 5730 (1968).

133. W. E. Lamb, Jr., "Approach to Thermodynamic Equilibrium (and Other Stationary States)," The Physicist's Conception of Nature, J. Mehra, editor (D. Reidel Publ. Co., Dordrecht, Holland, 1973), pp. 166-186.

134. I. Burak, Y. Noter, and A. Szöke. IEEE J. Quant. Electron. QE-9, 541 (1973).

135. K. R. Manes and H. J. Seguin, J. Appl. Plyss. 43, 5073 (1972).

136. J. F. Figueira, W. H. Reichelt. G. T. Schappert. T. F. Stratton, and C. A. Fenstermacher Appl. P/lys. Lett. 22, 5 (1973). 


\section{BIBLIOGRAPHY OF RECENT PUBLICATIONS}

Altwood D., and L. Coleman, "Holographic Interferometry of Laser Produced Plasmas," Bull. Amer. Phys. Soc. 18, 1342 (1973).

Boster, T. A., “High Resolution Absolute X-Ray Spectra from Laser-Produced Plasmas," Bull. Amer. Phys. Soc. 18, 1586 (1973). Paper presented at winter meeting of the American Physical Society, Berkeley, Calif., 1973.

Coleman, L. W., "Picosecond Image Converter Cameras for Laser Diagnostics," paper presented at meeting of Society of Photo-Optical Instrumentation Engineers, San Diego, Calif., Aug. 27-29, 1973. LLL Rept. No. UCRL74946 Preprint (1973).

Condreva, K.., A Photoconductive Detector Evaluation at $10.6 \mu$, LLL internal report LER-73-103701 (1973).

Dahlbacka. G. H., Investigation of the Effects of Self-Generated Magnetic Fields on the Implosion of DT Spheres, LLI. Rept. UCRL-74927 Preprint (Oct. 1973).

Dahlbacka, G. H., G. Chapline, and T. A. Weaver, Pion Gamma Ray Emission from Black Holes, LLL Rept. UCRL-75151 (Feb. 1974).

Gunn, S. R., “A Tubular Calorimeter for High-Power Laser Pulses," Rev. Sci. Instrum. 43, 15?3 (1972).

Gunn, S. R., "Calorimetric Measurements of Laser Energy and Power," J. Phys. E: Sci. Instrum. 6, 105 (1973).

Gunn, S. R., A Revieu of Calorimetric Measurements of Luser Energy and Power, LLL Rept. UCID-16214 (1973). This is an expanded version of the journal article immediately preceding; it contains more material than could be included in the journal article.

Gunn, S. R., Volume-Absorbing Calorimeters for High-Power Laser Puises, LLL Rept. UCRL-75223 Preprint (1973). To be published, Rev. Sci. Instrum.

Kruer. W. L., "Heating of Underdense Plasmas by Intense Lasers," Phys. Fluids 16, 1548 (1973).

Kruer, W. L., K. G. Estabrook, and J. J. Thomson, Nonlinear Behavior of Light-Driven Plasma Instabilities, LLL Rept. UCRL-74947 Preprint (1973). To be published in Laser Interaction and Related Plasma Phenomena, Vol. 3 (1973).

Langdon, A. B., and B. F. Lasinski, Filamentation and Subsequent Decay of Laser Light, LLL Rept. UCRL-75029 Preprint (1973).

Langdon, A. B., B. F. Lasinski, and W. L. Kruer, Plasma Heating at One-Quarter the Critical Density, LLL Rept. UCRL-75018 Preprint (1973).

Saroyan, R. Allyn, The Effective Resolution of Scanning Spectrometers, LLL Rept. UCRL-75072 Preprint (Aug. 24, 1973).

Shay, H. D., LNA Presentation, Battelle Memorial Institute, November 1973, LLL Rept. UCRL-75185 (1973).

Shay, H. D., F. Seward, T. Palmieri, G. B. Zimmerman, and J. Nuckolls, Comparison of X-Ray Paotographs and Calculations of Laser Generated Plasmas, LLL Rept. UCRL-74925 (1973).

Simnons. W. W., G. W. Leppelmeier, and B. C. Johnson, Optical Beam Shaping Devices Using Polarization Effects, LLL Rept. UCRL-75272 (1973). To be published in Applied Oprics.

Slivinsky, V. W., and Harry N. Kornblum, "Measurements of the High Energy X-Ray Spectrum from a LaserProduced Plasma," Bull. Amer. Phys. Soc. 18, 1256 (1973). Paper presented at alınual meeting of the Division of Plasma Physics of the American Physical Society, Philadelphia, Pa., 1973. 
Thomas, S. W., R. L. Carman, H. R. Spracklen, G. R. Tripp, and L. W. Coleman, "Ten Picosecond Streak Camera for the Laser Fusion Program at LLL," paper presented at Electro-Optical Systems Design Conference, New York, N.Y., Sept. 18-20, 1973. LLL Rept. UCRL-74909 Preprint (1973).

Thomson, J. J., R. J. Faehl, W. L. Kruer, and S. E. Bodner, Electron Heating Due to Parametric Instability Turbulence, LLL Rept. UCRL-74945 Preprint (1973). To be published in Phys. Fluids.

Thomson, J. J., and J. I. Ka:ush, Effects of Finite-Bandwidth Driver on the Parametric Instability, LLL Rept. UCRL-75374 Preprint (1973).

Valeo, E., Stability of Filamentary Structures, LLL Rept. UCRL-75226 Preprint (1973). Submitted to Phys. Fiuxis..

Violet, C. E., "Conventional Differential Absorbe Method Applied to a Temperature-Varying Plasma," paper presented at the annual meeting of the Division of Plasma Physics of the American Physical Society, Philadelphia, Pa. 1973.

Violet, C. E., J. Petruzzi, and S. W. Mead, "Laser t. X-Ray Conversion Efficiency," paper presented at the annual meeting of the Division of Plasma Physics of the American Physical Society, Philadelphia, Pa., 1973.

Violet, C. E., J. Petruzzi, S. W. Mead, J. E. Swain and R. A. Saroyan, X-Rays and Reflected Light from LaserProciuced Plasmas, LLL Rept. UCRL-74409 Preprat Rev. 1 (1973).

Zimmerman, G., Numerical Simulation of the High Density Approach to Laser-Fusion, LLL Rept. UCRL-75173 Preprint (Oct. 17, 1973).

Zimmerman, G., and A. Thiessen, Two-Dimensional Calculations of High Density Laser Induced Fusion: Study of Symmetry and Taylor Instability, LLL Rept. UCRL-75926 Preprint (Oct. 5, 1973). 